Doreen Müller

\title{
Flucht und Asyl in europäischen Migrationsregimen
}

Metamorphosen einer umkämpften Kategorie am Beispiel der EU, Deutschlands und Polens

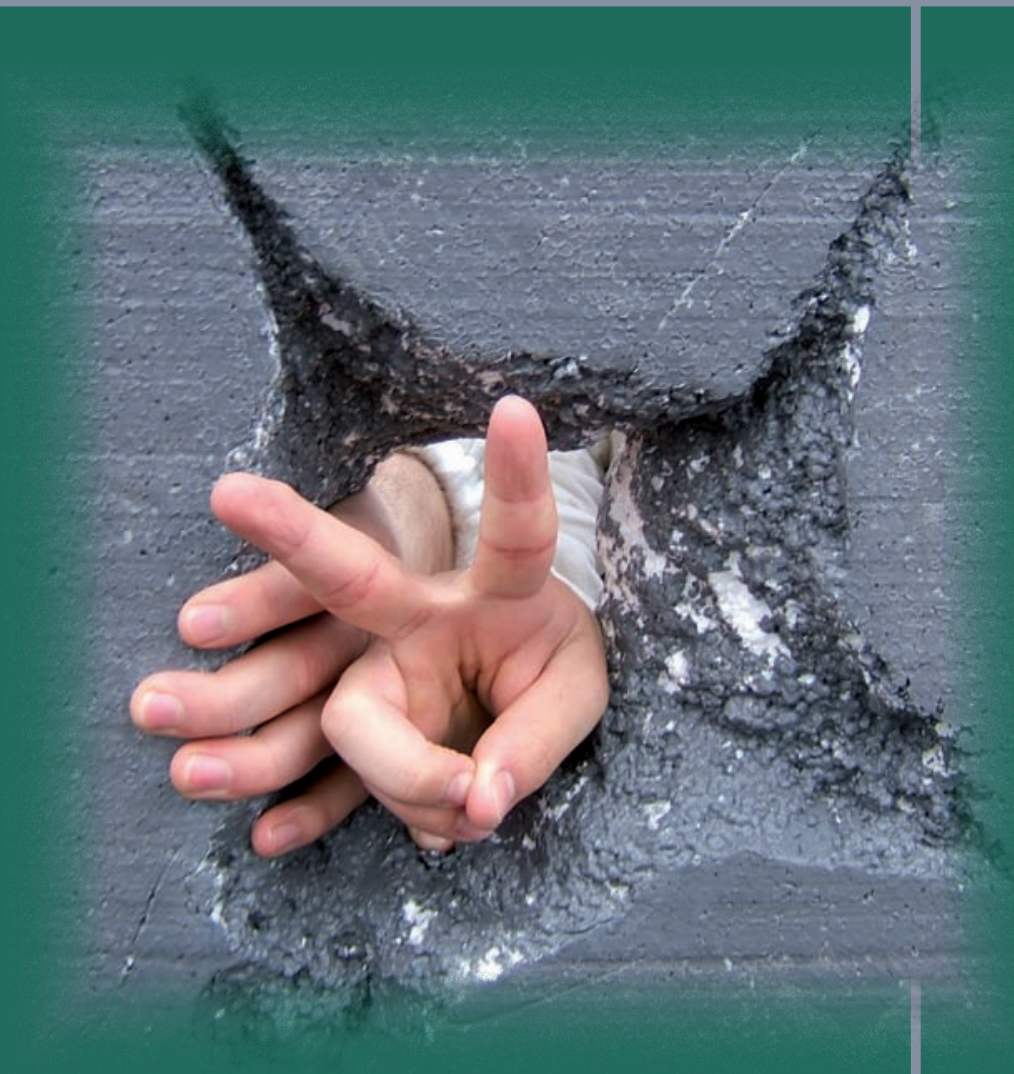



Flucht und Asyl in europäischen Migrationsregimen

This work is licensed under the Creative Commons License 3.0 "by-nd", allowing you to download, distribute and print the document in a few copies for private or educational use, given that the document stays unchanged and the creator is mentioned. You are not allowed to sell copies of the free version. 
erschienen im Universitätsverlag Göttingen 2010 
Doreen Müller

Flucht und Asyl in europäischen Migrationsregimen

Metamorphosen einer umkämpften Kategorie am Beispiel der EU, Deutschlands und Polens

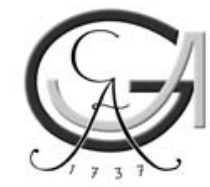

Universitätsverlag Göttingen 2010 


\section{Bibliographische Information der Deutschen Nationalbibliothek}

Die Deutsche Nationalbibliothek verzeichnet diese Publikation in der Deutschen Nationalbibliographie; detaillierte bibliographische Daten sind im Internet über $<$ http://dnb.ddb.de> abrufbar.

Dieses Buch ist auch als freie Onlineversion über die Homepage des Verlags sowie über den OPAC der Niedersächsischen Staats- und Universitätsbibliothek

(http://www.sub.uni-goettingen.de) erreichbar und darf gelesen, heruntergeladen sowie als Privatkopie ausgedruckt werden. Es gelten die Lizenzbestimmungen der Onlineversion. Es ist nicht gestattet, Kopien oder gedruckte Fassungen der freien Onlineversion zu veräußern.

Satz und Layout: Alexander Mirau Umschlaggestaltung: Franziska Lorenz

Titelabbildung: Fadl/Umbruch Bildarchiv, Symbolisch errichtete Mauer auf der Glienicker Brücke (Potsdam/Berlin) im Rahmen eines bundesweiten Aktionstages von Flüchtlingsorganisationen und antirassistischen Initiativen am 22.04.2006 anlässlich der bevorstehenden Innenministerkonferenz

(C) 2010 Universitätsverlag Göttingen http:/ / univerlag.uni-goettingen.de ISBN: 978-3-941875-71-5 


\section{Inhaltsverzeichnis}

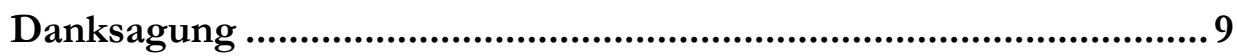

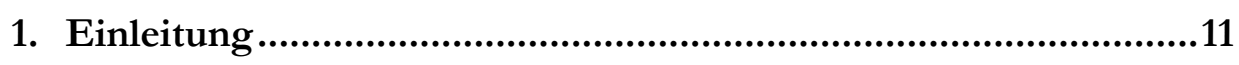

2. ,Flucht und Asyl' im Kontext von Migration und Migrationspolitik ...................................................................... 17

2.1 Zentrale Begriffe: Migration, Migrationspolitik und Migrationsregime ….....................................................................18

2.2 Ansätze zur Erklärung von Migrationspolitik ................................27

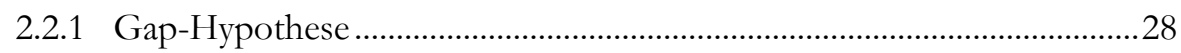

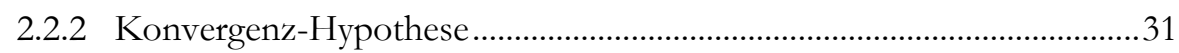

2.2.3 Scheitern der Migrationskontrollpolitik? ......................................................

2.2.4 „Autonomie“ oder „Eigensinnigkeit“ der Migration ...................................33

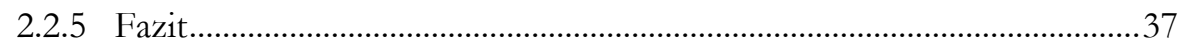

2.3 Kategorienkonstruktion als migrationspolitisches Fundament: das Beispiel Flucht und Asyl ...........................................................38

2.3.1 MigrantInnenkategorien in der Migrationsforschung..............................40

2.3.2 Politische Konstruktion von MigrantInnenkategorien..............................4 


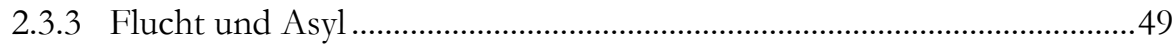

2.3.4 Flüchtlingskonstruktionen und ihre Bedeutung im Migratonsregime...60

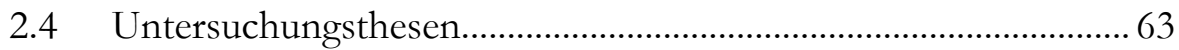

3. Untersuchungsdesign und Methoden.........................................66

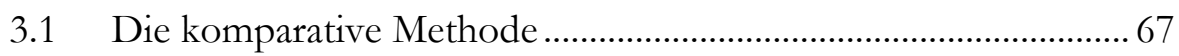

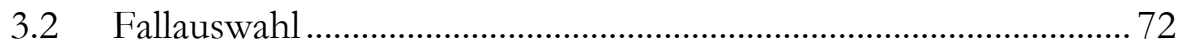

3.3 Erhebungsmethoden: Dokumentenanalyse und ExpertInnen-

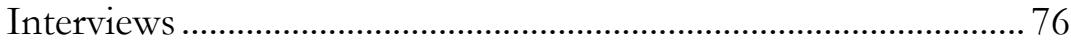

4. Asylpolitik der EU ................................................................83

4.1 Richtlinien, Verordnungen und weitere Maßnahmen..................... 88

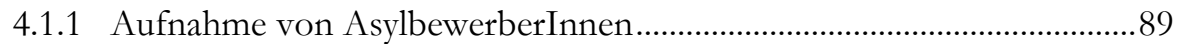

4.1.2 Anerkennung als Flüchtlinge................................................................95

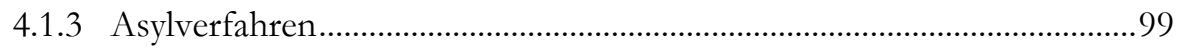

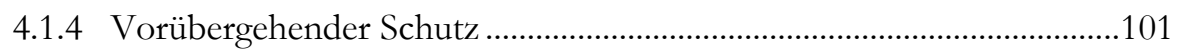

4.1.5 Dublin-II- und Eurodac-Verordnung ......................................................104

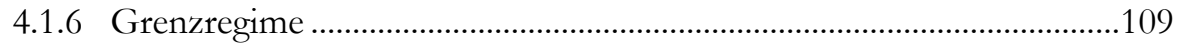

4.1.7 Außenpolitische Maßnahmen ...........................................................111

4.1.8 Rückkehr und Abschiebung .................................................................115

4.2 Analyse: Asyl im Migrationsregime auf EU-Ebene.....................119

4.2.1 Definition und Verfahren .....................................................................119

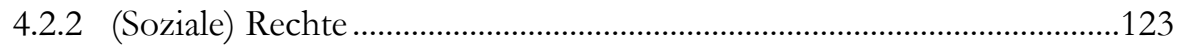

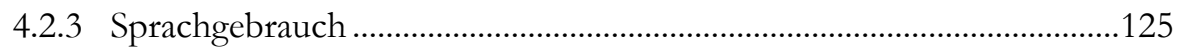

4.3 Supranationalisierung: Neue Möglichkeiten der Legitimation, Gestaltung und Umsetzung von Asylpolitiken..............................132

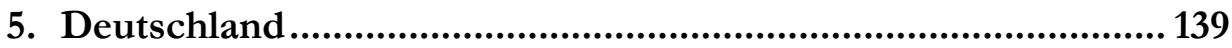

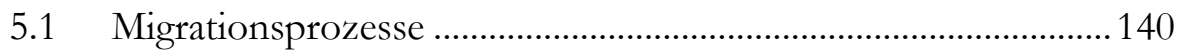

5.2 Migrationspolitik in der BRD ...................................................... 144

5.3 Kategorie Flucht und Asyl: AkteurInnen, Regulierungen und

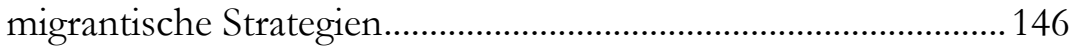

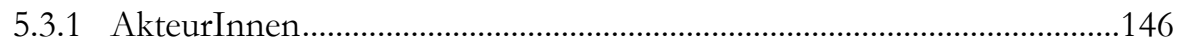

5.3.2 Phasen des asylpolitischen Wandels........................................................150

5.3.3 Flüchtlingsschutz zwischen Recht und Praxis.........................................185 
5.4 Kennzeichen des deutschen Flüchtlingsschutzregimes............... 204

5.5 Resümee der Einflussfaktoren .................................................... 206

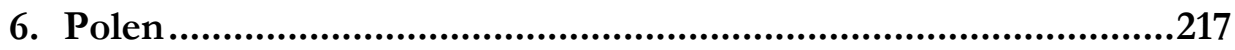

6.1 Migrationsprozesse: Zusammenspiel von Auswanderung und Rückkehr, Einwanderung und Transitmigration.......................... 219

6.1.1 Auswanderung und Rückkehr: Eine ,nationale Erfahrung' und

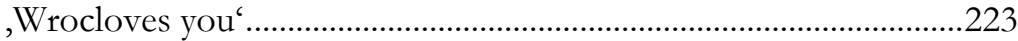

6.1.2 Einwanderung: „Polen ist der östlichste Westen“....................................235

6.2 Keine Migrationspolitik in Polen?.................................................... 244

6.3 Kategorie Flucht und Asyl: AkteurInnen, Regulierungen und migrantische Strategien ................................................................... 251

6.3.1 AkteurInnen.....................................................................................252

6.3.2 Phasen des asylpolitischen Wandels .....................................................260

6.3.3 Flüchtlingsschutz zwischen Recht und Praxis.......................................271

6.3.4 Kennzeichen des polnischen Flüchtlingsschutzregimes.........................288

6.3.5 Resümee der Einflussfaktoren ..............................................................290

7. Flüchtlingsschutz in Deutschland und Polen............................. 299

8. Fazit: Die Metamorphosen der Kategorie Flucht und Asyl........ 309

Anhang I: InterviewpartnerInnen ...................................................319

Anhang II: Tabellen und Diagramme ............................................. 320

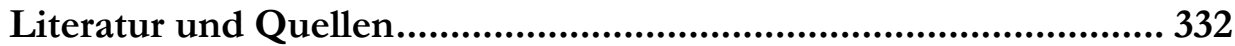





\section{Danksagung}

Die vorliegende Studie ist als Dissertation an der Universität Göttingen entstanden. Bei der Realisierung des Vorhabens bin ich von einer Vielzahl von Menschen unterstützt worden, denen ich an dieser Stelle herzlich danken möchte.

Zunächst möchte ich meinem Betreuer Peter Lösche danken, der mir mit Anregungen und Kritik stets konstruktiv zur Seite stand. Meiner Betreuerin Ursula Birsl danke ich, dass sie mich ermutigt und bestärkt hat, ein solches Projekt anzugehen, für die Offenheit, mit der sie mich bei der Entwicklung meiner Ideen begleitet hat und für manche pragmatische Orientierungshilfe in einem intensiven Forschungsprozess. Der von ihr ins Leben gerufene Arbeitszusammenhang ,Identity \& Belonging ${ }^{e}$ war und ist für mich ein wichtiger Ort des Austauschs und der gegenseitigen Unterstützung, sowohl auf wissenschaftlich-inhaltlicher Ebene als auch mit Blick auf vielerlei Höhen und Tiefen, die das (Doktorandinnen)Leben mit sich bringt. Dafür danke ich neben Ursula Birsl Renate Bitzan, Urte Böhm, Michaela Köttig, Daniela Marx, Erika von Rautenfeld und Agnieszka Zimowska. Renate Bitzan, die mir über viele Jahre Vorbild und Mentorin war, gilt mein besonderer Dank.

Dass ich diesen Weg eingeschlagen habe und zu Ende gehen konnte, habe ich ganz wesentlich Kai Marquardsen zu verdanken. Das Ausmaß und die Vielseitigkeit seiner Unterstützung sind eigentlich kaum in Worte zu fassen. Er hat mich bestärkt, gestützt und kritisiert, mit mir studiert, diskutiert, gelesen und vor allem: 
gelebt. Nach der Geburt von Louis hat er mir die nötigen Freiräume für die letzten Schliffe an der Arbeit geschaffen.

Meinen Eltern Franz und Anita Müller danke ich für das unerschütterliche Vertrauen, mit dem sie meinen bisherigen Lebensweg begleitet haben, für ihre großzügige finanzielle Unterstützung und dafür, dass sie immer für mich da sind.

Alexander Mirau sei für seine wahrlich multidimensionale Unterstützung gedankt: von der Beherbergung im Transit auf Forschungsreisen über Hilfe bei der Vorbereitung zur Disputation einschließlich Kinderbetreuung und Bügeln bis hin zur technischen Präparation des Manuskripts für die Abgabe und schließlich für den Druck.

Stephan Lessenich danke ich für seine Hilfe bei der Sondierung erster inhaltlicher Ideen vor dem Start und Christian Banse, Renate Bitzan, Anne Karrass, Daniela Marx, Holk Stobbe und Agnieszka Zimowska für intensives Korrekturlesen am Ende des Dissertationsprojekts.

Überdies konnte ich als Stipendiatin im DFG-Graduiertenkolleg „Die Zukunft des Europäischen Sozialmodells" in Göttingen von der materiellen Sicherheit eines Promotionsstipendiums und vom regen Austausch mit anderen DoktorandInnen profitieren. Unter ihnen möchte ich insbesondere Anne Karrass für ihre vielfältige und stets äußerst kompetente inhaltliche wie freundschaftliche Unterstützung danken.

Schließlich danke ich herzlich Sabine Kroner, mit der ich Forschungsreisen nach Polen unternommen habe. Der, Polen $A G^{e}$ bei der ari Berlin danke ich ebenfalls für gemeinsame Reisen nach Polen, während derer sie mir geholfen haben, Kontakte zu knüpfen und mir einen Teil des flüchtlingspolitischen Feldes zu erschließen. Auch bei den InterviewpartnerInnen in Polen möchte ich mich für ihre Bereitschaft zu vielen Gesprächen bedanken, in denen sie mir vielfältige Einblicke in die polnische Flüchtlingspolitik eröffnet haben. Und ganz besonders bin ich den Flüchtlingen in den polnischen Lagern zu Dank verpflichtet, die uns trotz widriger Umstände eingeladen und von ihren Erlebnissen berichtet haben. Ich wünsche ihnen allen, dass sie inzwischen an den Orten ihrer Wahl und nach ihren Vorstellungen leben können. 


\section{Einleitung}

Nach den Höchstständen in den 1990er Jahren befinden sich die AsylbewerberInnenzahlen in fast allen ,alten' Mitgliedstaaten der Europäischen Union auf einem historischen Tiefststand. Gleichzeitig entwickeln sich die neuen Mitgliedstaaten zu Zielländern von Migration und haben seit einigen Jahren nennenswerte AsylbewerberInnenzahlen zu verzeichnen. Insgesamt jedoch ersuchen immer weniger Menschen Asyl oder Flüchtlingsschutz in Europa. Im Jahr 2006 war die Zahl der AsylbewerberInnen so niedrig wie zuletzt vor 20 Jahren (vgl. UNHCR 2007, S. 4). Es scheint, als hätte die Kategorie ,Flucht und Asyl‘ ihre zentrale Bedeutung für Migrationsbewegungen in die EU verloren. Eine nahe liegende Erklärung dafür könnte ein Blick auf die aktuellen Flüchtlingsstatistiken liefern: So ist laut UNHCR (2006) die Zahl der Flüchtlinge, die sich außerhalb ihres Herkunftslandes befinden, weltweit zurückgegangen. Gleichzeitig wurden einige bedeutende EU nahe Konflikte, die Flüchtlingsbewegungen nach sich zogen, inzwischen (mehr oder minder) befriedet (zum Beispiel im ehemaligen Jugoslawien). Die Schlussfolgerung, dass die Anträge auf Flüchtlingsschutz zurückgehen, weil das Flüchtlingsphänomen überhaupt an Bedeutung verliert, weil zum Beispiel die Flucht- bzw. Migrationsursachen nicht mehr bestehen, wird jedoch durch drei Aspekte relativiert: Zum einen sind zu den fortbestehenden neue Konflikte und Fluchtursachen hinzugekommen, die zu neuen Flüchtlingsbewegungen führen. Dies spiegelt sich zum anderen auch darin, dass die Zahl der Flüchtlinge, die eine 
nationalstaatliche Grenze überschritten haben, zwar gesunken, die Zahl der Binnenflüchtlinge jedoch gestiegen ist. Und zum dritten gewinnt irreguläre Immigration nach Europa in den vergangenen Jahren rasant an Bedeutung.

Der Rückgang der AsylbewerberInnenzahlen bei gleichzeitig zunehmender irregulärer Immigration verweist auf die schwindende Bedeutung des Asylrechts als Einwanderungsoption in Europa. Ziel der vorliegenden Untersuchung ist daher herauszuarbeiten, wie sich dieser Bedeutungswandel in den vergangenen 20 Jahren vollzogen hat und wie er erklärt werden kann. Dabei bildet die politische Konstruktion von MigrantInnenkategorien als zentrales migrationspolitisches Instrument den analytischen Ausgangspunkt der Arbeit. In den Gesetzgebungen der Einwanderungsländer werden verschiedene „Dimensionen des Wanderungsprozesses“ (Pries 2001, S. 37) verknüpft und daraus Kategorien entwickelt, die die Voraussetzungen für eine legale Einreise und die Inanspruchnahme von Rechten definieren. Mittels dieser Kategorisierungen sollen MigrantInnen mit bestimmten ,erwünschten“ Merkmalen selektiert und mit einer Zutrittsmöglichkeit zum jeweiligen Territorium ausgestattet, während andere davon ausgeschlossen werden. Die unterschiedlichen Einwandererkategorien korrespondieren mit unterschiedlichen Rechtsstatus und abgestuften Rechten innerhalb des Territoriums - die Kategorisierung geht daher mit einer Hierarchisierung von Rechtslagen einher. Gleichzeitig bringt die Definition und Regulierung, legaler ${ }^{\star}$ Einwanderungsmöglichkeiten die irreguläre Immigration erst hervor. Die Einteilung von MigrantInnen zum Beispiel in ,Flüchtlinge', ,ArbeitsmigrantInnen“ oder ,Illegale' basiert also auf politischen Konstruktionen, die sich in erster Linie an nationalstaatlichen Interessen orientieren; als solche sind sie nicht statisch und unverrückbar - vielmehr können sich im Zeitverlauf Neu- und Umdefinitionen und ein Wandel der an die jeweiligen Kategorien geknüpften Maßnahmen ergeben.

Die Kategorie ,Asyl' stellt dabei insofern eine Besonderheit dar, als sie auf völkerrechtlichen Verpflichtungen basiert, die sich aus der Ratifikation der Genfer Flüchtlingskonvention ergeben. Sie kann daher nicht ohne Weiteres den Selektionsbestrebungen nach Erwünschtheitskriterien unterworfen werden. Dennoch wurde in den vergangenen Jahrzehnten durch zahlreiche Maßnahmen auch das Recht, Flüchtlingsschutz in einem EU-Mitgliedstaat zu erhalten, massiv eingeschränkt.

In der vorliegenden Studie soll also die Bedeutung der Kategorienkonstruktion für die migrationspolitischen Bemühungen der EU und ihrer Mitgliedstaaten sowie für die MigrantInnen selbst herausgearbeitet werden. Dabei sollen die sich wandelnde Bedeutung einzelner Kategorien sowie die Verschiebungen zwischen Kategorien und zwischen den EU-Mitgliedstaaten, die sich in den letzten Jahren im europäischen Migrationsregime vollzogen haben, am Beispiel der veränderten Rolle von ,Flucht und Asyl' in zwei ausgewählten Länderfallstudien aufgezeigt werden. Anhand des Beispiels wird illustriert, dass die Kategorien Gegenstand und Ergebnis von politischen Aushandlungsprozessen sind: Es wird dargelegt, wie der 
gewandelte Blick auf Flucht und Asyl dazu geführt hat, dass diese Kategorie ohne ihren völkerrechtlich verankerten Grundgehalt zu verändern - mit einer Fülle an Maßnahmen und Instrumenten verknüpft wurde, mittels derer sie im geografischen Kern Europas beinahe in die Bedeutungslosigkeit manövriert und die Verantwortung für Asylsuchende in die Länder an den Außengrenzen sowie die Nachbarstaaten der EU transferiert wurde.

Um also zu untersuchen, welche Bedeutung die Konstruktion von ,Flucht und

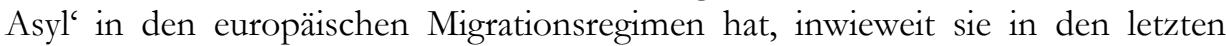
Jahren einem Wandel unterlag und welchen Einfluss sie auf die Realität und Strategien von MigrantInnen hat, sollen die Regelungen, Definitionen und Debatten, die sich in diesem Bereich aufspüren lassen, nachvollzogen werden. Daraus können abschließend auch Schlussfolgerungen über einige prominente Ansätze zur Analyse und Erklärung von Migrationspolitik - wie die gap- und die KonvergenzHypothese sowie die These vom ,Scheitern'von migrationspolitischen Strategien - gezogen werden.

Die zentrale These der Untersuchung ist, dass sich im Rückgang der Flüchtlingszahlen ein Wandel in der Bedeutung der Kategorie Flucht und Asyl manifestiert, der durch das Verschwinden der (regulär anwesenden) Flüchtlinge aus den ehemals bedeutenden Aufnahmestaaten wie der Bundesrepublik Deutschland gekennzeichnet ist. Dies ist als ein Ergebnis umfassender asylpolitischer Restriktionen zu begreifen. Die Verengung des Asylrechts vollzieht sich dabei weniger durch eine Neudefinition dessen, wer ein Flüchtling ist bzw. wer Asyl erhalten soll, sondern vor allem durch einen erschwerten Zugang zum Asylverfahren in den Ländern im geografischen Kern der EU. ${ }^{1}$ Dies führt zu einer Auslagerung asylpolitischer Verantwortung und Instrumente. Damit kann erklärt werden, warum immer weniger MigrantInnen die angestrebten Zielländer erreichen und diejenigen, die einwandern, vor allem irreguläre Wege nutzen. Dies impliziert auch, dass die schwindende Bedeutung der Kategorie Flucht und Asyl auf dem EUTerritorium nicht das Ende der Immigration in die EU bedeutet, sondern dass sich Immigration durch das Verschließen dieses und anderer regulärer Einwanderungswege hin zu anderen, einschließlich irregulären Wegen sowie in die Staaten

\footnotetext{
${ }^{1}$ In der vorliegenden Studie werden die asylpolitischen Restriktionen in den Zielländern als entscheidender Faktor betrachtet, der den Rückgang der Flüchtlingszahlen bedingt. Zwar ließe sich argumentieren, dass die niedrigen Flüchtlingszahlen in einigen Ländern mit relativ liberalen Migrationsregimen - wie bspw. Spanien - dadurch bedingt sind, dass weniger restriktiv gestaltete Einwanderungsmöglichkeiten zur Verfügung stehen (vgl. Birsl 2005, S. 214) oder ein Leben ohne Aufenthaltsdokumente vergleichsweise leicht organisiert werden kann. Dass anderen Einwanderungsoptionen der Vorzug gegeben wird, ist jedoch auch auf die Restriktionen im Asylbereich zurückzuführen. Auch ließe sich einwenden, dass die steigende Zahl der Binnenflüchtlinge weniger auf Abschottungstendenzen in den Zielländern als in einem Mangel an notwendigen Ressourcen begründet liegt, die für eine Flucht über größere Distanzen und über Staatsgrenzen hinweg notwendig wären. Auch hier spielen jedoch die migrationspolitischen Verschärfungen in den Zielländern eine Rolle: Durch hohe einwanderungsrechtliche Hürden wird auch ein höheres Maß an Ressourcen notwendig, um diese Hürden zu überwinden.
} 
an den Außengrenzen und die Nachbar- bzw. Transitstaaten verschiebt. Gleichzeitig lässt sich eine Zuspitzung der sozialen Situation von Asylsuchenden beobachten. Entgegen der These eines „Scheiterns der Migrationspolitiken“ (Castles 2005) wird hier davon ausgegangen, dass die politischen Rahmenbedingungen und rechtlichen Regulierungen insofern wirkungsmächtig für MigrantInnen sind, als sie die Bedingungen, unter denen die Migration stattfindet, verändern und damit auch die Formen der Migration. Insofern ist auch die These von der „Autonomie der Migration" (vgl. Karakayalı/Tsianos 2005) dahingehend zu relativieren, als dass sich für MigrantInnen je nach ihren individuellen Voraussetzungen in unterschiedlicher Weise Handlungsspielräume eröffnen oder verschließen, sie mit ihren widerständigen und ,eigensinnigen“ (Benz/Schwenken 2005) Praxen also Steuerungsversuche unterlaufen, diesen aber auch unterlegen sein können.

Um diese Entwicklungen aufzuzeigen, werden die Veränderungen in den letzten rund 20 Jahren im Asylbereich am Beispiel von Deutschland und Polen herausgearbeitet. Dafür wird zunächst ein begrifflicher und theoretischer Rahmen geschaffen, der die weitere Untersuchung verorten und die Prämissen transparent machen soll: Zunächst werden einige für die Untersuchung grundlegende Begriffe erläutert - Migration, Migrationspolitik und Migrationsregime. Des Weiteren werden die zentralen Ansätze vorgestellt, die derzeit die Debatte um Ziele, Ergebnisse und die Funktionsweisen von Migrationspolitik bestimmen. Auf dieser Basis wird diskutiert, welche Bedeutung der Konstruktion bestimmter Einwandererkategorien in der Migrationspolitik zukommt und dies schließlich anhand der Kategorie Flucht und Asyl eingehend illustriert. Aus diesen Betrachtungen heraus werden einige Untersuchungsthesen formuliert, die im Fortgang der Studie anhand der asylpolitischen Regulierungen auf EU-Ebene, in der Bundesrepublik Deutschland sowie in Polen überprüft und expliziert werden sollen. Auf der Basis dieser theoretischen Vorüberlegungen wird in einem zweiten Schritt das methodische Instrumentarium erläutert, bevor konkret mit der Darstellung der asylpolitischen Regulierungen anhand von Dokumentenanalysen und - im Falle Polens - mithilfe von ExpertInnen-Interviews begonnen wird. Den Ausgangspunkt bilden zunächst die asylpolitischen Bestimmungen auf EU-Ebene, da die Entwicklungen in den Mitgliedstaaten in diesem Politikbereich nicht mehr losgelöst von den fortschreitenden Europäisierungsprozessen betrachtet werden können. Die EU tritt in diesem Feld seit einigen Jahren als zunehmend wichtige Akteurin in Erscheinung, die bestrebt ist einen für alle Mitgliedstaaten einheitlichen asylpolitischen Rahmen zu konstituieren. Vor diesem Hintergrund werden in zwei Länderstudien die asylpolitischen Entwicklungen in Deutschland und Polen untersucht und im Fazit miteinander kontrastiert. Mit Polen und Deutschland wurden zwei Länder ausgewählt, die sich in der Entwicklung ihrer Asylregime, in der Bedeutung, die Asyl als Einwanderungsoption hat und in ihren Positionen im Europäischen Einigungsprozess unterscheiden. Beide Länder sind exemplarische Beispiele für den Wandel, den das europäische Asylregime derzeit vollzieht: Während die Länder im geogra- 
fischen Kern der EU - wie die Bundesrepublik Deutschland - seit Jahren rückläufige AsylbewerberInnenzahlen zu verzeichnen haben, sehen sich die Länder an den Außengrenzen und insbesondere die neuen Mitgliedstaaten wie Polen einer neuen Rolle als Aufnahmeländer von Asylsuchenden gegenüber. Am Beispiel Polens und Deutschlands kann also die Verschiebung von Migration aus dem geografischen Kern der EU an ihre Außengrenzen und weiter in die Nachbarstaaten und damit der zentrale Modus des Wandels des europäischen Asylregimes illustriert werden. Es wird daher nicht nur darum gehen, Ähnlichkeiten und Unterschiede zwischen den Beispielfällen zu identifizieren, sondern Ziel ist es auch Beziehungen, Wechselwirkungen und Verschiebungen zwischen den beiden Ländern mit Bezug auf die Kategorie Flucht und Asyl herauszuarbeiten. Aus den Erkenntnissen, die aus der Analyse der Entwicklungen auf EU-Ebene und aus den beiden Länderstudien gewonnen werden, werden abschließend - unter Rückbezug auf die im Theoriekapitel formulierten Untersuchungsthesen - Schlussfolgerungen über den Wandel der Kategorie Flucht und Asyl im europäischen Asylregime gezogen.

Damit erschließt die vorliegende Studie das Feld der Asylpolitik in dreierlei Hinsicht neu: Zum einen wird die Asylpolitik im Kontext migrationspolitischer Kategorienkonstruktionen diskutiert. Auf diese Weise wird die Bedeutung der Kategorisierung und Hierarchisierung von ,erwünschten' und ,unerwünschten MigrantInnen als zentraler Bestandteil des ,Migrationsmanagements' aufgezeigt. Zum anderen liefern die beiden Fallstudien ein umfängliches Bild der Asylpolitik in Deutschland und Polen. Für die Bundesrepublik wird damit ein seit einigen Jahren vernachlässigtes Forschungsfeld aufgegriffen und aktualisiert. Das polnische Asylregime ist noch recht jung und bislang weitgehend unerforscht - hier bietet die Untersuchung einen Einblick in die Entwicklung und Ausgestaltung der Asylpolitik in einem der neueren EU-Mitgliedstaaten. Indem die beiden Fallstudien schließlich in die Entwicklungen auf EU-Ebene eingebettet und aufeinander bezogen werden, eröffnet die Studie einen neuen und umfassenden Einblick in die Dynamiken, die den Wandel des Asylregimes in den vergangenen 20 Jahren bestimmt haben. 



\section{2. ,Flucht und Asyl' im Kontext von Migration und Migrationspolitik}

Im folgenden Kapitel sollen zum einen die für die Untersuchung zentralen Begriffe vorgestellt werden. Zum anderen werden überblicksartig die derzeit wichtigsten Ansätze zur Analyse und Erklärung von Migrationspolitik, wie sie in der politikwissenschaftlichen Migrationsforschung diskutiert werden, vorgestellt. In Auseinandersetzung mit diesen Ansätzen wird schließlich im dritten Teil des Kapitels die Bedeutung der Kategorisierung und Hierarchisierung von MigrantInnen im Migrationsregime herausgearbeitet. Im Mittelpunkt steht dabei die Kategorie Flucht und Asyl. Es wird gezeigt, dass es sich beim gegenwärtig vorherrschenden Verständnis von Flucht und Asyl um eine in politischen und wissenschaftlichen Debatten und Aushandlungsprozessen konstruierte Kategorie handelt und dass die Konstruktion von verschiedenen Einwandererkategorien (zum Beispiel Asylsuchende, ArbeitsmigrantInnen, Illegale) einen wichtigen Pfeiler der Migrationspolitik bildet.

Davon ausgehend werden die zentralen Thesen der Untersuchung entwickelt. Damit wird die begriffliche Grundlage und eine theoretische Perspektive geschaffen, entlang derer schließlich die Definitionen und Regulierungen im Wechselspiel zwischen der EU und den ausgewählten Mitgliedstaaten Deutschland und Polen bezüglich der Kategorie Flucht und Asyl und deren Bedeutungswandel untersucht werden können. 


\title{
2.1 Zentrale Begriffe: Migration, Migrationspolitik und Migrationsregime
}

Im Folgenden werden die Begriffe Migration, Migrationspolitik und Migrationsregime, die für die vorliegende Untersuchung zentral sind, mit Blick auf die aktuelle wissenschaftliche Debatte erläutert und definiert.

\section{Migration}

Es ist inzwischen eine Binsenweisheit, dass es weder in den Sozialwissenschaften noch auf politisch-administrativer Ebene in unterschiedlichen Staaten oder internationalen Organisationen einen Konsens darüber gibt, was unter dem Begriff Migration zu verstehen ist. Die Administrationen einzelner Länder haben jeweils eigene Vorstellungen von Migration, was die Vergleichbarkeit statistischer Daten erschwert. In den Sozialwissenschaften herrscht ebenfalls Uneinigkeit: Muss der Wohnort gewechselt und eine politische Grenze überschritten werden, damit man von Migration sprechen kann? Welche Distanz muss mindestens zurückgelegt worden sein? Kann man schon bei einem Aufenthalt in einer anderen Region von weniger als einem Jahr von Migration sprechen? Und lässt sich Migration auf bestimmte Zwecke eingrenzen? All diese Fragen deuten die Vielfalt möglicher Begriffsbestimmungen an und haben dazu geführt, dass verschiedene Dimensionen von Wanderungsprozessen unterschieden werden, die zur Klassifizierung von Migrationsbewegungen herangezogen werden können (vgl. z.B. Düvell 2006, S. 11; Pries 2001, S. 37; Treibel 2003). Anhand dieser Dimensionen kann ,geografische Mobilität ${ }^{6}$ differenziert und in Typologien zusammengefasst werden. Einige dieser Dimensionen zielen auch auf die nähere Bestimmung von Fluchtmigration ab, wie „Gründe und Umstände für den Ortswechsel (freiwillig, unfreiwillig; Arbeits- oder Fluchtmigration; [...]“ (Pries 2001, S. 37) bzw. „Zweck und Motiv“ oder „Charakter der Entscheidung“ (erzwungen oder freiwillig) (Düvell 2006, S. 11). ${ }^{2}$

Die gängigen Vorstellungen von Migration fasst Ludger Pries wie folgt zusammen:

\begin{abstract}
„Seit der weltweiten Durchsetzung der Nationalstaaten als der primären politischen Verfassung gesellschaftlichen Zusammenlebens in den letzten zwei Jahrhunderten wird externe oder internationale Migration allgemein als Wechsel von einem nationalstaatlichen,Container' [...] in einen anderen aufgefasst. Wenn der neue Wobnort zum festen Lebensmittelpunkt auf unbestimmte Zeit wird, spricht man üblicherweise von Emigration. Ist der neue Wohnort dagegen nur ein transitorischer, an dem ein Mensch
\end{abstract}

\footnotetext{
${ }^{2}$ Im Abschnitt 2.3 Kategorienkonstruktion als migrationspolitisches Fundament: das Beispiel Flucht und Asyl wird zum einen die Problematik der Abgrenzung von Fluchtmigration, von Freiwilligkeit und Unfreiwilligkeit etc. näher erläutert und zum anderen werden die wissenschaftlich erstellten Typologien mit den politischen Kategorienkonstruktionen ins Verhältnis gesetzt.
} 
- wegen politischer Verfolgung oder zum Zwecke der Erwerbsarbeit - nur eine befristete Zeit verbringt bzw. zu verbringen beabsichtigt, um anschließend in seine Herkunftsregion bzw. ,Heimat" zurückequkehren, so sprechen wir in der Regel von Pendelmigration oder Remigration. "(Pries 2001, S. 5f., Hervorh. i. Orig.)

In Abgrenzung zu den Verkürzungen dieses, Container-Denkens' in der Migrationsforschung wird Migration zunehmend auch als transnationales Phänomen begriffen. Dieser Ansatz geht auf Linda Basch, Nina Glick Schiller und Cristina Szanton Blanc zurück (vgl. Basch/Glick Schiller/Szanton Blanc 1994) und wurde im deutschsprachigen Raum zum Beispiel von Pries aufgegriffen. Er beschreibt Transmigration ,als eine moderne Variante der nomadischen Lebensform“. Sie stehe

„im Zusammenhang mit transnationalen Sozialräumen, die sich pluri-lokal zwischen und oberhalb von verschiedenen Wohn- und Lebensorten aufspannen. In dem Typus der Transmigration ist $W$ anderung als nicht mehr vorwiegend der - einmalige, zeitlich eng begrenzte - Übergang zwischen verschiedenen, örtlich eindeutig fixierten Lebenszusammenhängen. Vielmehr wird Wanderung selbst (wieder) zu einer Daseinsform. "(Pries 2001, S. 8f., Hervorh. i. Orig.)

Das Thema der Transmigration problematisiert gleichzeitig die grundlegende Voraussetzung, die den meisten aktuellen Vorstellungen von Migration zugrunde liegt: Das Denken in Nationalstaaten und die Konstruktion von Migration als Abweichung von einer unterstellten Norm der Sesshaftigkeit: „Nations-, Europa-, Kultur- und Wir-Gruppen-VorStellungen sind somit von fundamentaler Bedeutung für die Definition von ,Migration“ und ,MigrantInnen', Bilder von Migrationen verstärken Wir-Gruppenkonstruktionen“ (Benedikt 2004, S. 66). Während ein Ortswechsel innerhalb der Bundesrepublik, also ,innerhalb des Territoriums einer vorgestellten Gemeinschaft" (ebd., S. 65) nicht als Migration thematisiert wird, wird erst das Überschreiten einer nationalstaatlichen Grenze zur Migration, worin die Vorstellung einer imaginierten „Wir“- und einer „Sie-Gruppe“ (ebd., S. 66) eine zentrale Rolle spielt. Derzeit findet laut Benedikt ein diskursiver Wandel beim Sprechen über Migration von einer nationalen zu einer (EU-) europäischen WirGruppe statt. Die Verschiebung des Verständnisses von Migration als vor allem Bewegungen, bei denen EU-Außengrenzen überschritten werden, finde dabei nicht nur über legislatorische Definitionen wie durch den Begriff ,Drittstaatenangehöriger statt, sondern auch im öffentlichen Diskurs (vgl. ebd.). In der EU und ihren Mitgliedstaaten wird vor allem Migration aus Nicht-EU-Staaten (so genannte Drittstaaten) als Problem gesehen und in den Zuständigkeitsbereich der Justizund Innenminister verwiesen. Hingegen gilt die Migration zwischen EU-Staaten als erwünschte ,Mobilität des Humankapitals', deren legale Voraussetzung mit der ,Freizügigkeit von Personen` geschaffen wurde und die mit verschiedenen Maß- 
,Flucht und Asyl` im Kontext von Migration und Migrationspolitik

nahmen gefördert werden soll (Benedikt 2004, S. 67f.). ${ }^{3}$ So setzten sich auch zunehmend die beiden unterschiedlich konnotierten Begriffe Migration (für Bewegungen aus ,Drittstaaten“ in die EU) und grenzüberschreitende Mobilität (für Bewegungen zwischen EU-Staaten) durch.

Diese knappe Darstellung soll nur einen kurzen Einblick in die sozialwissenschaftliche Debatte geben. In der vorliegenden Studie wird Migration als „eine spezifische Form räumlicher Mobilität“" (Stobbe 2004, S. 8) begriffen und bezüglich der räumlichen und zeitlichen Dimension und der Wanderungsursache (vgl. Treibel 2003, S. 20) in einem sehr weiten Sinne verstanden. Hinsichtlich des Raumaspekts werden sowohl Binnen- als auch internationale Wanderung hinzugezählt, bezüglich der Zeit werden temporäre und dauerhafte Ortswechsel ebenso wie Pendelbewegungen mitgedacht. Und mit Blick auf die Migrationsmotive bzw. die Migrationsentscheidung wird von einem komplexen Mix an Ursachen ausgegangen, der nicht auf die Unterscheidung zwischen Arbeits- und Flucht- bzw. freiwilliger und unfreiwilliger Migration reduzierbar ist. Lediglich „,[e]in einmaliger und zeitlich befristeter Aufenthalt zu touristischen oder geschäftlichen Zwecken“ (Stobbe 2004, S. 8) wird hier nicht unter dem Begriff Migration subsumiert.

Ausgehend von einem Migrationsbegriff, der die Komplexität von Migration angesichts der verschiedenen möglichen Ausprägungen und Kombinationen der unterschiedlichen „Dimensionen des Wanderungsprozesses“ (Pries (2001) ${ }^{4}$ berücksichtigt, wird das Thema Flucht in der vorliegenden Studie daher nicht als Sonderfall von Migration, sondern als Teil davon betrachtet. Wie weiter unten gezeigt werden wird, ist das Kriterium der (Un)Freiwilligkeit der Wanderung in der politischen und teilweise auch noch in der wissenschaftlichen Debatte über Flucht von zentraler Bedeutung. Dabei wird ausgeblendet, dass sich Fluchtmigration ebenfalls durch einen Mix verschiedener Ausprägungen von Motiven, Zielen, zurückgelegter Distanz, Dauer usw. auszeichnet und nicht etwa auf die Ursache der individuellen politischen Verfolgung, die Voraussetzung des Überschreitens von Staatsgrenzen und einmalige, legale Süd-Nord-Migration reduzierbar ist. Versuche, unterschiedliche Grade an Freiwilligkeit zu definieren und verschiedene Fluchtgründe (ökonomische, politische, ökologische, soziale) fein zu differenzieren und zu hierarchisieren und auf diese Weise Migration und Flucht voneinander zu trennen, werden dem Phänomen deshalb nicht gerecht. Flucht als Bestandteil von Migration zu begreifen verweist stattdessen auf die fließenden Übergänge, die die Ausprägungen verschiedener Migrationsbewegungen miteinander verbinden. So entflieht eine Studentin aus Marokko, die in Frankreich ein Studium aufnimmt, womöglich auch den geringen Zukunftschancen und den einengenden Familien-

\footnotetext{
${ }^{3}$ So hat die EU-Kommission das Jahr 2006 zum „Jahr der Mobilität der Arbeitnehmer“ ausgerufen. Ziel ist die Erhöhung der Mobilität von ArbeitnehmerInnen in der EU (vgl. Newsletter Migration und Bevölkerung 2006, S. 4 sowie das Kapitel 4. Asylpolitik der EU).

${ }^{4}$ Vgl. etwas ausführlicher hierzu im Abschnitt 2.3.3, S. 53: Wissenschaftliche Auseinandersetzung mit dem Flüchtlingsbegriff.
} 
verhältnissen im Herkunftsland, während die kongolesischen Flüchtlinge Ende 2008/Anfang 2009 mit der Wanderung in den Südsudan ihr nacktes Leben zu schützen suchten. Und unter den griechischen ,GastarbeiterInnen' in der Bundesrepublik fanden sich auch solche, die die Gastarbeiteranwerbung zur Flucht vor der Militärdiktatur nutzten.

Fluchtmigration kennt also viele individuelle Ausprägungen, die aufgrund der Engführung des Themas in der Einwanderungspolitik der EU-Staaten unberücksichtigt bleiben. Die politische Definition des Flüchtlingsbegriffs ist jedoch entscheidend für Verlauf und Erfolg der Wanderung: ob sie legal oder, illegal' erfolgt, inwieweit Asyl und Flüchtlingsschutz als Einwanderungsoptionen zur Verfügung stehen, welche Ressourcen erforderlich sind usw. Ziel der Untersuchung ist es am Beispiel von Flucht aufzuzeigen, mit welchen Definitionen und Vorstellungen von Migration versucht wird, Einwanderung politisch zu regulieren. In den folgenden Abschnitten dieses Kapitels wird daher das Verständnis von Fluchtmigration im Rahmen migrationspolitischer Strategien genauer ausgeleuchtet. Dabei wird die politische Definition von Flucht als eine zentrale Säule migrationspolitischer Regulierungsversuche expliziert und mit wissenschaftlichen Deutungsangeboten kontrastiert.

\section{Migrationspolitik}

In der Literatur finden sich verschiedene Versuche Migrationspolitik zu definieren. So ist Migrationspolitik zum Beispiel für Klaus Barwig und Christoph Schuhmacher (2002, S. 300) der ,gezielte Versuch, von staatl. bzw. suprastaatl. Seite auf die räumliche Mobilität Einfluß zu nehmen“. Franck Düvell (2006, S. 113) fasst unter Migrationspolitik ,,alle politischen Maßnahmen [...], die explizit auf die geografische Mobilität von Menschen zielen“. Aristide R. Zolberg (1999, S. 81) führt dazu aus:

,the relevant policies encompass not only the regulation of outward and inward movement across state borders - including the movement of persons who are not, or declare that they are not, migrants - but also the rules governing the acquisition, maintenance, loss, or voluntary relinquishment of 'membership' in all its aspects, political, social, economic, and cultural."

Ursula Birsl (2005, S. 72ff.) diskutiert, ob es sich bei der Migrationspolitik überhaupt um ein scharf abgegrenztes, eigenständiges Politikfeld im engeren Sinne handelt. Denn sowohl die Motive als auch die Maßnahmen von Kontroll- und Steuerungsversuchen seien oft in anderen Politikfeldern angesiedelt, wie der Wirtschafts-, Arbeitsmarkt-, Sozial-, Innen-, Außen- und Sicherheitspolitik. Lediglich die Regulierung des Zugangs zum Territorium (Visapolitik und Grenzregime) und des rechtlichen Status innerhalb des Territoriums bildeten den Kern der Maßnahmen, die sich konkret auf Migrationsbewegungen konzentrieren. Laut Stephen 
,Flucht und Asyl‘ im Kontext von Migration und Migrationspolitik

Castles (2005, S. 16) „geht es bei Migrationskontrolle um die Regulierung von Nord-Süd-Beziehungen."

Neben der Frage der Abgrenzbarkeit von Migrationspolitik gegenüber anderen Politikfeldern werden Definitionen von Migrationspolitik wie die von Barwig und Schuhmacher (2002) dahingehend kritisiert, dass sie fast ausschließlich den (National-)Staat oder suprastaatliche Institutionen als relevante Akteure innerhalb dieses Politikfeldes diskutieren. Inspiriert durch Konzepte aus der Regime-Theorie wurde in einigen jüngeren theoretischen Auseinandersetzungen der Blick auf eine Vielzahl weiterer beteiligter Akteure gerichtet (vgl. Karakayalı/Tsianos 2005). ${ }^{5}$ Dazu gehören Internationale Organisationen (IOs), Unternehmen, NichtRegierungs-Organisationen (NGOs), Medien, MigrantInnen(-selbstorganisationen), Think Tanks und MigrationsforscherInnen. Diese Erweiterung des Akteursrahmens eröffnet ein breites Spektrum an Fragen, die sich an die gängige Definition, aber auch die gängige Untersuchung von Migrationspolitik stellen. Diese betreffen insbesondere die Entstehung neuer Formen des Regierens - als Stichworte seien Supranationalisierung, NGOisierung und Privatisierung genannt und den Stellenwert von MigrantInnen in wissenschaftlichen und politischen Debatten. In den neueren Migrationstheorien (vgl. Haug 2000; Pries 2001; Düvell 2006) kann unter anderem ein Trend zu einer stärkeren Fokussierung auf Strategien und Praxen von MigrantInnen festgestellt werden. Einige ForscherInnen plädieren dafür, dass - anstatt die wechselnden Migrationsregime der Aufnahmeländer zu analysieren - im Sinne eines „Transnationalismus von Unten“ die Handlungsperspektive der MigrantInnen in den Vordergrund zu stellen sei (vgl. Lutz 2005, S. 82). Ansätze wie die des Transnationalismus, der Transmigration, von Migrations-Netzwerken und -Kreisläufen sowie cumulative causation (vgl. Pries 2001) führen weg von einem Verständnis von Migration oder Migrationspolitik, innerhalb dessen MigrantInnen nur als Objekte und Gegenstände von Statistiken und Migrationen als zu steuernde, Naturabläufe' begriffen werden, welche in Form von Kurvenverläufen oder dicken Pfeilen abgebildet werden, die auf Landkarten von der ,Peripherie“ ins ,Zentrum weisen ${ }^{6}$. Die Lesart von MigrantInnen als eigenständige AkteurInnen verändert auch die Definition von Migrationspolitik. Aus diesen Überlegungen heraus wird das Verständnis von Migrationspolitik entwickelt, das dieser Studie zugrunde liegt: Die folgenden Stichworte umreißen diesen Migrationspolitik-Begriff und geben einen Überblick über die verschiedenen Aspekte, die darunter subsumiert werden: ${ }^{7}$

\footnotetext{
${ }^{5}$ Die zahlreichen AkteurInnen in diesem Bereich sind in der „Virtual Cartography of European Migration Policies“ anschaulich dargestellt (vgl. Forschungsgruppe Transit Migration/Labor k3000 o.J.). Neben je einer Karte, die „Diskurse“, „Europäisierung“ und „Orte und Praktiken“ darstellen, findet sich dort auch eine Karte, die die Vielzahl der AkteurInnen verdeutlicht.

${ }^{6}$ So zum Beispiel bei Fassmann/Münz 1996.

${ }^{7}$ Dieser Begriff bezieht sich nur auf das Einwanderungsgeschehen in die Zielländer der nördlichen Hemisphäre. Sowohl in den Ländern des ,Nordens' als auch des ,Südens' finden sich darüber hinaus Migrationspolitiken, die auf die Auswanderung und deren Regulierung zielen.
} 
Migrationspolitik

- ist der Versuch räumliche - vor allem grenzüberschreitende - Mobilität zu beeinflussen bzw. zu kontrollieren, durch die Regulierung des Zugangs zum Territorium (Bedingungen von Ein- und Ausreise: Formulierung legaler Einwanderungsoptionen, Grenzregime) und des rechtlichen Status innerhalb des Territoriums (vgl. Birsl 2005, S. 74) (Kategorisierung und Hierarchisierung, Bedingungen des Aufenthalts, Zugang zu sozialen und politischen Rechten, Minderheitenrechte, Antidiskriminierung).

- basiert auf der Definition von räumlich abgegrenzten, souveränen Territorien - die sich seit dem 19. Jahrhundert in Westeuropa als Nationalstaaten manifestieren - und damit verbundenen Konstruktionen von Zugehörigkeit, die nationalstaatlich über die Staatsbürgerschaft und verschiedene Konzepte zu deren Erlangung und Verleihung geregelt $\operatorname{sind}^{8}$.

- speist sich aus Motiven, Interessen und Maßnahmen anderer Politikfelder (Wirtschafts-, Arbeitsmarkt-, Sozial-, Außen- und Sicherheitspolitik).

- ist geprägt durch eine Vielzahl unterschiedlicher AkteurInnen auf nationaler, intergouvernementaler und supranationaler Ebene (Nationalstaaten ${ }^{9}$, MigrantInnenselbstorganisationen, IOs, NGOs, Initiativen, Unternehmen, intergouvernementale und supranationale Institutionen, WissenschaftlerInnen und Think Tanks), deren Interessen im Migrationsregime ${ }^{10}$ aufeinander treffen. Ein weiterer Akteur sind die Medien, die diese Interessen nicht nur ,neutral ${ }^{\varsigma}$ vermitteln, sondern eigenständig strukturieren - bedingt durch eigene, vor allem wirtschaftliche Interessen sowie ihre institutionellen Strukturen. Die Durchsetzung der Interessen der verschiedenen AkteurInnen ist abhängig von ihrer jeweiligen Macht- und Verhandlungsposition, die wiederum von weiteren Faktoren (zum Beispiel Zugang zu Ressourcen, Medien und Netzwerken, das jeweilige politische System und die politische Kultur) beeinflusst werden.

- ist durch Prozesse der Supra- und Transnationalisierung, NGOisierung und Privatisierung im Wandel begriffen.

- ist häufig gekennzeichnet durch eine Kluft zwischen proklamierten Zielen, Output und Outcome ${ }^{11}$.

- ist in den westeuropäischen Ländern eingebettet in einen kontroversen, häufig mit Ressentiments beladenen öffentlichen Diskurs ${ }^{12}$.

\footnotetext{
8 Zur Darstellung verschiedener Erklärungsansätze für die Entstehung staatlicher Versuche der Migrationssteuerung und Migrationsverwaltung vgl. Jochen Oltmer (2003, S. 10ff.). Er datiert die Herausbildung migrationspolitischer Interventionen im Wesentlichen auf den Zeitraum zwischen den 1880er und den 1920er Jahren (vgl. ebd.).

${ }^{9}$ Mit ,Nationalstaat' ist hier kein einheitlicher Akteur gemeint. Stattdessen können auch die Staaten in eine Vielfalt an AkteurInnen mit zum Teil widerstreitenden Interessen aufgefächert werden: Als Beispiele seien politische Parteien, Verwaltung und Behörden sowie WählerInnen genannt.

${ }^{10}$ Vgl. dazu den folgenden Abschnitt.

${ }^{11}$ Vgl. dazu den Abschnitt 2.2.1 zur Gap-Hypothese.
} 
Asylpolitische Regelungen sind Teil der Migrationspolitik und daher ebenfalls durch die genannten Aspekte gekennzeichnet. Durch asylpolitische Definitionen wurde die Kategorie des Asylsuchenden bzw. Flüchtlings geschaffen und mit verschiedenen, sich im Zeitverlauf wandelnden Maßnahmen verknüpft. Die jeweilige Konstitution der Kategorie Flucht und Asyl steht in einer Wechselbeziehung mit anderen Kategorien - sowohl hinsichtlich ihrer Gestaltung durch politische EntscheidungsträgerInnen als auch hinsichtlich ihrer Inanspruchnahme durch MigrantInnen. Asylpolitik ist damit ein wichtiger Teilbereich von Einwanderungspolitik. Aufgrund der völkerrechtlichen Verankerung des Asylrechts wird häufig unterstellt, es handele sich um einen migrationspolitischen Sonderfall. Wie die vorliegende Studie zeigen wird, unterliegt jedoch auch das Asylrecht migrationspolitischen Konjunkturen. Es ist ein wichtiger Bestandteil von Migrationspolitik und dient bspw. in den Nachbarstaaten der EU als erster Schritt hin zur Etablierung umfassenderer migrationspolitischer Regelungen und Institutionen.

\section{Migrationsregime}

Bei dem Versuch, das Zusammenspiel und die Vernetzung von AkteurInnen, Prozessen und Institutionen in der Migrationspolitik adäquat zu erfassen, gewinnt die Regime-Analyse an Bedeutung. Diese wurde von VertreterInnen des Interdependenzansatzes in den Internationalen Beziehungen angesichts der zunehmenden Verflechtung verschiedener und zum Teil neuer AkteurInnen entwickelt. Mit diesem neuen Analyse-Rahmen sollen die Beschränkungen der neo-realistischen Schule in den Internationalen Beziehungen überwunden werden. Nach Beate Kohler-Koch (1989) ist es das Ziel der Regime-Analyse zu bestimmen, wie und unter welchen Bedingungen sich autonome AkteurInnen vor dem Hintergrund diverser widerstreitender und paralleler Interessen, wie sie für die internationalen Beziehungen charakteristisch sind, auf eine Zusammenarbeit einigen können, die ihnen auf Dauer bestimmte Handlungsrestriktionen auferlegt. Regime sind daher die „institutionalisierte Form des norm- und regelgeleiteten Verhaltens bei der politischen Bearbeitung von Konflikten oder Interdependenzproblemen in unterschiedlichen Sachbereichen“ (Wolf 1994, S. 423). Wesentliche Strukturmerkmale von Regimen sind Prinzipien, Normen, Regeln und Entscheidungsverfahren (vgl. ebd.), die in hierarchischer Beziehung zueinander stehen (vgl. Kohler-Koch 1989, S. 35).

Auch in der Migrationsforschung wurde der Regime-Begriff aufgegriffen, um bisherige Verkürzungen und Schwachstellen der Analyse von Migrationspolitiken, -regulierungen und -praxen zu überwinden. Die Konzeptualisierung von migrationsbezogenen AkteurInnen, Prozessen und Institutionen und deren Vernetzung

12 Zur Frage, welche AkteurInnen diese Debatte bestimmen und welche Einstellungen (für oder gegen MigrantInnen) sie transportieren vgl. Statham 2003 für das Beispiel Großbritannien. Er interpretiert die Ergebnisse mit Bezug auf Freemans ,interest group approach“ und die so genannte „racist public thesis“. 
als Migrationsregime korrespondiert mit der Abkehr vom Konzept des ,Migrationssystems', das politische, ökonomische und rechtliche Strukturen fokussiert und dabei die kollektiven und individuellen Praktiken von MigrantInnen vernachlässigt (vgl. Karakayalı/Tsianos 2005, S. 35). Nach Serhat Karakayalı und Vassilis Tsianos

„ermöglicht der Regimebegriff eine Vielzahl von AkteurInnen einzubeziehen, deren

Praktiken aufeinander bezogen sind, nicht aber in Gestalt einer zentralen (systemischen)

Logik geordnet, sondern vielfach überdeterminiert sind." (Karakayall/Tsianos 2005, S.

46)

Die beiden Autoren wollen auf diese Weise „Aspekte der Autonomie der Migration" (ebd.) theoretisch fassen und die Aktivitäten der MigrantInnen und der Kontrollagenturen nicht als Objekt-Subjekt-Verhältnis begreifen. Dementsprechend sind „Regulationen als Effekte, als Verdichtungen von sozialen Handlungen“ zu verstehen und „nicht funktionalistisch vorauszusetzen“ (ebd., S. 48). Insofern sei auch der Staat nicht als „Ausgangspunkt gesellschaftlicher Handlungen“ (ebd.) zu konzipieren. Karakayalı und Tsianos schlagen vor das Verhältnis zwischen den AkteurInnen im Migrationsregime als „asymmetrisches Macht-Verhältnis“ (ebd., S. 49) zu sehen, in dem die AkteurInnen der Kontrollagenturen zwar überlegen seien, das Ergebnis der Überlegenheit jedoch nicht die Verhinderung von Mobilität sei, sondern die Regulation der grenzüberschreitenden Arbeitsmobilität. Insofern bestehe eine selektive Durchlässigkeit der Grenzen, die sie - in Anlehnung an Foucault - als ,Produktivität' des Grenzregimes bezeichnen. Nicht Abschottung, sondern Entrechtung sei demnach die zentrale Funktionsweise dieses Grenzregimes.

Die Überwindung der Staatsbezogenheit ist auch das Ziel von VertreterInnen der ,Governance'-Debatte, die auch ,Governance of Migration“ einschließt. Der Begriff Governance dient dazu eine neue Qualität des Regierens, der Politik angesichts von Globalisierungs- und Migrationsprozessen und damit verbundenen möglichen Souveränitätsverlusten für den Nationalstaat zu beschreiben. Laut Dirk Messner und Franz Nuscheler (1996, S. 5) meint Global Governance einen

„,breit angelegten, dynamischen und komplexen Prozess interaktiver Entscheidungsfindung, an dem viele Akteure beteiligt sind. Die Nationalstaaten bleiben zwar Hauptakteure der internationalen Politik. und behalten eine unverzichtbare Scharnierfunktion zwischen verschiedenen Handlungsebenen der Global-Governance-Architektur [...], aber sie sind nicht mehr die einqigen Akteure. Die, geteilte Souveränität' nimmt ihnen nicht das Gewaltmonopol nach innen, fordert ihnen aber Souveränitätsverzichte ab, um die kollektive Bearbeitung von Problemen zu ermöglichen. "

Global Governance wird gleichzeitig von WissenschaftlerInnen und politischen AkteurInnen normativ als Reformprojekt, als neuer Politiktypus und „Kontrastmodell zu hegemonialen Weltordnungskonzepten“ (ebd.) propagiert, mit dem Globalisierungsprobleme unter Einbeziehung nicht-staatlicher AkteurInnen und 
,Flucht und Asyl` im Kontext von Migration und Migrationspolitik

der ,Zivilgesellschaft' durch Konsensfindung gelöst werden könnten. Auch in der Governance-Debatte wird also betont, dass Nationalstaaten nicht (mehr) die einzigen und zentralen Akteure sind, dennoch werden auch in diesen Ansätzen - wie Karakayalı und Tsianos zutreffend kritisieren - MigrantInnen überwiegend lediglich als Objekte betrachtet und sind so gleichsam die „,abwesende Ursache“ des Governance-Diskurses“ (Karakayalı/Tsianos 2005, S. 48, Hervorh. i. Orig.). Gleichzeitig zeige eine kritische Betrachtung der Praxis von ,new governance ${ }^{6}$, dass die Einbindung transnationaler Organisationen und Think tanks einerseits (zum Beispiel die ,International Organization for Migration“ oder das ,International Center für Migration Policy and Development ${ }^{\star}$ in Wien) und von NGOs andererseits „einen migrationspolitischen Konsens" ermögliche, in dem Migration überhaupt erst als zu regulierendes Problem konstruiert werde (Hess/Tsianos 2003). Die Transit Migration Forschungsgruppe (2007) zeichnet diese Entwicklung anhand der Etablierung eines Migrationsregimes im Südosten Europas nach. So musste in der Türkei zunächst einmal ein Asyldiskurs etabliert werden, um migrationspolitische Maßnahmen als aus menschenrechtlicher Sicht unabwendbar einzuführen (vgl. Hess/Karakayalı 2007, S. 49ff.). Mittels dieser Maßnahmen könnten dann ,echte“ von ,illegalen' Flüchtlingen getrennt werden, um ersteren effektiven Schutz zu gewähren. Die auch von Menschenrechtsorganisationen unterstützte Etablierung eines Asylregimes bedeutet für jene, die den Asyl-Kriterien nicht entsprechen, Selektion, Inhaftierung und Abschiebung bzw. Illegalisierung (vgl. ebd.).

Die Betrachtung der migrationspolitischen Entwicklungen durch die ,RegimeBrille‘ eröffnet den Blick auf eine große Zahl an AkteurInnen, die in diesem Feld (inter-)agieren. Als grobe Kategorien lassen sich die folgenden nennen: ${ }^{13}$

- Nationalstaaten und nationalstaatliche Institutionen (Legislative, Exekutive, Judikative)

- EU-Institutionen (EU-Parlament, EU-Kommission, EU-Rat der Minister für Justiz und Inneres)

- Internationale und intergouvernementale Organisationen (IOM, UNHCR, UNO-Flüchtlingshilfe)

- NGOs auf internationaler, regionaler und nationaler Ebene (Wohlfahrtsverbände, Gewerkschaften, Menschenrechtsorganisationen, Kirchen)

- Forschungseinrichtungen

- Unternehmen

- Autonome Organisationen und Netzwerke von MigrantInnen und UnterstützerInnen (Flüchtlingsselbstorganisationen, antirassistische Gruppierungen)

- Medien

- MigrantInnen

\footnotetext{
${ }^{13}$ Die Grob-Strukturierung ist angelehnt an die Unterteilung, die in der „Migmap“ zu den AkteurInnen des Migrationsregimes dargestellt ist (vgl. Forschungsgruppe Transit Migration/Labor k3000 o.J.).
} 
In der vorliegenden Untersuchung liegt das Hauptaugenmerk auf einem ,SubRegime، des Migrationsregimes - dem Asylregime. Das Asylregime ist dem Migrationsregime jedoch nicht untergeordnet, sondern in dieses eingebettet und ein integraler Bestandteil dieses Gesamtregimes und wird ebenfalls durch die genannten AkteurInnen geprägt. Im Folgenden wird die Bedeutung des Asylregimes innerhalb des Migrationsregimes verdeutlicht. Ausgehend von einigen zentralen Ansätzen zur Erklärung von Migrationspolitik wird die Kategorisierung und Hierarchisierung von MigrantInnen als zentrales migrationspolitisches Instrument untersucht. Die Konstruktion hierarchisierter MigrantInnenkategorien kann als eine der ,Hauptleistungen“ von Migrationspolitik und zentraler Pfeiler von Migrationsregimen verstanden werden und ist gleichzeitig Voraussetzung und Ausdruck menschenrechtlich legitimierter Governance-Strategien.

\subsection{Ansätze zur Erklärung von Migrationspolitik}

Die Untersuchung der Entwicklung der Asylregime in der EU knüpft an einige derzeit einflussreiche Debatten der (politikwissenschaftlich orientierten) Migrationsforschung an. Die Politikwissenschaft hat sich erst relativ spät dem Thema Migration bzw. Migrationspolitik gewidmet. Die Forschung über Migration wurde vor allem durch SoziologInnen, AnthropologInnen und HistorikerInnen sowie oft in interdisziplinärer Perspektive durchgeführt. Politikwissenschaftliche Aspekte wurden von diesen ForscherInnen häufig ausgeblendet, während die Politikwissenschaft ihrerseits den Gegenstand lange Zeit ignorierte. Aus diesem Grund wird häufig bemängelt, dass fundierte theoretische Konzepte in der noch jungen politikwissenschaftlichen Migrationsforschung rar seien (vgl. Zolberg 1999; Freeman 2005, S. 111; Lahav/Guiraudon 2006). Die verschiedenen Ansätze befassen sich mit der Erklärung der Rolle staatlicher Regulierung von Migration (policies), der Willensbildungs-, Aushandlungs- und Implementierungsprozesse (politics) im Bereich Migrationspolitik, der Bedingungen der Integration bereits eingewanderter MigrantInnen in die politischen Strukturen (polity) und der Rolle von Migration in den internationalen Beziehungen. ${ }^{14}$

${ }^{14}$ Laut Gary P. Freeman (2005) ist es aber noch ein weiter Weg, bis die Erklärungskraft dieser Ansätze ausreichend getestet wurde und entsprechend ausgereift ist. Freeman (2005) verdeutlicht die Ignoranz der US-amerikanischen Politikwissenschaft gegenüber Migration durch den Verweis auf die geringe Zahl etablierter PolitologInnen, die sich diesem Thema widmen und die marginale Rolle, die politikwissenschaftlich inspirierte Artikel zum Thema Migration in den führenden Monografien, Sammelbänden und Zeitschriften der Disziplin spielen. Er sucht dies damit zu erklären, dass Migration sich an der Schnittstelle verschiedener Disziplinen befindet und entsprechende Forschung daher meist interdisziplinär angelegt sei. Interdisziplinäre Ansätze seien jedoch - trotz zunehmender Versuche, diese Trennungen aufzubrechen - meist weniger angesehen als solche, die sich mehr innerhalb einer einzigen Disziplin verorten. Hinzu komme das Fehlen verlässlicher und vergleichbarer Daten. Freeman moniert außerdem die oft normative Haltung von ForscherInnen in diesem Bereich, die oft selbst einen Migrationshintergrund hätten. Dies führe dazu, dass ver- 
Ein bedeutender Teil der Forschung wird von der Frage geleitet, inwieweit Nationalstaaten in der Lage sind, Migration zu steuern bzw. zu kontrollieren und bereits eingewanderte MigrantInnen zu ,integrieren'. ${ }^{15}$ Zwei zentrale Annahmen, die in der so genannten Gap-Hypothese und der Konvergenz-Hypothese zusammengefasst werden, sind Ausgangspunkt für höchst unterschiedliche Schlüsse das Scheitern von Migrationspolitiken auf der einen und die gleich bleibende, wenn nicht gestiegene Steuerungsfähigkeit von Nationalstaaten auf der anderen Seite. Das Scheitern wiederum wird aus unterschiedlichen Perspektiven betrachtet - mit Fokus auf einen steuerungs- und kontrollunfähigen Staat oder die widerständigen Praxen von MigrantInnen. Die beiden Hypothesen und die daraus abgeleiteten Schlussfolgerungen werden im Folgenden kurz umrissen und hinsichtlich ihrer Erklärungskraft für die Frage nach der Bedeutung der Kategorisierung in europäischen Migrationsregimen untersucht.

\subsubsection{Gap-Hypothese}

Gallya Lahav und Virginie Guiraudon (2006, S. 218) nennen drei Dimensionen von gaps: (1) die Kluft zwischen den Zielen und Interessen der Öffentlichkeit einerseits und der politischen EntscheidungsträgerInnen andererseits (bezüglich Entscheidungsfindung und Implementierung), (2) zwischen Policy-Zielen und -Outcomes sowie (3) zwischen internationaler und einzelstaatlicher Ebene. Vor dem Hintergrund der Überlegungen in den vorangegangenen Abschnitten kann diesen Dimensionen gewissermaßen als Pendant oder als (Teil-) Erklärung zu (2) noch eine weitere hinzugefügt werden: die Kluft zwischen politisch konstruierten Kategorien und Einwanderungsmöglichkeiten auf der einen sowie den Migrationsgründen bzw. den Zielen und Strategien der MigrantInnen auf der anderen Seite.

Ein prominenter Gegenstand politikwissenschaftlicher Untersuchungen ist die Dimension (2): Sie bezieht sich auf die Kluft zwischen offiziellen restriktiven Zielen

schiedene Fragen aufgrund ,politischen Korrektheits'- Empfindens nicht gestellt würden und ,leidenschaftslose', rigide Analyse vernachlässigt werde. All dies habe zu dem geringen Ansehen der Migrationsforschung beigetragen (ebd., S. 112ff.). Fast identische Ausführungen machen Lahav und Guiraudon (2006) zur Lage der europäischen politikwissenschaftlichen Migrationsforschung.

Der Kritik Freemans an der Normativität der Migrationsforschung kann entgegengehalten werden, dass seine eigenen Ausführungen keineswegs wertfrei oder objektiv sind. Freemans theoretische Ansätze sind durch die neoklassische politische Ökonomie inspiriert, womit eine spezifische Sicht auf den Forschungsgegenstand verbunden ist. So liegt sein Augenmerk in dem zitierten Artikel auf ,Kosten- und Nutzen'- Aspekten von Immigration. Die darin enthaltenen Vorannahmen sind allerdings im gegenwärtigen Mainstream der Sozialwissenschaften angesiedelt und werden daher als nicht-normativ und objektiv charakterisiert. Zu den Widersprüchen vermeintlich normativer bzw. objektiver Forschung vgl. Lothar Peter (2005).

${ }^{15}$ Die Frage nach der Steuerungsfähigkeit der Nationalstaaten zeigt die recht eingeengte Perspektive der Politikwissenschaft: Im Mittelpunkt steht der Nationalstaat und dessen Fähigkeit eigene migrationspolitische Leitlinien aufzustellen und umzusetzen, während MigrantInnen nur als Objekte der nationalstaatlichen Politik Erwähnung finden. 
oder Policy-Output (Entscheidungsinhalte der Politik, das heißt migrationspolitische Leitlinien und Maßnahmen) und tatsächlichem liberalem Outcome (Implementation der Maßnahmen und Situation von MigrantInnen). Policy-Outcomes sind nach Lahav und Guiraudon (2006, S. 209) das Ergebnis von

„(a) the struggles between actors in different fields (economy, politics, law), (b) the tradeoffs made by elected leaders that face varying pressures depending on the institutional characteristics of each field, and (c) implementation structures."

Die Kluft zwischen Zielen, Output und Outcome ist laut Wayne A. Cornelius und Takeyuki Tsuda (2004) ein empirischer Tatbestand, weshalb die Bezeichnung als ,Hypothese' irreführend sei. Die Kluft sei sowohl bei Abwehr- als auch bei Anwerbepolitiken beobachtbar. Cornelius und Tsuda (vgl. ebd.) identifizieren zwei Arten von gaps: (1) unintendierte Folgen und (2) inadäquate Implementation und Umsetzung der Politiken. Als Erklärung für dieses - wenngleich schwer messbare - Phänomen führen sie inhärente Fehler der Politiken, makrostrukturelle Faktoren, einzelstaatliche und internationale Bedingungen bzw. Zwänge und uneindeutige bzw. ambivalente Policy-Ziele an (vgl. ebd., S. 7ff.). Auch Stephen Castles (2005) schließt sich der gap-Hypothese an und versucht in einem Aufsatz zu erklären, „warum Migrationspolitiken scheitern“ (ebd., S. 10). Dieses ,Scheitern“ der nationalstaatlichen Migrationspolitik sei jedoch nicht normativ gemeint, da ineffiziente Politiken zur Entwicklung offenerer und integrativerer Gesellschaften beigetragen hätten. Vielmehr gehe es um die Analyse der Faktoren, die dafür verantwortlich seien, dass eine Politik ihre (offiziell) proklamierten Ziele nicht oder nur zum Teil erreiche oder nicht beabsichtigte Konsequenzen zeitige (vgl. ebd., S. 12). Dabei nimmt er mehr als Cornelius und Tsuda die Rolle von MigrantInnen selbst in den Blick. Er unterscheidet und erläutert drei Typen von „Faktoren der Migrationsausformungen“:

„Faktoren, die sich aus der sozialen Dynamik des Migrationsprozesses ergeben; Fak.toren, die mit Globalisierung, Transnationalismus und den Nord-Süd-Beziehungen verknüpft sind; Faktoren innerhalb der politischen Systeme" (ebd., S. 13).

Er macht deutlich, dass sich durch das Zusammenspiel dieser Faktoren und deren Wechselwirkung mit Migrationspolitik-Regimen ein höchst komplexes Bild ergibt. Es überrasche daher nicht, dass die Politiken, mit denen versucht wird, in diese komplexen Prozesse steuernd einzugreifen, ihrem Gegenstand häufig nicht gerecht werden und unintendierte Effekte hervorbringen (vgl. Castles 2005, S. 30). Auch wenn er davon ausgeht, dass aufgrund der Komplexität des Gegenstandes keine übergreifende Theorie entwickelt werden kann, sei es

„entscheidend wichtig zu untersuchen, wie diese Faktoren (und sicher noch andere) in bestimmten Migrations- und Gemeinschaftsbildungsprozessen zusammenwirken, will man zu ausgeglicheneren und realistischeren Politiken kommen" (Castles 2005, S. 30, Hervorh. i. Orig.). 
Dabei seien - in Übereinstimmung mit seiner Analyse der wichtigsten Faktoren vor allem drei Aspekte zu berücksichtigen: das Verständnis von Migration als langfristiger sozialer Prozess mit einer eigenen Dynamik, die Bedeutung von Wanderungen ,als integrale[r] Aspekt der Nord-Süd-Beziehungen in der gegenwärtigen Globalisierungsphase" und die Art und Weise der staatlichen und supranationalen Politikformulierung (ebd., S. 30f.). Die Analyse werde dadurch erschwert, dass die Staaten oft eine Politik zwar offiziell ausrufen, jedoch gleichzeitig - im Interesse der Aufrechterhaltung von Legitimität und um das Scheitern vorangegangener Maßnahmen zu verdecken - eine Politik betreiben, die der offiziellen mitunter konträr entgegensteht.

Auch Lahav und Guiraudon (2006, S. 203) konstatieren in ihrem Überblick über die gap-Debatte, dass der gap aus der Sicht der meisten SoziologInnen seine Ursache in der Irrelevanz von Politiken für die Migrationsdynamiken hat: „States are plainly misguided in their belief that policy can influence inevitable migration flows." (ebd.) Auch aus der Sicht der beiden AutorInnen sind Politiken nur ein Faktor bei der Ausformung von Migrationsprozessen. Zudem sind sie kein rationales Gebilde auf der Basis theoretisch fundierter Modelle, sondern entstanden in Aushandlungsprozessen, die selbst von verschiedenen Faktoren abhängig sind (vgl. Lahav/Guiraudon 2006, S. 204). Nicht zuletzt durch die Strategien der MigrantInnen selbst, die wiederum in ein komplexes Geflecht aus Motiven und Bedingungen eingebettet sind, verursachen viele Politiken Effekte, die den verkündeten Zielen entgegenstehen - etwa wenn durch stärkeres Abschließen der Grenzen vormals nicht-dauerhafte Migration zu dauerhafter Immigration wird, weil sich das Risiko für wiederholte Grenzübertritte stark erhöht hat.

Lahav und Guiraudon (2006, S. 207) plädieren dafür, stärker auf die AkteurInnen der Regulierung von Migration zu fokussieren. Auf diese Weise ließen sich Anhaltspunkte dafür sammeln, dass Input und Outcome viel kongruenter seien als bisher angenommen. Dazu sei die Behauptung eines ,embedded liberalism' in den Einwanderungsländern (vgl. Hollifield 1992), der - gestützt durch internationale Menschenrechts-Regelungen und begrenzte Fähigkeiten - zu liberalen Outcomes beigetragen habe, zu überprüfen. So seien die Thesen über das Verhältnis von Öffentlichkeit und Eliten zu überdenken: Lahav und Guiraudon verweisen auf Studien, die zeigen, dass die Öffentlichkeit weniger, die Eliten hingegen stärker restriktiv eingestellt seien, als bisher angenommen. Eliten tendierten darüber hinaus dazu, auf Druck seitens der extremen Rechten zu reagieren. Weiterhin sei die Vielzahl der AkteurInnen, die die Policy-Outcomes beeinflussen, zu berücksichtigen. ${ }^{16}$ Internationale AkteurInnen bzw. intergouvernementale Kooperationen, von denen meist angenommen wird, sie würden die Einzelstaaten mit Zwängen belegen, hätten vielmehr eine ermöglichende Funktion: Sie legitimierten und stütz-

\footnotetext{
${ }^{16}$ Lahav und Guiraudon (2006, S. 207) nennen organisierte Interessengruppen, Gerichte, ,ethnische Gruppen, Gewerkschaften, Ordnungsbehörden, Polizei- und Sicherheitsagenturen sowie lokale und private Akteure.
} 
ten restriktive Politiken, die auf nationaler Ebene andernfalls nicht durchsetzbar gewesen wären. Neben der internationalen bzw. intergouvernementalen und nationalen ist die lokale Ebene mit zu berücksichtigen, von der das ,Ob und ,Wie ${ }^{6}$ der Umsetzung der vorgegebenen Politiken im Wesentlichen abhängt.

\subsubsection{Konvergenz-Hypothese}

Während die Existenz der Kluft zwischen Policy-Output und -Outcome, zwischen den Zielen und Maßnahmen und den tatsächlichen Folgen der Politik nach Ansicht von Cornelius und Tsuda (2004) unumstritten ist, ist nach Ansicht der beiden Autoren unklar, inwieweit eine Konvergen ${ }^{17}{ }^{17}$ zwischen den Immigrationskontrollpolitiken der fortgeschrittenen Industriestaaten konstatiert werden kann (vgl. ebd., S. 15). So gebe es zwar kaum noch stark abweichende Fälle, es sei aber fraglich, ob diese Ähnlichkeiten Beispiele für tatsächliche Policy-Konvergenz seien. Denn dies würde im engeren Sinne eine Gruppe „Arbeitskraft-importierender Staaten“ implizieren, die sich in mehr oder minder koordinierter Weise in Richtung eines einzigen Immigrationspolitik-Typus entwickeln (ebd.). Die Frage, in Richtung welchen Modells die Politiken der Staaten konvergieren und die Bewertung des Ausmaßes, in dem sich die Immigrationspolitiken westeuropäischer und asiatischer Staaten an die ,klassischen Einwanderungsländer ${ }^{`}$ angleichen (USA, Kanada und Australien), ginge implizit von einem evolutionären Modell aus. Danach repräsentierten die traditionellen Einwanderungsländer die am meisten fortgeschrittene Stufe der Politikentwicklung, die die anderen Staaten schließlich auch erreichen. Einen solch unilinearen Ansatz lehnen die Autoren ab, da die Immigrationspolitiken der klassischen Einwanderungsländer sich selbst in wichtigen Punkten voneinander unterschieden und nicht stabil seien. Statt auf globalem Level sollten daher Policy-Konvergenzen auf regionaler Ebene, also zum Beispiel unter den Mitgliedstaaten der EU untersucht werden - und zwar eher auf der Ebene von ,Makro'- (grundsätzliche Charakteristika der Migrationssysteme einzelner Staaten) als von ,Mikro'-Politiken (detailliertere Regelungen, Verfahren und Mechanismen zur Umsetzung der Makro-Politiken), da die Makropolitiken stabiler seien. Zur Erklärung der Konvergenzen führen die Autoren „,parallel path development“ (parallele Pfadentwicklung), ,policy emulation“ (Politik-Nachahmung), „regional integration“ (regionale Integration), globale Ereignisse und Geopolitik sowie die öffentliche Meinung an (Cornelius/Tsuda 2004, S. 17ff.).

Ein weiterer Trend der Migrationspolitik, der derzeit in verschiedenen Ländern anzutreffen ist, ist ein Perspektiven- bzw. Paradigmenwechsel vom Selbstverständnis der Staaten als Nicht-Einwanderungsländer bzw. als Einwanderungs-

\footnotetext{
17 ,[T]he 'convergence bypothesis' claims that there is growing similarity among labor-importing countries in terms of: (1) the policies that their governments have adopted to control immigration; (2) policies designed to integrate immigrants into host societies by providing them with social services as well as political, economic, and social rights; and (3) attitudes towards immigrants and immigration policy preferences among general publics." (Cornelius/Tsuda 2004, S. 4)
} 
,Flucht und Asyl` im Kontext von Migration und Migrationspolitik

länder wider Willen hin zum Bekenntnis ein Einwanderungsland zu sein oder sich zumindest für bestimmte MigrantInnengruppen zu öffnen - und zwar sowohl für (hoch-)qualifizierte als auch zum Teil für unqualifizierte, für saisonale, temporäre und dauerhafte Einwanderung.

„Charakteristisch für den Paradigmenwechsel ist jedoch, dass nicht alle Migrantengruppen gleichermaßen in den OECD-Ländern willkommen sind-Armutsflüchtlinge, AsylbewerberInnen oder Familienangehörige, die nicht zur ,Kernfamilie‘ gehören, sollen nur eingeschränkt oder gar nicht die Möglichkeit erhalten, dauerhaft einzuwandern." (Stobbe 2004, S. 10)

Begründet wird dieser Perspektivwechsel überwiegend mit dem im Standortwettbewerb notwendigen ,Kampf um die besten Köpfe' und der demografischen Entwicklung in den Einwanderungsländern. Auch hinsichtlich dieser selektiven Öffnung unter Nützlichkeitsaspekten ist also eine Konvergenz zu beobachten, die sich in der Definition von Kategorien legaler Einwanderung niederschlägt.

\subsubsection{Scheitern der Migrationskontrollpolitik?}

Aus den beiden vorgestellten Hypothesen werden unterschiedliche Schlüsse gezogen. Auf der einen Seite wird davon ausgegangen, dass ,the nation state matters'. Danach sind die Nationalstaaten (weiterhin) in der Lage, ihre Souveränität gegen externe wie interne Einflüsse zu behaupten. Dabei wird zum Beispiel die Konvergenz-Hypothese als nicht zutreffend zurückgewiesen: So versucht Rogers Brubaker (1992) aufzuzeigen, dass es trotz Konvergenzanforderungen durch supranationale Einflüsse weiterhin divergierende migrationspolitische Zielsetzungen und Maßnahmen in den einzelnen Staaten gebe. Als Beispiel führt er Unterschiede in den Staatsbürgerschaftskonzepten an. Einmal etablierte Immigrations- und Staatsbürgerschaftspolitiken trotzen laut Brubaker relativ beharrlich möglichen Veränderungsversuchen. Dem entgegen stehen Ansätze, die eine zunehmende Konvergenz der Politiken konstatieren und diese als strategische Entscheidung zur Bewältigung von Herausforderungen sehen, mit denen die meisten reichen Industriestaaten derzeit in ähnlicher Weise konfrontiert sind. Sofern Kontrollmöglichkeiten eingeschränkt werden, wird dies vor allem auf endogene Faktoren zurückgeführt und als Ergebnis der Aushandlung widerstreitender politischer Ziele innerhalb der Staaten gesehen. Der policy-gap wird nicht als ein neues, sondern „ein der Migrationspolitik immanentes Phänomen“ (Stobbe 2004, S. 35) gedeutet, das sich daran zeige, dass „Nationalstaaten auch in der Vergangenheit nicht in der Lage gewesen sind, Migration lückenlos zu kontrollieren“ (ebd.). So hätten MigrantInnen zwar neue Strategien entwickelt und durch technischen Fortschritt entstünden neue Möglichkeiten vor allem in den Bereichen Transport und Kommunikation. Gleichzeitig seien jedoch auch die Kontrolltechniken modernisiert und verfeinert worden, mit denen die Nationalstaaten ihre migrationspolitischen Ziele heute womöglich eher noch effizienter umsetzen könnten als zuvor (vgl. ebd.). Zudem 
verkenne die Rede vom gap zwischen Policy-Zielen und -Ergebnissen, „that the goals themselves are often ambiguous as well as grossly unrealistic" (Zolberg 1999, S. 80). Zolberg (vgl. ebd.) zeigt am Beispiel der USA auf, dass in der Vergangenheit mithilfe migrationspolitischer Strategien immer wieder auf Immigrationsbewegungen Einfluss genommen werden konnte. Er macht zudem deutlich, dass

,[i]n the present climate, assessments of the capacity of states to control immigration also have important public policy implications, because widespread perceptions of a catastrophic 'loss of control' may precipitate and legitimize the enactment of draconian measures that conflict with other societal objectives and desirable values. "

(ebd., S. 74)

Auf der anderen Seite stehen AutorInnen, die diese Entwicklungen stärker als Anzeichen des wachsenden Kontrollverlusts der Nationalstaaten über die Migration interpretieren. Sie sehen die abnehmende Steuerungsfähigkeit der Staaten im zunehmenden Einfluss exogener Faktoren begründet, vor allem durch transnational gültige menschenrechtliche Normen (vgl. Soysal 1994), Prozesse der ökonomischen Globalisierung (vgl. Sassen 1996) und die Dynamik der Migrationsprozesse selbst (Migrationsnetzwerke und transnationale soziale Räume) (vgl. Pries 2001).

Martina Benz und Helen Schwenken (2005, S. 365f.) kritisieren die Staatszentriertheit solcher Analysen sowie ,die Trennung von Staat und Ökonomie und damit verbunden ein statisches Staatsverständnis“ bei gleichzeitiger „Nichtberücksichtigung des Wandels von Staatlichkeit“ (ebd.). Ferner fokussiere die Rede vom Scheitern meist lediglich auf Schwierigkeiten, das Überschreiten von territorialen Grenzen zu kontrollieren. Die verschiedenen anderen migrationspolitischen Bereiche, die „nichtsdestotrotz ihre Wirkung zeigen“ (ebd.), würden ausgeblendet. Es dürfe jedoch nicht außer acht gelassen werden, dass migrationspolitische Maßnahmen und Instrumente auch den Aufenthalt innerbalb des Territoriums, also die Situation nach dem Grenzübertritt, regulieren und damit einen „ständig prekären politischen und sozialen Raum“ für MigrantInnen schaffen (ebd.).

\subsection{4 „Autonomie“ oder „Eigensinnigkeit“ der Migration}

Einen Gegenentwurf zur Staatszentriertheit und zum Blick auf MigrantInnen als Objekte der Migrationspolitik in der ,herkömmlichen' Migrationspolitikforschung bietet die Diskussion um die ,Autonomie der Migration‘. Mit dieser Perspektive sollen die ,individuellen oder kollektiven Praktiken der Migration“ (Karakayalı/Tsianos 2005, S. 35) als solche (an)erkannt und ins Zentrum der Analyse gestellt werden. Die ProtagonistInnen dieses Diskussionsstranges bringen mit ihrem Blickwinkel mehrere Neuerungen in die Debatte ein. Mit dem Fokus auf MigrantInnen als zentrale AkteurInnen wird in erster Linie deren relative Autonomie gegenüber den Kontrollpolitiken betont. Dies zeige sich auch hinsichtlich des migrationspolitischen Instruments der Kategorisierung: $\mathrm{Da}$ die Motive der 
MigrantInnen in den vorgegebenen Kategorien keine Entsprechung finden, wandeln sie mitunter ihre ,Geschichten' in der erforderlichen Weise um und beschaffen die nötigen Beweise und Papiere (vgl. Hess/Tsianos 2004, S. 12). Sie überschreiten territoriale Grenzen und leben in den Ländern des Nordens, obwohl sie nicht erwünscht sind. Dies zeigt sich aktuell in der wachsenden Zahl irregulärer MigrantInnen. Die Migrationspolitik sei nicht in der Lage, Migrationsbewegungen nach Belieben zuzulassen oder zu stoppen, vielmehr versuche sie ,hinterherhinkend“, auf die Aktivitäten der MigrantInnen zu reagieren (vgl. ebd.). Die irreguläre Migration sei auch ein Hinweis darauf, dass die Metapher von der, Festung Europa' nicht zutreffe. Stattdessen seien territoriale Grenzen bzw. Grenzräume durchlässig, statt Abschottung sei Entrechtung bis hin zur Illegalisierung die zentrale Funktionsweise des Grenzregimes (vgl. ebd.). Mit seiner Hilfe würde migrantische Arbeit von den Orten ihrer Reproduktion, von Ressourcen und Rechten getrennt (vgl. Karakayalı/Tsianos 2005, S. 49). Für die Analyse der Migrationen und der Politiken schlagen die AnhängerInnen der Autonomie-These - wie bereits oben erwähnt - die Konzeptualisierung der AkteurInnenvielfalt und des Verhältnisses der AkteurInnen zueinander mittels des Regimebegriffs vor. Auf diese Weise würden MigrantInnen nicht länger als Objekte der Handlungen staatlicher Kontrollagenturen, sondern als eigenständige AkteurInnen begriffen, denen die staatlichen Kontrollorgane zwar überlegen seien, das Ergebnis der kontrollpolitischen Interventionen sei jedoch nicht Immobilität. Gleichzeitig verbinden die AutorInnen mit diesem Perspektivwechsel auch ein Verständnis von Migration als soziale Bewegung, indem sie „den Exodus, die Flucht, die Migration als kraftvolle Abstimmung mit den Füßen und als Selbstermächtigungsstrategie für ein besseres Leben in den Mittelpunkt der Analyse stellen" (Hess/Tsianos 2004). Ziel sei demzufolge die Entwicklung einer ,theoretische[n] Figur [...], innerhalb derer die soziale Bewegung der Migration als eine Größe im Kräfteparallelogramm auftauchen kann" (Karakayalı/Tsianos 2005, S. 50).

Ausgangspunkt dieser Beiträge ist der italienische Operaismus (vgl. Wright 2005). Mit diesem ,Arbeiterismus' (bzw. ,Arbeiterwissenschaft') grenzten sich radikale Linke in Italien in den sozialen Kämpfen der 1960er und 1970er Jahre von ihren traditionellen parteipolitischen und gewerkschaftlichen Zusammenhängen ab (vgl. Birkner/Foltin 2006). Mit ,Autonomie“ wird „die Unabhängigkeit der Arbeiterklasse sowohl von den Vorgaben der organisierten Arbeiterbewegung wie auch von den Diktaten des Kapitals" (Wright 2005, S. 13) umschrieben. Der Begriff blieb nicht auf den Bereich Arbeit und kapitalistische Produktionsweise beschränkt, sondern wurde weiterentwickelt und auf andere Bereiche - wie Migration - angewendet. Auch in Deutschland wurde dieser Ansatz aufgegriffen und schon zur Zeit der Migration der, GastarbeiterInnen' in theoretische wie politische Auseinandersetzungen eingespeist (vgl. Birkner/Foltin 2006, S. 66f.).

Die jüngste Wiederbelebung der Debatte um eine ,Autonomie der Migration“ ist jedoch nicht unwidersprochen geblieben. Innerhalb antirassistischer politischer 
Zusammenhänge in Deutschland erntete der Ansatz vor allem von Flüchtlingsselbstorganisationen Kritik. Gruppen wie die ,Karawane für die Rechte der Flüchtlinge und MigrantInnen' (vgl. www.thecaravan.org) beanstanden vor allem, dass dies ein privilegierter Zugang zu dem Thema sei, der die Besonderheiten der Situation von Flüchtlingen ausblende. So wird mit Blick auf die im AutonomieAnsatz unterstellten „effektiven Netzwerke“ der MigrantInnen und eine „Globalisierung von unten“ kritisiert, diese Sicht impliziere, „dass Flüchtlinge und MigrantInnen sanft nach Europa geleitet werden - der Horror und die Gründe der Flucht bleiben unerwähnt" (iz3w 2005, S. 20). Die Karawane macht unter dem Slogan „Wir sind hier, weil ihr unsere Länder zerstört“ auf die Rolle der Zielländer bei der Entstehung von Migration aufmerksam und lenkt den Blick dabei auf Migrationsursachen, die geprägt sind durch Kolonialismus, neokoloniale Strukturen und die Dominanz westlicher Interessen in Globalisierungsprozessen, die sich in der Behandlung der Flüchtlinge in der Bundesrepublik fortsetzen (vgl. The Caravan 2004). Sie betonen, dass die politischen Fluchtgründe nicht ausgeblendet werden können und eine andere Ausgangslage für die Flüchtlinge bedingen, als sie für die „MigrantInnen zweiter oder dritter Generation“ gelte (vgl. ebd.). So seien sie zum einen stärker durch Abschiebung bedroht und zum anderen sei eine Abschiebung für sie mitunter mit traumatisierenden oder lebensbedrohlichen Folgen verbunden (vgl. ebd.).

Nach Ansicht von ,kanak attak' unterwirft sich die ,Karawane' damit einer „homogenisierenden Identitätslogik“ (iz3w 2005, S. 19), die die Kategorisierung und Hierarchisierung verschiedener MigrantInnengruppen und einen humanistischen Diskurs reproduziere, der nur die Einwanderung und Anwesenheit bestimmter MigrantInnen als legitim anerkennt und damit in einer defensiven Praxis verhaftet sei (vgl. iz3w 2005, S. 19). Der Slogan der Karawane negiere das voraussetzungsfreie Recht auf Mobilität:

„[I]n der Perspektive der Migration als soziale Bewegung muss sich Migration nicht aus einer kolonialen Zerstörungsgeschichte ableiten, um Mobilität zu legitimieren. "(ebd.)

Eine weitere kritische Perspektive bringen Martina Benz und Helen Schwenken (2005) in die Diskussion ein. Sie beanstanden ebenfalls, dass unter dem undifferenzierten Begriff der Autonomie die verschiedenen Positionen von MigrantInnen unsichtbar würden. Sie kritisieren die Tendenz des Konzepts,

„aufgrund der Theorietradition und Begrifflichkeit [...] das komplexe Verbältnis von Staat, Migration und Gesellschaft als eine schlichte Gegenüberstellung Staat versus MigrantIn als autonomem (Kollektiv-)Subjekt qu konzeptionalisieren und gesellschaftliche Konflikte auf den Hauptwiderspruch zwischen Kapital und Arbeit zu reduzieren, ohne dass die viel weitreichenderen Rahmenbedingungen und Reproduktionskontexte problematisiert würden" (ebd., S. 370). 
Die Lesart von MigrantInnen als widerständige Subjekte komme einer Romantisierung der Migrationsprozesse gleich. Darüber hinaus stehe im Mittelpunkt des Autonomie-Ansatzes ein Verständnis von Migration, das in erster Linie Bewegungen von ErwerbsarbeiterInnen meine und die Analyse von deren Reproduktionskontexten vernachlässige (ebd., S. 373f.). Gerade Migrantinnen würden jedoch aufgrund dieser Trennung zwischen Produktion und Reproduktion prekarisiert. Ein Konzept, das erkenne, dass es auch in migrantischen Netzwerken hierarchische Machtverhältnisse und soziale Abhängigkeiten gibt, die oft entlang der Dimension Gender verlaufen, hätte auch Platz für Nicht-Autonomie und folglich auch für Solidarität (vgl. ebd., S. 374). Aufgrund dessen plädieren die Autorinnen für den Begriff der „Eigensinnigkeit von Migration“ (ebd.): „Dieses Eigensinnige ist weder von einer Autonomie noch von Determinismen bestimmt, vielmehr nicht-linear, aber auch nicht beliebig“ (ebd.). Darin sollen einige der Gesichtspunkte des Autonomie-Ansatzes, die in den Augen der Autorinnen einen wichtigen Perspektivwechsel für die Migrations(politik)forschung darstellen (Bedeutung illegalisierter Migration angesichts gegenwärtiger Produktionsverhältnisse und der Transformation von Staatlichkeit, Konzeption von MigrantInnen als AkteurInnen) aufgehoben sein. Gleichzeitig sollen jedoch ,eine normative Aufladung und drohende Vereinheitlichung ebenso wie eine Dichotomisierung Staat versus MigrantIn“ und „,androzentrische Verkürzungen“ (ebd., S. 375) vermieden und „die ermöglichenden und beschränkenden Faktoren und deren Kombination“ (ebd.) stärker einbezogen werden.

Die wichtige Kritik an dem Konzept, wie sie die ,Karawane' aus der Sicht selbstorganisierter Flüchtlinge und die beiden Autorinnen aus feministischer Perspektive insbesondere an der Romantisierung der Verhältnisse in der Migration formuliert haben, kann außerdem durch einige weitere Aspekte gestützt werden. Diese beziehen sich auf die Begriffe „Definitionsmächtigkeit“ und „Widerstand“. Aus ihrer These, dass die Migrationspolitik mit ihren Kontrollversuchen nur den MigrantInnen ,,hinterherhinkend“ agiere, ziehen Sabine Hess und Vassilis Tsianos (2004) den Schluss,
„, [a]uch die [...] mit allen zu Verfügung stehenden Mitteln zu kritisierende Exterrito- rialisierung von Lagern [...] ließe sich anders lesen: als Definitionsmächtigkeit der Mig- ration über politische Steuerungsversuche" (ebd., S. 12).

Diese These formulieren Sabine Hess und Vassilis Tsianos, obwohl sie sogleich verdeutlichen, dass diese Lager nichtsdestotrotz von Elend geprägt seien. Treffender wäre es, von der Wirkungsmächtigkeit der Handlungen der MigrantInnen zu sprechen, die sich jedoch nicht notwendig positiv auf die Migrationsbewegungen auswirken. Denn die Praxis der MigrantInnen erzeugt tatsächlich kontrollpolitische Wirkungen (Verschärfung der Zugangsregeln usw.), jedoch überwiegend nicht - wie es der Begriff Definitionsmächtigkeit suggeriert - in ihrem Sinne. Darüber hinaus bleiben die Begriffe „Widerstand“ und „soziale Bewegung“ vage 
und werden nicht weiter zu bisherigen Ansätzen in diesem Bereich ins Verhältnis gesetzt. $^{18}$

Trotz dieser Kritiken an dem Ansatz, wie ihn Karakayalı und Tsianos (2005) in ihrem Artikel beschreiben, hat die Debatte um die ,Autonomie der Migration“ einen wichtigen Perspektivwechsel in Abgrenzung zu bisherigen Ansätzen der Migrations(politik)theorie ermöglicht. Insbesondere drei Aspekte dieser Diskussion werden in die vorliegende Untersuchung einfließen: 1. die Konzeptualisierung der verschiedenen AkteurInnen und ihr Zusammenspiel als ,Migrationsregime“, 2. die Einbeziehung von MigrantInnen als zentrale AkteurInnen in diesem Regime, deren Autonomie allerdings von verschiedenen Faktoren abhängig ist (Geschlecht, Alter, Herkunftssituation etc.) und 3. die Infragestellung der Kategorisierung und Hierarchisierung von MigrantInnen.

\subsubsection{Fazit}

Die Untersuchung von Migrationspolitik ist in der Politikwissenschaft ein vergleichsweise neues Forschungsfeld. Die Ansätze, die sich bislang mit der Erklärung von Migrationspolitik befasst haben, gingen meist von einer Steuerungsperspektive aus. Das heißt sie nehmen vor allem die Aktivitäten der nationalstaatlichen Regierungen in den Blick und fragen, ob und wie es gelingt, Migration zu kontrollieren und in die gewünschten Bahnen zu lenken und bieten mitunter Vorschläge zur Entwicklung effizienterer Politiken an. Diesen Ansätzen werden zunehmend die Perspektiven der MigrantInnen entgegengesetzt und Migration bzw. die Praxis von MigrantInnen als eigenständige Kraft im migrationspolitischen Geschehen konzipiert. Die These vom ,Scheitern` der migrationspolitischen Steuerungsversuche erscheint so in einem neuen Licht: Sie ist dann nicht mehr - wie aus der Perspektive der Steuerung von Migration - eine vor allem negative Bewertung vermeintlich missglückter politischer Interventionen, sondern könnte - aus der Perspektive der Migration - im Gegenteil als positiver Beleg für den Erfolg migrantischer ,Projekte' bzw. Praxen quer zu allen entgegenstehenden Regulierungen und Maßnahmen gelesen werden. Gleichzeitig ist eine solche euphorische Sichtweise von der Realität vieler MigrantInnen weit entfernt, denn gerade aus

18 Den Versuch einer genaueren Untersuchung des migrantischen, antirassistischen Widerstands unternimmt Manuela Bojadžijev (2002). Dabei handelt es sich ihrer Ansicht nach meist um „Kämpfe gegen Autoritäten, die direkt auf die Individuen einwirken, und die im strukturellen und individuellen Sinn als unmittelbare Kämpfe gegen Machtinstanzen - zum Beispiel Vermieter, Behörden, Arbeitgeber - interpretiert werden können“ (ebd., S. 145). Es gehe meist um die kurz- und mittelfristige Verbesserung der Lebensbedingungen, häufig seien darin aber auch revolutionäre Ansätze enthalten. Auch „unorganisierte[...], individuelle[...] Handlungen“ (ebd.) im Alltag, die sich vor allem durch die alltäglichen Zumutungen aufgrund der rechtlichen Situation von MigrantInnen häufig ereignen, werden von Bojadžijevs Widerstandsbegriff erfasst. Um jedoch eine Verwässerung des Begriffs durch eine zu umfassende Ausweitung zu vermeiden, müssten die „Unterschiede zwischen Renitenz, alltagsbasiertem Widerstand und organisierter politischer Bewegung“ sowie verschiedenartige Lebensbedingungen und Interessen berücksichtigt werden (vgl. ebd.). 
,Flucht und Asyl` im Kontext von Migration und Migrationspolitik

ihrer Perspektive ist die These vom ,Scheitern' kaum aufrechtzuerhalten: Dies würde die Wirkungsmächtigkeit, die die Steuerungsversuche für viele MigrantInnen durchaus entfalten, ignorieren.

Die migrationspolitischen Steuerungsversuche erweisen sich also einerseits für MigrantInnen als wirkungsmächtig und verfehlen andererseits angesichts der widerständigen Strategien von MigrantInnen mitunter ihre Ziele. Beispielhaft für diese zwei Seiten migrationspolitischer Interventionen wird im Folgenden ein grundlegendes migrationspolitisches Instrument - die Konstruktion von verschiedenen, hierarchisierten Kategorien ,erwünschter ${ }^{6}$ und ,unerwünschter ${ }^{6}$ MigrantInnen - auf ihre Bedeutung im Migrationsregime hin untersucht.

\subsection{Kategorienkonstruktion als migrationspolitisches Fundament: das Beispiel Flucht und Asyl}

Ein bedeutendes migrationspolitisches Instrument, das darauf abzielt, Migrationen zu steuern, ist die Kategorisierung und Hierarchisierung von MigrantInnen. Die vorliegende Untersuchung greift mit ihrem Fokus auf ,Flucht und Asyl' beispielhaft einen Bereich der politisch vorgegebenen Kategorienkonstruktion auf, ohne diese jedoch einfach zu reproduzieren. Stattdessen ist es ein zentraler Teil der Analyse, anhand der Kategorie Flucht und Asyl den aktuellen Mustern und Trends der Migrationspolitik nachzugehen und die Bedeutung der Konstruktion dieser Kategorie im Migrationsregime sowie ihren Einfluss auf die Realität von MigrantInnen herauszuarbeiten. Dadurch kann - anknüpfend an die Kritik an den oben umrissenen vorherrschenden theoretischen Ansätzen - ein Beitrag dazu geleistet werden, Migrationspolitik nicht ausschließlich aus der (national)staatlichen Perspektive zu untersuchen, sondern die gängigen migrationspolitischen Raster, die oft auch Untersuchungen über Migrationspolitik unhinterfragt zugrunde gelegt werden, selbst zum Gegenstand der Analyse zu machen. Die folgende Auseinandersetzung mit der Kategorien- und Typologienbildung in der Migrationspolitik und in Teilen der Migrationsforschung am Beispiel Flucht und Asyl soll dafür die Grundlage bilden, bevor schließlich die Konstruktion von MigrantInnenkategorien und deren Wirkmächtigkeit am Beispiel Flucht und Asyl in den Länderstudien illustriert wird.

Im Folgenden werden zunächst einige einleitende Bemerkungen zur Bedeutung der Kategorienkonstruktion in der Migrationspolitik formuliert. Vor diesem Hintergrund wird an einigen Beispielen gezeigt, dass die politisch vorgegebenen Kategorien in der (politikwissenschaftlichen) Migrationsforschung mitunter unterhinterfragt übernommen werden. Daran anschließend wird - mithilfe der von Pries entwickelten „Dimensionen des Einwanderungsprozesses“ - dargestellt, wie die GesetzgeberInnen in den Einwanderungsländern durch die Verknüpfung mehrerer dieser Dimensionen Kategorien entwickeln, die die Bedingungen für 
eine legale Einreise und die Inanspruchnahme von Rechten für ,erwünschte‘ Einwanderer definieren.

Die Bildung von Kategorien und Typologien ist ein zentraler Bestandteil sowohl der Migrationsforschung als auch der Migrationspolitik: „A typologisation of migration is not only an analytical endeavor but above all a political and legal activity“ (Abiri 2000, S. 21). Dabei fällt auf, dass sich politikwissenschaftliche Untersuchungen häufig an den Kategorien (klassischerweise ,Gastarbeit‘, Flucht und ,illegale Einwanderung) orientieren, die von Ausländer- und Einwanderungsgesetzen vorgegeben werden. Hingegen weisen die in Einwanderungsbestimmungen $\mathrm{zu}$ findenden Kategorien nur wenige Übereinstimmungen mit den Migrationsursachen auf, wie sie vor allem MigrationsforscherInnen aus der Soziologie herausgearbeitet haben. Die durch den Gesetzgeber konstruierten Kategorien bilden daher die komplexen Wirkungszusammenhänge in der Migration nicht adäquat ab. Sie sind jedoch die Grundlage für die Erstellung von hierarchisch aufgebauten Ordnungsschemata, anhand derer sowohl reguläre Möglichkeiten des Zugangs zum nationalstaatlichen Territorium als auch Aufenthalts- und damit Rechtsstatus innerhalb des Territoriums definiert werden. Das Missverhältnis zwischen Migrationsursachen und Kategoriensystem sowie den damit verbundenen Einwanderungsmöglichkeiten produziert permanent Ausschlüsse und ein in den vergangenen Jahren an Bedeutung gewinnendes Phänomen: die irreguläre Einwanderung. Das erklärte Ziel, Migration durch Kategorisierung zu ,steuern“, indem „bestimmte Gruppen ermutigt, andere hingegen abgeschreckt werden sollen“ (Thränhardt 1997, S. 141), wird jedoch kaum erreicht. Denn MigrantInnen gehen unterschiedlich mit dem eingeschränkten ,Angebot ${ }^{`}$ von Kategorien, die reguläre Einwanderungsmöglichkeiten eröffnen, um: Sie schreiben ihre Geschichten mitunter entsprechend den Anforderungen immer wieder neu, um ihre Migrationsprojekte umzusetzen, gelangen außerhalb des Kategorienschemas - irregulär in die Zielländer oder nutzen reguläre Einreisemöglichkeiten (zum Beispiel Touristenvisa ${ }^{19}$ ) und kehren nach Ablauf der vorgegebenen Fristen nicht in die Herkunftsländer zurück oder organisieren Pendelmigrationen. Die politische Konstruktion von Kategorien führt aufgrund des Wechselspiels zwischen Migration und ,Kategorienangebot' zur Illegalisierung von Migration. Indem bestimmte, legale Einwanderungsoptionen geschaffen werden, die mit bestimmten Anforderungen verbunden sind, werden gleichzeitig all diejenigen ausgeschlossen, die diesen Anforderungen nicht entsprechen. Diese Wirkungsmächtigkeit der Kategorienkonstruktion lässt sich aktuell zum Beispiel in den Transitstaaten nachvollziehen, in denen auf Betreiben der EU und anderer AkteurInnen (zum Beispiel In-

\footnotetext{
19 Tourismus entspricht zwar nicht den gängigen Definitionen von Migration (vor allem aufgrund der nur kurzen Dauer des Ortswechsels), und Touristenvisa sind offiziell auch keine Einwanderungsmöglichkeit. Dennoch werden sie von vielen MigrantInnen als solche genutzt: Sie reisen mit Touristenvisa ein und bleiben nach Ablauf des Visums als so genannte „Visa-Overstayers“ irregulär im Land.
} 
,Flucht und Asyl` im Kontext von Migration und Migrationspolitik

ternationale Organisationen) neue Migrationsregime etabliert werden. Indem sie Kategorien und Verfahren für reguläre Einwanderung, also beispielsweise Asylverfahren, installieren, schaffen sie erst die irreguläre Migration.

„Mit der Einrichtung eines derartigen Verfahrens wird [...] ,irreguläre Migration“ zu einem real existierenden Objekt, ihre Evidenz verdichtet sich gleichsam in einer juridischen Figur. " (Andrijasevic et al. 2005, S. 353)

Aufgrund der weit reichenden Deutungsmächtigkeit politischer AkteurInnen in Bezug auf die Formen von Migration sollen die politischen Kategorien in der vorliegenden Studie nicht einfach übernommen werden. Sie werden stattdessen als Teil der Migrationspolitik begriffen und als solcher analysiert. Dies beinhaltet, die Kategorien zu hinterfragen, mit tatsächlichen Erscheinungsformen zu kontrastieren und die Wirkmächtigkeit der Kategorisierung sowie ihre Bedeutung im Migrationsregime zu untersuchen. Denn eine Forschung, „die sich nur an den politischen Zuordnungen orientiert, enthält der Analyse von Migrationspolitik wichtige Bewertungskriterien vor“ (Birsl 2005, S. 21). Gleichzeitig strukturieren die Kategorien die migrationspolitische Landschaft und gewähren so einen „Einblick in Muster, Mechanismen und Entwicklungen der Migrationspolitik sowie in politische Deutungen des Migrationsgeschehens“ (ebd.). Schließlich können anhand dieser Kategorisierungen - wie hier am Beispiel Asyl - Ähnlichkeiten, Unterschiede und Konvergenzen in der Migrationspolitik im Ländervergleich herausgearbeitet werden (vgl. ebd.).

\subsubsection{MigrantInnenkategorien in der Migrationsforschung}

(Politik)wissenschaftliche Texte bleiben mit ihren Kategorien mitunter sehr nah an den politisch konstruierten - dies wird im Folgenden an einigen Beispielen illustriert. So umfasse das Spektrum der Einwanderung in und nach Europa nach Heinz Fassmann und Rainer Münz (1996, S. 18ff.) koloniale und postkoloniale Wanderer ${ }^{20}$, ,ethnische ${ }^{6}$ Einwanderung ${ }^{21}$, ArbeitsmigrantInnen und deren Angehörige sowie Flüchtlinge. Unter ,Sonstige‘ fassen die Autoren die Wanderung von Eliten, RentnerInnen und RemigrantInnen zusammen. In der Aufzählung der von Fassmann und Münz identifizierten Kategorien zeigt sich eine Verknüpfung verschiedener Aspekte. Sie stellen eine Mischung aus Motiven, historischen Bezügen und Formen der Migration sowie ,persönlichem Profil' der MigrantInnen dar, die zum Teil ineinander greifen und kaum voneinander trennbar sind (vgl. Birsl 2005, S. 45). Im Grunde beschreiben die Autoren also das Migrationsgeschehen mit den politisch konstruierten Kategorien - die zwar an das tatsächliche Migrationsge-

\footnotetext{
20 Zuwanderer gleicher und anderer Nationalität aus den ehemaligen Kolonien (vgl. Fassmann/ Münz 1996, S. 18ff.).

21 Zuwanderer gleicher, ethnischer' Zugehörigkeit (vgl. ebd.).
} 
schehen anknüpfen, dieses jedoch analytisch keineswegs erfassen können (und wollen), wie weiter unten erläutert wird.

Ähnliches gilt für Barwig und Schumacher (2002). Sie führen in ihrem Beitrag über Migration/Migrationspolitik im „Kleinen Lexikon der Politik“ (Nohlen 2002) Arbeitsmigration, AussiedlerInnen, (post)koloniale Migration und ,illegale Einwanderung an. Damit beschränken sie sich noch mehr als Fassmann und Münz auf die als politisch bzw. rechtlich als relevant eingestuften Gruppen. Sie fügen der Aufzählung der anderen beiden Autoren noch eine weitere Kategorie hinzu, die in den letzten Jahren zunehmend an Bedeutung gewann - die so genannte, illegale‘ Immigration, worunter alle MigrantInnen subsumiert werden, die sich irregulär, also ohne gültige Aufenthaltsdokumente in der EU aufhalten.

Auch die von Petrus Han (2000, S. 63) genannte Einteilung der „,seit 1945 eintretenden unterschiedlichen internationalen Migrationsformen“ unterscheidet sich nur in Nuancen von den vorangegangenen und den politischen Kategorisierungen: „Arbeitsmigration, Familienzusammenführung, Flüchtlinge, Migration von Studenten, Migration ethnischer Minderheiten, illegale Migranten“ (vgl. ebd.).

Eine ähnliche „Systematik der Einwanderung“ bietet Dietrich Thränhardt (1997, S. 141ff.) an. Er nennt ,ethnisch`Zugehörige, postkoloniale MigrantInnen, angeworbene Arbeitskräfte, EU-BürgerInnen, Flüchtlinge und undokumentierte Einwanderer (vgl. ebd.). Allerdings verweist er im Vorfeld auf die Bedeutung der Kategorisierungen als Versuch Migrationen auf diese Weise zu steuern. Diese Steuerungsversuche „können in einigen Fällen aber kontraproduktive oder unvorhergesehene Wirkungen haben“ (Thränhardt 1997, S. 141). Die Kategorie ,illegale Migration“ ist letztlich ein ,Sammelbecken' für all jene, die die Anforderungen für die regulären Kategorien nicht erfüllen, deren Migrationsgründe bzw. ,persönliches Profil‘ also nicht durch die übrigen genannten Kategorien (und mithin die regulären Einwanderungsoptionen) abgedeckt sind.

Diese Übernahme der politisch konstruierten Kategorien deutet auf eine unkritische Sicht auf die Bedeutung und die Rolle des (National-)Staates hin, mitunter sogar auf die (nicht transparent gemachte) Übernahme der nationalstaatlichen Perspektive. Indem der Nationalstaat als quasi, natürlicher' Rahmen vorausgesetzt wird, wird auch die Organisation von Zugehörigkeit über den Nationalstaat als selbstverständlich unterstellt. Grenzüberschreitende Wanderung wird dann zur Ausnahmeerscheinung und Abweichung von einer eigentlich durch Immobilität und klare nationale Zugehörigkeiten gekennzeichneten Ordnung. Der Fokus auf den Staat und der damit einhergehende Fokus auf grenzüberschreitende Migration „accepts the perception of human beings as belonging to different states and their movement across borders as an anomaly" (Abiri 2000, S. 22).

Darüber hinaus finden sich jedoch Ansätze aus verschiedenen Disziplinen, unabhängiger von den politisch vorgegebenen Kategorien Migration in all ihren Ausprägungen, ihre Voraussetzungen, Ursachen, Formen, ihren Verlauf und ihre Folgen zu beschreiben und zu erklären - gleichwohl geschieht auch dies nicht 
herausgelöst aus politischen Diskursen und „Wirklichkeitsdefinitionen“ (Birsl 2005, S. 18). Die verschiedenen theoretischen Ansätze zur Erfassung und Erklärung von Migration sind inzwischen in einigen Überblickswerken dargestellt worden (vgl. Düvell 2006; Haug 2000; Pries 2001; Treibel 2003). Es wird immer wieder betont, dass es keine einheitliche, allumfassende ,Migrationstheorie ${ }^{6}$ als solche gebe. Stattdessen befassen sich verschiedene Disziplinen aus ihrer jeweiligen Perspektive 22 mit Ausschnitten des Themas und haben entsprechend eine Vielzahl an Ansätzen und Deutungsangeboten hervorgebracht, die einander ergänzen, sich aber mitunter auch widersprechen. Im Allgemeinen werden klassische und neuere Ansätze unterschieden. Die klassischen Theorien internationaler Migration befassen sich vor allem mit der Frage,

„warum welche Bevölkerungsgruppen in welcher Form grenzüberschreitend wandern; welche sozialen, kulturellen, ökonomischen und politischen Wirkungen dies auf die Herkunfts- und Ankunftsgesellschaften hat; und wie sich die Migranten in die Ankunftsgesellschaften integrieren" (Pries 2001, S. 12).

Dabei definieren die klassischen Ansätze Migrationen vor allem als ein- oder zweimalige Ortsveränderungen und untersuchen Begleitumstände und Fragen der Integration in den Ankunftsregionen (vgl. Pries 2001, S. 12). Demgegenüber verstehen neuere Ansätze Migration auch als dauerhaften Zustand, ,als eine neue soziale Lebenswirklichkeit für eine wachsende Anzahl von Menschen“ (ebd., S. 32). Sie gehen von der ausschließlichen Betrachtung der Mikro- oder Makroebene weg und fokussieren ,eine Meso-Analyseebene und [...] Bewegungen und Sozialräume zwischen bzw. oberhalb der Herkunfts- und Ankunftsregion“ (ebd., Hervorh. i. Orig.). Sie stellen damit sowohl Weiterentwicklungen früherer Ansätze als auch Interpretationen neuer Phänomene zum Beispiel vor dem Hintergrund von Globalisierungsprozessen dar. Dabei geht es vor allem darum aufzuzeigen, auf welche Weise die Migrationsbewegungen aufrecht erhalten werden und „eine neue und eigene Qualität“ aufweisen und inwieweit im Rahmen neuer internationaler Migrationsprozesse neue „transnationale soziale Wirklichkeiten“ entstehen (ebd., S. 32). Die zentralen Ansätze befassen sich mit ,Migrationsnetzwerken' und ,-kreisläufen', ,Cumulative Causation', ,Transnationalismus' und ,Transmigration‘.

Typologien und Kategorien spielen in fast allen Theorien eine Rolle, bleiben häufig jedoch implizit. Nach Pries ist Typenbildung in den Sozialwissenschaften nicht nur ein methodisches Hilfsmittel, sondern ein „Verfahren der Theoriebildung“ und eine Möglichkeit der „Komplexitätsreduktion“, wenn einfache Kausalzusammenhänge nicht identifiziert werden können und „Hypothesentests im Sinne des Kritischen Rationalismus nicht geboten erscheinen“ (Pries 2001, S. 38). Annette Treibel (2003, S. 20) zufolge beziehen sich die meisten Typologien auf die Aspekte Raum, Zeit, Ursache und Umfang der Migration. Die Liste der „Dimen-

22 Vgl. den Überblick bei Treibel 2003, S. 17 f. 
sionen des Wanderungsprozesses“, die sich laut Pries (2001, S. 37) in den verschiedenen Typologien finden, ist noch umfassender, wenn auch sicherlich nicht abgeschlossen:

- „Raumdistanzen“ „(interne/externe Migration, Nah- und Fernmigration)“

- „Zeitaspekte (Tages-, Wochen-, saisonale, langfristig-zyklische, einmalig-dauerhafte Migration)“"

- „Charakteristika von Herkunfts- und Ankunftsregion (Land-Stadt- oder DritteWelt-Erste Welt-Migration)“"

- „Häufigkeit von Ortsveränderungen (einmalige Emigration/Immigration, Rückkehrmigration, Pendelmigration)“

- „Gründe und Umstände“ „(freiwillig, unfreiwillig; Arbeits- oder Fluchtmigration; ökonomisch, politisch, religiös-kulturell bedingt)“"

- „Art des Zutritts zur Ankunftsregion (angeworben/ eingeladen, geduldet, unerwünscht; mit legalen Dokumenten, mit gefälschten Dokumenten, eingeschleust, gültige Dokumente; friedlich, unorganisiert; Invasion, Eroberung)“"

- „Form des zurückgelegten Weges und die Art des Transportmittels“ (direkte, indirekte, sequenzielle; zu Fuß, motorisiert, per Flugzeug)““

- „Charakteristika der Wanderungsform (individuelle, Gruppen-, Massenwanderung; organisiert, nicht organisiert; markt-, familien-, netzwerkvermittelt)“"

- „personenbezogene Merkmale der Migranten (unqualifiziert, hochqualifiziert, Experten; ethnische Zuschreibung)“

- „mit der Wanderung verbundene[...] grundlegende[...] Ziele[...] (Rettung des eigenen Lebens, Verhinderung einer Verschlechterung, Verbesserung der Lebensumstände)“ (ebd.).

MigrationsforscherInnen greifen sich aus diesem Katalog einzelne Kriterien heraus und konstruieren daraus Typologien - mitunter ohne dies offen zu legen. Manche beziehen sich, wie bereits erwähnt, gleich auf das Kategorienangebot, das politische EntscheidungsträgerInnen hervorgebracht haben. In diesem Fall können - wie die oben genannten Beispiele von Fassmann und Münz sowie Han zeigen, ganz unterschiedliche Dimensionen im Vordergrund stehen: Während der Begriff der ,Arbeitsmigration“ die Migrationsgründe in den Mittelpunkt stellt, fokussiert der Begriff der, illegalen Migration` auf die ,Art des Zutritts zur Ankunftsregion' und blendet die Vielfalt der Gründe dieser,Gruppe' aus, in der sich auch eine Vielzahl an ,ArbeitsmigrantInnen` befindet.

Pries (2001) hingegen verknüpft für seine Analyse die Dimensionen „Verhältnis zur Herkunftsregion“, „Verhältnis zur Ankunftsregion“, „Hauptmotiv für Wanderung“ und „Zeithorizont der Wanderung“ zu vier Idealtypen internationaler MigrantInnen: EmigrantInnen, RückkehrmigrantInnen, Diaspora-MigrantInnen und TransmigrantInnen (vgl. Pries 2001, S. 39). Andrijasevic et al. (2005, S. 347) plädieren dafür, nicht 
,Flucht und Asyl` im Kontext von Migration und Migrationspolitik

„an Kategorien anzusetzen, die von den Ausländer- und Migrationsgesetzen vorgegeben sind, sondern umgekehrt, Strategien in den Blick zu nehmen, die MigrantInnen im Umgang mit migrationspolitischen Kategorisierungen entwickelt haben".

\subsubsection{Politische Konstruktion von MigrantInnenkategorien}

Auf fast alle der von Pries aufgeführten Dimensionen beziehen sich auch die Kategorienkonstruktionen der GesetzgeberInnen. Sie verknüpfen verschiedene Dimensionen und entwickeln daraus Kategorien, die die Voraussetzungen für eine legale Einreise und die Inanspruchnahme von Rechten definieren. Dies sind, wie bereits angesprochen, die von Fassmann und Münz (1996) genannten Kategorien, die sie verkürzt mit dem tatsächlichen Migrationsgeschehen gleichsetzen: koloniale und postkoloniale Wanderer, ,ethnische` Einwanderung, ArbeitsmigrantInnen und deren Angehörige sowie Flüchtlinge. Diese korrespondieren mit bestimmten Interessen der ,Aufnahmeländer ${ }^{6}$ und spiegeln damit gleichzeitig den Mix aus verschiedenen Politikfeldern wider, die das Politikfeld Migrationspolitik beeinflussen. ${ }^{23}$ Dieses Zusammenspiel aus Interessen, Politikfeldern, Migrationsdimensionen, Kategorienkonstruktion und Einwanderungsmöglichkeiten wird im Folgenden in einem kurzen Überblick skizziert und soll schließlich auf den zentralen Dualismus zwischen ,erwünschter und ,unerwünschter sowie regulärer und irregulärer Immigration zugespitzt werden.

Bei der Definition von Einwanderungsmöglichkeiten durch die GesetzgeberInnen in den Einwanderungsländern spielen vor allem arbeitsmarkt- und wirtschaftspolitische Erwägungen eine Rolle, was sich an der Fokussierung auf ,erwünschte 'personenbezogene Merkmale (siehe die Dimensionen bei Pries) zeigt. Dies sind insbesondere bestimmte Qualifikationen, die auf dem jeweiligen Arbeitsmarkt gerade nachgefragt werden, aber auch andere soziale Merkmale, etwa wenn der Nachweis über ein bestimmtes Vermögen zur Einwanderungsvoraussetzung gemacht wird. Diese Argumentation entlang von Nützlichkeitskriterien korrespondiert mit der Tatsache, dass

„Einwanderung [...] fast ausschließlich unter dem Gesichtspunkt ibres betriebs- und volkswirtschaftlichen Nutzens für das als ,Wirtschaftsstandort" begriffene Aufnahmeland bewertet" wird (Butterwegge 2003, S. 67; Butterwegge 2006, S. 203f.). ${ }^{24}$

Hinsichtlich der Arbeitsmigration hat daher in den vergangenen Jahren ein Perspektivwechsel stattgefunden, weg vom Credo der ,Null-Immigration', wie es seit dem offiziellen Anwerbestopp in den meisten westeuropäischen Ländern Anfang der 1970er Jahre galt (tatsächlich aber nie der Realität entsprach), hin zur Öffnung

${ }^{23}$ Zum Zusammenspiel verschiedener Politikfelder in der Migrationspolitik vgl. Birsl (2005, S. 72ff.).

24 Thränhardt (2003, S. 16) führt hierzu aus: „Die Aggressivität gegenüber Einwanderern aus der armen Welt und insbesondere aus islamischen Ländern in vielen Ländern Europas ist die aggressive Unterseite des neuen Diskurses über die Einwanderung, der stark auf die ökonomischen Belastungen bzw. Vorteile der Einwanderung abstellt.“ 
für Personen mit bestimmten Qualifikationen. Dieser Perspektivenwechsel und seine Verknüpfung mit dem Nützlichkeitsdenken finden ihren Ausdruck in der Anwerbung gut ausgebildeter Fach- und Führungskräfte, die öffentlich als ,Kampf um die besten Köpfe“ ausgerufen wurde. Gleichzeitig gelten „Armutsmigration“ und Flucht als „Standortnachteil“" (ebd.). Das von der Unabhängigen Kommission Zuwanderung vorgeschlagene Punktesystem zur ,arbeitsmarktorientierte[n] Zuwanderung“ (Unabhängige Kommission 2001, S. 83) hat die „personenbezogenen Merkmale“ (Pries 2001, S. 37) zentral in den Mittelpunkt des Auswahlprozesses gestellt. Die personenbezogenen Merkmale werden auch bei der aktuell viel diskutierten Forderung nach ,Integrationsfähigkeit' angesprochen - etwa wenn die Anerkennung der nationalen Verfassungen und Sprachkenntnisse zur Einreisevoraussetzung gemacht werden. ${ }^{25}$ Bezüglich der Raumdistanzen als auch hinsichtlich der Herkunftsregion werden generell Personen aus als ,ähnlich` definierten ,Kulturkreisen' bzw. der ,ersten Welt' bevorzugt, was sich besonders deutlich anhand der Freizügigkeit innerhalb der EU im Vergleich zu den strengen Regelungen für die Einreise aus ,Drittstaaten' manifestiert. Wenn eine Einreisemöglichkeit eröffnet wird, wird häufig versucht, auf die Zeitdimension Einfluss zu nehmen, indem der erlaubte Aufenthalt begrenzt wird, wie dies zum Beispiel bei der Anwerbung von ,GastarbeiterInnen' und aktuell zum Beispiel bei der deutschen GreenCard-Regelung der Fall war. Bei solchen Anwerbeprogrammen werden gleichzeitig die Wanderungsform und der Umfang der Migration beeinflusst. Bei der Eröffnung von Einwanderungsmöglichkeiten für Personen mit ,erwünschten` personenbezogenen Merkmalen wird also versucht, an individuell vorliegende Migrationsgründe anzuknüpfen oder diese erst zu schaffen (wie zum Beispiel im Rahmen von Anwerbepolitiken). Es zeigt sich demzufolge, dass Arbeitsmigration mit Einwanderungsmöglichkeiten verbunden ist, allerdings nur unter sehr eng gefassten Voraussetzungen und Bedingungen. Reguläre Arbeitsimmigration geht häufig dem Familiennachzug voraus. Dessen Ausgestaltung lässt weniger Spielraum für die politische Formulierung von Bedingungen, da er ein völkerrechtlich verbrieftes Recht darstellt.

Die so genannte (post-)koloniale Einwanderung - wie sie beispielweise in Frankreich und Großbritannien vorzufinden ist -, die ebenfalls als Arbeitsmigration und Familienmigration auftreten kann, berührt vor allem außen- und bündnispolitische Erwägungen. Hingegen kann die Konstruktion von Einwanderungsmöglichkeiten aufgrund, ethnischer' Zuschreibungen - wie zum Beispiel in Deutschland und Polen - zwar keinem Politikfeld explizit zugeordnet werden, jedoch spiegelt sich darin zum Beispiel im Falle der Einwanderung der so genann-

\footnotetext{
25 So sollen zum Beispiel in den Niederlanden Einwanderungswillige bereits vor der Einreise ihre Sprachkenntnisse nachweisen (vgl. Spiegel online vom 14.03.2006). In der Bundesrepublik wird der Nachweis von Sprachkenntnissen seit Inkrafttreten des Zuwanderungsänderungsgesetzes von nachziehenden EhegattInnen verlangt - Länder wie die USA, Kanada und Japan sind davon allerdings ausgenommen (vgl. dazu den Abschnitt „Zuwanderungsänderungsgesetz,,, S. 182).
} 
ten ,Volksdeutschen“ oder ,AussiedlerInnen` deutlich der Einfluss nationalkonservativer Interessen.

In der Migrationspolitik finden sich demzufolge - sehr zugespitzt formuliert (national)konservative sowie wirtschaftsliberale Interessen, die die Konstruktion von Kategorien und deren Verbindung mit Einwanderungsmöglichkeiten beeinflussen: Erstere propagieren die Abwehr von MigrantInnen im Interesse des Schutzes einer vermeintlich homogenen nationalen Gemeinschaft, wobei die Einwanderung derjenigen befürwortet wird, die als Angehörige dieser Gemeinschaft definiert werden. Hingegen wird aus wirtschaftsliberaler Sicht die Einwanderung von Arbeitskraft - (hoch) qualifiziert und/oder durch Illegalisierung entrechtet positiv bewertet (vgl. Butterwegge 2003). Eine dritte (Argumentations-)Gruppe bilden diejenigen, die um arbeitsrechtliche und soziale Standards fürchten, welche durch illegalisierte MigrantInnen ausgehöhlt würden. Von diesen Standpunkten aus wird die Immigration von MigrantInnen, die nicht den Verwertungsmaßstäben genügen, oder von solchen, die nicht durch, ethnische' Zuschreibungen als ,erwünscht' gelten, abgelehnt. Das Zusammenspiel dieser Standpunkte hat in den Einwanderungsländern selektive Migrationsregime hervorgebracht, die entsprechend der Definition, erwünschter ${ }^{6}$ und ,unerwünschter ${ }^{6}$ Einwanderer reguläre Einwanderungsmöglichkeiten eröffnen oder verweigern.

Dem ,gate of entry“ (Birsl 2005, S. 204) Arbeitsimmigration, das auf nationalstaatliche, vor allem wirtschafts- und arbeitsmarktpolitisch begründete Eigeninteressen zurückgeht, stehen der Familiennachzug und die Kategorien Flucht und Asyl gegenüber, die auf Verpflichtungen aus völkerrechtlichen Verträgen oder nationalstaatlichen Verfassungen beruhen und die einstmals erfolgreiche Durchsetzung menschenrechtlicher Interessen widerspiegeln. Diese Interessen sind in den vergangenen Jahren zunehmend zurückgedrängt und die entsprechenden Einwanderungsoptionen mehr und mehr verengt worden. Mehr noch als die Familienzusammenführung ist die Kategorie Flucht und Asyl davon betroffen. Indem sie mit verschiedenen Maßnahmen - wie zum Beispiel der ,Drittstaatenregelung $^{26}$ - an die oben genannten Wanderungsdimensionen anknüpften, haben politische EntscheidungsträgerInnen die fast vollständige Schließung dieses gates herbeigeführt. Dabei sind die Art des Zutritts zur Ankunftsregion, faktisch die Form des zurückgelegten Weges und die Art des Transportmittels sowie die Gründe und Umstände entscheidend - zunächst für eine erfolgreiche Einreise, dann für die Anerkennung als Flüchtling oder Asylsuchende/r und schließlich für die damit verbundene Perspektive (dauerhafter oder temporärer Aufenthalt, soziale und politische Rechte). Die Einengung des Asylrechts, die sich nicht zuletzt an den stark zurückgegangenen Asylantrags- und Anerkennungszahlen zeigt ${ }^{27}$, wie auch der Familienzusammenführung und die selektive Öffnung für bestimmte

\footnotetext{
26 Zum Inhalt der Drittstaatenregelung vgl. das EU-Kapitel sowie die beiden Länderanalysen.

27 Die Asylantragszahlen lagen in Europa insgesamt sowie in den 25 EU-Mitgliedstaaten im Jahr 2006 auf dem niedrigsten Stand seit 20 Jahren (vgl. UNHCR 2007, S. 4).
} 
Gruppen mit ,erwünschten`Qualifikationen haben dazu geführt, dass sich eine wachsende Zahl von Menschen außerhalb dieses Kategorienschemas in der EU aufhält, deren Migrationsmotive, persönliches Profil oder auch Reiseweg nicht mit den Anforderungen für einen regulären Aufenthalt zusammenpassen. Die Kategorie Flucht und Asyl, deren Bedeutung als Einwanderungsmöglichkeit und als Schutzoption in den vergangenen Jahren massiv zurückgegangen ist, wird im nächsten Abschnitt ausführlicher erläutert.

Ihr Pendant findet die Kategorisierung zur Definition von Einwanderungsmöglichkeiten in den damit korrespondierenden, verschiedenen Rechts- bzw. Aufenthaltsstatus auf dem Territorium des jeweiligen Ankunftslandes. Diese können von kurzfristig verlängerten Duldungen bei gleichzeitiger Ausreiseaufforderung und Abschiebeandrohung über längerfristige Aufenthaltsbewilligungen bis hin zur Staatsbürgerschaft reichen. Mit diesen unterschiedlichen Status geht auch die unterschiedlich umfassende Gewährung von bürgerlichen, politischen und sozialen Rechten einher. In diesem Zusammenhang wird in den Sozialwissenschaften in den vergangenen Jahren eine intensive Debatte über universelle Menschenrechte auf der einen und exklusive Staatsbürgerrechte auf der anderen Seite und über die Bedeutung des Zusammenspiels dieser beiden Pole vor dem Hintergrund internationaler Migration für die Zukunft des Nationalstaates geführt. ${ }^{28}$ Für die hier interessierende Frage nach der Bedeutung der Kategorienkonstruktion für die Migrationspolitik und die Migrationsregime bietet Lydia Morris (2002) einen geeigneten Ansatz: Danach stellen die unterschiedlichen Grade von Mitgliedschaft ein „system of civic stratification“ bzw. ein „system of stratified rights“ dar, innerhalb dessen staatlich gewährte Rechte und Schutz mit unterschiedlichen Einreise-Kategorien verknüpft werden und eng mit Überwachung und Kontrolle verbunden sind. Damit identifiziert sie die Gewährung oder Vorenthaltung von

\footnotetext{
${ }^{28} \mathrm{Im}$ Vordergrund dieser Debatte steht die Frage, inwieweit Migration die Staatsbürgerschaft als Mitgliedschaftskonzept und damit auch die Bedeutung des Nationalstaates selbst in Frage stellt. Dabei wird einerseits argumentiert, die Staatsbürgerschaft stelle auch in Zukunft die zentrale Form der Mitgliedschaft und Voraussetzung für die Inanspruchnahme von Rechten dar. Damit einher geht die Auffassung, die Nationalstaaten behaupteten ihre Souveränität erfolgreich gegenüber externen und internen Einflüssen (vgl. stellvertretend Brubaker 1992). Demgegenüber konstatieren Yasemin Soysal (1994) und David Jacobson (1996) am Beispiel von ArbeitsmigrantInnen in Westeuropa und deren sozialer Gleichstellung mit StaatsbürgerInnen, dass sich aufgrund der wachsenden Bedeutung des Menschenrechtsregimes eine Form der „postnationalen Mitgliedschaft“ herausgebildet habe, die die Bedeutung der herkömmlichen nationalen Staatsbürgerschaft untergrabe, indem Rechte von dieser abgekoppelt würden. Eine vermittelnde Position nimmt Christian Joppke (1999) ein: Seiner Ansicht nach sei ein Beharren der Nationalstaaten auf Grenzkontrollen bei gleichzeitig steigender Bedeutung von Menschenrechten, eine Differenzierung von Mitgliedschaften beim gleichzeitigen Versuch, diese in einem einheitlichen Staatsbürgerschaftskonzept zusammenzuführen sowie ein Festhalten an nationalen Modellen des Umgangs mit Diversität bei gleichzeitigem multikulturellen Druck auf monokulturelle Nationenkonzeptionen zu beobachten. Eine Zusammenführung dieser Stränge und ihre Zuspitzung auf die Herausforderungen des Menschenrechtsregimes und der Nationalstaaten durch irreguläre Einwanderung am Beispiel der medizinischen Versorgung von Illegalisierten in der Bundesrepublik finden sich bei Müller (2004).
} 
,Flucht und Asyl` im Kontext von Migration und Migrationspolitik

Rechten als eine Basis des ,Migrationsmanagements'. Sie kritisiert die Abkopplung der Anti-Diskriminierungs-Politiken in der EU von den Einwanderungsbestimmungen:

„The conditions of entry are exempt from commitments to equal treatment; yet it has been argued that an endorsement of non-discrimination cannot be viewed independently from the laws on immigration. [...] We have at least the appearance within the EU of immigration regulations which discriminate at the point of entry, alongside explicit limitations of free movement and settlement, together with commitments to remove discrimination from the internal operations of the member states. "(Morris 2002, S. 23f.)

Ebenso deutlich hat Etienne Balibar (1991) formuliert, dass durch die Konstruktion von MigrantInnen-Kategorien mit unterschiedlichen Rechten Diskriminierung Teil der Europäischen Gemeinschaft und quasi in sie eingeschrieben sei: „In fact discrimination is written into the very nature of the European Community “ (ebd., S. 6).

Die Definition von ,erwünschten' und ,unerwünschten` MigrantInnen und die Eröffnung von legalen Einwanderungsmöglichkeiten für erstere erfolgt durch die legislative Festschreibung (die häufig - zum Beispiel im öffentlichen Diskurs dazu führt, diese Konstruktionen als quasi [natur-]gegeben anzuerkennen), die judikative Bestätigung (seltener auch Widerlegung) und die exekutive, das heißt behördliche Umsetzung. Ein zentraler Ort der Kategorienkonstruktion und -legitimation, der diese Unterscheidung ebenfalls überwiegend stützt bzw. diese Unterstützung erst herstellt, ist der öffentliche und medial vermittelte Diskurs (vgl. Butterwegge/Hentges 2006; Jung/Wengeler/Böke 1997).

Darüber hinaus machen die politischen Kategorisierungsaktivitäten, die - wie gezeigt - teilweise durch die Migrationsforschung reproduziert werden, auch die Sicht auf und den Umgang mit MigrantInnen in diesen beiden Bereichen offenkundig: MigrantInnen sind Objekte, Zahlen, Typen, die nach Kategorien sortiert und in diverse Ordnungsschemata eingeordnet werden. Wer in das Schema nicht hineinpasst, soll von der Einwanderung abgehalten, abgeschoben oder gar ,bekämpft ${ }^{\natural}$ werden, wie es das oftmals formulierte Ziel der Migrationspolitik - die ,Bekämpfung der illegalen Migration‘ - impliziert.

Die vorangegangene Reflexion über Migrationskategorien hat gezeigt, dass diese Kategorien ein Ergebnis politischer und diskursiver Konstruktionsprozesse sind. Die Kategorien basieren auf nationalstaatlichen Vorstellungen von Zugehörigkeit und verknüpfen verschiedene Dimensionen des Wanderungsprozesses zu einem Anforderungsprofil für Einwanderungswillige, das verschiedenen nationalstaatlichen Interessen entspricht, jedoch das Gros der MigrantInnen ausschließt. Damit wird allerdings die Einwanderung dieser ,unerwünschten 'MigrantInnen nicht verhindert, sondern in irreguläre Bahnen gelenkt, was mit einer massiven Entrechtung einhergeht. Die Kategorienkonstruktion ist daher nach Morris zentraler Bestandteil des Migrationsmanagements oder - mit den Begriffen von Kara- 
kayalı und Tsianos (2005, S. 49) gesprochen - eine wichtige Voraussetzung der „Produktivität“ des Grenz- und Migrationsregimes.

\subsubsection{Flucht und Asyl}

Vor dem Hintergrund der im vorangegangenen Abschnitt herausgearbeiteten Bedeutung der politischen Konstruktion von MigrantInnen-Kategorien soll nun die für die vorliegende Untersuchung zentrale Kategorie Flucht und Asyl näher beleuchtet werden. Es wird gezeigt, welche politischen, rechtlichen und wissenschaftlichen Wirklichkeitsdefinitionen dieser Kategorie zugrunde liegen. Daraus sollen einige Schlüsse über die Rolle dieser Kategorie aber auch der Kategorisierung selbst im Migrationsregime gezogen werden.

\section{Politische Definition des Flüchtlingsbegriffs}

Aristide R. Zolberg, Astri Suhrke und Sergio Aguayo (1989) geben einen sehr umfassenden historischen Überblick über Flüchtlingsdefinitionen. ${ }^{29}$ Die Darstellung führt bis ins 16. Jahrhundert zurück, als der Begriff Flüchtling (refugee) ${ }^{30}$ im Kontext von Asyl- und Schutzgewährung für ,Fremde', die vor Verfolgung flohen, benutzt wurde. Diese historische Einbettung der Definitionsversuche und -aktivitäten unterstreicht sehr eindrücklich die Bedeutung politischer Konstruktionen und deren Abhängigkeit von politischen Konjunkturen für die Beantwortung der Frage, wer ein Flüchtling ist und sich für Aufnahme und Schutz qualifiziert. Die Autoren zeigen,

,why only some populations and not others in similar situations were treated as refugees and thereby how the development of codes reflects broader political considerations and discretionary judgements by state authorities"

(Zolberg et al. 1989, S. 4).

Für die politische Definition dessen, was in der Gegenwart unter Flucht verstanden wird, sind das Abkommen über die Rechtsstellung der Flüchtlinge von 1951 und das dazu gehörige New-York-Protokoll von 1967 zentrale Referenzpunkte auch Genfer Flüchtlingskonvention (GFK) genannt. Es wird stets darauf hingewiesen, dass die Konvention als eine Reaktion auf den Nationalsozialismus und den Holocaust entstanden (vgl. Boswell 2002; Treibel 2003, S. 161) und daher „für die Erfassung der Flüchtlingsprobleme nach dem Zweiten Weltkrieg in Europa“ (Han 2000, S. 79) konzipiert worden sei. Nach Art. 1 A Nr. 2 dieser Konvention ist unter dem Begriff, Flüchtling“ jede Person zu verstehen, die

\footnotetext{
${ }^{29}$ Annette Treibel (1999, S. 168) bezeichnete das Buch von Zolberg, Suhrke und Aguayo als „Standardwerk der Fluchtforschung“"

${ }^{30}$ Das englische Wort ,refugee ist dabei abgeleitet aus dem Französischen ,réfugié (Zolberg et al. 1989, S. 5).
} 
,Flucht und Asyl` im Kontext von Migration und Migrationspolitik

„[...] aus der begründeten Furcht vor Verfolgung wegen ibrer Rasse, Religion, Nationalität, Zugebörigkeit zu einer bestimmten sozialen Gruppe oder wegen ibrer politischen Überzengung sich außerhalb des Landes befindet, dessen Staatsangehörigkeit sie besitrt, und den Schutz dieses Landes nicht in Anspruch nehmen kann oder wegen dieser Befürchtungen nicht in Anspruch nehmen will; oder die sich als Staatenlose infolge solcher Ereignisse außerhalb des Landes befindet, in welchem sie ibren gewöhnlichen Aufenthalt hatte, und nicht dorthin zurückkehren kann oder wegen der erwähnten Befürchtungen nicht dorthin zurückekehren will. "(Abkommen über die Rechtsstellung der Flüchtlinge)

Ein Flüchtling ist laut dieser Bestimmung also, wer verfolgt wird - dafür nennt die GFK genau fünf, den Flüchtlingsstatus rechtfertigende Gründe: ,Rasse‘, Religion, Nationalität, Zugehörigkeit zu einer sozialen Gruppe und politische Überzeugung. Darüber hinaus muss sich die Person außerhalb ihres Herkunftslandes befinden; die GFK erfasst mithin keine Binnenflüchtlinge. Dieser enge Flüchtlingsbegriff war das Ergebnis eines politischen Kompromisses bei der damaligen internationalen Konsensfindung. Bis zur Verabschiedung des Protokolls von 1967 war die Definition beschränkt auf EuropäerInnen und auf Personen, die vor dem 1. Januar $1951 \mathrm{zu}$ Flüchtlingen geworden waren. Mit dem Protokoll wurden diese auf Raum und Zeit bezogenen Begrenzungen aufgehoben (vgl. Zolberg et al. 1989, S. 25).

Neben dem Verbot der Ausweisung und Zurückweisung unter bestimmten Bedingungen - dem Non-Refoulement-Gebot ${ }^{31}$ des Art. 33 - legt die Konvention verschiedene Rechte und Pflichten für anerkannte Flüchtlinge fest. Damit sind sowohl die Rechtsstellung der AntragstellerInnen als auch das Verfahren der Statusbestimmung nicht in der Konvention geregelt. Die GFK begründet zudem kein Recht auf Asyl oder Schutz für den Einzelnen gegenüber dem Staat, sondern beinhaltet das Recht der Staaten, Asyl zu gewähren. Damit ist sie letztlich auf den ,Goodwill' der unterzeichnenden Staaten angewiesen tatsächlich Flüchtlinge anzuerkennen und aufzunehmen. Die mit dieser Definition verbundene Individualisierung des Flüchtlingsstatus brachte die Praxis der Einzelfallprüfung hervor, innerhalb derer Flüchtlinge gezwungen sind, ihre ,begründete Furcht vor Verfolgung ${ }^{6}$ nachzuweisen. Während dieses Anerkennungsverfahrens sind sie in den meisten europäischen Staaten in ihren Rechten nicht der übrigen Bevölkerung gleichgestellt. Von politischen Rechten wie dem Wahlrecht sind Flüchtlinge wie die meisten anderen MigrantInnen ausgeschlossen. Zudem wurden insbesondere die sozialen Rechte - Unterkunft, Sozialleistungen, Arbeitsrecht - und in Deutschland

31 Das Non-Refoulement-Gebot verbietet es den Vertragsstaaten, einen Flüchtling in ein Land oder Gebiet auszuweisen, in dem ,sein Leben oder seine Freiheit wegen seiner Rasse, Religion, Staatsangehörigkeit, seiner Zugehörigkeit zu einer bestimmten sozialen Gruppe oder wegen seiner politischen Überzeugung bedroht sein würde." (Art. 33 Abs. 1 GFK) Auf diese Klausel darf sich jedoch nicht berufen, wer eine Gefahr für die Sicherheit oder die Allgemeinheit des Aufnahmelandes darstellt oder wegen eines Verbrechens oder besonders schweren Vergehens verurteilt wurde (Art. 33 Abs. 2 GFK). 
zum Beispiel auch die Bewegungsfreiheit im Laufe der vergangenen Jahrzehnte massiv eingeschränkt. Die Konvention wurde von zahlreichen Staaten unterzeichnet. ${ }^{32}$ Einige haben zusätzlich in ihren Verfassungen ein Recht auf Asyl für politisch Verfolgte verankert.

Da der Fokus der GFK auf individuelle Verfolgung - wie im Weiteren näher ausgeführt werden wird - den tatsächlichen Ereignissen nicht gerecht werden konnte, sind neben den formalen Flüchtlingsstatus entsprechend der GFK die zahlenmäßig überlegenen so genannten de facto-Flüchtlinge getreten. Darunter werden in der Regel all diejenigen gefasst, die zwar keine individuelle Verfolgung nachweisen können, aber von anderen Menschenrechtsverletzungen - beispielsweise durch generalisierte Gewalt oder Bürgerkriege - oder von Naturkatastrophen betroffen sind. Wenn diesen Flüchtlingen Schutz gewährt wird, so erfolgt dies meist aufgrund ihrer Zugehörigkeit zu einer Gruppe, zum Beispiel von Menschen aus einem bestimmten Herkunftsland oder die gemeinsam einem bestimmten politischen Ereignis, das mit Menschenrechtsverletzungen verbunden war, ausgesetzt waren. In diesen Fällen findet entweder keine individuelle Statusbestimmung statt, oder es handelt sich um abgelehnte AsylbewerberInnen, die aus ,humanitären Gründen' nicht abgeschoben werden können und daher de factoFlüchtlinge sind. Damit hat sich zusätzlich zum GFK-Status eine informelle, weniger institutionalisierte und rechtlich weniger abgesicherte Form des Flüchtlingsschutzes entwickelt (vgl. Lavenex 2002, S. 33ff.). Bemühungen, den Status und die Rechte von de facto-Flüchtlingen international $\mathrm{zu}$ formalisieren, waren bisher nicht erfolgreich.

Der Bezug auf den engen Flüchtlingsbegriff der Konvention ermöglichte es den unterzeichnenden Staaten in Westeuropa Ende der 1970er und in den 1980er Jahren, als durch den Anwerbestopp 33 immer mehr Menschen das Asyl als letzte Einwanderungschance neben dem Familiennachzug zu nutzen versuchten, die Legitimität der Immigration, der keiner der Verfolgungsgründe zugrunde lag, öffentlichkeitswirksam anzuzweifeln. Wie im Folgenden noch am Beispiel Deutschlands genauer ausgeführt werden wird, verlagerte sich die öffentliche Debatte über Migration hin zu einer Asyldebatte, innerhalb derer sich eine Verschärfung der konstruierten Trennlinie zwischen Flucht und anderen Formen der Migration vollzog. Im Mittelpunkt steht dabei die Annahme, erstere erfolge ,unfreiwillig', die letzteren hingegen ,freiwillig' sowie die Differenzierung zwischen ,legitimen' (politische Verfolgung) und ,illegitimen' Gründen (Suche nach Arbeit, Verbesserung

\footnotetext{
32 Bis zum Oktober 2008 waren 141 Staaten sowohl der Genfer Flüchtlingskonvention als auch dem New Yorker Protokoll beigetreten (vgl. UNHCR 2008a). Hinzu kommen drei Staaten, die nur der Konvention und drei Staaten, die nur dem New Yorker Protokoll beigetreten sind (vgl. ebd.).

33 Der so genannte Anwerbestopp bedeutete jedoch keineswegs das Ende der regulären Arbeitsimmigration. Stattdessen haben seitdem fortwährend Anwerbungen stattgefunden, die in Deutschland ihren Ausdruck in zahlreichen ,Anwerbestoppausnahmeverordnungen` fanden.
} 
,Flucht und Asyl' im Kontext von Migration und Migrationspolitik

der individuellen Lebensbedingungen, geschlechtsspezifische Fluchtgründe). ${ }^{34}$ Diese Unterscheidungen sind die Grundlage für die Klassifizierung in ,unerwünschte', das Asylverfahren ,missbrauchende' ${ }^{6}$, Wirtschaftsflüchtlinge ${ }^{6}$ und ,tatsächlich politisch Verfolgte', denen - sofern sie verschiedene weitere Voraussetzungen erfüllen - ein Aufenthaltsstatus gewährt wird. ${ }^{35}$ Diese Tendenz, die Einwanderung von MigrantInnen, die die Voraussetzungen der GFK nicht erfüllen, als rechtswidrigen Missbrauch des Flüchtlingsschutzes und als unzumutbare Bürde für die Sozialsysteme zu diskreditieren, erhielt nach dem Fall des ,Eisernen Vorhangs' noch einmal neuen Auftrieb ${ }^{36}$ und mündete in vielen Ländern in der Verschärfung des Asylrechts, der Anerkennungsverfahren und der sozialen Rahmenbedingungen für AsylantragstellerInnen. Diese Sicht spiegelt auch eine Vorstellung von Migration wider, die „für die Ursachen der Einwanderung ausschließlich die individuellen Einwanderer verantwortlich macht und eine aktive Beteiligung der aufnehmenden Staaten an den Migrationsprozessen leugnet" (Sassen 2000, S. 168). Damit werde ausgeblendet, dass die Zielländer sowohl in staatlicher als auch in wirtschaftlicher Hinsicht Beziehungen zu den Herkunftsländern geschaffen haben, die Kapital- aber auch Migrationsbewegungen nach sich zogen (vgl. ebd., S. 170). So wandern MigrantInnen häufig in die Länder, die von den Herkunftsländern einst kolonisiert wurden oder die früher Arbeitskräfte in den Herkunftsländern angeworben haben. Neben der direkten Mitverantwortung vieler Zielländer für Fluchtursachen, zum Beispiel durch ihre aktive Beteiligung an Konflikten in den Herkunftsregionen, arbeitet Saskia Sassen auch einen Zusammenhang zwischen der Transnationalisierung der Wirtschaft und den Migrationsbewegungen heraus (vgl. Sassen 2000, S. 168ff.).

Inzwischen geht die Bedeutung der Kategorie Flucht und Asyl stark zurück. Flüchtlinge und Asylsuchende sind zwar nicht von der Agenda der politischen AkteurInnen und aus den öffentlichen Debatten verschwunden, wohl aber zu-

${ }^{34}$ Die Abgrenzung von Flucht und anderen Migrationsformen durch die Bestimmung des Grades der Freiwilligkeit beschäftigt auch die Fluchtforschung, wie im folgenden Abschnitt erläutert werden wird.

35 Der Topos des ,Missbrauchs des Asylrechts' tauchte im Asyldiskurs der Bundesrepublik bereits Anfang der 1960 Jahre auf und findet sich inzwischen auch in den Verlautbarungen der zuständigen EU-Institutionen sowie in Ansätzen in den neuen Aufnahmeländern für Flüchtlinge, wie zum Beispiel Polen (vgl. die Analyse des Asylrechts der EU in Kapitel 4 sowie die Länderstudien zu Deutschland (Kapitel 5) und Polen (Kapitel 6) in der vorliegenden Untersuchung).

36 Während des ,Kalten Krieges' herrschte zunächst eine liberale Stimmung gegenüber Flüchtlingen vor - zumindest gegenüber denjenigen aus dem, Ostblock'. Die Flucht von Menschen aus den Ostblock-Ländern in den,Westen“ wurde als Beweis für die Überlegenheit des westlichen Systems interpretiert, und die meisten wurden als Flüchtlinge anerkannt. „Im Wettstreit der Systeme demonstrierten diese Migranten die höhere Attraktivität des Westens." (Fassmann/Münz 1996, S. 25) Gleichzeitig waren diese Fluchtbewegungen zahlenmäßig durch die Einschränkung der Reisefreiheit auf der anderen Seite des ,Eisernen Vorhangs' eng umgrenzt - die seitens des Westens betriebene „Politik der ,offenen Tür' gegenüber Ostmitteleuropa“ sei daher „risikolos“ gewesen (ebd.). Viele Ostblockstaaten waren Ausgangspunkt für Flüchtlinge, wie zum Beispiel Polen nach der Ausrufung des Kriegsrechts durch General Jaruzelski im Jahr 1980. 
nehmend aus den Ländern der EU - zumindest aus den Statistiken. So befinden sich die Asylantrags- und Anerkennungszahlen seit Jahren im freien Fall. ${ }^{37}$ Nur ein Bruchteil der Flüchtlinge schafft es unter den Voraussetzungen der sicheren Dritt- und Herkunftsstaaten-Regelung (vgl. 4. Asylpolitik der EU) noch, ein Asylverfahren zu durchlaufen. Zusammen mit äußerst geringen Anerkennungsquoten führt dies dazu, dass kaum noch Personen den Flüchtlingsstatus erhalten.

\section{Wissenschaftliche Auseinandersetzung mit dem Flüchtlingsbegriff}

In wissenschaftlichen Ausführungen finden sich verschiedene Kritiken am Flüchtlingsbegriff der GFK. Allgemein wird darauf hingewiesen, dass er die komplexen Ursachen, Motive und Erscheinungsformen von Flucht, die sich seit dem Zweiten Weltkrieg verändert haben, nicht mehr abbilden kann. So wird bemängelt, dass die Verfolgung durch nicht-staatliche AkteurInnen nicht durch die GFK abgedeckt sei. Diese Sichtweise blende aus, ,that persecution is related to broad historical processes in which complex internal and external forces interact" (Zolberg et al. 1989, S. 25). Weiterhin wird angeführt, dass die wenigsten eine individuelle Verfolgung nachweisen könnten und viele zudem bei ihrer Flucht gar keine Landesgrenzen überschreiten - und daher als Binnenflüchtlinge ein wichtiges Kriterium der GFK nicht erfüllen. Stattdessen sei das Fluchtgeschehen geprägt durch Binnenflucht, Verschleppung, Vertreibung, Flucht vor Armut, (Bürger-)Kriegen, Naturoder Umweltkatastrophen (vgl. Han 2000, S. 79; Treibel 1999, S. 161). Darüber hinaus wird der Gender-Dimension in der Flüchtlingspolitik und im Flüchtlingsrecht zunehmend mehr Aufmerksamkeit geschenkt. Aus dieser Perspektive wird kritisiert, dass Politiken und Recht "fail to acknowledge the gendered reality of women's lives, including restrictive patriarchal 'protections' and private violence." (Calavita 2006, S. 112) Die Flüchtlingskonventionen seien daher noch immer "implicitly gendered“ (ebd.).

Einen Schritt weiter geht Anthony H. Richmond: Nach dessen Ansicht ist die Konvention ,anachronistic and needs to be replaced. [...] New international laws are needed that will protect all migrants, including contract workers, against discrimination [...]" (Richmond 2002, S. 718). Er macht deutlich, dass sowohl ,reine Flüchtlinge als auch, reine` WirtschaftsmigrantInnen idealtypische Konstrukte sind, die so in der Realität nicht vorfindbar sind. Laut Stephen Castles (vgl. 2005, S. 16) ist die Trennung zwischen, Wirtschaftsmigranten' und Flüchtlingen deshalb schwierig, weil ,schwache Volkswirtschaften' und ,schwache Staaten' in der Regel zusammen auftreten. Menschen versuchten daher durch Migration sowohl Verarmung als auch Menschenrechtsverletzungen zu entgehen. Das Zusammenspiel verschiedener Motive impliziere einen „Migrations-Asyl-Nexus“ und mache deutlich, dass die gegenwärtigen Entwicklungen mit dem gern verwendeten Begriff „Migrationskrise“ nicht zutreffend umschrieben seien, vielmehr handele es sich

37 Vgl. Fn. 27. 
um „eine Krise der Nord-Süd-Beziehungen, die durch ungleiche Entwicklung und krasse Ungleichheit verursacht ist.“ (Castles 2005, S. 16). Treibel (1999, S. 162) unterstreicht, dass die „Festlegung von Flüchtlingseigenschaften“ eine „politische Frage" sei. Auch sie verabschiedet sich von der Differenzierung zwischen Arbeitsund Fluchtmigration. Demzufolge ,ist die Unterscheidung zwischen Arbeitsmigration und Fluchtmigration nur noch bedingt hilfreich. [...] Zwang und Freiwilligkeit mischen sich [...]" (Treibel 1999, S. 21). Nach ihren Erkenntnissen sind die wichtigsten Ursachen von Migration die „Suche nach Arbeit“ und der „Schutz vor Verfolgung“ (ebd.). Migration umfasse daher beides: Arbeits- und Fluchtmigration. Die Begrenzung des Migrationsbegriffs auf ,freiwillige Migration', die sie in der ersten Auflage ihres Überblickswerkes zu ,Migration in modernen Gesellschaften' selbst noch vorgenommen hatte, sei daher ebenfalls inzwischen nicht mehr überzeugend. Sie verwendet in ihren Ausführungen einen Flüchtlingsbegriff, der an die Flüchtlingsdeklarationen der Organisation für Afrikanische Einheit und der mittelamerikanischen Staaten ${ }^{38}$ angelehnt ist und der all diejenigen einschließt, „die durch Kriege, Bürgerkriege, Katastrophen und andere Notlagen gezwungen werden, ihre Heimat zu verlassen“ (Treibel 1999, S. 162). Philip Marfleet (2006, S. 12) schlussfolgert aus der Auseinandersetzung um die Konventionsdefinition: „The only satisfactory answer for those who experience displacement is a policy of open borders $[\ldots]^{\text {“. }}$.

Die Trennung zwischen Flüchtlingen und sonstigen MigrantInnen wurde in den durch die Forschung entworfenen Typologien bislang überwiegend nachvollzogen. ${ }^{39}$ Zwei einflussreiche Texte, die über Typologien eine Theorie der Fluchtmigration zu entwickeln versuchten, waren die von Anthony H. Richmond (1988; 1993) und Zolberg et al. (1989). Laut Richmond lässt sich das tatsächliche Fluchtgeschehen in einem Kontinuum zwischen ,proaktiver' und ,reaktiver ${ }^{6}$ Migration nachzeichnen. Dabei sind proaktive MigrantInnen diejenigen, die ihren Nettonutzen zu steigern versuchen, während der Grad der Freiheit der reaktiven MigrantInnen stark eingeschränkt ist. Laut Richmond lassen sich alle internationalen MigrantInnen irgendwo zwischen diesen beiden Polen verorten und auch Flüchtlinge seien nicht nur reaktiv. Konventionsflüchtlinge ordnet er in die Kategorie

\footnotetext{
38 Die Organisation für Afrikanische Einheit (OAU) verständigte sich 1969 in Addis Abeba auf die ,OAU Convention governing the specific aspects of refugee problems in Africa'. Im Jahr 1984 wurde die ,Cartagena Declaration on Refugees' durch die mittelamerikanischen Staaten verabschiedet. Beide beinhalten einen umfassenderen Flüchtlingsbegriff als die GFK. So enthält Artikel 1 der OAU Convention neben der GFK-Definition folgende Erweiterung in Abs. 2: „The term 'Refugee' shall also apply to every person who, owing to external aggression, occupation, foreign domination or events seriously disturbing public order in either part of the whole of his country of origin or nationality, is compelled to leave his place of habitual residence in order to seek refuge in another place outside his country of origin or nationality." (OAU Convention, S. 47) Die Deklaration von Cartagena geht noch über diese Definition hinaus, entfaltet jedoch völkerrechtlich keine Verbindlichkeit (vgl. Cartagena Declaration).

${ }^{39}$ Für einen Überblick über einige Flüchtlings-Typologien vgl. Treibel (1999, S. 164ff.).
} 
,reaktiv` ein, während so genannte ,Wirtschaftsflüchtlinge` ungefähr in der Mitte des reaktiv/proaktiv-Kontinuums zu verorten seien. Aus seinem multivariaten Modell reaktiver Migration erstellt Richmond (1993) eine Typologie mit 25 Faktoren-Kombinationen, die seiner Ansicht nach nicht einfach eine Liste der traditionellen ,Push-Faktoren' darstellen, sondern die Interaktionen zwischen motivationalen und sozial-strukturellen Determinanten sowie die komplexen Interaktionen zwischen politischen, ökonomischen, umweltbedingten und sozialen Faktoren, die die Migrationsneigung beeinflussen, berücksichtigen. Damit werde vor allem gezeigt, wie inadäquat Flüchtlingsdefinitionen sind, die nur ein Element aus der Kausalkette herausgreifen, wie die Angst vor Verfolgung. Eine solche Angst sei oft nur ein Faktor in einer viel komplizierteren Beziehung zwischen prädisponierenden Faktoren, strukturellen Zwängen, verursachenden Ereignissen und ermöglichenden Umständen. Aus dieser Aufstellung leitet Richmond auch einige „,policy-conclusions“ ab, in denen er vor allem für eine Erweiterung der Flüchtlingsdefinition und ein generelles Umdenken hinsichtlich der „globalen Strukturen“ plädiert (ebd., S. 21f.).

Zolberg et al. (1989, S. 274) weisen auf die häufige unkritische Vermischung soziologischer und rechtlicher Definitionen hin. Als Beispiel führen sie die Flüchtlingsdefinition von Gil Loescher und Ann Dull Loescher (1982, S. 1) an: danach sind Flüchtlinge ,among the world's most disadvantaged people“ und „do not voluntarily leave their homes to seek economic opportunity in another country. [...] they flee because of persecution or fear of it." Laut Zolberg et al. ist die soziologische Existenz von Flüchtlingen unabhängig von deren rechtlicher Anerkennung. Daher gebe es legale und illegale Flüchtlinge - Flüchtlinge also, die soziologisch als solche zu bezeichnen seien, jedoch rechtlich nicht anerkannt würden. Ihre Flüchtlings-Typologie umfasst ,Aktivisten' (activists), ,Zielscheiben“ (targets) und ,Opfer (victims). ${ }^{40}$ Die ersten beiden Gruppen seien durch die GFK abgedeckt, während die ,Opfer' einen großen Teil der Flüchtlinge bilden, jedoch durch die Konvention nicht berücksichtigt werden (vgl. Zolberg et al. 1989, S. 30). Das verbindende Element, das die Flüchtlingstypen zu einer kohärenten Gruppe zusammenführe und sie von anderen unterscheide, sei Gewalt. Diese Gewalt könne aus Konflikten zwischen Staat und Zivilgesellschaft, zwischen gegnerischen Armeen, zwischen ethnischen Gruppen oder Klassenformationen entstehen, die der Staat entweder nicht kontrollieren wolle oder könne.

„We shall therefore define refugees as persons whose presence abroad is attributable to a well-founded fear of violence, as might be established by impartial experts with adequate information. "(ebd., S. 269)

$40,[\ldots]$ the classic activists are dissenters and rebels whose actions contribute to the conflict that eventually forces them to flee. The targeted refugees are individuals who, through membership in a particular group, are singled out for violent action. And the victims are randomly caught in the cross fire or are exposed to generalized social violence." (Zolberg et al. 1989, S. 269) 
Hier deutet sich bereits an, dass die Kritik an der Vermischung soziologischer und rechtlicher Definitionen von den Autoren selbst nicht beherzigt wird: Ihren eigenen Kriterien fügen sie den Hinweis an, dass diese durch Experten zu prüfen seien und verknüpfen damit die soziologische Definition von Flüchtlingen doch mit einer Legitimierung durch staatliche bzw. rechtliche Instanzen. Gleichzeitig gehen sie davon aus, dass der Flüchtlingsstatus eine ,privileged form of migration" sei (Zolberg et al. 1989, S. 270). Um zu verhindern, dass sich die Zahl der Flüchtlinge in den Zielländern endlos vervielfache, könne dieser Status nur einer begrenzten Zahl von Menschen gewährt werden. Sie schlagen daher zur Begrenzung der Zahl der Anspruchsberechtigten ein ,Ranking-Prinzip' vor, das sich an der Unmittelbarkeit und dem Grad der lebensbedrohlichen Gewalt orientieren müsse. Danach sollen vorrangig die am meisten Betroffenen Schutz erhalten, während weiter unten in dieser Liste Eingeordnete Hilfe erhalten können, soweit es die Ressourcen und der politische Wille zulassen. Repressionen im kulturellen Bereich - wie die Einschränkung der Religionsfreiheit - hätten in diesem Ranking einen untergeordneten Stellenwert, sofern sie nicht mit lebensbedrohlichen Formen von Gewalt verbunden sind. Auch Situationen, in denen die ökonomischen Voraussetzungen für die Lebenserhaltung plötzlich beseitigt werden, können laut den Autoren eine lebensbedrohliche Gewalt darstellen. Diesen Opfern ökonomischer, das heißt struktureller Gewalt sollte in ihren Herkunftsländern geholfen werden. Auch diese aus der Begriffsbestimmung abgeleiteten Ratschläge für eine Hierarchisierung stehen der zunächst vollzogenen Abgrenzung von der Vermischung rechtlicher und soziologischer Definitionen entgegen und stellen eine Basis für politische Kategorisierung und Ausschluss von Flüchtlingen und damit schließlich für deren Illegalisierung dar.

Ein Beispiel für die jüngere Auseinandersetzung mit dem Thema sind die Arbeiten von Elisabeth Abiri (2000). Sie stellt ihren Ausführungen den Begriff „reactive migration“ voran. Darunter versteht sie ,migration that is an involuntary reaction to specific events and developments" (Abiri 2000, S. 22). Reaktive Migration finde darüber hinaus meist über irreguläre Wege statt. Damit geht auch sie über die Definition der GFK hinaus, führt jedoch die Unterscheidung zwischen freiwilliger und unfreiwilliger Migration wieder ein, die Richmond, an den sie sich mit dem Begriff ,reactive migration' anlehnt, explizit zurückgewiesen hatte. Sie stimmt mit Richmond darin überein, dass die Trennlinie zwischen aktiver und reaktiver Migration unmöglich zu ziehen sei. Die beiden Begriffe seien stattdessen die Enden einer fließenden Skala. Sie hebt hervor, dass der Begriff ,reaktive Migration auf die Lebensbedingungen im Herkunftsland und weniger auf die potenziellen Möglichkeiten im Zielland abstellt. Daraus folgert sie, dass restriktive Politiken die Migration kaum verhindern können. Gleichzeitig sei es jedoch wichtig festzuhalten, dass eine Person, die auf das Geschehen im Herkunftsland reagiert, bei der Wahl des Ziellandes höchst aktiv sein kann. Diese aktive Wahl solle jedoch nicht 
darüber hinweg täuschen, dass Migration als eine Reaktion auf die Lebensbedingungen im Herkunftsland initiiert wird.

Diesen Versuchen der mehr oder weniger kritischen Bewertung und Erweiterung des Flüchtlingsbegriffs stehen die Ausführungen von Sandra Lavenex (vgl. 2002, S. 1) gegenüber, die sich in jüngerer Zeit in mehreren Publikationen mit der Europäisierung von Flüchtlingspolitiken auseinandergesetzt hat. Sie vollzieht die Trennung zwischen Migration und Flucht, Freiwilligkeit und Unfreiwilligkeit in ihren Untersuchungen noch immer nach:

„Unlike the broader phenomenon of migration, which occurs on a voluntary basis, the notion of asylum derives from international buman rights and liberal republican understandings about the relationship between the state and the citizen." (ebd.)

Lavenex denkt ihre Flüchtlingsdefinition gewissermaßen vom Staat aus. So sei der Staat sowohl die Ursache als auch die Lösung des ,Asylproblems'. Flüchtlinge seien einerseits definiert als Individuen, deren grundlegende Menschenrechte verletzt wurden und die den Schutz ihres Herkunftslandes verloren haben. Dieser Schutzverlust führe andererseits zur Verantwortung der internationalen Gemeinschaft - ein anderer Staat übernimmt durch die Asylgewährung den Schutz der grundlegenden Menschenrechte. Flüchtlinge seien daher Ausdruck der Spannung zwischen den zwei konstituierenden Prinzipien des modernen Staatensystems: dem Prinzip territorialer Souveränität und der Universalität der Menschenrechte.

Christina Boswell (2002) arbeitet heraus, dass das internationale Flüchtlingsrecht und die GFK in der wissenschaftlichen und politischen Diskussion von einigen für die Eingrenzung auf Flucht aufgrund individueller Verfolgung, von anderen aber auch für die mit der Konvention verbundenen nicht realisierbaren Anforderungen an die Staaten kritisiert würden. Die hohen Anforderungen resultierten vor allem aus dem ,exile bias' - der Gewährung dauerhaften Aufenthalts bzw. Exils statt Rückführung, was insbesondere den Entwicklungen nach dem Ende des ,Kalten Krieges‘ nicht mehr gerecht werde. Die ethische Diskussion sei geprägt von liberalem Universalismus auf der einen und national inspirierten Rechtfertigungen von Restriktionen auf der anderen Seite. Es fehle an alternativen Philosophien oder Konzepten der Flüchtlingsethik, die die Kluft zwischen diesen beiden Positionen überwinden und damit helfen könnten, den Flüchtlingsschutz zu fördern. Sie stellt vier Möglichkeiten vor, wie der universelle Ansatz, der in der Vergangenheit an den Rand der Debatte gedrängt worden sei, wieder gestärkt werden könnte, die aber vor allem eine Annäherung an national geprägte Positionen darstellen (vgl. Boswell 2002, S. 7ff.). ${ }^{41}$

\footnotetext{
${ }^{41}$ So bestünde eine Möglichkeit zur Überwindung des gaps zwischen nationalen und universalen Positionen darin, mit Verweis auf den sozio-ökonomischen Nutzen für die Mitglieder des Nationalstaats für eine liberalere Politik zu werben. Sie räumt ein, dass dies zum einen nur ein Ansatz sei, mit dem eventuell „welfare nationalists“, nicht aber ,ethnonationalists“ zu überzeugen seien. AuBerdem sei der Bezug auf die ökonomische Notwendigkeit von Einwanderung keine adäquate Lö-
} 
Eine explizite Verteidigung des Status Quo hat Matthew E. Price (2006) in seinem Artikel „Persecution Complex: Justifying Asylum Law's Preference for Persecuted People“ formuliert. Er unterscheidet eine „humanitäre“ und eine „politische“ Asylkonzeption. Unter die humanitäre Konzeption subsumiert Price die Bestrebungen, das Recht auf Asyl über die in der GFK genannten Gründe hinaus auszudehnen. Gleichzeitig würde in dieser Konzeption nicht zwischen Schutz und Mitgliedschaft getrennt, das heißt statt temporärer dominiere dauerhafte Schutzgewährung. Flüchtlingsschutz sei jedoch „a 'scarce resource“" (ebd., S. 416). Eine Ausweitung auf andere Fluchtursachen und die fehlende Trennung zwischen Mitgliedschaft und temporärem bzw. lokalem Schutz müsse daher laut Price zum Kollaps des Flüchtlingsschutzsystems führen. Deshalb plädiert Price für seine politische Asylkonzeption, die sich auf politisch Verfolgte beschränkt und zwischen ,a need for protection and a need for membership“ (ebd., S. 418) unterscheidet und daher auch „temporary protection“ (ebd., S. 431), „humanitarian military intervention“ (ebd., S. 444) und „overseas resettlement“ (ebd., S. 445) als Alternativen zur Mitgliedschaft kennt. Diese seien billiger als dauerhaftes Asyl in Europa oder den USA und könnten somit mehr Menschen zugute kommen als die dauerhafte Mitgliedschaft der humanitären Konzeption. Damit schreibt Price gegen die meisten der oben angeführten WissenschaftlerInnen an, die laut seiner Einteilung AnhängerInnen der humanitären Konzeption sind. Gleichzeitig liegt er im Trend politischer Regulierungen im Asylbereich, indem er bereits praktizierte Maßnahmen (,temporary protection“, „overseas resettlement“, „military intervention“) durch seine normative Theorie unterstützt.

sung für das ,Flüchtlingsproblem', da Flüchtlinge nicht unbedingt den ökonomischen Verwertungsansprüchen genügen würden. Es sei unwahrscheinlich, dass der „Pool“ an AsylbewerberInnen von den Staaten als Rekrutierungsmöglichkeit in Betracht gezogen wird, weshalb sie die Hoffnung, auf diesem Wege das ,Problem‘ zu lösen, als zu optimistisch einschätzt. Weitere Möglichkeiten seien die Konsenssuche durch Dialog, wie sie der UNHCR betreibe. Hier müssten allerdings die „Universalisten“ eine „pragmatische“ Position einnehmen. Damit könne freilich nur die noch radikalere Erosion des Flüchtlingsrechts, nicht jedoch der erhebliche Rückbau der Schutzstandards verhindert werden. Eine weitere Strategie sei die Lösung des ,Flüchtlingsproblems' in den Herkunftsländern: Menschenrechts-Monitoring, Schutz in den Herkunftsregionen, Prävention durch Bekämpfung der Fluchtursachen, etc. Damit könne allerdings nicht die Notwendigkeit von Asylgewährung in den industrialisierten Staaten beseitigt werden. Nach dem von ihr favorisierten Ansatz sollte stärker betont werden, dass , liberal responses to refugees [...] embody a tradition of western thought that is constitutive of the group identity and culture of most European nations. By articulating these liberal goals, communities can also celebrate and reinforce their shared norms. A generous attitude to helping outsiders can reaffirm group identities. [...] And, arguably, it offers a better strategy for mobilising support for more generous refugee policies through emphasising a group's pride in affirming a shared liberal tradition." (Boswell 2002, S. 9f.) Indem sie hier auf nationale Gruppenidentitäten abstellt, um die Aufnahme von ,Outsidern“ zu erreichen, verschärft sie eher Abgrenzungen zwischen ,Eigenem“ und ,Fremdem' und konzipiert den Flüchtlingsschutz als Gnadenakt. Es ist höchst zweifelhaft, inwieweit dies der Stärkung der universalistischen Position förderlich ist. 
Der kurze Überblick zeigt, dass sich fast alle AutorInnen darin einig sind, dass die Flüchtlingsdefinition der GFK zu kurz greift und die Komplexität der aktuellen Migrationsprozesse nicht zu erfassen vermag. Die aus dieser Kritik heraus entwickelten umfassenderen Definitionen sind nur zum Teil geeignet, die Verkürzungen der Konvention zu überwinden. Sofern weit gehende Definitionen angeboten werden, führt dies mitunter - wie das Beispiel Zolberg et al. zeigt - zu Vorschlägen, wie die unterstellte, damit verbundene ,Massenimmigration' vermieden werden kann. Damit ist ein wichtiges Problem angesprochen, von dem die wissenschaftliche Auseinandersetzung um Migration geprägt ist: Trotz vereinzelter kritischer Reflexion, wonach die Festlegung von Flüchtlingseigenschaften eine ,ppolitische Frage“ sei (Treibel 1999, S. 162) und soziologische und rechtliche Definitionen zu trennen seien (vgl. Zolberg et al. 1989, S. 274), und trotz der Aufgabe der im politischen Diskurs bedeutenden Unterscheidung zwischen ,freiwilliger ${ }^{\varsigma}$ und ,unfreiwilliger Migration', sind die wissenschaftlichen noch immer eng mit den rechtlichen Definitionen und dem politischen Diskurs verflochten. Wie bereits angeführt, sind die politisch festgelegten Kategorien und Definitionen in vielerlei Hinsicht zentral, dennoch sollten sie nicht als unhinterfragte Basis angelegt werden, auf die dann soziologische oder politologische Analysen aufbauen und damit die politische Kategorisierung zementieren. Indem Nationalstaaten und nationalstaatliches Handeln die Grundlage (politik)wissenschaftlicher Analyse bilden und selbstverständlich zum Beispiel vom Eigeninteresse der Nationalstaaten an der Bestimmung über den Zugang zum Territorium ausgegangen wird, indem sich viele Studien an den darauf basierenden politischen Zielsetzungen orientieren, zu deren Umsetzung Ratschläge erteilen oder die Perspektive von Nationalstaaten übernehmen, wird grenzüberschreitende Migration als Anomalität und vor allem als ,Problem` definiert. Sie wird zur Ausnahme in einer eigentlich von Sesshaftigkeit und nationalstaatlicher Zugehörigkeit geprägten Welt. Diese „,naturalization of the global regime of nation-states by the social sciences“ wird als „methodological nationalism“ (Wimmer/Glick Schiller 2003, S. 576) bezeichnet und ist vielen sozialwissenschaftlichen Untersuchungen inhärent. Der methodologische Nationalismus drücke sich unter anderem aus in der Annahme, Nationalstaaten seien die natürlichen Einheiten für vergleichende Studien, in der Gleichsetzung von Gesellschaft und Nationalstaat sowie der Gleichsetzung nationaler Interessen mit den Zielen der Sozialwissenschaften (Wimmer/Glick Schiller 2003, S. 576). Gerade die Migrationsforschung und die darin vorgenommene Auswahl von Untersuchungsgegenständen und Untersuchungsdesigns, die Formulierung von Problem- und Begriffsdefinitionen und von Untersuchungsthesen sind durch diese Naturalisierung der nationalstaatlich bedingten Prozesse sozialer und kultureller Schließung geprägt. Eine grundsätzliche Problematisierung und kritische Analyse von Migrationskontrollpolitiken kann unter diesen Prämissen nicht stattfinden. Daher ist die „politikwissenschaftliche Debatte [...] ein gutes Beispiel für die Reproduktion von Herrschaftswissen“ (Benz/Schwenken 2005, S. 366). Gleichwohl ist Andreas 
Wimmer und Nina Glick Schiller in ihrer wichtigen Auseinandersetzung mit dem 'methodologischen Nationalismus' darin zuzustimmen, dass

„[i]t would be certainly naïve to think that we will ever develop a theoretical language not profoundly influenced by the social and political forces around us. Most of us have come to understand that any observation is shaped by the positionality of the observer - including the ones unmasking methodological nationalism."

(Wimmer/ Glick Schiller 2003, S. 600)

\subsubsection{Flüchtlingskonstruktionen und ihre Bedeutung im Migratons- regime}

Im Folgenden werden aus den vorangegangenen Überlegungen einige Schlussfolgerungen über die Bedeutung der Kategorienbildung im Migrationsregime - mit besonderem Fokus auf die Konstruktion der Kategorie Flucht und Asyl - gezogen.

Die Kategorisierung (und Hierarchisierung) von MigrantInnen ist ein wichtiges Instrument der Migrationspolitik und ein zentraler Bestandteil des Migrationsregimes. Dabei erfassen die Kategorien nicht die Komplexität der individuellen Dimensionen des Wanderungsprozesses (Gründe, Umstände, Ziele), sondern sind vorwiegend an nationalstaatlichen Interessen ausgerichtet. Durch das Anknüpfen an Dimensionen wie zum Beispiel bestimmte Gründe (politische Verfolgung) und personenbezogene Merkmale etc. werden verschiedene ,Idealtypen“ von Einwanderern definiert und mit einer legalen Zutrittsmöglichkeit zum nationalstaatlichen Territorium ausgestattet oder explizit davon ausgeschlossen. Weitere Anforderungen werden in der EU durch die ,sichere Drittstaatenregelung' und die ,Dublin-IIVerordnung' zum Beispiel bezüglich der Form des zurückgelegten Weges und des Transportmittels gestellt. Gleichzeitig dient die Typisierung als Grundlage für die verschiedenen hierarchisierten Status innerhalb des Territoriums. Die Kategorienbildung ist daher Teil des Versuchs, Migration zu steuern. Die Kategorien sind nicht statisch und ein für allemal festgelegt. Je nach politischen Konjunkturen und öffentlichem Diskurs kann es Neu- und Umdefinitionen geben, die mitunter zum Verschwinden alter ,Einwanderertypen' und zum Entstehen neuer führen. Ein aktuelles und aufschlussreiches Beispiel ist die Kategorie Flucht und Asyl. Als völkerrechtlich verbrieftes Recht vor dem Hintergrund der Geschehnisse während des Nationalsozialismus und aufgrund politischer Interessenlagen im Kalten Krieg erst vor einigen Jahrzehnten geschaffen, verliert dieser Typus als Einwanderungsmöglichkeit inzwischen wieder an Bedeutung. Dies ist Teil des Wandels des Migrationsregimes in der EU, das zunehmend durch irreguläre Immigration gekennzeichnet ist. Denn mit dem Schließen des Asyl- (und anderer regulärer) gates wird Immigration kaum verhindert, sondern lediglich in andere - irreguläre - Bahnen gelenkt. Nach Andrijasevic et al. (2005, S. 346f.) ist das Sinken der AsylbewerberInnenzahlen daher zwar ,als Ende eines bestimmten Typs der Migration, nämlich 
des Asylregimes, zu verstehen, jedoch nicht als Ende der Migrationen." Ob tatsächlich bereits von einem ,Ende“ oder eher von schwindender Bedeutung des Asylregimes gesprochen werden kann, sollen die Länderstudien der vorliegenden Untersuchung zeigen. Jedoch verdeutlicht diese Erörterung der Bedeutung und Funktion der Kategorienkonstruktion, dass migrationspolitische Eingriffe den Kontext, innerhalb dessen Migration stattfindet und damit auch die Bedingungen, unter denen MigrantInnen wandern, verändern können. Doch ist das Ergebnis dieser Eingriffe nicht (vollständige) Abschottung, sondern ein neues Migrationsregime, in dem sich die Form der Migration verändert hat (vgl. ebd., S. 347). Inwieweit es sich dabei um intendierte oder nicht intendierte Effekte handelt, müsste weiter untersucht werden. ${ }^{42}$

Dass viele MigrantInnen nach der Verengung regulärer Einwanderungsmöglichkeiten irregulär einwandern, macht jedoch auch deutlich, „dass Migration kein mechanischer Prozess ist, dem durch das Zu- oder Aufdrehen gesetzlicher und anderer Wasserhähne ein Ende gesetzt werden kann" (Andrijasevic et al 2005, S. 346). MigrantInnen changieren zwischen den vorgegebenen Kategorien, setzen sich über sie hinweg oder definieren sich selbst entsprechend den Vorgaben immer wieder neu. Die jüngere Forschung hat gezeigt, dass MigrantInnen vielfältige Strategien einsetzen, um ihre Migrationsprojekte zu verwirklichen:

„Migrants have adopted new migration strategies which have often undermined the efforts
of restrictive immigration policies. [...] new restrictions can trigger new forms of resis-
tance. Although power and knowledge are unequally distributed between policy makers
and their targets, migrants are not simply victims and passive recipients of these policies;
they also have the capacity to mobilize resources and create or broaden their own spaces of
control." (Koser/Lutz 1998, S. 4)

Eine zentrale Rolle spielen dabei zum Beispiel Migrationsnetzwerke. Diese liefern MigrantInnen Informationen und helfen, die Risiken und Kosten der Wanderungsprozesse zu senken. Durch diese Netzwerke werden Kommunikations- und Transportstrukturen geschaffen, die Mehrfachmigrationen und damit Migration in Kreisläufen ermöglichen (vgl. Pries 2001, S. 34ff.). Mit diesen und anderen Praktiken werden Migrationen zumindest ein Stück weit von den legalen Einwanderungsmöglichkeiten abgekoppelt und fordern MigrantInnen letztlich auch die politischen EntscheidungsträgerInnen immer wieder heraus. ${ }^{43}$

Mit der rechtlichen Kategorisierung von MigrantInnen - als Flüchtlinge, ArbeitsmigrantInnen usw. - wird der Status des ,Illegalen` erst geschaffen. Die Hie-

\footnotetext{
42 Die Beantwortung dieser Frage ist Teil der Diskussion um die Wirksamkeit der Migrationspolitik und das Verhältnis von migrationspolitischen Zielen, Maßnahmen und Ergebnissen (vgl. Castles 2005, S. 10-24).

${ }^{43}$ Claudia Finotelli (2007) hat in ihrer Studie aufgezeigt, dass MigrantInnen mit ähnlicher Herkunftsstruktur sich in Deutschland als AsylbewerberInnen, in Italien hingegen als ,Illegale aufhielten, was mit den in beiden Ländern unterschiedlich definierten Handlungsspielräumen in den jeweiligen Kategorien zusammenhängt.
} 
,Flucht und Asyl` im Kontext von Migration und Migrationspolitik

rarchisierung der Rechtslagen entlang dieser Kategorien, die mit einer abgestuften Entrechtung bis hin zur Illegalisierung korrespondiert, ist aus Sicht der Einwanderungsländer zwar einerseits - mit Blick auf die irreguläre Migration - mit einem Verlust an Kontrolle über die auf dem Territorium Anwesenden verbunden. Andererseits ist sie aber ökonomisch funktional, da sich illegalisierte Arbeitskräfte gut in die arbeitsmarktpolitischen Deregulierungsbestrebungen und den in vielen Wirtschaftszweigen postulierten Bedarf nach flexiblen und billigen Arbeitskräften einpassen. Die Arbeit der Sans Papiers stellt in vielen Branchen einen zentralen Pfeiler dar (beispielsweise in der Bau- und Landwirtschaft, Gebäudereinigung, Haushalts- und Pflegedienstleistungen sowie Sexarbeit). Durch Visa- und Grenzpolitiken kann und soll daher der physische Zugang zum Territorium nicht vollständig kontrolliert werden, aber zusammen mit der umso effizienteren Regulierung des Zugangs zu sozialen und politischen Rechten innerhalb des Territoriums können hier die Interessen verschiedener nationaler AkteurInnen bedient werden: ${ }^{44}$ So wird die Nachfrage nach flexiblen Arbeitskräften befriedigt und gleichzeitig eine Teilhabe ,Anderer ${ }^{6}$ am national definierten Sozialstaat verhindert; zudem kann so die Konservierung des als homogen imaginierten Nationalstaats suggeriert werden.

Die zunehmende irreguläre Immigration zeigt auch, dass theoretische Erklärungsansätze, die MigrantInnen noch immer nicht als wichtige AkteurInnen im Migrationsregime verstehen, zu kurz greifen. ${ }^{45}$ Vielmehr muss stärker als bisher berücksichtigt werden, „dass Migration ein Moment der Selbstständigkeit gegenüber politischen Maßnahmen besitzt, die sie zu kontrollieren beabsichtigen." (Andrijasevic et al 2005, S. 347) Damit soll jedoch nicht einer gänzlichen ,Autonomie der Migration ${ }^{4} 46$ das Wort geredet werden, die tendenziell ausblendet, dass die migrationspolitischen Regulierungen für viele MigrantInnen wirkungsmächtig sind und die Umsetzung ihrer Migrationsprojekte verhindern, verzögern oder prekarisieren können. Eine differenziertere Perspektive muss davon ausgehen, dass MigrantInnen je nach Herkunftssituation, Motiven und Hintergründen, Bildungsniveau, Rückkehrmöglichkeit, Geschlecht, Alter, etc. unterschiedlich von

44 Zolberg (vgl. 1999, S. 77) zeigt dies unter anderem am Beispiel der US-Immigrationspolitik an der Grenze zu Mexiko auf: „The hybrid regulatory system that emerged along the southern border in the 1920s - an effectively enforced barrier to formal immigration - combined with laissez-faire regarding the movement of labor, makes sense if the objective was to deter, not the physical entry of Mexicans into the United States, but rather their social and political entry into American society."

45 Als Beispiel für die gänzliche Ausblendung des Akteurscharakters von MigrantInnen sei auf ein Zitat von Annette Treibel (1999, S. 158) verwiesen, die schreibt: „Das Thema Flucht weist einige Besonderheiten auf, die für die sonstige Migrationsforschung nicht gelten: Fluchtmigration ist ein Feld, das aufgeteilt ist zwischen internationaler Politik, Menschenrechtsgruppen, Hilfsorganisationen und politikwissenschaftlicher Analyse. Ein genuin soziologisches Feld ist es (bisher) nicht.“ Bei der Aufteilung des Feldes sucht man vergeblich nach den Flüchtlingen, die in der Lesart von Annette Treibel an der Fluchtmigration offenbar unbeteiligt sind.

${ }^{46}$ Vgl. den Abschnitt 2.2.4 „Autonomie“ oder „Eigensinnigkeit“ der Migration. 
den Maßnahmen betroffen sind und sich daher je unterschiedliche Handlungsspielräume eröffnen. Insofern gilt eine Selbstständigkeit gegenüber den migrationspolitischen Regulierungen nicht für alle MigrantInnen und alle Phasen des Migrationsprozesses gleichermaßen. Dies zeigt sich an denjenigen, denen die Netzwerk- und andere Ressourcen fehlen, die entweder gar nicht erst ihr Herkunftsland verlassen oder lediglich in Nachbarregionen migrieren können oder bei dem Versuch, nach Norden zu gelangen sterben, in Lagern inhaftiert werden oder unter prekären Bedingungen in der, Illegalität' leben.

Vor diesem Hintergrund ist es nicht das Ziel der vorliegenden Untersuchung, eine weitere Definition des Flüchtlingsbegriffs vorzunehmen und auf ihre politischen Umsetzungschancen hin abzuklopfen. Stattdessen soll konträr dazu die Kategorisierung von Migrationen in den Vordergrund der Analyse gerückt und aufgezeigt werden, welche Rolle die Kategorienkonstruktion in der Migrationspolitik spielt. Am Beispiel der Kategorie Flucht und Asyl wird gezeigt, welche Debatten, Definitionen und Regulierungen sich im Wechselspiel zwischen EU und Mitgliedstaaten bezüglich der Kategorie Flucht und Asyl aufspüren lassen, inwieweit die Kategorie in den letzten Jahren einem Wandel unterlag und inwieweit dies für Migrationsprozesse wirkungsmächtig war. Indem die Änderungen bei diesen Konstruktionen und der Umgang von Flüchtlingen mit diesen Änderungen nachgezeichnet werden, kann auch der Wandel der Migrationsregime anhand der Kategorisierungen gezeigt werden.

\subsection{Untersuchungsthesen}

Vor dem Hintergrund der Vorüberlegungen aus den vorangegangenen Abschnitten sollen im Folgenden zwei unterschiedliche europäische Asylregime - Deutschland als altes EU-Mitglied und Einwanderungsland sowie Polen als neues EUMitglied und Auswanderungsland - miteinander kontrastiert werden. Die folgenden Thesen werden die Untersuchung leiten und im Fazit abschließend diskutiert: Es wird davon ausgegangen, dass in den vergangenen zwei Jahrzehnten Verschiebungen zwischen migrationspolitischen Kategorienkonstruktionen und zwischen einzelnen Mitgliedstaaten stattgefunden haben, im Zuge derer die Bedeutung der Kategorie Flucht und Asyl für Migrationsbewegungen in (die alten) EU-Mitgliedstaaten deutlich zurückgegangen ist. Es haben sich neue migrationspolitische Präferenzen herausgebildet, aufgrund derer eine selektive Öffnung für bestimmte MigrantInnen nach ökonomischen Nutzen-Aspekten stattgefunden hat, während gleichzeitig versucht wird, ,unerwünschte' Immigration, die diesen Anforderungen nicht gerecht wird, zu verhindern bzw. weitestgehend zu entrechten. Im Zuge dessen hat sich ein entscheidender Wandel im Umgang mit Flüchtlingen und Asylsuchenden vollzogen. Die Restriktion der auf Flüchtlinge bezogenen Politiken hat zu einem Verschwinden' der Flüchtlinge aus dem geografischen Kern der EU 
durch a) Auslagerung in die neuen Mitgliedstaaten an den Außengrenzen und in die Transit- und Herkunftsländer sowie durch b) Illegalisierung geführt.

Anhand der konkreten Regulierungen in Deutschland und Polen lassen sich Aussagen über einige prominente Thesen über das Funktionieren und die Wirkung von Migrationspolitik treffen. So wird davon ausgegangen, dass es in den einzelnen Ländern eine zunehmend ähnliche Entwicklung (Konvergenz) hinsichtlich der Migrationspolitiken gibt. Dies betrifft insbesondere das migrationspolitische Instrument der Kategorienkonstruktion und Hierarchisierung von MigrantInnen. Gleichzeitig können die Länderbeispiele dazu dienen, die Stichhaltigkeit der gap-Hypothese und der zu ihrer Erklärung identifizierten Faktoren zu spezifizieren. Eine Kluft kann dabei zwischen den politisch konstruierten Einwandererkategorien einerseits und den Migrationsgründen, Zielen und Strategien der MigrantInnen andererseits vermutet werden, die wiederum aufgrund der ,Autonomie ${ }^{6}$ oder ,Eigensinnigkeit' der Migration eine Kluft zwischen den proklamierten Zielen der Migrationspolitik und den erzielten Effekten bedingt.

Dennoch zeigt sich anhand des Beispiels ,Flucht und Asyl', dass daraus nicht zwangsläufig ein Scheitern der (offiziellen) Migrationspolitiken abgeleitet werden kann: Zwar kann Migrationspolitik Migrationen nur in begrenztem Umfang tatsächlich zielgerichtet steuern. Sie ist jedoch wirkungsmächtig hinsichtlich der Bedingungen, unter denen sich Migrationsprozesse vollziehen und damit auch hinsichtlich der Sozialstruktur der Migrierenden (Geschlecht, Alter, sozialer Status der MigrantInnen, etc.), der Form der Migrationsprozesse und der Zuweisung von hierarchisierten Rechtsstatus innerhalb des Territoriums.

Dabei können über die Intendiertheit bzw. Nicht-Intendiertheit der Konsequenzen der Migrationspolitik nur Mutmaßungen angestellt und Indizien für möglicherweise „versteckte[...] Zielsetzungen“ (Castles 2005, S. 12) gesammelt werden. In dieser Hinsicht wird jedoch davon ausgegangen, dass die mit der Kategorisierung und Hierarchisierung verbundene abgestufte Entrechtung - in Form einer Hierarchisierung von Rechtslagen bis hin zur Illegalisierung - eine eigene Funktion im Migrationsregime im Interesse der Zielländer erfüllt. Bezüglich der Frage nach der Kontrollfähigkeit von Nationalstaaten soll daher ein stärkerer Fokus auf das Verhältnis von staatlichen Kontrollagenturen und Migrationsbewegungen selbst gelegt werden. Dies impliziert, sich nicht der einen (Kontrollverlust) oder anderen (Kontrollfähigkeit) Position anzuschließen. Während die Auslagerung von unerwünschten ImmigrantInnen aus der Sicht der Kontrollagenturen als erfolgreiche Kontroll- und Steuerungspolitik gewertet werden könnte, bedeutet die mit den hohen Zugangshürden erzeugte - und durchaus funktionale - Illegalisierung auch einen Kontrollverlust. Dies zeigt, dass die Rede vom Kontrollverlust oder Kontrollgewinn die Komplexität und Widersprüchlichkeit der Entwicklungen verkennt und sich das Migrationsgeschehen vielmehr durch eine Gleichzeitigkeit von Kontrollverlust und Kontrollgewinn auf Seiten der regulierenden AkteurInnen und damit gleichzeitig durch Verlust und Gewinn von Handlungsspielräumen auf 
Seiten der MigrantInnen auszeichnet. Es geht also nicht darum, mit der Diskussion um die Kontroll- und Steuerungsfähigkeit „Migration zum Problem [zu] stilisieren“ (Birsl 2005, S. 52), sondern im Gegenteil die Handlungsspielräume von MigrantInnen auszuloten, die sich mit dem Wandel des Asylregimes ergeben. 



\section{Untersuchungsdesign und Methoden}

In den folgenden Abschnitten wird das methodische Vorgehen dargelegt, das für diese Studie gewählt wurde. Ausgehend von einigen grundsätzlichen Überlegungen zur komparativen Methode wird das Untersuchungsdesign in Anlehnung an das von Theda Skocpol und Margaret Somers (1980) beschriebene „Contrast of Contexts"-Design entwickelt. Auf dieser Grundlage wird die Auswahl der beiden Länder Polen und Deutschland erläutert. Schließlich werden die verwendeten Erhebungsmethoden vorgestellt und insbesondere der Zugang zu empirischem Material mittels ExpertInnen-Interviews eingehend diskutiert.

\subsection{Die komparative Methode}

Die vergleichende Methode in den Sozialwissenschaften ist gekennzeichnet von der Auseinandersetzung zwischen variablen- und fallorientierten Herangehensweisen. Variablenorientierte KomparatistInnen (vgl. King et al. 1994; Lijphart 1971) testen theoriegeleitete Hypothesen. Dafür zerlegen sie ihren Untersuchungsgegenstand in Variablen und untersuchen die Beziehungen zwischen diesen mittels statistischer Verfahren bzw. durch Korrelation. Variablenorientierte Forschung bezieht größere Fallzahlen ein. Ziel ist es, verallgemeinerbare Aussagen zu treffen, die - bis zur Widerlegung - universelle, also raumzeitlich unabhängige Gültigkeit beanspruchen können. KritikerInnen beanstanden, dass die statistische Kontrolle 
auf der Basis stark vereinfachender Annahmen operiert. Zudem wird der Charakter der getroffenen Aussagen direkt durch den Charakter der Methode bestimmt: "That is, these methods culminate in probabilistic statements about a variable's average" (Ragin 1987, S. 64).

Fallorientierte KomparatistInnen (vgl. Ragin 1987; Rüschemeyer 2003) hingegen versuchen, der Komplexität ihrer Fälle gerecht werden und sie kontextbezogen und geschichtlich fundiert zu untersuchen. Dies macht Methoden erforderlich, die sensibel für Zeit, Ort, Akteure und Prozesse sind (vgl. Ragin 1987, S. 11). Fallorientierte Methoden verlangen eine Betrachtung der Fälle als Ganze (,as whole entities“, Ragin 1987, S. 52) und nicht als bloße Ansammlung von Variablen (,collections of variables“, ebd. S. 52). Daraus ergibt sich das „viele Variablen, kleine Fallzahlen"-Dilemma: Je größer die empirische oder theoretische Spezifität, umso kleiner ist die Zahl der Fälle, die für die Untersuchung einschlägig sind. Je geringer die Fallzahl, umso schwieriger wird es, eine erklärende Aussage zu treffen, die dem Standard der ,Mainstream-Sozialwissenschaft ${ }^{`}$ entspricht, insbesondere ihrem quantitativen Zweig (vgl. ebd., S. 10). Dies hat zur Folge, dass nur eine begrenzte Zahl an Fällen einbezogen und nur kontextbezogene Aussagen getroffen werden können. Mit dieser Forschungslogik können daher Theorien mittlerer Reichweite, die sich nur auf einen bestimmten Gegenstand beziehen, entwickelt oder bestimmte Muster aufgedeckt werden. Ziel der fallorientierten KomparatistInnen ist es nach Charles C. Ragin (1987), Ähnlichkeiten und Unterschiede zwischen makrosozialen Einheiten zu identifizieren. Mit diesem Wissen könne die Bedeutung historischer Ergebnisse und Prozesse für gegenwärtige institutionelle Arrangements verstanden, erklärt und interpretiert werden. Dabei beschränken sie sich nach Ragin nicht auf eine Katalogisierung und Erklärung zwischengesellschaftlicher Ähnlichkeiten und Unterschiede, sondern sind an den Fällen an sich interessiert, ihren unterschiedlichen historischen Bedingungen usw. Diese holistische Herangehensweise erfordert einen eher offenen Forschungsprozess, der einen Dialog zwischen Evidenz und Theorie, also eine Überarbeitung der These(n) während des Forschungsprozesses ermöglicht (vgl. Ragin 1987, S. 42, 52).

Zusammenfassend lässt sich konstatieren, dass die Grenzen der variablenorientierten Strategie und die Grenzen der fallorientierten Strategie „converge morphologically“ (Ragin 1987, S. 68): Die Anwendung der fallorientierten Strategie wird schwieriger, je größer die Fallzahl ist, weil sie holistisch operiert (Problem der erforderlichen Vertrautheit mit den Fällen, Zahl der Vergleiche steigt rasant an). Das Problem der variablenorientierten Strategie verläuft parallel dazu: Je komplexer das kausale Argument ist, umso größer die methodologischen Probleme. Komplexe kombinierte Annahmen können nicht getestet, aber auch keine Subtypen gebildet werden, wenn nicht genügend Fälle vorhanden sind (vgl. ebd.).

Auch wenn der Graben zwischen fallorientierten und variablenorientierten Verfahren oft unüberbrückbar scheint, gibt es inzwischen zahlreiche Versuche, kombinierte Ansätze zu entwickeln. Dabei spricht vieles für eine komplementäre 
Ergänzung qualitativer und quantitativer Komparatistik: Die holistische Exploration neuer Forschungsgegenstände ist eine Voraussetzung für fundierte quantitative Analysen mit großen Fallzahlen. Mittels qualitativer Verfahren können zum Beispiel für quantitative Studien infrage kommende Variablen zunächst einmal identifiziert werden. Andererseits können qualitative Studien zur Plausibilisierung kausaler Annahmen beitragen oder auch deren Begrenztheit illustrieren. Die Wahl des jeweiligen Verfahrens ist abhängig von der interessierenden Forschungsfrage und setzt ein Bewusstsein für die Grenzen und Chancen beider Herangehensweisen voraus, insbesondere im Hinblick auf die Reichweite der zu treffenden Aussagen:

„It is important to bear in mind, however, that the deeper into the individual case a study probes, the less able it is to make generalizations. All good comparative studies recognize the inferential limitation of their analyses. "(Landman 2002, S. 922)

Vor dem Hintergrund dieser grundsätzlichen Überlegungen zu den Möglichkeiten und Grenzen vergleichender Verfahren wird hier der Wandel der Kategorie Flucht und Asyl mittels fallorientierter Methoden bearbeitet. Die vorliegende Studie hat vor allem explorativen Charakter und fokussiert weniger auf kausale Analyse als auf die Aufdeckung von Mustern und Trends, die Grundlage weitergehender Forschung sein können. Angesichts dieses Anspruchs wird sich die Studie an das von Theda Skocpol und Margaret Somers (1980) vorgestellte „contrast of contexts“Design (Kontrastierung von Kontexten) anlehnen.

In ihrer systematischen Untersuchung der Methodologie vergleichender historischer Forschung identifizieren Skocpol und Somers drei Logiken: die parallele Demonstration von Theorie („Parallel Demonstration of Theory“), die Kontrastierung verschiedener Kontexte („Contrast of Contexts“) und die Makro-Kausalanalyse („Macro-Causal-Analysis“). Die Makro-Kausalanalyse hat den Autorinnen zufolge den Anspruch, kausale Hypothesen für Makrostrukturen und -prozesse zu bestätigen oder zu entkräften oder neue Generalisierungen zu entwickeln:

„Macro-analysts [...] tend to move back and forth between alternative explanatory bypotheses and comparisons of relevant aspects of the histories of two or more cases." (Skocpol/Somers 1980, S. 182)

Damit ähnele die Forschungslogik der makrokausalen Analyse der der statistischen Analyse und sei also ,a kind of multivariate analysis to which scholars turn in order to validate causal statements about macro-phenomena for which, inherently, there are too many variables and not enough cases" (ebd.). Die dieser Logik zugrunde liegenden analytischen Designs sind John Stuart Mills (1874, S. 278-284) induktive „Method of Agreement“ (Konkordanzmethode) und „Method of Difference“" (Differenzmethode). ${ }^{47}$

$47 \mathrm{Zu}$ Mills Methodenkanon gehört auch noch die Kombination dieser beiden Verfahren, die er als indirekte Differenzmethode bezeichnet (vgl. Mill 1874, S. 283f.). 
Der makro-kausalen Analyse mit ihrem kausalanalytischen Anspruch stellen Skocpol und Somers die „Parallel Demonstration of Theory“ und das „Contrast of Contexts“-Design gegenüber. Die „Parallele Demonstration von Theorie“ zielt darauf,

\section{„to persuade the reader that a given, explicitly delineated hypothesis or theory can repeat- edly demonstrate its fruitfulness - its ability convincingly to order the evidence - when ap- plied to a series of relevant historical trajectories. "(Skocpol/Somers 1980, S. 176)48}

Eine Theorie bzw. Hypothese wird also auf verschiedene Fälle, die möglichst viele Möglichkeiten abdecken sollen und deren Unterschiede vor allem in kontextbezogenen Besonderheiten liegen, angewendet (deduktiv). Dabei wird zuerst ein theoretisches Modell bzw. eine Hypothese formuliert und im zweiten Schritt zur Illustration anhand der Fälle übergegangen. Da die Fälle mit Blick auf die Theorie ausgewählt werden (positive Fallauswahl), kann mit diesem Design nur eine Illustration, nicht jedoch eine Bestätigung einer Theorie erfolgen (vgl. Skocpol/Somers 1980, S. 191).

Im Gegensatz dazu wird mit dem „Contrast of Contexts“-Design versucht, die einzigartigen Merkmale jedes einzelnen Falles herauszuarbeiten und zu zeigen, wie diese besonderen Eigenschaften die Entstehung genereller sozialer Prozesse beeinflussen (vgl. Skocpol/Somers 1980, S. 178). Die ausgewählten Fälle sollen ein möglichst breites Spektrum umfassen, noch wichtiger ist aber ihre klare Unterscheidbarkeit (vgl. ebd., S. 179). Die Kontraste zwischen den individuellen Fällen werden mit Bezug auf „broad themes“, „orienting questions“ oder Idealtypen entwickelt (ebd., S. 178). „Broad themes“ und „orienting questions“ bilden den Rahmen für das Aufzeigen von Unterschieden zwischen den Fällen. Idealtypen dienen als Benchmarks, gegen die die einzigartigen Eigenschaften jedes Falles herausgearbeitet werden können (vgl. ebd.). Dabei können „themes“, „questions“ oder Idealtypen zu Beginn des Forschungsprozesses formuliert werden oder aber währenddessen entstehen. Statt expliziter Theoretisierung strebt dieses Design vor allem danach, die historische Integrität jedes einzelnen Falles als Ganzem zu respektieren und anzuerkennen, dass jeder für sich eine nicht-reduzierbare Einheit und eine komplexe soziohistorische Konfiguration ,in its own right" (ebd.) darstellt. Damit sollen vor allem die Grenzen übermäßig generalisierender Theorien aufgezeigt werden. Die Erarbeitung neuer erklärender Generalisierungen wird zugunsten dieses deskriptiven Holismus nicht angestrebt. Das Verfahren eignet sich besonders gut für die Untersuchung von zwei Fällen: „Books based upon straightforward paired comparisons nicely illustrate the basic essence of Contrastoriented comparative history“ (ebd.). Irreführenderweise wird die „Parallele De-

\footnotetext{
48 „The Parallel comparativists seek above all to demonstrate that a theory similarly holds good from case to case; for them differences among the cases are primarily contextual particularities against which to highlight the generality of the processes with which their theories are basically concerned.“ (Skocpol/Somers 1980, S. 178)
} 
monstration von Theorie“ bisweilen mit der Konkordanz-, das „Contrast of Contexts“-Design mit der Differenzmethode gleichgesetzt. Diese Einordnung steht aber der expliziten Trennung, die Skocpol und Somers zwischen den beiden Designs und der Makro-Kausal-Analyse vornehmen, entgegen (vgl. kritisch Trampusch 2000, S. 68, Fn. 43). ${ }^{49}$

Für die vorliegende Untersuchung werden also zwei möglichst trennscharfe Untersuchungseinheiten in ihrer Komplexität dar- und einander gegenübergestellt. Die asylpolitischen Entwicklungen in Polen und Deutschland werden in zwei Fallstudien herausgearbeitet und schließlich aufeinander bezogen, um Ähnlichkeiten, Unterschiede und Verschiebungen in ihren jeweiligen Kontexten zu zeigen. Ziel ist es also, Entwicklung und Wandel der Konstruktion und Regulierung der Kategorie Flucht und Asyl in ein Verhältnis zu den Kontexten zu stellen, in denen sie etabliert werden, das heißt herauszufinden, ob sich dieser Wandel in den Ländern kontextspezifisch unterschiedlich vollzieht. Die Kontrastierung durch die Gegenüberstellung der zwei Untersuchungseinheiten soll dazu beitragen Abweichungen, also Variationen der Kategorisierungen, aber auch Wechselwirkungen zwischen den Asylregimen, also ihr Verhältnis zueinander, zu zeigen und theoretische Annahmen und Generalisierungen der Migrations(politik)theorie in Frage zu stellen. Beide Länderstudien basieren im Sinne der von Skocpol und Somers genannten ,broad themes' und ,orienting questions' auf einer weitgehend kongruenten Untersuchungsstruktur.

Dennoch handelt es sich nicht um eine ,reine' Anwendung des von Skocpol und Somers beschriebenen Designs. Indem die beiden Fälle jeweils eingehend untersucht und schließlich aufeinander bezogen werden, soll nicht nur nach Ähnlichkeiten und Unterschieden zwischen den Kontexten, sondern auch nach Beziehungen, Wechselwirkungen und Verschiebungen zwischen ihnen gefahndet werden. Darüber hinaus ist die Untersuchung nicht so theorieabstinent angelegt, wie es die ,reine Lehre' des Verfahrens fordert. Die verschiedenen theoretischen Ansätze, von denen die Studie inspiriert wurde, wurden am Anfang der Untersuchung transparent gemacht. Dies trägt der Einsicht Rechnung, dass es unmöglich ist, jegliche Annahmen und Theorien völlig auszublenden, die sich aus Studium und Forschungspraxis speisen und die deshalb jede Forschungsarbeit beeinflussen. Daher wird hier explizit gemacht, was Skocpol und Somers bei anderen Arbeiten als implizit vorhanden identifiziert haben - dass zum Beispiel spätestens bei

\footnotetext{
${ }^{49}$ Darüber hinaus veranschaulichen Skocpol und Somers in einer „Triangle of Comparative History“ die Möglichkeiten einer Kombination von je zwei der drei Logiken (vgl. Skocpol/Somers 1980, S. 188). Sie kommen aber zu dem Schluss, dass „, [c]omparative historical studies seem to work best when they are done primarily according to one logic or another" (ebd., S. 196). Dennoch bilden die drei Designs ein komplementäres System, in dem die Grenzen des einen durch die Möglichkeiten des anderen Verfahrens überwunden werden können, wie die Autorinnen in „a Cycle of Transitions“" zeigen (vgl. ebd., S. 196f.).
} 
Untersuchungsdesign und Methoden

der Formulierung von Idealtypen, „themes“ und „,questions“ theoretische Überlegungen eine Rolle spielen, allerdings nicht als solche deklariert werden. ${ }^{50}$

\subsection{Fallauswahl}

Voraussetzung für die Anwendung der komparativen Methode ist die Vergleichbarkeit der Fallbeispiele. Die Vergleichseinheiten - in diesem Falle Nationalstaaten - müssen deshalb eine minimale Homogenität in für die Fragestellung relevanten Aspekten aufweisen (vgl. Berg-Schlosser 2003, S. 107). Für die ausgewählten Länder Deutschland und Polen gilt dies insofern, als sie Teil der Grundgesamtheit der - seit dem 1. Januar 2007 - 27 EU-Mitgliedstaaten sind. Weitere verbindende Komponenten zwischen diesen Ländern liegen in der demokratischen Verfasstheit und in der Tatsache begründet, dass es sich um Länder handelt, die sich - zu unterschiedlichen Zeitpunkten - von ehemaligen Abwanderungsländern zu Einwanderungsländern entwickelt haben bzw. entwickeln (vgl. Currle 2004). Hinzu kommt im Hinblick auf die ,räumliche Taxonomie“ (Aarebrot/Bakka 2003, S. 67) die geografische Nähe der beiden Länder. Die Nachbarschaft von Deutschland und Polen bedingt „eine gemeinsame historische Erfahrung“ (ebd., S. 68), die sich insbesondere in der Verflechtung der beiden Staaten durch Migrationsprozesse manifestiert, welche sich in verschiedenen Richtungen vollzogen haben und noch immer vollziehen. Aufgrund der aktuellen Asylzuständigkeitsregelungen in der EU hat die geografische Nähe der beiden Staaten und ihre räumliche Positionierung innerhalb der EU einmal mehr spezifische migrationspolitische Dynamiken erzeugt, die zu einer wachsenden Zahl von Asylsuchenden in Polen geführt haben, wie im Folgenden noch näher erläutert wird.

Ansonsten zeichnen sich beide Fälle durch ihre Unterschiedlichkeit aus: Sie unterscheiden sich hinsichtlich

- der Entwicklung ihrer Asylregime,

- der Bedeutung, die Asyl als gate of entry hat sowie

- ihrer Rolle im Prozess der Europäischen Integration bzw. dem Einfluss, den die europäischen Vorgaben auf ihre Regime haben.

\footnotetext{
50 „Of course the price paid in Contrast-oriented comparative histories is that descriptive holism precludes the development of explanatory arguments, even when these are implicitly present, crying to be drawn out of the comparative-historical materials. [...] Worse yet, most (if not all) works of Contrast-oriented comparative history actually smuggle implicit theoretical explanations into their case accounts. Usually this happens through the device of posing common themes or questions to provide the framework for the case accounts and comparisons" (Skocpol/Somers 1980, S. 193). Sie kritisieren dies am Beispiel der Arbeiten von Reinhard Bendix, in dessen Einleitung und durch dessen Fallstudien hindurch sich bestimmte Erklärungsansätze fänden. Dies sei zwar wenig überraschend, da es schwierig sein dürfte, tausende Jahre Weltgeschichte ohne theoretische Leitlinie anzuschauen. Problematisch aber sei es, dass Bendix - „like any good Contrast-oriented comparative historian" - jegliche theoretische oder erklärende Zielsetzung abstreite (ebd.).
} 
Gemeinsam ist ihnen jedoch die Restriktivität der Asylpolitiken, die sich zum Beispiel in der geringen Quote anerkannter AsylbewerberInnen manifestiert. Die genannten Aspekte werden im Folgenden näher erläutert.

Asylregime

Das Grundgesetz der Bundesrepublik enthielt seit seiner Entstehung 1949 das Recht auf Asyl für politisch Verfolgte. Dieses wurde im Zuge des „Asylkompromisses“ 1993 insbesondere durch die „sichere Drittstaaten“- und die „sichere Herkunftsstaatenregelung“ stark eingeschränkt. Dies und die Verabschiedung des Asylbewerberleistungsgesetzes, das eine deutliche Senkung der Transferleistungen für AsylbewerberInnen unter das Sozialhilfeniveau beinhaltet sowie des Asylverfahrensgesetzes bilden zusammen eine fortan restriktive Asylgesetzgebung (vgl. Birsl 2004). In der Flüchtlingspolitik findet sich in der Bundesrepublik ein breites Spektrum unterschiedlich ausgerichteter und unterschiedlich mächtiger AkteurInnen, die beständig um die Deutungshoheit in diesem Politikfeld und um seine Ausgestaltung ringen.

Polen verankerte erst 1997 die Gewährung des Flüchtlingsstatus in seiner Verfassung (Art. $56 \mathrm{II}$ ) und regelte die Umsetzungsbestimmungen im neuen Ausländergesetz (vgl. Currle 2004, S. 364f.). Die Regelungen werden als restriktiv eingeschätzt (vgl. Bem 2004). Mit den steigenden Flüchtlingszahlen vergrößert sich auch sukzessive das im Augenblick noch recht überschaubare Feld der flüchtlingspolitischen AkteurInnen sowie das eher marginale öffentliche Interesse am Thema.

\section{Bedeutung von Asyl als gate of entry}

In absoluten Zahlen gemessen war die Bundesrepublik eines der bedeutendsten Aufnahmeländer für Asylsuchende in Europa ${ }^{51}$. Seit dem Anwerbestopp Anfang der 1970er Jahre gewann dieses gate of entry neben dem Familiennachzug als eine der wenigen verbliebenen Einwanderungsmöglichkeiten stark an Bedeutung. Angesichts der niedrigen Anerkennungsquoten stellt es heute allerdings lediglich ein ,Nadelöhr' dar.

Polen ist zwar - stellt man Ab- und Einwanderung gegenüber - weiterhin ein Abwanderungsland, seit Beginn der 1990er Jahre jedoch gleichzeitig Ziel von Einwandernden aus Osteuropa. Ein Großteil dieser Immigration ist temporär ausgerichtet und vollzieht sich irregulär, zum Beispiel die Pendelmigrationen ukrainischer ArbeiterInnen. Zudem hat Polen seit Mitte der 1990er Jahre deutlich steigende Asylantragszahlen zu verzeichnen, was auf eine zunehmend wichtigere Rolle von Asyl als gate of entry hinweist (vgl. Tabelle $9 \mathrm{im}$ Anhang). Dies ist nicht

\footnotetext{
${ }^{51}$ Betrachtet man das Verhältnis der Asylanträge zur Einwohnerzahl, relativiert sich das Bild: Für den Zeitraum von 1995-2004 steht Deutschland dann nur noch an 13. Stelle in Europa, während es in absoluten Zahlen die Liste anführt (vgl. UNHCR 2006, Annex 8, S. 225).
} 
Untersuchungsdesign und Methoden

zuletzt bedingt durch seine besondere geografische Rolle als Land, dass eine rund $1000 \mathrm{~km}$ lange EU-Außengrenze zu überwachen hat sowie durch die asylpolitischen Maßnahmen und Zuständigkeitsregelungen westeuropäischer Staaten und der EU, wodurch Polen für Flüchtlinge vom einstigen Transit- zum Zielland wurde (vgl. Currle 2004, S. 378f.).

Rolle der Länder im EU-Integrationsprozess, Einfluss auf EU-Ebene und Einfluss der EU-Vorgaben auf die Asylregime

Deutschland gehört zu der Ländergruppe, die am längsten am Integrationsprozess beteiligt sind und eine dominierende Rolle in diesem Prozess innehaben. Im Zusammenhang mit der Vereinheitlichung der Asylpolitik auf EU-Ebene zeichnete es sich insbesondere durch seine zum Teil massive Blockadehaltung aus.

Polen hat als neues EU-Mitglied bis zum Beitritt eine marginale Rolle im Integrationsprozess gespielt und war an der Aushandlung der asylpolitischen EURichtlinien bis dahin formal nicht beteiligt. In der Literatur wird davon ausgegangen, dass der EU-Beitritt ein maßgeblicher Faktor für die zeitliche sowie inhaltliche Gestaltung der polnischen Gesetzgebung im Migrationsbereich war (vgl. Currle 2004, S. 362; Geddes 2003, S. 173; Iglicka et al. 2005, S. 2; vgl. kritisch dazu Kicinger 2005).

Die ungleichen Positionen der Länder im Verhältnis zu den EU-Vorgaben spiegeln sich besonders deutlich im Bereich der Zuständigkeitsregelungen wider: So zeigt sich, dass ein Teil der Flüchtlinge, die aus dem geografischen, Kern' Europas ,verschwinden', in Polen sprichwörtlich wieder ,auftaucht'. Mehr noch, durch die prekäre soziale Situation von Flüchtlingen auf der polnischen Seite und die Anwendung der Dublin-II-Verordnung auf deutscher Seite kommt es zu einem ,Hin- und Her von Flüchtlingsbewegungen. Damit stehen die beiden Länder auch als exemplarisches Beispiel für die Verschiebung von Migrationen aus dem Kern der EU an die Ränder und von dort weiter in die Transit- und Herkunftsländer, der sich Flüchtlinge allerdings mitunter hartnäckig widersetzen.

Zusammenfassend betrachtet, zeichnen sich die Untersuchungsländer durch unterschiedliche Positionen im Integrationsprozess und hinsichtlich der Ausgestaltung ihrer Asylregime aus. Deutschland spielte und spielt eine zentrale Rolle im Integrationsprozess, Asyl ist ein wichtiges gate of entry, jedoch durch die restriktive Ausgestaltung der Asylgesetzgebung nur schwer zugänglich. Mit Deutschland wird zudem ein Land betrachtet, in dem das interessierende Politikfeld eine längere, stark politisierte Geschichte hat. Polen kontrastiert damit, indem es seine Flüchtlingspolitik erst in jüngerer Zeit und ohne starke Politisierung installiert hat. Polen ist Teil der neuen Mitgliedstaaten und war insofern an der Gestaltung der asylpolitischen Vorgaben kaum beteiligt, hat jedoch - in Vorbereitung auf den Beitritt seine Asylgesetzgebung bereits mit restriktiven Elementen ausgestattet. Die Aufnahme von AsylbewerberInnen gewinnt - nicht zuletzt aufgrund der Zuständigkeitsregelungen innerhalb der EU - in Polen an Bedeutung. Die beiden Länder 
stehen damit beispielhaft für die Verschiebungen, die sich zwischen den Asylregimen innerhalb der EU vollziehen.

Der Untersuchungszeitraum wurde für beide Länder entsprechend der Entwicklung ihrer Asylregime gewählt - so können beide Fälle in ihrer Gesamtheit betrachtet werden. Daher beginnen die Ausführungen zur Bundesrepublik mit der Entstehung des bundesdeutschen Asylrechts 1949 und zu Polen mit der Entstehung seines Asylregimes Anfang der 1990er Jahre. Beide Länderstudien enden mit dem Jahr 2007. Der Fokus der kontrastierenden Analyse liegt auf der Entwicklung der beiden Asylregime in den vergangenen knapp 20 Jahren seit 1990.

Den beiden Länderstudien wird ein Kapitel zu den asylpolitischen Entwicklungen auf EU-Ebene vorangestellt. Angesichts der Versuche, die asylpolitischen Regelungen in allen EU-Mitgliedstaaten $\mathrm{zu}$ vereinheitlichen und die operative Zusammenarbeit in diesem Bereich zu erweitern, werden auch die Vorgaben und Aktivitäten der EU in diesem Politikfeld dargestellt und analysiert. Sie spielen eine zunehmend wichtige Rolle für die Gestaltung der Asylregime in den Mitgliedstaaten und zeigen gleichzeitig, welche Konzepte und Maßnahmen im Zusammenspiel der Mitgliedstaaten mit der Kategorie Flucht und Asyl verknüpft werden. Hierfür werden die wichtigsten asylrechtlichen Richtlinien, Verordnungen und weitere Maßnahmen sowie Stellungnahmen von Kommission, Rat und Parlament hinsichtlich der Ausgestaltung der Kategorie Flucht und Asyl, ihrer sprachlichen Präsentation und der Bedeutung dieser Supranationalisierungsprozesse für den Wandel des Asylrechts untersucht. In den Länderstudien und in der abschließenden Analyse wird auf die Bedeutung der europäischen Mehrebenenpolitik für die politischen Prozesse und Entwicklungen im Asylbereich jeweils eingegangen.

Um den Einfluss der Zusammenarbeit auf EU-Ebene auf das Politikfeld Asyl im Einzelnen zu bestimmen, wäre allerdings eine weitere, separate Untersuchung notwendig. Zudem ist der Nachweis nicht leicht zu erbringen, dass es sich bei einer bestimmten Entwicklung tatsächlich um die Folge eines EU-Einflusses handelt (vgl. Falkner 2003; Haverland 2005); beinahe unbestritten ist jedoch, dass ,Europe matters' und durch den Einfluss der Europäischen Union Wandel in den Mitgliedstaaten herbeigeführt wird. Unklar ist allerdings weiterhin, wie: in welchem Ausmaß, in welche Richtung, in welchem Tempo und zu welchem Zeitpunkt. ${ }^{52}$ Gleichzeitig muss berücksichtigt werden, dass es sich bei Europäisie-

\footnotetext{
52 Während die Entwicklungen auf EU-Ebene, also das Ausmaß, in dem die Mitgliedstaaten die supranationale Institutionen-Bildung und den entsprechenden Policy-Output beeinflussen, bereits länger ein Thema politikwissenschaftlicher Arbeiten ist, ist der umgekehrte Prozess - das heißt inwieweit die Implementierung von EU-Policies Anpassungen in den Mitgliedstaaten hervorruft erst im Verlaufe der vergangenen Jahre zum Gegenstand der Forschung geworden. Empirisch zeigen sich divergierende Einflüsse der EU sowohl in verschiedenen Policy-Feldern als auch unterschiedlichen Ländern. Für die Beantwortung der Frage „How Europe matters“ (vgl. Knill/ Lehmkuhl 2002) sind inzwischen einige theoretische Konzepte entstanden, die den Einfluss der EU auf die Mitgliedstaaten zu erschließen suchen. So wird einerseits auf die institutionelle Kompatibilität europäischer und mitgliedstaatlicher Arrangements - also den Grad der Abweichung von und der
} 
Untersuchungsdesign und Methoden

rungsprozessen nicht um ein einseitiges top (EU) - down (Mitgliedstaaten)Phänomen handelt, sondern dass das Verhältnis zwischen der EU und den Mitgliedstaaten ein dynamisches und wechselseitiges ist und somit auch ständige Feedback-Prozesse zwischen den verschiedenen Ebenen stattfinden.

\subsection{Erhebungsmethoden: Dokumentenanalyse und ExpertInnen-Interviews ${ }^{53}$}

Zur Untersuchung der Fragestellung wird vor allem auf Dokumentenanalysen zurückgegriffen. Als Quellen werden Gesetzestexte und relevante Veröffentlichungen (Pressemitteilungen, Protokolle, Stellungnahmen) sowohl staatlicher als auch von NGO- und migrantischen AkteurInnen analysiert. Weiterhin werden einige quantitative Sekundärdaten zur Illustration von Entwicklungstrends herangezogen sowie zur Absicherung bzw. Untermauerung der Ergebnisse, bspw. zur Kontrastierung von Policy-Outputs und -Outcomes. Da die flüchtlingspolitische Situation für den polnischen Fall noch relativ unerforscht ist und Dokumente und Veröffentlichungen nur in unzureichendem Maße sowie nur von bestimmten AkteurInnen vorliegen, wird die Dokumentenanalyse hier zur Exploration des Feldes zusätzlich durch leitfadengestützte ExpertInnen-Interviews ergänzt. Die InterviewpartnerInnen sind Personen, die in Polen an der Politikimplementierung beteiligt, also in Behörden tätig sind, sowie MitarbeiterInnen von flüchtlingspolitischen NGOs, MigrationsforscherInnen und VertreterInnen von MigrantInnenselbstorganisationen. Des Weiteren wurden in Polen verschiedene Flüchtlingslager besucht und Gespräche mit Flüchtlingen geführt, die jedoch nur handschriftlich protokolliert $^{54}$ und nicht systematisch ausgewertet wurden, sondern lediglich als Hintergrundwissen in die Untersuchung eingeflossen sind.

erforderlichen Anpassungsleistung an die EU-Vorgaben („Misfit“) - abgestellt (vgl. Héritier et al. 1996; Knill/Lenschow 1999) und andererseits das Ausmaß, in welchem die europäischen Politiken die Möglichkeitsstrukturen und die Interessenkonstellationen in den Mitgliedstaaten beeinflussen (vgl. Schneider 2001), betont. Zunehmend werden die beiden Erklärungsmuster auch miteinander verknüpft (vgl. Green Cowles/Risse 2001).

${ }^{53}$ Renate Liebold und Rainer Trinczek (2005) merken an, dass der Begriff ,Experten-Interview` sehr unpräzise ist. Es sei ansonsten üblich, ein Verfahren durch den Verweis auf die Erhebungsmethode zu benennen, und nicht - wie beim ExpertInnen-Interview - durch die Bezeichnung des Interviewpartners.

${ }^{54}$ Mit einer systematischen und methodisch einwandfreien Einbeziehung von Interviews mit Flüchtlingen in beiden Ländern wäre die Untersuchung zwar ihrem Anspruch näher gekommen, MigrantInnen als AkteurInnen im Migrationsregime zu begreifen. Dies erwies sich aber mit Blick auf die zur Verfügung stehenden zeitlichen, personellen und finanziellen Ressourcen als zu umfassendes Projekt, das den bestehenden Rahmen weitaus überstiegen hätte. Die Untersuchung beschränkt sich deshalb auf die Analyse der politischen Prozesse und Regulierungen auf der Makro- und Meso-Ebene. Die MigrantInnen-Perspektive findet Eingang durch die Rezeption der Verlautbarungen von MigrantInnenselbstorganisationen, durch Statistiken, die Anhaltspunkte für migrantische Strategien liefern sowie durch die erwähnten Gespräche. Die Einflechtung der Mikro-Ebene durch 
Für die ExpertInnen-Interviews wird auf die Systematisierung dieser Methode durch Michael Meuser und Ulrike Nagel (1991; 1997) zurückgegriffen. ${ }^{55}$ Das ExpertInnen-Interview dient der „Rekonstruktion komplexer Wissensbestände“ und kann sowohl eigenständig als auch als Teil eines Methodenmix zur Anwendung kommen (Meuser/Nagel 1997, S. 481). In der Politikwissenschaft wird das ExpertInnen-Interview insbesondere zur Erforschung politischen Handelns und in der Policy-Forschung eingesetzt (vgl. Abels/Behrens 2005, S. 174).

Meuser und Nagel beziehen sich ,auf diejenigen ExpertInnen, die selbst Teil des Handlungsfeldes sind, das den Forschungsgegenstand ausmacht" und damit nicht auf solche, die „von außen - im Sinne eines Gutachters“ Aussagen über ein Handlungsfeld treffen (Meuser/Nagel 1991, S. 443). Das Verfahren konzentriert sich auf

„ExpertInnen als FunktionsträgerInnen innerhalb eines organisatorischen oder institutionellen Kontextes. Die damit verknüpften Zuständigkeiten, Aufgaben, Tätigkeiten und die aus diesen gewonnenen exklusiven Erfahrungen und Wissensbestände sind Gegenstände des ExpertInneninterviews" (Meuser/Nagel 1991, S. 444 sowie ausfübrlicher zum ExpertInnen-Begriff 1997, S. 483ff.).

Mit der Erhebung dieser Wissensbestände kann nach Meuser und Nagel (1997, S. 489f.) ein Beitrag zur Erklärung sozialen Wandels geleistet werden. Die AutorInnen differenzieren dabei zwischen Arbeiten, in denen das ExpertInnen-Interview einen zentralen Rang einnimmt, wo also die ExpertInnen die Zielgruppe der Untersuchung (Betriebswissen) oder eine zur Zielgruppe komplementäre Handlungseinheit (Kontextwissen) bilden und solchen, wo sie im Untersuchungsdesign eher eine Randstellung einnehmen. In letzterem Falle verbinden sich mit der Durchführung von ExpertInnen-Interviews eher explorativ-felderschließende Ziele wie die Beschaffung von Hintergrundinformationen und die „Illustrierung und Kommentierung der Aussagen der Forscherin“ (Meuser/Nagel 1991, S. 445).

Das ExpertInnen-Interview ist dem Verfahren nach ein qualitatives Interview, unterscheidet sich aber aufgrund anders gelagerter Erkenntnisinteressen von anderen Arten qualitativer Interviews (narrativ, fokussiert, biografisch, problemzent-

qualitative Interviews mit einer größeren Zahl an MigrantInnen über einen längeren Zeitraum hinweg könnte in einem auf dieser Analyse aufbauenden Projekt folgen.

55 Die Artikel der beiden AutorInnen sind in der derzeitigen Forschung zentrale Referenzpunkte, wenn es um das ExpertInnen-Interview geht. In ihrem grundlegenden Artikel von 1991 beanstanden Meuser und Nagel, dass das ExpertInnen-Interview zwar vielfach zur Anwendung kommt, die Methodik jedoch kaum systematisch rekapituliert wurde und insbesondere bis dato keine Verständigung hinsichtlich überzeugender Auswertungsverfahren stattgefunden habe. Ebenso weisen Liebold und Trinczek (2005) darauf hin, dass es sich beim leitfadengestützten ExpertInnen-Interview um eines der am häufigsten angewendeten Verfahren handele, wohingegen sich die methodologische und methodische Diskussion eher bescheiden ausnehme. Für einen ausführlichen Überblick über Theorie, Techniken und Anwendungsfelder kann inzwischen auf den Sammelband von Alexander Bogner, Beate Littig und Wolfgang Menz (2002) zurückgegriffen werden. Darin wurde der Meuser/Nagel-Artikel von 1991 als ,Klassiker‘ unverändert nochmals abgedruckt. 
riert etc.) in Gesprächsführung und Auswertung (vgl. Meuser/Nagel 1997, S. 482). Meuser und Nagel favorisieren ein leitfadengestütztes offenes Interview als Erhebungsinstrument, wobei der Leitfaden eher anzusprechende Themen als fertig ausgearbeitete Fragen enthalten soll (vgl. ebd., S. 486f.). Auf diese Weise haben die ExpertInnen Gelegenheit, das Thema vor dem Hintergrund ihres Wissens zu dimensionieren. Diese „Gratwanderung zwischen Strukturierung und Offenheit“ ist laut Liebold und Trinczek $(2005$, o. S.) ein voraussetzungsvolles Unternehmen.

Sowohl für die thematische Vorstrukturierung mittels Leitfaden als auch für die Auswahl der geeigneten ExpertInnen und nicht zuletzt, um von den ExpertInnen als kompetente Gesprächspartnerin wahrgenommen zu werden, ist es erforderlich, sich zunächst durch andere zugängliche Quellen Kenntnisse zum Untersuchungsgegenstand anzueignen.

Die Auswertung der ExpertInnen-Interviews „orientiert sich [...] an thematischen Einheiten, an inhaltlich zusammengehörigen, über die Texte verstreuten Passagen - nicht an der Sequenzialität von Äußerungen je Interview" (Meuser/Nagel 1997, S. 488; dies. 1991, S. 453). ${ }^{56}$ Auf die Identifikation dieser thematischen Einheiten (Paraphrasierung) folgt das Kodieren, also die thematische Ordnung der paraphrasierten Passagen, bei der die Terminologie der ExpertInnen einbezogen wird. Danach werden „thematisch vergleichbare Textpassagen aus verschiedenen Interviews gebündelt" (Thematischer Vergleich) (Meuser/Nagel 1997, S. 489). Im darauf folgenden Arbeitsschritt der „Soziologischen Konzeptualisierung" wird von den Texten und der darin befindlichen Terminologie im Sinne einer empirischen Generalisierung abstrahiert und Kategorien gebildet (vgl. Meuser/Nagel 1991, S. 662f.; Meuser/Nagel 1997, S. 489). Abschließend erfolgt die „Theoretische Generalisierung“. Dabei werden „Sinnzusammenhänge zu Typologien und zu Theorien verknüpft“ (Meuser/Nagel 1997, S. 489).

Auf der Grundlage dieser Bestimmung des Verfahrens durch Meuser und Nagel wurden für die vorliegende Untersuchung in Polen 17 ExpertInnen-Interviews durchgeführt (vgl. die Übersicht über die InterviewpartnerInnen im Anhang). ${ }^{57}$ Sie dienen, wie oben bereits angedeutet, in erster Linie der Exploration des Untersuchungsfeldes sowie der Ergänzung der Dokumentenanalyse und nehmen für die inhaltliche Fundierung der Studie einen wichtigen Stellenwert ein. Die ExpertInnen sind aber auch insofern selbst Zielgruppe biw. Gegenstand der Untersuchung als sie Teile des Asylregimes, das im Mittelpunkt dieser Studie steht, repräsentieren und damit gleichzeitig Aufschluss über die Beschaffenheit des Asylregimes und das Verhältnis der AkteurInnen zueinander geben. Als ExpertInnen wurden daher Personen angefragt, die als AkteurInnen im Asylregime aktiv sind und dieses mitbestimmen - bei der Auswahl wurde darauf geachtet, die Vielfalt der AkteurIn-

\footnotetext{
56 Zur ausführlichen Darstellung der einzelnen Auswertungsschritte vgl. Meuser/Nagel (1991).

57 Die meisten Interviews wurden gemeinsam mit Sabine Kroner durchgeführt, die ebenfalls im Rahmen einer Dissertation über polnische Migrationspolitik im weiteren Sinne - also nicht beschränkt auf den Asylbereich - forscht.
} 
nen, ihrer Funktionen und Interessen zu berücksichtigen und ein möglichst breites Akteursspektrum zu erreichen.

Da das AkteurInnenfeld des Asylregimes in Polen noch recht überschaubar ist, wurde mit den 17 geführten Interviews das Spektrum der AkteurInnen, wie es sich zum Zeitpunkt der Erhebung darstellte, im Wesentlichen erfasst. Dies zeigt sich auch darin, dass die Hinweise der Interviewten auf weitere GesprächspartnerInnen sich in den meisten Fällen mit der bereits ausgearbeiteten ExpertInnenliste überschnitten. In den wenigen Fällen, wo dies nicht der Fall war, vermittelten die GesprächspartnerInnen zum Teil sehr bereitwillig Kontakte und fungierten aktiv als ,Türöffner'. ${ }^{58}$

Der Zugang zu den ExpertInnen und die Interviewsituation selbst sind durch „sozialstrukturelle und situative Faktoren“ geprägt (Abels/Behrens 2005, S. 177). Hierzu zählen „Geschlecht, Alter, professioneller Status/Titel, Erfahrungshintergrund, Idiosynkrasien/Einstellungen und Organisationszugehörigkeit“, die miteinander verwoben sind (ebd.). Insbesondere für die vorliegende Untersuchung und sicherlich für viele andere länderübergreifende Studien lassen sich noch die Herkunft bzw. Staatsbürgerschaft und die damit verknüpften Zuschreibungen hinzufügen. Die Interviewerin kann also zwar versuchen, im Gespräch möglichst neutral aufzutreten oder auch strategisch eine bestimmte Haltung an den Tag zu legen, um bestimmte Informationen zu bekommen. Dennoch ist die Interviewsituation durch die oben genannten Faktoren vorgeprägt, denn diese ,sind in allen sozialen Interaktionen und damit auch in der Interviewsituation immer und unausweichlich präsent“ (ebd., S. 177). Für die Untersuchung des Asylregimes in Polen ist - je nach spezifischem Hintergrund des jeweiligen Interviewpartners variierend - davon auszugehen, dass mein Hintergrund als junge weibliche Doktorandin mit deutscher Staatsbürgerschaft mit den entsprechenden Zuschreibungen den Zugang zum Feld und die Interviewsituationen in unterschiedlicher Weise mit beeinflusst hat. Da dies hier nicht in aller Ausführlichkeit erörtert werden kann, seien nur einige Hinweise genannt, die über die Rolle dieser Faktoren Aufschluss geben. So hat sich der Feldzugang über ein auf Polnisch verfasstes Anschreiben mit Briefkopf der Universität Göttingen als recht mühelos erwiesen: Bis auf wenige Ausnahmen signalisierten die ExpertInnen schnell ihre Bereitschaft zum Interview. Sowohl die weitere Korrespondenz im Vorfeld der Interviews als auch die Interviews selbst wurden schließlich auf Englisch durchgeführt, auf das alle ExpertInnen auf hohem Niveau zurückgreifen konnten. Nichtsdestotrotz dürfte sich die polnische Ansprache beim Erstkontakt positiv ausgewirkt haben. Für die Interviewsituation selbst lässt sich resümieren, dass die Kombination der Faktoren

\footnotetext{
58 Diese ,Türöffner' erwiesen sich im Einzelfall als sehr hilfreich. So war es durch ihre Vermittlung teilweise möglich mit Personen zu sprechen, die auf offizielle Anfragen nicht reagiert hatten. Im Falle des polnischen Innenministeriums allerdings half auch die Intervention eines hochrangigen Mitarbeiters im Hauptquartier der Grenzschutzbehörde nicht weiter. Das Innenministerium war nicht zu einem Gespräch bereit.
} 
Geschlecht, Alter und professioneller Status besonders prägend war. Dabei zeigte sich, dass einerseits die Ähnlichkeit zwischen Interviewerin und GesprächspartnerIn in den Ausprägungen dieser Faktoren die Gespräche positiv beeinflusste als auch andererseits die Unterschiedlichkeit bereichernde Effekte zeitigte. Während also beispielsweise zu relativ jungen Wissenschaftlerinnen aufgrund des vergleichbaren Hintergrundes schnell ein für die Interviewsituation positives Vertrauensverhältnis aufgebaut werden konnte, wirkten sich die Zuschreibungen, die uns durch ältere männliche Personen mit hohem professionellen Status insbesondere auf der Ebene der Geschlechterverhältnisse zuteil wurden, ebenfalls vorteilhaft aus. Ähnliche Erfahrungen beschreiben Gabriele Abels und Maria Behrens (2005, S. 178):

\section{„Wir sind auf der Basis unserer Interviewerfahrungen der Meinung, dass das Geschlecht, obschon es hier eine diskriminierende Komponente gibt, erkenntnisbringend eingesetzt werden kann. Blockierende Effekte sind andererseits ebenfalls systematisch nicht auszu- schließen."}

Positive Effekte ergeben sich hier durch auf Profilierung zielende und paternalistische Verhaltensweisen ${ }^{59}$, die auf eigentlich überkommenen Geschlechterverhältnissen beruhen, gleichwohl jedoch den Output der Gespräche steigern können. Dies zeigt sich beispielsweise, wenn uns ein Interviewpartner an einen weiteren vermittelt und dessen Widerstreben mit der Bemerkung zu entkräften versucht, es handele sich doch ,nur um zwei junge Frauen, die ein bisschen reden wollen'. Damit wird uns - aufgrund unseres Alters und Geschlechts, die gleichzeitig die Ausblendung unseres professionellen Hintergrunds bedingen - eine geringe ,Gefährlichkeit' zugeschrieben, mit der hier auf Offenheit zum und im Gespräch appelliert wird. Die Erfahrungen mit den ExpertInnen-Interviews für diese Untersuchung stützen also im Wesentlichen die Hypothesen von Abels und Behrens. Die weitere Konkretisierung dieser Effekte im ExpertInnen-Interview harrt allerdings noch umfassenderer systematischer Bearbeitung.

Neben dieser Reflexion der Interviewsituation ist noch auf einige Abweichungen von der Methode, wie sie Meuser und Nagel konzipiert haben hinzuweisen, die angesichts der Spezifität des Untersuchungsfalles für die vorliegende Untersuchung notwendig wurden. Dazu gehört zum einen, dass die GesprächspartnerInnen in Polen kaum das von Meuser und Nagel aufgestellte Kriterium erfüllen, wonach ExpertInnen sich dadurch auszeichnen, dass sie bereits seit mindestens zehn Jahren in dem betreffenden Feld arbeiten. Das liegt daran, dass es sich hier-

\footnotetext{
59 Abels und Behrens (vgl. 2005, S. 181ff.) fügen den vier Interaktionseffekten von Berthold Vogel (1995) - dem Paternalismus-, dem Katharsis-, dem Eisberg- und dem Rückkoppelungseffekt - den Profilierungseffekt hinzu und beleuchten diese vor dem Hintergrund ihrer eigenen Forschungserfahrungen aus der Geschlechterperspektive. Dem Rückkoppelungs- und Eisbergeffekt begegneten wir wie Abels und Behrens ebenfalls kaum. Auch den Katharsiseffekt („Wechsel des Befragten von seiner Rolle als Experte zur Privatperson“ (ebd., S. 183)) konnten wir - allerdings im Gegensatz zu den beiden Autorinnen - kaum feststellen.
} 
bei um ein relativ junges Politikfeld in Polen handelt. Entsprechend sind die polnischen Institutionen und deren MitarbeiterInnen, die sich mit Flüchtlingspolitik und deren Umsetzung befassen, oft noch nicht seit einem so langen Zeitraum in dem Bereich aktiv.

Hinzu kommt, dass die Vorgabe, alle ExpertInnen auf der Basis desselben Leitfadens zu befragen aufgrund der explorativen Zielsetzung der Interviews nicht eingehalten werden kann. Allerdings formulieren Meuser und Nagel (1997, S. 488) dieses Kriterium relativ offen:

„Es ist der gemeinsam geteilte institutionell-organisatorische Kontext der ExpertInnen, der die Vergleichbarkeit der Interviewtexte weitgehend sichert; darüber hinaus wird die Vergleichbarkeit gewährleistet durch die leiffadenorientierte Interviewführung. "

Um das Feld der Flüchtlingspolitik in Polen zu erforschen ist die Erschließung und Befragung eines heterogenen AkteurInnenfeldes notwendig. Um diesem gerecht zu werden wurde ein Basis-Leitfaden entwickelt, der Themenfelder enthielt, die in allen Interviews angesprochen wurden. Diese umfassten neben Fragen zur Organisation bzw. Institution des Interviewten die Bereiche Migrationspolitik, AkteurInnen, öffentliche Debatte, konkrete Situation von MigrantInnen und Flüchtlingen sowie Erwartungen bezüglich der künftigen Entwicklungen. Ausgehend von diesen Themenfeldern wurden die Fragen den jeweiligen GesprächspartnerInnen angepasst, da zum Beispiel eine NGO-Mitarbeiterin oder das Mitglied einer Flüchtlingsselbstorganisation einen anderen Erfahrungshintergrund und eine andere Perspektive auf das Thema besitzt als ein Mitarbeiter der Zentralen Ausländerbehörde. Daher lassen sich zwar die Ergebnisse der Befragung nicht schablonenartig übereinander legen, bleiben jedoch aufgrund der gemeinsamen Basis-Struktur durchaus vergleichbar.

Auch das Vorgehen bei der Auswertung der Interviews wurde den Erfordernissen des Untersuchungsdesigns angepasst. Insbesondere, da Meuser und Nagel bei den von ihnen vorgeschlagenen Auswertungsschritten weniger die explorative Variante des ExpertInnen-Interviews im Blick hatten, sind einige Modifikationen erforderlich. Die ersten vier Auswertungsschritte (Transkription, Paraphrase, Überschriften und Thematischer Vergleich) wurden im Wesentlichen gemäß den Vorgaben von Meuser und Nagel angewendet. Die ,soziologische Konzeptualisierung' und ,theoretische Generalisierung' wurden hingegen nicht durchgeführt und stattdessen das durch die ersten vier Schritte systematisierte Material in der Länderstudie beschrieben.

Konkret wurde das Material zunächst recht ausführlich transkribiert. Obwohl Meuser und Nagel eine detaillierte Transkription nicht für notwendig erachten, wurde dieser Weg gewählt, da sich während der Transkription zeigte, dass so ein vollständigeres Bild gewonnen werden kann: Zum einen macht sich die Forscherin durch die intensive Bearbeitung stärker mit dem Material vertraut, zum anderen war es aufschlussreich zu sehen, an welchen Stellen sich einzelne Interview- 
partner wiederholen, wo sie zögern, wo sie stringent antworten und in welchen Bereichen Unsicherheiten bestehen. Indem Kontinuitäten, Auffälligkeiten und Widersprüche im Text ermittelt werden, kann ein differenziertes Bild dieses Politikbereichs gezeichnet werden. Gleichzeitig erhält man so einen Einblick in die Art, wie sich das Thema für die Interviewten darstellt und in ihre eigene Position innerhalb des ExpertInnenspektrums bzw. innerhalb des Asylregimes.

Die Paraphrasierung wurde - im Gegensatz zur Empfehlung von Peter Ullrich (2006, S. 105), der sich ebenfalls mit Auswertungsstrategien für explorative ExpertInnen-Interviews auseinandergesetzt hat - nicht weggelassen, da sie sowohl für die Tilgung von Redundanzen als auch - erneut - für eine intensive Auseinandersetzung mit dem Material nötig ist. Für eine textnahe Auswertung wäre die $\mathrm{Pa}$ raphrasierung im ersten Arbeitsschritt nach der Transkription problematisch, da man sich auf diese Weise schon zu Beginn der Auswertung deutlich vom Wortlaut des Textes entfernt. Für die Zwecke, die die Interviews und deren Auswertung für die vorliegende Studie erfüllen sollen, ist ein solches Vorgehen jedoch gerechtfertigt: Die Interviews dienen als Quelle für Kontextinformationen. Die enthaltenen Informationen werden durch die Paraphrasierung verdichtet und können so leichter systematisiert werden. Eine intensive Auswertung, die nah am Originaltext arbeitet, ist in diesem Zusammenhang nicht erforderlich und nicht zielführend.

Für die Bildung der Überschriften wurde der Vorschlag Ullrichs (2006, S. 106), eine Auswertungsmaske zu erstellen, gewinnbringend aufgenommen. Es wurde eine zweispaltige Tabelle je Interview erstellt, in die die relevanten Paraphrasen oder Zitate nach thematischen Bereichen eingeordnet und innerhalb eines thematischen Bereichs noch einmal mit Überschriften versehen wurden. Dabei wurde die Chronologie des Interviews aufgebrochen. Nachdem einige Interviews so durchgearbeitet waren, ergab sich bereits eine Maske mit angesprochenen Themen (die denen des Fragebogens im Wesentlichen entsprachen). Diese wurde als Grundlage für die Durcharbeitung der weiteren Interviews verwendet (und auch auf die vorherigen noch einmal aufgelegt), sodass erkennbar war, welche Interviewpartner sich zu welchen Themen geäußert oder nicht geäußert haben.

Im nächsten Schritt (thematischer Vergleich) wurde dieses Verfahren Interview-übergreifend angewendet. Das heißt es wurden thematisch vergleichbare Passagen aus verschiedenen Interviews zusammengestellt und die Überschriften vereinheitlicht. Die gewonnen Erkenntnisse werden in der Länderstudie dargestellt und gegebenenfalls durch weitere Quellen untermauert. Dabei werden die Aussagen der InterviewpartnerInnen nicht als ,Wahrheit ${ }^{`}$ wiedergegebenen, sondern mit Rücksicht auf ihren institutionellen Kontext und ihre Position im Asylregime in die Untersuchung aufgenommen. So können Aussagen, die nur einige ExpertInnen äußerten oder die von vielen übereinstimmend genannt wurden, entsprechend eingeordnet werden. 


\section{Asylpolitik der EU}

Im Vergleich zu anderen Politikfeldern recht spät, aber nun doch in zunehmendem Tempo wird auch der Bereich der Migrations- und Asylpolitik mehr und mehr Gegenstand der Vereinheitlichungsbestrebungen auf EU-Ebene. ${ }^{60}$ Um Aussagen über den Wandel der Kategorie Flucht und Asyl treffen zu können, müssen die asylpolitischen Entwicklungen auf EU- Ebene ${ }^{61}$ daher mit einbezogen werden.

${ }^{60}$ Zahlreiche AutorInnen haben sich im vergangenen Jahrzehnt mit der ,Europäisierung' einzelstaatlicher Politiken befasst - dabei wurde neben verschiedenen anderen Politikfeldern auch der Bereich der Migrations- und Asylpolitik untersucht. Arbeiten, die die EU-Entwicklungen in einen Kontext mit den Entwicklungen in verschiedenen Ländern stellen - mitunter durch Zugrundelegen von Fallstudien - sind zum Beispiel Birsl 2005; Byrne/Noll/Vedstedt-Hansen 2003; Holzer/Schneider 2002; Lavenex 2002 und Morris 2002. Daneben gibt es eine größere Zahl an Publikationen, die die Migrations- und Asylregime auf nationalstaatlicher Ebene erforschen und zum Beispiel in der Art von Nachschlagewerken herausgegeben werden (vgl. Currle 2004; Gieler/Fricke 2004) sowie Untersuchungen, die die Politikprozesse auf EU-Ebene untersuchen (vgl. Angenendt 1999; 2002; Geddes 2000; Givens/Luedtke 2004; Hatton 2004; Holzberger 2003).

${ }^{61}$ Wenn von ,der EU' die Rede ist, soll nicht unterstellt werden, dass es sich dabei um einen einzigen, einheitlichen Akteur mit einer bestimmten Interessenlage handelt. Vielmehr finden sich auch innerhalb des EU-Gebildes widerstreitende Interessenskonstellationen, wie beispielsweise zwischen den Regierungen der Mitgliedstaaten oder zwischen den und innerhalb der verschiedenen Organe (Kommission, Europäischer Rat, Ministerrat, Parlament). Diese traten gerade in dem hier betrachteten Politikfeld in den vergangenen Jahren sehr deutlich zutage. Gleichwohl wird hier der Entstehungsprozess der Regelungen, die auf EU-Ebene verabschiedet wurden, nicht untersucht. Stattdes- 
Sie bilden einen wichtigen Referenzpunkt der Entwicklungen in den zwei untersuchten Ländern Deutschland und Polen und geben gleichzeitig Auskunft über die aktuelle Konstruktion der Kategorie Flucht und Asyl, wie sie sich als Ergebnis der Aushandlungsprozesse zwischen den Mitgliedstaaten und schließlich auch des Zusammenspiels von Rat, Kommission, Parlament und weiteren AkteurInnen darstellt.

Zwar war bereits im Vertrag zur Gründung der Europäischen Wirtschaftsgemeinschaft von 1957 neben dem freien Verkehr von Waren, Dienstleistungen und Kapital auch die Freizügigkeit von Personen vorgesehen. Die Migrationspolitik war jedoch bis 1985 einzelstaatlich geregelt. Die fortschreitenden Bemühungen um die Konstituierung eines gemeinsamen Binnenmarkts erfordern auch einen Abbau der Binnengrenzen. Dies setzt nicht nur Reise- und Niederlassungsfreiheit von EG- bzw. EU-BürgerInnen, sondern auch von so genannten ,Drittstaatenangehörigen'voraus. Daher wird mit dem Wegfall der Binnengrenzen aus der Sicht der EU-Mitgliedstaaten eine gemeinsame Politik hinsichtlich des Zugangs von Drittstaatenangehörigen zum und ihres Status auf dem EG- bzw. EU-Territorium notwendig.

Da sich die Mitgliedstaaten auf EG-Ebene zunächst nicht auf den Abbau der Binnengrenzen und gemeinsame Regelungen zu Einreise und Aufenthalt der Drittstaatenangehörigen einigen konnten, unterzeichneten die Benelux-Staaten, Frankreich und die Bundesrepublik Deutschland im Jahr 1985 auf intergouvernementaler Ebene das Übereinkommen von Schengen. ${ }^{62}$ Ziel war es, den ,SchengenRaum', also einen Raum ohne Binnengrenzen und mit einheitlichen Einreisekontrollen an den Außengrenzen zu schaffen. Dieses Vorhaben wurde allerdings erst 1995 verwirklicht. Seitdem wuchs die Bedeutung der Migrations- und Asylpolitik auch als Teil der auf EG-Ebene verhandelten Politiken. Dem Schengener Übereinkommen (Schengen I) folgten das Schengener Durchführungsübereinkommen (Schengen II, 1990), das Dubliner Abkommen (1990) und schließlich der Maastrichter Vertrag (1993). Dies ist der Vertrag zur Gründung der Europäischen Union (EU) auf Basis des ,Drei-Säulen-Modells` der europäischen Zusammenarbeit. Mit dem Amsterdamer Vertrag (AV), der 1997 verabschiedet wurde und 1999 in Kraft trat, wurden wesentliche Elemente der Migrations- und Asylpolitik in die Gemeinschaftszuständigkeit, also aus der dritten Säule der intergouvernementalen Zusammenarbeit in den Bereichen Justiz und Inneres in das supranationale Rechtsetzungsverfahren der ersten Säule überführt (vgl. Angenendt 2002a; Geddes 2000). ${ }^{63}$ Gleichzeitig wurde der Schengen-Besitzstand, also die bis dahin gefassten Beschlüsse der Schengen-Mitglieder, in den Rahmen der EU aufgenommen.

sen werden die Richtlinien und Verordnungen sowie die operative Zusammenarbeit als vorläufiges Ergebnis der Aushandlung dieser Interessen betrachtet und damit als ,EU'-Standpunkt behandelt.

${ }^{62}$ Für einen Abriss der Entwicklungen auf EG- bzw. EU-Ebene vgl. Birsl 2005, S. $106 f f$.

${ }^{63}$ Die zweite Säule umfasst die Gemeinsame Außen- und Sicherheitspolitik (GASP) und ist intergouvernemental angelegt. 
Die Artikel 61 bis 69 des Amsterdamer Vertrages regelten das weitere Vorgehen in diesem Politikbereich auf EU-Ebene. Innerhalb eines Übergangszeitraums von fünf Jahren nach Inkrafttreten des Vertrages sollten demnach Maßnahmen zur Gewährleistung eines freien Personenverkehrs ergriffen werden. Während die Migrationspolitik im weiteren Sinne - und dabei insbesondere Regelungen bezüglich des Zugangs zum Arbeitsmarkt sowie Integration - von der Vergemeinschaftung (noch) weitgehend unangetastet bleibt, konzentrieren sich die im Amsterdamer Vertrag festgelegten Ziele neben gemeinsamen Regelungen und Maßnahmen bezüglich Visa, Familienzusammenführung, ,illegale‘ Einwanderung sowie ,Rückführung' und der Festlegung der Rechte von legal anwesenden Drittstaatenangehörigen stark auf den Bereich Asyl. Für die erste Phase (1999-2004), die der Schaffung einer, mit den Worten von Andrew Geddes gesprochen, ,,rather grandiosely entitled '[...] area of freedom, justice and security" (Geddes 2005, S. 265) dienen sollte, wurde im Bereich Asyl die Verabschiedung von Richtlinien über Minimalstandards für Aufnahmebedingungen, das Asylverfahren, die Bestimmung der Flüchtlingseigenschaft und des subsidiären und vorübergehenden Schutzes (Qualifizierungsrichtlinie) sowie die Zuständigkeit der einzelnen Staaten für die Durchführung von Asylverfahren (Dublin-II-Verordnung) und eine ,,ausgewogene Verteilung der Belastungen“ zwischen den Mitgliedstaaten angestrebt (Art. 63 AV). Die Vorschläge für diese Richtlinien erarbeitete die Kommission und legte sie dem Ministerrat zur Abstimmung vor. Während des Übergangszeitraums galt im Ministerrat das Einstimmigkeitsprinzip, das Parlament wurde lediglich angehört (Art. $67 \mathrm{AV}$ ).

Dabei zeigte sich, dass sich die Innenminister der Mitgliedstaaten in bestimmten Bereichen besonders schnell einigen konnten, während es in anderen zahlreiche Widerstände und zähe Verhandlungen gab, die durch das Einstimmigkeitsprinzip verschärft wurden, sodass einige Richtlinien erst in sprichwörtlich letzter Minute und die Asylverfahrensrichtlinie sogar erst Monate nach Ablauf der im Amsterdamer Vertrag gesetzten Frist verabschiedet wurden. ${ }^{64}$ Die langwierigen Verhandlungen über die Richtlinienvorschläge der Kommission hatten zur Folge, dass ,viele der ursprünglich liberalen Ideen verschlissen oder sogar in ihr Gegenteil verkehrt“" wurden (Bendel 2006, S. 125). Als Ursachen für die Restriktion identifiziert Petra Bendel (vgl. 2006, S. 125f.) die wirtschaftliche Situation in einigen großen Mitgliedstaaten, die Betonung sicherheitspolitischer Interessen nach dem 11.09.2001 und die konservativere Ausrichtung des Ministerrats nach den Wahlen in einigen Mitgliedstaaten. Dabei nutzten die Innen- und Justizminister „häufig genug den Mangel an Öffentlichkeit in ihren Verhandlungen aus, um in Brüssel Entscheidungen durchzusetzen, die ihnen zu Hause verwehrt waren, um diese

${ }^{64}$ Die Richtlinie über Mindestnormen für vorübergehenden Schutz wurde bereits im Juli 2001 verabschiedet. Über Mindestnormen für die Anerkennung als Flüchtlinge (Qualifikationsrichtlinie) einigten sich die Mitgliedstaaten im Rat erst am 30.04.2004. 
dann im eigenen Heimatland als „Brüsseler Oktroy“ hinzustellen.“ (Bendel 2006, S. 131)

Die Mitgliedstaaten sollen die ausgehandelten Vorgaben in der Regel binnen zwei Jahren nach Verabschiedung in nationalstaatliches Recht umsetzen, in vielen Fällen gibt es jedoch auch auf der mitgliedstaatlichen Ebene Verzögerungen. ${ }^{65}$ Durch den Vertrag von Nizza, der im Februar 2003 in Kraft trat, wurde für die Phase nach dem Inkrafttreten der Richtlinien (mit der Asylverfahrensrichtlinie im Dezember 2005) im Asyl- und Flüchtlingsbereich das Mehrheitsprinzip im Ministerrat und das Mitentscheidungsverfahren für das Europäische Parlament festgelegt. In den übrigen Bereichen regulärer Einwanderung gilt nach wie vor das Einstimmigkeitsprinzip.

Sowohl im Amsterdamer Vertrag (Art. 63) als auch von Seiten des Europäischen Rates wurde die Genfer Flüchtlingskonvention zunächst als zentraler Referenzpunkt der EU-Asylpolitik benannt. Bei der ,Sondertagung über die Schaffung eines Raums der Freiheit, der Sicherheit und des Rechts in der Europäischen Union' des Europäischen Rates in Tampere 1999 wurden die Eckpunkte des weiteren Vorgehens, das sich aus den Maßgaben des Amsterdamer Vertrages ergab, festgelegt. In den Schlussfolgerungen des Rates heißt es:

„Der Europä̈sche Rat bekräftigt die Bedeutung, die die Union und die Mitgliedstaaten der unbedingten Achtung des Rechts auf Asyl beimessen. Er ist übereingekommen, auf ein Gemeinsames Europäisches Asylsystem binzunirken, das sich auf die uneingescbränkte und allumfassende Anwendung der Genfer Flüchtlingskonvention stützt, wodurch sichergestellt wird, daß niemand dorthin zurückegeschickt wird, wo er Verfolgung ausgesetzt ist, das heißt der Grundsatz. der Nichtzurückweisung gewabrt bleibt." (SN 200/99)

Für diese Zusicherung der Achtung der GFK und des darin enthaltenen NonRefoulement-Gebots erhielt der Europäische Rat von vielen Menschenrechts- und Flüchtlingsorganisationen große Zustimmung (vgl. Hatzinger 2006), die jedoch in den darauf folgenden Jahren zunehmend von der Kritik an den schließlich verabschiedeten Richtlinien verdrängt wurde (vgl. Frankfurter Rundschau vom 30.03.2004). ${ }^{66}$ Denn „die Aufbruchstimmung Tamperes [schlug sich] kaum in entsprechender Rechtsetzung nieder" (Bendel 2006, S. 126).

\footnotetext{
${ }^{65}$ Werden die Richtlinien nicht innerhalb der vorgegeben Frist in innerstaatliches Recht umgesetzt, so werden einzelstaatliche Regelungen, die dem Inhalt der Richtlinien widersprechen, nichtig und die Richtlinie kommt direkt zur Anwendung, entfaltet also volle Rechtskraft. Außerdem kann die EU-Kommission gegen säumige Mitgliedstaaten ein Vertragsverletzungsverfahren beim Europäischen Gerichtshof einleiten, welcher Geldstrafen verhängen kann.

66 In einer gemeinsamen Stellungnahme hatten Amnesty International, Caritas, Pax Christi, Ärzte ohne Grenzen und Human Rights Watch insbesondere die Unvereinbarkeit der ,Sicheren Herkunftsstaaten-' und der ,Sicheren Drittstaatenregelung' mit geltendem Völkerrecht (der GFK, der EU-Grundrechtecharta und der Rechtsprechung des EU-Menschenrechtsgerichtshofs) kritisiert. Dem schloss sich auch UN-Flüchtlingskommissar Ruud Lubbers an, der befürchtete, einige EU-
} 
Die Bilanz des Tampere-Programms, die die Europäische Kommission 2004 zog, spricht bereits eine andere Sprache. Darin forderte sie neben der Einführung eines gemeinsamen Asylverfahrens und eines einheitlichen Status

„ein [en] integrierte [n] Ansatz, mit effizienten Verfahren für Verwaltungsentscheidungen über die Rückkehr, Wiedereingliederungsprogrammen und Einreiseverfahren zu entwickeln, die geeignet sind, Personen, deren Antrag unbegründet ist, abzuschrecken und zur Bekämpfung von Menschenhändler- und Schleppernetzen beitragen. Ein solches Vorgehen ist deshalb so wichtig, weil die Opfer des Missbrauchs der Asylverfahren häufig die echten Flüchtlinge sind "

(KOM(2004) 401, Hervorh. i. Orig.).

Hier wird der Schwerpunkt weniger auf den Flüchtlingsschutz selbst gelegt, als auf die Selektion der ,echten' aus der Masse der übrigen Flüchtlinge, die das Asylverfahren ,missbrauchen'. ,Rückkehr', ,Abschreckung' und ,Bekämpfung' als zentrale Stichworte bilden - nicht nur semantisch - einen starken Kontrast zu den Schlussfolgerungen von Tampere. ${ }^{67}$

Mit dem „Haager Programm zur Stärkung von Freiheit, Sicherheit und Recht in der Europäischen Union“" (2005/C 53/01) wurde im November 2004 die Umsetzung dieser Pläne und damit die Fortsetzung der ,Harmonisierung ${ }^{6}$ bis 2010 beschlossen. In dieser zweiten Phase sollen über die bisherigen Minimalstandards hinaus einheitliche Asylverfahren und ein einheitlicher unionsweit gültiger Status für Asylberechtigte und Personen mit subsidiären Schutzstatus eingeführt werden (vgl. 2005/C 53/01). Des Weiteren soll die Abschaffung der Binnengrenzen bei gleichzeitiger Verstärkung der Außengrenzen weiter vorangetrieben werden:

„Der Europäische Rat verweist auf die Bedeutung, die der raschen Abschaffung der Kontrollen an den Binnengrenzen, der weiteren schrittweisen Verwirklichung des integrierten Grenzschutzsystems für die Außengrenzen und dem Ausbau der Kontrollen an den Außengrenzen und deren Überwachung zukommt." (2005/C 53/01, S. 6)

Zur Vorbereitung der Verabschiedung der entsprechenden Maßnahmen bis 2010 hat die Kommission im Juni 2007 das Grünbuch über das künftige Gemeinsame Europäische Asylsystem vorgelegt (vgl. $\operatorname{KOM}(2007) 301)$.

Im Haager Programm wird überdies eine neuere Entwicklung, die sich in den vergangenen Jahren in der europäischen Asylpolitik abzeichnete, explizit:

„Ferner sollten in einer gesonderten, in enger Absprache mit dem UNHCR durchzuführende [n] Studie die Vorteile, die Zweckmäßigkeit und die Durchfübrbarkeit einer gemeinsamen Bearbeitung von Asylanträgen außerhalb der EU geprüft werden, wobei die-

Mitglieder würden versuchen, „ihre eigenen restriktivsten und umstrittensten Praktiken auf alle künftigen 25 Mitgliedstaaten zu übertragen“ (zit. n. Frankfurter Rundschau vom 30.03.2004).

67 Vgl. zur Bedeutung dieser Begriffe 4.2.3 Sprachgebrauch. 
ses Verfahren die gemeinsame europäische Asylregelung ergänzen und den einschlägigen internationalen Normen entsprechen würde. "(2005/C 53/01, S. 4)

Hierin zeigen sich die zunehmenden Bestrebungen der EU, ihre Maßnahmen im Bereich Migration stärker auf die Herkunfts- und Transitregionen zu konzentrieren. Unter den Stichworten, externe Aspekte der Asylpolitik' und ,Verbesserung des Schutzes in Drittländern' werden verstärkt migrations- und außenpolitische Aktivitäten der EU miteinander verknüpft, wie sich bspw. in der Einflechtung migrationspolitischer Ziele in die Europäische Nachbarschaftsstrategie zeigt (vgl. Banse/Müller/Stobbe 2007).

Trotz fortbestehender Widerstände in verschiedenen Bereichen - wie hinsichtlich des Zugangs von Nicht-EU-BürgerInnen zum Arbeitsmarkt und der Integrationspolitik - schreitet die migrationspolitische Zusammenarbeit auf EU-Ebene also voran. Dabei gehört der Asylbereich zu den derzeit am weitesten regulierten auf EU-Ebene. Allerdings hat sich die zunächst als ,Aufbruchstimmung' gefeierte liberale Tendenz der Sondertagung in Tampere nicht durchgesetzt. Den seit Jahren stark rückläufigen AsylbewerberInnenzahlen zum Trotz wird auch auf EUEbene die ,Bekämpfung des Asylmissbrauchs' als zentrale Leitlinie formuliert.

Im Folgenden werden die wichtigsten Regelungen im Asylbereich auf EUEbene vorgestellt. In einem zweiten Schritt werden diese daraufhin untersucht, welche Konstruktion der Kategorie Flucht und Asyl dem Migrationsregime auf EU-Ebene zugrunde liegt, mit welchen Rechten sie verknüpft und wie sie in den Texten sprachlich präsentiert wird. Aus diesen Aspekten werden schließlich Schlussfolgerungen hinsichtlich der Bedeutung der Europäisierung dieses Politikfeldes für den Wandel des Asylregimes gezogen.

\subsection{Richtlinien, Verordnungen und weitere Maßnahmen}

Für die Untersuchung werden die derzeit vorliegenden Asylrichtlinien ${ }^{68}$, die Dublin-II-Verordnung sowie einige weitere Maßnahmen (Grenzregime, außenpolitische und ,Rückkehr'-Maßnahmen) im Migrationsbereich vorgestellt. Der gesamte Umfang der auf EU-Ebene initiierten bzw. koordinierten migrationspolitischen Aktivitäten kann hier nicht abschließend erörtert werden. Es ist zudem wichtig festzuhalten, dass nicht nur die unmittelbar im Bereich Asyl- und Flüchtlingspolitik angesiedelten Maßnahmen das Asylregime bestimmen. Auch Maßnahmen mit

\footnotetext{
${ }^{68}$ Die Reihenfolge der Darstellung der Regelungen begründet sich wie folgt: Zunächst werden die zentralen Richtlinien in der zeitlichen Abfolge ihrer Verabschiedung vorgestellt. Darauf folgt - in Abweichung der zeitlichen Chronologie - die als erste verabschiedete Richtlinie zum vorübergehenden Schutz, da diese in ihrer praktischen Bedeutung als weniger zentral eingeschätzt wird. Schließlich folgen die Dublin-II-Verordnung sowie ein Überblick über weitere für den Asylbereich relevante Maßnahmen.
} 
Bezug auf anderweitig kategorisierte ,Drittstaatenangehörige ${ }^{69}$, die ,Bekämpfung illegaler Migration“ und des ,Menschenhandels“ (vgl. Rat der Europäischen Union 2002) sowie die Visapolitik haben Einfluss auf die Entwicklung der Kategorie Flucht und Asyl - auf ihre Inanspruchnahme und damit auf ihre derzeitige und zukünftige Bedeutung. Denn die Asylantragstellung stellt für viele MigrantInnen die einzige Möglichkeit einer Legalisierung ihres Aufenthalts dar, da es kaum noch andere legale Einwanderungswege, zum Beispiel zur Erwerbsarbeit, gibt. Da auch das Asylrecht - wie zu zeigen sein wird - durch den Visumszwang und andere Maßnahmen zunehmend verengt wird, nutzen auch potentielle AsylantragstellerInnen illegale Einreisewege, oft mithilfe von Schleusern, und sind damit den entsprechenden ,Bekämpfungsstrategien“ der EU ausgesetzt. Ein weiterer wichtiger Aspekt dieser Strategien ist die Erhebung, Speicherung und Vernetzung von Daten - vor allem im Visa-Informationssystem (VIS), Schengen-Informationssystem (SIS) und im Eurodac-System. Dieser Aspekt wird ebenfalls jeweils mit angesprochen, kann aber im Rahmen der Arbeit nicht en detail dargestellt und diskutiert werden.

\subsubsection{Aufnahme von AsylbewerberInnen ${ }^{70}$}

Die Aufnahmerichtlinie ${ }^{71}$ gibt Mindestnormen für die Aufenthaltsbedingungen von AsylbewerberInnen in den EU-Mitgliedstaaten vor. Sie bezieht sich dabei auf diejenigen, die einen Status nach der GFK beantragen bzw. sich im Folge- oder Berufungsverfahren befinden. Die Anwendung der Richtlinie auf andere Gruppen - zum Beispiel solche, die subsidiären Schutz ${ }^{72}$ beantragen - obliegt den Mitgliedstaaten. Die Richtlinie regelt die Bedingungen für den Zugang zu Informationen, Wohnsitz und Bewegungsfreiheit, Einheit der Familie, Zugang zum schulischen und beruflichen Bildungssystem und zum Arbeitsmarkt, soziale Leistungen einschließlich Gesundheitsversorgung, Unterbringung und Verpflegung (Kap. II). Darüber hinaus werden Kriterien festgelegt, aufgrund derer die Mitgliedstaaten die „gewährten Vorteile“, die sich aus der Richtlinie ergeben, einschränken können (Kap. III). Schließlich werden auch Regelungen für „,besonders bedürftige Personen“ (Kap. IV) getroffen. Ferner werden Vorgaben hinsichtlich des Einlegens von Rechtsmitteln im Zusammenhang mit der Gewährung der in der Richtlinie festge-

69 Dazu gehören zum Beispiel Familienangehörige (vgl. 2003/86/EG), Studierende und Auszubildende (vgl. 2004/114/EG), ForscherInnen (vgl. 2005/71/EG), hochqualifizierte ArbeitnehmerInnen und SaisonarbeiterInnen (vgl. KOM(2005) 669).

70 2003/9/EG: Richtlinie des Rates vom 27.01.2003 zur Festlegung von Mindestnormen für die Aufnahme von Asylbewerbern in den Mitgliedstaaten.

71 Alle Richtlinien enthalten einen Absatz über ,günstigere Bestimmungen', wonach die Mitgliedstaaten die Vorgaben der Richtlinie nicht unterschreiten dürfen, wohl aber günstigere Bestimmungen verabschieden oder beibehalten können, wenn sie der jeweiligen Richtlinie nicht entgegenstehen. Damit wird der Charakter der Richtlinie als Regelung von Mindestnormen betont.

72 Vgl. zum subsidiären Schutz den Abschnitt 4.1.2. 
legten Aufnahmebedingungen (Kap. V) und zur „Verbesserung der Effizienz des Aufnahmesystems“ (Kap. VI) gemacht.

Ziel ist es laut Präambel, AsylbewerberInnen ein menschenwürdiges Leben zu ermöglichen und vergleichbare Standards in allen Mitgliedstaaten zu schaffen, um damit so genannte, sekundäre Migration' zu verhindern. Im Folgenden werden die zentralen Aspekte der Richtlinie herausgestellt - unter dem Gesichtspunkt des Verhältnisses, in das die Asylsuchenden mit den StaatsbürgerInnen gestellt werden sowie kursorisch im Vergleich zur bisherigen Rechtslage in den Mitgliedstaaten.

Eine Ausweitung der Rechte von Flüchtlingen gegenüber der derzeitigen Rechtslage in vielen Mitgliedstaaten stellt die Regelung dar, dass Asylsuchende über Aufnahmebedingungen und soziale Leistungen sowie über Unterstützungsund Beratungsorganisationen eingehend und in einer für sie verständlichen Sprache informiert werden müssen (Art. 5). Weiterhin sollen die Mitgliedstaaten die

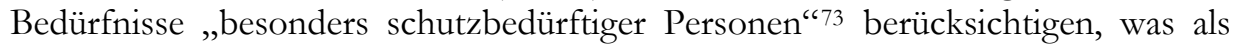
„Allgemeiner Grundsatz“ (Art. 17) in die Richtlinie aufgenommen wurde. Insbesondere Minderjährige (Art. 18) und Opfer von Folter und Gewalt (Art. 20) sollen psychologische Betreuung und eine qualifizierte Beratung erhalten. Für Minderjährige sollen Verwandte ausfindig gemacht und die Minderjährigen möglichst dort untergebracht werden. In einer Rangordnung für die Unterbringung steht die Unterbringung bei Verwandten an erster Stelle, gefolgt von Pflegefamilien, Aufnahmezentren mit speziellen Einrichtungen für Minderjährige und schließlich andere geeignete Unterkünfte. Allerdings hat diese Vorgabe lediglich Empfehlungscharakter; die Entscheidung über die Art der Unterbringung wird den Mitgliedstaaten überlassen.

Eine solche Beschränkung auf Empfehlungen sowie uneindeutige Formulierungen finden sich auch bei zahlreichen anderen Vorgaben. Auf diese Weise werden den Mitgliedstaaten umfangreiche Handlungsspielräume bei der konkreten Ausgestaltung der Aufnahmebedingungen eingeräumt. Sehr offen formuliert sind zum Beispiel der Zugang zur beruflichen Bildung („Mitgliedstaaten können [...] Zugang zur beruflichen Bildung gestatten“; Art. 12, Hervorh. D.M.), die Wahrung der Einheit der Familie (,die Einheit der Familie [...] so weit wie möglich zu wahren“; Art. 8, Hervorh. D.M.), die Unterbringung in „Unterbringungszentren, die einen angemessenen Standard gewährleisten“ (Art. 14 Abs. 1b, Hervorh. D.M.) sowie der Umgang mit unbegleiteten Minderjährigen („Geschwister sollen möglichst zusammen bleiben“; Art. 19 Abs. 2, Hervorh. D.M.).

Neben diesen Bestimmungen, die sich auf die Situation von Asylsuchenden in einigen Mitgliedstaaten positiv auswirken könnten, gleichzeitig aber vage formu-

\footnotetext{
73 Dazu zählen laut Richtlinie: Minderjährige, Behinderte, ältere Menschen, Schwangere, Alleinerziehende mit minderjährigen Kindern und Personen, die Folter, Vergewaltigung oder sonstige schwere Formen psychischer, physischer oder sexueller Gewalt erlitten haben. Die Schutzbedürftigkeit soll im Einzelfall festgestellt werden.
} 
liert sind, gibt es zahlreiche Vorgaben, die die Einführung oder Beibehaltung restriktiver Standards ermöglichen. Dazu gehören:

- Bewegungsfreiheit: Die bisher nur im deutschen Recht enthaltene Regelung der Residenzpflicht, wonach AsylbewerberInnen ein ihnen zugewiesenes Gebiet nicht ohne Genehmigung verlassen dürfen, hat auch Eingang in die Richtlinie gefunden: „Asylbewerber dürfen sich im Hoheitsgebiet des Aufnahmemitgliedstaats oder in einem ihnen von diesem Mitgliedstaat zugewiesenen Gebiet frei bewegen“ (Art. 7 Abs. 1, Hervorh. D.M.). In Absatz 2 heißt es: „Die Mitgliedstaaten können - aus Gründen des öffentlichen Interesses, der öffentlichen Ordnung oder wenn es für eine reibungslose Bearbeitung und wirksame Überwachung des betreffenden Asylantrags erforderlich ist - einen Beschluss über den Wohnsitz des Asylbewerbers fassen“ und ihm „einen bestimmten Ort zuweisen“ (Abs. 3). Die Gewährung der materiellen Aufnahmebedingungen kann an die Bedingung geknüpft werden, dass der Asylbewerber sich an die Residenzpflicht hält.

- Zugang zu Bildungseinrichtungen: Laut Richtlinie sollen Minderjährige einen Zugang zum Bildungssystem haben, der dem der Staatsangehörigen des betreffenden Staates entspricht (Art. 10 Abs. 1). Allerdings erlaubt die Richtlinie auch die Durchführung des Unterrichts in den „Unterbringungszentren“. AuBerdem ist es mit der Richtlinie vereinbar, wenn der Zugang zum Bildungssystem ab dem Zeitpunkt der Asylantragstellung um maximal drei Monate verzögert wird.

- Zugang zum Arbeitsmarkt: Art. 11 der Richtlinie ermöglicht den Mitgliedstaaten einen Zeitraum zu bestimmen, in dem der/die AsylbewerberIn keinen Zugang zum Arbeitsmarkt hat, dieser darf ein Jahr nicht überschreiten. Danach bleibt es den Mitgliedstaaten überlassen, den Zugang an weitere Voraussetzungen und Bedingungen zu knüpfen, die auch ausdrücklich das Vorrangprinzip vorsehen, demzufolge UnionsbürgerInnen und regulär anwesende Drittstaatenangehörige ein Vorrecht auf die Vermittlung von Arbeitsplätzen haben. Ungeachtet dessen können die Mitgliedstaaten Zugang zu beruflicher Bildung gewähren.

- Sach- oder Geldleistungen: Die materielle Unterstützung der AsylbewerberInnen kann nach Ermessen des Mitgliedstaates in Sach- oder Geldleistungen oder in Form von Gutscheinen ausgezahlt werden.

- Unterbringung in Lagern, Haft: Für die Unterbringung sind neben Privathäusern und Wohnungen auch „Räumlichkeiten zur Unterbringung von Asylbewerbern für die Dauer der Prüfung eines an der Grenze gestellten Asylantrags“ und „Unterbringungszentren“ vorgesehen (Art. 14 Abs. 1). Allerdings sieht der Artikel auch diverse Gründe vor, aufgrund derer Ausnahmen von diesen Modalitäten möglich sind (Art. 14 Abs. 8), zum Beispiel wenn die materiellen Aufnahmebedingungen ,in einer bestimmten Region nicht zur Verfügung stehen“ oder „sich der Asylbewerber in Gewahrsam oder in Grenzgebäuden be- 
findet, die er nicht verlassen darf" (ebd.). Hier wird also die Inhaftierung von AsylbewerberInnen erwähnt, ohne dass die Bedingungen näher erläutert werden. ${ }^{74}$ Die Frage der Inhaftierung ist inzwischen in der ,Rückführungsrichtlinie ${ }^{6}$ konkretisiert worden. Unbegleitete Minderjährige können schon ab einem Alter von 16 Jahren in Aufnahmezentren für Erwachsene untergebracht werden (Art. 19 Abs. 2).

- Medizinische Versorgung: Die Richtlinie sieht eine medizinische Versorgung vor, die ,zumindest die Notversorgung“ und „die unbedingt erforderliche Behandlung von Krankheiten" umfasst.

- Entzug dieser „Vorteile“: Die durch die Richtlinie gewährten „Vorteile“ können eingeschränkt oder entzogen werden. Hierfür legt die Richtlinie einen längeren Kriterienkatalog vor, wie zum Beispiel die Verletzung der Residenzpflicht, von Melde- und Auskunftspflichten, Verschweigen von eigenen Finanzmitteln oder falls die Asylantragstellung nicht umgehend nach Ankunft im Aufnahmestaat erfolgte oder gegen Vorschriften in Unterbringungszentren verstoßen wurde.

- Familienbegriff: Der Richtlinie liegt ein sehr enger Familienbegriff zugrunde, der nur Ehegatten und nicht verheiratete Partner, die eine dauerhafte Beziehung mit dem Asylbewerber führen (sofern diese im Ausländerrecht des jeweiligen Mitgliedstaates ähnlich wie verheiratete behandelt werden) umfasst sowie die minderjährigen, abhängigen Kinder des Paares, wenn die Familie bereits im Herkunftsland bestanden hat (Art. 2 d). Andere vom Asylsuchenden abhängige Personen werden ausgeschlossen.

Die Richtlinie regelt also nur wenige Sachverhalte konkret und bindend. Stattdessen eröffnet sie den Mitgliedstaaten einen recht weiten Interpretationsspielraum, der sowohl gleichberechtigte Aufnahmebedingungen im Verhältnis zu den StaatsbürgerInnen als auch eine deutliche Beschränkung der Rechte von Asylsuchenden zulässt. In einigen Bereichen unterschreitet die Richtlinie die bisherigen Standards in vielen alten und zum Teil den neuen Mitgliedstaaten (zum Beispiel Residenzpflicht, Sachleistungsprinzip, Beschränkung des Arbeitsmarktzugangs, Lagerschulen). ${ }^{75}$ Diese Regelungen fanden sich bislang nur in einigen, die Residenzpflicht nur in einem Mitgliedstaat - der Bundesrepublik Deutschland - und können nun im Einklang mit und unter Berufung auf EU-Vorgaben europaweit eingeführt werden.

\footnotetext{
${ }^{74}$ Bereits jetzt ist die Inhaftierung von Asylsuchenden eine weit verbreitete Praxis, die immer stärker ausgebaut wird. Die für diese Arbeit untersuchten Länder Polen und Deutschland sind dafür zwei herausragende Beispiele. In Polen werden fast alle Asylsuchenden, die über keine Einreiseerlaubnis verfügen und an der Grenze einen Asylantrag stellen, zunächst routinemäßig inhaftiert.

$75 \mathrm{Zu}$ dieser Einschätzung kommt auch der Europäische Flüchtlingsrat ECRE: „Preliminary assessment of the impact of the Directive on reception policies in individual Member States indicates that many of the Articles fall short of the current standard of reception in many member states." (ECRE 2003, S. 3)
} 
Viele Bestimmungen, die nach der Verabschiedung von Flüchtlingsorganisationen kritisiert wurden, waren im Richtlinien-Entwurf der Kommission aus Flüchtlingsperspektive noch liberaler ausgestaltet. Das Verhältnis von Kommission und Rat bei der Aushandlung der Richtlinie kann hier nicht im Einzelnen erörtert werden. Es sei lediglich zusammenfassend auf verschiedene für Flüchtlinge günstigere Regelungen hingewiesen, die sich im Kommissionsvorschlag fanden, die aber vom Rat nicht übernommen wurden. Dazu gehört insbesondere der Arbeitsmarktzugang, der den zentralen Streitpunkt bei den Verhandlungen im Rat darstellte: Der Kommissionsvorschlag sah eine Begrenzung des Ausschlusses vom Arbeitsmarkt auf sechs Monate vor (jetzt: ein Jahr) und erwähnte das Vorrangprinzip nicht explizit. Darüber hinaus enthielt der Entwurf keine Möglichkeit der Einrichtung von Lagerschulen und keine Residenzpflicht. Vorgesehen war hingegen die Möglichkeit, mit Erlaubnis des Mitgliedstaates eine eigene Unterkunft zu suchen. Die Familiendefinition umfasste weitere Familienmitglieder, die vom Asylsuchenden abhängig sind oder traumatische Erfahrungen gemacht haben. Die medizinische Untersuchung zum Schutz der, öffentlichen Gesundheit' sollte im Kommissionsentwurf noch im Einklang mit der Menschenwürde stattfinden. Dieser Passus findet sich nun nicht mehr in der Richtlinie. Die Sozialleistungen sollten laut Entwurf so ausgerichtet sein, dass sie eine Verarmung der Asylsuchenden verhindern (jetzt: „einem Lebensstandard entsprechen, der die Gesundheit und den Lebensunterhalt der Asylbewerber gewährleistet $\left.{ }^{\circ}\right) .{ }^{76}$

Die gemeinsamen EU-Regeln zur Aufnahme von AsylbewerberInnen ermöglichen den Mitgliedstaaten, AsylbewerberInnen deutlich schlechter zu stellen als legal anwesende Drittstaatenangehörige, Unions- und StaatsbürgerInnen: Sie können materiell auf niedrigem Niveau versorgt, durch Lagerunterbringung, Lagerschulen, Residenzpflicht und Ausschluss vom Arbeitsmarkt von der übrigen Gesellschaft des Aufnahmelandes weitestgehend abgeschirmt und durch das Sachleistungsprinzip (Gutscheine, Lagerunterbringung, Versorgung mit Altkleidern) stigmatisiert werden. Die Aufnahmerichtlinie enthält daher eine Reihe von Möglichkeiten, die Asylsuchenden auszugrenzen. Auffallend ist dabei, dass die meisten Strategien nicht mit der Einsparung von Kosten begründbar sind, wie dies häufig suggeriert wird. Im Gegenteil: Der Ausschluss vom Arbeitsmarkt, die Lagerunterbringung und die Versorgung mit Sachleistungen und Gutscheinen sind weitaus teurer als eine gleichberechtigte Teilhabe durch die Möglichkeit zu arbeiten, eine eigene Wohnung zu suchen und mit Bargeld einzukaufen.

Die Richtlinie sollte bis zum Februar 2005 in das Recht der Mitgliedstaaten umgesetzt werden. Gegen die Mitgliedstaaten, die dieser Vorgabe nicht nachgekommen sind oder die die Kommission nicht über die entsprechenden Umsetzungsmaßnahmen informiert hatten, leitete diese Vertragsverletzungsverfahren ein (vgl. $\operatorname{KOM}(2007)$ 745). In ihrem Bericht setzt sich die Kommission mit der Um-

${ }^{76}$ Vgl. dazu auch ECRE (2003). 
setzung der Richtlinie auseinander (vgl. ebd.). Entsprechend den weiten Handlungsspielräumen, die den Mitgliedstaaten durch die Vorgaben gelassen werden, hat die Kommission kaum Möglichkeiten, an den Zuständen in den Mitgliedstaaten Kritik zu üben. Dennoch beanstandet sie in ihrem Bericht, dass die Richtlinie in einigen Mitgliedstaaten nicht im Abschiebegewahrsam und in Transitzonen angewendet werde (vgl. $\operatorname{KOM}(2007) 745$, S. 3). Sie resümiert ferner, dass die AsylbewerberInnen in den meisten Mitgliedstaaten in Sammelunterkünften untergebracht und auch Bekleidung und Lebensmittel überwiegend nicht als Geldsondern als Sachleistungen gewährt würden. Wenn Geldleistungen gezahlt würden, so seien diese überwiegend unterhalb des Sozialhilfeniveaus angesiedelt und damit zu niedrig, um den Lebensunterhalt zu bestreiten (vgl. ebd., S. 6). Der Ermessensspielraum bezüglich der Einschränkung der Bewegungsfreiheit werde nur von Deutschland und Österreich grundsätzlich zuungunsten der AsylbewerberInnen genutzt, indem diese generell eine Residenzpflicht für alle AsylbewerberInnen vorsehen. Des Weiteren bemängelt die Kommission die automatische Ingewahrsamnahme von AsylbewerberInnen und eine lange Dauer des Gewahrsams in einigen Mitgliedstaaten. Viele Mitgliedstaaten gewährten Minderjährigen, die sich in Gewahrsam befinden, keinen Zugang zu Bildungseinrichtungen (vgl. ebd., S. 9). Die größten Probleme liegen nach Ansicht der Kommission bei der Umsetzung der Rechte besonders schutzbedürftiger Personen. Trotz dieser Beanstandungen schlussfolgert die Kommission: „Alles in allem ist die Richtlinie in den meisten Mitgliedstaaten zufrieden stellend umgesetzt worden.“ (ebd., S. 11)

Auch Flüchtlingsorganisationen haben die Aufnahmebedingungen in den Mitgliedstaaten mit Blick auf die EU-Vorgaben untersucht (vgl. ECRE 2005; ICF 2005). Der Report des Europäischen Flüchtlingsrates (ECRE) zeigt ebenfalls, dass die Aufnahmebestimmungen und -praxen der einzelnen Mitgliedstaaten auch nach der Umsetzung deutlich variieren (vgl. ECRE 2005). Dies gelte insbesondere für die Bestimmungen über den Zugang zum Arbeitsmarkt. Hier fänden sich sowohl zur Dauer des Ausschlusses vom Arbeitsmarkt zahlreiche unterschiedliche Regelungen (zwischen drei und 12 Monaten), als auch verschiedene Bestimmungen, die den Zugang zum Arbeitsmarkt faktisch behindern (Beschränkung auf bestimmte Arbeitsbereiche, Festlegung einer Arbeitszeithöchstdauer pro Woche oder Jahr, Vorrangprinzip, Antragstellung durch den Arbeitgeber). Nur in wenigen Fällen sei die Richtlinie zum Anlass genommen worden, die soziale Situation für AsylbewerberInnen zu verbessern (zum Beispiel automatischer und unbeschränkter Zugang zum Arbeitsmarkt nach sechs Monaten in Spanien, statt wie bisher bei Vorlage eines Arbeitsangebots), stattdessen habe es in zahlreichen Fällen eine Anpassung ,nach unten' gegeben (zum Beispiel haben Österreich und Litauen die Möglichkeit der Reduktion von sozialen Leistungen mit verschiedenen Begründungen erst eingeführt). Es ist davon auszugehen, dass sich die Situation in den neuen Mitgliedstaaten durch die Richtlinien-Vorgaben verbessert, während die alten Mit- 
gliedstaaten kaum von der Möglichkeit Gebrauch machen, eventuell vorhandene höhere Standards beizubehalten oder solche einzuführen.

\subsubsection{Anerkennung als Flüchtlinge ${ }^{77}$}

Die Qualifikations- oder Anerkennungsrichtlinie regelt die Kriterien für die Anerkennung von MigrantInnen als Flüchtlinge oder als Personen, denen subsidiärer Schutz gewährt wird sowie den Inhalt des Schutzstatus, also die damit verbundenen Rechte. Ziel ist laut Kommissions-Vizepräsident Franco Frattini „,die Angleichung der derzeit sehr unterschiedlichen Anerkennungsraten und somit zur Verringerung des so genannten Asylshopping beizutragen“ (EU-Pressemitteilung vom 10.10.2006). Bisher gibt es in den Mitgliedstaaten deutlich variierende Anerkennungschancen für Menschen, die ähnliche Fluchtgeschichten und -gründe geltend machen (vgl. Neumayer 2005). Als Beispiel seien AntragstellerInnen aus der Russischen Föderation genannt: Die Zahl der Schutzsuchenden aus der Russischen Föderation, meist TschetschenInnen, deren Anträge auf Flüchtlingsstatus positiv beschieden werden, geht in den EU-Mitgliedstaaten deutlich auseinander. Sie reicht von 0 Prozent in der Slowakei bis zu 73 Prozent in Österreich im Jahr 2007 (vgl. UNHCR 2008b). ${ }^{78}$

Die Flüchtlingsdefinition in Artikel 2c der Richtlinie ist an die GFK und das dazugehörige New Yorker Protokoll von 1967 angelehnt. ${ }^{79}$ Allerdings schränkt sie den Kreis derer, die als Flüchtlinge anerkannt werden können, auf Drittstaatenangehörige und Staatenlose ein, während die GFK eine solche Einengung nicht kennt. ${ }^{80}$ Der subsidiäre Schutzstatus soll laut Richtlinie AntragstellerInnen verliehen werden, die die Kriterien der Flüchtlingsdefinition nicht erfüllen, bei denen aber andere Gründe gegen eine Rückkehr ins Herkunftsland sprechen. ${ }^{81}$

\footnotetext{
77 2004/83/EG: Richtlinie des Rates vom 29.04.2004 über Mindestnormen für die Anerkennung und den Status von Drittstaatsangehörigen oder Staatenlosen als Flüchtlinge oder als Personen, die anderweitig internationalen Schutz benötigen, und über den Inhalt des zu gewährenden Schutzes.

78 Die vom UNHCR ausgewiesene Anerkennungsquote für die Slowakei berücksichtigt erstinstanzliche Entscheidungen. Die Quote für Österreich beinhaltet zusätzlich die Entscheidungen nach der Einlegung von Rechtsmitteln. Allerdings liegt auch die Gesamtschutzquote in der Slowakei, also die Schutzquote unter Einbeziehung anderer Schutzstatus, bei 0 Prozent, in Österreich bei 85 Prozent (vgl. UNHCR 2008b).

79 „Im Sinne dieser Richtlinie bezeichnet der Ausdruck [...] ,Flüchtling ‘ einen Drittstaatsangehörigen, der aus der begründeten Furcht vor Verfolgung wegen seiner Rasse, Religion, Staatsangehörigkeit, politischen Überzeugung oder Zugehörigkeit zu einer bestimmten sozialen Gruppe sich außerhalb des Landes befindet, dessen Staatsangehörigkeit er besitzt, und den Schutz dieses Landes nicht in Anspruch nehmen kann oder wegen dieser Furcht nicht Anspruch nehmen will, oder einen Staatenlosen, der sich aus denselben vorgenannten Gründen außerhalb des Landes befindet und nicht dorthin zurückkehren kann oder wegen dieser Furcht nicht dorthin zurückkehren will und auf den Artikel 12 keine Anwendung findet [...]“ (Richtlinie 2004/83/EG, Art. 2c).

$80 \mathrm{Zu}$ weiteren Unterschieden im Verhältnis zur GFK vgl. UNHCR 2005.

${ }^{81}$ Dies gilt insbesondere, wenn „er bei einer Rückkehr in sein Herkunftsland oder, bei einem Staatenlosen, in das Land seines vorherigen gewöhnlichen Aufenthalts tatsächlich Gefahr liefe, einen
} 
Die Richtlinie erkennt für den Flüchtlingsstatus grundsätzlich auch Nachfluchtgründe an (also Aktivitäten wie ein Religionswechsel, die erst nach der Flucht und im Aufnahmeland stattgefunden haben) (Art. 5), dies gilt jedoch nicht für Folgeanträge. Die GFK hingegen sieht eingeschränkten Schutz auch für diejenigen vor, denen aufgrund exilpolitischer Aktivitäten Verfolgung droht. Neben dem Staat und Parteien oder Organisationen, die den Staat oder einen wesentlichen Teil davon beherrschen, werden in der Richtlinie auch nichtstaatliche Akteure als solche anerkannt, von denen Verfolgung oder ein ernsthafter Schaden ausgehen kann. Besteht eine inländische Fluchtalternative (,interner Schutz'), so wird erwartet, dass die AntragstellerInnen dort Schutz suchen. Die Mitgliedstaaten können deshalb die Gewährung eines Schutzstatus verwehren, auch wenn „praktische Hindernisse für eine Rückkehr bestehen“. UNHCR (2005) wendet hier ein, eine Fluchtalternative müsse sicher und erreichbar sein, überdies bestehe bei einer Verfolgung durch staatliche Akteure generell keine interne Fluchtalternative. Des Weiteren definiert die Richtlinie Verfolgungshandlungen (Art. 9) und -gründe (Art. 10). Als Verfolgungshandlung gelten unter anderem auch sexuelle Gewalt (Art. 9 Abs. 2a) sowie unter bestimmten Umständen Strafverfolgung und Bestrafung wegen Militärdienstverweigerung (,wenn der Militärdienst Verbrechen und Handlungen umfassen würde, die unter die Ausschlussklauseln des Artikels 12 Absatz 2 fallen“) (Art. 9 Abs. 2e) und „Handlungen, die an die Geschlechtszugehörigkeit anknüpfen oder gegen Kinder gerichtet sind" (Art. 9 Abs. 2f). Bei den Verfolgungsgründen sind insbesondere das öffentliche Glaubensbekenntnis und die Definition der „sozialen Gruppe“ hervorzuheben, die sich auch auf das gemeinsame Merkmal der sexuellen Ausrichtung gründen kann. Die Aufnahme geschlechterspezifischer Aspekte ermöglicht nun auch die Anerkennung von Asylanträgen von Frauen, „die z.B. mit Akten sexueller Gewalt, häuslicher Gewalt und Genitalverstümmelung begründet werden; Homosexualität kann ebenfalls als Verfolgungsgrund gelten“ (EU-Pressemitteilung vom 10.10.2006).

Der Flüchtlingsstatus erlischt, wenn die anerkannte Person zum Beispiel eine neue Staatsbürgerschaft angenommen hat, freiwillig zurückkehrt oder wenn die Umstände entfallen, die den Flüchtlingsstatus begründet haben. Die Veränderung

ernsthaften Schaden im Sinne des Artikel 15 zu erleiden [...].“ Artikel 15 definiert „ernsthaften Schaden“ als „die Verhängung oder Vollstreckung der Todesstrafe“ (Art. 15a) oder „Folter oder unmenschliche oder erniedrigende Behandlung oder Bestrafung eines Antragstellers im Herkunftsland“ (Art. 15b) oder „eine ernsthafte individuelle Bedrohung des Lebens und der Unversehrtheit einer Zivilperson infolge willkürlicher Gewalt im Rahmen eines internationalen oder innerstaatlichen Konflikts" (Art. 15c). Dabei bleibt offen, inwieweit der Schutzanspruch auch dann besteht, wenn es sich um Gefahren handelt, denen die Bevölkerung oder die Bevölkerungsgruppe, der der Schutzsuchende angehört, allgemein ausgesetzt ist. Diese Problematik wird in dem Artikel selbst nicht angesprochen, der Erwägungsgrund Nr. 28 (Erwägungsgründe sind Auslegungsgrundsätze zur Interpretation der Richtlinien-Vorgaben) stellt allerdings fest: „Gefahren, denen die Bevölkerung oder eine Bevölkerungsgruppe eines Landes allgemein ausgesetzt ist, stellen für sich genommen normalerweise keine individuelle Bedrohung dar, die als ernsthafter Schaden zu beurteilen wären." 
der Situation im Herkunftsland soll dabei erheblich und nicht nur vorübergehend sein. Unter verschiedenen Umständen kann eine Person auch von vornherein von der Anerkennung als Flüchtling ausgeschlossen werden: wenn sie zum Beispiel den Schutz einer Organisation oder einer UN-Institution (außer UNHCR) genießt oder Verbrechen gegen den Frieden, Kriegsverbrechen oder Verbrechen gegen die Menschlichkeit oder eine schwere nichtpolitische Straftat außerhalb des Aufnahmelandes oder Handlungen begangen hat, die den Zielen und Grundsätzen der UN (Art. 1 und 2 der UN-Charta) entgegenstehen. Der Flüchtlingsstatus kann unter anderem aberkannt, beendet oder seine Verlängerung verweigert werden, wenn im Anerkennungsverfahren Tatsachen falsch dargelegt oder verschwiegen oder gefälschte Dokumente verwendet wurden; oder wenn die Person eine „Gefahr für die Sicherheit des Mitgliedstaates“ oder die „Allgemeinheit dieses Mitgliedstaates" darstellt (Art. 14). Ähnliche Grundsätze für Ausschluss, Erlöschen und Aberkennung gelten für den subsidiären Schutzstatus (Art. 16, 17 und 19). Diese Regelungen werden damit begründet, dass die EU ,asylsuchenden Terroristen keinen Unterschlupf bieten“" will (ebd.).

Der Unterschied zwischen dem Flüchtlingsstatus und dem subsidiären Status besteht im Wesentlichen in den damit verbundenen Rechten, wie die folgende Übersicht zusammenfassend zeigt. 
Übersicht 1: Vergleich Flüchtlingsstatus - subsidärer Status

(Zusammenstellung und Hervorh. D.M.).

\begin{tabular}{|c|c|c|}
\hline & Flüchtlingsstatus & Subsidiärer Status \\
\hline $\begin{array}{l}\text { Familie } \\
\text { (Art. 23) }\end{array}$ & $\begin{array}{l}\text { Familienangehörige, die selbst } \\
\text { keinen Flüchtlingsstatus haben, } \\
\text { haben Anspruch auf Ver- } \\
\text { günstigungen aus Art. 24-34 wie } \\
\text { der anerkannte Flüchtling }\end{array}$ & $\begin{array}{l}\text { Mitgliedstaaten können Bedingun- } \\
\text { gen festlegen, unter denen Famili- } \\
\text { enangehörige von subsidiär } \\
\text { Schutzberechtigten die Vergünsti- } \\
\text { gungen aus Art. 24-34 erhalten }\end{array}$ \\
\hline $\begin{array}{l}\text { Aufenthaltstitel } \\
\text { (Art. 24) }\end{array}$ & $\begin{array}{l}\text { mind. } 3 \text { Jahre gültig und verlänger- } \\
\text { bar }\end{array}$ & $\begin{array}{l}\text { mind. } 1 \text { Jahr gültig und verlänger- } \\
\text { bar }\end{array}$ \\
\hline $\begin{array}{l}\text { Arbeitsmarkt- } \\
\text { zugang } \\
\text { (Art. 26) }\end{array}$ & $\begin{array}{l}\text { nach Anerkennung Zugang zum } \\
\text { Arbeitsmarkt }\end{array}$ & $\begin{array}{l}\text { Zugang ja, dabei kann Arbeits- } \\
\text { marktlage berücksichtigt werden / } \\
\text { Vorrangprüfung für begrenzten } \\
\text { Zeitraum möglich }\end{array}$ \\
\hline $\begin{array}{l}\text { Zugang zu } \\
\text { berufsbildenden } \\
\text { Maßnahmen } \\
\text { etc. } \\
\text { (Art. 26) }\end{array}$ & $\begin{array}{l}\text { zu gleichwertigen Bedingungen wie } \\
\text { Staatsangehörige }\end{array}$ & $\begin{array}{l}\text { Bedingungen können von Mitglied- } \\
\text { staaten festgelegt werden }\end{array}$ \\
\hline $\begin{array}{l}\text { Zugang zu } \\
\text { Bildung } \\
\text { (Art. 27) }\end{array}$ & \multicolumn{2}{|c|}{$\begin{array}{l}\text { für Minderjährige zu denselben Bedingungen wie Staatsangehörige } \\
\text { für Erwachsene entsprechend den Bedingungen für andere reguläre } \\
\text { Drittstaatenangehörige }\end{array}$} \\
\hline $\begin{array}{l}\text { Sozialhilfe } \\
\text { (Art. 28) }\end{array}$ & entsprechend Staatsangehörigen & $\begin{array}{l}\text { kann auf Kernleistungen be- } \\
\text { schränkt werden }\end{array}$ \\
\hline $\begin{array}{l}\text { Med. Versor- } \\
\text { gung } \\
\text { (Art. 29) }\end{array}$ & $\begin{array}{l}\text { zu Bedingungen wie Staatsange- } \\
\text { hörige }\end{array}$ & $\begin{array}{l}\text { kann auf Kernleistungen be- } \\
\text { schränkt werden }\end{array}$ \\
\hline $\begin{array}{l}\text { Wohnraum } \\
\text { (Art. 31) }\end{array}$ & \multicolumn{2}{|c|}{$\begin{array}{l}\text { zu Bedingungen, die auch für andere reguläre Drittstaatenangehörige } \\
\text { gelten }\end{array}$} \\
\hline $\begin{array}{l}\text { Freizügigkeit } \\
\text { (Art. 32) }\end{array}$ & \multicolumn{2}{|c|}{$\begin{array}{l}\text { zu Bedingungen, die auch für andere reguläre Drittstaatenangehörige } \\
\text { gelten }\end{array}$} \\
\hline $\begin{array}{l}\text { Integration } \\
\text { (Art. 33) }\end{array}$ & $\begin{array}{l}\text { Mitgliedstaaten sehen „,von ihnen } \\
\text { als sinnvoll erachtete Integrations- } \\
\text { programme vor [...]" } \\
\text { (Art. } 33 \text { Abs. 1) }\end{array}$ & $\begin{array}{l}\text { Zugang zu Integrations- } \\
\text { programmen, wenn „die Mitglied- } \\
\text { staaten es für sinnvoll erachten“ } \\
\text { (Art. } 33 \text { Abs. 2) }\end{array}$ \\
\hline
\end{tabular}

Die Übersicht verdeutlicht, dass es überwiegend den Mitgliedstaaten überlassen wird, den subsidiären Schutz auszugestalten und dass ihnen dafür die Möglichkeit 
der Beschränkung von Leistungen und Rechten, die die Schutzberechtigten in Anspruch nehmen können, eingeräumt wird. Dies gilt insbesondere für Sozialhilfe, medizinische Versorgung und den Zugang zum Arbeitsmarkt.

Der der Richtlinie zugrunde liegende Familienbegriff ist vom Inhalt her identisch mit dem der Aufnahmerichtlinie, also auf die Kernfamilie beschränkt. Dabei wird es den Mitgliedstaaten überlassen, ob sie die für Familienangehörige geltenden ,Vergünstigungen' auch auf andere enge Verwandte, die auf den Schutzberechtigten angewiesen sind, ausdehnen wollen (Art. 23 Abs. 5).

Vor allem der subsidiäre Schutz und der Zugang zum Arbeitsmarkt waren Streitpunkte bei den Verhandlungen im Rat. Die Richtlinie sollte bis 10.10.2006 ins Recht der Mitgliedstaaten umgesetzt sein. Bis dahin hatten erst sechs Mitgliedstaaten dieser Vorgabe Folge geleistet: Estland, Litauen, Österreich, Frankreich, Slowenien und Luxemburg.

\subsubsection{Asylverfahren ${ }^{82}$}

Um die Asylverfahrensrichtlinie gab es die meisten Konflikte zwischen Kommission, Rat und Parlament. Sie wurde als einzige nicht rechtzeitig vor Mai 2004 vom Rat verabschiedet. Die Richtlinie enthält Mindestnormen für Verfahren zur Zuerkennung und Aberkennung der Flüchtlingseigenschaft, das heißt unter anderem den Zugang zum Verfahren (Art. 6), Anforderungen an die Prüfung von Anträgen (Art. 8) und an Entscheidungen (Art. 9) sowie Garantien ${ }^{83}$ (Art. 10) und Verpflichtungen $^{84}$ (Art. 11) für AsylbewerberInnen im Rahmen des Verfahrens. Des Weiteren enthält die Richtlinie Garantien für unbegleitete Minderjährige (Art. 17), wonach die Mitgliedstaaten für diese einen Vertreter bestellen sollen. Dies können sie allerdings verweigern, wenn der Minderjährige 16 Jahre und älter oder verheiratet ist. Die Mitgliedstaaten können ferner ärztliche Untersuchungen zur Altersbestimmung vorsehen. Zur Ingewahrsamnahme führt die Richtlinie aus, dass die Mitgliedstaaten Personen nicht allein, weil sie AsylbewerberInnen sind, in Gewahrsam nehmen dürfen (Art. 18).

Die zentralen und umstrittensten Instrumente der Richtlinie sind die Konzepte der sicheren Herkunfts ${ }^{85}$ - und der sicheren Drittstaaten ${ }^{86}$. Die damit verbundenen

82 2005/85/EG: Richtlinie des Rates vom 1.12.2005 über Mindestnormen für Verfahren in den Mitgliedstaaten zur Zuerkennung und Aberkennung der Flüchtlingseigenschaft

$83 \mathrm{Zu}$ den Garantien gehören: Aufklärung über Rechte und Pflichten, Zugang zu Dolmetschern und dem UNHCR, Inkenntnissetzung über das Ergebnis des Verfahrens, gleichwertige Garantien für Grenzverfahren.

${ }^{84}$ Die Verpflichtungen beinhalten: Zusammenarbeit mit Behörden, Meldepflicht und persönliches Vorsprechen, die Vorlage von Dokumenten, die Angabe des Aufenthaltsortes, die Durchsuchung des Bewerbers und seiner Sachen, Lichtbildaufnahme und Aufzeichnen von Aussagen.

${ }^{85}$ Nach Anhang II der Richtlinie gilt ein Herkunftsstaat als sicher, „wenn sich anhand der dortigen Rechtslage, der Anwendung der Rechtsvorschriften in einem demokratischen System und der allgemeinen politischen Lage nachweisen lässt, dass dort generell und durchgängig weder eine Verfolgung im Sinne des Artikels 9 der Richtlinie 2004/83/EG noch Folter oder unmenschliche oder 
Ziele werden in der Erwägung 22 der Richtlinie deutlich: Danach sollen die Mitgliedstaaten zwar alle Asylanträge in der Sache prüfen, aber nur dann, wenn nicht ein anderer Staat den Antrag prüfen und Schutz geben würde. Asylsuchende, die aus sicheren Herkunfts- oder aus bzw. über sichere Drittstaaten einreisen, können nach einem beschleunigten Verfahren (Art. 23 Abs. 4c) in diese zurückgeschoben werden. Die Mitgliedstaaten können auf EU-Ebene eine gemeinsame Minimalliste sicherer Herkunftsstaaten erstellen (Art. 29), zusätzlich steht es den Mitgliedstaaten frei durch innerstaatliche Rechts- und Verwaltungsvorschriften selbst weitere Staaten als sicher zu erklären (Art. 30). Während bei der Drittstaatenregelung nach Art. 27 auch Regeln festgelegt werden sollen, „die es ermöglichen, in Form einer Einzelprüfung festzustellen, ob der betreffende Drittstaat für einen bestimmten Asylbewerber sicher ist, und die dem Asylbewerber zumindest die Möglichkeit bieten, die Anwendung des Konzepts der sicheren Drittstaaten mit der Begründung anzufechten, dass er der Folter oder einer grausamen, unmenschlichen oder erniedrigenden Behandlung oder Bestrafung unterworfen würde" (Art. 27 Abs. 2 c), so ist dies beim Konzept der so genannten ,supersicheren' Drittstaaten nicht der Fall (Art. 36). Bei diesem „Europäischen Konzept der sicheren Drittstaaten“ können die Mitgliedstaaten gänzlich auf die Prüfung des Asylantrags und der Sicherheit des Asylbewerbers in seiner spezifischen Situation verzichten und in den betreffenden Drittstaat abschieben. Artikel 35 erlaubt es den Mitgliedstaaten, Verfahren an der Grenze oder in Transitzonen - etwa an Flughäfen - durchzuführen (Abs. 1) und andere Sonderverfahren, die von den Garantien des Kapitels II der Richtlinie abweichen, beizubehalten. Dies ermöglicht es, die Einreise von Asylsuchenden zunächst zu verweigern. Erst wenn das Grenz- oder Transitverfahren die Frist von vier Wochen überschreitet, soll der Asylbewerber in das Hoheitsgebiet des Mitgliedstaates einreisen dürfen (Abs. 4).

Obgleich der erste Kommissions-Entwurf der Richtlinie schon im Jahr 2000 vorlag, wurde sie erst im Dezember 2005 verabschiedet - Rat, Kommission und Parlament konnten sich jahrelang nicht einigen. ${ }^{87}$ Das Parlament stimmte dem ersten Kommissionsvorschlag von 2000 erst nach der Einarbeitung von über 100 Änderungen zu. Da sich der Rat auf dieser Basis nicht einigen konnte, wurde die Kommission vom Europäischen Rat durch die Erklärung von Laeken aufgefordert, einen neuen Vorschlag auszuarbeiten. Dieser wurde im Juni 2002 veröffentlicht. Nach zwei Jahren Verhandlungen konsultierte der Rat mit seinem Vorschlag

erniedrigende Behandlung oder Strafe noch Bedrohung infolge willkürlicher Gewalt im Rahmen eines internationalen oder innerstaatlichen bewaffneten Konflikts zu befürchten sind.“

86 Als sicher gilt ein Drittstaat, in dem für den Asylbewerber „,keine Gefährdung von Leben und Freiheit aus Gründen der Rasse, der Religion, der Nationalität, der Zugehörigkeit zu einer bestimmten sozialen Gruppe oder der politischen Überzeugung" besteht, das Non-RefoulementGebot eingehalten wird und ein Antrag auf Anerkennung als GFK-Flüchtling möglich ist (Art. 27 Abs. 1 a-d).

${ }^{87} \mathrm{Vgl}$. zu den Etappen der Entstehung der Richtlinie:

http://ec.europa.eu/prelex/detail_dossier_real.cfm?CL=de\&DosId=159479 
erneut das Parlament. Dieses legte einen Katalog von 174 Änderungsvorschlägen vor, die ausnahmslos vom Rat ignoriert wurden; im Amtsblatt der Europäischen Union wird die erneute Konsultation des Parlaments gänzlich verschwiegen (vgl. Kreissl-Dörfler 2006).

Wichtigster Streitpunkt ist das Konzept der so genannten ,supersicheren“ Drittstaaten, das keine Einzelfallprüfung mehr vorsieht. Darüber hinaus erklärt sich das Parlament nur mit einer Liste sicherer Herkunftsstaaten auf EU-Ebene einverstanden und lehnt die Ergänzung um weitere Drittstaaten durch die Mitgliedstaaten ab. An der Entscheidung über diese Liste will das Parlament - anders als es die Richtlinie derzeit vorsieht - im Mitentscheidungsverfahren beteiligt werden. Es hat daher vor dem Europäischen Gerichtshof eine Nichtigkeitsklage erhoben, die sich gegen die Bestimmungen der Richtlinie richten, die seine bloße Anhörung vorsehen. Laut Richtlinie sollte der Rat nach Anhörung des Europäischen Parlaments mit qualifizierter Mehrheit eine Minimalliste der Drittstaaten, die von den Mitgliedstaaten als sichere Herkunftsstaaten zu betrachten sind, sowie eine Liste sicherer europäischer Drittstaaten erstellen und ändern können. Der EuGH erklärte die entsprechenden Bestimmungen in seinem Urteil vom Juni 2008 für nichtig und verfügte, dass das Parlament entgegen der bisherigen Regelung im Mitentscheidungsverfahren an der Erstellung der Listen zu beteiligen sei (vgl. EuGH 2008).

Die Richtlinie sollte bis zum 1. Dezember 2007 in innerstaatliches Recht umgesetzt werden. Laut Grünbuch zum Gemeinsamen Europäischen Asylsystem strebt die Kommission eine weitere Angleichung der Asylverfahren in den Mitgliedstaaten an - so müsse zukünftig an die Stelle einer „Reihe verfahrensrechtlicher Standards“ ein gemeinsames „Standardverfahren“ treten (KOM(2007) 301, S. 3).

\subsubsection{Vorübergehender Schutz ${ }^{88}$}

In der Begründung für den Erlass der Richtlinie für vorübergehenden Schutz bezieht sich der Rat bzw. die Kommission auf die Flüchtlingsbewegungen im Zusammenhang mit den Kriegen im ehemaligen Jugoslawien. Die Richtlinie regelt Mindestnormen bezüglich der Rechte und Pflichten der Vertriebenen, die vorübergehend Schutz erhalten und eine ,solidarische“ Verteilung der Schutzberechtigten bzw. der mit der Aufnahme verbundenen „Belastungen“ zwischen den Mitgliedstaaten. Vorübergehender Schutz soll laut Richtlinie im Falle eines „Massenzustroms" von Vertriebenen gewährt werden, die nicht in ihr Herkunftsland zurückkehren können und mit deren Aufnahme das Asylsystem überfordert wäre.

\footnotetext{
88 2001/55/EG: Richtlinie des Rates vom 20. Juli 2001 über Mindestnormen für die Gewährung vorübergehenden Schutzes im Falle eines Massenzustroms von Vertriebenen und Maßnahmen zur Förderung einer ausgewogenen Verteilung der Belastungen, die mit der Aufnahme dieser Personen und den Folgen dieser Aufnahme verbunden sind, auf die Mitgliedstaaten.
} 
Vertriebene sind demnach Drittstaatenangehörige oder Staatenlose, die ihr Herkunftsland verlassen mussten oder evakuiert wurden und die aufgrund der Lage im Herkunftsland nicht sicher und dauerhaft zurückkehren können. Damit sind vor allem Personen gemeint, die aus Regionen geflohen sind, in denen bewaffnete Konflikte oder dauernde Gewalt herrschen oder die systematischen oder weit verbreiteten Menschenrechtsverletzungen ausgesetzt sind (Art. 2c). Darunter können auch Flüchtlinge gemäß GFK sein. Das Bestehen eines solchen „Massenzustroms“ wird per Ratsbeschluss festgestellt (Art. 5). Der vorübergehende Schutz ist auf ein Jahr beschränkt und kann sich um maximal ein Jahr verlängern bzw. durch den Rat verlängert werden (Art. 4). Der Schutz endet entweder mit Erreichen der Höchstdauer oder jederzeit mit Beschluss des Rates, der sich daran orientieren soll, ob eine sichere und dauerhafte Rückkehr möglich ist (Art. 6). Die Mitgliedstaaten können auch außerhalb des Ratsbeschlusses vorübergehenden Schutz gewähren, das Verteilungssystem zwischen den Mitgliedstaaten greift dann allerdings nicht (Art. 7).

Die Mitgliedstaaten sind verpflichtet den Schutzberechtigten die VisaErlangung zu erleichtern (Art. 8 Abs. 3), ihnen Zugang zum Arbeitsmarkt zu gewähren (Vorrangprinzip ist möglich) (Art. 12) sowie für Unterbringung und Sozialleistungen zu sorgen und dabei Personen mit besonderen Bedürfnissen besonders zu berücksichtigen (Art. 13). Dabei kann die medizinische Versorgung auf eine Notversorgung beschränkt werden. Unter 18jährige sollen einen den StaatsbürgerInnen ähnlichen Zugang zum öffentlichen Bildungssystem erhalten (Art. 14 Abs. 1). Der Familienbegriff der Richtlinie geht über den der bereits genannten Richtlinien hinaus: Auch ,andere enge Verwandte, die zum Zeitpunkt der den Massenzustrom auslösenden Ereignisse innerhalb des Familienverbands lebten und zu diesem Zeitpunkt für ihren Unterhalt vollständig oder größtenteils auf den Bürgen angewiesen waren“ (Art. 15 Abs. 1b), werden als Familienangehörige definiert. Die Schutzberechtigten sollen ungehinderten Zugang zum Asylverfahren haben (Art. 17). Nach Ablauf des Schutzes sind „freiwillige Rückkehr“" (Art. 21) als auch Zwangsmaßnahmen zur Rückkehr (Art. 22) vorgesehen. Dabei soll ausdrücklich keine Abschiebung stattfinden, falls und solange es der Gesundheitszustand der Person nicht zulässt (Art. 23 Abs. 1).

Die Verteilung zwischen den Mitgliedstaaten erfolgt gemäß den von diesen angegebenen Aufnahmekapazitäten (Art. 25). Die Richtlinie ermöglicht es den Mitgliedstaaten, Personen vom vorübergehenden Schutz auszuschließen (Art. 28), die Gründe sind ähnlich wie in der Anerkennungsrichtlinie: wenn Verdacht auf ein Verbrechen gegen den Frieden, Kriegsverbrechen oder Verbrechen gegen die Menschlichkeit besteht oder vor der Aufnahme ein anderes schweres Verbrechen begangen wurde. ${ }^{89}$ Gegen den Ausschluss vom vorübergehenden Schutz oder von

89 Bei letzterem soll die Schwere der zu erwartenden Verfolgung gegen die Art der begangenen Straftat abgewogen werden. Weitere Ausschlussgründe sind Handlungen, die den Grundsätzen der 
der Familienzusammenführung können die Betroffenen im betreffenden Mitgliedstaat Rechtsbehelfe einlegen (Art. 29).

Unklar bleibt in der Richtlinie das Verhältnis zwischen der Konzeption des vorübergehenden Schutzes und dem Recht auf Asyl. In der Richtlinie wird betont, dass letzteres durch die Richtlinie nicht angetastet werden soll - Schutzberechtigte sollen die Möglichkeit haben, einen Asylantrag zu stellen. Die Trennung zwischen „vorübergehend Schutzberechtigten“ und GFK-Flüchtlingen zeigt sich auch in der Benennung als Vertriebene statt als Flüchtlinge. UNHCR hält die Definition des Begriffs „,vertriebene Staatsangehörige“ für „missverständlich“, da der Eindruck entstehen könne, es handele sich nicht um Flüchtlinge. Stattdessen könnten aber von der Richtlinie erfasste Personen in den Geltungsbereich der GFK fallen (vgl. UNHCR 2003).

Es ist zu vermuten, dass es sich hier nicht um ein ,Missverständnis ' handelt, sondern um eine beabsichtigte stärkere Ausdifferenzierung innerhalb der Kategorie ,Flüchtling‘ auf EU-Ebene. Auf die Defizite des GFK-Status angesichts aktueller Migrationen wird nicht mit dessen Ausweitung, sondern mit der Konstruktion einer neuen Kategorie reagiert, die mit deutlich weniger Rechten - insbesondere hinsichtlich der Aufenthaltsdauer - verbunden ist und deren Aktivierung darüber hinaus von einem eigenen Rats-Beschluss abhängig ist. Mit der Einführung dieser Kategorie findet sich auf EU-Ebene nun ein dreistufig hierarchisiertes Kategoriensystem, das die Inanspruchnahme von Schutz durch - laut Definition - ,unfreiwillige' MigrantInnen strukturiert:

1. GFK-Flüchtlinge (Anerkennungsrichtlinie)

2. Personen mit subsidiärem Schutz (Anerkennungsrichtlinie)

3. vorübergehend Schutzberechtigte (Richtlinie zum vorübergehenden Schutz) Das Interesse an einer solchen weiteren Differenzierung, mit deren Hilfe viele Schutzbegehren außerhalb der Asylverfahren gehalten werden können, könnte eine mögliche Erklärung dafür sein, dass die Richtlinie die erste war, die verabschiedet wurde. Sie forderte offenbar kaum Kontroversen heraus. Eine weitere Erklärung für die vergleichsweise schnelle Einigung könnte sein, dass die Wirksamkeit der Richtlinie von einem weiteren Ratsbeschluss abhängig gemacht wurde. Es ist fraglich, ob der Rat angesichts der aktuellen Tendenzen, den Flüchtlingsschutz aus der EU auszulagern, jemals einen Beschluss über das Bestehen eines „Massenzustroms“ treffen wird. Bislang hat die Richtlinie jedenfalls noch keine Anwendung gefunden.

UN zuwiderlaufen oder die begründete Annahme, dass die Person eine Gefahr für die Sicherheit oder die Allgemeinheit des Aufnahmestaates darstellt. 


\subsubsection{Dublin-II- und Eurodac-Verordnung ${ }^{90}$}

Eine für die Architektur des europäischen Asylregimes besonders folgenreiche und seitens Flüchtlingsorganisationen und EU-Parlament stark kritisierte Regelung ist die Dublin-II-Verordnung. Die Verordnung löst das Dubliner Übereinkommen von $1991 \mathrm{ab}$. Sie ist seit dem 17.09.2003 anzuwenden und regelt, welcher Mitgliedstaat für die Prüfung eines Asylantrags zuständig ist. Sie hat ohne Umsetzung in einzelstaatliches Recht verbindliche und unmittelbare Wirkung in den Mitgliedstaaten. Die Verordnung hebelt innerbalb der EU die ,sichere Drittstaatenregelung' aus.

Nach der Verordnung ist - sehr grob zusammengefasst - derjenige Staat für die Prüfung des Asylantrags zuständig, in dem entweder bereits ein Familienmitglied als Flüchtling anerkannt wurde (Art. 7) oder einen noch nicht entschiedenen Antrag gestellt hat (Art. 8) oder der Mitgliedstaat, der die Einreise des Asylsuchenden zum Beispiel durch Ausstellung eines Aufenthaltstitels (Art. 9 Abs. 1) oder eines Visums (Art. 9 Abs. 2) ermöglicht bzw. den illegalen Grenzübertritt nicht verhindert hat (Art. 10). Die Kriterien für die Zuständigkeit werden sehr detailliert in der Verordnung beschrieben und in einer Rangfolge aufgeführt, in der sie zur Anwendung kommen sollen. Auch wenn die Anwesenheit eines Familienmitglieds der Kernfamilie mit Flüchtlingsstatus an vorderster Stelle der Rangfolge genannt wird, bleibt es laut Art. 15 den Mitgliedstaaten überlassen, ob sie eine Familienzusammenführung aus humanitären Gründen herbeiführen, auch wenn sie nicht für die Anträge aller Familienmitglieder zuständig sind (,Selbsteintrittsrecht ${ }^{\circ}$ ). Dies entspricht der gleich zu Beginn konstatierten Unterordnung der Einheit der Familie unter die Erreichung der Ziele der Verordnung: So heißt es in der Erwägung 6, die Einheit der Familie solle gewahrt werden, ,soweit dies mit den sonstigen Zielen vereinbar ist, die mit der Festlegung von Kriterien und Verfahren zur Bestimmung des für die Prüfung eines Asylantrags zuständigen Mitgliedstaats angestrebt werden".

Die Mitgliedstaaten, die für das Verfahren zuständig sind, können die Asylsuchenden dann entsprechend ihren einzelstaatlichen Rechtsvorschriften in einen (sicheren) Drittstaat (außerhalb der EU) abschieben (Art. 3 Abs. 3). Die Verordnung enthält auch abseits der Familienzusammenführung ein ,Selbsteintrittsrecht ${ }^{6}$ für die Mitgliedstaaten, wonach diese freiwillig die Prüfung eines Asylantrages übernehmen können, auch wenn sie nach den Kriterien der Verordnung nicht dafür zuständig wären (Art. 3 Abs. 2 und Art. 15).

Das Verfahren sieht folgendermaßen aus (Kap. V): Kann ein Mitgliedstaat Indizien ${ }^{91}$ oder Beweise dafür anführen, dass ein Asylbewerber sich bereits in einem

\footnotetext{
90 2003/343/EG: Verordnung (EG) Nr. 343/2003 des Rates vom 18. Februar 2003 zur Festlegung der Kriterien und Verfahren zur Bestimmung des Mitgliedstaats, der für die Prüfung eines von einem Drittstaatsangehörigen in einem Mitgliedstaat gestellten Asylantrags zuständig ist.

91 Unter Indizien werden in der Richtlinie „einzelne Anhaltspunkte“ verstanden, „die, obwohl sie anfechtbar sind, in einigen Fällen nach der ihnen zugebilligten Beweiskraft ausreichen können“
} 
anderen Mitgliedstaat aufgehalten hat - zum Beispiel auf der Durchreise - oder bereits in einem anderen Mitgliedstaat einen Asylantrag gestellt hat, ersucht er diesen Mitgliedstaat, den Antragsteller (wieder-)aufzunehmen und sein Asylverfahren durchzuführen. Für die Umsetzung der Verordnung wurde das EurodacSystem geschaffen, mithilfe dessen Fingerabdrücke von AsylantragstellerInnen oder Personen gespeichert werden, die illegal Grenzen überschritten haben oder sich illegal in einem Mitgliedstaat aufhalten und aufgegriffen wurden (vgl. 2000/2725/EG). Wurden die Fingerabdrücke des Asylbewerbers bereits in einem anderen Mitgliedstaat im Eurodac-System erfasst ${ }^{92}$, so ergeht an diesen Mitgliedstaat ein Aufnahmeersuchen. Entscheidet der andere Mitgliedstaat nicht innerhalb einer Frist über das Ersuchen, wird davon ausgegangen, dass dem Aufnahmegesuch stattgegeben wurde und es entsteht eine Rücknahmeverpflichtung. ${ }^{93}$ Ein vom Antragsteller eingelegter Rechtsbehelf hat keine aufschiebende Wirkung. ${ }^{94}$

Der Verordnung liegt unter anderem die Annahme zugrunde, dass die Aufnahme-, Anerkennungs- und Menschenrechtspraxis in allen Mitgliedstaaten auf gleich hohem Niveau - also bereits angeglichen - ist. Da dies nicht der Fall ist, haben die Asylsuchenden nicht in allen Staaten die gleichen Chancen auf Anerkennung ihres Gesuchs. Menschenrechtsorganisationen kritisieren, dass gerade Folteropfer und Traumatisierte nicht in allen Mitgliedstaaten den gleichen Zugang $\mathrm{zu}$ angemessener medizinischer Versorgung haben (vgl. ECRE 2006; Pro Asyl 2008).

UNHCR hat eine Studie zur Anwendung der Verordnung in den Mitgliedstaaten vorgelegt (vgl. Kok 2006), die deutlich macht, dass zahlreiche Differenzen zwischen den Praxen in den verschiedenen Mitgliedstaaten bestehen, die mit Differenzen zwischen dem Wortlaut der Verordnung und dem Behördenhandeln korrespondieren. Die Verordnung hat daher in der Praxis schon verschiedene

(Art. 18 Abs. 3b, i). „Ihre Beweiskraft hinsichtlich der Zuständigkeit für die Prüfung des Asylantrags wird von Fall zu Fall bewertet“ (Art. 18 Abs. 3b, ii). Diese Indizien sollen - sofern sie „kohärent, nachprüfbar und hinreichend detailliert sind“ (Art. 18 Abs. 5) genügen, damit der ersuchte Mitgliedstaat seine Zuständigkeit anerkennt.

92 Die Zahlen aus dem Eurodac-System verdeutlichen laut Kommission, dass „das Dublin-System nicht genügend abschreckend war, um ,Asyl-Shopping zu verhindern“, denn im Jahr 2005 wurde per Eurodac noch ein Anteil von 16 Prozent Mehrfachantragstellungen unter den Asylanträgen ermittelt (KOM(2007) 299, S. 11).

${ }^{93} \mathrm{Zu}$ den verschiedenen Fristen vgl. Kap. V der Verordnung.

${ }^{94}$ Dies hat laut einer UNHCR-Studie (vgl. Kok 2006) zur Folge, dass - zum Beispiel in Deutschland - Asylsuchende, die unmittelbar nach der Inkenntnissetzung über den Transfer in einen anderen Mitgliedstaat direkt dorthin abgeschoben werden, damit faktisch keine Chance auf die Inanspruchnahme des Rechtsbehelfs haben. Infolgedessen werden die Rechtsbehelf-Anträge entweder direkt nach der Abschiebung als unbegründet abgelehnt (Deutschland) oder dem Rechtsbehelf wird - wie in Österreich geschehen - nach der Abschiebung des Asylsuchenden nach Polen stattgegeben und es entsteht schließlich Streit darüber, ob Polen oder Österreich die Kosten für die erneute Überstellung nach Österreich übernehmen soll, der in dem Beispielfall mit der Kostenzuweisung an den Asylbewerber endete (vgl. Kok 2006, S. 19f.). In Tschechien haben die Asylsuchenden nach der Übermittlung der Entscheidung 30 Minuten Zeit, einen Rechtsbehelf einzulegen (vgl. ebd., S. 20). 
Fälle hervorgebracht, anhand derer dokumentiert werden konnte, dass die Anwendung der Verordnung zu Kettenabschiebung, Refoulement oder Hin- und Herschieben von Asylsuchenden zwischen den Mitgliedstaaten geführt hat. Zu ähnlichen Ergebnissen kommt eine ausführliche Studie des Europäischen Flüchtlingsrates ECRE zur Umsetzung der Verordnung in 20 Mitgliedstaaten (vgl. ECRE 2006). ECRE kritisiert insbesondere die Praxis der Mitgliedstaaten, Asylsuchende oft monatelang zu inhaftieren und Familienmitglieder voneinander zu trennen ${ }^{95}$, bis das Übernahmeersuchen gestellt und die Entscheidung darüber gefallen ist. Ein weiterer Hauptkritikpunkt ist, dass viele Mitgliedstaaten den überstellten AntragstellerInnen ein faires Verfahren verweigern. ${ }^{96}$ Damit einher gehen die Verweigerung elementarer Aufnahmebedingungen und der Entzug grundlegender sozialer Rechte. Weiterhin machen die Staaten laut dieser Studie nur sehr selten von ihrem Selbsteintrittsrecht im Interesse der Familienzusammenführung Gebrauch. Der Europäische Flüchtlingsrat schlägt als Alternative zu Dublin II vor, die Asylanträge in dem jeweiligen Land zu prüfen, in dem sie gestellt wurden und eine Vereinbarung zur Lastenteilung für diejenigen Staaten zu finden, die eine überproportional hohe Zahl an Asylanträgen zu verzeichnen haben.

Ein Ergebnis der Verordnung ist die Auslagerung der Verantwortung für Asylanträge in die Mitgliedstaaten an den EU-Außengrenzen: Trotz der Betonung darauf, dass es um „die Erreichung eines Gleichgewichts der Zuständigkeitskriterien im Geiste der Solidarität" (Erwägung 8) ginge, weist die Richtlinie die Verantwortung für die Asylanträge durch das Kriterium des ,illegalen Grenzübertritts' faktisch den Staaten an den östlichen und südlichen Außengrenzen zu. Dies bestätigen auch die von UNHCR gesammelten Daten. Danach sind diese Staaten häufiger das Ziel von ,Überstellungen', als sie selbst ,Überstellungen durchführen. Demgegenüber überwiegen in Großbritannien, Tschechien und Finnland die Überstellungen die Übernahmen, während das Verhältnis in Österreich, Deutschland und den Niederlanden ausgeglichen ist (vgl. Kok 2006, Annex II).

Die Überstellungen werden allerdings nur in 30 Prozent der Fälle auch tatsächlich durchgeführt (vgl. ebd.). ${ }^{97}$ Diese Ergebnisse der UNHCR-Studie werfen auch die Frage nach Sinn und (Kosten-)Effizienz der Verordnung auf. Angesichts des hohen administrativen Aufwands, der für das Eurodac-System, die Zuständigkeitsprüfungen, die Übernahme-Ersuchen und die Überstellungen selbst betrieben

\footnotetext{
95 So zum Beispiel in der Bundesrepublik: In der ZAST Eisenhüttenstadt werden Familien, bei denen die Zuständigkeit nach Dublin II geprüft wird, häufig getrennt - die Väter werden in Haft genommen, Mütter und Kinder in der Erstaufnahme untergebracht.

96 Dies geschieht, wenn im nationalen Recht Klauseln enthalten sind, die eine Ausreise als Abbruch des Verfahrens werten, das nach der Rückkehr nicht mehr aufgenommen oder nur als beschleunigtes Folgeverfahren durchgeführt wird.

${ }^{97}$ Laut Bericht der EU-Kommission zur Bewertung des Dublin-Systems (vgl. KOM(2007) 299, S. 4) wurden im Zeitraum von September 2003 bis Dezember 200555.310 Übernahmegesuche gestellt, davon 40.180 Gesuche genehmigt, aber nur 16.842 Menschen, rücküberstellt‘.
} 
wird, stellt sich die Frage, inwieweit mit der Verordnung ein bürokratisches System geschaffen wurde, das ausschließlich zusätzliche Kosten erzeugt. Dies gilt insbesondere für Länder wie Deutschland, wo fast ebenso viele Ersuchen um Rücküberstellung an andere Mitgliedstaaten gestellt werden wie Übernahmeersuchen an Deutschland. ${ }^{98}$ Insgesamt wurden nach Schätzungen des UNHCR im Jahr 2004 für 15 Prozent der in den EU-25 gestellten Asylanträge Zuständigkeitsbestimmungsverfahren gemäß Dublin II durchgeführt. Das Kostenargument wird von der Kommission jedoch mit Verweis auf übergeordnete politische Ziele zurückgewiesen:

„Ungeachtet der finanziellen Auswirkungen halten die Mitgliedstaaten aber die Verwirklichung der politischen Ziele des Systems für sehr wichtig. "

(KOM(2007) 299, S. 14)

Da viele MigrantInnen versuchen, sich diesen Auslagerungsbestrebungen zu entziehen, ist ein zweites Ergebnis der Dublin-II-Praxis in der zunehmenden Illegalisierung von Migration zu sehen. Viele MigrantInnen wandern irregulär weiter nach Westen, um den schlechten Lebensbedingungen und der Abschiebungsgefahr zum Beispiel in (süd-)osteuropäischen Mitgliedstaaten zu entkommen oder weil sie Anknüpfungspunkte zu anderen Zielländern haben (Verwandte, Freunde, Sprache). ${ }^{99}$ Zudem ist zu vermuten - auch wenn dazu naturgemäß wiederum keine Zahlen vorliegen -, dass Flüchtlinge vermehrt Anstrengungen unternehmen, direkt und unbemerkt in das Zielland zu gelangen, was eine aufwändigere und damit kostenintensivere Schleusung erforderlich macht. Zudem tauchen zahlreiche Flüchtlinge, die aufgrund der Verordnung, rücküberstellt' werden sollen, unter (vgl. KOM(2007) 299). Dies erklärt - neben der Ablehnung von Überstellungsanträgen durch den angefragten Mitgliedstaat und der Vernichtung von Hinweisen auf den Einreiseweg - die geringe Zahl der tatsächlich durchgeführten ,Rücküberstellungen“. Vor dem Hintergrund, „dass Asylbewerber nach Erhalt eines Überstellungsbeschlusses häufig nicht mehr auffindbar sind“ (KOM(2007) 299, S. 8), werden Asylsuchende zunehmend inhaftiert, bis über die Zuständigkeit für ihren Antrag entschieden ist (vgl. ebd., S. 9), was - wie bereits erwähnt - auf scharfe Kritik von Flüchtlingsorganisationen stößt (vgl. Pro Asyl 2008).

98 Über die konkrete Höhe der Kosten liegen keine genauen Daten vor. Dennoch wird zumindest die Tatsache, dass einige Staaten ebenso viele MigrantInnen überstellen, wie an sie selbst überstellt werden, auch von der Kommission als unsinniger Kostenfaktor angesehen (vgl. $\operatorname{KOM}(2007)$ 299, S. 8). Sie will daher eine Regelung finden, die in solchen Fällen eine „Begrenzung der Zahl der Überstellungen“ ermöglicht. Damit solle auch weitere Sekundärmigration verhindert werden womit die Kommission eine weitere Folge der Verordnung erkennt, die allerdings nicht nur bei ausgeglichenen Überstellungszahlen zwischen den Mitgliedstaaten auftritt, sondern ein generelles Ergebnis der Verordnung ist.

${ }^{99}$ Berichte von tschetschenischen Flüchtlingen, mit denen ich in Polen gesprochen habe, bestätigten dies: Viele von ihnen waren bereits in einem EU-Mitgliedstaat westlich von Polen, sind dann aufgrund der Dublin-II-Verordnung zurückgeschoben worden und versuchen es anschließend erneut. 
Das Ungleichgewicht bei der Zuweisung der Zuständigkeiten zuungunsten der Mitgliedstaaten an den südlichen und östlichen Außengrenzen kritisiert auch das Europäische Parlament. In einer Entschließung zur gemeinsamen Einwanderungspolitik der Europäischen Union vom 28.9.2006 fordert es die Kommission

„eindringlich auf, im Hinblick auf die Änderung der Verordnung (EG) Nr. 343/2003 (,Dublin II') so schnell wie möglich tätig zu werden und dabei den Grundsatz dieser Verordnung in Frage zu stellen, nämlich, dass es sich bei dem Mitgliedstaat, der für die Behandlung des Asylantrags zuständig ist, um das erste Land handelt, in das die Asylbewerber einreisen, was den im Süden und im Osten der Europäischen Union gelegenen Ländern eine unerträgliche Last aufbürdet, und einen gerechten Mechanismus für eine gemeinsame Verantwortung der Mitgliedstaaten einzufübren". (BR Drs. 799/06, S. 6)

Während in der Verordnung selbst noch - wie oben angesprochen - erklärt wurde, es handele sich um eine Regelung ,im Geiste der Solidarität‘, weist nun selbst die Kommission diese Begründung für die Verordnung zurück und stellt gleichzeitig ihre Wirksamkeit infrage. So heißt es in ihrem „Grünbuch über das Gemeinsame Europäische Asylsystem“ (KOM(2007) 301):

„Das Dublin-System (Dublin- und Eurodac-Verordnungen) war nicht als Lastenteilungsinstrument konzipiert. Sein vorrangiges Ziel bestand darin, auf der Grundlage gerechter und objektiver Kriterien rasch festzulegen, welcher Mitgliedstaat für die Prüfung eines im Gebiet der EU eingereichten Asylantrags zuständig ist, und Sekundärbewegungen zwischen den Mitgliedstaaten vorzubeugen. Wie der am 6. Juni veröffentlichte Bewertungsbericht gezeigt hat, wurden diese Ziele des Dublin-Systems weitgehend erreicht, obwohl Fragen in Bezug auf die Wirkesamkeit des Systems bei der Verringerung von Sekundärbewegungen weiterbin offen bleiben."

Damit bekräftigt die Kommission ihre bereits im Jahr 2000 in einem Arbeitspapier geäußerte Kritik an den Zielen und der Wirksamkeit des Dublin-Systems (vgl. SEK(2000) 522). Dennoch ist sie in ihrem Verordnungsentwurf nicht von diesem Verfahren abgewichen. In ihren Überlegungen zur Fortentwicklung des Instruments resümiert die Kommission im Grünbuch die vorgeschlagenen „Alternativsysteme“:

„Dazu gebörte beispielsweise ein System zur Übertragung der Zuständigkeit auf das Land, in dem der Asylantrag eingereicht wurde, das Herkunftsland des Asylbewerbers oder das letzte bekannte Transitland. "(KOM(2007) 301, S. 12)

Die konkreten Auswirkungen der Verordnung werden im Rahmen der Länderstudien noch näher erläutert. 


\subsubsection{Grenzregime}

Ausgangspunkt der Etablierung eines gemeinsamen europäischen Grenzregimes ist das Übereinkommen von Schengen. Dieses zunächst auf intergouvernementaler Ebene ausgehandelte Vertragswerk definierte eine gemeinsame europäische Außengrenze, während die Binnengrenzen nach und nach abgebaut werden sollten. Mit Schengen wurde vereinbart, die Einreisekontrollen bereits an den Außengrenzen durchzuführen, während man innerhalb der EU kontrollfrei reisen können sollte. Da die Mitgliedstaaten, die über keine Außengrenze verfügen, aufgrund des Wegfalls der Binnengrenzen ebenfalls von den migrationspolitischen und Grenzsicherungsmaßnahmen der Staaten an den Außengrenzen abhängig sind, sollte die Schaffung des gemeinsamen Schengen-Raumes flankiert werden durch eine Vereinheitlichung der Visaregelungen und Einreisebestimmungen sowie der Maßnahmen gegen irreguläre Einwanderung und grenzüberschreitende Kriminalität. Der Grenzschutz ist zwar weiterhin Sache der einzelnen Mitgliedstaaten, zunehmend wird aber auch in diesem Bereich eine stärkere Zusammenarbeit angestrebt. Schengen bildet damit den Grundstein für eine Vor- bzw. Auslagerung der Kontrollen und für eine Ausweitung der Grenzzonen im Innern (vgl. Hess / Tsianos 2007, S. 28). Mit dem Abkommen war die Entstehung von verschiedenen EU-Institutionen verbunden: Europol, das Schengener Informationssystem (SIS), das Center for Information, Discussion and Exchange on the Crossing of Frontiers and Immigration (CIREFI), die External Borders Practioners Common Unit und Frontex (vgl. ebd., S. 29). Das Abkommen wurde mit dem Amsterdamer Vertrag auf supranationale Ebene gehoben und gilt damit in allen Mitgliedstaaten. Allerdings sind noch nicht alle Staaten der letzten beiden Erweiterungsrunden 2004 und 2007 volle Mitglieder des Abkommens. Sie müssen erst alle SchengenStandards erfüllen (vor allem hinsichtlich Grenzsicherung und SIS), bevor der Rat entscheidet, dass die Kontrollen auch an den gemeinsamen Binnengrenzen mit diesen Staaten entfallen können. Hingegen sind Island, Norwegen und die Schweiz Mitglieder des Schengen-Raums, ohne jedoch EU-Mitglieder zu sein. Großbritannien und Irland sind nur partiell Mitglieder des Abkommens: Sie halten ihre Passkontrollen weiter aufrecht.

Um die Vereinheitlichung des Grenzregimes voranzutreiben, wurde durch Verordnung des Rates die Europäische Grenzschutzagentur Frontex (frontières extérieures) geschaffen, die am 1. Mai 2005 in Warschau ihre Arbeit aufnahm (vgl. 2004/2007/EG). Sie soll zur „Verbesserung der Koordinierung der operativen Zusammenarbeit zwischen den Mitgliedstaaten im Bereich des Schutzes der AuBengrenzen der Mitgliedstaaten“ beitragen (ebd., Erwägung 3). Die Aufgaben zeigen, dass es sich bei Frontex um ein Instrument handelt, das im polizeilichen Maßnahmenbereich der EU-Migrationspolitik angesiedelt ist: Ihr Auftrag umfasst die Koordination der operativen Zusammenarbeit der Mitgliedstaaten und deren technische und operative Unterstützung beim Schutz der Außengrenzen. Zudem soll sie die Mitgliedstaaten bei der Ausbildung von GrenzbeamtInnen (zum Bei- 
spiel durch Vereinheitlichung der Ausbildungsnormen) unterstützen und Risikoanalysen anfertigen. Außerdem soll die Agentur ein Zentralregister über technische Ausrüstungsgegenstände der Mitgliedstaaten führen, die diese bereit sind, anderen Mitgliedstaaten im Bedarfsfall vorübergehend zur Verfügung zu stellen. Frontex selbst verfügt über keine eigene technische Ausrüstung. Auch die Koordination gemeinsamer Abschiebungen und die Ermittlung bewährter Praktiken für die Beschaffung von Reisedokumenten gehören zum Aufgabenbereich von Frontex. Bisher haben die BeamtInnen der Agentur in dem jeweiligen Land, in das sie entsendet wurden, nur beratende Funktion - sie können weder jemanden festnehmen, noch verhören. Inzwischen liegt ein Vorschlag der EU-Kommission für eine Verordnung vor, die eine Ausweitung der Befugnisse der Frontex-MitarbeiterInnen vorsieht. ${ }^{100}$

Nach einigen Verzögerungen ist die Agentur im September 2006 im Rahmen der von der EU zugesagten Unterstützung Spaniens bei der Abwehr von Bootsflüchtlingen, die sich aus Westafrika auf die Kanarischen Inseln aufmachen, tätig geworden. Mit Militärpatrouillen unter Beteiligung mehrerer Mitgliedstaaten versucht Frontex, die Seegrenzen zwischen der EU und Afrika zu,sichern' (,HERA II' vor den Kanaren). Zunächst konnten die Frontex-Patrouillen den Anstieg der Zahl der MigrantInnen, die im Jahr 2006 auf den Kanarischen Inseln ankamen, nicht bremsen. Es wurden bald Beschwerden über die Schwerfälligkeit der Agentur laut. ${ }^{101}$ Der Frontex-Direktor Ilkka Laitinen (ein General mit 25 Jahren Grenzschutzerfahrung) verwies angesichts der lauter werdenden Kritik auf die mangelnde finanzielle Basis der Agentur, die eine eigene technische Ausstattung nicht ermögliche. Daher sei die Agentur von der Zusammenarbeit mit den Mitgliedstaaten abhängig. Außerdem seien die Aktivitäten an der südlichen EU-Außengrenze nur eine von 25 Operationen, mit denen Frontex befasst sei (vgl. Tagesspiegel vom 30.10.2006). Der Etat der Agentur lag im Jahr 2005 bei 6,157 Millionen Euro. Die für 2006 und 2007 vorgesehenen Beträge (11,754 und 21,1 Millionen Eu-

\footnotetext{
${ }^{100}$ Laut Verordnungsentwurf kann Frontex auf Anfrage eines Mitgliedstaates, der an seinen Grenzen einer ,besonderen Belastung' ausgesetzt ist, gemeinsame grenzpolizeiliche Teams entsenden. Diese erhalten grenzpolizeiliche Befugnisse, die denen der BeamtInnen vor Ort entsprechen und agieren unter Leitung der dortigen Grenzpolizei. Im Arbeitsprogramm des Bundesinnenministeriums der Bundesrepublik für die deutsche EU-Ratspräsidentschaft heißt es: „Wir wollen deshalb Frontex wesentlich stärken. Dazu gehört die zügige Verabschiedung und Umsetzung der Rechtsgrundlagen für die Einrichtung schneller Eingreifteams und die Übertragung exekutiver Befugnisse auf Gastbeamte bei gemeinsamen Grenzkontrollen.“ (Bundesministerium des Innern 2006a)

101 So hieß es im Tagesspiegel vom 30.10.2006: „Eine schlechtere Presse hatten in den Sommermonaten nur George Bush und Osama bin Laden. Politiker suchten Schuldige für die verheerenden Zustände und wurden in Warschau fündig." Laut Tagesspiegel kritisierte die spanische Regierung, Frontex sei „langsam und uneffektiv“. Auch Bundeskanzlerin Angela Merkel habe der Agentur „mehr Praxisnähe“ anempfohlen (vgl. Tagesspiegel vom 30.10.2006).
} 
ro, vgl. BT Drs. 16/1752) wurden mit Hinweis auf den Handlungsbedarf angesichts der Ereignisse auf den Kanarischen Inseln noch einmal deutlich erhöht. ${ }^{102}$

Während von Seiten der Regierungen der Mitgliedstaaten vor allem Kritik an der Schwerfälligkeit der Agentur geäußert wurde, kritisieren Menschenrechts- und MigrantInnen(selbst)organisationen die Einrichtung von Frontex als Ausdruck der Militarisierung der europäischen Migrationspolitik (vgl. Transnational Newsletter Crossing Borders 2006). Die Agentur beruft sich angesichts solcher Kritik zum einen darauf, Menschenleben zu retten ${ }^{103}$ und bedient sich zum anderen des Bildes von der ,illegalen Einwanderung;, um die Abwehrstrategien zu rechtfertigen. ${ }^{104}$ Hier zeigt sich neben der humanitären Begründung von Frontex-Einsätzen auch die Bedeutung der Kategorisierung als Legitimationsinstrument im öffentlichen Diskurs. Mit der Aussage: „Das sind keine Flüchtlinge, sondern illegale Migranten“ (Laitinen zit. n. Der Standard vom 21.12.2006), sollen die gewaltförmigen Abwehrmaßnahmen gerechtfertigt werden. Gewaltsames Vorgehen gegen ,Illegale` erscheint so nicht nur legitim, sondern angesichts der Allgegenwart des Topos ,Bekämpfung der illegalen Migration“ sogar geboten. Gleichzeitig lässt die Pauschalität der Äußerung erkennen, dass das militärische Vorgehen gegen MigrantInnen auch keine Filterung derjenigen ,boat people ${ }^{6}$ vorsieht, die die Kriterien der GFK erfüllen, deren Zugang also gemäß Kategorienschema eigentlich zulässig wäre.

\subsubsection{Außenpolitische Maßnahmen}

Mit dieser fortschreitenden Institutionalisierung der militarisierten migrationspolitischen Abwehrmaßnahmen korrespondiert der Fokus der EU auf die ,externen Aspekte der Asylpolitik', der darauf abzielt, MigrantInnen und Flüchtlinge bereits außerhalb der EU aufzufangen bzw. eine Weiterwanderung aus den Transitregionen zu verhindern. Die Aktivitäten in diesem Bereich begannen bereits Mitte der 1980er Jahre mit der Einführung der Drittstaatenregelung in einigen Mitgliedstaaten, der Vereinbarung von Rückübernahmeabkommen und der Unterstützung von Transitstaaten bei der ,Sicherung' ihrer Grenzen. Inzwischen ist dieser Bereich weiter ausgebaut und -differenziert worden. Dies zeigt sich in den Vorschlägen der Kommission zur „Verbesserung des Zugangs zu dauerhaften Lösungen“ (KOM(2004) 410), zu „regionalen Schutzprogrammen“ (KOM(2005) 388) und

102 Für das Jahr 2006 wurde eine Erhöhung um 3,5 Millionen Euro 2006 angesetzt; auf Initiative des Europäischen Parlaments sollte der Etat für 2007 um 14 Millionen Euro aufgestockt werden (vgl. Tagesspiegel vom 30.10.2006).

103 So zum Beispiel der Frontex-Direktor Ilkka Laitinen: „Im August und September [2006; D.M.] haben wir 2.300 Menschen rechtzeitig in ihren Booten aufgespürt und zurückbegleitet. [...] Wir haben deren Leben gerettet, verstehen Sie? In zwei Monaten haben wir 2.300 Menschenleben gerettet.“ (zit. n. Tagesspiegel vom 30.10.2006)

${ }^{104}$ So wird Laitinen im österreichischen ,Standard“ vom 21.12.2006 mit den Worten zitiert: „,Flüchtlinge? - Der Frontex-Direktor hebt zum ersten Mal seine Stimme: „Das sind keine Flüchtlinge, sondern illegale Migranten.',, (vgl. Der Standard vom 21.12.2006) 
der finanziellen Rahmung dieses Bereichs zum Beispiel durch das AENEASProgramm sowie in der Einflechtung migrationspolitischer Fragen in die neue Europäische Nachbarschaftsstrategie. Über die Nachbarschaftspläne, die mit den einzelnen Anrainern vereinbart werden, wird versucht, asylrelevante Politiken und Instrumente in die Nachbarstaaten zu exportieren. Indem dort die rechtlichen und institutionellen Strukturen für den Flüchtlingsschutz installiert werden, können Instrumente wie die ,sichere Drittstaatenregelung ${ }^{`}$ effizienter angewendet werden. Denn die Definition als sicherer Drittstaat erfordert es, dass dieser Staat die GFK unterzeichnet hat und bestimmte menschenrechtliche Mindeststandards erfüllt. Die Unterstützung der Nachbar- bzw. Transitstaaten beim Aufbau von Institutionen und Strukturen zur Durchführung von Asylverfahren zielt darauf ab, dass die Flüchtlinge bereits dort einen Status erhalten und gar nicht erst in die EU weiterreisen. Andernfalls können sie mit Verweis auf die Drittstaatenregelung aus der EU dorthin abgeschoben werden.

Das AENEAS-Programm (2004/491/EG) „für die finanzielle und technische Hilfe für Drittländer im Migrations- und Asylbereich“ soll „eine bessere Steuerung der Migrationsströme in all ihren Dimensionen [...] unterstützen“ und dabei vorrangig die Staaten der Europäischen Nachbarschaftspolitik berücksichtigen (ebd.). ${ }^{105}$ Gleichzeitig sollen durch dieses Programm in erster Linie diejenigen Länder gefördert werden, die sich hinsichtlich des Abschlusses von Rückführungsabkommen kooperativ zeigen (vgl. ebd.).

„Die Nachbarschaftspolitik - soweit zum gegenwärtigen Zeitpunkt schon eine Beurteilung möglich ist - lässt keine Abkehr von der bisher eher restriktiven europäischen Politike erkennen. Im Mittelpunkt stehen nach wie vor die Grenæsicherung und der Abschluss von Rückübernabmeabkommen. In den Aktionsplänen ist zwar immer wieder von einer umfassenden Zusammenarbeit die Rede, konkretisiert werden jedoch vornebmlich die restriktiven Aspekte. "(Angenendt 2006, S. 204)

Die Kategorisierung der MigrantInnen in Flüchtlinge und Illegale soll also durch den Export migrations- und grenzpolitischer Maßnahmen bereits an den Außengrenzen stattfinden, wie die folgende Aussage der Kommission zur „Steuerung gemischter Migrationsströme" einmal mehr illustriert:

„Ein weiteres Kernelement der externen Dimension der Asylproblematik ist die Notwendigkeit der Steuerung gemischter Migrationsströme, d.h. Migrationsströme, die an den Außengrenzen eines Mitgliedstaats ankommen und sowobl illegale Zuwanderer als auch schutzsuchende Personen umfassen. Die Antwort auf diese Herausforderung impliziert einen garantierten und verstärkten Zugang zum Schutz an den Außengrenzen. "(KOM(2007) 301, S. 15, Hervorh. i. Orig.)

105 Das Programm wurde im März 2004 angenommen und lief bis 2008 mit einem Gesamtbudget von 250 Millionen Euro. 
Auch über die von der Kommission vorgeschlagenen ,regionalen Schutzprogramme' sollen Asylsuchende bzw. Flüchtlinge möglichst außerhalb der EU aufgefangen und ihnen nur in Einzelfällen die Weiterreise in die EU gestattet werden. Der überwiegende Teil soll ,zurückgeführt' oder in der jeweiligen Region angesiedelt werden:

\section{„D]ie drei dauerhaften Lösungen sind Rückkeehr, örtliche Eingliederung oder Neuan- siedlung in einem Drittstaat, sofern die ersten zwei dauerhaften Lösungen nicht möglich sind." $(\mathrm{KOM}(2005)$ 388)}

Im Haager Programm (2005/C 53/01), das unter anderem die nächsten Schritte der Vergemeinschaftung festlegt, wird angestrebt Asylanträge künftig bereits auBerhalb der EU zu prüfen. Damit werden die Vorschläge, die unter anderen Tony Blair (UK Government 2003: „New Vision for Refugees“) und Otto Schily (2004: „Afrikas Probleme in Afrika lösen“) eingebracht hatten und die zunächst auf Empörung in der Öffentlichkeit und Ablehnung im Rat gestoßen waren, nun verwirklicht. Ohne vergleichbare mediale Öffentlichkeit wie sie im Sommer 2004 nach den Ereignissen um das Rettungsschiff der Hilfsorganisation, Cap Anamur' Otto Schily zuteil wurde, als er seine Idee von EU-Asylbewerberlagern in Nordafrika präsentierte, arbeiten Kommission und Rat inzwischen an der Umsetzung der Vorschläge. ${ }^{106}$

Die ,Zusammenarbeit ${ }^{\star}$ mit den Herkunfts- und Transitstaaten im Migrationsbereich erhielt in den vergangenen Jahren einen immer bedeutenderen Stellenwert. Unter diesem Vorzeichen steht auch die ,deutsch-französische Initiative für eine neue europäische Einwanderungspolitik ${ }^{6}$, die der deutsche Innenminister Schäuble und sein damaliger französischer Amtskollege Sarkozy am 26.10.2006 bei einem Treffen der Innenminister der sechs großen EU-Staaten vorgelegt haben. Neben einer Optimierung des Grenzregimes, Frontex-Einsätzen, gemeinsamen Grenzschutzpatrouillen und Abschiebeflügen soll verstärkt auf Rückübernahmeabkommen hingearbeitet werden. ${ }^{107}$ Doch die meisten Anrainer haben kein eigenes Interesse an der Aufrüstung ihrer Grenzen und der Installierung von Asylregimen.

\footnotetext{
106 Für Otto Schily war mit seinem Vorschlag das Ziel verbunden, dass MigrantInnen die Rechte, die ihnen auf EU-Territorium eingeräumt werden müssten, dann nicht mehr in Anspruch nehmen könnten. Damit können nach Schilys Ansicht aus ,rechtlichen' ,faktische` Fragen gemacht werden, wie der folgende Ausschnitt eines Interviews mit der Süddeutschen Zeitung zeigt: „SZ: Nehmen wir an, die geretteten Flüchtlinge wollen Asyl in Deutschland oder anderswo in der EU beantragen. Schily: Das können sie nicht. Auf dem Schiff oder in der Aufnahmeeinrichtung können sie kein Asyl für Deutschland oder ein anderes EU-Mitgliedsland beantragen. Das geht nur in Deutschland oder in einem anderen EU-Mitgliedsland. SZ: Dahin kommen sie aber nicht. Schily: Das ist aber dann nicht eine rechtliche, sondern eine faktische Frage." (Süddeutsche Zeitung vom 2.08.2004)

107 Der Rat hat die Kommission seit 2001 beauftragt, Rückübernahmeabkommen mit Albanien, Algerien, Hongkong, Macao, Marokko, Pakistan, Russland, Sri Lanka, der Ukraine, der Volksrepublik China, den Westbalkanstaaten und der Türkei auszuhandeln. Die Abkommen mit Hongkong, Macao, Sri Lanka und Albanien sind bereits in Kraft getreten; die Abkommen mit Russland und der Ukraine wurden unterzeichnet, sind jedoch noch nicht in Kraft getreten.
} 
(Im-)Migration spielt dort weder im öffentlichen noch im politischen Diskurs eine herausragende Rolle und wird stattdessen meist lediglich in Bezug auf die Emigration der eigenen StaatsbürgerInnen thematisiert, die zudem aufgrund der Rücküberweisungen eine bedeutende Einnahmequelle darstellt (vgl. Cuttitta 2004). Gleichzeitig ist Transitmigration vielerorts normaler Bestandteil der gesellschaftlichen Realität (vgl. Andrijasevic et al. 2005). Als Anreize für die Transit- und Herkunftsstaaten werden daher nicht mehr nur die Aufstockung oder Kürzung von Mitteln im Rahmen der Entwicklungszusammenarbeit diskutiert, sondern auch Visa-Erleichterungen für Studierende und Wissenschaftler sowie ein Kontingent für ,zirkuläre Migration‘. Damit ist ein quotierter und zeitlich befristeter Zugang von ArbeitnehmerInnen zum europäischen Arbeitsmarkt gemeint. ${ }^{108} \mathrm{Ob}$ und in welchem Umfang sie sich daran beteiligen, sollen die Mitgliedstaaten allerdings jeweils selbst entscheiden können. KritikerInnen sehen darin eine Neuauflage des ,Gastarbeiter'-Regimes, dessen Unzulänglichkeiten in der Vergangenheit bereits ausführlich diskutiert wurden. ${ }^{109}$

Die außenpolitischen Maßnahmen weisen ein deutliches Übergewicht im Bereich der Abwehr von Migrationen auf, wobei die Folgen der Auslagerung dem Blick der europäischen Öffentlichkeit weitgehend entzogen sind. ${ }^{110}$ Im Vergleich

108 Der Vorschlag für die EU-weite Einführung eines Quotensystems wurde von Italien bereits 2003 eingebracht. Dagegen gab es von deutscher Seite Bedenken. Italien nutzte zu diesem Zeitpunkt bereits seit einigen Jahren Einwanderungsquoten als Druckmittel bzw. Anreize - 1998 richtete die Mitte-Links-Regierung ein System der quotierten Einwanderung ein. „Durch Erhöhen bzw. Herabsetzen der Quoten werden Herkunfts- und Transitländer für ihre Kooperationsbereitschaft belohnt bzw. wird dadurch versucht, sie zu einer Kursänderung zu veranlassen. So war es etwa im Jahr 1998 in erster Linie dem italienischen Versprechen zu verdanken, Einwanderungsquoten zugunsten tunesischer Staatsbürger einzuführen, dass Tunesien ein Abkommen zur Rückübernahme von Personen ohne legalen Aufenthaltsstatus mit Italien abschloss“ (Cuttitta 2004). Nachdem das Abkommen 2001 abgelaufen war und die Einwanderungsquoten für Tunesien 2002 und 2003 herabgesetzt worden waren, ließen auch die Kontrollen auf tunesischer Seite nach. Im Jahr 2004 wurde die Quote wieder erhöht und Italien verpflichtete sich zur Unterstützung Tunesiens bei Ausbildung und technischer Ausrüstung von Grenzpolizei und Küstenwache. Daraufhin erließ das tunesische Parlament ein Gesetz mit harten Strafen für SchleuserInnen, HelferInnen und zufällige MitwisserInnen (vgl. ebd.).

109 Auch von den so genannten,GastarbeiterInnen', die seit Mitte der 1950er Jahre durch die Bundesrepublik Deutschland, aber auch durch andere Staaten angeworben wurden, wurde erwartet, dass sie nach einiger Zeit wieder in ihre Herkunftsländer zurückkehren würden - spätestens dann, wenn sie auf dem Arbeitsmarkt des Anwerbelandes nicht mehr gebraucht würden. Diese Vorstellung hat sich nicht bestätigt. Zahlreiche, GastarbeiterInnen' sind geblieben und haben ihre Familien nachgeholt.

110 Die Folgen der Kooperation zwischen der EU und ihren Nachbarstaaten für TransitmigrantInnen, die auf dem Weg in die EU in den Anrainerstaaten festsitzen, zeigen sich unter anderem in Marokko, das zum Beispiel Ende Dezember 2006 ca. 400 subsaharische MigrantInnen an der algerischen Grenze ausgesetzt hat - wo sie von den algerischen Grenzschützern ebenso zurückgewiesen wurden wie von den marokkanischen. Unter den bei einer nächtlichen Razzia Festgenommenen befanden sich Menschen mit verschiedensten Aufenthaltsstatus, einige hatten einen Flüchtlingsausweis des UNHCR (vgl. ABCDS et al. 2007; Pro Asyl 2007). 
zu den Abwehrpolitiken haben entwicklungspolitische Aktivitäten in den Herkunftsländern in diesem Zusammenhang einen deutlich geringeren Stellenwert. ${ }^{111}$ Es wird zwar immer wieder auf Bemühungen verwiesen, ,Fluchtursachen' bekämpfen zu wollen. Dieser Ansatz wird jedoch nicht nur der Komplexität der Bedingungen in den Herkunftsländern und der Verflechtung zum Beispiel mit den Handelspolitiken in den Zielländern nicht gerecht, sondern in diesem Bereich wird auch faktisch kaum etwas unternommen. So haben Regierungsvertreter der EUStaaten auf der europäisch-afrikanischen Migrationskonferenz im Juli 2006 in Rabat eine Reihe von Ideen zur Bekämpfung von Migrationsursachen vorgeschlagen, allerdings ohne einen konkreten Plan zu deren Umsetzung und Finanzierung $\mathrm{zu}$ entwickeln. Stattdessen werden Mittel bereits existierender Entwicklungshilfeprogramme an Bedingungen geknüpft, die sich aus migrationspolitischen Zielen der EU speisen. Die Umwidmung von Entwicklungshilfe in Maßnahmen zur Externalisierung von Asylpolitiken wird von der Kommission auch in ihrem „Grünbuch über ein Gemeinsames Asylsystem“ - wenn auch verklausuliert benannt:

„Seitdem die Kommission die Bedeutung der Erreichung dauerhafter Lösungen für Flüchtlinge und Asylbewerber für ibre Entwicklungshilfe erkannt hat, hat sie in den letzten Jabren begonnen die Asylproblematik systematisch in ibre Strategien zur Entwicklungszusammenarbeit einzubetten und weist diesem Bereich beträchtliche Finanzmittel aus den einschlägigen externen Hilfsprogrammen zu." (KOM(2007) 301, S. 14, Hervorh. i. Orig.)

Dieses Verhältnis von Entwicklungs- und Migrationspolitik wird auch von MigrationsforscherInnen in den Herkunfts- und Transitstaaten kritisiert:

„Die EU will vor allem in den Transitländern der Immigranten eingreifen und nicht in ihren Herkunftsländern. Auch wenn von Entwicklung die Rede ist: Europa setzt auf die Wabrung der Sicherheitsinteressen. "(Lablou 2006)112

\subsubsection{Rückkehr und Abschiebung}

Die dargestellten außenpolitischen Maßnahmen, die auf die Aushandlung von Rückübernahmeabkommen im Rahmen der ,Zusammenarbeit mit den Transitund Herkunftsstaaten' zielen, sind Teil der Strategien der EU die ,Rückkehr' von

Über die zahlreichen MigrantInnen, die nach der Abschiebung aus Marokko in der algerischen Wüste umgekommen sind, gibt es verschiedene Berichte, aber auch hier ist es schwierig, belegbare Informationen zu erhalten - schon allein deshalb, weil kaum Angaben darüber möglich sind, ob es sich bei den Toten um Zurückgeschobene oder um MigrantInnen handelt, die sich auf dem Hinweg nach Marokko befanden (vgl. Cuttitta 2004).

111 Vgl. zum Verhältnis von Entwicklungshilfe, Agrar-, Fischerei- und Handelspolitik und Migrationspolitik und ihrer Bedeutung im Sprachgebrauch auch den Abschnitt 4.2.3.

112 Mehdi Lahlou ist Professor am Nationalinstitut für Statistik und angewandte Ökonomie (INSEA) in Rabat/Marokko. 
MigrantInnen durchzusetzen. Um diesen Bereich, der für die „Bekämpfung der illegalen Einwanderung “ als zentral erachtet wird (vgl. $\operatorname{KOM}(2002) 175)$, weiter zu vereinheitlichen und zu effektivieren, wurde bereits 2004 auf Grundlage einer Ratsentscheidung die Zusammenarbeit bei Abschiebungen verstärkt (vgl. 2004/573/EG). Auf dieser Grundlage organisieren die EU-Staaten gemeinsame Abschiebeflüge.

Im Sommer 2008 wurde mit der Rückführungsrichtlinie schließlich eine erste gemeinschaftsrechtliche Basis für Abschiebungen und Abschiebehaft geschaffen (vgl. Europäisches Parlament 2008b). Sie ist gleichzeitig die erste Richtlinie im Bereich Einwanderung, die vom Europäischen Parlament im Juni 2008 im Verfahren der Mitentscheidung angenommen wurde. ${ }^{113}$ Zentral und zudem besonders umstritten an dieser Richtlinie sind die Regelungen zur Abschiebehaft, zum Einreiseverbot und zu den Rechtsbehelfen. Im Einzelnen sieht die Richtlinie vor, dass die Mitgliedstaaten gegen jeden Drittstaatenangehörigen, der sich illegal in ihrem Hoheitsgebiet aufhält, eine „Rückkehrentscheidung“ erlassen - sie haben allerdings die Möglichkeit, zum Beispiel aus verschiedenen humanitären Gründen, darauf zu verzichten (Art. 6). Die Mitgliedstaaten können einen Zeitraum von sieben bis 30 Tagen festlegen, innerhalb dessen der Betroffene „freiwillig“ ausreisen soll (Art. 7). Es steht ihnen frei, die Frist nur auf Antrag des Betroffenen zu gewähren und darüber hinaus in solchen Fällen auf die Fristsetzung zu verzichten, in denen „Fluchtgefahr" oder „eine Gefahr für die öffentliche Sicherheit, die öffentliche Ordnung oder die nationale Sicherheit" besteht. Wird keine Frist eingeräumt oder kommt die Person der Ausreiseaufforderung nicht nach, soll ,die Vollstreckung der Rückkehrentscheidung", also die Abschiebung, erfolgen (Art. 8). Wer abgeschoben wurde, unterliegt einem Wiedereinreiseverbot, das individuell festgelegt wird und maximal fünf Jahre dauern soll (Art. 11). Es bleibt den Mitgliedstaaten überlassen, in Einzelfällen von diesem Einreiseverbot abzusehen. Gegen Rückkehrentscheidungen, Abschiebungen und Einreiseverbote können Rechtsbehelfe eingelegt werden (Art. 12). Allerdings sind die Möglichkeiten, rechtlich gegen eine Abschiebung oder eine Inhaftierung in eine Abschiebehaftanstalt vorzugehen, eng umgrenzt worden.

Die Richtlinie macht auch Vorgaben zur Abschiebehaft: In Fällen, in denen entweder „Fluchtgefahr" besteht oder jemand die Abschiebung „behindert" oder zu umgehen versucht, kann die/der Betreffende in Haft genommen werden (Art. 15). Die Abschiebehaft soll maximal sechs Monate dauern, kann aber in bestimmten Fällen („,mangelnde Kooperationsbereitschaft“ des Betroffenen oder „Verzögerungen bei der Übermittlung der erforderlichen Unterlagen durch Drittstaaten“) auf 18 Monate ausgedehnt werden. Die Inhaftnahme muss von einer Justiz- oder Verwaltungsbehörde angeordnet werden. Es wird ebenfalls darauf verwiesen, dass

113 Im Mitentscheidungsverfahren muss der Rat die Mehrheitsmeinung des Parlaments berücksichtigen. Ist das Parlament mit dem Vorschlag des Rates nicht einverstanden, so muss dieser mit den ParlamentarierInnen einen Kompromiss erarbeiten, dem beide Seiten zustimmen können. 
die Haft beendet werden soll, sobald sich herausstellt, dass die Abschiebung nicht durchführbar ist. Die Inhaftierung soll in „speziellen Hafteinrichtungen“ und nur bei mangelnden Kapazitäten auch in ,gewöhnlichen Haftanstalten“, dann aber „gesondert von den gewöhnlichen Strafgefangenen“ erfolgen (Art. 16). Auch unbegleitete Minderjährige und Familien können - „im äußersten Falle“ - inhaftiert werden (Art. 17). Der Überblick zeigt bereits, dass auch diese Richtlinie einen weiten Interpretationsspielraum für die Mitgliedstaaten eröffnet. So können sie bspw. bei einer „unvorhersehbaren Überlastung der Kapazitäten der Hafteinrichtungen" (Art. 18) von den vorgegebenen Haftbedingungen und den Fristen für die gerichtliche Überprüfung der Haft abweichen. Weiterhin ist die kostenlose Rechtsberatung und -vertretung als Kann-Bestimmung formuliert, die Gewährung kostenloser Prozesskostenhilfe wird von den jeweiligen einzelstaatlichen Rechtsvorschriften abhängig gemacht.

Streitpunkt zwischen Kommission, Rat und Parlament und Gegenstand der Kritik von Menschenrechtsorganisationen ${ }^{114}$ und Kirchen ${ }^{115}$ war vor allem die Dauer der Haft von bis zu 18 Monaten und die Möglichkeit, auch Kinder und Familien zu inhaftieren. Die GegnerInnen der Regelung fürchten unter anderem eine Ausweitung der Abschiebehaft in Staaten, die bislang geringere Haftzeiträume vorsahen, was bspw. Italien bei der Verabschiedung der Richtlinie angekündigt hat. BefürworterInnen setzen dem entgegen, auf diese Weise werde die Haftdauer in den Staaten begrenzt, die bislang gar kein Limit vorsahen (wie Dänemark, Estland, Finnland, Litauen, die Niederlande, Großbritannien und Schweden). Der ursprüngliche Kommissionsvorschlag hatte die Haftdauer auf maximal sechs Monate begrenzt, viele ParlamentarierInnen forderten eine noch kürzere Haft und sprachen sich gegen die Inhaftierung Minderjähriger aus. Beide Vorschläge fanden jedoch nicht die Zustimmung des Ministerrates. Die Regelung, wonach die Dauer der Haft unter bestimmten Umständen bis auf 18 Monate ausgedehnt werden kann, ist das Ergebnis eines Kompromisses zwischen Ministerrat und Parlament, dem das Parlament mit den Stimmen von Konservativen, Liberalen, der Union für ein Europa der Nationen und einiger SozialistInnen und SozialdemokratInnen schließlich mit 369 zu 197 Stimmen zustimmte. ${ }^{116}$ Damit hat sich unter anderem Deutschland einmal mehr durchgesetzt, das in seinem nationalen Recht ebenfalls eine Haftdauer von bis zu 18 Monaten vorsieht. ${ }^{117}$

\footnotetext{
${ }^{114}$ In einer europaweiten Kampagne wurden 40.000 Unterschriften gegen die Richtlinie gesammelt (vgl. www.outrageousdirective.org). Vgl. auch die gemeinsame Stellungnahme von Amnesty International und dem Europäischen Flüchtlingsrat (ECRE 2008).

115 So wandten sich die Vertreter verschiedener Kirchen und christlicher Organisationen am 30.05.2008 mit einem Brief an das Europäische Parlament, in dem sie die Dauer der Haft und das Wiedereinreiseverbot kritisierten (vgl. COMECE 2008).

116106 ParlamentarierInnen enthielten sich.

117 Die Rolle Deutschlands bei der Aushandlung der Richtlinie wird auch durch die Aussage des Staatssekretärs im Bundesinnenministerium, Peter Altmaier (CDU), illustriert, wonach die wesentlichen deutschen Forderungen in der Richtlinie umgesetzt wurden: „Wir haben im Sinne Deutsch-
} 
Kritik an der Annahme der Richtlinie durch das Parlament kam auch aus zahlreichen lateinamerikanischen Herkunftsländern von MigrantInnen. Die PräsidentInnen mehrerer lateinamerikanischer Staaten befürchteten Konsequenzen für ihre StaatsbürgerInnen in der EU, die dort nur zu einem Teil über Aufenthaltsgenehmigungen verfügen, und verurteilten die Richtlinie scharf. In einer Erklärung des südamerikanischen Wirtschaftsbündnisses Mercosur, dem sich weitere südamerikanische Staaten anschlossen, wurde die Kritik an der Richtlinie bekräftigt (vgl. Die Welt vom 03.07.2008). Die Staatschefs verwiesen bei ihrem Protest unter anderem auf die Migrationsgeschichte, in deren Verlauf Millionen EuropäerInnen ungehindert nach Lateinamerika eingewandert seien, betonten den Beitrag, den die Arbeit der MigrantInnen in der EU zu deren wirtschaftlicher Prosperität leiste und verwiesen auf die Bedeutung der Rücküberweisungen für ihre eigene wirtschaftliche Situation, die das finanzielle Volumen der Entwicklungshilfe deutlich übersteigen. ${ }^{118}$

Der Bereich der Abschiebung und Rückkehr wird durch verschiedene Programme unterstützt, wie bspw. durch AENEAS, den Europäischen Flüchtlingsfonds, ARGO ${ }^{119}$ und durch den im EU-Rahmenprogramm „Solidarität und Steuerung der Migrationsströme“ angesiedelten Rückkehrfonds (vgl. 2007/575/EG). Über den Rückkehrfonds sollen im Zeitraum zwischen 2008 und 2013676 Millionen Euro abrufbar sein.

\section{„Mit diesen Mitteln können u.a. die freiwillige Rück.kehr gefördert und die Mitgliedstaa- ten bei der Beratung aussichtsloser Antragsteller unterstützt werden. Um dem Problem der jährlich 500.000 Menschen, die sich unrechtmäßig in der EU aufhalten, Herr zu werden, sollen auch Rückführungsflüge gemeinsam mit anderen Mitgliedstaaten organi- siert und aus dem Fonds bezablt werden ". (Bundesministerium des Innern 2007a) ${ }^{120}$}

In den Länderstudien wird noch näher auf das Problem der ,Freiwilligkeit' bei der Rückkehr und auf die konkrete Praxis von Abschiebung und Abschiebehaft einzugehen sein.

lands erreicht, dass die Abschiebungen von denen, die wir loswerden wollen, in Zukunft erleichtert werden“ (vgl. Tagesspiegel vom 05.06.2008).

118 So z.B. der bolivianische Präsident Evo Morales Ayma in einem offenen Brief vom Juni 2008 (vgl. Morales Ayma 2008).

119 ARGO war das bis 2006 gültige Aktionsprogramm für Verwaltungszusammenarbeit in den Bereichen Asylpolitik, Einwanderung und Außengrenzen.

${ }^{120}$ Um die hier als Problem angeführten 500.000 MigrantInnen ins Verhältnis zu den weltweiten Migrationen zu setzen, sei ein Kommentator der ,Deutschen Welle“ zitiert: „Etwa 500.000 illegale Einwanderer schaffen es jährlich, in die Festung Europa einzudringen. Sie stellen für eine EUBevölkerung von 450 Millionen keine Bedrohung dar. Von ,Flut ${ }^{`}$ oder ,Schwemme` kann keine Rede sein. Weltweit, so die Vereinten Nationen, leben 200 Millionen Menschen als Migranten, also gerade einmal 0,25 Prozent davon kommen nach Europa. Die allermeisten Flüchtlinge wandern aus armen Ländern in andere arme Länder“" (vgl. Riegert 2005). 


\subsection{Analyse: Asyl im Migrationsregime auf EU-Ebene}

Im Folgenden soll es darum gehen zu erörtern, welche Schlussfolgerungen sich aus diesen Entwicklungen hinsichtlich der Konstruktion der Kategorie Flucht und Asyl im Migrationsregime der EU ziehen lassen. ${ }^{121}$ Dafür werden die oben dargestellten Regulierungen und Maßnahmen entlang der folgenden Fragen zusammenfassend analysiert: Wie wurde die Kategorie Flucht und Asyl im Zuge der Bestrebungen, ein gemeinsames europäisches Asylregime auf EU-Ebene zu konstruieren, definiert und mit welchen Verfahren und Rechten wurde sie verknüpft? Um auch jenseits der rechtlichen Regelungen einen Eindruck davon zu gewinnen, welches Bild von Flucht und Asyl in den Diskussionen um das Thema auf EUEbene vermittelt wurde, werden einige wichtige Topoi herausgearbeitet, die die Debatte rund um die Entwicklung des Asylregimes geprägt haben. Im letzten Abschnitt wird schließlich diskutiert, welche Bedeutung den Europäisierungsprozessen beim derzeitigen Wandel der Asylregime in der EU zukommt.

\subsubsection{Definition und Verfahren}

Die Darstellung der Richtlinien zeigt, dass die Flüchtlingsdefinition der GFK durch das EU-Recht nicht angetastet wurde. Dennoch findet ein grundlegender Wandel der Kategorie Flucht und Asyl statt, der sich über die Verfahrensinstrumente - also die Zuständigkeitsregelungen - vollzieht, mithilfe derer der Zugang zu den Mitgliedstaaten und deren Asylverfahren erschwert wird.

So ist die Flüchtlingsdefinition, die nun auf EU-Ebene verankert wurde, im Wortlaut fast identisch mit der der Genfer Flüchtlingskonvention. Die Qualifikationsrichtlinie geht insofern über die GFK hinaus, als sie explizit auch geschlechtsspezifische Verfolgung als Asylgrund definiert und als Verfolger auch nichtstaatliche Akteure anerkennt. Damit konkretisiert die Richtlinie die GFK, in deren Wortlaut diese Kriterien nicht enthalten waren, weshalb es von weitergehenden Flüchtlingsdefinitionen in den jeweiligen Ländergesetzgebungen bzw. der GFK-Interpretation durch die Gerichte abhängig war, ob solche Verfolgungsgründe erfolgreich geltend gemacht werden konnten. Gleichzeitig enthält die Flüchtlingsdefinition der Richtlinie aber auch eine deutliche Eingrenzung der GFK-Definition, indem sie den Geltungsbereich des Schutzes auf Drittstaatenangehörige beschränkt. Die Ausschlussgründe nach Artikel 12 hingegen sind mit

\footnotetext{
121 Dabei ist zu berücksichtigen, dass es sich bei den Richtlinien allein um Rechtsakte handelt, die noch nichts über die tatsächliche Umsetzung sagen - weder über die Implementierung in das Recht der Mitgliedstaaten, noch über den Umgang der dortigen Behörden mit den Gesetzesvorgaben und auch nicht über die konkreten Auswirkungen auf MigrantInnen. Auf diese Fragen soll im zweiten Teil der Arbeit - in den Länderstudien - näher eingegangen werden. Hier werden lediglich einige Anhaltspunkte zur Beantwortung dieser Fragen angesprochen.
} 
denen in der GFK nahezu identisch. Ferner fand eine deutliche Ausdifferenzierung der Flüchtlingsstatus in den Richtlinien statt. ${ }^{122}$

Für die Entwicklungen auf EU-Ebene kann also resümiert werden, dass die Kategorie Flucht und Asyl, wie sie bislang durch die GFK definiert wurde, weitgehend unangetastet geblieben ist. Die Definition, wer ein Flüchtling ist, hat sich also fast nicht verändert. Trotz dieser Kontinuität der Definition, also des materiellen Rechts, lässt sich ein grundlegender Wandel des Flüchtlingsrechts konstatieren.

Der Wandel der Kategorie Flucht und Asyl findet über das Verfahren, und zwar über die Zuständigkeitsregelungen, statt. Durch sie wird es immer schwieriger, Zugang zu den Mitgliedstaaten und deren Asylverfahren zu erhalten. Durch die Dublin-II-Verordnung müssen auf dem Landweg einreisende MigrantInnen ihre Asylanträge bereits in den Ländern an den Außengrenzen stellen. Diese können von der sicheren Dritt- und Herkunftsstaatenregelung, wie sie nun auch in der Asylverfahrensrichtlinie verankert sind, Gebrauch machen und die MigrantInnen in die Transit- (also meist die Nachbar-)länder oder die Herkunftsstaaten zurückschieben. Um dies zu erleichtern, schließen nicht mehr nur die einzelnen Mitgliedstaaten selbst Rückübernahmeabkommen mit den Nachbar-, Transit- bzw. Herkunftsländern; inzwischen engagiert sich auch die Kommission verstärkt für den Abschluss solcher Abkommen zwischen der EU und den betreffenden Staaten. Auf diese Weise wird der migrationspolitische Filter zunehmend bereits, vor den Toren' der EU wirksam. Damit „befindet sich der Vorverlagerungsprozess schon in einem fortgeschrittenen Stadium“ (Cuttitta 2004). So bestätigte auch der damalige Parlamentspräsident Josep Borrell beim Europäischen Rat im März 2006 in Brüssel, dass „wir das Problem in Länder jenseits unserer Grenzen verlagern, während die Beschlüsse im Rat für Justiz und Inneres blockiert sind“ (zit. n. Bendel 2006, S. 131).

Um die Anreize für diese Staaten zu erhöhen, wurden verschiedene Konzepte der ,Zusammenarbeit` mit den Anrainern (Europäische Nachbarschaftsstrategie) und den Herkunftsstaaten (im Entwicklungshilfebereich) entwickelt. Doch diese „Kooperation wurde letztlich vorwiegend dahingehend interpretiert, dass die EU die Herkunftsländer befähigen solle, selbst aktiv gegen Fluchtbewegungen vorzugehen“ (Bendel 2006, S. 127, vgl. auch Banse/Müller/Stobbe 2007).

Die erfolgreiche Anwendung der Zuständigkeitsregelungen und des migrationspolitischen Filters im Allgemeinen erfordert eine Ausweitung der Kontrollen und zwar sowohl an den Außengrenzen als auch im Innern der EU. Auch hier intensiviert sich die Zusammenarbeit zwischen den Mitgliedstaaten, obwohl häufig betont wird, dass es sich um besonders sensible und den Kernbereich nationalstaatlicher Souveränität berührende Bereiche handelt. Das zeigt sich sowohl beim

\footnotetext{
122 Siehe Abschnitt 4.1.4 Vorübergehender Schutz, S. 101ff.: GFK-Flüchtlinge (Anerkennungsrichtlinie), Personen mit subsidiärem Schutz (Anerkennungsrichtlinie) und vorübergehend Schutzberechtigte (Richtlinie zum vorübergehenden Schutz).
} 
Grenzregime (Frontex) als auch bei den verschiedenen Bemühungen um EUweite Datenbanken und deren Vernetzung (VIS, SIS, Eurodac).

Als Begründung für die Notwendigkeit der Zuständigkeitsregelungen werden vor allem zwei Argumente angeführt: Zum einen soll auf diese Weise das so genannte ,Asylum Shopping ${ }^{6123}$ verhindert werden - also dass Asylsuchende ihre Anerkennungschancen durch Antragstellung in verschiedenen Mitgliedstaaten zu erhöhen versuchen bzw. dass sie selbst über das Zielland entscheiden. Zum anderen sollen die Regelungen zu einer, gerechten Verteilung der Belastungen“ zwischen den Mitgliedstaaten beitragen. Auch vor ihrem Inkrafttreten war jedoch bereits absehbar, dass sie nicht zu einer gleichmäßigeren Verteilung der Asylanträge, sondern im Gegenteil, zu einer Übertragung der Verantwortung auf die Staaten an den Rändern der EU führen würden.

Will man die Wirkung dieser Regelungen bestimmen, stößt man schnell an die Grenzen empirischer Forschung. Die Vermutung, dass sich die Migration unter diesen erschwerten Bedingungen auf klandestine Wege verlegt, kann nur schwer belegt werden. Es handelt sich um Prozesse, die mit den gängigen Techniken nicht erfasst werden können, da sie sprichwörtlich undokumentiert stattfinden. Die Forschung über diese unsichtbaren Prozesse hat zum einen das Problem, das Phänomen zu quantifizieren ${ }^{124}$, wie es häufig im Interesse der Begründung der Relevanz der Forschung gefordert wird. Zum anderen ist auch ein qualitativer Feldzugang schwierig, da die ,unsichtbaren AkteurInnen' schwer erreichbar sind. ${ }^{125}$ Die vorliegenden Untersuchungen machen die Folgen der Regelungen

${ }^{123}$ Die Rede vom ,Asylum Shopping‘ ist ein eindrückliches Beispiel dafür, wie Politiken über einen bestimmten Sprachgebrauch legitimiert werden. Der Begriff knüpft an die verbreitete Vorstellung von den bogus refugees bzw. Asylbetrïgern an. Er suggeriert, dass sich Flüchtlinge mit der Leichtigkeit und Unbeschwertheit von KonsumentInnen gewissermaßen in den Supermärkten der bumanitären Flüchtlingshilfe bedienen und dabei die Angebote genau prüfen und das beste auswählen. Diese Sichtweise verschleiert, dass die Regale in diesen Geschäften längst leer sind - die Anerkennungszahlen in Europa sind seit Jahren auf einem konstant niedrigen Niveau. Selbst wenn man es schafft, sich Zutritt zum Shop zu verschaffen, ist die Wahrscheinlichkeit groß, mit leeren Händen wieder herauszukommen und dabei macht es kaum noch einen Unterschied, welchen Laden man betritt. Davon abgesehen, dass der Begriff des ,Asylum Shopping' die zahlreichen weiteren Gründe ausblendet, die MigrantInnen bei der Entscheidung für ein Zielland leiten, suggeriert er, dass das Weiterwandern auf der Suche nach einer Bleibechance vollkommen unberechtigt und eine Last für die EU ist. Damit taucht der Rückbau des Flüchtlingsschutzes als Ursache für die Weiterwanderung gar nicht mehr auf, sondern wird im Gegenteil als deren Folge präsentiert. Mit der Dublin-IIVerordnung soll nun vorgeblich die $W$ abl des Shops beeinflusst werden. Zu den konkreten Auswirkungen vgl. die Länderstudien zu Deutschland und Polen. Vgl. zu dem Begriff auch Abschnitt 4.2.3.

${ }^{124}$ Es werden verschiedene Möglichkeiten diskutiert, zu einem Schätzwert zu gelangen - zum Beispiel über die Hochrechnung von Statistiken über amtliche Kontrollen im Inland oder der Polizeilichen Kriminalstatistik. Je nach verwendetem ,Schätzprinzip“ variiert die angegebene Zahl zum Beispiel für die Bundesrepublik zwischen 100.000 und einer Million Statuslosen (vgl. Sinn et al., S. 45ff.).

125 Gleichwohl gibt es inzwischen Publikationen von Forschern, die sich von diesen Schwierigkeiten nicht abschrecken ließen (vgl. z.B. Alt 2003; Stobbe 2004). 
sichtbar: Einerseits erhöhen sich die Kosten für die Einreise, insbesondere um einen Asylantrag im angestrebten Land stellen zu können. Finanzielle Ressourcen, soziale Netzwerke und Schleuser werden immer wichtiger, wenn die Migration gelingen soll. Dies bedingt eine erhebliche soziale Selektion, die sich auch in der Repräsentation der Herkunftsländer und den Merkmalen der erfolgreichen MigrantInnen (insbesondere hinsichtlich Alter und Geschlecht) niederschlagen dürfte. Andererseits führen die Schwierigkeiten, im Rahmen des Asylregimes selbst das Zielland wählen zu können und das Wissen um die geringen Anerkennungschancen dazu, dass vermehrt irreguläre Wege genutzt werden. Sabine Hess und Serhat Karakayalı (2007, S. 45) zitieren den entsprechenden Ratschlag eines Transitmigranten in der Türkei an seinen Freund bezüglich der Asylantragstellung: „,Stay away from this system! “. Zur Begründung heißt es:

\section{„Alle seine Bekannten, selbst diejenigen, die aus Bürgerkriegsländern wie Liberia oder Sierra Leone kommen, waren skeptisch. Denn was sie von denjenigen, die Asyl bean- tragt hatten, erfubren, erinnerte sie an die Polizei: Unfreundliche Interview-Verböre, ri- gide Definition politischer Verfolgung, Beweislast auf der Seite des Flüchtlings, lange Verfahrensdauer und Unsicherbeit, niedrige Anerkennungsquote, keine finanæielle Un- terstützung"(Hess/Karakayah 2005, S. 45f.).}

Doch nicht alle MigrantInnen wandern irregulär weiter - diejenigen, denen die Möglichkeiten fehlen, verbleiben in den Staaten an den Außengrenzen und in den Transitstaaten. Damit vollzieht sich - entgegen den offiziellen Zielen der DublinII-Verordnung und der Drittstaatenregelung - eine Auslagerung , unerwünschter Immigration in diese Staaten. Diese Entwicklung ist nicht nur Ergebnis der Asylrichtlinien und -verordnungen, sondern auch des außenpolitischen Engagements für eine ,Zusammenarbeit ${ }^{`}$ mit den Herkunfts- und Transitstaaten. Es wird zwar versucht, durch das Beharren auf Einhaltung der GFK in diesen Ländern eine menschenrechtliche Basis für die Flüchtlingsaufnahme zu schaffen. Damit soll die Kooperation als die bessere Lösung für MigrantInnen präsentiert werden, die vor Ort geschützt werden könnten und sich dann nicht mehr auf den gefährlichen Weg in die EU machen müssten. Der Realität in den Anrainerstaaten entspricht dieses Bild jedoch ebenso wenig wie den Migrationsvorhaben vieler MigrantInnen. Die Exterritorialisierungsstrategie führt also nicht nur zum faktischen Rückgang der Zahl derer, die Asylanträge stellen und Ansprüche auf soziale Teilhabe geltend machen können, sondern trägt auch dazu bei, die Folgen der EU-Migrationspolitik möglichst nicht mehr bis in die europäische Öffentlichkeit dringen zu lassen. Denn während die Bilder von MigrantInnen, die bei der Überfahrt nach Europa ertrunken, erfroren, verdurstet oder verhungert sind, hin und wieder Aufmerksamkeit und Proteste gegen die Migrationspolitiken der EU und ihrer Mitgliedstaaten erregen, entzieht sich das, was in der Ukraine, in Libyen, Tunesien und Marokko mit den MigrantInnen geschieht, fast vollständig den Blicken der 
Öffentlichkeit: „Hauptsache, das Sterben geschieht abseits der europäischen Öffentlichkeit diskret.“ (Cuttitta 2004)

Mit den Zuständigkeitsregelungen und der damit verbundenen Datenerfassung und Antragsbearbeitung ist ein mächtiger bürokratischer Apparat entstanden, der hohe Kosten verursacht. Deren (Mis-) Verhältnis zum Output bzw. Outcome lässt sich zwar aufgrund mangelnder Daten nicht ermitteln, wird aber - nach Aussagen der Kommission - von den Mitgliedstaaten aufgrund übergeordneter politischer Zielstellungen in Kauf genommen (vgl. $\operatorname{KOM}(2007)$ 299, S. 14).

\subsection{2 (Soziale) Rechte}

Die Durchsicht der Richtlinien verleitet dazu, lediglich die sozialen Rechte und ihre Einschränkungen im Vergleich zum Rest der Bevölkerung in den Blick zu nehmen, denn politische und bürgerliche Rechte werden in den Richtlinien nicht erwähnt. Bevor also näher auf die Einschränkung der sozialen Rechte eingegangen wird, sei hier noch darauf hingewiesen, dass insbesondere AsylbewerberInnen in den meisten Mitgliedstaaten der EU viele politische Rechte nicht für sich reklamieren können bzw. diese faktisch verwehrt werden. Dies spiegelt den humanitären Charakter des Asylrechts wider ${ }^{126}$, das nie politisch ,von unten' erkämpft, sondern ,von oben' installiert und als Recht der Staaten, Asyl zu gewähren, verstanden worden ist. ${ }^{127}$

Die unterschiedlichen Flüchtlingsstatus, wie sie in den Richtlinien definiert werden, sind mit einem differenzierten Umfang an sozialen Rechten verknüpft. Zudem besteht hinsichtlich der Gewährung sozialer Rechte ein breiter Gestaltungsspielraum für die Mitgliedstaaten. Die Richtlinien legitimieren eine massive soziale Schlechterstellung von Flüchtlingen im Verhältnis zu StaatsbürgerInnen, EU-BürgerInnen und anderen Drittstaatenangehörigen mit sicheren Aufenthaltsstatus: Die Aufnahmerichtlinie, die hierfür zentral ist, erlaubt unter anderem Einschränkungen der Bewegungsfreiheit, (zeitlich begrenzten) Ausschluss vom Arbeitsmarkt, Sachleistungen, Unterbringung und Schulbildung in Lagern und die Beschränkung der Gesundheitsversorgung auf medizinische Notversorgung. So wird eine Beibehaltung restriktiver Regelungen in einigen Mitgliedstaaten, in anderen, bisher liberaleren Asylregimen eine Absenkung der Standards ermöglicht. Gegenüber der Ausgangssituation in den neuen Mitgliedstaaten stellen die niedrigen sozialen Minimalstandards der Richtlinien zum Teil jedoch eine Verbesserung dar.

\footnotetext{
126 Humanitäre Konzepte orientieren sich meist nur an dem Fakt der Hilfsbedürftigkeit der Betroffenen, stellen aber die Ursachen der Bedürftigkeit nicht in Frage. Dies bedingt ein naturalisiertes Verständnis von sozialen und politischen Problemen. Darüber hinaus sehen humanitäre Ansätze überwiegend nur eine Grundversorgung der Betroffenen vor, die sich am Grad der unmittelbaren Bedürftigkeit orientiert.

127 Zur Geschichte des Rechts auf Asyl vgl. Kimminich (1982).
} 
Die Beschneidung der sozialen Rechte wird häufig damit begründet, dass auf diese Weise eine abschreckende Wirkung erzielt werden könne. Es sollen möglichst keine ,Anreize für eine ,Einwanderung in die Sozialsysteme' geschaffen werden. Die Abschreckungsmaßnahmen im sozialen Bereich sind dabei oft teurer als eine Gleichbehandlung mit den StaatsbürgerInnen. Dies gilt auch für die in den Richtlinien enthaltenen Maßnahmen: Die Mitgliedstaaten nehmen zusätzliche Kosten für die soziale Schlechterstellung und Abschreckung von Flüchtlingen in Kauf. Dies zeigt sich im Verbot den eigenen Lebensunterhalt mit Erwerbsarbeit zu verdienen oder in der Lagerunterbringung, die weitaus kostspieliger ist als eine dezentrale Unterbringung sowie in den sonstigen Ausformungen des Sachleistungsprinzips, das mit einem hohen administrativen Aufwand verbunden ist. Viele neue Mitgliedstaaten hingegen verfügen häufig (noch) nicht über die Ressourcen für eine bessere Versorgung. Dort sind Flüchtlinge mitunter in der schlechten Versorgung mit den BürgerInnen gleichgestellt. Gleichwohl wird mit diesen Maßnahmen in beiden Fällen eine soziale Stigmatisierung erzeugt, die entlang des Merkmals der ,Herkunft' verläuft und einen gesellschaftlichen Konsens über das Nicht-Willkommensein von Asylsuchenden erleichtert. Eine solche Konstruktion der ,Anderen' durch ihre sichtbare Ausgrenzung (Lagerunterbringung, Arbeitsverbot, Bezahlen mit Gutscheinen im Supermarkt) ermöglicht es Mitgliedern der Mehrheitsgesellschaft, sich nach unten bzw. außen abzugrenzen. Indem MigrantInnen im Zielland auf die untersten sozialen und Arbeitmarktpositionen verwiesen werden, soll der Status der ,Einheimischen' gesichert werden. Dieses von Hans-Joachim Hoffmann-Nowotny (1973) als „Unterschichtung“ und „neofeudale Absetzung“" bezeichnete Phänomen, bei dem sich die Statuszuweisung an ethnischen, also an zugeschriebenen und nicht an erworbenen Kriterien (wie bspw. Qualifikation) orientiert, wird durch die explizite sozialrechtliche Schlechterstellung und Ausgrenzung von Asylsuchenden durch den Gesetzgeber gestützt und auf institutionellem Wege verfestigt.

Dass MigrantInnen im Allgemeinen und Asylsuchende im Besonderen nicht die gleichen Rechte innehaben wie StaatsbürgerInnen, sondern entsprechend ihres jeweiligen Aufenthaltsstatus in abgestufter Form automatisch schlechter gestellt sind, speist sich aus dem Denken in Kategorien nationalstaatlicher Zugehörigkeit. Dieses Denken befindet sich auch durch die Schaffung des EU-BürgerInnenstatus nicht in Auflösung, denn die EU basiert nach wie vor auf nationalstaatlichen Abgrenzungen. Eine Diskussion der vielfältigen Ursachen gesellschaftlicher Probleme zum Beispiel bei der Verteilung von Ressourcen wird verhindert, solange ein Konsens darüber besteht, dass die ,Anderen', qua nationalstaatlichem Verständnis als nicht-zugehörig Definierten, diese Ressourcen unrechtmäßig in Anspruch nehmen - und deshalb auch für die soziale Situation der Einheimischen verantwortlich zu machen sind. Gesellschaftliche Verteilungskonflikte können so ein Stück weit ,um'- bzw. ,abgeleitet' werden. Nach Hoffmann-Nowotny spiegeln sich 
in den ablehnenden Einstellungen der ,Einheimischen“ gegenüber MigrantInnen vor allem strukturelle Probleme der Einwanderungsgesellschaft:

„Auf einen Satz gebracht sind die Fremden nur der, Aufhänger" für interne Probleme der hochentwickelten Einwanderungsgesellschaft, die in der Auseinandersetzung über die ,Überfremdung' lediglich sichtbar werden. "(Hoffmann-Nowotny 1973, S. 151)

Indem Asylsuchenden per Gesetz grundlegende Rechte vorenthalten werden, werden diese Einstellungen bestätigt und reproduziert - wobei genauer zu untersuchen wäre, inwieweit die Forderungen nach solchen Politiken der Ab- und Ausgrenzung von den Eliten selbst gesetzt werden oder aus der WählerInnenschaft kommen, die den Eliten derlei Zugeständnisse abringt. ${ }^{128}$ Es ist anzunehmen, dass hier eine Wechselwirkung zwischen den Ressentiments der Mehrheitsgesellschaft und den politisch-administrativen Maßnahmen gegenüber Einwanderern besteht. Auf die Funktion der sozialen Schlechterstellung wird in den Länderstudien noch etwas näher eingegangen.

\subsubsection{Sprachgebrauch}

Die flüchtlingspolitischen Maßnahmen sind auch auf EU-Ebene durch einen spezifischen Sprachgebrauch gekennzeichnet, mit dem die politischen AkteurInnen das Thema präsentieren und für öffentliche Unterstützung ihrer Vorhaben werben. Um die zentrale Rolle des Sprachgebrauchs bei der Präsentation und Begründung asyl- und migrationspolitischer Maßnahmen umfassend zu analysieren, ist eine eigene Untersuchung erforderlich. Hier sollen lediglich entsprechend der erkenntnisleitenden Fragestellung einige Beispiele für die starke Bedeutung der Kategorisierung von MigrantInnen und insbesondere die Darstellung der Kategorie Flucht und Asyl bei der sprachlichen Begründung und Legitimierung der Maßnahmen herausgearbeitet werden. Hierzu zählen

- die Rahmung des Politikfeldes unter dem Leitsatz der Schaffung eines ,Raums der Freiheit, der Sicherheit und des Rechts', der im Wesentlichen lediglich EUBürgerInnen einschließt,

- die damit einhergehende sprachliche Differenzierung zwischen (erwünschter) Mobilität von EU-BürgerInnen innerhalb dieses Raumes und (unerwünschter) Migration von Drittstaatenangehörigen in diesen Raum hinein,

- die starke Hierarchisierung von MigrantInnen, die sich auch sprachlich in deren unterschiedlicher Bewertung ausdrückt (Förderung der Einwanderung von

\footnotetext{
128 Diese Frage erörtert Paul Statham (2003) auf der Basis einer empirischen Untersuchung in Großbritannien. Er schlussfolgert, dass ,the nature of the asylum debate - at present restrictive and stigmatising - to a large extent comes politically from the top down, rather than in response to mobilised public pressure." (ebd., S. 174) Eine Übertragbarkeit der Ergebnisse auf andere Länder oder den EU-Kontext ist sicherlich nicht ohne Weiteres möglich. Dennoch stellt die Untersuchung die weit verbreitete Annahme in Frage, ausgrenzende Politiken seien lediglich eine Antwort auf die ängstliche oder ImmigrantInnen gegenüber ablehnend eingestellte Bevölkerung.
} 
„Freizügigkeit ist ein Grundrecht der EU-Bürgerinnen und -Bürger, das bestmöglich genutzt werden sollte, da es neue Wege zum Lernen, zum Arbeiten und zur Weiterbildung eröffnet. "(zit. n. Migration und Bevölkerung 2006)

Freizügigkeit als Grundrecht wird hier also auf den EU-BürgerInnenstatus beschränkt. ${ }^{130}$ Gleichzeitig zeigt das ebenfalls aus diesem Zusammenhang stammende Zitat von Kommissionspräsident Barroso: „Europa muss darüber nachdenken, wie es seine Arbeitskräfte effizienter und anpassungsfähiger machen kann“ (ebd.), dass es hier weniger um die Inanspruchnahme von Grundrechten als um die effiziente Verwertung von Arbeitskraft bzw. des ,Humankapitals‘ geht.

Die Freizügigkeit ist für EU-BürgerInnen also einerseits ein exklusives Grundrecht, andererseits aber auch mit Ansprüchen und Anforderungen hinsichtlich ihrer Flexibilität und Anpassungsfähigkeit auf dem EU-Arbeitsmarkt verbunden. Benedikt (vgl. 2004, S. 68) stellt fest, dass nach dieser Logik ein Süditaliener systemkonform handelt, wenn er dem Arbeitsplatz folgt, während ein Nordafrikaner mit demselben Ziel systemwidrig handelt. ${ }^{131}$ Er illustriert anhand einer Umkehrung der Begriffe den Unterschied in der Bedeutung zwischen Migration und Mobilität: So würde eine Kommissionspapier, in dem von „mobilen afrikanischen Arbeitnehmern“ statt ,afrikanischen Wirtschaftsmigranten oder -flüchtlingen“ die Rede ist, zumindest ungewohnt klingen (ebd., S. 68). Dabei betont Benedikt (2004, S. 67), dass nicht allein die rechtlichen Definitionsakte für den Wandel des Vokabulars verantwortlich sind:

„Die Bedeutungsverschiebung von ,Migration“ in Richtung einer Kategorie zur Beschreibung von EU-Außengrenzen überschreitenden Bewegungen ergibt sich in einer breiteren öffentlichen Thematisierung, die nicht unbedingt mit dem Anspruch geschieht, diese Begriffe von ,Migration",Migranten" von einer vorausgesetzten nationalen auf eine (unions-),europäische' Ebene zu übertragen. "

130 Diese Freizügigkeit genießen EU-BürgerInnen jedoch nicht nur auf dem Territorium der EU, sondern auch in den meisten anderen Regionen der Welt. Ein Umstand, der von den aus der EU Ausgeschlossenen nicht notwendig als naturgegeben wahrgenommen wird. So betonte der marokkanische Ökonomie-Professor Mehdi Lahlou auf einer Tagung der Friedrich-Ebert-Stiftung in Berlin (,Die Europäische Dimension deutscher Flüchtlingspolitik' am 22.11.2006), dass Freizügigkeit als Menschenrecht verankert und es kaum nachvollziehbar sei, dass die EuropäerInnen Reisefreiheit in Anspruch nehmen und uneingeschränkt in die Herkunftsländer der MigrantInnen reisen könnten, während der Zugang zur EU für Menschen aus der südlichen Hemisphäre versperrt werde.

131 Benedikt schlussfolgert daraus, dass „,[d]ie Neuordnung der Zugehörigkeiten zwischen Migration und Mobilität am unionseuropäischen Arbeitsmarkt [...] somit keineswegs rein marktökonomischen Grundsätzen [gehorcht], obwohl sie in den EU-Dokumenten im Politikfeld Migration so gut wie nie explizit kulturell begründet wird" (Benedikt 2004, S. 68). Hier ist jedoch einzuwenden, dass gerade mit der Entrechtung, des Nordafrikaners' ein Angebot an besonders flexibler und billiger Arbeitskraft geschaffen wird, das durchaus nachgefragt wird und daher einer marktökonomischen Logik zunächst nicht widerspricht. Erst die Hierarchisierung und abgestufte Entrechtung von Arbeitskräften entlang des Kriteriums ,Herkunft' legt dann kulturalistische Muster offen. 
Dennoch schätzt er die Bedeutung von Elitendiskursen als hoch ein, da der EUIntegrationsprozess nicht auf Basisbewegungen und der Aktivität breiter Massen gründe (vgl. Benedikt 2004, S. 67).

Der ,Raum der Freiheit, der Sicherheit und des Rechts' ist also schon per definitionem weitgehend auf EU-BürgerInnen beschränkt. Inwieweit er auch MigrantInnen einschließt, ist abhängig von ihrem Status: So sind Hochqualifizierte bzw. AkademikerInnen aus Drittstaaten gegenüber Flüchtlingen und anderen ,unerwünschten` MigrantInnen relativ besser gestellt. Die Immigration von Hochqualifizierten wird in der Nützlichkeitslogik des ,Standortwettbewerbs' positiv konnotiert, erfährt dabei aber auch eine deutliche Abgrenzung von der ,Gruppe` der ,Unerwünschten‘, den ,illegalen“ ImmigrantInnen. Ziel des Asylregimes ist es, die ,tatsächlich Schutzberechtigten‘, aus der großen Zahl der ,Unberechtigten` herauszufiltern. Während, echte Flüchtlinge geschützt' werden sollen, soll die ,illegale Immigration bekämpft werden. So heißt es im innenpolitischen Programm zur deutschen Ratspräsidentschaft: „Unser Ziel ist, Flüchtlinge zu schützen und Missbrauch zu verhindern“ (Bundesministerium des Innern 2006a). Ein ähnlicher Sprachgebrauch findet sich im Grünbuch der Kommission zum Gemeinsamen Asylsystem:

„Ebenso müssen den nationalen Asylbehörden geeignete Instrumente bereitgestellt werden, damit sie Asylströme effizient bewältigen und Betrug und Missbrauch effektiv verhindern können, um so die Integrität und Glaubwürdigkeit des Asylsystems zu wahren. " (KOM(2007) 301, S. 3)

Auch Steffen Angenendt und Roderick Parkes folgen dem Grünbuch in seiner Missbrauchsargumentation:

„Eine Kernfrage für das künftige Gemeinsame Asylsystem lautet daher, wie die ungerechtfertigte Inanspruchnabme von Asyl reduziert werden kann. Realistischerweise wird der Flüchtlingsschutz nur dann gestärkt werden können, wenn der Missbrauch minimiert wird, denn nur unter dieser Bedingung werden die Mitgliedstaaten den Zugang zu den Verfabren lockern. "(Angenendt/Parkes 2007, S. 3)

Flüchtlingsschutz und der ,Missbrauch“ dieses Schutzes durch Unberechtigte bilden in der Rhetorik inzwischen ein festes Begriffspaar. Auf EU-Ebene hat sich der Begriff des ,Asylum Shopping ${ }^{6132}$ als Bezeichnung für eine Spielart dieses ,Missbrauchs` durchgesetzt. Die Verhinderung des ,Asylum Shopping taucht als Ziel in den Erwägungsgründen einiger Richtlinien auf. Gemeint ist die mehrfache Asylantragstellung in verschiedenen Mitgliedstaaten. Aus der Perspektive der ,Autonomie der Migration'133 ist das Phänomen lediglich Ausdruck der Versuche von MigrantInnen, die Hürden des Einwanderungsrechts zu überwinden - um auf dem Landweg einzureisen, müssen sie in einem der Staaten an den Außengrenzen

132 Vgl. zu den Implikationen dieses Begriffs auch Fn. 123.

133 Vgl. Kap. 2.2.4 „Autonomie“ oder „Eigensinnigkeit“ der Migration 
einen Asylantrag stellen. Wenn dieses jedoch nicht ihr Zielland ist oder sie dort schlechte Lebensbedingungen vorfinden, reisen sie weiter und stellen anderswo erneut einen Antrag - mitunter unter einem anderen Namen. Der Begriff, Asylum Shopping ' diskreditiert die eigenständige Wahl des Ziellandes als grundsätzlich unberechtigt, legitimiert gleichzeitig die Zuständigkeitsregelungen als übergeordnetes Prinzip und setzt zudem Flucht und Migration mit einem Einkaufsbummel gleich, bei dem sich MigrantInnen einfach bedienen.

Die Exklusivität und der Ausschlusscharakter des ,Raumes der Freiheit, der Sicherheit und des Rechts` zeigen sich auch darin, dass gerade die ,Bekämpfung illegaler Migration' als wichtige Voraussetzung für die ,Sicherheit' in Europa und damit als Teil der Errichtung dieses Raumes gesehen wird. ,Illegale' Migration wird dementsprechend häufig in einem Atemzug mit ,Bekämpfung des Terrorismus' und ,grenzüberschreitender Kriminalität' genannt. Dass der Sicherheitsaspekt bereits an prominenter Stelle in dem dreiteiligen Leitmotiv enthalten ist, unterstreicht einmal mehr seine herausragende innenpolitische Bedeutung, die spätestens seit den Anschlägen vom September 2001 viele andere Interessen in den Schatten gestellt hat.

Parallel zum ,Missbrauchsthema' setzt sich zunehmend ein Argumentationsmuster durch, wonach Flüchtlingsschutz nicht zwangsläufig innerhalb der EU gewährt werden muss. Dabei gewinnt die ,Zusammenarbeit ${ }^{`}$ mit Herkunfts- und Transitstaaten, die auch den Aufbau von Asylregimen beinhaltet, einen zentralen Stellenwert in der Außendarstellung der EU-Migrationspolitik. Hier wird oft mit humanitären Aspekten argumentiert, die insbesondere dann Konjunktur haben, wenn die Medien in den Sommermonaten Bilder von MigrantInnenbooten oder angeschwemmter Leichen zeigen. Die Aufrüstung der Grenzen und die Errichtung von Auffanglagern in den Transitregionen werden mit der Begründung, das Sterben an den Grenzen verhindern und MigrantInnen von den gefährlichen Fahrten auf seeuntüchtigen Holzbooten abhalten zu wollen, vorangetrieben. Das Argument, wonach sowohl die Auslagerung des Flüchtlingsschutzes als auch eine effiziente Überwachung der Grenzen im Interesse der Flüchtlinge sind, ist inzwischen ein wichtiger Topos geworden und lässt sich an zahlreichen Zitaten illustrieren. ${ }^{134}$ Hier ,wird das Narrativ des neuen Humanitarismus angestrengt, welches

134 Vgl. zum Beispiel das oben bereits angeführte Zitat des Frontex-Direktors Ilkka Laitinen (siehe Fn. 103), sowie die Argumentation aus dem Programm des deutschen Innenministeriums zur deutschen Ratspräsidentschaft: „Angesichts der im Frühjahr wieder stärker werdenden Migrationsbewegungen über das Meer in Richtung Europa und den damit verbundenen Gefahren für das Leben vieler Menschen sehen es die Innenminister und der Vizepräsident der Europäischen Kommission, Franco Frattini, als vordringlich an, in Migrationsfragen eng mit den Herkunfts- und Transitländern an den Süd- und Ostgrenzen der EU zusammenzuarbeiten." (Bundesministerium des Innern 2007b) Ähnlich äußerte sich Otto Schily 2004 in der von ihm angestoßenen Debatte um Auffanglager der EU in Nordafrika: „Wir müssen dafür sorgen, dass Menschen sich nicht mutwillig in Gefahr begeben. Die Anrainerstaaten müssen dazu gebracht werden, Menschen davor zu bewahren, sich mit seeuntüchtigen Schiffen aufs Mittelmeer zu begeben. Und die brutalen Aktivitäten der 
fordert, die Migration abzuhalten, um menschliche Tragödien zu vermeiden“ (Hess/Tsianos 2007, S. 34). Eine solche menschenrechtliche Legitimation der Ausgrenzungspolitiken stellt die Wirkungszusammenhänge auf den Kopf. Zum einen sind die verstärkten Patrouillen sowohl auf EU-Seite als auch auf Seiten der Anrainer kein Schutz für die MigrantInnen, sondern im Gegenteil eine Ursache für die Todesfälle an den Grenzen. Denn die meisten lassen sich durch solche Maßnahmen kaum aufhalten. Vielmehr weichen sie auf längere und gefährlichere Routen aus, um ihr Ziel zu erreichen. Aus Angst vor Querelen mit Behörden oder vor einer Anklage wegen Schlepperei hüten sich die Kapitäne von anderen Schiffen, MigrantInnen in Seenot an Bord zu nehmen. ${ }^{135}$ Zum anderen ist auch die Inanspruchnahme der Dienste der oft als eigentliches Problem dargestellten Schleuser eine Folge der Hürden auf dem Weg in die EU:
„Je höher die Zäune an den Außengrenzen der EU gebaut werden und je ausgefeilter die Überwachungstechnik wird, desto lukerativer wird das Geschäft mit dem organisierten Grenzübertritt, weil immer weniger MigrantInnen in der Lage sind, obne professionelle Hilfe in die EU einzureisen. "(Banse/Müller/Stobbe 2007, S. 194)

Neben dieser menschenrechtlichen Begründung der auf Ab- und Ausgrenzung zielenden Maßnahmen wurde mit der Formel der ,Bekämpfung der Migrationsursachen' eine weitere zentrale Forderung vieler NGOs diskursiv eingebunden. Doch im Gegensatz zur Prominenz dieses Instruments bei öffentlichen Erklärungen spielt es faktisch eine sehr untergeordnete Rolle:

„Es wird viel von Entwicklung geredet, aber das ist alles nur Theorie. Auf dem Gebiet der Sicherheit werden Mittel bereitgestellt, werden die entsprechenden Institutionen ins

kriminellen Schleuserbanden müssen mit äußerster Härte bekämpft werden.“ (Süddeutsche Zeitung vom 2.08.2004) Mit einem Gastbeitrag in der Frankfurter Allgemeinen Zeitung wandte Schily sich „an jene, die sich berechtigte Sorgen um die Menschen machen, die Opfer von Schleusern werden oder auf eigene Faust versuchen, mit seeuntüchtigen Booten von Nordafrika über das Mittelmeer nach Europa zu gelangen. Viele Menschen hat dieser Versuch das Leben gekostet. Das kann und das darf niemand gleichgültig lassen." (Schily 2004) Der Sprecher des Innenministeriums, Rainer Lingenthal, sagte im Zusammenhang mit Schilys Vorschlag, es ginge darum, ,das Karussell des Elends zu durchbrechen" (Frankfurter Rundschau vom 22.07.2004). Der CSU-Europaabgeordnete Manfred Weber, Berichterstatter des Europäischen Parlaments bei der Diskussion um die umstrittene Rückführungsrichtlinie sagte, durch Abschiebungen würden Millionen Menschen ohne Papiere „aus der Illegalität herausgeholt“: „Das Sklaventum, das wir heute dort haben, muss beendet werden" (Europäisches Parlament 2008a). Auch die Abschiebung wird hier also als Mittel zur Wahrung der Menschenrechte präsentiert.

135 Vgl. zu den möglichen Folgen der Rettung von MigrantInnen den prominenten Fall der Cap Anamur vom Sommer 2004. Der Kapitän Stefan Schmidt, der damalige Leiter der Organisation Cap Anamur, Elias Bierdel, und der Erste Offizier, Vladimir Daschkewitsch, wurden wegen Beihilfe zur illegalen Einreise angeklagt, weil die Cap Anamur vor der Küste Italiens 37 schiffbrüchige MigrantInnen aufnahm. 


\section{Leben gerufen. In der Entwicklungspolitik hingegen gibt es weder Geldmittel im nötigen} Umfang noch die notwendigen Strukturen" (Lablou 2006)"136.

Doch es ist nicht nur aufschlussreich darauf zu schauen, welche Argumentationen von Seiten der EntscheidungsträgerInnen wie verknüpft und verwendet werden, sondern auch, was in den öffentlichen Verlautbarungen nicht angesprochen wird. Dazu gehört zum Beispiel die Mitverantwortung der EU und ihrer Mitgliedstaaten für die Situation in den Herkunftsländern. Die Probleme in der so genannten ,Dritten Welt ${ }^{6}$ werden oft als ,hausgemacht ${ }^{6}$ dargestellt - die wirtschaftliche und soziale Situation, Korruption und Diktaturen sind allein ,Afrikas Probleme ${ }^{6137}$. Das Missverhältnis zwischen den teils ineffizienten Entwicklungshilfestrategien ${ }^{138}$ einerseits und zum Beispiel der protektionistischen Agrar- und expansiven Fischereipolitik andererseits wird hingegen nicht thematisiert. Die Entwicklungshilfen werden als großzügige Spenden und Teil der Bemühungen um die Beseitigung der Migrationsursachen dargestellt. Häufig werden sie jedoch in den Dienst bestimmter EU-Interessen gestellt und zeitigen dann Effekte, die dem Ziel der ,Entwicklung' im Sinne einer Verbesserung der Lebensbedingungen vor Ort zuwider laufen. So wird die Vergabe von Mitteln vielfach an Bedingungen geknüpft, etwa um der EU Zugang zu bestimmten Ressourcen zu verschaffen - wie den Fischgründen im Senegal ${ }^{139}$ - oder um die Staaten zur Öffnung ihrer Märkte im Rahmen von so genannten ,Wirtschaftspartnerschaftsabkommen' zu bewegen. Die Fischerei- und Handels-, aber auch die Agrarpolitik der EU und ihre Auswirkungen auf die Situation in den Herkunftsstaaten der MigrantInnen laufen so auch den migrationspolitischen Zielen entgegen. Sie tragen dazu bei, dass traditionelle ökonomische Strukturen aufgebrochen und zerstört werden und so Teilen der Bevölkerung die Lebensgrundlage entzogen wird. Insofern bekämpfen InnenpolitikerInnen auch die Folgen der Maßnahmen aus anderen Politikbereichen wie der Agrar- und Fischereipolitik. Überspitzt formuliert, werden Milliarden in den Grenzschutz investiert, um diejenigen aufzuhalten, die ihre Boote nach dem Abfischen der

136 Zu Mehdi Lahlou vgl. Fn. 112.

137 Dies suggeriert nicht zuletzt der Titel von Otto Schilys Gastbeitrag in der Frankfurter Allgemeinen Zeitung vom 23.07.2004: „Afrikas Probleme in Afrika lösen“ (vgl. Schily 2004).

138 Vgl. zur Kritik an der aktuellen Ausrichtung und Umsetzung der Entwicklungshilfen die Diskussionen um die ,Pariser Erklärung، über Grundprinzipien effizienter Entwicklungshilfe und die Konferenzen des High Level Forum on Aid Effectiveness (HLF) (vgl. u.a.: Alliance2015 2008).

139 Durch Abkommen mit dem Senegal sicherte sich die EU seit 1981 die Rechte an großen Teilen der Fischbestände an der senegalesischen Küste. Fisch ist im Senegal Grundnahrungsmittel, für viele auch die Haupteinnahmequelle und zudem das wichtigste Exportgut des Landes. Die senegalesischen Kleinfischer können jedoch mit den hochmodernen EU-Fischfangflotten nicht konkurrieren, die die Fischbestände stark dezimiert haben: In den westafrikanischen Gewässern sind in den letzten 20 Jahren die Grundfischbestände um die Hälfte zurückgegangen. Der Senegal erhält im Gegenzug 16 Millionen Euro jährlich von der EU, ein Teil davon stammt aus den Mitteln der europäischen Entwicklungszusammenarbeit - „Entwicklungsgelder werden dazu benutzt, europäischen Trawlern den Zugang zu den Beständen der senegalesischen Kleinfischer zu ermöglichen“ (Germanwatch 1997). 
Gewässer durch die europäischen Fischfangflotten für die Beförderung von MigrantInnen nutzen. Diese konkurrierenden politischen Interessen und die widersprüchlichen Folgen ihres Zusammenspiels finden in den öffentlichen Stellungnahmen und Papieren jedoch keine Erwähnung. Indem die Bedeutung europäischer Politiken für die Situation in den Herkunftsländern - sowohl bei ihrer Konzeption und Umsetzung als auch in ihrer öffentlichen Darstellung - und damit die Mitverantwortung für die Lebensbedingungen vor Ort ausgeblendet wird, kann Migration überhaupt erst in der vorherrschenden Weise als exogen produziertes ,Problem ' und Migrationspolitik entsprechend als humanitäre Geste für Asylsuchende und als ,Kampf ' gegen, illegale Einwanderung' präsentiert werden.

Zusammenfassend lässt sich sagen, dass der ,Raum der Freiheit, der Sicherheit und des Rechts' nur EU-BürgerInnen und eingeschränkt auch einigen Drittstaatenangehörigen offen steht. Diese faktische Trennung wurde sprachlich nachvollzogen durch unterschiedliche Begriffe für die Bewegungen innerhalb dieses Raumes und von außen in diesen Raum hinein: Die positiv konnotierte und erwünschte ,Mobilität' steht der negativ besetzten und überwiegend unerwünschten ,Migration' gegenüber. Zur Kluft zwischen MigrantInnen und EU-BürgerInnen kommt eine starke Ausdifferenzierung und Hierarchisierung innerhalb der Gruppe der MigrantInnen. Diese schlägt sich in dem entsprechend besetzten Vokabular nieder: Flüchtlinge schützen, Missbrauch verhindern, Einwanderung von Hochqualifizierten fördern, illegale Immigration bekämpfen. Die mit dem ,Migrationsmanagement ${ }^{t}$ derzeit verbundenen Maßnahmen wie etwa die Vorverlagerung der Kontrollen werden mit menschenrechtlichen Argumenten und der formelhaft wiederholten Zusicherung, Migrationsursachen bekämpfen zu wollen, diskursiv abgesichert. Während die tatsächlichen Aktivitäten bei der Beseitigung der Ungleichheiten zwischen nördlicher und südlicher Hemisphäre sehr begrenzt sind, wird der gegenläufige Einfluss verschiedener EU-Politiken zum Beispiel im Agrarund Fischereibereich nicht thematisiert.

\subsection{Supranationalisierung: Neue Möglichkeiten der Legitimation, Gestaltung und Umsetzung von Asylpolitiken}

Wie gezeigt wurde, findet der Wandel der Kategorie Flucht und Asyl auf EUEbene über Verfahrensinstrumente - das heißt über die Zuständigkeitsregelungen - statt. So wird der Zugang zu den Mitgliedstaaten und deren Asylverfahren erschwert. Gleichzeitig enthalten die Richtlinien ein abgestuftes System verschiedener Flüchtlingsstatus, die jeweils mit einem unterschiedlichen Umfang an sozialen Rechten verbunden sind. Dabei ermöglichen die Richtlinien eine starke soziale Schlechterstellung von Flüchtlingen im Verhältnis zur übrigen Bevölkerung - die konkrete Ausgestaltung wird jedoch den Mitgliedstaaten überlassen. All diese 
Maßnahmen werden mithilfe eines bestimmten Sprachgebrauchs und bestimmter Argumentationsmuster präsentiert, die sich der Kategorisierung und Hierarchisierung von MigrantInnen bedienen. Dies zeigt sich zum Beispiel durch die Differenzierung zwischen (erwünschter) Mobilität von EU-BürgerInnen und (unerwünschter) Migration von Drittstaatenangehörigen sowie durch die Hierarchisierung von MigrantInnen, die über Topoi wie ,Förderung der Einwanderung von Hochqualifizierten' und ,Bekämpfung illegaler Migration' zum Ausdruck kommt.

Der Wandel des Asylrechts durch seine teilweise Supranationalisierung korrespondiert mit veränderten Möglichkeiten der Legitimation, Gestaltung und Umsetzung von Politiken. Im Folgenden soll daher resümierend der Frage nachgegangen werden, welche diesbezüglichen Neuerungen durch die teilweise Supranationalisierung des Politikfeldes entstanden sind.

Aus Sicht der Mitgliedstaaten ging es bei den Anfängen der Vergemeinschaftung der Migrationspolitiken zunächst um die Durchsetzung eines vor allem ökonomisch motivierten Projekts - des gemeinsamen Binnenmarkts. Auf dem Weg zur Verwirklichung dieses Projekts hat sich gezeigt, dass dieser auch gemeinsame Regelungen in anderen Politikfeldern wie der Migrationspolitik erfordert. Denn durch die Abschaffung der Binnengrenzen, die für EG-BürgerInnen mit Reiseund Niederlassungsfreiheit einherging, wurde aus der Sicht der Mitgliedstaaten auch eine gemeinsame Regelung des Zugangs von Drittstaatenangehörigen zum und ihres Status auf dem EU-Territorium notwendig. Das ökonomische Interesse am gemeinsamen Binnenmarkt überwiegt demzufolge offenbar deutlich das Interesse an der gänzlich eigenständigen Festlegung von Einwanderungsbestimmungen. Allerdings kann die Europäisierung der Migrationspolitiken nicht nur als Nebenprodukt der Konstruktion des Binnenmarktes gesehen werden. Stattdessen ergeben sich durch die Übertragung des Politikfeldes auf EU-Ebene neue Möglichkeiten bei der Legitimation, der Formulierung und der Umsetzung der Politiken.

Hinsichtlich der Legitimationsbasis der asylpolitischen Europäisierung ist zunächst festzustellen, dass sich das AkteurInnenfeld in diesem Bereich nicht nur um die Akteurin ,EU' erweitert hat, sondern ein breites Spektrum an Gremien und Arbeitsgruppen entstanden ist, das auch (N)GOs, IOs und wissenschaftliche Think Tanks umfasst. Auch auf den Flüchtlingsbereich spezialisierte private Unternehmen wie die Betreiber von Flüchtlingsunterkünften oder private Wachdienste in Abschiebehaftanstalten bieten ihre Dienstleistungen zunehmend europaweit an. ${ }^{140}$ Damit bewegt sich die Migrationspolitik nicht nur ein Stück weit weg

\footnotetext{
140 Vgl. zum Beispiel das Unternehmen ,European Homecare', das in verschiedenen europäischen Staaten nach eigener Auskunft, soziale Dienstleistungen` im Flüchtlingsbereich anbietet, was unter anderem auch den Betrieb von Flüchtlingsunterkünften einschließlich Versorgung mit Lebensmitteln und ,Rückkehrberatung' umfasst (vgl. www.eu-homecare.com). In der größten Abschiebehaftanstalt der Bundesrepublik - in Büren - ist das Unternehmen mit der ,psychosozialen Betreuung der Häftlinge beauftragt.
} 
vom nationalstaatlichen Entscheidungsbereich, sondern wird auch in eine neue Form des Regierens - Governance - überführt.
„Im Gegensatz. zu ,Government' sei Politik im Rahmen der Governance ,dezentrali- siert;, auf ,multiplen Ebenen', in "Netzwerken', unter ,strategischer Partizipation der Zivilgesellschaft' und der starken Einbeziehung von ,Experten-Wissen' zu gestalten, wie auch im Weißbuch über ,Europäisches Regieren' der EU-Kommission von 2001 nach- zulesen ist." (Hess/ Karakayal 2007, S. 47)

Die Multiplizierung und Ausdifferenzierung der AkteurInnen ist aber nicht mit einem Plus an demokratischer Kontrolle verbunden. Im Gegenteil: Die Legitimationsbasis der EU-Asylpolitik ist schmal, denn sowohl der Entscheidungsmodus im Rat und die geringen Mitwirkungsmöglichkeiten anderer Organe wie der Kommission, des Parlaments und des EuGH bis 2004 als auch die geringe Beteiligung der EU-BürgerInnen an Wahlen zum Europäischen Parlament bedingen ein starkes Demokratiedefizit und eine geringe legitimatorische Absicherung der Politiken. Insbesondere das Europäische Parlament stand bei den meisten Entscheidungen in diesem Bereich bislang außen vor oder wurde selbst bei energischer Intervention, wie im Falle der Asylverfahrensrichtlinie, schlichtweg ignoriert.

Es zeigt sich, dass dies von den nationalstaatlichen Akteuren genutzt wird, um Themen, deren Aushandlung auf nationaler Ebene ein hohes Konflikt- und Widerstandspotential birgt, auf EU-Ebene zu regeln und so die Durchsetzung auf einzelstaatlicher Ebene schließlich mit Hinweis auf die zwingend umzusetzenden EU-Vorgaben zu erleichtern. So können die Innenminister der Mitgliedstaaten relativ unbehelligt Entscheidungen treffen und diese im eigenen Land wahlweise als „Brüsseler Oktroy“ (Bendel 2006, S. 31) oder - im Gegensatz dazu - als Ergebnis der eigenen Durchsetzungsstärke präsentieren. Sandra Lavenex (vgl. 2006) interpretiert die Internationalisierung ebenfalls als eine Strategie der für die Immigrationsregulierung zuständigen Minister, ihre Autonomie gegenüber politischen, normativen und institutionellen Zwängen des Policy-Making zu erhöhen. Diese Zwänge haben sich nach Ansicht von Lavenex im Laufe des Vergemeinschaftungsprozesses bereits von der nationalen auf die EU-Ebene verlagert, nun vollziehe sich eine Verschiebung der Immigrationskontrollpolitiken nach außen, in die EU-Außenbeziehungen ${ }^{141}$. Durch diese Verschiebung suchen die politischen Akteure den Gewinn, der aus der Europäisierung gezogen wird, zu maximieren und die Zwänge, die mit der zunehmenden Supranationalisierung verbunden sind, zu minimieren (vgl. ebd.).

Wie auch auf nationalstaatlicher Ebene sind zudem die von den Entscheidungen unmittelbar Betroffenen - die MigrantInnen - nicht in die Entscheidungsprozesse einbezogen. Dennoch beschränkt sich die Ausweitung und Europäisierung des AkteurInnenfeldes nicht auf die, die als BeraterInnen im Gesetzgebungspro-

141 Vgl. zu den außenpolitischen Maßnahmen S. $111 \mathrm{ff} .$. 
zess angehört werden - auch MigrantInnen und andere, auf Migrationen bezogene autonome Initiativen vernetzen sich zunehmend EU-weit (vgl. Schwenken 2006) über Internetseiten und Mailinglisten, organisieren länderübergreifende Treffen und versuchen ihre Forderungen durch Aktionen und Kampagnen einzubringen, die mehr und mehr auch die Herkunfts- und Transitländer der MigrantInnen einbeziehen.

Hinsichtlich der Politikformulierung bietet die Europäisierung ein Forum zum Austausch über migrationspolitische Regelungen und Praxen in den Mitgliedstaaten, die von den zuständigen Ministerien und Behörden als effektiv erachtet werden, sowie für die Vernetzung dieser Akteure. Welche Regelungen sich schließlich durchsetzen, wird durch die Machtverhältnisse in der EU bestimmt - das Einstimmigkeitsprinzip während der fünfjährigen Übergangsphase nach Inkrafttreten des Vertrages von Amsterdam führte immer wieder zu Blockaden einzelner Mitgliedstaaten, darunter auch Deutschland, die sich gegen einen Konsens in bestimmten Bereichen stellten. So stellt Petra Bendel (2006, S. 127) fest, dass „[p]olicy spillovers von einem Land zum nächsten [...] oftmals in der Bundesrepublik losgetreten [wurden], [...]. Zusehends restriktivere Regelungen wurden schließlich in EU-Recht gegossen." So spiegeln die den Richtlinien zugrunde liegenden Konzepte den Versuch der Mitgliedstaaten im geografischen Kern, Asylsuchende möglichst nicht bis auf ihr Territorium vordringen zu lassen. Die Einordnung in den Bereich ,Justiz und Inneres'statt bspw. unter ,Soziales', macht die starke Sicherheitsorientierung in diesem Politikbereich deutlich. Auch im Haager Programm wurde der Schwerpunkt nachdrücklich auf Sicherheit und Kontrolle gelegt. ${ }^{142}$ Anfänglich liberaler eingestellte Mitgliedstaaten wie Schweden wurden mit der Aussicht, Ausweichoption für all diejenigen zu werden, die anderswo keine Aufnahme finden, von der gemeinsamen Linie ,überzeugt'.

Neue Inhalte und Konzepte lassen sich hingegen weniger finden. Durch den besonderen Druck, der auf den neuen Mitgliedstaaten bei der Umsetzung der Vorgaben lastet und die neuen Herausforderungen, vor denen diese aufgrund der Zuständigkeitsregelungen stehen, ist ein Policy-Transfer von den alten Mitgliedstaaten zu den neuen zu erwarten und - wie das Beispiel Polen zeigen wird - teilweise auch schon zu beobachten. Einem , laisser faire', wie es den südeuropäischen Staaten vor deren EU-Beitritt und darüber hinaus vorgeworfen wurde, wird hier von Seiten der EU bereits relativ früh entgegengearbeitet. Rund um den Beitritt neuer Mitgliedstaaten lässt sich zudem gut beobachten, dass - wie bereits angesprochen - Austausch und Vernetzung auf EU-Ebene eine wichtige Rolle spielen.

\footnotetext{
142 Petra Bendel (2006, S. 128) geht davon aus, dass es sich dabei um eine Antwort auf die ,ängstlich eingestellte Öffentlichkeit" handelt. Der damit unterstellte ,direkte Draht' zwischen BürgerInnen und EntscheidungsträgerInnen, zumal auf EU-Ebene, ist jedoch fraglich. Zudem ist auch die Richtung der postulierten ,Ängstlichkeit' umstritten. Zu der Frage, ob es die Eliten oder die WählerInnen sind, die für restriktive Politiken mobilisieren vgl. für das Beispiel Großbritannien Statham (2003) und Fn. 128.
} 
Denn die Übernahme des Acquis communautaire, also der bereits bestehenden Regelungen durch die neuen Mitglieder, wird zum Beispiel flankiert durch die gezielte Einrichtung von Partnerschaften zwischen den Ausländerbehörden alter und neuer Mitgliedstaaten. Im Rahmen dieser Partnerschaften sollen die neuen Mitgliedstaaten bei der Etablierung ihrer Migrationsregime vom ,Know How der alten Mitglieder lernen.

Letzteres ist gleichzeitig ein Beispiel dafür, dass die Zusammenarbeit der Mitgliedstaaten auch eine Optimierung der Um- und Durchsetzung der Politiken durch verstärkte Kooperation auf operativer Ebene ermöglicht: Dies zeigt sich auch beim Aufbau der europäischen Grenzschutzagentur Frontex, bei der gemeinsamen Organisation von europaweiten Sammelabschiebungen oder beim Datenaustausch, der die Mehrfachstellung von Asylanträgen verhindern soll. Zudem sind die Staaten im EU-Verbund deutlich machtvoller, wenn es darum geht Druck auf die Nachbar- und Herkunftsstaaten auszuüben, um die Installierung von Asylregimen außerhalb der EU und die Vereinbarung von Rückübernahmeabkommen voranzubringen, die wiederum für die Umsetzung der Zuständigkeitsregelungen zentral sind.

Die - wenn auch eher zögerlich - zunehmende Konvergenz der Politiken auf EU-Ebene lässt jedoch nicht den Schluss zu, dies sei das Ende der nationalstaatlichen Regelungskompetenz in der Migrationspolitik. Stattdessen ergibt sich ein vielschichtiges Bild: Auf der einen Seite stehen die widerwillige Übertragung von Kompetenzen, der Versuch der einzelstaatlichen Einflussnahme auf die Politiken und die einzelstaatlich divergierende Umsetzung, die bisher eine umfassendere Supranationalisierung verhindern und die Rolle der Mitgliedstaaten als zentrale Akteure innerhalb der EU unterstrichen haben. Die Schwierigkeiten bei der Aushandlung der Richtlinien seit Amsterdam und ihre eher unscharfe Ausgestaltung mit großen Interpretations- und Umsetzungsspielräumen für die Mitgliedstaaten deuten darauf hin, dass die einzelnen Staaten recht erfolgreich versuchen, ihre jeweiligen Interessen und Konzepte auf EU-Ebene zu übertragen. Damit behalten sie weiterhin eine wichtige Rolle auch bei der gemeinschaftlichen migrationspolitischen Regulierung.

Andererseits bietet der Vergemeinschaftungsprozess den einzelstaatlichen AkteurInnen aber auch viele Vorteile: So kann die Inter- bzw. Supranationalisierung in diesem Politikbereich als Strategie begriffen werden, sowohl den nationalstaatlichen Zwängen für die Aushandlung und Umsetzung zu entkommen als auch die Migrations(kontroll)politiken durch Austausch und Zusammenarbeit effektiver zu gestalten.

Gleichwohl erhöht sich auf diese Weise der Aufwand für Absprachen und Koordination. Ein Beispiel für diese Problematik ist die Dublin-II-Verordnung. Wie oben bereits anhand der Zahlen dargestellt, wird das Ziel, die Staaten an den Außengrenzen für die Asylanträge von MigrantInnen, die auf dem Landweg eingereist sind, verantwortlich zu machen, nicht im angestrebten Maße erreicht. In der 
Bundesrepublik halten sich Anträge auf Rücküberstellungen an und von Deutschland die Waage. Lediglich die skandinavischen Staaten und Großbritannien ,überstellen' tatsächlich mehr Personen, als sie selbst wieder übernehmen müssen. Nur ein Teil der MigrantInnen wird allerdings tatsächlich zurückgeschoben. Diesem geringen Outcome stehen der aufwändige Aufbau des Eurodac-Systems und das umständliche Antragsverfahren auf Rückübernahme gegenüber. Die Neuerungen im Verfahren werden also selbst aus der Perspektive der Mitgliedstaaten bei der Betrachtung der tatsächlichen Ergebnisse - und vor allem mit Blick auf das Verhältnis zum Aufwand - relativiert. Dieses Missverhältnis von Kosten und Ergebnissen einiger Strategien wird aber offenbar aufgrund übergeordneter politischer Zielsetzungen vernachlässigt.

Hess und Tsianos (2007, S. 36) resümieren:

„Die EU-europäischen Migrationspolitiken haben mit einer Autonomie der Migration zu rechnen, die gelernt hat, mit ihnen umzugehen, ibnen auszuweichen und sie ins Leere laufen zu lassen. Deshalb spricht die EU auch nicht mehr die Sprache der Abschottung, sondern des Migrationsmanagements. Auch die Politik der Erweiterung und der Vorverlagerung der Grenzkontrollen lässt sich als eine Reaktion auf diese Selbstbehauptungskräfte der Migration lesen."

Doch für MigrantInnen bedeutet das ,Migrationsmanagement ${ }^{\star}$ auf EU-Ebene auch steigende Kosten für eine erfolgreiche Einreise und einen irregulären Aufenthalt. Zudem werden die Bestimmungen immer komplexer und die Verflechtungen unter den Mitgliedstaaten zunehmend undurchschaubarer, was Einreise und Aufenthalt ebenfalls erschwert. Auch wenn Regelungen wie die Dublin-IIVerordnung tatsächlich ein Beispiel für die Ineffizienz der EU-Politiken sind, sorgen sie andererseits in konkreten Fällen auch dafür, dass MigrantInnen hinund hergeschoben werden und in den Nachbarstaaten festsitzen.

Die Untersuchung der Vergemeinschaftungsprozesse auf EU-Ebene illustriert daher die in der Auseinandersetzung mit den theoretischen Konzepten vertretene These der Gleichzeitigkeit von Kontrollverlust und -gewinn auf Seiten der Mitgliedstaaten, die mit einem Verlust und Gewinn von Handlungsspielräumen auf Seiten der MigrantInnen korrespondiert. 



\section{Deutschland}

$\mathrm{Zu}$ den asylpolitischen Entwicklungen in der Bundesrepublik gab es vor allem Ende der 1980er bis Mitte der 1990er Jahre - parallel zur politischen Hochkonjunktur des Themas - eine Reihe von Veröffentlichungen, die den öffentlichen und politischen Diskurs, die rechtlichen Änderungen und die Auswirkungen auf Asylsuchende in den Blick nahmen. Seitdem gibt es - bis auf einige Ausnahmen ${ }^{143}$ - kaum systematische Analysen der fortlaufenden Entwicklungen in diesem Politikfeld. Die AsylbewerberInnen verschwinden zunehmend aus den Statistiken des Bundesamtes für Migration und Flüchtlinge und tauchen nun außerhalb behördlicher Register als irreguläre MigrantInnen auf. So hat sich der öffentliche Fokus und mit ihm die Forschung - hin zu anderen Themen verschoben: Die Anwerbung von Hochqualifizierten, Integrationschancen und -wille der bereits Eingewanderten sowie sicherheitspolitische Diskussionen und die ,illegale Migration dominieren nun öffentliche Debatten und wissenschaftliche Analysen. Zwar werden - ungeachtet der Zahl der AsylbewerberInnen - einige asylpolitische Argumentationsmuster im öffentlichen Diskurs immer wieder gern reaktiviert: So gibt es hier und da Schlagzeilen über ,Asylbetrüger` und werden Sommerlöcher mit asylpolitischen Vorschlägen ausgefüllt - von der Einrichtung von Flüchtlingslagern in Nordafrika bis hin zur Forderung nach der gänzlichen Abschaffung des

143 Vgl. mit je unterschiedlichen Schwerpunkten: Kühne/Rüßler 2000, Lavenex 2002 und Schuster 2003. 
Asylrechts. Aber aus wissenschaftlicher Sicht scheint das Thema nicht mehr ,en vogue'. Dies blendet jedoch die Bedeutung der asylpolitischen Entwicklungen der letzten 15 Jahre für die heutige Konstitution der einwanderungspolitischen Landschaft und der Migrationsprozesse aus. Eine Fortschreibung der Geschichte der bundesdeutschen Asylpolitik ist daher auch ein wichtiger Beitrag zum Verständnis des heutigen Migrationregimes.

Die Untersuchung der deutschen Asylpolitik wird im Folgenden zunächst eingebettet in die Migrationsprozesse und die migrationspolitischen Prämissen und Strategien, die für die Bundesrepublik prägend waren. Anschließend wird kurz das Feld der AkteurInnen skizziert, die die Asylpolitik in der BRD bestimmen. Auf dieser Grundlage werden Wandel und Kontinuität des deutschen Asylregimes anhand der zentralen Etappen der asylpolitischen Entwicklung in der Bundesrepublik untersucht: vom individuellen Grundrecht auf Asyl über seine Aushöhlung durch Verfahrensrestriktionen bis zur Verfassungsänderung und der Konsolidierung und Europäisierung der Restriktionen im Zuwanderungsgesetz. AnschlieBend wird die aktuelle Ausgestaltung des Flüchtlingsschutzes im Einzelnen dargestellt: Asylverfahren, soziale Rechte, Unterbringung in Lagern sowie Abschiebungen werden im Spannungsverhältnis von Recht und Praxis erläutert. Anhand der politischen Konjunkturen, die das Thema erlebt hat, und anhand der konkreten Gestaltung des Flüchtlingsschutzes werden danach die zentralen Kennzeichnen des bundesdeutschen Asylregimes herausgearbeitet. Schließlich werden die Faktoren, die den Wandel der Asylpolitik bestimmt haben, zusammenfassend diskutiert und analysiert.

Mit Blick auf die Literaturlage kann die Darstellung der Entwicklungen bis Mitte der 1990er Jahre relativ knapp und im Wesentlichen unter Rückgriff auf vorliegende Studien erfolgen. Die Phase danach sowie die aktuelle Gestaltung des Asylregimes werden ausführlicher und unter Hinzuziehung von Primärquellen dargestellt und analysiert.

\subsection{Migrationsprozesse}

Die asylpolitischen Entwicklungen und die Einwanderung von Asylsuchenden in die Bundesrepublik sind eingebettet in komplizierte Migrationsprozesse, die das Migrationsgeschehen in der Bundesrepublik bestimm(t)en. Jedoch begann die Migrationsgeschichte auf dem Territorium der heutigen Bundesrepublik nicht erst nach dem Zweiten Weltkrieg, wie viele Beiträge vermuten lassen, sondern ist bereits Jahrhunderte alt. ${ }^{144}$ Dabei spielten neben friedlichen Migrationsbewegungen „auch aggressive Grenzüberschreitungen, Flucht über Grenzen und die Ausgren-

\footnotetext{
144 Vgl. für einen Überblick seit der Frühen Neuzeit Bade/Oltmer 2007, seit dem 19. Jahrhundert Herbert 2003 und Oltmer 2003 sowie die Chronologie der Geschehnisse seit 1945 von Ohlinger/ DOMiT 2004.
} 
zung von Minderheiten innerhalb der Grenzen [...] sowie, nach der gewalttätigen Expansion der deutschen Grenzen im Zweiten Weltkrieg, auch in anderen europäischen Räumen“ (Bade/Oltmer 2007, S. 141) eine wichtige Rolle. Zudem „bewegten sich in der deutschen Geschichte aber nicht nur Menschen über Grenzen, sondern auch Grenzen über Menschen hinweg“" (ebd.).

Ein Blick in historische Studien zum Migrationsgeschehen im deutschsprachigen Raum ermöglicht auch für das Beispiel Deutschland von aktuellen politischen Konjunkturen des Themas Migration zu abstrahieren und gewissermaßen aus der Vogelperspektive zahlreiche aktuelle Diskussionen zu relativieren. Dabei lassen sich die folgenden Aspekte herausarbeiten: Zum einen haben Wanderungen auf dem Territorium der Bundesrepublik schon immer existiert - und zwar nicht nur als Einwanderung - sondern bis nach dem Zweiten Weltkrieg in weiten Teilen als Auswanderung, die - zum anderen - überwiegend ökonomische und soziale (sowie religiöse) Gründe hatte. Die heute im öffentlichen Diskurs als illegitim wahrgenommenen wirtschaftlichen Motive bildeten also für lange Zeit die wichtigste Grundlage der Auswanderung aus dem Territorium der heutigen Bundesrepublik. Darüber hinaus zeigt der weitergehende historische Rückblick auch frühe Versuche, Bevölkerungsbewegungen zu steuern, also zu fördern oder zu bremsen - sei es, um den herrschaftlichen Einfluss in einer bestimmten Region zu sichern, aus konfessionellen oder ökonomischen Erwägungen. Bei all dem illustriert das deutsche Länderbeispiel in historischer Perspektive - ebenso wie das polnische - die Konstruiertheit und damit die Wandelbarkeit von Grenzen und Zugehörigkeiten und ihre gewachsene Bedeutung seit der Entstehung von Nationalstaaten.

Vor diesem Hintergrund können schließlich die Entwicklungen seit dem Zweiten Weltkrieg betrachtet werden, die ihrerseits zur Einbettung der aktuellen asylpolitischen Ereignisse dienen sollen. Die Migrationsprozesse der Nachkriegszeit waren im Wesentlichen geprägt durch die Migrationen infolge des Zweiten Weltkrieges (vgl. Birsl 2005, S. 201f.). Hier ist zum einen die Rückkehr von Displaced Persons - vor allem ehemalige ZwangsarbeiterInnen und Überlebende der nationalsozialistischen Lager - zu nennen. Hinzu kommen Zwangsumsiedlungen infolge von Grenzverschiebungen und Ost-West-Fluchtbewegungen von Personen ,deutscher Abstammung (entweder Nachfahren früherer Ostsiedlungsbewegungen oder im Rahmen der ,NS-Volkstumspolitik‘ im Osten Angesiedelte). Die OstWest-Migration setzte sich auch nach der unmittelbaren Nachkriegszeit fort, als weiterhin so genannte AussiedlerInnen vor allem aus Polen und später auch aus den Nachfolgestaaten der Sowjetunion in die BRD einwanderten. Darüber hinaus fand bis zum Mauerbau eine Migrationsbewegung aus der Sowjetischen Besatzungszone (SBZ) in die westlichen Zonen statt (vgl. ebd., S. 202). Bis 1961 konnte der wachsende Arbeitskräftebedarf in der BRD noch durch die Einwandernden aus der DDR gedeckt werden. Danach begann die Bundesrepublik verstärkt mit der Anwerbung von Arbeitskräften aus Südeuropa. Der erste Anwerbevertrag wurde bereits 1955 mit Italien abgeschlossen, es folgten Griechenland und Spa- 
nien (1960), die Türkei (1961), Marokko (1963), Portugal (1964), Tunesien (1965) und Jugoslawien (1968) (vgl. ebd., S. 203f.). Mit dem Anwerbestopp 1973 veränderten sich die Einwanderungschancen und -prozesse grundlegend. Zwar war das ,Rotationsprinzip', wonach die so genannten ,GastarbeiterInnen' nur für einen begrenzten Zeitraum einreisen, in der Bundesrepublik arbeiten und anschließend durch neue Arbeitskräfte ersetzt werden sollten, ohnehin nicht vollständig umgesetzt worden. Doch viele derjenigen, die zwischen Herkunftsland und Bundesrepublik ,pendelten', entschieden sich nun, da durch den Anwerbestopp der Rückweg in die Bundesrepublik versperrt war ${ }^{145}$, zu bleiben und holten nach einiger Zeit auch ihre Familien nach. „Aus ,Gastarbeitern“ mit Daueraufenthalt wurden faktische Einwanderer." (Bade/Oltmer 2007, S. 160) Mit dem Anwerbestopp konnten Arbeitskräfte nicht mehr regulär in die BRD einwandern. Ein wichtiges ,gate of entry" war versperrt worden (Birsl 2005, S. 204).

Von nun an blieben für Einwanderungswillige neben dem Nachweis der deutschen ,Abstammung ${ }^{6}$ und der Anerkennung als AussiedlerInnen nur noch der Familiennachzug und das Recht auf Asyl als Einwanderungsoptionen. Die letzteren beiden sind nicht aus migrationspolitischen Steuerungsversuchen entstanden, sondern ergeben sich aus völkerrechtlichen Verpflichtungen und im Grundgesetz verankerten Rechten. Nichtsdestotrotz wurde seither auch versucht, den Zugang durch diese beiden Einwanderungsoptionen zu erschweren. Beim Familiennachzug wurde ein sehr enger Familienbegriff zugrunde gelegt, der den Familienvorstellungen in den meisten der Herkunftsländer zuwiderlief. Inzwischen gibt es zahlreiche weitere Restriktionen beim Nachzug von Familienangehörigen, die unter anderem eine Herabsetzung des Nachzugsalters von Kindern, Nachweise über zuvor erworbene Deutschkenntnisse der Nachziehenden sowie über ausreichend Wohnraum und Einkommen zur Versorgung der Angehörigen unabhängig von Sozialleistungen betreffen. Darüber hinaus gibt es verschiedene Bestrebungen, so genannte ,Scheinehen ${ }^{6}$ und inzwischen auch ,Scheinvaterschaften ${ }^{146}$ zu verhindern. Dabei geraten binationale Partnerschaften, in denen ein/e PartnerIn nur über einen prekären Aufenthaltsstatus verfügt, mehr und mehr unter den Generalverdacht, allein aus Gründen der Aufenthaltsverfestigung zu heiraten und Kinder anzuerkennen.

Auch das Recht auf Asyl gewann nach dem Ende der Arbeitskräfteanwerbung als Einreisemöglichkeit an Bedeutung und wurde mit zahlreichen Einschränkungen belegt, wie das folgende Kapitel zeigen wird. Einen bedeutenden Einschnitt für die Wanderungsprozesse stellte das Ende des Kalten Krieges dar:

\footnotetext{
145 Dies galt nicht für alle Herkunftsländer gleichermaßen. Sofern es sich um EG-Mitglieder handelte, galt das Wiedereinreiseverbot für Arbeitskräfte aus diesen Ländern nicht.

${ }^{146}$ Vgl. zum Beispiel Frankfurter Allgemeine Zeitung vom 29.07.2006: „Scheinvaterschaft. Schwerhörige Gesetzgeber“. Ende 2007 hat der Bundestag ein Gesetz verabschiedet, dass es Behörden erlaubt, die Vaterschaftsanerkennung für ein Kind anzufechten.
} 
„Mit der Öffnung des ,Eisernen Vorhangs", dem Wandel der politischen Systeme in den ehemaligen Staaten des Ostblocks und dem Ende der DDR 1989/90 wandelten sich die Migrationsmuster in Europa und in Deutschland. Das vereinigte Deutschland wurde erneut zum Ziel und zur Drehscheibe der Ost-West-Migration. Das zeigte sich vor allem bei der Zuwanderung von Asylsuchenden, Aussiedlern und jüdischen Kontingentflüchtlingen. "(Bade/Oltmer 2007, S. 163)

Diese Entwicklungen - die weitgehende Versperrung der Möglichkeit, als Arbeitskraft einzuwandern sowie die Verschärfungen beim Familiennachzug und im Asylrecht - brachten ein neues Phänomen hervor: die irreguläre Einwanderung bzw. den irregulären Aufenthalt. Dass sich MigrantInnen, auch wenn sie nicht die geforderten Voraussetzungen für eine Einreise erfüllen, über Kontrollen und Verbote hinwegsetzen und dennoch einreisen und sich irregulär in Deutschland aufhalten, zeigt, dass migrationspolitische Kontroll- und Steuerungsversuche teilweise ins Leere laufen. Sie sind deshalb aber nicht gleich dysfunktional: Durch ihre Entrechtung nehmen Illegalisierte die untersten Ränge auf dem Arbeitsmarkt ein und bedienen damit die große Nachfrage nach billigen und hochflexiblen Arbeitskräften, die faktisch keine Ansprüche an Arbeitgeber und Sozialleistungssystem haben. Nachdem also die GastarbeiterInnen von den Asylsuchenden und nachziehenden Familienangehörigen als zentrale Einwandererkategorie abgelöst wurden, treten an die Stelle der Asylsuchenden nun irreguläre MigrantInnen. Dabei handelt es sich weiterhin um Menschen, die mit einem komplexen Hintergrund an Motiven, Reisewegen und Plänen ihren Weg in die Bundesrepublik finden, allein ihre politische bzw. verwaltungstechnische Benennung und damit auch ihr Status - also ihre Chancen, ihren Aufenthalt zu legalisieren und Rechte in Anspruch zu nehmen haben sich gewandelt.

In der öffentlichen Auseinandersetzung wird vor allem die Einwanderung in die Bundesrepublik thematisiert. Aber mit Blick auf das hier ebenfalls untersuchte Beispiel Polen, das in erster Linie als Auswanderungsland bekannt ist, stellt sich die Frage, inwieweit Auswanderung auch aus Deutschland stattfindet. Laut Erhebungen des Statistischen Bundesamtes vollziehen sich auch heute noch Auswanderungsprozesse in nennenswertem Ausmaß. So ist die Zahl der Auswandernden in den letzten gut zehn Jahren relativ konstant geblieben - zwischen rund 600.000 und 760.000 Menschen haben in diesem Zeitraum jährlich das Land verlassen (vgl. Tabelle 1 im Anhang). Allein das Verhältnis zur Zahl der ,legalen‘ EinwanderInnen hat sich in den vergangenen Jahren gewandelt: Die Zahl der Einwandernden ist seit der zweiten Hälfte der 1990er Jahre zurückgegangen (vgl. ebd.). Damit hat sich auch der Saldo aus Zu- und Fortgezogenen verkleinert: Er lag im Jahr 2006 bei einem Rekordtief von 22.791 (vgl. ebd.). Eine genaue Analyse der Daten unter Berücksichtigung der zugrunde gelegten Definition von Ein- und Auswanderung sowie der Wanderungsmotive und -ziele, die sich hinter den Zahlen verstecken, kann hier nicht geleistet werden. Es soll lediglich illustriert werden, dass die Migra- 
tionsprozesse in der Bundesrepublik Deutschland nicht nur durch Einwanderung, sondern auch heute noch durch Auswanderung geprägt sind.

\subsection{Migrationspolitik in der BRD}

Der vorangegangene Abschnitt hat bereits einige Strategien gezeigt, mit denen die Bundesrepublik versucht, Migrationsprozesse zu steuern. Im Gegensatz zum weiter unten besprochenen polnischen Beispiel besteht daher kein Zweifel an der Existenz einer deutschen Migrationspolitik - auch wenn sie, angesichts des lange verweigerten Bekenntnisses, ein Einwanderungsland zu sein, nicht als kohärente Strategie bezeichnet werden und vor allem keine integrationspolitischen Maßnahmen zum Abbau von (institutioneller) Diskriminierung aufweisen kann. Begreift man Einwanderungspolitik jedoch als ,alle politischen Maßnahmen [...], die explizit auf die geografische Mobilität von Menschen zielen“ (Düvell 2006, S. 113), so kann eine solche für Deutschland bereits lange vor 1949 konstatiert werden. ${ }^{147}$

Bei der Untersuchung der Migrationspolitik in der Bundesrepublik wird mit Blick auf deren Entwicklung im Zeitverlauf häufig auf starke Kontinuitäten abgestellt. In der Tat lassen sich auch im historischen Rückblick bereits vor der Entstehung der Bundesrepublik bis heute ähnliche Konflikte und Strategien erkennen. Hier sind der Widerstreit zwischen wirtschaftlichen bzw. arbeitsmarktpolitischen Interessen an ausländischen Arbeitskräften auf der einen sowie nationale Bestrebungen zur Erhaltung einer vermeintlich ethnisch homogenen Gemeinschaft auf der anderen Seite zu nennen. Dieser Widerstreit spiegelt sich auch im öffentlichen Diskurs, der im Changieren zwischen der Stigmatisierung der Einwandernden und der Warnung vor drohender ,Überfremdung ${ }^{6}$ einerseits sowie der Betonung der Nützlichkeit der ausländischen Arbeitskräfte andererseits bereits bei der Diskussion um ausländische Arbeitskräfte im Deutschen Kaiserreich und in der Weimarer Republik dem heutigen nicht unähnlich war. Auch die Maßnahmen, die ergriffen wurden, um beide Belange in den Griff zu bekommen, ähneln sich im Zeitverlauf: So wurden Arbeitskräfte angeworben, eine dauerhafte Ansiedlung jedoch durch das Konzept der ,Karenzzeit ${ }^{\star}$ - ähnlich dem später eingesetzten Rotationsprinzip - zu verhindern versucht. Zudem wurde mithilfe der sich ausdifferenzierenden

\footnotetext{
147 Bade und Oltmer (2007, S. 169) legen offenbar einen engeren Begriff von Einwanderungspolitik zugrunde, da sie davon ausgehen, dass ein ,informelles Einwanderungsland“, wie es Deutschland darstellt, Einwanderern zwar „trotz seiner Selbstbeschreibung als Nichteinwanderungsland, möglicherweise fließende Übergänge von Arbeitswanderungen über Daueraufenthalte zu formeller Einwanderung bis hin zum Erwerb der Staatsangehörigkeit [bietet]. Es fehlen aber reguläre Einwanderungsgesetzgebung und Einwanderungspolitik, die für ein formelles Einwanderungsland charakteristisch sind“. Sie sehen den Übergang zum „formellen Einwanderungsland“ erst mit den einbürgerungsrechtlichen Änderungen des Ausländerrechts 1990, dem Wandel des Staatsangehörigkeitsrechts 2000 und dem Zuwanderungsgesetz von 2005 gegeben. Insofern stellen sie die Rolle des Staatsbürgerschaftsrechts in der Einwanderungspolitik besonders heraus.
} 
modernen Arbeitsverwaltung ein ,Legitimationszwang - als Vorläufer der heutigen Arbeitserlaubnis - und ein Inländervorrang in das Maßnahmenrepertoire aufgenommen. Doch trotz des Versuches der Erfassung durch Karteien und Register scheiterte man schon damals nicht selten mit der Vorstellung der Kontrollierbarkeit der Wandernden, denn diese versuchten mit vielerlei Mitteln, die Restriktionen zu unterlaufen. ${ }^{148}$

Karen Schönwälder (2006) plädiert dafür, im Hinblick auf die Migrationspolitik der Bundesrepublik die Kontinuitäten nicht überzubetonen. Auch wenn die Wandlungsprozesse „überlagert [sind] durch Kontinuitäten“, sollte man die Veränderungen „politische[r] Prioritäten und maßgebliche[r] Interventionsziele, zum Teil auch zentrale[r] Denkmuster" nicht verkennen, um dem Gegenstand gerecht zu werden (Schönwälder 2006, S. 10). Zwar sind Veränderungen meist nicht sehr tiefgreifend, insbesondere, wenn nicht nur der Wandel der Politikziele, sondern auch deren Umsetzung in den Blick genommen wird (vgl. Green 2006), dennoch können mit Schönwälder (2006, S. 10) im Hinblick auf die Migrationspolitik in der Bundesrepublik drei „umfassende Umorientierungen“ identifiziert werden: die Umsetzung von Menschenrechtsnormen nach 1945, der Versuch der Verhinderung von Einwanderung seit dem Anwerbestopp 1973 und die Anerkennung der Einwanderungsprozesse mit einem „Prioritätenwechsel hin zur Integration der Einwanderer“. Inwieweit diese ,Umorientierungen` ebenfalls für den Asylbereich konstatiert werden können, wird im vorliegenden Kapitel untersucht und schließlich im Abschnitt 5.5 Resümee der Einflussfaktoren diskutiert.

Die oben bereits angesprochenen migrationspolitischen Kontinuitäten, die diese Wandlungsprozesse überlagern, korrelieren mit zwei zentralen Prinzipien im Umgang der Bundesrepublik mit Einwandernden: Zum einen der Konstruktion des Staatsvolkes als nationale Gemeinschaft mit gemeinsamer Abstammung und dem sich daraus ableitenden ius sanguinis als bis zum Jahr 2000 vorherrschende Staatsbürgerschaftskonzeption, die davon ausging, dass man Deutsche/r nicht werden kann, sondern als solche/r geboren wird (vgl. Brubaker 1992). Damit eng verknüpft ist das zweite zentrale Prinzip im Umgang mit Einwanderungsprozessen: das Nicht-Bekenntnis zum Status als Einwanderungsland (vgl. auch Lavenex 2002). Aus dem Zusammenspiel dieser zwei Prinzipien mit der oben erwähnten Nachfrage nach ausländischen Arbeitskräften ergibt sich ein dritter einwanderungspolitischer Leitgedanke - die Beurteilung von Einwandernden nach Nützlichkeitskriterien. Heute zeigt sich dies insbesondere bei der Diskussion der Frage, was (hochqualifizierte) Einwanderer zur Positionierung Deutschlands im ,Standortwettbewerb، beitragen können. Diese Prämissen sind relativ beständig und werden inzwischen mehr oder weniger aufgeweicht (Nicht-Bekenntnis zum Einwanderungsland), fortgeschrieben (Vorstellung eines homogenen ,Volkes') oder

148 Die hier aufgezeigten Kontinuitäten können sehr schön anhand der ausführlichen Darstellung zur Geschichte der ,Ausländerpolitik` bei Herbert (2003) herausgearbeitet werden (vgl. außerdem Bade/Oltmer 2007, S. 149ff.). 
verfestigt (Nützlichkeitskriterien). Sie bilden den Hintergrund des politischen Prozesses und werden durch diesen immer wieder aktualisiert und lediglich kleineren Veränderungen unterzogen. Die Langlebigkeit dieser Prämissen ist unter anderem auf die mehr oder minder spezifische institutionelle Beschaffenheit des politischen Systems der Bundesrepublik zurückzuführen (vgl. auch Green 2006). Hier ringen Bund und Länder, die darin agierenden Parteien (als Fraktionen und in Koalitionen), die Judikative, eine verhältnismäßig dezentrale Verwaltung und außerparlamentarische AkteurInnen wie NGOs, Kirchen, Verbände, migrantische Initiativen und Unternehmen um Deutungs- und Gestaltungsmacht.

Einen Strang der Migrationspolitik bildet die Asylpolitik. Wie bereits zu Beginn dieses Kapitels angesprochen, besteht hinsichtlich der aktuellen asylpolitischen Entwicklungen in der Bundesrepublik zunehmend Forschungsbedarf. In den folgenden Abschnitten sollen die bestehenden Forschungslücken ein Stück weit geschlossen werden. Dafür wird zunächst ein kurzer Überblick über die AkteurInnen gegeben, die sich in diesem Feld bewegen, bevor die einzelnen Etappen der asylpolitischen Entwicklung in Deutschland bis heute dargestellt und analysiert werden.

\subsection{Kategorie Flucht und Asyl: AkteurInnen, Regulierungen und migrantische Strategien}

\subsubsection{AkteurInnen}

Das Asylregime der Bundesrepublik ist - wie oben bereits angesprochen - durch eine Vielzahl von AkteurInnen geprägt, die unterschiedlich stark institutionalisiert und mit unterschiedlich viel Deutungs-, Definitions- und Gestaltungsmacht ausgestattet sind. Im Gegensatz zum später dargestellten polnischen Beispiel findet sich hier sowohl eine starke Politisierung als auch eine hohe Ausdifferenzierung des Feldes. Zudem befassen sich viele AkteurInnen schon seit Jahrzehnten mit dem Thema Flucht und Asyl. Grob lassen sich staatliche AkteurInnen - auf nationaler und supranationaler (EU) Ebene - und die so genannten, gesellschaftlichen AkteurInnen' wie NGOs, (Wohlfahrts)Verbände, Kirchen, Gewerkschaften, MigrantInnenselbstorganisationen und UnterstützerInnengruppen sowie die Medien unterscheiden. Die staatlichen AkteurInnen können wiederum differenziert werden in die Parteien, die mit ihren Fraktionen in Bundestag und Bundesrat die Legislative bilden und im Bundestag und in den Landtagen meist in Koalitionen regieren. Gerade für das hier angesprochene Politikfeld sind die zuständigen Innenminister - sowohl auf Bundes- als auch auf Landesebene sowie in Form der Bundesinnenministerkonferenz - von herausragender Bedeutung (Ressortprinzip). Für den Migrationsbereich spielen auch die Gerichte eine wichtige Rolle - sowohl als Korrektiv für die Entscheidungen der Verwaltung (Verwaltungsgerichte), als 
auch mit Blick auf die Normenkontrolle (Bundesverfassungsgericht). Hinzu kommt das Bundesamt für Migration und Flüchtlinge. Es war zunächst unter dem Namen ,Bundesamt für die Anerkennung ausländischer Flüchtlinge` für die Durchführung der Asylverfahren zuständig. Mit dem Rückgang der AsylbewerberInnenzahlen wurde sein Aufgabenbereich ausgeweitet: Inzwischen begreift es sich als ,Kompetenzzentrum für Asyl, Migration und Integration'. Das heißt, es soll Integration einerseits und ,freiwillige Rückkehr ${ }^{6}$ andererseits fördern sowie als Think Tank Forschungsaufgaben im Bereich Einwanderung übernehmen.

In beiden Bereichen - Legislative und Judikative - wird die EU zu einer immer bedeutenderen Akteurin. Die dort zwischen den Innenministern der Mitgliedstaaten getroffenen EU-weit gültigen Vorgaben (Richtlinien und Verordnungen) müssen in einzelstaatliches Recht umgesetzt werden. Dabei ist die gemeinschaftliche Regelung des Asylbereichs - verglichen bspw. mit dem Zugang von Nicht-EUMigrantInnen zu den einzelstaatlichen Arbeitsmärkten - bereits relativ weit fortgeschritten. Nach einer Übergangsphase ist nun die Macht der einzelstaatlichen Innenminister auf EU-Ebene in diesem Politikfeld nicht mehr uneingeschränkt. Die Möglichkeiten einzelner Minister, bestimmte Vorhaben zu blockieren, wurden durch die Ersetzung des Einstimmigkeitsprinzips durch das Mitentscheidungsverfahren im Rat beschränkt. EU-Kommission und -Parlament kommen inzwischen zunehmend umfassendere Kompetenzen zu.

Das oben genannte Spektrum staatlicher AkteurInnen auf legislativer und judikativer Ebene muss ergänzt werden durch die Verwaltung selbst in Form von Ämtern und Behörden (Exekutive). Auf dieser Ebene wird ganz wesentlich bestimmt, welche Folgen die beschlossenen Politiken für die Betroffenen tatsächlich haben, ob etwa eine Arbeitserlaubnis erteilt, ein sicherer Aufenthaltsstatus verliehen oder eine Ehe als, echt ${ }^{\star}$ anerkannt wird. Dabei kann es zu sehr unterschiedlichen Bedingungen für Flüchtlinge in verschiedenen Bundesländern, aber auch Städten und Gemeinden kommen. Hier spielen die unterschiedlichen Länderregierungen ebenso eine Rolle wie der Ermessenspielraum der Behörden, dessen Nutzung auch von den politischen Verhältnissen vor Ort abhängig ist: Inwieweit bspw. das Sachleistungsprinzip durchgesetzt oder Bargeld ausgezahlt wird, hängt von den politischen Konstellationen in den Kommunen ab - und zwar sowohl von den parteipolitischen Kräfteverhältnissen als auch von der Aktivität und dem Einfluss von Initiativen und Unterstützungsstrukturen vor Ort. Gerade auf kommunaler Ebene können solche Initiativen durch die Mobilisierung von Protest relativ unmittelbar Druck auf die jeweiligen EntscheidungsträgerInnen ausüben. Konträr zu MigrantInnen- und Unterstützungsinitiativen ist hier allerdings auch an Nachbarschaftsinitiativen zu denken, die aus Gründen der Ablehnung gegen AsylbewerberInnen zum Beispiel gegen die Einrichtung von Gemeinschaftsunterkünften in ihrem Viertel aktiv werden.

Mit MigrantInnen- und Unterstützungsinitiativen sind bereits einige der AkteurInnen angesprochen, die zumeist unter den Oberbegriff ,gesellschaftliche 
AkteurInnen' subsumiert werden. Hier sind zunächst karitative Wohlfahrtsverbände zu nennen, die in der Flüchtlingsberatung aktiv sind und sich auch in die migrationspolitische Diskussion einbringen, wie der Caritas-Verband. ${ }^{149}$ Während die einen zum Beispiel die Unterbringung in Lagern kritisieren, sind andere - wie die Arbeiterwohlfahrt (AWO) - am Betrieb von Gemeinschaftsunterkünften beteiligt und haben hierin eine wichtige Einkommensquelle (vgl. Pieper 2008). Weiterhin sind die Ärztekammern zu nennen, die in jüngster Zeit immer wieder durch Beschlüsse des Deutschen Ärztetages gegen die Schlechterstellung von Flüchtlingen im Gesundheitssystem durch das Asylbewerberleistungsgesetz und gegen die den ÄrztInnen zugewiesene Rolle in der Einwanderungspolitik protestiert haben. ${ }^{150}$

Die Kirchen haben in der Bundesrepublik im Asylbereich vor allem mit dem Kirchenasyl, das bis heute in einigen Kirchen immer wieder gewährt wird, eine wichtige Rolle gespielt. Im Sinne christlicher Beistandspflicht sollen auf diesem Wege besondere Härten im Einzelfall verhindert werden. Auf diese Weise hat die Kirchenasylbewegung neben dem Einsatz für einzelne Flüchtlinge auch immer wieder viel Öffentlichkeit erreicht und ihren Einfluss in diesem Bereich geltend gemacht. Zudem bringen sich kirchliche AmtsträgerInnen aller Hierarchieebenen immer wieder in die Diskussion um die Bedingungen für Einwanderer in der Bundesrepublik ein - sei es im Zusammenhang mit Einzelfällen oder mit gestalterischen Absichten, wie bspw. die zahlreichen Beiträge von Kirchen und kirchlichen Wohlfahrtsverbänden mit Forderungen zur Verbesserung der Situation von Personen ohne Aufenthaltsstatus zeigen (vgl. z.B. Die deutschen Bischöfe 2001).

$\mathrm{Zu}$ den flüchtlingspolitischen NGOs gehören Pro Asyl151, das im Zusammenhang mit den ersten Schritten zur Verschärfung des Asylrechts im Jahre 1986

\footnotetext{
149 Als Beispiel sei die unabhängige Rechts- und Gesundheitsberatung genannt, die die Caritas wöchentlich in einem Bus im Lager Bramsche/Niedersachsen als Gegenpol zur dort von den Behörden durchgeführten ,Rückkehrberatung' anbietet (vgl. Pieper 2008, S. 314f.).

${ }^{150}$ So wird die Abschaffung eines Passus im AufenthG gefordert, wonach öffentliche Stellen den Ausländerbehörden den irregulären Aufenthalt von Personen bekannt machen sollen. Hierbei ist umstritten, inwieweit zum Beispiel öffentliche Krankenhäuser zur Übermittlung von Daten illegalisierter PatientInnen verpflichtet sind. Außerdem fordern die ÄrztInnen die Sicherstellung, dass ihre Arbeit nicht unter den Straftatbestand der Beihilfe zur illegalen Einreise bzw. zum illegalen Aufenthalt ( $\ 96$ AufenthG) fällt. Des Weiteren lehnen sie die Beteiligung von ÄrztInnen bei der Feststellung des Alters von AusländerInnen ab (vgl. Deutscher Ärztetag 2007). Im Zusammenhang mit Gutachten im Vorfeld von Abschiebungen spricht sich der Ärztetag dagegen aus, die bloße ,Reisefähigkeit‘ zu beurteilen (vgl. Deutscher Ärztetag 2004).

151 Pro Asyl wird wiederum von Kirchen und Wohlfahrtsverbänden unterstützt sowie unter anderem von der bundesdeutschen Sektion von Amnesty International, dem Republikanischen Anwältinnen- und Anwälteverein, der Arbeitsgemeinschaft Ausländer- und Asylrecht im Deutschen Anwaltverein und vielen anderen. Im vorliegenden Kapitel werden verschiedene Texte von Pro Asyl als Quellen herangezogen. Zum einen kann auf diese Weise der rechtliche Rahmen mit Beispielen und Erkenntnissen aus der Flüchtlingsberatungspraxis angereichert werden. Zum anderen werden so verschiedene Kritiken am aktuellen Asylrecht aus flüchtlingspolitischer Perspektive in der Analyse berücksichtigt.
} 
entstanden ist sowie die über Pro Asyl vernetzten Flüchtlingsräte, die in den Bundesländern angesiedelt sind. Diese betreiben dort - wiederum im Austausch mit flüchtlingspolitischen Initiativen vor Ort - Öffentlichkeits- und Lobbyarbeit für Flüchtlinge und führen eigene Projekte zur Verbesserung der Situation von Flüchtlingen durch. Bisweilen in enger Vernetzung mit den Flüchtlingsräten gibt es eine ganze Reihe weniger institutionalisierte flüchtlingspolitische und antirassistische Initiativen, die - zum Teil als eingetragene Vereine, aber ohne feste MitarbeiterInnenstellen, zum Teil auch gänzlich ohne Institutionalisierung - vor Ort Flüchtlingspolitik machen. Das Spektrum reicht

- von der Nachbarschaftsinitiative, die sich spontan zusammenfindet, um gegen die Abschiebung langjähriger NachbarInnen oder SchulfreundInnen zu protestieren und

- Flüchtlingsgruppen, die gegen die Bedingungen in den Unterkünften revoltieren,

- über antirassistische Gruppen und Flüchtlingsselbstorganisationen, die versuchen, die konkrete Situation Einzelner nicht allein mit Rechtshilfe und Spendensammlungen, sondern mit weitergehenden politischen Forderungen und Kampagnen zu verbinden,

- bis hin zu bundesweiten Netzwerken, die die politischen Forderungen zu bündeln suchen.

Die Übergänge zwischen diesen Gruppierungen sind fließend - aus Nachbarschaftsinitiativen entwickeln sich mitunter dauerhafte Einrichtungen, aus einzelnen Unterstützungsaktionen werden längerfristige Kampagnen. Zwischen den Initiativen existieren mitunter personelle Überschneidungen und eine enge Zusammenarbeit. Viele Gruppen beteiligen sich an bundesweiten oder auch europaweiten Vernetzungsaktivitäten und organisieren bundesweite oder grenzüberschreitende Aktionstage und Kampagnen. Gleichzeitig gibt es Kooperationen mit den institutionalisierteren Einrichtungen wie Pro Asyl und den Flüchtlingsräten, die mit Informationen, Rechtshilfe, finanzieller Unterstützung und Öffentlichkeitsarbeit die Arbeit vor Ort unterstützen.

In den Aushandlungsprozessen zwischen diesen verschiedenen AkteurInnen spielen die Medien eine zentrale Rolle. Wie die Untersuchung zeigen wird, handelt es sich bei der Asylpolitik um ein stark politisiertes Feld, das zum Beispiel in Wahlkämpfen immer wieder eine bedeutende Rolle eingenommen hat. Sowohl für PolitikerInnen als auch für Verwaltungen und lokale Initiativen sind die Medien wichtige Akteure im Thematisierungs- und Aushandlungsprozess. Sie sind dabei allerdings keine ,neutralen Mittler', sondern sie können ein Thema verschweigen, Missstände aufdecken oder die Wirklichkeitsinterpretationen bestimmter AkteurInnen in den Vordergrund stellen. Sie können entscheidend dazu beitragen, ein Thema an die Öffentlichkeit zu bringen und so Druck auf EntscheidungsträgerInnen auszuüben oder aber deren Wirklichkeitsinterpretationen einer breiten Masse zugänglich zu machen. In diesem Bereich gab und gibt es viel Kritik an 
verschiedenen Medien, die die symbolische und emotionale Aufladung des Themas gern mit sprachlichen und visuellen Mitteln verstärken um ihre Auflagen bzw. Einschaltquoten zu steigern und dabei häufig nicht versuchen, der Komplexität des Themas gerecht zu werden. Statt Informationen nur zu, vermitteln' treten sie mit eigenen Deutungsangeboten bis hin zur eigenen Inszenierung von Geschehnissen auf (vgl. zum Beispiel Butterwegge/Hentges 2006; Jung/Wengeler/Böke 1997; Jäger/Link 1993). Nach diesem kurzen Überblick über Migrationsprozesse und Migrationspolitik in der Bundesrepublik sowie über die AkteurInnen, die das Politikfeld Asyl wesentlich mitbestimmen, werden nun die einzelnen Etappen der asylpolitischen Entwicklung in der Bundesrepublik seit ihrer Gründung dargestellt.

\subsubsection{Phasen des asylpolitischen Wandels}

Der Wandel der Asylpolitik in der Bundesrepublik wird im Folgenden in fünf Phasen unterteilt: Die erste Phase bildet die Verankerung des Grundrechts auf Asyl im Grundgesetz und seine relativ unangefochtene Stellung von 1949 bis 1972. Die zweite Phase (von 1973 bis 1984) ist durch die Einschränkung des Asylrechts mittels Restriktionen im Asylverfahren gekennzeichnet und geht über in die dritte Phase (1985 bis 1993), in der zunehmend eine Änderung des Asylgrundrechts diskutiert und schließlich 1992/93 auch umgesetzt wurde. Die vierte Phase (1994 bis 1999) steht im Zeichen der konkreten Folgen der Grundgesetzänderung und die fünfte Phase (2000 bis 2007) umfasst die aktuellen Entwicklungen der Asylpolitik im Rahmen der Debatten um das Zuwanderungsgesetz.

\section{9 - 1972: Grundrecht auf Asyl als Menschenrecht}

Bereits der Zeitraum zwischen dem Ersten und dem Zweiten Weltkrieg war von Flucht, Umsiedlungen und Vertreibungen geprägt. Ca. eine Million Menschen wanderten nach dem Ersten Weltkrieg bis Mitte der 1920er Jahre aus den nicht mehr zu Deutschland gehörenden Gebieten nach Deutschland ein (vgl. Bade/Oltmer 2007, S. 153). Durch die gewaltförmige Expansion des nationalsozialistischen Deutschlands im Zweiten Weltkrieg wurde eine Bevölkerungsbewegung in bis dahin ungekanntem Ausmaß ausgelöst. Dabei „war Deutschland Motor und Zentrum der europäischen Massenzwangswanderungen“ (Bade/Oltmer 2007, S. 155). Nach dem Krieg wurden die meisten der Displaced Persons (DPs) überwiegend frühere ZwangsarbeiterInnen und Überlebende der nationalsozialistischen Lager - mittels Rückkehrprogrammen der Alliierten in ihre Herkunftsländer gebracht. Der Status derjenigen, die nicht zurückkehren konnten, wurde mit dem „Gesetz über die Rechtsstellung heimatloser Ausländer“ am 25.04.1951 geregelt. Die öffentliche Wahrnehmung und der Umgang mit den DPs zeigten den von Vergessen und Verdrängen geprägten Umgang mit dem Nationalsozialismus in der Bundesrepublik. Der Begriff, heimatlose Ausländer' spiegelt nach 
Ansicht von Karen Schönwälder (1999, S. 132f.) die Ausblendung der Ursache für die Anwesenheit der DPs:

„Was der deutsche Staat und die deutsche Gesellschaft diesen Zwangsarbeitern und
Häftlingen angetan hatte, versuchte schon der 1951 eingefübrte Begriff des ,Heimatlosen
Ausländers" zu verschleiern; ,heimatlos' hieß es nun neutral, und nicht etwa verschleppt.
Gleichzeitig wurden die DPs bzw. „Heimatlosen Ausländer" recht pauschal mit Krimi-
nalität identifiziert."

Die Diskussion über die Verankerung eines Grundrechts auf Asyl in der bundesdeutschen Verfassung wurde vor dem Hintergrund der deutschen Geschichte und der persönlichen Erfahrungen einiger der Mütter und Väter der Verfassung geführt. ${ }^{152}$ Viele der vom NS-Regime Verfolgten und schließlich Deportierten hatten vergebens versucht, Zuflucht im Ausland zu finden (vgl. für das Beispiel USA Klein 2007). Einige der Mitglieder des Parlamentarischen Rates, der über die Ausgestaltung der Verfassung der Bundesrepublik beriet ${ }^{153}$, hatten die NS-Zeit überlebt, weil sie rechtzeitig das Land verlassen und anderswo Aufnahme gefunden hatten (vgl. Münch 1992, S. 17). Das Grundrecht auf Asyl wurde mit dem Wortlaut „Politisch Verfolgte genießen Asyl“ im Februar 1949 einstimmig im Parlamentarischen Rat angenommen. Diese als großzügig wahrgenommene Formulierung war unter den Mitgliedern des Rates zunächst nicht unumstritten. Um den Kreis der AntragstellerInnen zu begrenzen, wurden verschiedene Vorschläge unterbreitet, etwa Menschen mit bestimmter politischer Gesinnung auszuschlieBen ${ }^{154}$ oder das unbeschränkte Asylrecht nur Deutschen ${ }^{155}$ zu gewähren. Diese

${ }^{152}$ Das kommt zum Beispiel bei der Diskussion darüber zum Ausdruck, ob zum Recht auf Asyl auch ein Recht auf Arbeit hinzugefügt werden soll, wie Heinz Renner von der KPD vorschlug: „Politisch Verfolgte genießen Asylrecht einschließlich des Rechts auf Arbeit.“ (44. Sitzung des Parlamentarischen Rates am 19.01.1949, zit. n. Spaich 1982, S. 27) Er fügte hinzu: „Die Praxis, die wir politischen Emigrierten hinter uns haben, läßt es wünschenswert erscheinen, diesen Zusatz einzufügen.“ (ebd.) Friedrich Wilhelm Wagner (SPD) ergänzte: „Was er gesagt hat, hat seine Begründung in einer zum Teil sehr bitteren Erfahrung, die wir draußen gemacht haben. Wir waren sehr glücklich, daß wir draußen unterkamen und daß wir dadurch Hitler und seinen Henkersknechten entkommen konnten. Aber es war sehr bitter für die Tausenden, als sie draußen waren mit Asylrecht, aber ohne die Möglichkeit zu arbeiten und sich dadurch zu ernähren.“ (ebd., S. 30)

${ }^{153}$ Für die Gestaltung der Grundrechte war der Ausschuss für Grundsatzfragen innerhalb des Parlamentarischen Rates zuständig.

154 So zum Beispiel die Intervention von Dr. Fecht (CDU): ,„[... ] wo es sich um Leute handelt, die nach ihren Grundsätzen undemokratisch sind. Wir wären unter Umständen genötigt, in Massen Leute aufzunehmen, die mit unserer Auffassung und mit unserem Gesetz vollständig in Widerspruch stehen." (18. Sitzung des Parlamentarischen Rates am 4.12.1948, zit. n. Spaich 1982, S. 20) oder Dr. von Brentano (CDU): „Aber ich frage mich [...] ob es richtig und notwendig ist, daß wir das Asylrecht so weit ausdehnen, daß wir etwa in Deutschland zur Oase auch derjenigen politisch Verfolgten werden, die ihre Tätigkeit, die sie zum Abwandern aus ihrer Heimat veranlaßt hat, auch hier fortsetzen werden, nämlich den Kampf gegen die Demokratie." (44. Sitzung des Parlamentarischen Rates am 19.01.1949, zit. n. Spaich 1982, S.32)

155 So zum Beispiel Dr. von Brentano (CDU): „Das unbeschränkte Asylrecht soll den Deutschen gegeben werden, die sich wegen ihres Eintretens für die Demokratie auf dieses Asyl zurückziehen. 
Versuche schlugen jedoch fehl, und der „Parlamentarische Rat hat das Asylrecht allen politisch Verfolgten ohne Ansehen der Person, Herkunft oder politischen Überzeugung eingeräumt“ (Nuscheler 2004, S. 140). Durch die Konzeption als Teil der Grundrechte (Art. 1- 19) und damit als ein individuell einklagbares Recht unabhängig von der Staatsbürgerschaft der asylsuchenden Person, hatte das Asylrecht im Grundgesetz eine starke Stellung. Zudem war es als Teil der Grundrechte vor schneller einfachgesetzlicher Revision geschützt.

Allerdings blieb die Formulierung vage, da die Definition dessen, was unter politischer Verfolgung zu verstehen ist, nicht präzisiert wurde. Diese Präzisierung oblag den Gerichten, die Art. 16 Abs. 2 Satz 2 GG in verschiedenen Urteilen in den folgenden Jahren und Jahrzehnten mit Inhalt füllten. Die Interpretation von Bundesverwaltungs- und Bundesverfassungsgericht kann wie folgt zusammengefasst werden: Erstens bezog sich der Begriff der politischen Verfolgung nur auf Verfolgung durch staatliche Akteure (bis auf wenige Ausnahmen, bspw. wenn die Verfolgung dem Staat zugerechnet werden kann, weil dieser nicht willens oder in der Lage ist, Schutz zu bieten). Verfolgung durch nichtstaatliche Akteure wurde daher nicht erfasst. Zweitens muss die Verfolgung politisch motiviert sein (aus Gründen der ,Rasse‘, Religion, Nationalität oder Zugehörigkeit zu einer bestimmten sozialen Gruppe oder politischen Meinung). Drittens muss die Verfolgung einen bestimmten Grad an Intensität und Partikularität aufweisen. Generelle Menschenrechtsverletzungen begründen danach keinen Schutzanspruch - selbst wenn es sich um Folter handelt (vgl. Lavenex 2002, S. 41). ${ }^{156}$ Die höchstrichterliche Auslegung des Grundrechts, das eigentlich über das internationale Recht hinausgehen sollte, entsprach realiter einer im Vergleich mit Art. 1 der GFK engeren Auffassung (vgl. ebd.): Es erfordert erstens einen höheren Grad an Objektivität der Verfolgung und erkennt keine subjektive Angst vor Verfolgung an. Zweitens wird Verfolgung durch nichtstaatliche Akteure nicht anerkannt (Bürgerkrieg) und drittens soll auch die Darlegung von Nachfluchtgründen ${ }^{157}$ keine Verleihung des Flüchtlingsstatus begründen. Insofern handelte es sich zwar um „ein über das Völkerrecht und das Recht anderer Staaten hinausgehendes individuelles und einklagbares Grundrecht für Ausländer“ (Nuscheler 2004, S. 139), die zu erfüllenden

Ein Ausländer, der wegen entgegengesetzter Bestrebungen hierher nach Deutschland kommt, kann dieses unbeschränkte Asylrecht nicht in Anspruch nehmen." (44. Sitzung des Parlamentarischen Rates am 19.01.1949, zit. n. Spaich 1982, S. 29)

156 Eine Argumentation, die wiederum im eklatanten Widerspruch zum Umgang mit den so genannten ,Ostblockflüchtlingen“ stand: „Für das Gericht war nicht der bloße Tatbestand der Folter, sondern ihr ,politischer Zweck ${ }^{\varsigma}$ entscheidend. Während es in der Strafverfolgung von kommunistischen Regimen den ,politischen Zweck ${ }^{\varsigma}$ der Herrschaftssicherung erkannte, beurteilte es die Folter in der Türkei (einem NATO-Land) als ,übliches Mittel' zur Einhaltung der staatlichen Ordnung und als ,nicht asylrelevant', weil die Folter nicht auf die politische Gesinnung des/der Gefolterten abziele." (Nuscheler 2004, S. 142).

157 ,Nachfluchtgründe ' bezeichnen Schutzgründe, die durch exilpolitische Betätigung nach der Flucht entstanden sind. 
Bedingungen erfuhren jedoch in der Rechtsprechung in Vergleich zum Völkerrecht eine Engführung. Daher relativiert auch Nuscheler seine Einschätzung:

„Deutschland zeichnete sich zwar durch ein weltweit einmaliges subjektiv-einklagbares Asylrecht aus, das jedoch auf dem Verwaltungs- und Gerichtswege und im administrativen Vollzug seine Einmaligkeit verlor. "(Nuscheler 2004, S. 148)

In den ersten zwei Jahrzehnten nach seiner Entstehung fristete das Grundrecht auf Asyl ein Schattendasein. Die 1953 erlassene Asylverordnung beantwortete grundlegende Fragen zur Anerkennung und verfügte die Einrichtung einer zentralen Anlaufstelle für Asylsuchende - die Bundesdienststelle für die Anerkennung ausländischer Flüchtlinge. Doch in der Asylverordnung spielte Art. 16 Abs. 2 Satz 2 GG keine Rolle - die Verordnung bestimmte, dass als Flüchtling anerkannt wird, wer die Voraussetzungen nach Art. 1 der GFK erfüllt. Insofern bezog sich die Asylgewährung in den ersten Jahren vor allem auf die GFK und kaum auf das grundgesetzliche Recht auf Asyl. Die Anträge derer, die das Grundrecht auf Asyl in Anspruch nehmen wollten, wurden auf Basis der wieder eingesetzten Ausländerpolizeiverordnung von 1938 von Ausländerämtern der Länder entschieden, was eine länderspezifisch stark variierende Anerkennungspraxis zur Folge hatte (vgl. Dickel 2002, S. 281).

Erst das Ausländergesetz von 1965 enthielt Regelungen zum Asylverfahren, die eine Inanspruchnahme des Art. 16 Abs. 2 GG ermöglichten und bundesweit einheitlich regelten - es bezog sich sowohl auf das Grundgesetz als auch auf die GFK. Die Asylverordnung wurde damit abgelöst. Aus der Bundesdienststelle in Zirndorf wurde das Bundesamt für die Anerkennung ausländischer Flüchtlinge. Obwohl in dieser Phase die Anwerbung ausländischer Arbeitskräfte auf Hochtouren lief, waren weder das Ausländergesetz selbst noch die Debatten darum von einem rein liberalen, der Einwanderung zugewandten Geist geprägt. Karen Schönwälder (1999) arbeitet für die ausländerpolitischen Debatten und Entscheidungen der ersten zwei Jahrzehnte nach dem Nationalsozialismus sowohl Kontinuitäten zu Argumentation und Gesetzgebung der NS-Zeit als auch die bereits damals anzutreffende Popularität aktueller Argumentationsmuster heraus. Beispiel für die Kontinuität der Politik ist die 1951 erfolgte Wiedereinsetzung der Ausländerpolizeiverordnung (APVO) des Naziregimes von 1938, „lediglich offen rassistische Elemente galten als aufgehoben“ (Schönwälder 1999, S. 129). Ziel war es, AusländerInnen erfassen, kontrollieren und nötigenfalls ausweisen zu können. Gleichzeitig gaben die Debatten um das an die APVO anschließende Ausländergesetz einen Ausblick auf Argumentationsmuster, die sonst vor allem den Einwanderungsdiskursen späterer Jahrzehnte zugeordnet werden:

„die pauschale Abstempelung des ,Ausländers“ als Bedrohung, die Vorstellung von Zuwanderung als einem stetig anwachsenden Proze $\beta$, als illegal, als ,Unterwanderung' oder ,Überflutung" und die Selbstdarstellung als Opfer" (ebd., S. 132). 
Auch wenn es als Ausdruck der Weltoffenheit der Bundesrepublik gefeiert wurde, war es doch vor allem

„motiviert durch das Bestreben, den Behörden möglichst effektive Instrumente an die Hand zu geben, mit denen sie die Zusammensetzung der Bevölkerung kontrollieren und auch Liberalisierungsbestrebungen der Nachkriegszeit entgegenwirken konnten" (Schönwälder 2006, S. 11).

In Gesetzgebung und administrativer Praxis der Phase bis 1973 wurden vor allem Flüchtlinge aus den Staaten des damaligen Ostblocks relativ bereitwillig aufgenommen. Hier wirkten die Schemata des Kalten Krieges zusammen mit arbeitsmarktpolitischen Überlegungen. Laut eines Beschlusses der IMK von 1966 sollten daher auch Flüchtlinge aus Ostblockstaaten, deren Asylgesuch negativ beschieden wurde, nicht abgeschoben werden.

\section{3 - 1984: Einschränkungen auf dem Verfahrenswege}

In der zweiten Phase rückt das bis dahin relativ unumstrittene Asylrecht stärker in den Mittelpunkt der öffentlichen Debatten. Die Gründe dafür, die im Folgenden dargelegt werden, stehen im Zusammenhang mit dem Anwerbestopp 1973, der veränderten Herkunftsstruktur und der wachsenden Zahl der Flüchtlinge.

Bis 1973 waren es vor allem arbeitsmarktpolitische Ziele, die den Umgang mit MigrantInnen in der Bundesrepublik bestimmten. Der Anwerbestopp für ausländische Arbeitskräfte geht in seiner Wirkung jedoch weit über die Frage der Einwanderung und Rückkehr von Arbeitskräften hinaus. Er ist ein Einschnitt in das gesamte migrationspolitische Feld. Er verschloss eine zentrale Einwanderungsmöglichkeit, die bisher auch im eigentlichen Sinne politisch Verfolgte zum Beispiel aus Griechenland und der Türkei genutzt hatten, ohne sich auf das Asylrecht zu beziehen. Wer nun aus welchen Gründen auch immer in die Bundesrepublik einwandern wollte, konnte sich nur noch auf das Recht auf Familienzusammenführung oder das Recht auf Asyl berufen. So wurde relativ schnell die Vermutung laut, das Asylrecht werde zur Ausweichoption für diejenigen, die ansonsten über die Anwerbemaßnahmen Zugang gefunden hätten (vgl. Roos 1991, S. 50). ${ }^{158}$

Der Blick auf das Asylrecht wandelte sich zudem durch die Veränderung der Herkunftsstruktur der Asylsuchenden (vgl. Münch 1992, S. 63f.). Bis dahin war die Herkunftsstruktur der Flüchtlinge relativ konstant - die meisten kamen aus osteuropäischen Staaten. Wie Simone Wolken (1988) aufzeigt, hatte zwar die Zahl der Flüchtlinge im Vergleich zwischen den Jahren 1972 und 1973 - als das Thema Gegenstand öffentlicher Debatten wurde - nicht wesentlich zugenommen, aber

\footnotetext{
158 Tatsächlich wurde jedoch das zunächst verhängte Arbeitsverbot bereits 1975 aufgrund des weiterhin bestehenden Arbeitskräftebedarfs durch eine Weisung der Bundesanstalt für Arbeit wieder gelockert (vgl. Dickel 2002, S. 283f.; Münch 1992, S. 66ff.). Damit sollten gleichzeitig die Kommunen entlastet werden, die durch die eingeführte Umverteilung der Flüchtlinge auf die Länder aufgrund des Arbeitsverbots die soziale Unterstützung der Flüchtlinge übernehmen mussten.
} 
ihre Herkunftsstruktur hatte sich seit 1971 verändert: Der Anteil derer, die aus außereuropäischen Ländern kamen, nahm zu. Durch die veränderte Herkunftsstruktur wurden zwei Fundamente der bisherigen bundesrepublikanischen Flüchtlingspolitik erschüttert: Die bis dato bestehende Bereitschaft zur Flüchtlingsaufnahme fußte - im Hinblick auf die Herkunftssituation der Flüchtlinge - auf Eurozentrismus und Antikommunismus (vgl. Roos 1991, S. 49f.): Solange die Flüchtlinge aus (ost)europäischen Staaten kamen und als Flüchtlinge vor den ,realsozialistischen' Regimen - also als AntikommunistInnen - gesehen werden konnten, passten sie ins Weltbild des ,Kalten Krieges“ und untermauerten die Überlegenheit von Marktwirtschaft und Demokratie westlicher Prägung. Außerdem zeichneten sie sich durch vermeintliche ,kulturelle Nähe' aus. Die Entscheidung zur Aufnahme chilenischer Flüchtlinge nach dem Militärputsch von 1973, „die als links bis linksextrem eingestuft" (Wichert 1998, S. 109) wurden, passte bereits nicht mehr in dieses Muster. ${ }^{159}$

Der dritte Aspekt der Veränderung war die zunehmende Zahl der AsylbewerberInnen. Ab 1974 stieg auch die Zahl der Antragszahlen, wohingegen die Anerkennungszahlen bereits seit Anfang der 1970er Jahre zurückgingen. Auch wenn im Vergleich der Jahre 1973 und 1974 eine Verdopplung der Antragszahlen festgestellt werden kann, so ist der allgemeine Aufruhr angesichts der immer noch unspektakulären Zahl von knapp 9.500 AntragstellerInnen unverständlich. In einer ähnlichen Größenordnung bewegen sich derzeit die Flüchtlingszahlen in Polen, ohne dass dieses Thema größere öffentliche Aufmerksamkeit erfährt (vgl. dazu das folgende Kapitel 6. Polen).

Unter dem Einfluss dieser Entwicklungen - der arbeitsmarktpolitischen Unerwünschtheit weiterer Einwanderung, der veränderten Herkunftsstruktur bei gleichzeitig wachsenden Antragszahlen und sinkenden Anerkennungszahlen rückte das Thema Asyl in der zweiten Phase stärker auf die politische Agenda. Die beschriebenen Veränderungen wurden als Beleg für einen ,Missbrauch des Asylrechts $^{6}$ gewertet, der als zentraler Topos Eingang in den öffentlichen Diskurs fand und diesen über Jahrzehnte hinweg beherrschte ${ }^{160}$ : „Seit Mitte der siebziger Jahre hat die Asylpolitik ihren angestammten Platz in den Wahlkämpfen und Sommerlöchern“ (Roos 1991, S. 50). Hauptakteure der Politisierung des Themas waren die

159 Wichert (1998, S. 109f.) dokumentiert einige der sicherheitspolitischen Bedenken, die in zahlreichen Anfragen an die Bundesregierung im Zusammenhang mit der Aufnahme von ChilenInnen geäußert wurden.

160 Die Rede vom ,Missbrauch des Asylrechts' tauchte erstmals bereits in der vierten Legislaturperiode (1961-1965) in den Protokollen des Deutschen Bundestages auf und erfreute sich fortan wachsender Popularität. Vgl. zur Entwicklung der ,Missbrauchs'-Diskussion Münch 1992, S. 148ff., sowie zum ,Missbrauch' und weiteren Schlagwörtern und Argumentationsketten in der Asyldebatte Meyer 1997; zu verschiedenen Argumenten im Einwanderungsdiskurs und zur Popularität des ,Missbrauchs'-Topos seit den 1970ern und insbesondere Anfang der 1980er Jahre vgl. Wengeler 1997. Vermeintlich humanitär gewendet wurde (und wird) der ,Missbrauchs'-Topos, indem meist argumentiert wird, dass der ,Asylmissbrauch“ vor allem auf Kosten der, wirklich Verfolgten' gehe. 
CDU-regierten Länder, die damit in Opposition zur sozialliberalen Bundesregierung traten (vgl. Lavenex 2002, S. 45). Die zentralen Begründungen der Missbrauchsthese bezogen sich auf die bereits angesprochene veränderte Herkunftsstruktur, die ein Ausweichen von ArbeitsmigrantInnen auf das Asylrecht belegen sollte. ${ }^{161}$ Auch die bis heute immer wieder bemühte These, die niedrigen Anerkennungszahlen würden einen ,Missbrauch“ belegen, wurde in die Debatte eingeführt. ${ }^{162}$ Gleichzeitig veränderte sich der Sprachgebrauch: Wassermetaphorik ${ }^{163}$ zur Beschreibung der Entwicklung der AsylbewerberInnenzahlen wurde salonfähig (vgl. Münch 1992, S. 152). Wolken (1988, S. 65) beschreibt den Zeitraum von 1973 bis 1976 als „Vorbereitungs- und Thematisierungsphase“, die einer Phase zahlreicher Gesetzesnovellen vorausging, die einschneidende Veränderungen des Asylverfahrens zum Ziel hatten.

Ab 1977 wurde mit einer Fülle von Maßnahmen versucht, den zuvor diskursiv konstruierten ,Missbrauch des Asylrechts' zu beenden. ${ }^{164}$

161 Die veränderte Herkunftsstruktur lässt sich stattdessen mit einer Veränderung der politischen Situation in verschiedenen Regionen begründen (vgl. Dickel 2002, S. 282).

162 Davon abgesehen, dass die niedrigen Anerkennungsquoten stattdessen als Indiz dafür gedeutet werden können, dass das Asylrecht in der Bundesrepublik für den Umgang mit den Einwanderungswilligen ungeeignet ist, werden bei dem unterstellten Zusammenhang (niedrige Anerkennungsquote als Beleg für Asylmissbrauch) auch diverse Informationen vorenthalten. Dazu gehört etwa die Tatsache, dass die tatsächlichen Anerkennungszahlen höher liegen, weil zum einen die Gerichte häufig Entscheidungen des Bundesamtes revidieren (was ein Beleg für die mangelnde Qualität der Bundesamtsentscheidungen ist). Zum anderen werden häufig noch andere Aufenthaltstitel bis hin zur Duldung verliehen, weil eine Abschiebung - zum Beispiel aufgrund drohender Gefahr für Leib und Leben - nicht möglich ist. Letzteres verweist auf die enge Ausgestaltung und Auslegung des Flüchtlingsbegriffs und den politischen wie behördlichen Unwillen, Aufenthaltstitel mit einer Perspektive zu vergeben, worin sich zudem eine verfehlte Integrationspolitik spiegelt. Andererseits ist die tatsächliche Zahl der AntragstellerInnen geringer einzuschätzen, da das Bundesamt erst Mitte der 1990er Jahre begann, in der Statistik zwischen Erst- und Folgeanträgen zu unterscheiden; zudem gehen auch diejenigen, die das Verfahren gar nicht zu Ende führen, zunächst als AntragstellerInnen in die Statistik ein. Aktuell ergibt sich mit Inkrafttreten des Zuwanderungsgesetzes eine weitere Überschätzung der ohnehin nur noch geringen Antragszahlen: So wird für minderjährige Kinder von AsylbewerberInnen, von Amts wegen` ein Asylantrag gestellt. Seit dem 1.01.2005 bis zum Oktober 2006 betraf dies 27 Prozent der gestellten Anträge, wie auf Anfrage der Bundestagsabgeordneten Ulla Jelpke mitgeteilt wurde (vgl. Jelpke 2006). „Diese Anträge haben keine Chance auf Anerkennung, weil eine individuelle politische Verfolgung neu Geborener auszuschließen ist." (ebd.)

163 Der Einwanderungsdiskurs ist unter anderem durch den Gebrauch bestimmter Metaphern gekennzeichnet, mit Hilfe derer Einwanderungsfragen präsentiert werden (vgl. Böke 1997). Eine zentrale Metapher ist die Darstellung von Einwanderungsprozessen als, Wasserlauf', zum Beispiel als Asylantenstrom, -welle, -schwemme, -flut usw.

164 Vgl. zum Zusammenhang von Sprachgebrauch in der Asyldebatte und politischem Handeln Meyer 1997. Evelyn Meyer arbeitet unter anderem heraus, dass „,der Sprachgebrauch in der Asyldiskussion [...] das politische Handeln“ determiniert, denn „,[d]urch Sprache erzeugte Wirklichkeitsinterpretationen geben den Rahmen des Machbaren vor". Durch den spezifischen Sprachgebrauch in der Asyldebatte würden politische Handlungen „vorbereitet und herbeigeführt“, gleichzeitig legitimiere der Sprachgebrauch das politische Handeln (ebd., S. 161f.). 
„Hauptansatzpunkt für Neuregelungen ist dabei die Annahme, dass das lange Asylanerkennungsverfabren den Anreiz schaffe, aus asylfremden Gründen in die Bundesrepublik zu kommen" (Münch 1992, S. 155).

Daher richteten sich die Maßnahmen in den kommenden Jahren vor allem auf eine Verkürzung des Verfahrens und eine Verschlechterung der sozialen Bedingungen. Der erste Schritt wurde 1977 mit einer Änderung des Ausländergesetzes unternommen, die die Grenz- und Ausländerbehörden ermächtigte, vorab zu entscheiden, ob es sich bei einem Asylantrag um einen Missbrauchsversuch handelt. Sie sollten einen als unbegründet und daher als missbräuchlich qualifizierten Antrag nicht an das Bundesamt übersenden und stattdessen den Aufenthalt beenden. Das Bundesverfassungsgericht stufte die Regelung 1981 als verfassungswidrig ein. Nichtsdestotrotz war diese Gesetzesänderung Ausgangspunkt zahlreicher Verschärfungen. So wurde 1978 das erste Gesetz zur Beschleunigung des Asylverfahrens verabschiedet, das in Verbindung mit der zeitgleich verabschiedeten Novelle zur Verwaltungsgerichtsordnung Widerspruchs- und Berufungsmöglichkeiten reduzierte (vgl. Wolken 1988, S. 66). Im Vorfeld der Wahlen zum neunten Bundestag wurde das Thema Asyl schließlich durch den von der CDU/CSUOpposition im Bundestag eingebrachten Entwurf für ein Zweites Beschleunigungsgesetz zum Wahlkampfthema. Der nach Ablehnung des Oppositionsentwurfs eingebrachte Entwurf der Regierungskoalition wurde 1980 als Zweites Beschleunigungsgesetz verabschiedet und zielte ebenfalls auf eine Verkürzung des Asylverfahrens ${ }^{165}$ (vgl. Wolken 1988, S. 66; Nuscheler 2004, S. 144f.). Gleichzeitig wurde die Visapflicht für weitere Herkunftsländer von Flüchtlingen eingeführt, um den Zugang zur Bundesrepublik zu erschweren. ${ }^{166}$ Diese Maßnahmen wurden eingerahmt durch Bestimmungen zur Verschlechterung der sozialen Situation: Sozialhilfe sollte in Sachleistungen ausgezahlt und der Anspruch auf Kindergeld während des Asylverfahrens gestrichen werden. Während der ersten zwei Jahre des Verfahrens durften AsylbewerberInnen nicht arbeiten und sollten bevorzugt in so genannten Gemeinschaftsunterkünften untergebracht werden (vgl. Wolken 1988, S. 69).

Obwohl die Antragszahlen nach dem Beschleunigungsgesetz 1980 zurückgingen, brachten die CDU-regierten Länder einen neuen Gesetzentwurf mit zahlreichen weiteren Verschärfungen ein, der über den Vermittlungsausschuss schließlich zur Verabschiedung des Asylverfahrensgesetzes 1982 führte (vgl. Lavenex 2002, S. 46). ${ }^{167}$ Mit dem Asylverfahrensgesetz wurde wiederum eine Beschleunigung der Verwaltungs- und Gerichtsverfahren angestrebt sowie die Einschrän-

\footnotetext{
165 Dies sollte durch die Ersetzung des Anerkennungsausschusses beim Bundesamt durch EinzelentscheiderInnen erreicht werden. Darüber hinaus wurde die Residenzpflicht nach Ermessen der Ausländerbehörden eingeführt.

166 So wurden MigrantInnen aus Afghanistan, Äthiopien, Sri-Lanka, Iran, Türkei, Bangladesh und Indien visapflichtig.

167 Vgl. zu den Details des Gesetzgebungsverfahrens und der Regelungen auch Wolken 1988, S. 69ff.
} 
kung des Rechts auf Einreise bei ,offensichtlich unbegründeten` Anträgen; die bereits vorher eingeleitete Verschlechterung der sozialen Situation wurde gesetzlich festgeschrieben (vgl. Wolken 1988, S. 70f.; Dickel 2002, S. 285ff.). Die Komplexität des Asylverfahrens und somit auch die Unübersichtlichkeit für die AntragstellerInnen erhöhten sich durch die Fülle an Novellen und unterschiedlichen Verfahren entscheidend (vgl. Wolken 1988, S. 70f.).

Nach der Verabschiedung des Asylverfahrensgesetzes wurde es für kurze Zeit etwas ruhiger um das Thema Asyl. Als Gründe für diese „Dethematisierung“ nennt Wolken (1988, S. 71ff.) zum einen, dass die nun in der Regierungsverantwortung stehenden Unionsparteien das ,Asylproblem ' bis dahin stets als hausgemachtes Problem dargestellt hatten und ihre Vorschläge in das Asylverfahrensgesetz eingearbeitet worden waren. Zum anderen gingen die Antragszahlen zurück, während gleichzeitig der Anteil der Flüchtlinge aus Osteuropa wieder anstieg. Einen direkten Zusammenhang zwischen den eingeleiteten Maßnahmen und der Veränderung der Antragszahlen relativiert Wolken (1988, S. 73) jedoch durch einen Vergleich mit den USA, die ohne die entsprechenden Maßnahmen eine ähnliche Flüchtlingsstatistik aufwiesen. Darin zeige sich die große Bedeutung anderer Faktoren, wie die Lage in den Herkunftsregionen. Bis 1984 wurden zwar weitere legislative Maßnahmen ergriffen, sie standen aber im Zeichen der bereits zuvor eingeleiteten Politik und erregten kaum Aufsehen.

Aus der Vielfalt der hastig entwickelten Novellen und Nachbesserungen in dieser Phase kristallisieren sich im Wesentlichen drei Strategien heraus:

- Abschreckung durch Verschlechterung der sozialen Situation,

- Zugangserschwerung durch Visumszwang und

- Einschränkung der Widerspruchsmöglichkeiten durch Verkürzung des Rechtsweges.

Diese drei Hebel erweisen sich auch in Zukunft als zentral: An ihnen wurde auch in den kommenden Jahren immer wieder angesetzt, wenn es darum ging, die Zahl der AsylbewerberInnen öffentlichkeitswirksam zu senken.

Aufgrund der verschärften Bedingungen für MigrantInnen bzw. Flüchtlinge in der Bundesrepublik entwickelte sich in dieser Phase ein Netz von flüchtlingsunterstützenden Initiativen und Selbstorganisationen. Zum Teil aus dieser Arbeit heraus entstanden in dieser Phase auch die ersten Flüchtlingsräte in den Bundesländern, die die flüchtlingspolitischen Initiativen vernetzen, wie bspw. der Flüchtlingsrat Berlin 1981 und der Niedersächsische Flüchtlingsrat 1984. Zudem traten die Kirchen als AkteurInnen in diesem Feld verstärkt in Erscheinung: Sie nehmen Flüchtlinge in ihre Räume auf, um sie vor Abschiebung zu schützen. Die ersten Kirchenasyle wurden im Jahre 1983 eingerichtet. Dabei ging und geht es meist darum Zeit zu gewinnen, um alle Rechtsmittel für die Betroffenen ausschöpfen zu können. 


\section{5 - 1993: Grundgesetzänderung}

Bis Mitte der 1980er Jahre wurden die Lösungen zur Bekämpfung des ,Asylmissbrauchs' vor allem auf der Ebene des Asylverfahrens diskutiert. Das Grundrecht auf Asyl in der Verfassung war bis dato nur vereinzelt von einigen Kommunalund Landespolitikern infrage gestellt worden (vgl. Wolken 1988, S. 82). Dies sollte sich nun ändern: Das Thema Asyl wurde ab 1985 wieder prominenter auf die öffentliche Agenda gebracht - unter anderem im Berliner Wahlkampf. Um das ,Problem` zu beschreiben, konnte man sich auf bereits etablierte ,Missbrauchs'Argumente beziehen, neu war jedoch die immer häufiger propagierte Ursache des Problems: Die Generosität des individuellen Grundrechts auf Asyl im Grundgesetz.

Zunächst wurde diese Argumentationslinie jedoch nur von einzelnen CDUoder CSU-PolitikerInnen vertreten, eine Grundgesetzänderung schien in weiter Ferne. Daher konzentrierte man sich vorerst weiter auf Verschärfungen auf einfachgesetzlicher Ebene: Im Jahre 1986 wurde das „Gesetz zur Änderung asylverfahrensrechtlicher, arbeitserlaubnisrechtlicher und ausländerrechtlicher Vorschriften“ verabschiedet. Es enthielt ein fünfjähriges Arbeitsverbot für AsylbewerberInnen (ein Jahr für Flüchtlinge aus Osteuropa) und schloss selbstgeschaffene Nachfluchtgründe aus dem Begriff der politischen Verfolgung aus. Des Weiteren sollten Anträge von AsylbewerberInnen, die sich mehr als drei Monate in einem sicheren Drittland aufgehalten hatten, entweder (bei Einreise aus Nicht-EG-Ländern) als ,unbeachtlich' eingestuft oder ihnen sollte (bei Einreise aus EG-Staaten, der Schweiz, Österreich, Schweden und Norwegen) die Einreise verweigert werden. Hierbei handelte es sich also bereits um einen Vorläufer der späteren sicheren Dritt- und Herkunftsstaatenregelung. Anträge, in denen sich AsylbewerberInnen auf wirtschaftliche Not, Krieg oder allgemeine Notsituationen beriefen, sollten als ,offensichtlich unbegründet' klassifiziert werden. ${ }^{168}$ Lavenex (2002, S. 48) arbeitet mit Blick auf die Parlamentsdebatten zu diesem Gesetz zwei diskursive Änderungen heraus: Zum einen sei die Einreise von AsylbewerberInnen zunehmend als illegaler und krimineller Akt aufgefasst und zum anderen seien Bürgerkriegsflüchtlinge zunehmend mit, Wirtschaftsflüchtlingen' gleichgesetzt worden.

Durch Beschluss der Innenministerkonferenz (IMK) von 1985 wurden außerdem die Bedingungen für Osteuropaflüchtlinge erschwert. Der pauschale Abschiebungsschutz, der zuvor für sie galt, wurde abgeschafft, womit Osteuropaflüchtlinge in das Asylverfahren gedrängt wurden. Zudem sollte die damals unter Flüchtlingen verbreitete Einreise über Ost-Berlin eingeschränkt werden: Die Bundesregierung wirkte bei den DDR-Behörden darauf hin, dass diese nur noch Personen mit gültigem Anschlussvisum in die BRD weiterreisen ließen, woraufhin die Zahl der Einreisen über Ost-Berlin stark zurückging (vgl. Wolken 1988, S. 84). Des Weiteren wurden Fluggesellschaften verpflichtet, Passagiere nur mit den er-

168 Vgl. zu den Details der Aushandlung und des Inhalts Wolken 1988, S. 77ff. 
forderlichen Visa zu transportieren, andernfalls drohten Geldstrafen und der Rücktransport der jeweiligen Person auf Kosten der Fluggesellschaft. Im Jahre 1989 wurde die Visumspflicht auf weitere Herkunftsländer von Flüchtlingen ausgeweitet - auch für Minderjährige unter 16 Jahren. Im darauffolgenden Jahr legte die Bundesrepublik zudem bei der Ratifizierung der Kinderrechtskonvention einen Vorbehalt ein, wonach in der BRD nicht alle Kinderrechte auch für Flüchtlingskinder gelten sollten. Dieser Vorbehalt wurde erst 2010 zurückgenommen und hat(te) unter anderem zur Folge, dass jugendliche Flüchtlinge ab dem 16. Lebensjahr im Asylverfahren wie Erwachsende behandelt und z.B. in Abschiebehaft genommen werden können.

Mit der nicht abreißenden Kette an Novellen zum Thema Asyl seit 1977 war der Art. 16 Abs. 2 Satz 2 GG nach und nach ausgehöhlt worden: Der Zugang zum Asylverfahren wurde erschwert; ein erfolgreicher Ausgang des Verfahrens wurde angesichts der eingeschränkten Widerspruchsmöglichkeiten immer unwahrscheinlicher. Während vor allem SPD und FDP noch nach Möglichkeiten suchten, das Thema weiter auf diesem Wege zu bearbeiten, wurden die Forderungen der Unionsparteien nach einer Verfassungsänderung lauter. Hatte diese Forderung 1980 - bei höheren Antragszahlen als 1985 - noch eine untergeordnete Rolle gespielt, so wurde die Infragestellung des Asylgrundrechts ab Mitte der 1980er Jahre mehr und mehr hoffähig: Durch die dauerhafte Anprangerung des vermeintlichen ,Asylmissbrauchs ‘ durch, Wirtschaftsflüchtlinge' war der Boden für weitergehende Forderungen bereitet. Vor den Bundestagswahlen 1987 brachten CDU und CSU das Thema Asyl - trotz Protesten aus den anderen Parteien - als Wahlkampfthema auf die Agenda. Der damalige Innenminister Friedrich Zimmermann (CSU) proklamierte die Infragestellung des Asylgrundrechts als ,einzige Lösung' des ,Problems'.

Durch die Entwicklungen am Anfang der 1990er Jahre änderten sich die Rahmenbedingungen nachhaltig: Mit dem Fall des Eisernen Vorhangs wurden Befürchtungen laut, dass angesichts des Wegfalls der engmaschigen Ausreisekontrollen in den Ländern des ehemaligen Ostblocks die Zahl der MigrantInnen, die sich von dort in Richtung, Westen' aufmachen würden, rapide zunehmen würde. Tatsächlich stieg die Zahl der AsylbewerberInnen stark an - in erster Linie eine Folge des Bürgerkrieges in Jugoslawien.

„Die Krisenentwicklung in Ost-, Ostmittel- und Südosteuropa fübrte, zusammen mit den Abwehrmaßnahmen gegen Armutsflüchtlinge aus der, Dritten Welt; zu einer kompletten Umkehr der Relationen: 1986 waren noch rund 74,8 Prozent der Asylsuchenden aus der ,Dritten Welt' gekommen. 1993 stammten 72,1 Prozent aus Europa und vor allem aus Ost-, Ostmittel- und Südosteuropa. " (Bade/Oltmer 2007, S. 164$) 169$

169 Peter Kühne und Harald Rüßler (2000, S. 30f.) erklären die Zunahme der Flüchtlingszahlen ebenfalls mit der sich zuspitzenden Situation in vielen Herkunftsländern. Diese sei seit Ende der 1980er Jahre geprägt gewesen durch ,imperialen Zerfall und Re-Ethnisierung ehemals realsozialis- 
Dass es sich nun in der Mehrzahl wieder um europäische und insbesondere ,osteuropäische ${ }^{6}$ Flüchtlinge handelte, war jedoch nach dem Ende des ,Kalten Krieges' kein Argument mehr für eine Vorzugsbehandlung. Gleichzeitig nahm die Zahl der AussiedlerInnen aus der ehemaligen Sowjetunion zu (während die Zahl derer aus Polen zurückging). Hinzu kam eine größere Zahl von ÜbersiedlerInnen aus der ehemaligen DDR. Während die Unionsparteien bei der Aushandlung des Asylkompromisses auf hohe Kontingente für ,deutschstämmige‘ AussiedlerInnen beharrten und auch die übersiedelnden Ex-DDR-BürgerInnen kaum als Zielscheibe in der politischen Debatte geeignet waren, konzentrierte sich diese im Wesentlichen auf die AsylbewerberInnen. Die Rede vom ,Asylmissbrauch w wurde verknüpft mit Belastungsgrenzen (,Das Boot ist voll'), Kostenfaktoren und Flutmetaphern (vgl. Wichert 1998).

Vor dem Hintergrund dieser Entwicklungen müssen noch weitere Argumente und Faktoren berücksichtigt werden, um die tatsächliche Durchsetzung der lange anvisierten Verfassungsänderung erklären zu können. Dazu gehören zum einen die Verknüpfung des Themas mit der Europäischen Integration bzw. - in den Worten von Sandra Lavenex (2002) - die "Europeanization of the discourse arena", die zunehmenden Wahlerfolge rechter Parteien und die massiven öffentlichen Ausschreitungen gegen MigrantInnen, wie im Folgenden noch kurz erläutert wird.

Insbesondere durch die jeweiligen Bundesinnenminister, die das Amt in den Jahren vor und während der Grundgesetzänderung innehatten - Friedrich Zimmermann (CSU), Wolfgang Schäuble (CDU) und Rudolf Seiters (CDU) -, wurde die Änderung des Artikels 16 GG zunehmend in einen Kontext mit der Europäischen Integration gestellt. Diese Diskussion erreichte ihren Höhepunkt rund um die Ratifizierung des Schengener Abkommens: Vor allem aus den Reihen der Unionsparteien wurde argumentiert, dass man mit den vermeintlich höheren Standards in der Bundesrepublik in einem Europa ohne Binnengrenzen, wie es das Schengener Abkommen vorsah, zum ,Reserveasylland' werden würde. ${ }^{170}$ Ar-

tischer Gesellschaften Südost- und Osteuropas“ und den daraus folgenden Kriegen und Bürgerkriegen sowie durch die „sozialen und politischen Krisenherde an der inneren und äußeren Peripherie Europas: Autonomiebestrebungen der Kurden in der Türkei, aber auch im Irak, und der Krieg, den die Regierung der Türkei gegen die kurdische Guerilla und deren soziales Umfeld führt; sodann die Zerklüftung der Gesellschaften Afghanistans, des Libanon und Algeriens mit der Folge eines Zerfalls staatlicher Autorität und sowohl staatlichen sowie nicht-staatlichen Terrors." Darüber hinaus nennen sie „politische Verfolgung und schwere Menschenrechtsverletzungen in weiteren, mehr als hundert Staaten der Welt“ (ebd., S. 31), darunter Iran und mehrere afrikanische Staaten, als Ursachen für Fluchtmigration.

170 „Wir wollen mit unserer Regelung, mit der wir uns anpassen an das Niveau der Schutzgewähr aller anderen zivilisierten Staaten, insbesondere der europäischen Staaten, ja nichts anderes als eine faire Lastenteilung in Europa erreichen, die wir aber erst erreichen können, wenn wir eben nicht mehr Schutz gewähren als alle anderen." (Schäuble in der Debatte des Bundestages am 26.05.1993, zit. n. Das Parlament vom 11.06.1993) „Wenn nur ein einziges Land, die Bundesrepublik Deutschland, in seiner verfassungsrechtlichen Schutzgewähr über die Schutzgewähr der Genfer Konvention hinausgeht - es gibt keine zweite Verfassung auf dieser Erde, die dies tut -, dann braucht man 
gumentiert wurde, die Bundesrepublik könne aufgrund des in ihrer Verfassung verankerten individuellen Grundrechts auf Asyl nicht von den Zuständigkeitsregelungen des Schengener Durchführungsübereinkommens (SDÜ) Gebrauch machen. Die CDU/CSU-Parlamentsfraktion machte die Ratifizierung des SDÜ von einer Änderung des Asylgrundrechts abhängig (vgl. Lavenex 2002, S. 156). Dieses Argument des über die Maßen großzügigen individuellen Grundrechts auf Asyl stellt Bernhard Santel (1995, S. 109) infrage:
„Ob allerdings, wie in der Bundesrepublike vor der Änderung des Grundgesetzes immer wieder behauptet, die besondere deutsche Verfassungslage für die überdurchschnittlich ho- be Zuwanderung verantwortlich war, erscheint zweifelhaft. Zwar garantierte die Bundes- republik bis zur umfassenden Neuordnung des Asylrechts zum 1.7. 1993 als einziger Staat der Welt politisch verfolgten Ausländern ein subjektiv-öffentliches Recht auf Asyl. Obwohl damit die rechtsnormative Grundlage in Deutschland vom Standard der übrigen Aufnahmeländer, die sich stärker an der Genfer Flüchtlingskonvention orientierten, ab- wich, bedeutete dies keineswegs, dass auch die deutsche Asylpraxis der anderer europäi- scher Staaten überlegen war."

Als weiteres Argument für eine Verfassungsänderung wurden die Wahlerfolge rechter Parteien, wie der Republikaner 1989 in Berlin (7,5 Prozent), ins Feld geführt: „Die Abwehr des Rechtsextremismus wurde schließlich zur zentralen Legitimationsbasis für die Abwehr von Fluchtmigranten“ (Kühne/Rüßler 2000, S. 36). ${ }^{171}$ Während die Dramatisierung des Themas und sein Einsatz zu Wahlkampfzwecken zunächst auch in den Reihen der CDU noch auf Widerspruch gestoßen waren, wurde die Grundgesetzänderung Ende der 1980er Jahre in den Unionsparteien zunehmend konsensfähig. Noch sperrten sich jedoch der Koalitionspartner (FDP) und die Opposition (SPD) gegen dieses Vorhaben.

In der Hochphase der öffentlichen Auseinandersetzung über das Thema kam es zu einer Vielzahl von zum Teil tödlichen (Brand-)Anschlägen auf AsylbewerberInnen in Sammelunterkünften, teilweise auch auf AussiedlerInnen und Wohnungen türkischer Familien. ${ }^{172}$ Wie die oben angesprochenen Wahlerfolge rechter

sich hinterher nicht zu wundern, wenn zwei Drittel aller Asylbewerber in Europa nach Deutschland kommen." (ebd.)

171 „In Teilen der politischen Klasse, in den Medien und anderen gesellschaftlichen Institutionen wollte und will man den Rechtsextremismus dadurch entschärfen, indem man Teile des Gedankenguts in sein eigenes Gedankengut übernimmt. Symptomatisch dazu ist die Äußerung des Innenministers, der eine ,durchrasste' Gesellschaft nicht zulassen will. Die Ergebnisse dieser Vorgänge sind fatal: Es findet eine Normalisierung des Rechtsextremismus statt.“ (Heitmeyer 1992, S. 6)

172 Zum Beispiel in Hoyerswerda (September 1991), Hünxe (Oktober 1991), Greifswald (November 1991), Mannheim (Mai 1992), Rostock (August 1992), Mölln (November 1992) und Solingen (Mai 1993). Dies ist nur eine kleine Auswahl der zahlreichen Übergriffe und Brandanschläge auf MigrantInnen zwischen 1990 und 1993. Allein im Jahr 1991 wurden gemäß Bundeskriminalamt 338 Brandanschläge gegen Unterkünfte von AusländerInnen - 247 in den alten und 91 in den neuen Ländern - und 291 Körperverletzungen gegen AusländerInnen aus rassistischen und rechtsextremistischen Motiven verübt (vgl. Frankfurter Allgemeine Zeitung vom 18.01.1992). 
Parteien wurden auch die Anschläge durch zweifelhafte Thesen zu Ursache und Wirkung für die Grundgesetzänderung instrumentalisiert. So seien die PolitikerInnen aufgrund der Eskalation unter Druck geraten ihre Handlungsfähigkeit zu beweisen. Die Antwort auf die Ausschreitungen wurde in der Reduktion der Einwandererzahlen gesucht, nicht etwa im Vorgehen gegen diejenigen, die die Anschläge verübt hatten oder gegen rassistische Tendenzen in der Gesellschaft. ${ }^{173}$ In dieser Sichtweise sind die Anschläge Ausdruck des Volkswillens, der ein Handeln der PolitikerInnen erzwang - dieses Handeln richtete sich jedoch weniger gegen die TäterInnen als vielmehr gegen ihre Opfer - die AsylbewerberInnen. Deren gewachsene Zahl und mangelnde Integration sei - so landläufige Interpretationen insbesondere auch von PolitikerInnen ${ }^{174}$ - Auslöser für die Aggressionen. Diese Sichtweise blendet aus, dass die Verfassungsänderung bereits seit 1985 Gegenstand einer öffentlichen Diskussion war, die soziale Probleme (Wohnungsnot, Arbeitslosigkeit) in einen Kontext mit der Anwesenheit von Flüchtlingen stellte. Das Anfang der 1990er Jahre Flüchtlingen gegenüber feindlich gesinnte gesellschaftliche Klima wurde stattdessen teilweise erst durch die von den Unionsparteien vor allem in Wahlkämpfen verwendeten Argumentationsmuster geschürt. Damit wurde an vorhandene rassistische Einstellungen in der Bevölkerung angeknüpft ${ }^{175}$ und gesellschaftliche Probleme auf diejenigen projiziert, die aufgrund mangelnder politischer Partizipations- und Repräsentationsrechte am politischen Willensbildungsprozess in seinen klassischen Formen nicht teilnehmen und sich entsprechend kaum zur Wehr setzen können. Die Struktur der vorgebrachten Argumentationsmuster konstruiert ein gemeinsames ,Eigenes', ein ,Wir', dem das ,Andere', ,Fremde ${ }^{6}$ als Bedrohung gegenübergestellt wird. So werden rassistische Eskalationen nicht durch gestiegene Zahlen von AsylbewerberInnen ausgelöst, sondern

\footnotetext{
173 Vgl. zum Beispiel Schwarz (2001, S. 239): „Trotz der heftigen [parteipolitischen] Auseinandersetzungen waren sich die Verhandlungspartner darin einig, daß sie gemeinsam eine mögliche Lösung schnell finden mußten, um die fremdenfeindlichen Ausschreitungen einzudämmen und das in der Bevölkerung vorhandene Gewaltpotential gegenüber Ausländern zu entschärfen.“ und: „Die zunehmende Unzufriedenheit in der Aufnahmegesellschaft machte sich in fremdenfeindlich motivierten Straf- und Gewalttaten sowie den Wahlerfolgen rechtsextremistischer Parteien bei Landtagswahlen bemerkbar. Infolgedessen gerieten die Bundestagsparteien unter politischen Handlungsdruck, der deutschen Bevölkerung eine Lösung zur Senkung der Zuwanderungszahlen zu präsentieren.“ (Schwarz 2001, S. 248)

174 Dass die Flüchtlinge als Ursache für die Stimmung in der Bevölkerung und schließlich für die Übergriffe und Anschläge identifiziert wurden, zeigt Wichert (1998, S. 115f.) am Beispiel einiger Zitate von Unions- und SPD-PolitikerInnen. Besonders bezeichnend erscheint das von ihm angeführte Beispiel des Ministerpräsidenten Mecklenburg-Vorpommerns Bernd Seite und des Bundesinnenministers Rudolf Seiters, die nach den tagelang andauernden Angriffen gegen MigrantInnenUnterkünfte in Rostock-Lichtenhagen auf Pressekonferenzen vor Ort den ,Asylmissbrauch anprangerten und eine Verfassungsänderung forderten (vgl. Wichert 1998, 120f.).

175 Vgl. die Untersuchung von Jäger (1995), wonach ein Großteil der Bevölkerung in der Bundesrepublik in rassistische Diskurse verstrickt ist. Wichert (1998, S. 118) stellt deshalb fest: „Angesichts solcher Haltungen und Einstellungen in der Bevölkerung scheint die Verlockung für Politiker, auf dieser Klaviatur zu spielen, enorm groß.“
} 
durch deren Darstellung in der Öffentlichkeit als Ursache verschiedener gesellschaftlicher Probleme, die mehr oder weniger latent vorhandene rassistische Einstellungen aktiviert ${ }^{176}$ :

„Die ausfübrliche Darstellung des,Asylantenstroms' durch die Medien und seine öffentliche Interpretation durch Politiker war dazu geeignet, ausländerfeindliche Prädispositionen in der Bevölkerung zu verstärken. "(Münch 1992, S. 177f.)

Durch die Darstellung der legislativen Maßnahmen als Antwort auf die rassistische Eskalation werden die AttentäterInnen rehabilitiert: Ihre Forderungen werden als legitim und sogar politikleitend dargestellt; die Art und Weise, mit der diese Forderungen transportiert wurden sowie deren rassistische Inhalte, geraten in den Hintergrund. ${ }^{177}$ Nachdem AsylbewerberInnen seit Jahren in der öffentlichen Auseinandersetzung als ,Scheinasylanten“ und Belastung der Sozialsysteme präsentiert wurden, wurde schließlich die auch daraus resultierende Eskalation als Legitimation für die Einschränkung des Asylrechts verwendet:

,A vicious circle was created in which the political discussions of the crisis heightended tension on the streets, and violence on the streets ensured louder calls for 'sometbing to be done'."(Schuster 2003, S. 207)

Diese Entwicklungen und Argumentationen bildeten den Rahmen für die sich nun durchsetzende Grundgesetzänderung. Die CDU/CSU-Fraktion brachte 1991 einen Änderungsantrag zur Änderung des Grundgesetzes ein. Noch opponierten Koalitionspartner FDP und die Oppositionsfraktion der SPD gegen die Pläne. Die FDP schwenkte im Juni 1992 auf den Kurs von CDU/CSU ein - offiziell mit Blick auf die bevorstehende Harmonisierung des Asylrechts in Europa. Auch die SPD-Führung zog zunehmend eine Grundgesetzänderung in Erwägung, hatte dabei aber noch keine Unterstützung in der Parteibasis. Kurz nach den Ausschreitungen in Rostock-Lichtenhagen stimmte der SPD-Parteivorsitzende Björn Engholm in der Petersberger Erklärung vom August 1992 einer Verfassungsänderung zu. Auch hier wurde Bezug genommen auf die Implementierung des Europäischen Acquis - unter der Bedingung, dass für Bürgerkriegsflüchtlinge ein eigener Status geschaffen und die Möglichkeiten des Bundesamtes erweitert würden. Dieser als ,Petersburger Wende' bezeichnete Richtungswechsel der SPD verstärkte zwar die innerparteilichen Konflikte um diese Frage (der linke Parteiflügel, der ,Frankfurter Kreis‘, war gegen eine Grundgesetzänderung), bahnte aber dennoch den Weg zur Grundgesetzänderung mit der erforderlichen Zustimmung der SPD.

\footnotetext{
176 „Nicht die faktische Zuwanderung sondern deren öffentliche Darstellung und Wahrnehmung strukturieren den Spielraum auch zukünftiger Flüchtlingspolitik. Die ,Asylantenfrage' wurde zum Spielball parteipolitischer und wahlkampftaktischer Instrumentalisierungen bereits zu einer Zeit, als der Flüchtlingszugang aus heutiger Sicht lächerlich niedrig war.“ (Roos 1991, S. 48)

177 So postulierte Oskar Lafontaine (damals SPD), wer die Probleme der Integration der Ausländer übersehe, leiste „rechtsradikalen Stimmungen Vorschub“ und gefährde so das Asylrecht (zit. n. Frankfurter Rundschau vom 4.08.1990).
} 
Auf dem Sonderparteitag der SPD im November 1992 wurde die asylpolitische Wende der Partei gegen den Widerstand der Parteilinken formal abgesegnet. Am 6.12.1992 wurde schließlich der Asylkompromiss zwischen CDU, FDP und SPD ausgehandelt - er enthielt auch zahlreiche über den Asylbereich hinausreichende Maßnahmen, unter anderem zum Beispiel zur Aufnahme von AussiedlerInnen und zur Einbürgerung, weshalb er nach Ansicht von Klaus J. Bade und Jochen Oltmer (2007, S. 166) ,in Wirklichkeit ein umfassender Migrationskompromiß war". Begründet wurde der Kompromiss mit

- der Verhinderung des ,Asylmissbrauchs“ und dem Schutz tatsächlich politisch Verfolgter,

- der Verminderung von Ängsten in der Bevölkerung durch Zuwanderungssteuerung,

- dem Ziel der europäischen Zusammenarbeit im Asylbereich und

- der angestrebten Bekämpfung der Fluchtursachen.

Der von Rudolf Seiters Ende November 1992 vorgelegte Gesetzentwurf stellte jedoch eine verschärfte Version des ,Kompromisses` dar. Zahlreiche Forderungen, die die SPD gestellt hatte (zum Beispiel die Anerkennung von Vergewaltigung als Verfolgungsmerkmal, die Legalisierung der doppelten Staatsbürgerschaft und ein kommunales Wahlrecht für AusländerInnen), wurden nicht übernommen (vgl. Schwarze 2001, S. 238f.). Aufgrund von Protesten seitens der SPD wurde daher ein interfraktioneller Entwurf erarbeitet, der nach langwierigen Nachverhandlungen im Mai 1993 mit der erforderlichen Zweidrittelmehrheit im Bundestag angenommen wurde. Dabei stimmten die CDU/CSU einstimmig, die FDP ganz überwiegend (73 zu 6 Stimmen) und bei der SPD mehr als die Hälfte der Abgeordneten dafür. ${ }^{178}$ Bündnis 90/Die Grünen und PDS votierten einstimmig gegen die Änderung. ${ }^{179}$ Gleichzeitig wurde das Asylbewerberleistungsgesetz mit Zweidrittelmehrheit angenommen. Am Tag darauf stimmte der Bundesrat ebenfalls mit Zweidrittelmehrheit ab, woraufhin die Änderungen am 1. Juli 1993 in Kraft traten. Das Bundesverfassungsgericht erklärte die Verfassungsmäßigkeit der neuen Regelungen.

Der neue Art. 16 a GG lautete in Absatz 1 wie sein Vorgänger Art. 16 Abs. 2 Satz 2 GG a.F.: „Politisch Verfolgte genießen Asylrecht“. Doch in den Absätzen 2 bis 4 wurde dieses Recht deutlich eingeschränkt. ,Herzstück ' dieser Einschränkungen sind die sichere Dritt- (Art. 16 a Abs. 2) und die sichere Herkunftsstaatenrege-

\footnotetext{
$178 \mathrm{Zu}$ den Gründen für das Einlenken der SPD vgl. Schwarze (2001, S. 238): „Diese Zustimmung beweist, daß die SPD zu diesem Zeitpunkt mehrheitlich besonders daran interessiert war, der Bevölkerung ein gemeinsames Verhandlungsergebnis als politische Lösung präsentieren zu können und dadurch ihre politische Handlungsfähigkeit unter Beweis zu stellen. Wäre der ,Niklauskompromiß` von der SPD-Bundestagsfraktion nicht akzeptiert worden, hätte die Regierungskoalition die SPD für den weiteren Anstieg der Asylbewerberzahlen und der fremdenfeindlich motivierten Straf- und Gewalttaten verstärkt verantwortlich gemacht.“

179 Sehr ausführlich zu den Verhandlungen um den ,Asylkompromiss' und der Entwicklung der Positionen der einzelnen AkteurInnen zwischen 1989 und 1993 vgl. Schwarze (2001).
} 
lung (Art. 16 a Abs. 3). Danach kann sich auf dieses Recht nicht berufen, wer aus einem als ,sicher' deklarierten Herkunfts- oder über einen als ,sicher' deklarierten Drittstaat einreist. Als sicher gelten die Mitgliedstaaten der EG/EU und solche Staaten, die die GFK ratifiziert haben und anwenden. Wird dennoch ein Asylantrag gestellt, so gilt dieser als ,offensichtlich unbegründet' ${ }^{\varsigma}$ und eine Abschiebung kann ohne Rücksicht auf einen eingelegten Rechtsbehelf durchgeführt werden. Die Drittstaatenregelung hatte für die Bundesrepublik deshalb zentrale Bedeutung, weil sie alle angrenzenden Staaten zu ,sicheren Drittstaaten` erklärte und sich damit mit einem so genannten ,Cordon Sanitaire‘ umgab. Obwohl sie sich zunächst dagegen gesträubt hatte, stimmte die SPD im Rahmen des ,Asylkompromisses' zu, auch Polen und die übrigen östlichen Nachbarstaaten zu sicheren Drittstaaten zu erklären. Flankiert wurde die Regelung durch den Abschluss von Rückübernahmeabkommen mit den entsprechenden Staaten - denn nur auf der Basis solcher Abkommen kann eine Abschiebung in die jeweiligen Länder auch tatsächlich stattfinden. Für das hier ebenfalls betrachtete Länderbeispiel Polen bedeutete die Einordnung als sicherer Drittstaat und das 1993 mit Deutschland abgeschlossene Rückübernahmeabkommen ${ }^{180}$ den Beginn der Entwicklung zum Aufnahmeland von Asylsuchenden, wie sich auch anhand der seitdem wachsenden Zahl der Anträge in Polen und der Rücküberstellungen dokumentieren lässt. Polen selbst schloss daraufhin ebenfalls Rückübernahmeabkommen mit den Nachbarn ab.

Wie bereits im Vorfeld und seitdem immer wieder von KritikerInnen unterstrichen wurde, sorgen diese beiden Regelungen dafür, dass MigrantInnen nur dann noch auf Aufnahme ins Asylverfahren der Bundesrepublik hoffen können, wenn sie nicht auf dem Landweg, sondern auf direktem Wege - also in der Regel mit dem Flugzeug - in die Bundesrepublik einreisen. Dazu sind allerdings - aufgrund der Inverantwortungnahme der Beförderungsunternehmen - gültige Einreisepapiere (Visa) notwendig, deren Vergabe ebenfalls eingeschränkt wurde. Seit dem Inkrafttreten der Regelung sind die Behörden deshalb mit einem sich ausbreitenden Phänomen konfrontiert: MigrantInnen, die keine Papiere vorweisen können. Die Papiere werden oft vernichtet, damit der Reiseweg nicht mehr rekonstruierbar und eine Abschiebung nicht durchführbar ist.

180 Das Rückübernahmeabkommen beruhte auf einem Übereinkommen zwischen den SchengenStaaten und Polen von 1991 und war mit der Zahlung von 120 Mio. DM für 1993 und 1994 verknüpft, die unter anderem zur Grenzsicherung und Ausbildung von BeamtInnen verwendet werden sollten (vgl. Dickel 2002, S. 299). 
Zusammen mit dem neuen Asylverfahrensgesetz wurden damit die oben bereits herausgearbeiteten Strategien fortgesetzt, allerdings auf neuem Niveau:

- Abschreckung durch Verschlechterung der sozialen Situation (Asylbewerberleistungsgesetz),

- Zugangserschwerung durch sichere Dritt- und Herkunftsstaatenregelung (und entsprechende Rückübernahmeabkommen) und damit Auslagerung der Verantwortung an die Nachbarstaaten,

- Einschränkung der Widerspruchsmöglichkeiten: bei Einreise über sichere Drittstaaten Abschiebung unabhängig vom eingelegten Rechtsbehelf

Mit dieser einschneidenden Restriktion des Asylrechts differenzierte sich auch das Feld der flüchtlingspolitischen Initiativen weiter aus. Das Spektrum reicht von Nachbarschaftsinitiativen, die versuchen, die Abschiebung von langjährigen NachbarInnen zu verhindern, bis hin zu Kampagnen gegen Flüchtlingslager und Abschiebehaft, gegen die Ausgabe von Wertgutscheinen anstelle von Bargeld ${ }^{181}$, gegen die Residenzpflicht sowie für ein Bleiberecht. Im Jahr 1986 wurde die Bundesarbeitsgemeinschaft Pro Asyl gegründet, in der zahlreiche Organisationen einschließlich der Flüchtlingsräte zusammengeschlossen sind.

Die sich an die Grundgesetzänderung anschließende Phase von 1994-2007 wird im Folgenden in zwei Abschnitte aufgeteilt: Der erste umfasst die Umsetzung des neuen Asylrechts nach 1993 und weitere Verschärfungen auf der Verfahrensebene im Vorfeld der Bundestagswahl 1998. Der zweite Abschnitt wird eingeleitet durch die im Jahr 2000 begonnene ,Zuwanderungsdebatte' und die Diskussionen um das Zuwanderungsgesetz, das 2005 verabschiedet wurde. Schließlich gehören in diese Phase auch eine Reihe von so genannten ,Altfallregelungen ${ }^{6}-$ die letzte wurde im Jahr 2007 von der Innenministerkonferenz beschlossen.

\section{4 - 1999: Flüchtlingspolitik und -praxis mit dem neuen Art. 16a GG}

Wie bereits ausgeführt, wurde durch die Änderung des Grundgesetzes der Reiseweg der Asylsuchenden zum zentralen Gegenstand bei der Entscheidung über den Antrag. Wer über ein sicheres Drittland einreist, hat keine Chance mehr auf Anerkennung. Die Drittstaatenregelung sowie die sichere Herkunftsstaatenregelung und das Asylverfahren sind Gegenstand zahlreicher Kritik an der Novelle. Das Bundesverfassungsgericht, das aufgrund der Klagen mehrerer AsylbewerberInnen über die Verfassungsmäßigkeit dieser drei Kernpunkte zu entscheiden hatte, machte in seinem Beschluss im Jahre 1996 lediglich einige minimale Vorgaben zur Umsetzung der Regeln, erklärte sie in der Sache jedoch für verfassungsgemäß (vgl. BVerfGE 94, 49).

181 Gutscheininitiativen in verschiedenen Städten informieren über die soziale Situation von Flüchtlingen, die unter das Asylbewerberleistungsgesetz fallen, organisieren Gutscheinumtausch und ,antirassistische Einkäufe' und verhandeln teilweise auch mit den Städten bzw. Landkreisen über eine Abschaffung des Sachleistungsprinzips. 
Trotz des mit dem Asylkompromiss geschaffenen Sonderstatus für Bürgerkriegsflüchtlinge mussten auch diese weiterhin das Asylverfahren durchlaufen mit entsprechend geringen Anerkennungschancen. Aufgrund von Streitigkeiten zwischen Bund und Ländern über die Übernahme der Kosten wurde der entsprechende $\ 32$ a AuslG erst 1999 erstmals (und bisher das einzige Mal) - im Zusammenhang mit der Aufnahme von rund 15.000 Flüchtlingen aus dem Kosovo angewendet (vgl. Beauftragte 2004).

Die Antragszahlen gingen nach Inkrafttreten der Verfassungsänderung zurück. Dies ist auch auf die Stabilisierung der politischen Situation in den europäischen Herkunftsregionen zurückzuführen. Ein Großteil der AntragstellerInnen in dieser Phase kam aus Europa und der Türkei. Mit der Konsolidierung der (süd-)osteuropäischen Staaten und dem Ende der Jugoslawienkriege entfiel für die Menschen aus diesen Ländern die Möglichkeit, sich auf das Asylrecht oder den Status als Bürgerkriegsflüchtling zu beziehen. Gleichwohl kann kein Wegfall der Wanderungsgründe konstatiert werden - sowohl Roma in den Staaten des ehemaligen Jugoslawien als auch KurdInnen in der Türkei sind weiterhin von Menschenrechtsverletzungen betroffen. Auch außerhalb Europas gibt es eine Vielzahl von Menschen, die sogar die engen Kriterien des Asylgrundrechts in der Bundesrepublik erfüllen, die aber die hohen Hürden auf dem Weg dorthin nicht überwinden können. Hinzu kommen diejenigen, deren Migrationsmotive in den engen Grenzen des europäischen Einwanderungs- und Flüchtlingsrechts keine Einreise rechtfertigen. Sie verbleiben in den Herkunfts- und Transitregionen, während in der Bundesrepublik Flüchtlingsunterkünfte aufgrund mangelnder ,Nachfrage‘ geschlossen werden und sich auch das für die Asylanträge zuständige Bundesamt für Migration und Integration (damals noch ,Bundesamt für die Anerkennung ausländischer Flüchtlinge') nach neuen Aufgabenfeldern umsehen muss. Die gesunkenen Antragszahlen können also nicht allein auf eine veränderte Situation in den Herkunftsländern zurückgeführt werden, sondern sind auch ein Ergebnis der rechtlichen Verschärfungen. Es ist anzunehmen, dass die verbleibende Einwanderung auf andere Einwanderungswege umgeleitet wird.

Neben der Frage nach der Einwanderung von Asylsuchenden stellte sich in den 1990er Jahren vermehrt die Frage, was mit denjenigen geschehen soll, die das Territorium der Bundesrepublik erreicht haben und sich seit Jahren im Asylverfahren befinden oder abgelehnt wurden und dennoch nicht abgeschoben werden können. In der Behördenpraxis wird diese Frage überwiegend mit kurzfristigen Duldungen beantwortet. Dabei handelt es sich um einen Rechtstitel, mit dem lediglich die Abschiebung ausgesetzt, jedoch kein Aufenthaltsrecht begründet wird. Die ,Geduldeten' sind vielfach vom Zugang zum Arbeitsmarkt ausgeschlossen und erhalten häufig lediglich abgestufte Sozialleistungen nach dem Asylbewerberleistungsgesetz. In dieser Situation verharren in der Bundesrepublik mehre- 
re Zehntausend MigrantInnen, viele bereits länger als zehn Jahre. ${ }^{182}$ Für sie wurden in dem betrachteten Zeitraum mehrere bundesweite Altfallregelungen ausgehandelt (1996, 1999, 2001 für Personen aus Bosnien-Herzegowina und der Bundesrepublik Jugoslawien sowie 2007), die jedoch aufgrund der strengen Kriterien jeweils nur den Aufenthalt eines kleinen Teils der Betroffenen absicherten. ${ }^{183}$ Gleichzeitig wurden diese Regelungen meist verknüpft mit der Ankündigung, nach aufenthaltsrechtlicher Anerkennung der ,Berechtigten“ die übrigen verstärkt abzuschieben.

Während Bundesinnenminister Kanther eine positive Bilanz der Grundgesetzänderung von 1993 zog (vgl. Bundesministerium des Innern 1995), folgte auf die Umsetzung der Novellen eine Reihe von Protesten von Kirchen, Wohlfahrts- und Menschenrechtsorganisationen, UNHCR, Initiativen und Juristen, die sowohl die Dritt- und Herkunftsstaatenregelung kritisierten als auch das Asylbewerberleistungsgesetz und die Bedingungen in der Abschiebehaft. Trotz dieser Proteste wurde seit 1995 eine weitere Verschärfung des Asylbewerberleistungsgesetzes diskutiert, die schließlich 1997 in Kraft trat. ${ }^{184}$

Der Bundestagswahlkampf im Vorfeld der Bundestagswahlen von 1998 verhalf dem Thema Ausländerrecht im Allgemeinen und Asylrecht im Besonderen wieder auf die politische Agenda. So sollte mit einer im Juni 1997 beschlossenen Novelle des Ausländergesetzes die Abschiebung ausländischer Straftäter erleichtert werden. Damit wurde eine Forderung aufgegriffen, die in Wahlkämpfen gern von Republikanern und anderen im rechten Spektrum gestellt wird. Zudem verabschiedete der Bundestag im Juni 1998 eine weitere - auf Initiative Berlins zurückgehende - Verschärfung des Asylbewerberleistungsgesetzes. ${ }^{185}$ Nachdem der Entwurf von Wohlfahrtsverbänden und Menschenrechtsorganisationen als „Poli-

182 Im Jahr 2006 lebten rund 156.593 Geduldete in der Bundesrepublik, davon 26 Prozent bereits seit 1995. Unter den Geduldeten sind 64.238 Jugendliche unter 18 Jahren (vgl. BT Drs. 16/3446 vom 20.11.2006).

${ }^{183}$ Es handelt sich dabei meist um ,Stichtagsregelungen': InhaberInnen einer Duldung, die vor einem bestimmten Stichtag in die Bundesrepublik eingereist sind, können einen Antrag stellen. Dann wird eine Reihe weiterer Voraussetzungen geprüft, die sich vor allem auf die finanzielle Unabhängigkeit von Sozialleistungen beziehen. Beispielsweise konnten sich nach der Regelung von 1996 Familien, die vor dem 01.07.1990 und Alleinstehende, die vor dem 01.01.1987 nach Deutschland eingereist sind, um ein Bleiberecht bewerben. Zu den Bedingungen gehörte die Erfüllung bestimmter ,Integrationsmerkmale, wie der Nachweis einer Wohnung, ausreichend Lebensunterhalt, Schulbesuch der Kinder, Strafffreiheit, etc. Von dieser - vom damaligen Bundesinnenminister Manfred Kanther (CDU) als ,Gnadenregelung' bezeichneten - Maßnahme konnten jedoch nur wenige Tausend der damals rund 350.000 Geduldeten profitieren. Die Altfallregelung sollte gleichzeitig mit der verstärkten Abschiebung von abgelehnten AsylbewerberInnen einhergehen (vgl. Frankfurter Allgemeine Zeitung vom 30.03.1996; Süddeutsche Zeitung vom 30.03.1996).

184 PDS und Grüne hatten eigene Gesetzentwürfe eingebracht, mit denen AsylbewerberInnen die üblichen Leistungen nach dem Bundessozialhilfegesetz erhalten sollten.

185 Die zweite Verschärfung des AsylbLG wurde laut Classen (1999) mit durch die Berliner Ausländerbeauftragte Barbara John angestoßen. Das Land Berlin hatte nach entsprechenden Äußerungen Johns einen Gesetzentwurf im Bundesrat eingebracht (vgl. BR Drs. 691/97). 
tik des Aushungerns" 186 scharf kritisiert wurde, wurden einige Verschärfungen abgemildert. ${ }^{187}$ Tenor der Novelle ist der ebenfalls stigmatisierende Topos, dass AsylbewerberInnen in erster Linie einwandern, um Sozialleistungen zu ,erschleichen'. Mit der Gesetzesänderung wurde einmal mehr der Spielraum der Behörden ausgeweitet, die ohnehin schon unter dem Sozialhilfeniveau liegenden Leistungen für AsylbewerberInnen weiter zu kürzen - bis auf das (nicht näher definierte) ,unabweisbar Gebotene'. Diese Aktivitäten auf gesetzlicher Ebene im Vorfeld der Bundestagswahl wurden gerahmt durch zahlreiche wahlkampfstrategische Wortmeldungen, die sich gegen AusländerInnen richteten. Führend hierbei waren wiederum die Unionsparteien, die das Thema Einwanderung auf Initiative der CSU offiziell als Wahlkampfthema ausriefen. ${ }^{188}$ Aber auch in der SPD wurden entsprechende Positionen vertreten. So sagte der damalige Ministerpräsident Niedersachsens, Gerhard Schröder, wer das Gastrecht missbrauche, „für den gibt es nur eins: raus, und zwar schnell“. Zur Begründung fügte er eine Reihe von Stereotypen an:

\section{„Beim organisierten Autodiebstabl sind Polen nun einmal besonders aktiv, das Geschäft der Prostitution wird dominiert von der Russen-Mafia, Drogenkriminelle kommen be- sonders häufig aus Südosteuropa und Schwarzafrika" (vgl. Frankfurter Allgemeine Zei- tung vom 21.07.1997).}

Als Aufhänger für die Bundesratsinitiative Berlins hatte das Thema der Bürgerkriegsflüchtlinge aus dem ehemaligen Jugoslawien gedient. Diese würden sich weigern auszureisen und in der Bundesrepublik Deutschland bleiben, um Sozialhilfe zu empfangen. Ihre Rückkehr bzw. Abschiebung wurde unterdessen intensiv vorbereitet und kontrovers diskutiert. Insbesondere zur von den Länderinnenministern vorangetriebenen Rückkehr bosnischer Flüchtlinge gab es eine Reihe von Diskussionen - um den geeigneten Beginn der ,Rückführungen' sowie um ein Rückübernahmeabkommen mit der bosnischen Regierung. Die Länder lobten zusätzliche Prämien für frühe RückkehrerInnen aus. Seit dem Frühjahr 1997 ist ein Großteil der rund 340.000 Flüchtlinge zurückgekehrt, teils freiwillig, teils unter Zwang, teils versuchten sie in andere EU-Länder weiterzureisen.

Im Hinblick auf die Unterstützungsstrukturen spielte die Kirchenasylbewegung in dieser Phase eine zunehmend wichtigere Rolle und erlebte in den 1990er

186 Vgl. zum Beispiel Amnesty International (1998); vgl. auch Frankfurter Rundschau vom 21.03.1998 und Frankfurter Rundschau vom 29.04.1998.

187 Die Novelle trat am 01.09.1998 mit folgendem Wortlaut in Kraft: „Flüchtlinge, die zugewandert sind, um Leistungen nach dem AsylbLG zu erlangen oder geduldete und vollziehbar ausreisepflichtige Ausländer, bei denen aus Gründen, die sie selbst zu vertreten haben, aufenthaltsbeendende Maßnahmen nicht durchgeführt werden können, erhalten nur noch Leistungen in Höhe des - je nach Einzelfallsituation - unabweisbar Gebotenen". (\$ 1a AsylbLG)

188 Dabei ging es vor allem um die Verhinderung eines weiteren Zuzugs von Einwanderern und die Bekämpfung des ,Asylmissbrauchs‘ (vgl. Süddeutsche Zeitung vom 25.07.1998). Die CSU konnte sich nicht damit durchsetzen, den Satz ,Deutschland ist kein Einwanderungsland‘ in das Programm aufzunehmen. 
Jahren ihren Höhepunkt. Sie wurde 1994 mit der Gründung der ,Ökumenischen Arbeitsgemeinschaft Asyl in der Kirche' institutionalisiert. In der Ankündigung zur Ausstellung anlässlich 20 Jahren ,Asyl in der Kirche‘ im Jahr 2004 heißt es:

„Das Kirchenasyl war und ist eine Reaktion auf die ungelösten menschenrechtlichen Probleme des deutschen Asylrechts. Als ultima ratio im Einzelfall verweist jedes Kirchenasyl exemplarisch auf die schwerwiegenden Defizite. [...] Dabei geht es nicht darum, den Rechtsstaat in Frage zu stellen, sondern eine erneute Prüfung zu erreichen. Diese Einschätzung findet sich auch in den überwiegend unterstützenden Verlautbarungen von katholischen und evangelischen Kirchenleitungen, in denen das Kirchenasyl als Ausübung christlicher Beistandspflicht gewertet und befürwortet wird." (vgl. Asyl in der Kirche, o.J.)

Doch auch das Kirchenasyl bietet keinen hundertprozentigen Schutz: Mehrfach wurden Kirchenasyle von der Polizei geräumt und Strafverfahren gegen PfarrerInnen und Kirchenvorstände eingeleitet. Gleichzeitig hat es aber auch Vorschläge für ,Kirchenkontingente' gegeben, wie beispielsweise von der bayerischen Staatsregierung, die Kirchen könnten so „Härtefälle` vor der Abschiebung bewahren, müssten aber für die gesamten Kosten aufkommen. ${ }^{189}$

Aus der gewandelten Situation nach der Grundgesetzänderung und der zunehmenden Bedeutungslosigkeit des Asylrechts als Einwanderungsoption hat sich auch der Fokus vieler flüchtlingspolitischer Initiativen hin zu den Sans Papiers Menschen in prekären Aufenthaltsverhältnissen bzw. ohne Aufenthaltsdokumente - verschoben: So ist in dieser Phase beispielsweise das Netzwerk ,kein mensch ist illegal' entstanden, aber auch zahlreiche Anlaufstellen, die MigrantInnen in prekären Aufenthaltsverhältnissen oder ohne Papiere medizinische Versorgung vermitteln und oft gleichzeitig für eine Aufnahme in die Regelversorgung und Zugang zu Bildungseinrichtungen streiten. ${ }^{190}$

\section{0 - 2007: Flüchtlingsschutz im Zuwanderungsgesetz}

Mit dem Regierungswechsel von 1998 waren von Seiten flüchtlingspolitischer Organisationen auch Erwartungen an einen Wandel der Einwanderungspolitik verknüpft. Diese wurden jedoch - im Hinblick auf die Asylpolitik - kaum erfüllt, wie Liza Schuster in ihrer Analyse feststellt: „The most striking feature of asylum policy under the new government was its continuity with its predecessor." (Schuster 2003, S. 218) Obwohl die rot-grüne Regierung nun die Gelegenheit hatte, viele

\footnotetext{
189 Vgl. Frankfurter Allgemeine Zeitung vom 21.07.1995; Die Zeit vom 21.07.1995. Die Kirchen lehnten dies zwar ab, realiter hat es aber immer wieder Fälle gegeben, in denen UnterstützerInnengruppen eine Abschiebung nur verhindern konnten, wenn sie Sponsoren für die vollständige Versorgung der Flüchtlinge gewinnen konnten. Dies geht so weit, dass Kinder keine öffentliche Schule besuchen dürfen, sondern ein Platz in einer Privatschule finanziert werden muss.

${ }^{190}$ Entsprechende Medizinische Flüchtlingshilfen gibt es in verschiedenen Städten wie Berlin, Bonn, Bielefeld, Bochum, Freiburg, Göttingen, Hamburg, Hannover, Heidelberg und München.
} 
der aus der Oppositionsrolle heraus geforderten einwanderungspolitischen Veränderungen in die Tat umzusetzen, blieb sie hinter den einst gestellten Forderungen zurück, oder ihre Forderungen wurden aufgrund der Konstellationen im Bundesrat deutlich aufgeweicht. Insbesondere die asylpolitischen Forderungen wurden nicht realisiert. ${ }^{191}$ Der sozialdemokratische Innenminister Otto Schily stellte gar die Reste des Asylrechts in der Bundesrepublik infrage - mit dem schon zehn Jahre zuvor seitens der Unionsparteien bemühten Verweis auf die EU:

„Ein subjektives Recht auf Asylgewährung wird die EU nicht akzeptieren. [...] Auch binsichtlich der Frage, ob unser Verfassungsrecht und die einfachen Asylgesetze auf diesem Gebiet die ideale Lösung sind, darf kein Denkverbot verbängt werden. "(vgl. Die Zeit vom 28.10.1999) $)^{192}$

In der Praxis des Umgangs mit Asylsuchenden ist die restriktive Kontinuität auch auf die zentrale Rolle der Länder und der ausführenden Behörden zurückzuführen. So identifiziert Wolfgang Bosswick (2000) die bürokratischen Strukturen als zentralen Faktor für das Beharrungsvermögen in der asylpolitischen Praxis.

Der asylpolitischen Kontinuität stand ein Wandel des öffentlichen und politischen Diskurses im Hinblick auf die Einwanderung im Allgemeinen gegenüber. Während Roland Koch noch 1999 mit seiner Kampagne gegen die doppelte Staatsbürgerschaft Ministerpräsident in Hessen wurde, begann im Jahr 2000 eine Einwanderungsdebatte, die sich in erster Linie darum drehte, dass Deutschland Einwanderung brauche - und zwar Einwanderung von Hochqualifizierten. Nachdem die Bertelsmann Stiftung im Februar 2000 konstatierte, in Deutschland fehlten 80.000 qualifizierte Fachkräfte in der Informationstechnologie-Branche, kündigte der damalige Bundeskanzler Gerhard Schröder bei der Eröffnung der CeBIT eine branchenbezogene Öffnung des Arbeitsmarktes für SpezialistInnen aus dem Ausland an, die in der Green-Card Initiative mündete. Aufgrund des Widerstands der Unionsparteien, der seinen Ausdruck im Wahlkampf insbesondere in der „Kinder statt Inder“-Kampagne von Jürgen Rüttgers (CDU) fand (vgl. Der Spiegel vom 09.03.2000), wurde die Green Card allerdings so unattraktiv gestaltet, dass sie sich als ,Ladenhüter entpuppte (vgl. Der Spiegel vom 15.03.2004). Der Ver-

\footnotetext{
${ }^{191}$ Laut Koalitionsvertrag sollten im Asylbereich das Flughafenverfahren und die Dauer der Abschiebehaft unter dem Verhältnismäßigkeitsgrundsatz überprüft werden. Stattdessen wurden sowohl das neue Staatsangehörigkeitsrecht als auch das Zuwanderungsgesetz durch die starke Stellung der Unionsparteien im Bundesrat erheblich eingedampft.

192 Otto Schily führt weiter aus: „Wir müssen uns aber mit Blick auf Europa und auf die europäische Asylrechtsdebatte dem Gedanken öffnen, dass nicht jede Wohltat, die wir einem Menschen zuwenden, einklagbar sein muss. Ein Gast hat Hilfsbereitschaft verdient, aber in allen Ländern auBerhalb Europas kann er diese nicht gerichtlich erzwingen. [...] die Gerechtigkeitsgewähr der Entscheidung über ein Asylgesuch sollte sich meiner Meinung nach stärker an moralischen Maßstäben als an juristischen Klauseln orientieren." (vgl. Die Zeit vom 28.10.1999)
} 
such, mit den Konzepten der Gastarbeiterära 50 Jahre später Hochqualifizierte zu gewinnen ${ }^{193}$, stieß bei der Zielgruppe auf Desinteresse.

Dennoch zeichnete sich um die Jahrtausendwende ein einwanderungspolitischer Wandel ab: Die diskursiv bereits lange etablierte Unterscheidung zwischen erwünschten und unerwünschten Einwanderern wurde nun auch migrationspolitisch nachvollzogen. Ausgehend von den Ergebnissen der von Bundesinnenminister Schily eingesetzten Unabhängigen Kommission Zuwanderung (so genannte Süßmuthkommission) und den Arbeiten weiterer Kommissionen der einzelnen Parteien ${ }^{194}$ ging die Debatte von der Frage weg, ob Deutschland Einwanderungsland ist oder nicht, und drehte sich nunmehr um die optimale Steuerung von ,erwünschter ${ }^{6}$ und ,unerwünschter ${ }^{6}$ Einwanderung ${ }^{195}$ :

„Following the reasoning of the Independent Commission, political and societal elites in all fields recognised that immigration is an inevitable aspect of globalisation and should not be prevented in general but managed properly - at least for some categories. "(Cyrus/ Vogel 2003, S. 2)

Im Folgenden wird aufgezeigt, welchen Stellenwert die Asylpolitik im Rahmen der Zuwanderungsdebatte hatte, welche asylrechtlichen Änderungen im Zuwanderungsgesetz verankert wurden und wie sich diese in die bis dahin verfolgte Flüchtlingspolitik einordnen lassen.

\section{Unabhängige Kommission Zuwanderung}

Zunächst war umstritten, ob sich die ,Unabhängige Kommission Zuwanderung unter dem Vorsitz von Rita Süßmuth auch mit dem Thema Asyl befassen sollte.

193 Vgl. Schuster 2003, S. 221: „This policy option is a clear continuation of the guestworker policy of the 1960s and 1970s. Migrants are to be admitted to fill Germany's economic needs so long as they exist and for a temporary period only."

194 So legte auch die CDU im November 2000 den Bericht einer eigenen Zuwanderungskommission vor, die unter dem Vorsitz des saarländischen Ministerpräsidenten Peter Müller gearbeitet hatte. Der Bericht enthielt zunächst einige bemerkenswert liberale Passagen, diese hielten jedoch der Rückkopplung mit der Parteibasis nicht stand. Die CSU erarbeitete unter Leitung des damaligen bayerischen Innenministers Günther Beckstein ein eigenes Konzept. Im Zuge dessen wurde wiederum eine Änderung des Asylgrundrechts andiskutiert. Schließlich einigten sich CDU und CSU auf dessen vorläufige Beibehaltung. Die FDP stellte die überarbeitete Fassung eines Gesetzentwurfs vor, den die Bundestagsfraktion schon in der vorangegangenen Legislaturperiode entworfen hatte. Der Bericht der Kommission der Grünen wurde beim Parteitag im März 2001 um die Forderung nach Wiederherstellung des Asylrechts erweitert. Die PDS brachte sich mit, Eckpunkten für eine menschenrechtliche Zuwanderungspolitik' ein. Die SPD entzog sich zunächst diesem Kommissions-Boom mit Verweis auf die von ihrem Innenminister eingesetzte Süßmuth-Kommission, legte jedoch nach der Veröffentlichung des Süßmuth-Berichts ein eigenes Konzept vor (vgl. Reißlandt 2003, S. 129f.).

195 Peter Müller (2001), Unionspolitiker und Ministerpräsident des Saarlands, fasst zusammen, was diese Zielsetzung mit Blick auf Asylsuchende bedeutet: „Zu den eher unerwünschten Zuwanderungskategorien zählen also nicht die tatsächlich Asylberechtigten, sondern die unter missbräuchlichem Rückgriff auf das Asylrecht ins Land kommenden Wirtschaftsflüchtlinge.“ 
Bundespräsident Johannes Rau hatte davor gewarnt, die Themen Asyl und Einwanderung im Allgemeinen miteinander zu vermischen. Doch der damalige Innenminister Otto Schily wollte keine thematischen ,Tabus' in der Kommission von einer weiteren Eingrenzung des Asylrechts erhoffte er sich „Spielraum für Zuwanderer, die wir aus eigenem Interesse nach Deutschland holen wollen“ (vgl. Focus vom 03.07.2000). Der gesamte Bericht basiert auf einer Verknüpfung von Einwanderung mit der „Verwendbarkeit“ (Unabhängige Kommission 2001, S. 90, 92, 93) ${ }^{196}$ der Einwandernden auf dem Arbeitsmarkt. Das impliziert die genannte Differenzierung zwischen Einwandernden, die die auf dem Arbeitsmarkt geforderten Profile (langfristig) erfüllen und den übrigen, unerwünschten MigrantInnen. Unter dieser Prämisse wird schließlich auch das Thema Asyl diskutiert. Es wird - unter dem Hinweis, dass verlässliche Quellen fehlen - konstatiert, dass AsylbewerberInnen vor allem gering qualifiziert seien und deshalb hauptsächlich als Arbeitskräfte „im unteren Qualifikationsbereich“ zur Verfügung stehen (ebd., S. 45). Die Kommission schlägt für die künftige Gestaltung der Einwanderung ein „Modell der arbeitsmarktorientierten Zuwanderung“ (ebd., S. 83ff.) vor, über das auch „,befristeten Zuwanderern Wege zum Daueraufenthalt“ (ebd., S. 95ff.) eröffnet werden sollen. Für abgelehnte AsylbewerberInnen sollte diese Option über ein Punkteverfahren aber nur gelten, wenn sie das Land freiwillig verlassen haben und vom Ausland aus einen entsprechenden Antrag stellen (vgl. ebd., S. 95). So will man „Sogwirkungen für das Asylverfahren vermeiden und klarstellen, dass $\mathrm{Zu}$ wanderung, die aus humanitären Gründen gestattet wurde, und arbeitsmarktorientierte Zuwanderung nicht miteinander verzahnt sind“ (ebd., S. 96f.). ${ }^{197}$ Zur Abwendung dieser ,Sogwirkung' spricht sich die Kommission auch für die Beibehaltung des einjährigen Arbeitsverbots aus (ebd., S. 119).

Nach dieser Ausklammerung der AsylbewerberInnen und der Abgelehnten aus der Gruppe der unter arbeitsmarktpolitischen Erwägungen erwünschten Einwanderer werden im Asylkapitel des Kommissionsberichts die Eckpunkte für eine „effektive Flüchtlingspolitik“ umrissen. Zur Begründung der Empfehlungen wird das Thema unter zwei widerstreitenden Prämissen diskutiert: „Humanität“ auf der einen und „innerer Frieden“ (ebd., S. 123), „Leistungsfähigkeit der Aufnahmegesellschaft" (ebd., S. 124) und „Aufnahmebereitschaft“ (ebd., S. 124) auf der anderen Seite. Mit diesen Stichworten werden längst bekannte Topoi der Asyldebatte Belastungsgrenzen und Gefährdung des inneren Friedens durch Einwandernde angesprochen, deren Stichhaltigkeit zu belegen oder zu plausibilisieren die Kom-

\footnotetext{
196 „Erfahrungen klassischer Einwanderungsländer zeigen, dass die positive Wirkung von Zuwanderung für die Wirtschaft und die sozialen Sicherungssysteme vom Profil der Zuwanderer und ihrer Verwendbarkeit für den Arbeitsmarkt abhängt, und dass dabei aus ökonomischen Gründen sowie im Sinne einer Akzeptanz für die Zuwanderung eine Verdrängung einheimischer Arbeitskräfte vermieden werden muss. Der Zielgenauigkeit von Auswahlverfahren kommt daher entscheidende Bedeutung zu." (Unabhängige Kommission 2001, S. 90)

${ }^{197}$ Das von der Kommission vorgeschlagene Punktesystem fand bei der Diskussion um das Zuwanderungsgesetz keine Mehrheit.
} 
mission offenbar nicht für erforderlich hielt. Um diese beiden Aspekte (Humanität und innerer Frieden) in Einklang zu bringen, wird zunächst eine Reihe von ,Problemlösungen“ diskutiert. Dabei wird eine Verfassungsänderung als Option verworfen - sowohl eine Rückkehr zum alten Art. 16 Abs. 2 Satz 2 als auch eine Umwandlung des individuellen Grundrechts auf Asyl in eine institutionelle Garantie oder eine Änderung des Art. 19 Abs. 4 (Rechtsweggarantie) werden vor allem unter Effizienzerwägungen als untauglich eingeschätzt. Stattdessen werden, wie schon in den Jahrzehnten zuvor, verschiedene Strategien zur Verfahrensbeschleunigung, zur „Bekämpfung missbräuchlichen Verhaltens ${ }^{` 198}$ und zur konsequenten ,Rückführung‘ Abgelehnter empfohlen.

Erst am Ende des Kapitels wird diskutiert, inwieweit eine „Verbesserung der Schutzgewährung“ (ebd., S. 159ff.) erreicht werden könnte - hier konnte in der Kommission aber keine Einigung über konkrete Empfehlungen erzielt werden. Sie verwies daher auf die bevorstehende Europäisierung des Politikbereichs, der man nicht vorgreifen wolle. ${ }^{199}$ Die Reaktionen auf den Bericht waren dementsprechend gespalten: Zwar gab es auch viel positive Resonanz - insbesondere aus dem Innenministerium, dem Auftraggeber der Studie. Von Kirchen und Wohlfahrtsverbänden wurde er hingegen als an verschiedenen Punkten zu restriktiv kritisiert,

198 Der Topos des ,Asylmissbrauchs' taucht an mehreren Stellen des Kommissionsberichts auf: „Gleichzeitig muss aber einem Missbrauch des Asylrechts zu Einwanderungszwecken entgegengewirkt werden. Dieser verursacht nicht nur erhebliche Kosten, sondern mindert auch die Akzeptanz von Flüchtlingen in der einheimischen Bevölkerung." (Unabhängige Kommission 2001, S. 19) Die Verwendung des Begriffs wird interessanterweise ausführlich reflektiert, sich dann aber für seinen Gebrauch entschieden: „Das Wort ,Asylmissbrauch“ fällt ein ethisch begründetes Negativurteil. Der Begriff ist umfassend und pointiert, zugleich griffig, aber auch pauschalisierend. Er eignet sich gleichermaßen dazu, Asylbewerber, die nicht als politisch Verfolgte anerkannt werden, abzuwerten oder gar zu diffamieren und bestimmte missbilligenswerte Verhaltensweisen von Asylbewerbern zutreffend zu charakterisieren. Außerdem verdeckt diese Wortwahl, dass zu missbilligende Verhaltensweisen nicht nur bei Asylbewerbern, sondern auch bei anderen Zuwanderern auftreten, die sich ihrer Rückführung unzulässigerweise widersetzen, ohne zuvor ein Asylbegehren gestellt zu haben. In diesem Zusammenhang sollte auch nicht ganz übersehen werden, dass die missbräuchliche Inanspruchnahme von Rechten ein allgemeines und kein auf Asylbewerber beschränktes Phänomen ist. Die Kommission zieht aus der Mehrdeutigkeit des Begriffs Asylmissbrauch jedoch nicht etwa den Schluss, auf ihn zu verzichten. Dies verbietet sich schon, weil er in der öffentlichen und veröffentlichten Meinung sehr verbreitet ist, und weil er - richtig gebraucht - auf eine durchaus existierende Problematik hinweist. Es muss also darum gehen, diesen Begriff hinreichend differenziert zu verwenden. [...]“ (ebd., S. 145) Um ,missbräuchlichen Verhaltensweisen' entgegenzuwirken, werden vor allem Maßnahmen im Bereich der Datensammlung und arbeits- und sozialrechtliche Sanktionen vorgeschlagen.

199 Weiterhin werden Vorschläge zur Verbesserung der Aufenthaltsbedingungen für Konventionsflüchtlinge und der Situation Geduldeter unterbreitet. Die Kommission spricht sich gegen eine allgemeine Härtefallregelung aus und überlässt die Einrichtung von Härtefallkommissionen der Entscheidung der Bundesländer. Bezüglich der Aufnahme von Bürgerkriegsflüchtlingen kritisiert die Kommission die mangelnde Inanspruchnahme des $\ 32$ a AuslG. Die sei darauf zurückzuführen, dass in der Bestimmung die Frage der Kostenaufteilung nicht geregelt sei. Die Kommission empfiehlt daher, dass die Kosten je zur Hälfte vom Bund und von den Ländern zu tragen seien sowie eine EU-weite ,Lastenteilung' (vgl. Unabhängige Kommission 2001, S. 175f.). 
während die Unionsparteien ihn als „Zuwanderungserweiterungskonzept“ (CDUPressestelle 2001) ${ }^{200}$ zurückwiesen (vgl. Reißlandt 2003, S. 132).

\section{Entstehung des Zuwanderungsgesetzes}

Während die Parteien nach und nach die Berichte ihrer eigenen Zuwanderungskommissionen präsentierten, wurde im Innenministerium bereits der Referentenentwurf des Zuwanderungsgesetzes erarbeitet. Diesen stellte Otto Schily am 3. August 2001 der Öffentlichkeit vor. Er lehnte sich - nach der Einarbeitung der Vorschläge des grünen Koalitionspartners - an die Empfehlungen der Unabhängigen Kommission Zuwanderung an (vgl. Davy 2002). Nach den Vorstellungen des Ministeriums sollte das Gesetz binnen drei Monaten den Bundestag passiert haben. Doch die ,Aufbruchstimmung' in der Zuwanderungsdebatte hatte nicht alle Parteien gleichermaßen erfasst. Zwar war man sich über die Parteigrenzen hinweg einig, dass die Einwanderungspolitik neu zu gestalten sei, aber wie genau dies zu geschehen hatte, war höchst umstritten (vgl. Angenendt 2002b). Die Haltung der Unionsparteien in der Debatte wurde durch den Bericht der unionseigenen Zuwanderungskommission vorgezeichnet: Während die erste Fassung des Berichts der CDU-Kommission zur Einwanderung noch ein wenig die einwanderungspolitische Aufbruchstimmung spiegelte, musste das Papier nach Abstimmung mit der Basis korrigiert werden. Die zweite Fassung betonte, dass es explizit auch um die Begrenzung von Zuwanderung ginge und dass „Deutschland kein klassisches Einwanderungsland ist und es aufgrund seiner historischen, geographischen und gesellschaftlichen Gegebenheiten auch nicht werden kann".201

Durch die Ereignisse des 11. September 2001 veränderte sich die Zuwanderungsdebatte: Einwanderung wurde verstärkt als Sicherheitsrisiko diskutiert. Mit seinen zwei ,Anti-Terror-Paketen' brachte Otto Schily bereits zahlreiche ausländerrechtliche Verschärfungen durch den Bundestag. In der Diskussion um das Zuwanderungsgesetz hingegen gingen die Unionsparteien in eine Blockadehaltung. Obwohl Otto Schily schließlich auch einige Vorschläge der Unionsparteien in das Zuwanderungsgesetz aufnahm, ging der Entwurf am 1. März 2002 nur mit

200 Die CDU kritisiert: „Der Bericht der Süssmuth-Kommission setzt einseitig auf Zuwanderungserweiterung. Es werden ausschließlich die positiven, bereichernden Aspekte der Zuwanderung gesehen.“ (CDU-Pressestelle 2001) Dennoch finden sich auch Übereinstimmungen: „Gleichzeitig stehen einige Vorstellungen der Süssmuth-Kommission, die zu einer Verschärfung des geltenden Rechts führen, durchaus in Übereinstimmung mit der Konzeption der Union.“ (ebd.) Zu den asylpolitischen Vorschlägen heißt es: „Zudem fehlt eine klare Absage an die Erweiterung des Anwendungsbereiches von Artikel 16a GG. Unzureichend sind die Vorschläge zur Straffung der Asylverfahren.“ (ebd.) Positiv findet die Union: „die Forderungen nach einer Erweiterung der abgesenkten Leistungen nach dem Asylbewerberleistungsgesetz" (ebd.). Die Union kündigt an, dass nur dann ein parteiübergreifender Konsens zu erreichen sei, wenn ihr eigener Kommissionsbericht zur Grundlage der neuen Regelungen gemacht wird (vgl. ebd.).

201 Zunächst lautete der Titel des Papiers „Zuwanderung steuern. Integration fördern.“ Nach Abstimmung mit den CDU-Regionalkonferenzen wurde es umbenannt: „Zuwanderung steuern und begrenzen. Integration fördern“ (vgl. CDU 2001). 
den Stimmen von Rot-Grün durch den Bundestag - der Kanzlerkandidat der Union, Edmund Stoiber, wollte das Thema noch für die bevorstehenden Wahlen nutzen - der Unionspolitiker Friedrich Merz erklärte es dann offiziell zum Wahlkampfthema der Unionsparteien. ${ }^{202}$ Wieder einmal spielten also auch wahlkampftaktische Erwägungen eine zentrale Rolle: „In Anbetracht der anstehenden Landtags- und Bundestagswahlen wurde parteipolitisches Kalkül wichtiger als die zuvor vertretenen Sachargumente." (Angenendt/Kruse 2004, S. 176)

Bei der Abstimmung im Bundesrat Ende März 2002 wertete der Bundesratspräsident Klaus Wowereit die Stimmabgabe des Landes Brandenburg nach mehrmaliger Nachfrage an Ministerpräsident Manfred Stolpe als einheitlich für den Entwurf, obwohl es erklärtermaßen einen Dissens in der brandenburgischen GroBen Koalition gab und der brandenburgische Innenminister Jörg Schönbohm zunächst dagegen votiert hatte (vgl. Angenendt/Kruse 2004, S. 178f.; Reißlandt 2003, S. 133). Trotz der Skandalisierung dieses Vorgehens des Bundesratspräsidenten durch die Unionsparteien unterschrieb Bundespräsident Johannes Rau das Gesetz nach längerer Prüfung, empfahl aber eine Überprüfung durch das Bundesverfassungsgericht. Sechs Bundesländer ${ }^{203}$ strengten schließlich ein Normenkontrollverfahren beim Bundesverfassungsgericht an. Am 18. Dezember 2002 gab das Gericht seine Entscheidung bekannt, wonach das Gesetz nicht verfassungsgemäß zustande gekommen sei, da keine einheitliche Stimmabgabe des Landes Brandenburg vorgelegen habe.

Die Regierungskoalition brachte das Zuwanderungsgesetz im Januar 2003 in der gleichen Fassung nochmals im Bundestag ein ${ }^{204}$, wo es im Mai wiederum verabschiedet wurde. Der Innenausschuss des Bundesrates legte eine Liste mit 137 Änderungsanträgen vor, die im Vermittlungsausschuss verhandelt werden sollten. Dabei sollten zentrale Regelungen des Zuwanderungsgesetzes, einschließlich der Staatsangehörigkeitsreform, verschärft werden. Die dabei angesprochenen Punkte zeigen die jahrzehntelange Kontinuität der Debatte:

„Vergleicht man die genannten Kritikpunkte mit den Diskursen, die seit den 1980er Jabren die ausländerpolitische Debatte dominieren, bleibt festzustellen, dass die zentralen Streitpunkte der Zuwanderungsdebatte die gleichen geblieben sind, obne dass eine Annäberung in den (Sach-)Fragen - wie Zuwanderung, Integration und das Zusammenleben in Deutschland - unter globalisierten Rahmenbedingungen künftig gestaltet werden soll, gefunden werden konnte. "(Reißlandt 2003, S. 143f.)

\footnotetext{
202 Zudem hatte Friedrich Merz die ,Leitkulturdebatte' angestoßen, die nicht zuletzt Anknüpfungspunkte für die extreme Rechte bot (vgl. Hentges 2002).

${ }^{203}$ Dies waren Baden-Württemberg, Bayern, Hessen, Saarland, Sachsen und Thüringen.

204 Dies geschah mit dem Verweis darauf, dass die Verfassungswidrigkeit vom Bundesverfassungsgericht mit dem Zustandekommen - also der uneinheitlichen Abstimmung eines Bundeslandes nicht aber mit den Inhalten des Gesetzes begründet worden war. Schließlich sollte ein Vermittlungsverfahren bei der Konsenssuche helfen.
} 
Nach den Terroranschlägen von Madrid im März 2004 forderte die Union erneut Verschärfungen. Schließlich erklärte Kanzler Gerhard Schröder das Zuwanderungsgesetz zur ,Chefsache' und handelte mit der Opposition einen Kompromiss aus - andernfalls wollte die Regierung das Gesetz in Einzelgesetzen verabschieden. Der Vermittlungsausschuss stimmte im Juni 2004 dem entstandenen Kompromiss zu, der daraufhin im Juli von Bundestag und Bundesrat verabschiedet wurde und am 1. Juli 2005 in Kraft trat (vgl. Gesetz zur Steuerung und Begrenzung der Zuwanderung). Otto Schily fasste schließlich die Errungenschaften des Gesetzes aus seiner Sicht zusammen:

„Das Gesetz, ist vor allem ein Gewinn für unser Land, für Deutschland. Es stärkt unsere Position im internationalen Wettbewerb um die besten Köpfe und dient den wirtschaftlichen Interessen unseres Landes. Es mildert die Folgen der demographischen Entwicklung [...], es bremst den Zuzug in die sozialen Sicherungssysteme und es gibt uns die Möglichkeit, mit menschlichen Schicksalen auch menschlich umzugehen. Es verbessert die Integration all derer, die zu uns kommen, um bier zu leben und zu arbeiten. Nicht zuletzt erhöht es die Sicherheit unseres Landes, indem es jene, die hier Unfrieden stiften und Hass säen wollen, in die Schranken weist." (Plenarprotokoll 15/118, S. 10718f.)

Für Peter Müller (CDU) stellen sich die Verhandlungsergebnisse so dar:

„Dieses Gesetz ist ein Zuwanderungsbegrenzungsgesetz. Es macht Schluss mit der Vorstellung, Deutschland könne zu einer multikulturellen Einwanderungsgesellschaft umgestaltet werden. Deshalb ist dieses Gesetz. zustimmungsfähig. " Plenarprotokoll 15/118, S. 10723)

\section{Auf alten Pfaden: Asylrechtliche Änderungen im Zuwanderungsgesetz}

Das Zuwanderungsgesetz regelt umfassende Änderungen bereits bestehender Gesetze, darunter das Asylverfahrensgesetz, das Aufenthaltsgesetz und das Asylbewerberleistungsgesetz. Mit Blick auf das Flüchtlingsrecht haben sich einschneidende Veränderungen ergeben. Kleinen Fortschritten stehen eine Reihe uneingelöster Versprechen und eine Vielzahl von Verschlechterungen gegenüber. Zu den von Menschenrechtsorganisationen begrüßten Verbesserungen gehört die Anerkennung nichtstaatlicher und geschlechtsspezifischer Verfolgung. Obwohl von CDU und CSU immer wieder als Einfallstor für eine ,Flut von Asylanträgen“ kritisiert, wurde sie dennoch im neuen Regelwerk verankert. ${ }^{205}$ Dies geht auf die Vorgaben aus der EU-Qualifikationsrichtlinie zurück, die hiermit implementiert wurden. Die Umsetzung in die Praxis erweist sich allerdings als schleppend (vgl. Pro Asyl 2006b).

Zudem wurde eine Angleichung der Status von Asylberechtigten und Anerkannten nach der GFK vorgenommen - beide Gruppen erhalten nun eine (befris-

$205 \mathrm{Zu}$ weiteren zentralen Regelungen, die für Flüchtlinge in der BRD gelten, vgl. Abschnitt 5.3.3 Flüchtlingsschutz zwischen Recht und Praxis. 
tete) Aufenthaltserlaubnis, was für die GFK-Flüchtlinge eine Verbesserung darstellt. Für Asylberechtigte ist dies eine Verschlechterung, da sie bislang nach ihrer Anerkennung ein unbefristetes Aufenthaltsrecht erhielten. Sie bekommen nun nur noch einen befristeten Aufenthaltstitel. Nach drei Jahren soll routinemäßig ein Widerrufsverfahren stattfinden, in dem das Fortbestehen der Verfolgungsgründe überprüft und der Aufenthalt entsprechend verlängert oder beendet wird. ${ }^{206}$ Des Weiteren wurden die Möglichkeiten des Familienasyls erweitert.

Allerdings wurden die Bedingungen im Antragsverfahren verschärft: Die Anträge derer, die sich nicht unverzüglich bei der Aufnahmestelle melden, werden als Folgeanträge behandelt. Zudem gelten verschärfte Zulassungsvoraussetzungen und verkürzte Fristen für Widersprüche. Bei Folgeanträgen, die nach Ablehnung eines Erstantrages gestellt werden, werden seit Inkrafttreten des Zuwanderungsgesetzes keine so genannten ,selbstgeschaffenen Nachfluchtgründe' mehr anerkannt - das heißt, exilpolitische Tätigkeiten nach der Flucht spielen bei der Feststellung, ob jemandem politische Verfolgung droht, de facto keine Rolle mehr.

Die Einschätzung von Bade und Oltmer (2007, S. 168): „Beim Asylrecht wurden der ungewisse Status der Duldung und damit auch die de facto oft eintretenden Kettenduldungen abgeschafft" - wie es auch von Otto Schily angekündigt wurde $^{207}$ - trifft allerdings nicht zu. Denn neben der Einmündung der bisherigen Fülle von Aufenthaltsstatus in die (dauerhafte) Niederlassungs-, die (befristete) Aufenthaltserlaubnis, die Aufenthaltsgestattung für Asylsuchende sowie das Visum, besteht die Duldung als „,vorübergehende Aussetzung der Abschiebung“ weiter fort ( $\int 60$ a AufenthG). ${ }^{208}$ Zwar sollen Personen, die aufgrund humanitärer oder persönlicher Härten nicht abgeschoben werden können, eine Aufenthaltserlaubnis statt einer Duldung erhalten ( $\int 25$ Abs. 4 AufenthG) - es handelt sich jedoch überwiegend um Kann-Bestimmungen. Einschränkend heißt es, dass eine Aufenthaltserlaubnis nicht ausgestellt wird, wenn die ,freiwillige Ausreise' möglich ist oder ,der Ausländer das Ausreisehindernis selbst zu vertreten hat' (Bundesmi-

\footnotetext{
206 Die Widerrufsverfahren haben bereits vor dem Inkrafttreten des Zuwanderungsgesetzes stark an Bedeutung gewonnen: So hat die Zahl der Aberkennungen die Zahl der Anerkennungen in den vergangenen Jahren bereits überstiegen. So standen im Jahr 2003 8.345 Widerrufen 3.136 Anerkennungen nach Art. 16a GG und \51 AuslG gegenüber, im Jahr 2004 verschärfte sich dieses Verhältnis (14.972 Widerrufe und 2.067 Anerkennungen). Dabei sind insbesondere Flüchtlinge aus dem Kosovo und dem Irak betroffen (vgl. Pro Asyl 2005). Ein Rechtsgutachten des UNHCR kommt zu dem Schluss, dass die Widerrufspraxis in der Bundesrepublik - insbesondere in Bezug auf irakische Flüchtlinge - nicht mit der GFK und der EU-Qualifikationsrichtlinie vereinbar ist (vgl. UNHCR 2008c).

207 So verkündete Otto Schily am 01.07.2004 bei der Bundestagsdebatte zum Abschluss der Verhandlungen über das Zuwanderungsgesetz: „Dass wir die Kettenduldungen, die mit Recht immer als besonders schlimmer Zustand angeprangert wurden, abschaffen, ist, finde ich, ein großer Fortschritt.“ (Plenarprotokoll 15/118, S. 10720)

${ }^{208}$ Die als Vereinfachung dargestellte Regelung, wonach es statt fünf nur noch zwei Aufenthaltstitel geben soll, ist außerdem insofern irreführend, als nun 20 verschiedene Arten von Aufenthaltserlaubnissen entsprechend des Aufenthaltszwecks eingeführt wurden (vgl. Hügel 2004).
} 
nisterium des Innern 2006b, S. 83).209 Wie die Praxis zeigt, halten die Ausländerbehörden auf dieser Grundlage sehr zäh an dem gewohnten Vorgehen fest und haben bislang kaum Duldungen in Aufenthaltserlaubnisse umgewandelt. Diese Praxis der Ausländerbehörden wurde im Evaluationsbericht zum Zuwanderungsgesetz durch das - inzwischen CDU-geführte - Innenministerium dokumentiert und gestützt (vgl. Bundesministerium des Innern 2006b, S. 83ff.).

Zudem enthielt das Zuwanderungsgesetz von 2005 keine gesetzliche Bleiberechtsregelung. Eine solche wurde aufgrund nachhaltiger öffentlicher Forderungen zunächst 2007 durch die Innenministerkonferenz beschlossen und schließlich im Zuwanderungsänderungsgesetz als Altfallregelung aufgenommen $(\$ 104 \mathrm{a}, \mathrm{b}$ AufenthG). Erste Ergebnisse zeigen, dass aufgrund der umfangreichen Ausschlusskriterien sowie der Voraussetzungen zur Aufenthaltsdauer und zur selbstständigen Sicherung des Lebensunterhaltes auch mit dieser Regelung nur ein Bruchteil derer legalisiert wird, die sich seit Jahren in prekären Aufenthaltsverhältnissen in der Bundesrepublik befinden. ${ }^{210}$ Entgegen den von Wohlfahrtsverbänden und Kirchen gestellten Forderungen wurde auch keine allgemeine Härtefallregelung in das Gesetz aufgenommen. Die Bundesländer erhalten allerdings die Möglichkeit, Härtefallkommissionen einzurichten ( $\mathbb{2 3 a}$ AufenthG). Die Härtefallkommission kann die oberste Landesbehörde um die Erteilung einer Aufenthaltserlaubnis ersuchen. Ihre Zusammensetzung, die Ausschlussgründe sowie die Entscheidungspraxis sind in den einzelnen Bundesländern unterschiedlich. Dies spiegelt sich auch in den stark variierenden Zahlen zwischen den Bundesländern sowohl die Zahlen der zur Entscheidung angenommenen ,Fälle‘ als auch der positiv entschiedenen Anträge differieren erheblich (vgl. Bundesministerium des Innern 2006b, S. 85ff.).

Neben dem oben angesprochenen weiten Ermessensspielraum bei der Entscheidung über die Ausstellung von Aufenthaltserlaubnissen haben die Ausländerbehörden auch in anderen Bereichen zahlreiche Entscheidungsfreiheiten, da verschiedene Klauseln zur Bewertung des Wohlverhaltens der AntragstellerInnen durch die Behörde in das Gesetz aufgenommen wurden. Leistungen nach dem

${ }^{209}$ So soll eine Aufenthaltserlaubnis ,aus humanitären Gründen“ im Falle eines Abschiebungsverbotes ( $\ 25$ Abs. 3 AufenthG) nicht erteilt werden, wenn „der Ausländer wiederholt oder gröblich gegen entsprechende Mitwirkungspflichten verstößt". Eine Aufenthaltserlaubnis aufgrund von Ausreisehindernissen darf nur erteilt werden ,wenn der Ausländer unverschuldet an der Ausreise gehindert ist" ( $\$ 25$ Abs. 5). Zudem sind einige Gruppen weiterhin von vornherein von der Erteilung der Aufenthaltserlaubnis ausgeschlossen - zum Beispiel diejenigen, deren Antrag als „offensichtlich unbegründet" abgelehnt wurde ( $\mathbb{1} 10$ Abs. 3 AufenthG).

210 Ende Oktober 2006 lebten knapp 180.000 Geduldete bereits seit sechs bzw. acht Jahren (zeitliche Voraussetzung der IMK-Bleiberechtsregelung) in der Bundesrepublik. Davon erhielten knapp 20.000 nach dem IMK-Beschluss eine Aufenthaltserlaubnis. Im Jahr 2007 erhielten nach der gesetzlichen Altfallregelung insgesamt 11.765 AntragstellerInnen eine Aufenthaltserlaubnis, davon allerdings 9.088 Personen nur ,auf Probe', da sie die Voraussetzungen für eine vollständig eigenständige Sicherung des Lebensunterhaltes durch Erwerbstätigkeit nicht erfüllten (vgl. BT Drs. $16 / 8362 ; 16 / 7089)$. 
Asylbewerberleistungsgesetz erhalten Leistungsberechtigte nur, wenn es „unabweisbar geboten ist“, falls sie „sich in den Geltungsbereich dieses Gesetzes begeben haben, um Leistungen nach diesem Gesetz zu erlangen“, oder wenn „,aus von ihnen zu vertretenden Gründen aufenthaltsbeendende Maßnahmen nicht vollzogen werden können“ ( $\ 1$ a AsylbLG). Des Weiteren erhalten nur diejenigen nach drei (bzw. mit den Zuw ÄndG vier) Jahren Leistungen nach dem Sozialhilfegesetz, die „die Dauer des Aufenthalts nicht rechtsmissbräuchlich selbst beeinflusst haben“ ( 2 AsylbLG Abs. 1). Pro Asyl (2004) weist darauf hin, dass häufig schon die Ausschöpfung aller Rechtsmittel öffentlich als ,rechtsmissbräuchliches ${ }^{6}$ Verhalten dargestellt wird. All diese Regelungen bieten also einerseits weiten Interpretationsspielraum für die Behörden und verlangen andererseits von den Betroffenen die ständige aktive Mitwirkung an der eigenen Abschiebung. In der Praxis führt das regelmäßig zu einem Kampf um Deutungen und Interpretationen zwischen Behörden, AnwältInnen, Gerichten und Betroffenen.

Des Weiteren werden die bis dato nur in der Form von ,Modellprojekten“ existierenden ,Ausreisezentren‘ auf eine gesetzliche Grundlage gestellt. In \61 Abs. 2 AufenthG heißt es:

„,[...] In den Ausreiseeinrichtungen soll durch Betreunng und Beratung die Bereitschaft zur freiwilligen Ausreise gefördert und die Erreichbarkeit für Behörden und Gerichte sowie die Durchführung der Ausreise gesichert werden. "211

Ferner wurde der Kreis derjenigen, die unter das Asylbewerberleistungsgesetz fallen, erweitert. Danach bekommen auch diejenigen nur noch gekürzte Sozialleistungen - oft in Form von Sachleistungen -, die nach $\int 25$ Abs. 4 Satz 1 und 5 AufenthG ein Aufenthaltsrecht erhalten haben (also teilweise auch diejenigen, die eine Aufenthaltserlaubnis aus humanitären Gründen erhalten). Die Dauer des Leistungsbezugs nach AsylbLG wurde von drei auf vier Jahre erweitert ${ }^{212}$. Nach Ablauf dieser Frist sollen die Betroffenen allgemeine Sozialleistungen erhalten, sofern sie - wie oben bereits angesprochen - „die Dauer des Aufenthalts nicht rechtsmissbräuchlich selbst beeinflusst haben“ (\$ 2 Abs. 1 AsylbLG).

Die wenigen rechtlichen Verbesserungen unterliegen also in der Praxis dem Interpretationsspielraum der Bundesländer und der Ausländerbehörden (Anerkennung nichtstaatlicher und geschlechtsspezifischer Verfolgung, Ersetzung der Duldung durch eine Aufenthaltserlaubnis, Entscheidungen über ,Härtefälle). Die übrigen Änderungen bewegen sich im Wesentlichen im Bereich des bekannten Maßnahmenrepertoires, das erneut verschärft wurde - die Ausweitung der Lagerunterbringung (,Ausreisezentren'), die Verschlechterung der sozialen Lage (erweiterter Kreis von BezieherInnen und längerer Bezugs abgesenkter Sozialleistungen)

\footnotetext{
${ }^{211}$ Vgl. dazu auch im Abschnitt 5.3.3 S. 195ff., Unterbringung in Lagern, Residenzpflicht und Inhaftierung,.

212 Dies geschah allerdings erst mit der am 28. August 2007 in Kraft getretenen Änderung des Asylbewerberleistungsgesetzes.
} 
sowie die Einschränkung von Perspektiven (routinemäßige Widerrufsverfahren). Auch hier gibt es jeweils weite Auslegungs- und Handlungsspielräume für die Behörden.

\section{Zuwanderungsänderungsgesetz}

Die mit dem Zuwanderungsgesetz vorgezeichnete Linie setzt sich im Zuwanderungsänderungsgesetz, das 2007 verabschiedet wurde, fort. Offiziell geht es um die Umsetzung von elf EU-Richtlinien in nationales Recht. Diese Umsetzung ist jedoch nur unvollständig erfolgt oder die Mindestvorgaben werden durch das deutsche Recht unterschritten (vgl. Hoffmann 2007). Letzteres ist insbesondere im Hinblick auf die Versorgung hilfsbedürftiger und traumatisierter Personen und die medizinische Versorgung von Asylsuchenden zutreffend. Hierzu enthalten die Richtlinien günstigere Regelungen als das Zuwanderungsgesetz (vgl. Hoffmann 2007).

Zudem wurde der Grenzbehörde die Aufgabe übertragen denjenigen AsylantragstellerInnen die Einreise zu verweigern, die über einen sicheren Drittstaat oder einen gemäß Dublin-II-Verordnung zuständigen Staat eingereist sind ( $\mathbb{1} 18$ Abs. 2 AsylfG). Die Feststellung dieses Tatbestands obliegt ebenfalls der Grenzbehörde, bei der der Antrag gestellt wird. Parallel dazu wurden die Inhaftierungsbestimmungen ausgeweitet: Während Haft für AsylbewerberInnen bislang nur in Ausnahmefällen und maximal für vier Wochen verhängt werden durfte, können sie nun für die gesamte Zeit, in der der laut Dublin-II-Verordnung zuständige Staat ermittelt wird, in Haft genommen werden ( $\$ 14$ Abs. 3 AsylVfG), einstweiliger Rechtsschutz ist ausgeschlossen ( $\$ 34 a$ AsylVfG). Die Möglichkeit der freiwilligen Ausreise, wie sie in der Dublin-II-Verordnung selbst vorgesehen ist, besteht nach dem Asylverfahrensgesetz aufgrund dieser Inhaftierungsregel nicht, und wird zusätzlich durch \34a Abs. 1 AsylVfG verhindert, in dem es heißt: „Einer vorherigen Androhung und Fristsetzung [der Abschiebung] bedarf es nicht“".

Neben diesen (lediglich beispielhaft aufgeführten) Regelungen hat es im Zuwanderungsänderungsgesetz auch zahlreiche Verschärfungen gegeben, die nicht im Zusammenhang mit den EU-Richtlinien stehen. So wurde der Nachzug von EhegattInnen aus bestimmten Herkunftsländern erschwert. Nach $\int 27$ Abs. 1a Satz 1 Aufenthaltsgesetz soll der Familienachzug verweigert werden, wenn ,feststeht, dass die Ehe oder das Verwandtschaftsverhältnis ausschließlich zu dem Zweck geschlossen oder begründet wurde, dem Nachziehenden die Einreise in das und den Aufenthalt im Bundesgebiet zu ermöglichen“. Zudem müssen Nachziehende nun bereits vor der Einreise einfache Deutschkenntnisse nachweisen dies gilt ausdrücklich nicht für Personen bspw. aus den USA, Kanada, Israel und Japan. Begründet wird dies mit dem Ziel, ,Zwangsheiraten' verhindern zu wol- 
len. ${ }^{213}$ Die Frauenrechtsorganisation Terre des femmes kritisiert dies als Vorwand, um weitere einwanderungsrechtliche Verschärfungen durchzusetzen, während tatsächliche Maßnahmen zur Stärkung der Rechte von Migrantinnen fehlten oder durch die Regelungen sogar unterlaufen würden (vgl. Terre des femmes 2007). Darüber hinaus wurden die Hürden für die Einbürgerung wieder erhöht.

\section{Zwischenfazit: ,Steuern' gegen den ,Missbrauch'}

Das Zuwanderungsgesetz und das Zuwanderungsänderungsgesetz zeigen das Ringen der EntscheidungsträgerInnen um eine ,tatsächliche' Steuerung der Einwanderung, das aber immer wieder am ,missbräuchlichen Verhalten` der Einwanderungswilligen scheitert. Schon die Empfehlungen der Süssmuth-Kommission, die eine Liste ,missbräuchlicher Verhaltensweisen` aufgestellt und jeweils mit Vorschlägen für Gegenmaßnahmen versehen hatte, illustrieren diese Bemühungen. Und auch mit dem Zuwanderungsgesetz wird versucht, verbliebene ,Schlupflöcher ${ }^{6}$ im Einwanderungsrecht zu stopfen, durch welche, unerwünschte ${ }^{6}$ MigrantInnen einen Aufenthalt in der Bundesrepublik organisieren konnten. Die Rede vom Missbrauch, die auch hier - ähnlich wie zum Beispiel in der Debatte über Arbeitslose - die im öffentlichen Diskurs zentrale Unterscheidung, guter ${ }^{\varsigma}$ und ,schlechter Ausländer' hervorbringt, muss in zweierlei Hinsicht gewendet werden: Zum einen ist auf das von Pro Asyl angeführte Argument zu verweisen, wonach zum Beispiel auch die Ausschöpfung des Rechtsweges häufig bereits einen Missbrauchsverdacht mit sich bringt. Zum anderen sind auch die als ,missbräuchliches Verhalten' bezeichnete Vernichtung von Papieren, das Untertauchen vor der Abschiebung oder das Heiraten, um bleiben zu können, nichts anderes als der Ausdruck der „Eigensinnigkeit von Migration“ (Benz/Schwenken 2005, S. 374), die den Steuerungsversuchen zuwiderläuft. Es handelt sich um Strategien von MigrantInnen, die sich aufgrund eines komplexen Geflechts von Motiven der Beschränkung ihrer Bewegungsfreiheit widersetzen. So beklagte Peter Müller (CDU) in seinem Positionspapier vom Juli 2001:

, [T] rotz restriktiver Bestimmungen und Kontrollen ist es bisher nicht gelungen, das unkoordinierte und weitgehend ungesteuerte Nebeneinander unterschiedlichster Zuwanderungsgruppen zurück zuführen, geschweige denn in einem bedarfsgerechten, arbeitsmark.tund sozialverträglichen, Gesamtkonzept der Einwanderung aufgehen zu lassen. Die Gesamtschau der Einwanderungspolitik in Deutschland ergibt vielmehr ein unbefriedigendes Missverhältnis der erwünschten gegenüber eher unerwünschten Zuwanderungstatbeständen“. (Müller 2001, S. 7)214

\footnotetext{
${ }^{213}$ Den Vorwurf der Diskriminierung weist die Bundesregierung mit folgender Begründung zurück: „Die Privilegierung ist dadurch gerechtfertigt, dass der Zuzug der Angehörigen dieser Staaten im besonderen migrationspolitischen Interesse Deutschlands liegt." (BT Drs. 16/5498)

214 Speziell zum Asylbereich illustriert Peter Müller (2001, S. 13), ganz ähnlich wie die SüssmuthKommission, den gap zwischen ,policy-Output und ,policy-Outcome 6 , der auf die eigensinnigen Praxen
} 
Das Zitat zeigt, dass auch bei einigen UnionspolitikerInnen ein Wandel von Strategien der Abschottung hin zur Steuerungsperspektive stattgefunden hat, mit der versucht wird, Einwanderung zum Nutzen der Bundesrepublik zu kanalisieren. Wie schon von der Zuwanderungskommission unter Rita Süssmuth vorgegeben, wird auch das Asylrecht unter die zentrale Prämisse wirtschafts- und arbeitsmarktpolitischer Zielsetzungen subsumiert. Während es in den ersten Jahren seines Bestehens kaum denkbar war, dieses Recht infrage zu stellen, wurde ab Anfang der 1970er Jahre mit den gestiegenen AsylbewerberInnenzahlen argumentiert, die die ,Belastungsgrenze ‘ der Bundesrepublik überschreiten würden - eine Argumentation, mit der das Asylrecht 1993 drastisch eingeschränkt werden konnte. Rund sechs Jahre später wird Einwanderung für notwendig erklärt - im Interesse der Wettbewerbsfähigkeit der Bundesrepublik. Zwar ist die zuvor in der Diskussion so zentral gestellte Zahl der AsylbewerberInnen drastisch zurückgegangen. Doch nun werden die verbliebenen AsylbewerberInnen daraufhin überprüft, inwieweit sie etwas zum Standortwettbewerb beitragen können. Mit der Begründung, AsylbewerberInnen seien unqualifiziert und daher unter diesen Gesichtspunkten nicht im nationalen Interesse der Bundesrepublik, wurde eine neue Argumentationslinie in das asylpolitische Aushandlungsfeld eingeführt. Dabei handelt es sich nicht um eine spezifisch bundesrepublikanische Erscheinung:
„Während die entwickelten Industrienationen der Triade [...] gut ausgebildete Fach- bzw. Führungskräfte aus aller Herren Länder im ,Kampf um die besten Köpfe' zu ge- winnen suchen, gilt Armutsmigration bzw. Flucht den Bewohnern solcher reichen Länder als ,Standortnachteil', den man tunlichst zu vermeiden oder wenigstens zu verringern sucht" (Butterwegge 2003, S. 67).

Unter diesen Vorzeichen findet sich unter den EntscheidungsträgerInnen kaum noch jemand, der die Kämpfe von Asylsuchenden zur Kenntnis nimmt und sich ihre Forderungen zu eigen macht: Konservative Forderungen nach allgemeiner Begrenzung der Einwanderung stehen zwar liberalen Ideen einer arbeitsmarktori-

der AsylbewerberInnen zurückgeht, sehr anschaulich: „Kennzeichnend für die bisherige Einwanderungspolitik in Deutschland ist weiterhin das Problem der krassen Diskrepanz zwischen Rechtgebung und Rechtswirklichkeit, insbesondere im Asylrecht. Wir haben es bisher zugelassen, dass hier eine weitgehend ungesteuerte Einwanderung stattfindet, weil die Rückführung illegal eingereister Flüchtlinge und abgelehnter Asylbewerber aus tatsächlichen oder rechtlichen Gründen oftmals nicht mehr möglich ist. Hinderungsgründe können auf der einen Seite von dem betroffenen Ausländer selbst verursacht sein (zum Beispiel durch die verbreitete Praxis, Ausweispapiere zu vernichten und damit die wahre Identität/Nationalität des Antragstellers zu verschleiern, um so eine Abschiebung faktisch unmöglich zu machen). Abschiebungshindernisse können aber auch in den Zuständen des Herkunftsstaates begründet liegen.“ Und weiter: „Ein wesentlicher Grund für das Auseinanderklaffen von Anspruch und Wirklichkeit im deutschen Asylrecht ist jedoch die Dauer der verwaltungsrechtlichen und gerichtlichen Verfahren bei der Anerkennung von Asylbewerbern. Eine systematische Verfahrensverzögerung wird zum Beispiel oft dadurch bewirkt, dass innerhalb einer Familie durch nachgeschobene Anträge der Kinder auf Asyl die asylrechtliche Ablehnung der Eltern unterlaufen wird.“ (Müller 2001, S. 13f.) 
entierten Einwanderung gegenüber, einig ist man sich aber, dass AsylbewerberInnen nicht zu den ,Erwünschten“ zählen. Auch aus sozialdemokratischen Kreisen sind - seit der Ära Otto Schily - keine Appelle mehr zu asylpolitischen Liberalisierungen zu vernehmen. Bündnis 90/Die Grünen sind ihrerseits als Koalitionspartner in der Regierung unter Kanzler Gerhard Schröder selbst an der Aushandlung um das Zuwanderungsgesetz beteiligt gewesen. Nachdem sie wieder die Oppositionsrolle eingenommen hatten, stellten sie bei der Verabschiedung des Zuwanderungsänderungsgesetzes verschiedene Forderungen zur Verbesserung der Situation von Asylsuchenden, die jedoch die im Gesetz enthaltenen grundlegenden Strategien im Umgang mit Einwandernden nicht infrage stellten. ${ }^{215}$

Das weiterhin bemühte Schlagwort ,Asylmissbrauch“ und die Rede von den ,Sogwirkungen' des deutschen Sozialsystems halten sich dabei relativ zäh, ungeachtet der im Asylbereich offenkundigen Entwicklungen: Die Zahl der AntragstellerInnen ist massiv zurückgegangen - auf einen Stand, den sie zuletzt zu Beginn der Asyldebatte Anfang der 1970er Jahre hatte. Im europäischen Vergleich lag die Bundesrepublik im Jahr 2006 nur hinsichtlich der absoluten Zahlen an vierter Stelle nach Frankreich, Großbritannien und Schweden. Setzt man die absoluten Zahlen ins Verhältnis zur Bevölkerungszahl verändert sich das Bild: So liegt die Zahl der Asylanträge in Deutschland nur noch an 19. Stelle in Europa (vgl. Bundesamt für Migration und Flüchtlinge 2006) und bereits seit 1999 kontinuierlich deutlich unter dem EU-Durchschnitt (vgl. Tabellen 2 und 3 im Anhang).

\subsubsection{Flüchtlingsschutz zwischen Recht und Praxis}

Vor dem Hintergrund der verschiedenen Etappen, durch die die Asylpolitik der Bundesrepublik in den vergangenen Jahrzehnten geprägt wurde, wird es in den kommenden Abschnitten darum gehen, die aktuelle Gestaltung des Flüchtlingsschutzes im Spannungsfeld zwischen Recht und Praxis genauer auszuleuchten. Dafür wird das Asylverfahren ebenso untersucht wie die sozialen Rechte der Asylsuchenden, wobei die Unterbringung in Lagern, die Regelung der Residenzpflicht und die Abschiebehaft sowie das Instrument der Abschiebung genauer in den Blick genommen werden. Im Anschluss daran werden die zentralen Kennzeichen des deutschen Asylregimes herausgearbeitet.

\section{Asylverfahren}

Wird ein Asylantrag in der Bundesrepublik gestellt, wird er in Bezug auf zwei Status geprüft: Die Anerkennung als Asylberechtigter nach dem Grundgesetz oder

\footnotetext{
215 Vgl. das Statement des migrationspolitischen Sprechers von Bündnis 90/Die Grünen, Josef Winkler, zur Abschiebehaft in der Debatte um das Zuwanderungsänderungsgesetz: „Wir vertreten die Position, dass Abschiebehaft lediglich der Sicherung einer Abschiebung dienen darf. Das heißt, nur dann, wenn sich jemand der Abschiebung erkennbar entziehen will, darf Abschiebehaft verhängt werden.“ (Winkler 2008)
} 
die Zuerkennung der Flüchtlingseigenschaft nach der GFK ( $\$ 13$ AsylVfG). Dabei wird - als Ergebnis der Umsetzung der EU-Qualifikationsrichtlinie - der Begriff der Verfolgung im AufenthG dahingehend konkretisiert, dass eine „Verfolgung wegen der Zugehörigkeit zu einer bestimmten sozialen Gruppe" auch dann vorliegen kann, „wenn die Bedrohung des Lebens, der körperlichen Unversehrtheit oder der Freiheit allein an das Geschlecht anknüpft" und dass die Verfolgung auch von nichtstaatlichen Akteuren ausgehen kann ( $\$ 60$ Abs. 1 AufenthG). Damit wurde nach Ansicht vieler Flüchtlingsorganisationen zumindest formal eine große Schutzlücke geschlossen. Zwar war durch die Rechtsprechung, einschließlich des Bundesverwaltungsgerichts, bereits vor dem Inkrafttreten des Zuwanderungsgesetzes verschiedentlich anerkannt worden, dass Verfolgung aufgrund des Geschlechts zu einer asylrechtlichen Anerkennung führen kann, in der Praxis setzte sich die Ansicht jedoch nicht einheitlich durch (vgl. Pelzer/Pennington 2006). Allerdings gibt es auch nach der gesetzlichen Klarstellung noch Probleme bei der Umsetzung in die Praxis: So ist das Bundesamt der Ansicht, dass eine geschlechtsspezifische Verfolgung nur dann gegeben ist, wenn sie alle Frauen zum Beispiel eines Staates oder einer Ethnie betrifft. Andernfalls könne nicht von der Anknüpfung an eine ,soziale Gruppe‘ ausgegangen werden (vgl. Pelzer/Pennington 2006; Pro Asyl 2006a, S. 29). Auch die Ausweitung auf nichtstaatliche Verfolger erweist sich laut Pro Asyl in der Praxis als schwierig: So legen manche Gerichte das Kriterium an, die nichtstaatliche Verfolgung müsse von Gruppen ausgehen, die dem Staat, Parteien oder Organisationen ähnlich sind. Damit wird Verfolgung, die aus dem familiären Umfeld heraus stattfindet, nicht unter nichtstaatliche Verfolgung subsumiert. So wird zum Beispiel Gewalt gegen Frauen in traditioneller Sphärentrennung zwischen Öffentlichkeit und Privatheit als Privatsache abgetan und asylrechtlich letztlich wieder auf die herkömmliche „Zurechnungslehre“ abgestellt (vgl. Pelzer/Pennington 2006; Pro Asyl 2006a, S. 30).216

Doch bevor sich das Bundesamt und Gerichte mit einem Asylantrag befassen, muss zunächst einmal die Asylantragstellung gelingen. Wie bereits oben angesprochen, gibt es zahlreiche Hürden, die eine Einreise in die Bundesrepublik mit Hilfe eines Asylantrages verhindern. So sind auf dem Luftweg Eingereiste mit dem Flughafenverfahren konfrontiert. Die Anträge derer, die aus einem, sicheren Herkunftsstaat' per Flugzeug einreisen, sollen vor der Entscheidung über die Einreise geprüft werden ( $(18 \mathrm{a}$ AsylVfG). Wird der Antrag als ,offensichtlich unbegründet" abgelehnt, wird die Einreise in die Bundesrepublik verweigert. Reist man auf dem Landweg ein, greift häufig die „sichere Drittstaatenregelung“ ( $(26$ a AsylVfG) - der Antragsteller soll, wenn er auf seinem Reiseweg einen als sicher deklarierten Staat durchquert hat, dorthin zurückgewiesen werden. Der Antrag gilt dann als ,unbeachtlich“ (\$29).

216 Pro Asyl führt hier ein Urteil des VG Regensburg vom 17.01.2005 an. 
Ergänzt wird das Konzept der sicheren Herkunfts- und Drittstaaten um die Dublin-II-Verordnung der EU. Die EU-Mitgliedstaaten sind nicht nur alle als sicher deklariert, sondern mittels der Dublin-II-Verordnung ist auch die Zuständigkeit für Asylanträge genau geregelt (vgl. das Kapitel 4 Asylpolitik der EU). In den vergangenen zehn Jahren ist der Anteil der Dublin-Fälle an den Asylerstanträgen kontinuierlich gestiegen - von 3,5 Prozent im Jahr 1998 auf 28,1 Prozent im Jahr 2007 (vgl. Pro Asyl 2008, S. 6). Ein bedeutender Teil der Asylanträge, die in der Bundesrepublik gestellt werden, werden also als Dublin-Fälle nicht zur Prüfung angenommen. Die AsylbewerberInnen können bis zur Klärung der Zuständigkeit in ,Zurückweisungshaft ' genommen werden. Obwohl es zwischenzeitlich Differenzen über die asylrechtlichen und -faktischen Bedingungen in zwei Mitgliedstaaten gab - Norwegen hatte die Überstellung von Asylsuchenden nach Griechenland, Frankreich die Überstellung von TschetschenInnen nach Polen vorübergehend eingestellt - zeigt sich die Große Koalition unter Kanzlerin Angela Merkel von der Verordnung überzeugt (vgl. BT Drs. 16/8861). ${ }^{217}$ Auch die vielfach geäußerte Kritik, es handele sich bei dieser Zuständigkeitsregelung um eine Abwälzung asylpolitischer Aufgaben an die Länder an den Außengrenzen, teilt die Bundesregierung nicht:

„Aus Sicht der Bundesregierung haben sich die Vorschriften zu Solidarität und Verantwortungsteilung unter den Mitgliedstaaten bei der Aufnabme von Flüchtlingen grundsätzlich bewährt. "(BT Drs. 16/8861, S. 3)

Dass inzwischen das Verhältnis von Anträgen auf Rücküberstellung an die Bundesrepublik, denen die Bundesrepublik zugestimmt hat und von Anträgen der Bundesrepublik an andere Mitgliedstaaten, denen diese zugestimmt haben, fast ausgeglichen ist (vgl. Tabelle $4 \mathrm{im}$ Anhang), wird ebenfalls nicht als Ausdruck einer unnötigen Verordnung interpretiert, auf den mit einem Überstellungsstopp reagiert werden könnte: „Soweit erkennbar, stünde ein Saldierungsmechanismus zumindest im Spannungsverhältnis zu den objektiven Zuständigkeitskriterien der Dublin-Verordnung“ (BT Drs. 16/8861, S. 8). Dass der enorme bürokratische Aufwand zudem letztlich in einer vergleichsweise geringen Zahl an tatsächlichen Rücküberstellungen mündet, lässt sich an den Zahlen seit 2004 ablesen (vgl. Tabelle 5 im Anhang). Die Bundesregierung spricht dennoch von „Einzelfällen“, in

\footnotetext{
217 Die Bundesregierung hatte die Rücküberstellungen nach Griechenland nicht eingestellt, obwohl dort nach Rücküberstellung keine inhaltliche Überprüfung des Asylantrags mehr stattfand. Die EU-Kommission hatte im Januar 2008 entschieden, gegen Griechenland ein Vertragsverletzungsverfahren vor dem EuGH einzuleiten. Die Bundesregierung geht stattdessen grundsätzlich von der Einhaltung der Menschenrechte in den übrigen Mitgliedstaaten aus: Sie argumentiert, „dass die Menschenrechte in den Mitgliedstaaten der Europäischen Union, die als Rechts- und Wertegemeinschaft verfasst ist, eingehalten werden, sodass insoweit nicht gegenseitig Menschenrechtsverletzungen unterstellt werden sollten.“ (BT Drs. 16/8861) Und weiter heißt es: „Im Übrigen entspricht es der Politik der Bundesregierung, sich gegenüber Staaten, in denen die Menschenrechte verletzt werden, nachdrücklich für die Einhaltung der Menschenrechte einzusetzen.“ (ebd.)
} 
denen Rücküberstellungen nicht möglich sind (vgl. BT Drs. 16/8861, S. 10). Von dem so genannten Selbsteintrittsrecht ${ }^{218}$ macht die Bundesrepublik gleichwohl nur sehr selten Gebrauch - konkrete Zahlen hierzu existieren allerdings nicht (vgl. BT Drs. 16/8861, S. 11).

Wird ein Antrag zur Prüfung angenommen, kann er als „offensichtlich unbegründet" abgelehnt werden, wenn die „Voraussetzungen für die Zuerkennung der Flüchtlingseigenschaft offensichtlich nicht vorliegen“, das heißt im Einzelnen, wenn

- jemand „nur aus wirtschaftlichen Gründen oder um einer allgemeinen Notsituation oder einer kriegerischen Auseinandersetzung zu entgehen“ Zuflucht gesucht hat,

- der Vortrag über die Fluchtgründe „widersprüchlich“, „nicht substantiiert“ und auf gefälschte Beweise gestützt ist,

- jemand „über seine Identität oder Staatsangehörigkeit täuscht“, mit anderen Personalien einen weiteren Antrag gestellt hat, der Antrag nur gestellt wurde, um einer Abschiebung zu entgehen oder Mitwirkungspflichten verletzt wurden, usw.

Die Fluchtgründe müssen also bei der Anhörung vollständig, substantiiert und kohärent dargestellt werden - später vorgebrachte Aspekte werden nicht berücksichtigt ( $\int 25$ AsylVfG). Ebenfalls unberücksichtigt bleiben so genannte „Nachfluchttatbestände“, also wenn die Verfolgungsgefahr nach Ansicht der EntscheiderInnen ,auf Umständen beruht, die er nach Verlassen seines Herkunftslandes aus eigenem Entschluß geschaffen hat, es sei denn, dieser Entschluß entspricht einer festen, bereits im Herkunftsland erkennbar betätigten Überzeugung" (\ 28 Abs. 1 AsylVfG). Im Asylfolgeverfahren werden Nachfluchtgründe grundsätzlich nicht anerkannt ( $\int 28$ Abs. 2 AsylVfG). Exilpolitische Tätigkeiten, aufgrund derer jemand nach der Rückkehr Verfolgung fürchtet, werden also aus der Flüchtlingsanerkennung ausgeklammert. Diese Ausklammerung ist hinsichtlich ihrer Vereinbarkeit mit der Genfer Flüchtlingskonvention umstritten: In der Rechtsprechung wurde bislang sowohl in die eine (Vereinbarkeit) als auch in die andere Richtung (Unvereinbarkeit) argumentiert (vgl. Pro Asyl 2006a, S. 40). Pro Asyl unterstreicht, dass die GFK nicht danach fragt, „ob die Verfolgung durch eigenes Handeln ,provoziert' oder wo und wann sie entstanden ist, sondern fragt nur nach der Schutzbedürftigkeit des Flüchtlings“ (ebd.).

Wird der Antrag als „unbeachtlich“ oder „offensichtlich unbegründet“ klassifiziert, gilt eine Ausreisefrist von nur einer Woche (\$36 Abs. 1 AsylVfG).

Die AntragstellerInnen erhalten für die Dauer des Verfahrens eine „Aufenthaltsgestattung“ und sind verpflichtet, bis zu drei Monaten in einer Erstaufnahmeeinrichtung zu wohnen. Von dort aus sollen sie in „Gemeinschaftsunterkünfte“ umverteilt werden ( $\int 53$ AsylVfG). Die Aufenthaltsgestattung ist räumlich auf den

218 Vgl. Art. 3 Abs. 2 Dublin-II-Verordnung. 
Bezirk der Ausländerbehörde beschränkt, der nur mit Erlaubnis verlassen werden darf ( $\int 56,58$ AsylVfG). Diese räumliche Beschränkung - die so genannte Residenzpflicht - kann auch mit Zwang durchgesetzt werden ( $\int 59$ AsylVfG). In der Praxis führt dies dazu, dass Flüchtlinge, die Freunde oder Verwandte in anderen Bezirken besuchen, an (politischen) Veranstaltungen teilnehmen oder der Isolation und Abgeschiedenheit vieler Gemeinschaftsunterkünfte entkommen wollen, entweder einen bürokratischen Hürdenlauf um eine Erlaubnis auf sich nehmen müssen oder gegen die Residenzpflicht verstoßen. Bei mehrmaligem Verstoß gegen die Residenzpflicht drohen entweder Freiheitsstrafe bis zu einem Jahr ( $\int 85$ Satz 2 AsylVfG) oder ein Bußgeld bis zu 2.500 Euro (\$ 86 AsylVfG). Durch das Arbeitsverbot und den Sachleistungsvorrang können Flüchtlinge solche Bußgelder auf legalem Wege nur durch ,gemeinnützige Arbeit‘ abbezahlen, die mit einem Euro pro Stunde ,abgegolten' wird - für die Verletzung der Residenzpflicht wird so nicht selten ein monatelanger Arbeitseinsatz fällig. Zudem

„geht der Verstoß gegen die Residenapflicht und andere ausländerrechtliche Regelungen tatsächlich als eine Form der, Ausländerkriminalität' in die Statistik ein. Vorurteile gegen angeblich ,kriminelle Ausländer' werden damit scheinbar bestätigt, obwobl der Verstoß gegen die Residenzpflicht ein Deliket ist, das Deutsche gar nicht begehen können." (Kothen 2002, S. 57)

Die Residenzpflicht hat bereits eine längere Geschichte in Deutschland: Schon in der Ausländerpolizeiverordnung von 1938 war eine solche Regelung zu finden. Inzwischen hat die Bundesrepublik dafür gesorgt, dass die Residenzpflicht in die EU-Aufnahmerichtlinie (Art. 7) aufgenommen wurde und so als Instrument allen Mitgliedstaaten offen steht. Rund um die Residenzpflicht gibt es immer wieder Proteste und Widerstandsaktionen vor allem von betroffenen MigrantInnen.

In der Erstaufnahmeeinrichtung und im gesamten ersten Jahr des Aufenthaltes gilt ein Arbeitsverbot, das erst nach Ablauf dieses Jahres durch eine Beschäftigungserlaubnis aufgehoben werden kann. Diese Erlaubnis erteilen nicht mehr die Arbeitsämter, sondern die Ausländerbehörden - die damit nun für die Erteilung des Aufenthaltstitels und der Beschäftigungserlaubnis zuständig sind. In neuer bürokratietheoretischer Semantik nennt sich diese Zusammenlegung, One-StopGovernment'. In der Praxis werden AsylbewerberInnen damit auch beim Zugang zum Arbeitsmarkt vollständig dem Gutdünken der Ausländerbehörden überlassen, die nach Ansicht von Pro Asyl ,ihre neuen Kompetenzen zur Gestaltung einer restriktiven Anwendungspraxis missbrauchen“ (Pro Asyl 2006a, S. 22).

In den seltenen Fällen, in denen jemand als asylberechtigt oder als Konventionsflüchtling anerkannt wird, besteht dieser Status nicht zwangsläufig auf Dauer. Es wird eine Aufenthaltserlaubnis erteilt. Eine unbefristete Niederlassungserlaubnis - wie sie vor dem Inkrafttreten des Zuwanderungsgesetzes die Asylberechtigten erhielten - ist erst nach drei Jahren vorgesehen. Davor ist jedoch eine weitere Hürde geschaltet worden. Während bereits in den Jahren vor dem Inkrafttreten 
des Zuwanderungsgesetzes die Zahl der Widerrufe der Anerkennungen in die Höhe geschnellt ist, wurde diese Praxis nun als routinemäßige Überprüfung gesetzlich verankert ( $\$ 73$ AsylVfG). Spätestens nach drei Jahren soll das Bundesamt das Fortbestehen der Fluchtgründe überprüfen (Regelprüfung). Dies hängt auch damit zusammen, dass nach einer drei Jahre andauernden Verfolgungssituation eigentlich eine Niederlassungserlaubnis gewährt werden soll (\$ 26 Abs. 3 AufenthG) - will die Ausländerbehörde eine solche vergeben, muss sie zunächst das Fortbestehen des flüchtlingsrechtlichen Status beim Bundesamt erfragen.

Das Asylverfahrensgesetz enthält außerdem eine Reihe von Strafvorschriften. In verschiedenen Abstufungen wird dort unter Strafe gestellt, wer jemanden dazu verleitet im Asylverfahren unrichtige oder unvollständige Angaben zu machen, um damit die Erlangung eines Aufenthaltstitels zu ermöglichen. Die Strafen reichen von Geldstrafen bis hin zu mehrjährigen Haftstrafen ( $\$ \int 84 f$. AsylVfG). Der Verstoß gegen die Aufenthaltsbeschränkung wird als Ordnungswidrigkeit mit einer Geldbuße geahndet ( $(86$ AsylVfG). Schließlich wird klargestellt, dass mit dem Asylverfahrensgesetz Grundrechte eingeschränkt werden: die körperliche Unversehrtheit und die Freiheit der Person ( $\$ 89)$. Die Durchsetzung einwanderungspolitischer Ziele wird also höher gewertet als die Wahrung dieser Grundrechte.

In der Praxis ist das Asylverfahren nach Ansicht von KritikerInnen

„zum ,Ort eines verdichteten Misstrauens' geworden. Das staatliche Interesse an einer Abschiebung der Flüchtlinge überlagert das Prüfungsverfabren bis in die in der Anbörung gestellten Fragen binein. "(Pro Asyl 2006a, S. 34)

In einem Memorandum hat Pro Asyl (2005) die Mängel der Anerkennungspraxis aufgezeigt. Demnach stehe im Mittelpunkt der Befragung nicht die Darstellung der Fluchtursachen, sondern die Klärung des Reiseweges: Um Informationen über Schlepperorganisationen zu erhalten oder einen anderen Staat als zuständig für den jeweiligen Bewerber zu identifizieren, werde zunächst ausführlich untersucht, wie der Antragsteller in die Bundesrepublik gelangt ist.

Darüber hinaus wird kritisiert, dass die Anhörung, ,das Herzstück des Asylverfahrens', und die Formulierung des Bescheides meist zwei verschiedenen BeamtInnen beim Bundesamt obliegen. Viele Bescheide bestünden zu einem großen Teil aus Textbausteinen, also standardisierten Begründungen, die für die individuellen Motive der AntragstellerInnen keinen Raum ließen. Pro Asyl geht davon aus, dass die Anhörung selbst auf die anschließende Verwendung dieser Textbausteine hin fokussiert ist. Die Organisation ist immer wieder auch mit fehlerhaften Entscheidungen konfrontiert:

„Eine Prüfung, ob die handwerkliche Seite des Bescheides stimmt, findet offenbar nicht statt, sonst wäre es nicht erklärlich, dass Bescheide verschickt werden, in denen Textbausteine enthalten sind, die sich offenbar nicht auf den konkreten Fall beriehen, dass im Resümee des Sachverhaltes wesentliche Angaben, die der Antragsteller gemacht hat, nicht mehr zu finden sind" (Pro Asyl 2006a, S. 34). 
Zudem würden die Bescheide häufig auf die Widersprüchlichkeit der gemachten Angaben gestützt, ohne dass die AntragstellerInnen Gelegenheit hatten, diese Widersprüche aufzuklären.

In einer späteren Studie, die 77 Asylverfahren von EritreerInnen untersucht, wird ebenso deutliche Kritik geübt. In vielen der untersuchten Fälle waren die Bescheide des Bundesamtes durch Verwaltungsgerichtsurteile aufgehoben worden - zum Beispiel mit der Begründung, die „Art und Weise der Anhörung“ sei „fragwürdig“ (VG Würzburg, Az. W 7 S 04.30625, zit. n. Welge 2006, S. 124) oder es handele sich um eine „Aneinanderreihung von allgemein gültigen Bausteintexten“ und auf die ausführlich geschilderten Gründe der Antragstellerin sei „so gut wie nicht eingegangen“" worden (VG Darmstadt, Az.4 E 2165/03. A(3), zit. n. Welge 2006, S. 71). ${ }^{219}$ Die Lektüre der dargestellten ,Fälle‘ illustriert die Umsetzung des Asylverfahrensgesetzes durch das Bundesamt sehr anschaulich. Dieses hatte auf die Kritik reagiert, indem es die empirische Basis der Studie von 77 Fällen als zu schmal bezeichnete. ${ }^{220}$

\section{Soziale Rechte: Asylbewerberleistungsgesetz}

Die soziale Schlechterstellung von Asylsuchenden im Vergleich zu anderen Bevölkerungsgruppen wird in der Regel aus heutiger Sicht auf das Inkrafttreten des Asylbewerberleistungsgesetzes (AsylbLG) 1993 datiert. Die meisten der darin enthaltenen Regelungen reichen aber viel weiter zurück. Bereits 1982 wurde eine Reihe von Maßnahmen ergriffen, die auf eine Verschlechterung der sozialen Situation von AsylbewerberInnen abzielten: So sollten Sozialleistungen möglichst als Sachleistungen vergeben werden - laut Wolken (1988, S. 69) „eine Maßnahme, die gegenüber Obdachlosen immer wieder von den Gerichten als rechtswidrig beurteilt worden war“. Damit einher sollte die Unterbringung in ,Gemeinschaftsunterkünften' gehen. Die meisten Kommunen machten in den folgenden Jahren jedoch kaum vom Sachleistungsvorrang und den Möglichkeiten der Kürzung der Sozialhilfe Gebrauch. Nur in Bayern wurden diese Maßnahmen schon damals in relativ großem Umfang angewendet (vgl. Classen 1999). Dem im selben Jahr erlassenen zweijährigen Arbeitsverbot stand auf der anderen Seite ein Zwang zu gemeinnüt-

${ }^{219}$ Im Fazit der Untersuchung heißt es: „Obwohl im Falle der Ablehnung eines Asylantrags als ,offensichtlich unbegründet' eine erschöpfende Ermittlung des Sachverhaltes, damit , an der Richtigkeit der tatsächlichen Feststellungen vernünftigerweise kein Zweifel' mehr besteht, und eine besonders sorgfältige Prüfung und Begründung dieser Beurteilung vorliegen müssten, legen die hier untersuchten Verfahren nahe, dass es gerade dann zu einer Ablehnung als offensichtlich unbegründet kommt, wenn in der Anhörung erhebliche Probleme aufgetreten sind, hier namentlich Dolmetscherprobleme, mangelhafte Sachverhaltsaufklärung, Defizite bei den Anforderungen an ein faires Anhörungsverfahren, massive Missverständnisse zwischen Anhörer und Antragsteller. Diese wirken sich im Falle einer offensichtlich unbegründeten Ablehnung angesichts der damit verbundenen Rechtsfolgen besonders fatal für den Antragsteller aus." (Welge 2006, S. 125, Hervorh. i. Orig.)

220 Es handele sich nur um 2,8 Prozent der 2700 Asylverfahren von Eritreern in dem angesprochenen Zeitraum (vgl. Frankfurter Rundschau vom 16.03.2007). 
ziger Arbeit gegenüber. Wer diese nicht leisten wollte oder konnte, dem sollten die Sozialleistungen weiter gekürzt oder gänzlich gestrichen werden. ${ }^{221}$ Wolfgang Schuth (1988, S. 187) weist auf dieses widersprüchliche Verhältnis von Arbeitsverbot und Arbeitszwang hin:

„Nicht nur für Laien stellt sich die Frage, wie es rechtlich haltbar ist, daß man Menschen auf der einen Seite verbietet, für ibren eigenen Unterhalt aufaukommen, die Arbeit untersagt, und sie auf der anderen Seite im Rabmen der Sozialhilfe zur gzA [gemeinnützigen und zusätzlichen Arbeit, DM] zwingen kann."

Schuth (1988, S. 174f.) verdeutlicht auch, dass hier ein für die Sozialarbeit bis dato unbekanntes Phänomen geschaffen wurde: Deren Ziel, „Selbsthilfemöglichkeiten zu fördern oder zur Entwicklung der Persönlichkeit des Individuums beizutragen “ werde durch die künstlich erzeugte Abhängigkeit der Asylsuchenden von Sozialleistungen ad absurdum geführt. Die für diese Abhängigkeit verantwortlichen Faktoren fasst Georg Classen (1999) wie folgt zusammen: Arbeits- und Ausbildungsverbot und damit verbunden Ausschluss von der Kranken- und Pflegeversicherung, fehlende Sprachförderung, Zwangsumverteilung, Ausschluss von Kinder- und Erziehungsgeld sowie die Unterbringung in Gemeinschaftsunterkünften.

Das entscheidend neue und einschneidende am AsylbLG von 1993 war die Entkoppelung der Sozialleistungen für Asylsuchende vom Bundessozialhilfegesetz (BSHG). Die Leistungen wurden rund 25 Prozent unterhalb des Sozialhilfeniveaus und damit unterhalb dessen angesiedelt, was laut Zwölftem Sozialgesetzbuch „der Würde des Menschen entspricht“"(\1 SGB XII). Indem der Betrieb der ,Gemeinschaftsunterkünfte $^{6}$ und die Versorgung zum Beispiel mit Kantinenessen und Nahrungsmittelpaketen teilweise privatisiert - also an Unternehmen ausgelagert wurden, die auf diesem Wege Gewinne erzielen wollen, wurde das Leistungsniveau faktisch noch weiter abgesenkt. In der Begründung der damaligen Bundesregierung zum Gesetzentwurf heißt es:

„In einem Asylbewerberleistungsgesetz wird die Sicherstellung des Lebensunterhaltes für Asylbewerber den speziellen Bedürfnissen dieser Personengruppe angepasst." (vgl. BT

Drs. 12/4451, S. 1)

${ }^{221}$ Diese Heranziehung zur Arbeit kannte man in den Flüchtlingslagern also schon vor der Einführung der Ein-Euro-Jobs im Rahmen arbeitsmarktpolitischer Aktivierungsmaßnahmen - für eine DMark pro Stunde wurden Flüchtlinge zur gemeinnützigen Arbeit, meist direkt in den Unterkünften, herangezogen. Das Instrument hat - ähnlich wie für EmpfängerInnen des Arbeitslosengelds II oft zwei widerstreitende Bedeutungen für die Flüchtlinge: Einerseits müssen sie - vielfach unter Androhung einer Leistungskürzung - für einen Lohn fern jeglicher tarifvertraglicher Festlegungen arbeiten. Andererseits sind sie durch die niedrigen Sozialleistungen auf die zusätzlichen Einnahmen oft dringend angewiesen. Für Flüchtlinge, die dem Sachleistungsprinzip unterliegen, gilt dies noch mehr als für Arbeitslosengeld-II-BezieherInnen: Die Ein-Euro-Jobs sind oft die einzige legale Möglichkeit, dringend benötigte Dinge des täglichen Bedarfs finanzieren zu können. 
Darüber hinaus wurden die Leistungen seit Inkrafttreten des Gesetzes 1993 trotz Anstiegs der allgemeinen Verbraucherpreise um über 20 Prozent nicht mehr erhöht (vgl. BT Drs. 16/7574). Sie liegen daher inzwischen bei rund 35 Prozent unter dem Sozialhilfeniveau (vgl. auch Bundesministerium des Innern 2006b, S. 242). Statt der Grundsicherungsleistung für erwerbsfähige Hilfebedürftige nach dem Zweiten Sozialgesetzbuch (SGB II), die derzeit 345 Euro monatlich für Alleinstehende beträgt, erhalten Flüchtlinge nach dem AsylbLG maximal 224,97 Euro, wovon häufig nur 40,90 Euro als Taschengeld in bar und der Rest in Sachleistungen gewährt werden. Trotz dahingehender Forderungen von verschiedenen Seiten lehnt die Große Koalition unter Kanzlerin Merkel eine Erhöhung ab (vgl. BT Drs. 16/7574). Die Forderungen nach Anpassung des Leistungsniveaus an das Existenzminimum, also an die Regelleistungen nach dem Zwölften Sozialgesetzbuch (SGB XII), werden zum einen damit begründet, dass mit dem AsylbLG eine ungerechtfertigte Ungleichbehandlung geschaffen wurde. Zum anderen wird argumentiert, dass sich die Zahl der nach AsylbLG Leistungsberechtigten und damit auch die Ausgaben seit 1994 mehr als halbiert haben. Dieses Argument wird von der Bundesregierung ignoriert, obwohl bei der Einführung des Asylbewerberleistungsgesetzes die detaillierte Darstellung der Einsparpotenziale dieses Gesetzes gegenüber dem Bundessozialhilfegesetz eine bedeutende Rolle spielte, wie die Begründung der damaligen Bundesregierung zum Gesetzentwurf zeigt: „Es wird daher vorsorglich ein Einsparungsbetrag von rd. 2 Mrd. DM jährlich angenommen." (BT Drs. 12/4451, S. 6)

Mit dem Zuwanderungsgesetz haben diese Regelungen nicht an Aktualität verloren - vielmehr wurde der Kreis der Betroffenen ausgeweitet: Neben Asylsuchenden, Geduldeten und Ausreisepflichtigen fallen nun auch diejenigen unter das Gesetz, die eine Aufenthaltserlaubnis (aus humanitären Gründen) erhalten haben. Im Evaluationsbericht des Bundesinnenministeriums zum Zuwanderungsgesetz heißt es:

\section{„Die bestehenden Regelungen im Asylbewerberleistungsgesetz, haben sich im Wesentlichen als ausreichend und praktikabel erwiesen. Forderungen nach einer Erweiterung des Leis- tungsumfangs nach $\int 1$ AsylbLG erveisen sich als unbegründet. " Bundesministerium des Innern 2006b, S. 9, S. 241ff.)}

Dementsprechend wurden die Leistungen auch mit dem Zuwanderungsänderungsgesetz von 2007 nicht erweitert. Stattdessen wurde die Dauer des Bezuges der abgesenkten Leistungen von drei auf vier Jahre erhöht, was auch Konsequenzen für viele derjenigen hat, die bereits nicht mehr auf Leistungen nach dem AsylbLG angewiesen waren:

„Da keine Übergangsregelung im Gesetz vorgesehen ist, wird diese Formulierung in der Verwaltungspraxis [einiger Bundesländer, DM] offenbar so verstanden, dass auch Personen, die bereits Anspruch auf Leistungen analog zur Sozialhilfe hatten, wieder auf die reduzierten Leistungen zurückgesetzt werden sollen. "(BT Drs. 16/7365, S. 1) 
Begründet wird die Beschränkung der sozialen Rechte von Flüchtlingen damit, dass keine Anreize zur ,Einwanderung in die Sozialsysteme“ geschaffen werden sollen. Arbeitsverbot und Abhängigkeit von sehr geringen Sozial- bzw. Sachleistungen sollen davon abschrecken, das Asylrecht als Einwanderungsoption zu nutzen. Dass das Abschreckungsargument nicht an Popularität verloren hat, zeigt der Evaluationsbericht des Innenministeriums zum Zuwanderungsgesetz, in dem die abgesenkten (Sach-) Leistungen als sinnvoll erachtet werden „um einerseits keine Anreize zu schaffen, aus rein wirtschaftlichen Gründen in die Bundesrepublik Deutschland zu kommen, und um andererseits potentiellen Schlepperbanden den Nährboden zu entziehen“ (Bundesministerium des Innern 2006b, S. 243). ${ }^{222}$ Die Rechtfertigung für den Ausschluss einer bestimmten Gruppe von der sozialen Teilhabe per soziokulturellem Existenzminimum beschränkt sich im Wesentlichen auf das Argument,

„,dass sich Leistungsberechtigte nach diesem Gesetz typischerweise nur vorübergehend in der Bundesrepublik. Deutschland aufhalten und deshalb keine Leistungen für eine Integration in die deutsche Gesellschaft notwendig sind. [...] Eine Versorgung von Flüchtlingen unterhalb des Existenzminimums durch das AsylbLG ist daher nicht gegeben." (BT Drs. 16/7574, S. 3; vgl. auch Bundesministerium des Innern 2006b, S. 242; BT Drs. 12/4451, S. 5).

Diese Argumentation geht freilich an der Realität vorbei - zum einen aufgrund der in der Regel langen Dauer der Asylverfahren und der anschließend oft über Jahre ausgestellten Duldungen. Zum anderen ist die lange Bezugsdauer der abgesenkten Leistungen nunmehr auch strukturell - das heißt im Gesetz selbst - angelegt: mit der Ausweitung des Leistungsbezugs nach AsylbLG von drei auf vier Jahre sowie des Kreises der Betroffenen auf diejenigen mit Aufenthaltserlaubnis aus humanitären Gründen. Zugleich wird die Verfestigung eines faktisch jahrelangen Aufenthalts zu einem regulären Status auf diesem Wege ebenfalls verhindert: Um ein Daueraufenthaltsrecht zu erhalten, zum Beispiel über eine der seltenen Bleiberechtsregelungen, müssen regelmäßig ausreichendes Einkommen und Unabhängigkeit von Sozialleistungen nachgewiesen werden. Wer jedoch jahrelang vom Arbeitsmarkt fern- sowie durch soziale Isolation und fehlendes Geld für Sprachkurse vom Deutschlernen abgehalten wurde, wird es schwer haben, solche Bedingungen zu erfüllen. Durch diesen „Teufelskreis“ (Classen 1999) verfestigt sich die Abhängigkeit und auch nach jahrelangem Aufenthalt entsteht keine siche-

\footnotetext{
222 Ähnlich lautet die Begründung in der Antwort der Bundesregierung auf eine Große Anfrage der Fraktion Die Linke im Bundestag: „Der vom AsylbLG verfolgte Zweck, den Missbrauch des Asylverfahrens einzuschränken, rechtfertigt es, Asylbewerbern ohne Verstoß gegen den Gleichheitsgrundsatz die erforderliche Hilfe zum Lebensunterhalt in der Form von Sachleistungen zu gewähren“ (BT Drs. 16/9018, S. 26). In dieser Antwort der Bundesregierung in der 16. Legislaturperiode taucht mehrmals der bereits seit Jahrzehnten bemühte Missbrauchs-Topos auf. Er behält offenbar - unabhängig von den konkreten EmpfängerInnenzahlen oder nachgewiesenen ,Missbrauchsfällen` - uneingeschränkt seine Aktualität.
} 
re Bleibeperspektive. Darüber hinaus liegt es im Ermessen der Ausländerbehörden, MigrantInnen allein aufgrund des Sozialhilfebezugs auszuweisen. Eine solche „Ermessensausweisung“ ist unter anderem möglich, wenn jemand „,ür sich, seine Familienangehörigen oder für sonstige Haushaltsangehörige Sozialhilfe in Anspruch nimmt“ (\$55 Abs. 2 S. 6 AufenthG).

Der vielfach postulierte Wandel migrationspolitischer Konzepte in der Bundesrepublik hin zu integrativen Maßnahmen findet also keine Anwendung auf Flüchtlinge. Allerdings wird Integration, wie sie das Zuwanderungsgesetz impliziert, auch für die übrigen MigrantInnen nicht verstanden als Ermöglichung von Teilhabe, sondern beschränkt sich auf die Einrichtung sanktionsbewehrter ,Integrationskurse'. Flüchtlingen, die sich im Asylverfahren befinden oder über Jahre nicht abgeschoben werden können, wird soziale und gesellschaftliche Teilhabe durch die weitgehende Vorenthaltung sozialer Rechte jedoch gänzlich verwehrt. Dies zeigt sich auch im folgenden Abschnitt, der die räumliche Ausgrenzung von Flüchtlingen näher darstellt.

\section{Unterbringung in Lagern, Residenzpflicht und Inhaftierung}

Ein zentrales Element des ,Sachleistungsvorrangs' ist die Verweigerung der Übernahme von Mietkosten und die Unterbringung in einem der verschiedenen ,Lager'. Auch wenn der Lagerbegriff nach anfänglicher Benutzung durch PolitikerInnen ${ }^{223}$ und Behörden sowie im Beschleunigungsgesetz von 1980 inzwischen durch andere Begriffe ersetzt wurde, hat seine Verwendung in diesem Zusammenhang einige entscheidende Vorteile: Die mit Begriffen wie ,Gemeinschaftsunterkunft ${ }^{\circ}$ oder ,Ausreisezentrum` verbundene semantische Verschleierung der tatsächlichen Verhältnisse in den Unterkünften und der mit diesen Verhältnissen verfolgten politischen Ziele kann so vermieden werden. Gleichwohl soll die Verwendung des Begriffs keine Gleichsetzung mit den nationalsozialistischen Lagern und also auch keine Verharmlosung der dortigen Verhältnisse implizieren. Tobias Pieper (2008) macht deutlich, dass es aufgrund der Bandbreite dessen, was unter einem Lager verstanden werden kann, darauf ankommt, bei der Verwendung des Lagerbegriffs die mit dem jeweiligen Lager verbundene politische Absicht zu explizieren:

\section{„Das Lager als Begriff und Herrschaftskonzept bedarf also einer zusätzlichen Spezifi-} zierung, die die politische Zielsetzung deutlich macht. Durch diese Spezifizierung wird

\footnotetext{
${ }^{223}$ Eine gewisse Berühmtheit hat die Aussage des damaligen Ministerpräsidenten Baden-Württembergs - Lothar Späth - erlangt, der im Schwäbischen Tagblatt (vom 5.05.1982) resümiert, dass die AsylbewerberInnen durch die Einrichtung von Sammellagern erfolgreich abgeschreckt worden seien. So seien die AsylbewerberInnenzahlen erst zurückgegangen, nachdem „die Buschtrommeln signalisiert haben: geht nicht nach Baden-Württemberg, dort müßt ihr ins Lager“ (ebd.). Neben der offenen Verwendung des Lagerbegriffs und dem Bekenntnis zum Abschreckungsziel wird mit der Aussage auch ein rassistisch konnotiertes Bild von AsylbewerberInnen als ,Wilde` aus dem ,Busch expliziert.
} 
auch die Abgrenzung heutiger zu den Lagern des NS-Faschismus deutlich." (Pieper 2008, S. 529)

Unter Berücksichtigung dieser Spezifität der politischen Absichten und der damit verbundenen wesentlich unterschiedlichen Lebensbedingungen und Folgen des Lageraufenthalts kann dennoch eine gewisse Kontinuität der Unterbringung von „sozial schlechter gestellten Menschen“ (Pieper 2008, S. 34) in Barackenlagern in Deutschland konstatiert werden:
„Ein Beispiel aus dem Rubrgebiet: Die Baracke war Anfang des 20. Jabrbunderts als Übergangswobnheim für ledige Bergarbeiter gebaut worden. Wäbrend des NS- Fascbismus wurde sie zuerst als Lager für den Reichsarbeitsdienst benutฉt, später im Krieg wurden dort ZwangsarbeiterInnen untergebracht. In der BRD wurde die Baracke zuerst von KZ-Überlebenden genut₹t, dann von, deutschstämmigen' EmigrantInnen und anschließend von ,GastarbeiterInnen" (Pieper 2008, S. 34).

Derzeit können für die Bundesrepublik folgende Lagertypen unterschieden werden: ,Erstaufnahmeeinrichtungen', ,Gemeinschaftsunterkünfte', ,Ausreisezentren und - als geschlossene Lager - der Abschiebegewahrsam bzw. das Abschiebegefängnis. Die zentral organisierten Erstaufnahmeinrichtungen werden in der Regel nur kurzzeitig bewohnt bis eine Umverteilung auf eine der, Gemeinschaftsunterkünfte' stattfindet. Diese wiederum sind dezentralisiert in den Kommunen angesiedelt und können von ihrem Erscheinungsbild erheblich variieren - von ,normalen' Wohnhäusern bis hin zu Baracken ehemaliger Kasernen, Containern und Containerschiffen. Dabei sind die konkreten Bedingungen in den jeweiligen ,Gemeinschaftsunterkünften' stark von der politischen Situation in den Kommunen und insbesondere von der Existenz von Unterstützungsstrukturen und -initiativen abhängig:

„Ob Sacbleistungen ausgezablt werden oder das Lager tief versteckt in einem Wald oder im Zentrum einer Kleinstadt liegt, bängt direkt von den kommunalen politischen Kräfteverbältnissen und den lokalen Auseinandersetzungen und Kämpfen ab." (Pieper 2008, S. 514f.)

Eine neuere Entwicklung stellen die so genannten ,Ausreisezentren ' dar. Sie wurden Ende der 1990er Jahre zunächst als ,Modellprojekte` einiger Bundesländer eingerichtet (1998: Nordrhein-Westfalen und Niedersachsen, 1999: RheinlandPfalz). Das Konzept wurde schließlich von weiteren Bundesländern übernommen und hat inzwischen Eingang in das Zuwanderungsgesetz gefunden. Mit Hilfe der ,Ausreisezentren' sollen zum einen die durch die sinkenden Zahlen von AsylantragstellerInnen ungenutzten Lagerkapazitäten wieder einer neuen Aufgabe zugeführt werden. Zum anderen wird auf diese Weise versucht der großen Zahl von MigrantInnen zu begegnen, die trotz Ablehnung ihres Asylgesuchs oder sonstiger ,Ausreiseverpflichtung ${ }^{6}$ nicht abgeschoben werden können. Sie sollen durch die Einweisung in die Ausreisezentren und eine Reihe von Maßnahmen, denen sie 
sich dort zu unterziehen haben, zur ,freiwilligen Ausreise gedrängt werden. Da der Inhaftierung in Abschiebehaftanstalten durch das Bundesverfassungsgericht enge Grenzen gesetzt wurden, wurde von der Bund-Länder-Arbeitsgruppe ,Rückführung' (AG Rück) ${ }^{224}$ das Konzept der ,Ausreisezentren' nach holländischem Vorbild entworfen. Ziel soll es sein, faktische ,Ausreisehindernisse ${ }^{6}$ zu beseitigen, was in der überwiegenden Zahl der Fälle bedeutet, Passersatzpapiere zu beschaffen. Um dies zu erreichen und die MigrantInnen zur, Mitarbeit' zu bewegen, wird in den Ausreisezentren eine Fülle an Strategien angewendet: Sie reichen von dem Herausreißen aus dem gewohnten Umfeld durch die Einweisung in die ,Zentren“, über eine drastische Verschärfung der ohnehin prekären sozialen Situation (absolutes Arbeitsverbot, Streichung des ,Taschengeldes', Beschränkung der Bewegungsfreiheit auf das Stadtgebiet, Meldepflichten, Zimmerdurchsuchungen) bis hin zu einer „Kombination von ausländerrechtlicher Beratung und psychosozialer Betreuung“ (Martini-Emden 2000)225. Durch die „Beratung“ sollen die MigrantInnen über die ,ausländerrechtliche Perspektivlosigkeit was Integration und selbstbestimmte Lebensführung anbetrifft, aufgeklärt“ werden und durch „die psycho-soziale Betreuung soll den Betroffenen geholfen werden, die durch die Perspektivlosigkeit ihres Aufenthaltes in Deutschland allgemein und in der Einrichtung speziell auftretenden Probleme und Frustrationen in positive Ansätze für eine Reintegration in ihrer Heimat umzuwandeln.“ (ebd.) Des Weiteren soll versucht werden „objektive Hinweise auf die tatsächliche Identität und den Herkunftsstaat zu gewinnen“ (ebd.). Dietmar Martini-Emden, der für ein solches Modellprojekt in Rheinland-Pfalz verantwortlich zeichnet, stellt zufrieden fest, dass durch all diese Maßnahmen ,die Menschen in eine gewisse Stimmung der Hoffnungs- und Orientierungslosigkeit versetzt" werden (ebd.). Im Ergebnis reist nur ein kleiner Teil der MigrantInnen unter diesen Bedingungen aus, stattdessen taucht über die Hälfte der Betroffenen vor oder während der Unterbringung in den ,Ausreisezentren' unter. Dies wird von Seiten der Behörden unter der Rubrik ,undokumentierte Ausreise‘ als Erfolg verbucht. Die Jury des Unwortes des Jahres war der Ansicht, dass unter diesen Voraussetzungen von der ,Freiwilligkeit ${ }^{\star}$ der Ausreise nicht mehr gesprochen werden kann und hat den Terminus, freiwillige Ausreise daher aus 1130 Vorschlägen ausgewählt und zum Unwort des Jahres $2006^{226}$ erklärt. Bereits im Jahr 2002 war der Begriff ,Ausreisezentrum‘ als ein Unwort gerügt worden.

\footnotetext{
224 Die Arbeitsgruppe Rückführung wurde auf Grundlage eines Beschlusses der Innenministerkonferenz vom 14.05.1993 gegründet.

225 Dietmar Martini-Emden ist Leiter des Amtes für Ausländerangelegenheiten und der Clearingstelle Rheinland-Pfalz für Flugabschiebung und Passbeschaffung bei der Stadtverwaltung Trier.

226 „Gesucht werden Wörter und Formulierungen aus der öffentlichen Sprache, die sachlich grob unangemessen sind und möglicherweise sogar die Menschenwürde verletzen. Die Vorschläge können aus allen Bereichen der öffentlichen Kommunikation stammen, aus Politik, Verwaltung, Wirtschaft, Technik, Wissenschaft, Kulturinstitutionen oder Medien [...].“ (vgl. Unwort des Jahres o.J.)
} 
Im Gegensatz zu Polen, wo die Lagerunterbringung auch der akuten Wohnungsnot geschuldet ist, sind die Lager für Flüchtlinge in der BRD Teil einer migrationspolitischen Strategie, die - wie das genannte Zitat von Lothar Späth verdeutlicht - vor allem auf Abschreckung zielt (vgl. Fn. 223) und den Aufenthalt möglichst unattraktiv machen soll. Zudem wird durch die Isolation eine Abschiebung der Abgelehnten erleichtert: Es besteht keine Gefahr, dass sich FreundInnen und NachbarInnen aus der ,Mehrheitsgesellschaft ${ }^{\star}$ solidarisieren und versuchen, Abschiebungen zu verhindern. Auch in diesem Sinne ist die Ausweitung der Lagerunterbringung zu verstehen: Inzwischen ist das System so weit ausdifferenziert, dass sich Flüchtlinge mitunter von der Aufnahme über das Verfahren bis hin zur Vorbereitung der Ausreise bzw. Abschiebung ausschließlich in Lagern bzw. bisweilen in einem einzigen Lager aufhalten. Solche „multifunktionalen Großlager“ sind nach Pieper (2008, S. 515) Ausdruck eines Wandels des dezentralen Lagerregimes:

„So kristallisiert sich ein enger Lagerkreislauf heraus, in dem die Erstaufnahmeeinrichtung, die Gemeinschaftsunterkunft und die Ausreiseeinrichtung in einem Lagerkomplex untergebracht sind und die BewohnerInnen nur noch als Akte die Lagertypen wechseln. "(ebd.)

Damit werden Kontakte zur Außenwelt und ein Ankommen im ,normalen Alltag in der Bundesrepublik weitgehend ausgeschlossen.

Neben der Abschreckungs- und Desintegrationsfunktion dient die Lagerunterbringung auch der Kontrolle der Flüchtlinge, wie dies besonders für die Ausreisezentren auch als Ziel formuliert wurde. Außerdem ist mit der Lagerunterbringung auch eine Legitimierungsfunktion verbunden: Hier werden Handlungsund Kontrollfähigkeit des Staates angesichts einwanderungspolitischer Herausforderungen demonstriert. Tobias Pieper arbeitet außerdem heraus, dass die dezentrale Verteilung der Lager auf die Kommunen nach dem Anwerbestopp auch mit einem ökonomischen Ziel verbunden war bzw. ist:

„Die Politik reagierte auf die Forderungen der Arbeitgeberverbände nach weiteren ,billigen' migrantischen Arbeitskräften mit der dezentralen Verteilung von Asylsuchenden und deren Zulassung zum Arbeitsmarkt, abgestimmt nach den sektoralen Anforderungen. "(Pieper 2008, S. 41)

All diese Funktionen haben einen erheblichen Preis - und zwar nicht nur für die Betroffenen im Hinblick auf die Demontage grundlegender Rechte sowie für die Gesellschaft, die damit strukturelle Inseln der Exklusion produziert, sondern auch in finanzieller Hinsicht: Gerade bei der Lagerunterbringung werden die hohen Kosten des Sachleistungsprinzips besonders plastisch. Die Aufwendungen für Betrieb und Verwaltung der Lager, das Personal, die Zubereitung und Ausgabe der Mahlzeiten sowie nicht zuletzt für die Gewinne der BetreiberInnen etc. sind in der Summe rund doppelt so hoch wie im Vergleich mit einer dezentralen Unter- 
bringung in Wohnungen mit Selbstversorgung (vgl. Classen 1999; Schuth 1988, S. 177). Dies wird am Beispiel des Lagers Blankenburg in Niedersachsen deutlich: Das Land Niedersachsen zahlte hier statt einer Pauschale von 4.270 Euro pro Person und Jahr, die bei einer dezentralen Unterbringung in den Kommunen fällig würde, im Jahr 2005 pro Person 9.662 Euro. Laut Niedersachsens Innenminister Uwe Schünemann (CDU) sei dies langfristig günstiger, da die Lagerunterbringung zu einer Steigerung der Zahl der Ausreisenden führe (vgl. Jungle World vom 25.10.2006).

Die derzeitige Bundesregierung hat hierzu „keine aktuellen Erkenntnisse“, weist aber die ,pauschale Feststellung, dass die Gewährung von Sachleistungen etwa wegen eines erhöhten Verwaltungsbedarfs mit einem finanziellen Mehraufwand verbunden ist" zurück (BT Drs. 16/9018, S. 16). Da die Bundesregierung eine aussagekräftige eigene Berechnung schuldig bleibt, ist davon auszugehen, dass die soziale Schlechterstellung von AsylbewerberInnen durch den Sachleistungsvorrang keine Sparmaßnahme ist. Es wird nicht versucht, die Kosten, die rund um die Aufnahme von Flüchtlingen entstehen, möglichst gering zu halten. Im Gegenteil, die Bundesrepublik setzt erhebliche Mittel ein, um eine möglichst flächendeckende und umfassende Ausgrenzung von Flüchtlingen zu gewährleisten. Dies geschieht unter migrationspolitischen Steuerungsannahmen, die die Komplexität von Wanderungsfaktoren und -motiven auf die sozialstaatliche Attraktivität des Ziellandes reduzieren. Die Bedeutung der wohlfahrtsstaatlichen Leistungen des Ziellandes für die Entscheidung zur Wanderung und bei der Wahl des Ziellandes wird damit überschätzt. Im Vordergrund stehen bei diesen Maßnahmen eher populistische Ziele und die Demonstration von Handlungsfähigkeit als ein theoretisch fundiertes Verständnis von Migrationsprozessen.

Flankiert wird das Sachleistungsprinzip in seiner Ausformung als Lagerunterbringung durch die oben bereits angesprochene so genannte ,Residenzpflicht': Danach dürfen AsylbewerberInnen und Geduldete den ihnen zugewiesenen Landkreis nicht ohne Genehmigung der Ausländerbehörde verlassen - das Entkommen aus den oft abgelegenen Unterkünften ist damit offiziell verwehrt. Nicht zuletzt die Bedingungen in den Lagern treiben viele Flüchtlinge jedoch zu einer Verletzung dieser Vorschrift.

Sowohl die Residenzpflicht als auch die soziale Situation der MigrantInnen durch das Asylbewerberleistungsgesetz sind Gegenstand von politischen Mobilisierungen von MigrantInnen und ihrer UnterstützerInnen. Der Widerstand richtet sich gegen die Einschränkung der Bewegungsfreiheit, die Unterbringung und die Situation in den Lagern sowie gegen das Verbot, den Lebensunterhalt eigenständig zu verdienen.

Im Gegensatz zu den weitgehend offenen Erstaufnahmelagern, Gemeinschaftsunterkünften und Ausreisezentren ist die Abschiebehaft ein geschlossenes Lager, das ebenfalls mit der Verschärfung des Asylrechts etabliert wurde. Auf diesem Wege soll verhindert werden, dass MigrantInnen sich der Abschiebung 
,entziehen'. In der juristischen Interpretation handelt es sich nicht um eine Strafoder Beugehaft, sondern um eine „Verwaltungshaft“"227. Dafür werden sie entweder im normalen Strafvollzug oder in eigens eingerichteten Abschiebehaftanstalten interniert. Die derzeit größte dieser Art in der BRD findet sich in Büren (Nordrhein-Westfalen) mit einer Kapazität von 530 Plätzen. ${ }^{228}$ Zur Zahl der Inhaftierten liegen keine bundesweiten Statistiken vor, da die Justizvollzugsanstalten Ländersache sind. Laut Schätzungen von Amnesty International werden jährlich 20.000 bis 30.000 Menschen in Abschiebehaft interniert (vgl. Lochbihler 2006, S. 10). Obwohl es sich um eine, Verwaltungshaft ${ }^{\prime}$ handelt, sind die Haftbedingungen überwiegend identisch mit denen im normalen Strafvollzug: Die Häftlinge erhalten Haftnummern, eine Stunde Hofgang am Tag, ein bis zwei Stunden Besuchszeit im Monat, abends wird um 22 Uhr das Licht ausgeschaltet. Die persönlichen Gegenstände werden vom Bewachungspersonal verwaltet, das jeweils darum gebeten werden muss. Gleichzeitig besteht die ständige Angst vor der Abschiebung - kurz: die Inhaftierten erleben ein Maximum an Kontrolle und Fremdbestimmung (vgl. Holz 2007). Der stellvertretende Anstaltsleiter der Abschiebehaft Berlin Köpenick formulierte die Bedingungen aus seiner Sicht so: „Wir versuchen hier, das normale Leben für die Insassen zu ermöglichen - nur ohne Freiheit." (Berliner Woche vom 04.02.2004, zit. n. Holz 2007, S. 8) Ein nicht unerheblicher Teil der von den Amtsgerichten verhängten Haftbeschlüsse wird von höheren Gerichtsinstanzen wieder aufgehoben - die Betroffenen werden also teils monatelang inhaftiert und schließlich wieder entlassen. ${ }^{229}$ Laut der jährlich aktualisierten Dokumentation der Antirassistischen Initiative Berlin haben zwischen 1993 und 2006 mindestens 400 Menschen in Abschiebehaft versucht sich das Leben zu nehmen. Sie überlebten teilweise schwer verletzt, mindestens 50 Abschiebehäftlinge sind so gestorben.

\section{Abschiebung}

Wenn ein Asylantrag - wie in der weit überwiegenden Zahl der Fälle - negativ beschieden wird und auch kein Flüchtlingsschutz nach GFK oder subsidiärer Schutz zuerkannt wird, sollen die Betreffenden die Bundesrepublik verlassen. Da dies nur selten freiwillig geschieht, sind Abschiebungen ein Kernelement der Migrationspolitik. So heißt es im Evaluationsbericht zum Zuwanderungsgesetz:

\footnotetext{
227 So die Erläuterung der Abschiebehaftanstalt Neuss der JVA Düsseldorf in ihrer Selbstdarstellung unter der Rubrik ,Wir über uns' - ,Behördenpräsentation` - ,Abschiebehaft': http://www.jvaduesseldorf.nrw.de/wir/behoerdenpraes/hh_neuss/index.php

228 Weitere Abschiebehaftanstalten befinden sich in Ingelheim (Rheinland-Pfalz), Neuss (für Frauen, Nordrhein-Westfalen), Berlin-Köpenick, Eisenhüttenstadt (Brandenburg), Offenbach am Main (Hessen), Langenhagen (Niedersachsen) und Rendsburg (Schleswig-Holstein).

${ }^{229}$ Für das Beispiel Berlin vgl. Steffi Holz (2007, S. 19): „Ein Blick auf die Zahlen für Berlin zwischen 2001 und 2006 zeigt, dass durchschnittlich 50\% der Inhaftierten entlassen werden." Als Gründe nennt Holz erfolgreiche Haftbeschwerden, ärztlich attestierte Haftunfähigkeit oder Wegfall der Haftgründe, wenn zum Beispiel die Abschiebung nicht durchführbar ist.
} 


\begin{abstract}
„Zentrales Element einer konsequenten Ausländerpolitik, die einerseits der Integration der rechtmäßig in Deutschland lebenden Ausländer und andererseits der Begrenzung des Zurugs aus Staaten außerhalb der Europäischen Union verpflichtet ist, ist dass bestebende Ausreiseverpflichtungen durchgesetzt werden. Zwar setzen Bund und Länder hierbei auf den Vorrang der freiwilligen Rückkehr. Auf den Einsatz des Mittels der zwangsweisen Rückführung kann jedoch nicht verzichtet werden. " Bundesministerium des Innern 2006b, S. 140)
\end{abstract}

Die Alltäglichkeit der Abschiebungen zeigt sich anhand der Statistik: Zwar ist die Zahl der Abschiebungen seit einigen Jahren rückläufig (vgl. Tabelle 6 im Anhang) - im Jahr 2006 fanden dennoch 13.060 Abschiebungen auf dem Luftweg, 829 auf dem Landweg und fünf auf dem Seeweg statt (vgl. BT Drs. 16/4523). ${ }^{230}$ Dies korrespondiert auf den ersten Blick mit dem Rückgang der AsylbewerberInnenzahlen - konkretisiert werden kann der Zusammenhang jedoch aufgrund der Datenlage nicht: Eine differenzierte Statistik darüber, inwieweit es sich bei den Abgeschobenen um abgelehnte AsylbewerberInnen, Personen mit widerrufenem Asylrecht, ,Sans Papiers' oder Personen mit anderen Status handelte, liegt nach Aussage der Bundesregierung nicht vor (vgl. BT Drs. 16/4523). Die meisten der Abschiebungen verlaufen - der Statistik nach - reibungslos. In einigen Fällen müssen Abschiebungsversuche wegen des Widerstands der Betroffenen (2006: 301), wegen medizinischer Bedenken (2006: 76) oder wegen der Weigerung der Fluggesellschaft bzw. des Flugzeugführers (2006: 111), den Betreffenden gegen seinen Willen zu transportieren, abgebrochen werden (vgl. ebd.). Diese Zahlen sagen jedoch nichts darüber aus, in wie vielen Fällen Flüchtlinge sich gewehrt haben und dennoch abgeschoben wurden. Denn über den Einsatz von körperlicher Gewalt werden ebenfalls keine Statistiken geführt. Da immer wieder Fälle von Abschiebungen an die Öffentlichkeit dringen, in denen die ,begleitenden BeamtInnen“ Gewalt anwenden - bis hin zum Tod der Person, die abgeschoben werden soll ${ }^{231}$ - kann vermutet werden, dass viele Abschiebungen trotz Widerstands der Betroffenen und unter Einsatz von Gewalt sowie mit Hilfe von Beruhigungsmitteln 232 durchgesetzt werden.

${ }^{230}$ Hinzu kommen Zurückschiebungen (2006: 4.729) und Zurückweisungen (2006: 20.329) auf dem Luft-, Land- und Seeweg (vgl. BT Drs. 16/4523).

231 So starb im Mai 1999 Aamir Ageeb bei seiner Abschiebung in den Sudan. Da er sich gegen die Abschiebung gewehrt hatte, wurde er von drei BGS-Beamten an Armen und Beinen gefesselt. Sie setzten ihm einen Motorradhelm auf und drückten ihn während des Startens der Maschine mit dem Kopf nach unten. Aamir Ageeb verlor dabei das Bewusstsein und erstickte (vgl. Frankfurter Rundschau vom 05.02.2004).

232 So erhielt ein Flüchtling, der nach Pakistan abgeschoben werden sollte, gegen seinen Willen zwei Beruhigungsspritzen durch eine Krankenschwester der Gulf Air. Die Abschiebung wurde aufgrund des Protests von Passagieren abgebrochen, der Betroffene stellte Strafanzeige wegen Körperverletzung. Er wurde dennoch zwei Wochen später - beim fünften Versuch - abgeschoben (vgl. Frankfurter Rundschau vom 24.04.2008). 
Neben diesen Widerständen direkt bei der Abschiebung gibt es vor allem im Vorfeld der Abschiebungen bereits diverse Hindernisse. Als solche so genannte ,Rückführungshindernisse' identifiziert das Bundesinnenministerium „mangelndes Kooperationsverhalten der Herkunftsländer“ und die „Verweigerungshaltung der ausreisepflichtigen Personen“ (Bundesministerium des Innern 2006b, S. 7). Das Problem für die Ausländerbehörden liegt meist darin, einerseits herauszufinden, aus welchem Land die Betreffenden kommen und andererseits die für die Abschiebung erforderlichen Pässe zu beschaffen. So hieß es beim ,Praktikeraustausch` im Vorfeld der Evaluierung des Zuwanderungsgesetzes:

\section{„Der Nichtbesitz oder der vorgebliche Nichtbesitz, von Identitätsunterlagen wird strate- gisch zur Rückführungsverbinderung eingesetzt und gehört zur Standardinstruktion von Schleusern für die Geschleusten. Die Anzabl der Asylbewerber, die vorgeben, keinerlei Identitätsnachweise zu besitzen, liegt durchschnittlich zwischen 80 und $90 \%$ " (Bun- desministerium des Innern 2006c, S. 2062233}

Die zahlreichen Maßnahmen, die zur Beendigung der, Verweigerungshaltung der Betroffenen ergriffen werden, sind bereits angesprochen worden (zum Beispiel Ausreisezentren, Abschiebehaft etc.). ${ }^{234}$ Daneben sind noch Botschaftsvorführungen zu nennen, bei denen Delegationen der Herkunftsländer herausfinden sollen, ob die ihnen vorgestellten Personen StaatsbürgerInnen ihres Landes sind und dann entsprechend Papiere ausstellen.

Dazu kommt, dass im Zuwanderungsgesetz die Nichtkooperation bei der Identitätsfeststellung nun unter Strafe gestellt wurde ( $\mathbb{9} 95$ Abs. 1 Nr. 5; Abs. 2 Nr. 2 AufenthG). ${ }^{235}$ Diese Vorschrift wird in Ausreisezentren bereits dazu genutzt, die dort Festgehaltenen zusätzlich unter Druck zu setzen. Gegen rund ein Drittel der BewohnerInnen der Ausreiseeinrichtung in Bramsche sei Strafanzeige „wegen illegalem Aufenthalt und der Nichtkooperation bei der eigenen Ausreise und der dazu notwendigen Passbeschaffung gestellt" worden (Pieper 2008, S. 308). Die MigrantInnen erhalten dann eine Vorladung der Polizei zu einem Verhör, bei dem versucht wird, sie zur Unterschrift unter eine ,Freiwilligkeitserklä-

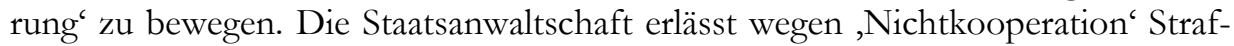
befehle in Höhe von 200-600 Euro. Wird das Geld nicht aufgebracht - was auf-

233 Die Zitate sind einem Statement zum Thema Rückführung von Dietmar Martini-Emden (vgl. Fn. 225) entnommen, das dieser zum Praktikeraustausch über das Zuwanderungsgesetz (vgl. Bundesministerium des Innern 2006c) beigetragen hat.

234 Dabei wird nicht versucht, den Charakter der Strategien zu beschönigen: „Da bei fehlenden Beweisen ohne wahre Angaben durch die Betroffenen eine Rückführung dauerhaft verhindert ist, muss zudem die Rückkehrmotivation durch geeignete Maßnahmen in allen Bereichen, auch im Repressiven, gefördert werden.“ (Bundesministerium des Innern 2006c, S. 207)

235 Die Begründung im Gesetzentwurf dazu lautete: „Die Einfügung von Nummer 5 ist erforderlich geworden, um der wachsenden Tendenz zur Verschleierung der Identität und Staatsangehörigkeit entgegenzuwirken. Die Folge ist, dass - oftmals über Jahre hinweg - vollziehbare Rückführungsentscheidungen nicht durchgesetzt werden können und in dieser Zeit Sozialleistungen in Anspruch genommen werden.“ (BT Drs. 15/420, S. 98) 
grund der sozialen Situation in der Regel nur mit Hilfe von Verwandten oder durch irreguläre Arbeit möglich ist - droht Gefängnis (vgl. Pieper 2008, S. 308f.).

Das Bundesinnenministerium hatte darüber hinaus im Evaluationsbericht zum Zuwanderungsgesetz gefordert, Abschiebungen künftig nicht mehr anzukündigen, so wie es in Niedersachsen bereits praktiziert wird. Dies wurde in den Verhandlungen zum Zuwanderungsänderungsgesetz aber offenbar nicht durchgesetzt.

Auf die zweite genannte Hürde neben der „Verweigerungshaltung der ausreisepflichtigen Personen“ (Bundesministerium des Innern 2006b, S. 7) - die Kooperationsbereitschaft der Herkunftsländer - kann mit gesetzgeberischen Mitteln jedoch nicht eingewirkt werden. Das Bundesinnenministerium strebt deshalb eine Erhöhung der Zahl der Rückübernahmeabkommen an, die eine effizientere Zusammenarbeit versprechen. Dafür wird „ein ganzheitlicher Politikansatz“ gefordert (Bundesministerium des Innern 2006b, S. 7). Die Herkunftsländer sollen mit einer Mischung aus Anreizen und Druck zur Identifizierung und Übernahme ihrer StaatsbürgerInnen und mitunter auch anderer StaatsbürgerInnen bewegt werden:

„Dies bedeutet, gewisse Unterstützungsleistungen an diese Staaten (₹. B. technische Ausstattungshilfe) ins Verbältnis zu ibrer Kooperationsbereitschaft im Rückführungsbereich zu setzen, einschließlich der Einbeziehung der Visaerteilungspraxis. " (Bundesministerium des Innern 2006b, S. 168)

Die Entwicklungshilfe wird damit in den Dienst der deutschen Einwanderungspolitik gestellt. Der Begriff der ,Erpressung، wurde allerdings beim ,Praktikeraustausch` zum Zuwanderungsgesetz gebraucht, um das Verhalten der Herkunftsländer zu charakterisieren. So schilderte Dietmar Martini-Emden die Strategien Nigerias in diesem Zusammenhang wie folgt:

„Ein Staat wie Nigeria geht beispielsweise so weit, dass er, wenn einem einzelnen Nigerianer aus seiner Sicht ,Unrecht" geschehen ist, die gesamte Kooperation mit den deutschen Behörden über Monate einstellt, was bei über 4.000 offenen Identifizierungsfällen für vermutlich nigerianische Staatsangehörige man auch als Erpressung bezeichnen könnte. "(Bundesministerium des Innern 2006c, S. 205)

Dass die Herkunftsstaaten auf die Freiwilligkeit der Ausreise ihrer Staatsangehörigen Wert legen, stößt ebenfalls auf Unverständnis:

„Eine Reibe von Staaten setzen sich zudem über das Völkergewohnheitsrecht völlig hinweg, indem sie auch bei nachgewiesener Staatsangebörigkeit der betroffenen Personen direkt oder indirekt auf nachgewiesener Freiwilligkeit zur Ausreise durch den Betroffenen bestehen. Allein diese eindeutig völkerrechtswidrige Verfahrensweise wird aktuell von 8 Staaten praktiziert und betreffen ca. 22.000 Ausreisepflichtige." Bundesministerium des Innern 2006c, S. 205)

Aus diesem Überblick über die aktuelle Konstitution des Flüchtlingsschutzes und unter Rückbezug auf die zuvor dargestellte Entwicklung der Kategorie Flucht und 
Asyl in der Bundesrepublik Deutschland werden im folgenden Abschnitt die zentralen Kennzeichen des deutschen Flüchtlingsschutzregimes herausgearbeitet.

\subsection{Kennzeichen des deutschen Flüchtlingsschutzregimes}

Die Entwicklung des Flüchtlingsschutzes in der Bundesrepublik ist seit den 1970er Jahren von einer fortschreitenden Restriktion gekennzeichnet. Um auf die Einwanderung von Asylsuchenden Einfluss zu nehmen, wurde an zwei Hebeln angesetzt:

- der präventiven Abschreckung potentieller Asylsuchender und

- der Beschränkung ihres Zugangs zum Territorium der Bundesrepublik bzw. zum Asylverfahren.

Mit diesen beiden Ansatzpunkten korrespondiert das Repertoire der ergriffenen Maßnahmen, das von Beginn der Restriktionsbemühungen an eine klare Kontinuität aufweist. So wurden einerseits - mit dem Ziel der Senkung der Einwanderungsanreize bzw. der Abschreckung - die soziale Situation der Asylsuchenden verschlechtert, die Asylverfahren verkürzt und die Durchführung von Abschiebungen erleichtert. Andererseits wurden konkrete Hürden aufgebaut, um den Zugang zum Asylverfahren zu beschränken. Dies begann bereits 1977, als die Grenz- und Ausländerbehörden ermächtigt wurden, vorab zu bestimmen, ob es sich um einen ,missbräuchlich“ gestellten Asylantrag handelt. Diese Regelung wurde zwar 1981 vom Bundesverfassungsgericht gekippt, sie ist aber der Vorläufer einer ganzen Reihe ähnlicher Maßnahmen, mit denen bereits von vornherein die Stellung von Asylanträgen verhindert werden sollte: die Ausweitung des Sichtvermerkszwangs, die Kooperation mit den DDR-Behörden bei der Beschränkung der Einreise über Ost-Berlin, die Involvierung der Transportunternehmen usw. Den bislang weitgehendsten Einschnitt beim Zugang zum Asylverfahren stellt die Grundgesetzänderung von 1993 mit der Einführung der sicheren Herkunfts- und Drittstaatenregelung dar.

Bis heute sind diese beiden Bereiche - soziale Schlechterstellung und Verfahrensrestriktionen - die zentralen Dreh- und Angelpunkte asylpolitischer Intervention von Seiten der Bundesregierungen und der Länder, wie die Untersuchung der Vorschläge der Zuwanderungskommission und der Bestimmungen im Zuwanderungs- und Zuwanderungsänderungsgesetz gezeigt haben.

Das deutsche Flüchtlingsschutzregime ist demnach durch folgende Merkmale geprägt:

- den so genannten ,Cordon-Sanitaire', durch den Deutschland alle auf dem Landweg Eingereisten auf die Zuständigkeit der Nachbarstaaten verweisen kann (sofern das zuständige Land ermittelt werden kann) und eine deshalb auf die Ermittlung des Reiseweges ausgerichtete Anhörung der AntragstellerInnen, 
- einen fortgesetzten Rückgang der Zahl der AntragstellerInnen, eine konstant niedrige Anerkennungsquote und eine hohe Zahl (faktisch dauerhaft) Geduldeter,

- eine nicht näher bezifferbare Zahl ,Untergetauchter` ohne gültige Aufenthaltsdokumente,

- die soziale Schlechterstellung von AsylbewerberInnen und Geduldeten durch Entkoppelung der Sozialleistungen für Asylsuchende vom Bundessozialhilfegesetz bzw. Zwölften Sozialgesetzbuch (SGB XII) bei gleichzeitig künstlich geschaffener Abhängigkeit von Unterstützungsleistungen durch das Verbot zu arbeiten; damit verknüpft sind medizinische, psychologische und finanzielle Unterversorgung, Zwang zum Nichtstun und Perspektivlosigkeit,

- den Sachleistungsvorrang: Leben in Heimen, ggf. Einkauf mit Wertgutscheinen bzw. Chipkarten oder Fremdversorgung durch Lebensmittelpakete und Kantinenessen,

- die Differenzierung des Lagersystems durch neue Konstrukte wie die ,Ausreisezentren' zur ,Förderung der freiwilligen Ausreise“ und Umbau der bestehenden Kapazitäten zu „multifunktionalen Großlagern“ (Pieper 2008, S. 515), die die Asylsuchenden mitunter während ihres gesamten Aufenthaltes - von der Ankunft über das Verfahren bis zur Abschiebung - nicht verlassen,

- Inhaftierung in Form von Zurückweisungshaft bis zur Klärung der Zuständigkeit gemäß Dublin-II-Verordnung sowie von Abschiebehaft zur Durchsetzung der Abschiebung, sowie

- eine hohe Zahl von Abschiebungen.

Die sozialen Probleme ergeben sich hier nicht aus der Not der Bundesrepublik, die Flüchtlinge zu versorgen, sondern sind Teil asylpolitischer Strategien zur Abschreckung von Asylsuchenden, die teils mit hohen Kosten verbunden sind. Die Schwierigkeiten, das Asylverfahren in Anspruch nehmen zu können und die soziale Schlechterstellung sind also gewollte Merkmale des deutschen Asylregimes. Nichtsdestotrotz gibt es an verschiedenen Stellen eine Kluft zwischen Recht und Praxis: Sie manifestiert sich aus der Perspektive der staatlichen AkteurInnen in den als ,missbräuchlich deklarierten Strategien der MigrantInnen zur Umgehung zahlreicher Regelungen bzw. im Widerstand gegen diese Regelungen (Nichtkooperation bei der Beseitigung von Ausreisehindernissen, Widerstand bei Abschiebungen, Bildung von Unterstützungsnetzwerken, Gutscheinumtausch, Verletzung der Residenzpflicht usw.). Aus der Perspektive der MigrantInnen zeigt sich diese Kluft vor allem zwischen dem Recht einerseits und der Machtfülle der Behörden bei dessen Auslegung andererseits und damit in der Abhängigkeit vom Wohlwollen einzelner BehördenmitarbeiterInnen (bei der Entscheidung über Arbeitserlaubnisse, Einweisung in Gemeinschaftsunterkünfte, Ausnahmegenehmigungen zur Residenzpflicht, die Behandlung von Krankheiten und natürlich bei der Entscheidung über den Asylantrag usw.). Der Ermessenspielraum der Behörden ist - wie an vielen Beispielen illustriert wurde - bereits in den gesetzlichen Regelungen sehr 
weit angelegt. Werden diese Handlungsspielräume zuungunsten der Betroffenen genutzt, so ist dies kein Verstoß gegen geltendes Recht. Um gegebenenfalls einen Verstoß nachzuweisen, ist der Weg durch die Gerichtsinstanzen für MigrantInnen, sofern sie die dafür erforderlichen Ressourcen aufweisen, mitunter versperrt (zum Beispiel weil die Abschiebung bereits vollzogen wurde). Oder die meist lange Dauer der Verfahren bedeutet angesichts der Dringlichkeit vieler Anliegen eine besondere Härte, zum Beispiel wenn es um Familientrennungen geht.

Aus dem Wechselspiel zwischen alltäglichen widerständigen Praxen der MigrantInnen und den Regulierungsversuchen durch politische EntscheidungsträgerInnen und Behörden, in dem letztere die machtvollere Position einnehmen, ergeben sich meist negative Konsequenzen für die MigrantInnen. Doch es gelingt ihnen immer wieder auch, kleine Erfolge zu erkämpfen, insbesondere dann, wenn durch die Mobilisierung von Unterstützungsstrukturen öffentlicher Druck erzeugt werden kann. Kollektive Strategien sind jedoch gerade für Flüchtlinge schwer zu entwickeln und umzusetzen. Dies liegt einerseits an ihrer Isolation in den Heimen, die eine Kontaktaufnahme zur Außenwelt erschwert, andererseits an der im Asylregime angelegten Individualisierung: Da jeder in erster Linie seinen eigenen Fall verfolgt und diesen im Zweifelsfall nicht durch politische Aktivitäten oder Verstöße gegen das Ausländerrecht (zum Beispiel die Residenzpflicht) gefährden will, sind den Kollektivierungschancen enge Grenzen gesetzt.

\subsection{Resümee der Einflussfaktoren}

Der Rückblick auf die Etappen der Entwicklung des deutschen Flüchtlingsschutzregimes hat bereits die verschiedenen Einflüsse illustriert, denen die Ausgestaltung dieses Politikfeldes unterlag. Entlang der Etappen lässt sich ein Politikwandel nachvollziehen, in dessen Rahmen ein individuelles Grundrecht auf Asyl in die Verfassung aufgenommen, wenige Jahrzehnte einer vergleichsweise geringen Zahl vor allem osteuropäischer Flüchtlinge gewährt und dann nach und nach auf einfachgesetzlicher und schließlich auch auf Verfassungsebene eingeschränkt wurde. Den gesunkenen AsylbewerberInnenzahlen zum Trotz wurden auch in den vergangenen Jahren weitere Restriktionen eingeführt, denen lediglich einige - durch EU-Vorgaben bedingte - Verbesserungen gegenüberstehen.

Im Folgenden soll zunächst erläutert werden, wie sich die asylpolitischen Veränderungsprozesse in die übrigen migrationspolitischen Entwicklungen in der Bundesrepublik einordnen lassen. Durch diese Kontextualisierung von Kontinuität und Wandel der Asylpolitik können die Besonderheiten aufgezeigt werden, die diesen Teilbereich der Migrationspolitik kennzeichnen. Vor diesem Hintergrund wird das Set an Einflussfaktoren, das den Asylbereich bestimmt hat, zusammenfassend erörtert.

Um die asylpolitischen in die sonstigen migrationspolitischen Wandlungsprozesse einzuordnen, werden sie nun vor der Folie der drei im Abschnitt 
5.2 Migrationspolitik in der BRD dargestellten Umorientierungen ${ }^{236}$ betrachtet. Es wird also der Frage nachgegangen, ob die allgemeinen migrationspolitischen Umorientierungen auch für den Asylbereich gelten. Es zeigt sich, dass die ersten beiden Phasen auch für den Asylbereich relevant sind. So war der Bezug auf menschenrechtliche Normen ein zentraler Faktor bei der Entstehung des Grundrechts auf Asyl. Nichtsdestotrotz weist Schönwälder darauf hin, dass diese menschenrechtliche Orientierung von außen aufgezwungen war, weshalb rassistische Denkmuster - obgleich öffentlich tabuisiert - fortbestanden und „bis weit in die 1970er Jahre eine systematische rassistische Ausgrenzungspolitik gegenüber potenziellen Einwanderern aus Afrika und Asien betrieben" wurde (Schönwälder 2006, S. 11). Im Verlaufe dieses Kapitels konnte aufgezeigt werden, dass diese menschenrechtliche Orientierung eng verknüpft war mit dem Antikommunismus des Kaltes Krieges und dem damit verbundenen Eurozentrismus: Die Veränderung der Herkunftsstruktur der Flüchtlinge (weniger aus Osteuropa und mehr aus Afrika und Asien) war mit ausschlaggebend für den asylpolitischen Wandel ab Anfang der 1970er Jahre. Daher fand das „Leitbild des demokratischen und humanitären Deutschlands“, das Schönwälder für die frühen 1970er Jahre feststellt und ,in dessen Licht die Lebensumstände der Gastarbeiter skandalisiert wurden“ (Schönwälder 2006, S. 15) kaum Anwendung auf die AsylbewerberInnen, die sich zu diesem Zeitpunkt schon mit dem Vorwurf des ,Missbrauchs' des Asylrechts konfrontiert sahen.

Auch der zweite von Schönwälder identifizierte „Politikwechsel“ hin „,zur aktiven Bekämpfung von Einwanderungsprozessen“" ist für den Asylbereich gleichermaßen als Wendepunkt relevant. Mit dem Anwerbestopp wurden auch die ersten Versuche einer Begrenzung der Einwanderung von Flüchtlingen unternommen. Die Einschränkung des Asylrechts 1992/93 schließlich lässt sich nach Ansicht von Schönwälder (2006, S. 14f.) ,in diese längerfristige Linie eines Vorherrschens restriktiver Intentionen einordnen“. Tatsächlich wurde bereits lange vorher mit einfachgesetzlichen Verschärfungen zur Grundgesetzänderung hingeleitet, gleichzeitig wurde mit der Einschränkung des Asylgrundrechts eine neue Qualität der Intervention erreicht, die noch einige Jahre zuvor nicht konsensfähig erschien.

Die dritte ,Umorientierung, die aktuelle Anerkennung der erfolgten Einwanderung und die Hinwendung zu Integrationsmaßnahmen, bleibt - wie auch Schönwälder verdeutlicht - unvollständig. Dies kann auch für den Asylbereich konstatiert werden, der durch diesen Politikwechsel kaum tangiert wird: Die Bleiberechtsregelung von 2007 schließt nur einen kleinen Teil der seit Jahren Geduldeten ein und versperrt den meisten von ihnen damit weiter eine gleichberechtigte

\footnotetext{
236 1. die Umsetzung von Menschenrechtsnormen nach 1945, 2. der Versuch der Verhinderung von Einwanderungsprozessen seit dem Anwerbestopp 1973 und 3. die Anerkennung der Einwanderungsprozesse mit einem „Prioritätenwechsel hin zur Integration der Einwanderer“ (Schönwälder 2006, S. 10).
} 
Teilhabe. Zudem wird die Notwendigkeit, AsylbewerberInnen Integrationschancen zu eröffnen, mit exakt den gleichen Argumenten (zeitliche Begrenztheit des Aufenthalts) wie vor 15 Jahren - bei der Verabschiedung des Asylbewerberleistungsgesetzes - verneint, obwohl viele sich realiter über Jahre, wenn nicht dauerhaft, in der BRD aufhalten. Das Gleiche gilt hinsichtlich der anvisierten Abschaffung der Kettenduldungen, die zwar als Ziel formuliert, letztlich aber im Interesse des Fortbestands der Abwehrpolitik nicht umgesetzt wurde. Eine Anerkennung der Einwanderungsprozesse auch von AsylbewerberInnen und die Eröffnung von Integrationschancen für diese Einwanderergruppe sind daher nicht zu erkennen. Vielmehr wird im Asylbereich an der Abschreckungs- und Abwehrpolitik festgehalten.

Für die Identifizierung von Paradigmenwechseln muss daher für verschiedene Subfelder der Migrationspolitik und also für verschiedene MigrantInnenkategorien unterschieden werden: Für den Asylbereich war nach der menschenrechtlichen Orientierung in der jungen Bundesrepublik, die stark von antikommunistischen und eurozentristischen Prämissen geleitet wurde, der im Zuge des Anwerbestopps vollzogene Wechsel hin zur Verhinderung von Einwanderungsprozessen zentral. Dieser Anfang der 1970er Jahre eingeschlagene Pfad der Restriktion erlebte einen Höhepunkt 1992/93 und wurde bis heute - ungeachtet des inzwischen erfolgten Bekenntnisses Einwanderungsland zu sein und der stark gesunkenen AsylbewerberInnenzahlen - nicht mehr verlassen.

Betrachtet man die Einwanderungspolitiken der Bundesrepublik in historischer Perspektive, tritt ein zentrales Paradigma hervor, das sich bereits lange vor 1949 finden lässt und das sich über all die angesprochenen Phasen und Politikwechsel hinweg bis heute durch eine bemerkenswerte Kontinuität auszeichnet: Die Unterscheidung der MigrantInnen nach Nützlichkeitskriterien bzw. die Ausrichtung des Maßes an Offenheit an den Erfordernissen des Arbeitsmarktes. Diese Ausrichtung war bereits seit der Reichsgründung 1870/71 immer gekoppelt mit dem letztlich kollidierenden Bestreben, die Staatsbevölkerung als ethnisch homogene Einheit zu konstruieren und zu erhalten, also keine dauerhaften Einwanderungsprozesse zuzulassen. Das Changieren zwischen Anwerbepolitiken aufgrund von „„Leutenot" in Industrie und Landwirtschaft" und Abwehrstrategien zum „Schutz des nationalen Arbeitsmarktes“ bei gleichzeitigem Wunsch, den angeworbenen Arbeitskräften keine dauerhafte Niederlassung zu gestatten, lässt sich daher bis ins späte 19. Jahrhundert zurückverfolgen (vgl. Bade/Oltmer 2007, S. 149ff.; Herbert 2003). Flüchtlinge bzw. AsylbewerberInnen fallen sowohl aus der Vorstellung von Nützlichkeit (wie zuletzt die Einschätzung der Süßmuthkommission gezeigt hat) als auch aus der Imagination einer homogenen Gemeinschaft heraus. Der menschenrechtliche Fokus kollidierte in den ersten knapp drei Jahrzehnten nach 1949 nicht mit diesem Nützlichkeitsparadigma, da in dieser Zeit eine hohe Nachfrage nach Arbeitskräften herrschte. Erst mit dem Anwerbestopp gewann dieses Kriterium an Bedeutung und vereinigte sich im öffentlichen Diskurs mit 
dem ethnokulturellen Konzept der Mitgliedschaft insofern, als nur nach diesem exklusiven ethnokulturellen Muster als zugehörig konstruierte Personen legitime TeilhaberInnen sozialstaatlicher Leistungen sein konnten. Dieser „'welfare chauvinism', the unwillingness of natives to share welfare state benefits with certain immigrant groups and asylum seekers who are perceived as intruders" (Faist 1994, S. 61), kumulierte in der Bundesrepublik in der Rede von ,Wirtschaftsflüchtlingen' bzw. ,Armutsflüchtlingen', die das ,Asylrecht missbrauchen', um Sozialleistungen in Anspruch zu nehmen. Diese Sichtweise findet sich im öffentlichen Diskurs, aber auch in Gesetzestexten (zum Beispiel im Asylbewerberleistungsgesetz, Asylverfahrensgesetz).

Um den beschriebenen spezifischen Wandel, aber auch die Kontinuität der Asylpolitiken vor dem Hintergrund der Entwicklung des bundesdeutschen Migrationsregimes zu verstehen, sollen im Folgenden einige zentrale Einflussfaktoren erörtert werden. Dabei geht es vor allem um den Zeitraum seit Anfang der 1990er Jahre, allerdings vor dem Hintergrund, dass die Geschehnisse jenes Zeitabschnitts bereits zuvor über zwei Jahrzehnte schrittweise eingeleitet wurden und zudem auf historisch gewachsenen Prämissen und Leitvorstellungen beruhen.

Den Hintergrund für den Umgang der Bundesrepublik mit Einwandernden bildet zunächst zum einen die Konstruktion der deutschen Nation als ethnisch homogene Gemeinschaft, die sich in der bis ins Jahr 2000 bestehenden Konzeption der Staatsbürgerschaft als Abstammungsprinzip spiegelt (vgl. Brubaker 1992). Zum anderen - und damit verknüpft - ist das lange vorherrschende Selbstverständnis der Bundesrepublik als Nicht-Einwanderungsland zu nennen (vgl. auch Lavenex 2002, S. 39f.). Vor dieser Folie ist der politische Prozess zu betrachten, in dessen Verlauf die bundesdeutsche Flüchtlingspolitik zunehmend restriktiver gestaltet wurde. Dieser Prozess ist durch einige Spezifika des politischen Systems der Bundesrepublik gekennzeichnet. Dazu gehört die im föderalen System hohe Frequenz von Wahlkämpfen. Der Überblick über die einzelnen Etappen hat die starke Verkoppelung der Konjunkturen des Themas und der gesetzgeberischen Aktivitäten mit Wahlkämpfen illustriert. Im Wettbewerb der Parteien um WählerInnenstimmen hat sich das Thema als Mobilisierungsgarant erwiesen. Dabei ist die Frage nach der Rolle politischer EntscheidungsträgerInnen einerseits und der Öffentlichkeit andererseits in diesem Feld hoch umstritten:

„In the literature on whether immigration politics is liberal or restrictive, an important explanatory factor is whether policies are seen as the outcome of political elite decision or, alternatively, as an outcome of populist politics. " (Statham 2003, S. 167)

Einerseits argumentiert Gary Freeman in seiner ,client politics' thesis - wie es Statham treffend zusammenfasst - ,that whatever noise may be made about the issue of migration during elections, the real business of migration policy takes place behind closed doors in the arena of organised politics" (Statham 2003, S. 168). Diese ,Politik hinter verschlossenen Türen“ wird demnach von Arbeitge- 
bern und deren Interesse an billigen Arbeitskräften sowie von starken Minderheitengruppen beeinflusst. Eine zweite Position, die Statham (2003, S. 168) „racist public thesis" nennt, betont

\section{"the importance of the social construction of immigration as a problem in public discourse, arguing that this cultural framing and politicisation of the issue shapes and influences the decisions taken by the executive in policy arenas" (Statham 2003, S. 168).}

Statham hat mit einer Diskursanalyse für Großbritannien herausgefunden, dass die restriktiven Einstellungen von den EntscheidungsträgerInnen gleichsam als normativer und diskursiver Rahmen vorgegeben werden, der schließlich in der Bevölkerung auf Resonanz trifft:

,the nature of the asylum debate - at present restrictive and stigmatising - to a large extent comes politically from top down, rather than in response to mobilised public pressure " (Statham 2003, S. 174).

Hier stellt sich die Konstruktion von ,bogus asylum seekers' bzw. Asylmissbrauch als zu bekämpfendes Problem also als Top-Down-Prozess dar.

Für den bundesdeutschen Kontext stellt sich ebenfalls die Aufgabe, das $\mathrm{Zu}$ sammenspiel von politischen Parteien, WählerInnen, weiteren AkteurInnen, Wahlkampfdynamik und Policy-Output genauer zu ergründen. Zwar wird die Instrumentalisierung des Themas in Wahlkämpfen aufgrund seiner hohen Symbolkraft häufig in der Literatur konstatiert, genaue Analysen stehen jedoch noch aus. Eine eingehende Untersuchung dieser Zusammenhänge kann in der vorliegenden Arbeit nicht geleistet werden, es sollen jedoch einige Anhaltspunkte genannt werden. In Verknüpfung der Thesen von Paul Statham (2003) und Thomas Faist (1994) wird hier von einem Wechselspiel ausgegangen: Die vor allem in Wahlkämpfen aufkommende Rede vom ,Asylmissbrauch` traf in weiten Teilen der Öffentlichkeit auf fruchtbaren Boden und verstärkte und verfestigte die dort bereits vorfindbaren Ressentiments ${ }^{237}$ - stigmatisierende und stereotypisierende Diskurse in der Öffentlichkeit wurden so gleichsam mobilisiert und legitimiert. Die Parteien konnten schließlich die sich radikalisierenden Forderungen der Öffentlichkeit, die sich zum Teil in gewalttätigen Ausschreitungen entluden, aufgreifen und mit rechtlichen Verschärfungen für Asylsuchende als ,MacherInnen` in

\footnotetext{
${ }^{237}$ Selbstverständlich soll hier nicht von der restriktiv eingestellten Öffentlichkeit ausgegangen werden. Es handelt sich vielmehr um ein komplexeres Feld, in dem eine Vielzahl an AkteurInnen und Positionen sowie Interessen vorzufinden ist. Gleichzeitig ist jedoch entscheidend, wie die wahlkämpfenden AkteurInnen die Öffentlichkeit wabrnebmen. Es genügt, dass sie davon ausgehen, mit diesen Themen WählerInnen erreichen zu können, sie bestimmen dann die Problemwahrnehmung in der Öffentlichkeit mit und das hier beschriebene Wechselspiel wird in Gang gesetzt. Für Großbritannien formuliert Statham (2003, S. 167) die Problematik der Erwartungen der PolitikerInnen: „Politicians in Britain appear to believe in the 'racist public theses', namely, that there are untapped resources of public grievances against asylum seekers, verging in many cases on racism or outright xenophobia, and that their policy proposals must compete for this political territory."
} 
einem Feld auftreten, in dem die von der Politik Betroffenen sich zumindest nicht als WählerInnen zur Wehr setzen können. Die Funktion der wahlkämpferischen Konjunkturen des Themas liegt in der identitätsstiftenden Konstruktion eines exklusiven ,Wir', die von anderen Themen und gesellschaftlichen Problemen, zum Beispiel von Verteilungskonflikten innerhalb der ,einheimischen Bevölkerung', ablenkt, was man nach Faist (1994) als symbolische Politik bezeichnen kann. ${ }^{238}$ Dieses Rekurrieren auf ein ,Wir ${ }^{6}$ kann wiederum anknüpfen an die tief verankerte Vorstellung der Bundesrepublik als ethnokulturelle bzw. ethnonationale Gemeinschaft. Im Zuge der Vereinigung der BRD und DDR wurde dieses Verständnis einer gemeinsamen Identität als nationale Identität intensiviert (vgl. Faist 1994, S. 62). In klarer Abgrenzung zu diesem ,Wir ${ }^{6}$ werden die AsylbewerberInnen als (spätestens seit der Ausweitung der Herkunftsländer auf asiatische und afrikanische Staaten) nicht dazu gehörig und kulturell andersartig identifiziert. Die „Identifikation und Negativklassifikation des ,Fremden“" ermöglicht es, das ,deutsche Volk‘ als „Opfer der Überfremdung, Ausbeutung bzw. Ausplünderung durch ,die anderen“" zu imaginieren (Butterwegge 2003, S. 396). Das Selbstverständnis als ,homogenes Volk' allein kann die Restriktionen natürlich nicht erklären, die ja auch in Staaten vollzogen wurden, die andere Nations- und Staatsbürgerschaftsverständnisse aufweisen, wie bspw. Frankreich. In der Bundesrepublik tritt dieses Selbstverständnis allerdings als Verstärker des konstruierten, Wir'-Gefühls auf, das auch in anderen Staaten vorfindbar ist. Es ist sowohl verankert im Denken der ,Einheimischen' als auch eingelassen in die Institutionen der Bestimmung über Zugehörigkeit und Staatsbürgerschaft und damit ausgesprochen beständig.

Für den Umgang mit dem Thema in den Wahlkämpfen muss zwischen den Parteien unterschieden werden - wie in den ,Etappen' bereits im Einzelnen gezeigt wurde. ${ }^{239}$ Die Unionsparteien machen sich klar für eine Begrenzung der Einwanderung stark und haben in der Vergangenheit immer wieder dazu beigetragen, den öffentlichen Diskurs mit stigmatisierenden Begriffen und Metaphern aufzuladen. Sie haben sich dabei mit ,ihren sprachlich konstruierten Wirklichkeiten durchsetzen können“ (Meyer 1997). Das Plädoyer für die Zulassung von Einwanderung unter Nutzenaspekten taucht zwar immer wieder auf, stößt aber auf

238 „Symbolic politics meant that immigration gained the status of a meta-issue. [...] Immigration and asylum could be referred to as a cause of manifold problems. For conservative parties and the emerging right-wing populist Republikaner, the symbolic politics of asylum was appealing because certain groups of immigrants and asylum seekers could be easily connected to a host of domestic issues.” (Faist 1994, S. 52) „Ultimately, immigration as a meta-issue means that the reference to immigration as a root cause of policy failures, socio-economic and political problems has gained currency to such an extent that it can be used to legitimate changes in the constitution without having to draw upon and present substantive policy solutions." (ebd., S. 68)

239 Dabei können die Parteien nicht als homogene, starre Gebilde gesehen werden: Vielmehr gibt es immer wieder abweichende Einzelmeinungen innerhalb einer Partei, Konflikte zwischen Parteiführung und -basis oder zwischen verschiedenen Parteiflügeln und Fortentwicklungen bestimmter Positionen im Zeitverlauf. 
Widerstände vor allem an der Parteibasis, wie die Auseinandersetzung um das Papier der Unionskommission in Vorbereitung auf das Zuwanderungsgesetz gezeigt hat. Das Nicht-Bekenntnis zum Status der Bundesrepublik als Einwanderungsland wurde hier beharrlich aufrecht erhalten. Die Sozialdemokraten vertreten eine etwas liberalere Position, die aber seit Anfang der 1990er Jahre an Kontur verloren hat. Es zeigt sich, dass auch aus ihren Reihen die öffentlichkeitswirksame Diffamierung von Asylsuchenden populärer wird ${ }^{240}$ bzw. die Forderung nach einer Abschaffung des Grundrechts auf Asyl prominente Fürsprecher gefunden hat. ${ }^{241}$ Bündnis 90/Die Grünen treten für eine humanere Flüchtlingspolitik ein, was sich aber - wie sich spätestens bei ihrer Beteiligung am Zuwanderungsgesetz gezeigt hat - in der Forderung nach einigen Korrekturen des restriktiven (Dis)Kurses erschöpft. Die angestrebten sicherheitspolitischen Verschärfungen haben sie schließlich veranlasst, sich aus den Verhandlungen im Vermittlungsausschuss zurückzuziehen. Die Linke stellt zwar auf Bundesebene deutlich radikalere Forderungen als die Bündnisgrünen und pflegt gute Kontakte zu NGOs in diesem Bereich, allerdings zeigt sich bei ihrer Regierungsbeteiligung in den Kommunen keine radikal alternative Politik auf der Umsetzungsebene. ${ }^{242}$ Die FDP ist nahe der CDU zu verorten, ,jedoch auf abgeschwächtem Niveau“ (Green 2006, S. 120). Rechte Parteien haben zwar den Einzug in den Bundestag im betrachteten Zeitraum nicht geschafft ${ }^{243}$, ihre zeitweiligen Erfolge auf Landesebene bestimmten aber das Agendasetting der anderen Parteien mit, indem diese - und insbesondere die Union - sich die Forderungen der Rechten teilweise zu eigen machten.

Doch im „semisouveränen“ politischen System der Bundesrepublik (Katzenstein 1987) kommt es aufgrund der Verteilung der Macht auf verschiedene Institutionen und AkteurInnen schließlich auf das Zusammenspiel an. Zwar nehmen die politischen Parteien bzw. die Bundestagsfraktionen eine starke Rolle ein, sind aber gleichzeitig gezwungen, sich in Koalitionen miteinander zu einigen und mit dem jeweiligen Gesetzesvorhaben den Bundesrat zu passieren und damit auch die Machtverhältnisse in den Ländern zu berücksichtigen. Es hat sich gezeigt, dass viele einwanderungspolitische Vorhaben parteipolitischen Scharmützeln im Wahl-

\footnotetext{
240 Vgl. zum Beispiel das oben bereits angeführte Zitat von Gerhard Schröder aus der Frankfurter Allgemeinen Zeitung vom 21.07.1997: Wer das Gastrecht missbrauche, ,für den gibt es nur eins: raus, und zwar schnell [...]. Beim organisierten Autodiebstahl sind Polen nun einmal besonders aktiv, das Geschäft der Prostitution wird dominiert von der Russen-Mafia, Drogenkriminelle kommen besonders häufig aus Südosteuropa und Schwarzafrika“.

${ }^{241}$ Vgl. Otto Schily in Die Zeit vom 28.10.1999.

242 Vgl. zum Beispiel die Kritik an der - im Vergleich mit anderen Berliner Bezirken - großen Zahl an MigrantInnen, die in Marzahn-Hellersdorf, unter der Leitung einer Sozialstadträtin und Bürgermeisterin der Linken, in Wohnheimen statt in eigenen Wohnungen untergebracht wurden (vgl. Die Tageszeitung vom 18.04.2007).

243 Ausgenommen sind die drei Personen in den 1980er Jahren, die als Unionsmitglieder in den Bundestag gelangten und schließlich zu den Republikanern wechselten.
} 
kampf zum Opfer gefallen sind. Diese Entwicklung ist auch auf den Umstand zurückzuführen, dass

„[a]ngesichts knapper Ergebnisse auf Bundesebene in den Wablen seit 1994 [...] die Volksparteien viel stärker zu einem Dauerwablkampf [neigen], in dem Kompromisse und Zugeständnisse an den politischen Gegner in hochsymbolischen Bereichen wie der Migrationspolitik naturgemäß schwieriger sind. "(Green 2006, S. 132)

Letztlich sind aber die konkreten Ergebnisse dieser Aushandlungen von der Umsetzung in der dezentralisierten Verwaltung abhängig, die sich in der Bundesrepublik „,immer wieder als strukturell konservativ erwiesen“ hat (Green 2006, S. 131), wie im Verlaufe des Kapitels verschiedentlich gezeigt wurde. Zudem werden die Ausrichtung der Verwaltung und die Umsetzung in den Kommunen wiederum stark von der Ausrichtung des jeweiligen Landesinnenministeriums und insbesondere von der Person des Innenministers beeinflusst. ${ }^{244}$ Die Gerichte nehmen in diesem Feld ebenfalls eine wichtige Position ein - dabei bilden die Verwaltungsgerichte häufig ein Korrektiv zu den Entscheidungen der Behörden.

Andere - vor allem (zivil-)gesellschaftliche - Akteure sind in diesem institutionalisierten Rahmen weniger machtvoll. Zwar werden Wohlfahrtsverbände, Kirchen und Gewerkschaften angehört und damit öffentlichkeitswirksam versucht die Legitimität bestimmter Gesetzesvorhaben zu erhöhen, ihre Vorschläge werden aber nicht selten parteipolitischen Erwägungen untergeordnet. Das hat sich zum Beispiel bei der Evaluierung des Zuwanderungsgesetzes gezeigt, für die eine Vielzahl an Expertisen aus diesem Bereich eingeholt wurde, deren Forderungen und Vorschläge haben sich aber im Zuwanderungsänderungsgesetz so gut wie gar nicht niedergeschlagen. Die weniger institutionalisierten Initiativen und Gruppie-

\footnotetext{
${ }^{244}$ Dies sei hier beispielhaft für Niedersachsen und den Streit zwischen Landesinnenministerium und Kommunen um flüchtlingspolitische Leitlinien aufgezeigt: So wurden die Versuche verschiedener Städte, das Gutscheinsystem zugunsten der Auszahlung von Bargeld an Flüchtlinge abzuschaffen, vom niedersächsischen Innenminister Uwe Schünemann mehrfach scharf zurückgewiesen. Auch in anderen flüchtlingspolitischen Fragen weist Schünemann die Einmischung der Städte zurück. So erklärte er in einer Rede vor dem Niedersächsischen Landkreistag am 6. März 2008 in Bad Zwischenahn zu den Widerständen gegen seine Flüchtlingspolitik in den Kommunen: ,[...] Ich bin hier für Klarheit, weil einige Kreistage und Räte größerer Städte die Durchführung der betreffenden Gesetze in einem bestimmten Sinn zu beeinflussen suchen. Es ist aber nicht Aufgabe der kommunalen Parlamente, sich durch entsprechende Beschlüsse einzusetzen, z. B.

- für ein großzügiges Bleiberecht von abgelehnten Asylbewerbern;

- für die Versorgung dieser Personen mit Bargeld statt der Ausgabe von Wertgutscheinen;

- für eine sofortige Weiterleitung der neu ankommenden Asylbewerber auf die Gemeinden;

- für ihre sofortige Unterbringung in eigenen Wohnungen.

Auch ist es nicht Aufgabe von Amtsärzten, eine gebotene Rückführung ins Heimatland dadurch zu verhindern, indem sie die geringeren Standards des dortigen Gesundheitssystems zum Anlass nehmen, durch entsprechende Atteste den Betroffenen einen Verbleib in Deutschland auf Kosten der Allgemeinheit zu ermöglichen. Die Fachaufsicht in meinem Hause muss und wird darauf achten, dass die in Bundestag und Bundesrat mit großer Mehrheit geschaffenen Regeln weiterhin wirksam bleiben. [...]““ (zit. n. Niedersächsischer Flüchtlingsrat 2008).
} 
rungen selbst organisierter Flüchtlinge, von denen es in der Bundesrepublik eine Vielzahl gibt, machen mit vielfältigen Aktionen auf die Situation von MigrantInnen und die Folgen der Einwanderungspolitik aufmerksam. Es finden Kampagnen und Aktionen statt gegen die Unterbringung in Lagern, die Residenzpflicht, gegen das Grenzregime und für Bewegungsfreiheit im Allgemeinen. Viel Energie wird jedoch auch für Abwehrkämpfe auf lokaler Ebene aufgewendet: Einzelne Abschiebungen werden verhindert, die Folgen des Handelns der Ausländerbehörden an die Öffentlichkeit gebracht und Widerstand gegen die alltägliche Ausgrenzung zum Beispiel durch das Asylbewerberleistungsgesetz organisiert. Oft konkretisieren sich die Ergebnisse dieser Kämpfe jedoch nur in kurzfristigen Korrekturen der Verhältnisse, wie der Sanierung von Lagern, der verbesserten Versorgung mit Lebensmitteln oder der Umverteilung Einzelner in Wohnungen. Dennoch konnten neben der konkreten Verbesserung der Lebenssituation der Betroffenen auf diesem Wege auch immer wieder Alternativen und Gegenreden in die vorherrschenden Diskursmuster eingespeist werden. Ein radikaler Diskurs- und Politikwechsel wurde durch diese Interventionen jedoch bislang nicht erreicht.

Darüber hinaus hat die EU an Bedeutung für das Politikfeld gewonnen. Ihre Rolle für den bundesdeutschen Kontext ist vielfältig: Mit den Richtlinien-Vorgaben im Asylbereich sind aktuell einige kleinere Änderungen im deutschen Asylrecht notwendig geworden, wie die Verankerung geschlechtsspezifischer und nichtstaatlicher Verfolgung als Asylgrund. Zudem wurde die EU - wie gezeigt - in der Argumentation der politischen EntscheidungsträgerInnen an unterschiedlichen Stellen herangezogen: Beispielsweise wurden die Verschärfungen bei der Grundgesetzänderung 1992/93 damit begründet, dass Deutschland mit der Umsetzung des Vertrages von Schengen angesichts seines grundgesetzlich verankerten Individualrechts auf Asyl zum ,Reserveasylland“ in einer Union ohne Binnengrenzen werde, oder es hieß schlicht: „Ein subjektives Recht auf Asyl wird die EU nicht akzeptieren“"245. In diesem Zusammenhang steht die EU für Sachzwänge, denen sich die Bundesrepublik zu beugen hat, während sich die deutschen Innenminister andererseits immer wieder mit ihrer restriktiven Haltung bei der Aushandlung der EU-Vorgaben hervorgetan haben. Damit diente der Topos der Europäisierung den nationalstaatlichen AkteurInnen je nach Bedarf dazu, Restriktionen auf nationalstaatlicher Ebene entweder als Politiken im Namen der Europäisierung darzustellen oder sich mit der Durchsetzung nationalstaatlicher Interessen bei der Verabschiedung von EU-Richtlinien zu profilieren.

Zusammenfassend betrachtet begann der Wandlungsprozess in der bundesdeutschen Asylpolitik mit dem Anwerbestopp. Mit dem sinkenden Bedarf an Arbeitskräften waren auch Flüchtlinge, deren Schutz zunächst aus historischer Perspektive und angesichts des Kalten Krieges als notwendig erachtet wurde, zunehmend weniger erwünscht. Sie wurden dem Nützlichkeitsparadigma, das bei der

245 Vgl. die Aussage von Otto Schily, zit. n. Die Zeit vom 28.10.1999. 
Betrachtung von MigrantInnen im deutschsprachigen Raum eine herausragende Kontinuität darstellt, subsumiert. Dies verschärfte sich mit der Diversifizierung der Herkunftsländer der Flüchtlinge und dem Ende der Blockkonfrontation: Nachdem nun zunehmend Flüchtlinge aus afrikanischen und asiatischen Staaten in Deutschland Aufnahme suchten und die ideologische Begründung für die Aufnahme von Osteuropa-Flüchtlingen entfiel, erreichte die Einschränkung des Asylrechts ihren Höhepunkt mit der Grundgesetzänderung von 1992/93. Bis heute setzen sich die Restriktionstendenzen im Asylbereich auf zwei Ebenen fort: bei der Abschreckung durch die fortgesetzte soziale Schlechterstellung von Asylsuchenden und bei der Beschränkung ihres Zugangs zum Territorium der Bundesrepublik. Diese Entwicklung lässt sich durch das Wirken einiger zentraler Einflussfaktoren erklären: Dazu gehört die Konstruktion der deutschen Nation als ethnisch homogene Gemeinschaft und das damit verknüpfte lange vorherrschende Selbstverständnis der Bundesrepublik kein Einwanderungsland zu sein. Diese beiden Leitvorstellungen bilden den Hintergrund für den politischen Prozess, in dessen Rahmen sich die Verschärfung des Asylrechts vollzog. Dieser politische Prozess zeichnet sich durch die im föderalen System stattfindenden Dauerwahlkämpfe aus, in deren Rahmen sich das Thema Asyl als Mobilisierungsgarant erwiesen hat sowie durch das im semisouveränen System komplizierte Zusammenspiel von Bund, Ländern, Verwaltung, Gerichten, zivilgesellschaftlichen Akteuren und der EU. 



\section{Polen}

"Writing about international migration as it affects the territory of a given
country is an extremely challenging and difficult task. It involves writing not
only about the population flows from and into that country, but also about
the country's history, geography, economy, internal and external policies,
issues surrounding immigrants' integration or alienation, etc. "

(Iglicka 2001b, S. 1)

Polens aktuelles Migrationsregime ist einerseits geprägt durch seine Nationalgeschichte und die mit der Besatzung durch verschiedene andere Staaten verbundenen Siedlungspolitiken. Nach dem Systemumbruch 1989 erlebte die Republik eine

„Renaissance des nationalen Unabhängigkeitsdenkens in Kategorien der Vormoderne mit der Übersteigerung von Ethnizität und der Abgrenzung von ,fremden "Elementen " (Bingen 1992, S. 72). „Die Nationalen zehrten (und zehren noch) von den historischen Verletzungen der Nation in den vergangenen zweihundert Jahren, den historischen Legenden und Heldenmythen, der Instrumentalisierung der Geschichte für polnische Interessen und Herrschaftslegitimierung " (ebd., S. 58).

Unter der Macht dieser Strömungen ist „eine andere Tradition der polnischen politischen Mentalität" in den Hintergrund geraten: 
„das Erbe der multinationalen, multikonfessionellen und multikulturellen ersten Republike, in der einer auf die Frage: ,Wer bist du?', antworten konnte: einer von bier, und ein Gebildeter, der sich seiner vielschicbtigen Identität bewusst war, vielleicht auch: Canonicus cracoviensis, nationae polonus, gente rutenus, origine judaeus - ich bin ein Krakauer Kanonikus, gehöre (als Adliger) der (politischen) polnischen Nation an, stamme aus Ruthenien und babe jüdische Vorfahren..." (Kržeminski 2004).

Die dieser Denkart seit 1989 überlegene Betonung eines homogenen, nationalen Kollektivs spiegelt sich unter anderem in dem lange Zeit einzigen migrationspolitischen Bereich, der in Polen geregelt war: die Repatriierungspolitik, die vergleichbar ist mit der deutschen Politik gegenüber ,AussiedlerInnen' bzw. ,Volksdeutschen'. Auch in der polnischen Bevölkerung herrscht ein solches Bild von Polen vor. Dies zeigt eine Untersuchung von Ewa Nowicka, in der sie unter anderem untersucht hat, inwieweit PolInnen bereit sind, AusländerInnen als Teil der „Polish national community“ zu akzeptieren (Nowicka 2005, S. 269). Dabei fand sie heraus, dass in den Augen der befragten PolInnen
„[b]onds of nationality are [...] largely based here on predestined foundations meaning that the Polish ethnic group or nation is viewed as a tribal community rather than a cul- tural-political community." (ebd., S. 272)

Ein zweiter wichtiger Faktor ist Polens Geschichte als ein von Wanderungsbewegungen geprägtes Land: Neben zwangsweisen Siedlungspolitiken der Besatzer ${ }^{246}$ bestimmten auch individuelle Wanderungen in größerem Maßstab die Geschichte des Landes. Zwischen dem 13. und dem 19. Jahrhundert kamen (deutsche) Einwanderer nach Polen, während sich in diesem Zeitraum sehr viel weniger Polen in Richtung Deutschland aufmachten (vgl. Borodziej/Lemberg 2004, S. 52). Doch seit der zweiten Hälfte des 19. und Anfang des 20. Jahrhunderts dominiert die Auswanderung aus Polen das polnische Migrationsgeschehen.

Der dritte Faktor, der die jüngere polnische Migrationspolitik beeinflusst und Motor insbesondere der Politik gegenüber Flüchtlingen und Asylsuchenden ${ }^{247}$ ist, ist der EU-Beitritt Polens im Jahr 2004. Offenbar ohne die starke Stellung des Denkens in nationalen Kategorien sowie die Erinnerung an die eigene Geschichte als Herkunftsland von Flüchtlingen zu tangieren, ist das polnische Flüchtlingsschutzregime in nahezu rein bürokratischer Weise konstruiert worden. Mit der Entwicklung flüchtlings- und grenzpolitischer Regulierungen und dem Aufbau der

\footnotetext{
246 Vgl. hierzu auch den Abschnitt 6.1.1 Auswanderung und Rückkehr: Eine ,nationale Erfahrung' und ,Wrocloves you'.

${ }^{247}$ Hier wird im Interesse der Lesbarkeit der im Deutschen übliche Begriff ,Asylsuchende/ $\mathrm{r}^{\varsigma}$ verwendet, auch wenn dieser für Polen eigentlich nicht zutreffend ist. Die meisten Flüchtlinge beantragen hier Schutz gemäß Genfer Flüchtlingskonvention, das verfassungsrechtlich verankerte Asyl spielt in Polen hingegen faktisch so gut wie keine Rolle (vgl. im Abschnitt 6.3.3, S. 271: Verfahren und Ausgestaltung des Flüchtlingsschutzes).
} 
notwendigen Infrastruktur wurde eine wichtige Vorbedingung für den EU-Beitritt erfüllt - praktisch ohne öffentliche Debatte und Auseinandersetzung.

Im Folgenden soll es darum gehen, Polens neue Rolle als Aufnahmeland von Asylsuchenden zu untersuchen. Dafür werden die Entstehung, die Ausgestaltung und die Folgen des Asylregimes in Polen dargestellt und analysiert. Am Ende des Kapitels werden zusammenfassend die zentralen Kennzeichen des polnischen Flüchtlingsschutzregimes herausgearbeitet und die wichtigsten Einflussfaktoren seiner Entstehung im Einzelnen erörtert. Zu Beginn werden die Entwicklungen zunächst eingebettet in das allgemeine Migrationsgeschehen in Polen in historischer Perspektive, das durch die Gleichzeitigkeit von Auswanderung und Rückkehr, Einwanderung und Transitmigration geprägt ist. Diese Ausführungen sind etwas umfangreicher als der entsprechende Abschnitt zu den Migrationsprozessen im Kapitel über die Bundesrepublik. Dies ist der Tatsache geschuldet, dass es für die Bundesrepublik Deutschland zu diesem Bereich bereits einige einschlägige Überblickswerke gibt, während der polnische Fall - vor allem die aktuelleren Entwicklungen - noch weniger gut dokumentiert ist. ${ }^{248}$

\subsection{Migrationsprozesse: Zusammenspiel von Auswanderung und Rückkehr, Einwanderung und Transitmigration}

„Although Poland is still a country of emigration, it has recently experienced an inflow of asylum seekers, movement of transit migrants and permanent immigration both from the East and the West. Indeed, a new ethnic diversity and creation of a new ethnic consciousness can now be observed. " (Iglicka 2005a, S. 5)

Das polnische Beispiel zeigt sehr eindrücklich den konstruierten Charakter von Maßnahmen rund um die Migration: Die Bedeutung von Grenzen, Zugehörigkeiten und Mobilitäten hat sich binnen kurzer Zeit grundlegend gewandelt. Migrationen, die unter der kommunistischen Herrschaft illegal waren, sind nun erlaubt, vormals geschlossene Grenzen geöffnet. Plötzlich gab es zum Beispiel in Deutschland keine Asylbewerber mehr aus Polen und inzwischen auch kaum noch ,Aussiedler - dafür ersuchen mehr und mehr Menschen Asyl in Polen. Und was in der Transformationsphase legal war - die massenhafte Einreise von HändlerInnen - wurde zum kriminellen Akt, seit die Grenzen zu den Nachbarn im Zuge des EU-Beitritts (wieder) geschlossen wurden.

\footnotetext{
248 Soweit nicht anders gekennzeichnet, wurden im Folgenden alle Zitate aus dem Polnischen (vor allem aus Zeitungsartikeln) von mir selbst übersetzt.
} 
Nach dem Zweiten Weltkrieg bis 1989 fand Migration in Polen fast ausschließlich unidirektional - als Auswanderungsbewegung - statt, die das kommunistische Regime überwiegend zu verhindern suchte. Die Auswanderung in dieser Phase wurde seinerzeit sowohl von westeuropäischen als auch von polnischen ForscherInnen kaum untersucht. Zum einen standen nahezu keine Daten und Informationen zur Verfügung. Zum anderen galt für die polnischen ForscherInnen im Einklang mit der herrschenden Ideologie, dass das Phänomen internationale Migration gar nicht existieren und so auch keinen Forschungsgegenstand darstellen durfte. „Therefore, the notion of the Central and Eastern European migration system did not even exist in the theoretical and research literature on international migration." (Iglicka 2001b, S. 4) Gleichwohl gab es staatlich unterstützte Migrationen zwischen den Ländern des Ostblocks, wenn auch in geringem Umfang: Dazu gehörten die ,Repatriierung ${ }^{6}$ ethnischer Minderheiten, Familienzusammenführungen sowie die streng überwachte Einwanderung von ,GastarbeiterInnen (meist aus anderen Ostblockstaaten) (vgl. Kaczmarczyk/Okólski 2005, S. 4).

Mit dem Systemwechsel hat sich das Migrationsgeschehen stark gewandelt:

„Since 1989, and hence over a relatively short time, old mechanisms of migration in Poland have been shaken up and replaced or significantly supplemented by entirely new mechanisms. "(Iglicka 2001b, S. 4)

In den westeuropäischen Staaten wurden Befürchtungen laut, dass mit dem Fall des ,Eisernen Vorhangs' eine Massenmigration von Ost nach West stattfinden würde. Diese Erwartung wurde jedoch nicht bestätigt: Zwischen 1989 und 1999 sind lediglich ca. 2,5 Millionen Menschen aus der Region (den mittel- und osteuropäischen Staaten und der GUS) nach Westen migriert (vgl. ebd., S. 5). Durch die Mobilität der Menschen aus der früheren Sowjetunion einerseits und die ihnen gegenüber verschärften Einwanderungsgesetze durch die westeuropäischen Staaten andererseits wurde in Mitteleuropa eine Pufferzone geschaffen: „Poland is probably the best example of a buffer zone country" (Iglicka 2001a, S. 5). Dabei vollzog Polen eine vergleichbare Entwicklung wie die südeuropäischen Staaten Italien, Spanien und Portugal, die sich in den 1980er Jahren langsam von Aus- zu Einwanderungsländern wandelten. Auch in Polen verkleinert sich inzwischen der Abstand zwischen Aus- und Einwanderungszahlen.

Heute ist das Migrationsgeschehen in Polen gekennzeichnet durch die Gleichzeitigkeit von Auswanderung, Einwanderung und Transitmigration. Neben dauerhaften und vor allem temporären Auswanderungsbewegungen von Arbeitskräften und Pendelmigrationen (,Arbeitstourismus', Kleinhandel) sowie Transitmigrationen lassen sich auch (temporäre) Einwanderung von Arbeitskräften - vor allem aus den östlichen aber auch aus den westlichen Nachbarstaaten - sowie (in geringem Umfang) die Rückkehr von früheren EmigrantInnen oder Vertriebenen beobachten. Diese Entwicklungen gehen innerhalb Polens einher mit der Entstehung neuer MigrantInnen-Communities einerseits und andererseits mit einer starken 
Präsenz der Emigration im öffentlichen Bewusstsein, da fast jede/r Verwandte oder Bekannte hat, die im Ausland leben. Zu den neuen Entwicklungen gehört ferner, dass Polen seit Anfang der 1990er Jahre erstmals auch Aufnahmeland für Flüchtlinge ist. Viele von ihnen sehen Polen noch immer als Transitland, werden aber durch die EU-weiten Zuständigkeitsregelungen gezwungen, in Polen zu bleiben.

In den folgenden beiden Abschnitten wird ein Überblick über die aktuellen Migrationsbewegungen aus, nach und durch Polen gegeben. In diesem Rahmen ist auch ein Blick auf die lange Geschichte der Bevölkerungsbewegungen auf dem heutigen polnischen Territorium notwendig. Durch diese historische Kontextualisierung kann das aktuelle Geschehen besser beschrieben und analysiert werden:

„Unfortunately, most experts treat 1989 as a starting point for studies of changes in population mobility dynamics in the region. This often leads to ad-hoc and primarily short-perspective analyses of East-West migration. Such an approach does not permit full understanding of the complexity of new population movements in the region, nor does it belp to understand their dynamics. "(Iglicka 2001b, S. 119)

Für diese Auseinandersetzung mit den Migrationsprozessen in Polen und vor allem mit den damit verbundenen Maßnahmen müssen zunächst einige zentrale Begriffe erläutert werden. ,Minderheiten', ,Ethnizität' und ,Nation' sind in den Darstellungen der polnischen Geschichte und insbesondere im Zusammenhang mit Aus- und Einwanderung, Umsiedlungen, Vertreibungen usw. stets wichtige Referenzpunkte. Dabei wird oft nicht erklärt, wie zum Beispiel die Begriffe ,ethnische' oder ,nationale Minderheit' verstanden werden (vgl. z.B. Owczarek 2002) oder es wird betont, dass es sich um völkerrechtlich nicht klar definierte und so in ihrer Verwendung oft unscharfe Begriffe handelt (vgl. z.B. Ziemer 1998). ${ }^{249} \mathrm{Nach}$ Ziemer (1998) habe sich in der Literatur durchgesetzt, von einer ,nationalen “ Minderheit zu sprechen, wenn es eine ,Titularnation' außerhalb des Siedlungsgebietes der Minderheit gibt (zum Beispiel die dänische Minderheit in Deutschland). Von einer ,ethnischen' Minderheit werde gesprochen, wenn die Minderheit nicht über eine ,Titularnation` außerhalb ihres Siedlungsgebietes verfügt (zum Beispiel Sorben in Deutschland).

Auch auf der Basis einer so differenzierten Definition können ,Ethnizität‘, ,Nation“ und ,Minderheiten“ jedoch nur mit Einschränkungen als analytische Kategorien zur Anwendung kommen. Denn es handelt sich um konstruierte Katego-

\footnotetext{
249 So verweist Ziemer (1998) auf unterschiedliche Definitionen im Internationalen Pakt über bürgerliche und politische Rechte von 1966, der europäischen Menschenrechtskonvention, in den Verfassungen einzelner Staaten und entsprechenden bilateralen Abkommen. Umstritten seien Fragen der Gruppengröße, des subjektiven Zugehörigkeitsgefühls und der Dauer der Anwesenheit im jeweiligen Land. Werde von ,nationaler ${ }^{\star}$ Minderheit gesprochen, so sei damit streng genommen zwischen unterschiedlichen Nationen-Konzepten zu unterscheiden (Kulturnation, Staatsnation), die sich wiederum teilweise mit der Frage von Ethnizität überschnitten.
} 
rien und nicht zuletzt „,(sozial-)wissenschaftlich erzeugte Effekte“ (Dittrich/Radtke 1990, S. 21):

„Während in der Auseinandersetzung mit dem Konzept ,Rasse“ die Brauchbarkeit der Kategorie selbst als Unterscheidungsmerkmal bestritten wird und der darauf gegründete Antirassismus sich politisch gegen die Rassisierung sozialer Konflikte wendet, wird in der Auseinandersetzung mit dem Nationalismus und der in seinem Gefolge stattfindenden Unterdrückung von ethnisch definierten Minderheiten bislang die Kategorie in ibrer Angemessenheit nicht in Frage gestellt [...] Aber so wenig die Kategorie ,Rasse' eine natürliche Entsprechung in der Wirklichkeit hat, so wenig sind ethnische Identität und ethnisches Bewußtsein natürliche Tatsachen, die jenseits historischer Konstruktionen bestehen oder gar konstitutiv für menschliches Leben wären. Sie sind entstanden in einer bestimmten historischen Konstellation als bestimmte bistorische Konstruktion eines konstanten sozialen Problems, an dem sich nach den Natur-nun auch die Sozial-Wissenschaften maßgeblich beteiligt haben. Nur in diesem Sinne haben sie Realität. Die einmal in die Welt entlassene wissenschaftliche Konstruktion eines Volksgeistes bzw. einer nationalen oder ethnischen Identität wurde und wird im politischen Alltag als Sinnstiftungsangebot ergriffen, benutzt und vor allem geglaubt. "(Dittrich/Radtke 1990, S. 23, Hervorh. i. Orig.)

Wenn man die Kategorien nicht als natürliche Einheiten, sondern als konstruiert begreift, so ermöglicht diese Perspektive zahlreiche Problemstellungen aufzudecken, die ansonsten als quasi ,naturgegeben' angenommen würden. Gerade an der wechselvollen Geschichte des polnischen Nationalstaates und der Zusammensetzung seiner Bevölkerung, in den Versuchen, diese wahlweise zu ,germanisieren', zu ,russifizieren', zu ,entdeutschen' oder zu ,polonisieren' bzw. zu ,verifizieren“ oder zu ,homogenisieren' kommt eine enorme Konstruktionsleistung seitens der verschiedenen historischen und aktuellen AkteurInnen zum Ausdruck. So wurden immer wieder neue Kategorien, Kriterien und Zugehörigkeiten produziert und (zwangsweise) angewendet, die wiederum im Zusammenhang mit Konflikten über Grenzen und Staatssouveränität standen oder dazu beitrugen, solche Konflikte zu entfachen und zu legitimieren. Die Konstruktionen und die damit verbundenen Vorstellungen von ,homogenen Nationen` hatten oft gewaltförmige Folgen für die Bevölkerung: Sie war betroffen von Vernichtung, Vertreibung, Verschleppung, Umsiedlung, Enteignung und Entwurzelung. 


\subsubsection{Auswanderung und Rückkehr: Eine ,nationale Erfahrung' und ,Wrocloves you'}

,[F] or more than a century Poland has been one of the biggest sending areas

in Central and Eastern Europe and a vast reservoir of labour for many countries in Western Europe and North America." (Iglicka 2001b, S. 53)

Die Geschichte der Bewegungen von Menschen aus (und nach) Polen ist eng mit der Geschichte der Kämpfe um das polnische Territorium und den damit verknüpften Bevölkerungspolitiken verwoben. Polen war in der Vergangenheit durch ,extern' initiierte territoriale Neuordnungen für lange Zeit als unabhängiger, souveräner Staat gänzlich von der Landkarte ,verschwunden‘. Von 1795-1918, mit einer kurzen Unterbrechung von 1807 bis 1815, war Polen zwischen Österreich, Preußen und Russland aufgeteilt. Die Besatzungsmächte haben jeweils unter anderem durch Siedlungs- und Vertreibungsmaßnahmen und die Unterdrückung des ,Polentums“ (zum Beispiel durch das Verbot, den Unterricht in den Schulen in polnischer Sprache abzuhalten) versucht, ihren Einfluss in der Region zu festigen. Durch territoriale Neuordnungen und damit verbundene Grenzverschiebungen wurden immer wieder neue Minderheiten erzeugt. Polen war später seinerseits bestrebt, seine vor allem auf diese Weise diversifizierte Bevölkerungsstruktur zu ,homogenisieren'. So wurde über Jahrhunderte ein komplexes Gefüge von Zugehörigkeiten und Identitäten erzeugt, innerhalb dessen nach wie vor um die Bedeutung und den Inhalt von Konstruktionen wie ,Abstammung', ,Herkunft ${ }^{6}$ und ,Staatsbürgerschaft' gerungen wird.

Betrachtet man Polen durch diese Brille der Konstruktionen von ,ethnischen und ,nationalen' Minderheiten, von Zugehörigkeit und Identität, die bis heute stets umkämpft waren und sind, so lässt sich Vorkriegspolen als ,multiethnisch und multireligiös“ (Majcherek 1999) oder „Vielvölkerstaat“ (Owczarek 2002, S. 710) bezeichnen, in dem unter anderem die größte jüdische Gemeinde Europas lebte. Diese Bevölkerungsstruktur hat sich grundlegend gewandelt: Während 1931 noch ein Drittel der polnischen Bevölkerung - auf dem damaligen Territorium - als einer ,ethnischen' oder ,nationalen' Minderheit zugehörig definiert wurde, sind nach Schätzungen heute nur noch zwei bis vier Prozent der Bevölkerung Minderheitenangehörige, wovon die deutsche Minderheit die größte Gruppe bildet (vgl. Alscher 2008; Owczarek 2002). „Polen [...] war ethnisch [...] radikal homogenisiert worden." (Bingen 1992, S. 47) Diese tiefgreifende Veränderung der Bevölkerungszusammensetzung war vor allem das Ergebnis der Vernichtungs- und Vertreibungspolitik der deutschen Besatzer während des Zweiten Weltkrieges: der Vernichtung der jüdischen Bevölkerung, der massenhaften Vertreibung und Ermordung von PolInnen und der Verschleppung von Kriegsgefangenen und ZivilistInnen als ZwangsarbeiterInnen in das, Deutsche Reich'. Mit dem Ende des 
Krieges und der deutschen Besatzung hinterließen Grenzverschiebungen und Umsiedlungen nach Deutschland, in die und aus der damaligen UdSSR sowie innerhalb Polens deutliche Spuren in der Bevölkerungsstruktur des Landes.

Bei der Emigration nach Deutschland, das über Jahrzehnte Hauptzielland von EmigrantInnen aus Polen war, spielten Zugehörigkeitskonstruktionen nach 1945 eine wichtige Rolle. Der Mix aus ,ethnischer', ,kultureller' und staatsbürgerlicher Zugehörigkeit, der im Laufe der Geschichte immer wieder neu definiert und zugewiesen wurde und deren Verhältnis zu den Selbstzuschreibungen der Einzelnen wird am Beispiel der ,AussiedlerInnen' deutlich:

„AussiedlerInnen begreifen sich nicht unbedingt ausschließlich als ,Deutsche. Sie haben den gleichen oder einen ähnlichen kulturellen und sprachlichen Hintergrund wie PolInnen, beide Gruppen überschneiden sich aufgrund persönlicher Beqiehungen zueinander. Jede Festlegung [...] läuft Gefahr, die Kategorie ,Ethnizität‘ bzw. ,MigrantInnen“ oder ,Minderheit' als einzige und permanente Ordnungskategorie zu akzeptieren. Die Herkunft brw. Ethnizität ist dennoch gesellschaftlich wirksam. Sie wird von außen - in der staatlichen Politik und durch die Mitglieder der Aufnabmegesellschaft - als Zuordnungskategorie angewandt. Unter bestimmten gesellschaftichen Voraussetzungen wählen MigrantInnen sie selbst als Bequgsgröße. Diese Fremd- und Selbstzuschreibungen sind nicht unbedingt deckungsgleich. Weiterhin stimmen die Motive der MigrantInnen und ihre Selbstzuschreibungen häufig nicht mit den durch die beteiligten Staaten in der Ausund Einreise- und Integrationspolitik vorgenommenen Kategorien überein, zumal sich die administrativen Kategorien von Entsende- und Aufnabmeland unterscheiden [...]" (Miera 2007, S. 11).

Für die Ausreise wurde die Staatsangehörigkeit als zentrales Kriterium definiert. Allerdings waren sich Polen und Deutschland bis Ende der 1980er Jahre uneinig darüber, wer die jeweilige Staatsbürgerschaft hatte bzw. erhalten sollte (vgl. Miera 2007, S. 27). Dabei war zentral, dass Polen lange abstritt, dass es auf seinem Territorium eine deutsche Minderheit gibt (vgl. ebd.). Die deutsche Seite hingegen forderte eine Ausreisemöglichkeit für die Angehörigen dieser Minderheit. Die Definition dessen, was als ,deutsch ' und was als ,polnisch' gelten sollte, erwies sich angesichts der deutsch-polnischen Geschichte als komplexes Unterfangen (vgl. Miera 2007, S. 27ff.).

Die historischen Ereignisse sind mithin vor dem Hintergrund dieser Konstruktionen von ,ethnischen' und ,nationalen' Minderheiten, von Zugehörigkeit und Identität in der polnischen Geschichte zu betrachten: Aufgrund der mit dem polnisch-sowjetischen Grenzvertrag vom August 1945 festgelegten Westverschiebung Polens kam es an der Ostgrenze zu großen Umsiedlungen (vgl. Alscher 2004, S. 215ff.). So wurden rund 500.000 Personen ukrainischer, weißrussischer und litauischer Herkunft von der polnischen Seite auf das Territorium der damaligen Sowjetunion umgesiedelt. UkrainerInnen (mit polnischer Staatsbürgerschaft), die einen unabhängigen ukrainischen Staat anstrebten und gegen die Zwangsum- 
siedlung Widerstand leisteten, wurden 1947 gewaltsam auf die neuen polnischen West- und Nordgebiete verteilt (,Aktion Wisła) und sollten dort assimiliert werden (vgl. Ziemer 1998; Owczarek 2002; Alscher 2004). Im Gegenzug wurden rund eineinhalb Millionen PolInnen, die bisher östlich der neuen Grenze gelebt hatten, überwiegend gewaltsam auf das neue polnische Staatsgebiet umgesiedelt.

Zudem kehrten (300.000) polnische JüdInnen nach dem Zweiten Weltkrieg zurück, verließen das Land (220.000) aber aufgrund starker antisemitischer Tendenzen in den Folgejahren wieder Richtung Israel, Westeuropa und USA (vgl. Alscher 2008, S. 1). Ferner wurden auf Grundlage des 1945 geschlossenen Potsdamer Abkommens bis 1950 rund drei Millionen Deutsche aus Polen ausgesiedelt; hinzu kommen ca. eine halbe Million Deutsche, die direkt zu Kriegsende in unorganisierten Bewegungen das Land verließen.

Neben diesen und weiteren (Um)Siedlungs- und Vertreibungsmaßnahmen hat es immer in größerem Umfang aus unterschiedlichen Gründen individuelle Emigrationen von PolInnen gegeben. „For centuries, Polish lands served as a vast reservoir of labour for many countries, most notably for Germany but also for overseas countries settled by Europeans.“ (Iglicka 2001b, S. 13) Bereits seit den 1870er Jahren migrierten PolInnen aufgrund mangelnder Arbeitsmöglichkeiten in Polen hauptsächlich ins Ruhrgebiet und nach Berlin, wo sie in der Industrie, im Handwerk, im Bergbau, als Dienstbotinnen oder Näherinnen arbeiteten (vgl. Miera 2007, S. 25). ${ }^{250}$ Während des ersten Weltkrieges und zwischen 1918 und 1939 wanderten jeweils zwei Millionen PolInnen aus (vgl. Okólski 1999, S. 16f.).

Nach dem Zweiten Weltkrieg bestimmte die Emigration aufgrund ,ethnischer Zugehörigkeit zur deutschen Minderheit die Auswanderungssituation. Seit „Abschluss der Vertreibungsmaßnahmen“" (Miera 2007, S. 33) bis zur Transformation verfolgte Polen überwiegend eine restriktive Auswanderungspolitik und verschloss seine Grenzen nach Westen fast völlig: „Between 1945 and 1989, all legislation on foreign migration reflected isolationist principles: they were simply repressive." (Iglicka 2001b, S. 14) Allerdings kam es phasenweise immer wieder zu Liberalisierungen. Diese unterteilt Frauke Miera (2007, S. 33f.) (mit Fokus auf die Auswanderung nach Deutschland) in fünf Phasen:

- „restriktive Ausreisepolitik in den 50er und 60er Jahren mit der Tauwetterperiode und der ,Aktion Familienzusammenführung'von 1956/57“" (Miera 2007, S. 33)

- „Abschluss des Warschauer Vertrages 1970, in dessen Rahmen sich die polnische Seite auf Erteilung von Ausreiseerlaubnissen für AussiedlerInnen bereit erklärte“ (ebd.)

\footnotetext{
${ }^{250}$ In Westfalen und den Rheinprovinzen schlug sich diese Bewegung in einem hohen polnischen Bevölkerungsanteil in einigen Städten nieder (Herne: 22 Prozent, Recklinghausen: 23 Prozent, um 1910) (vgl. Borodziej/Lemberg 2004, S. 53).
} 
- „Phase der Verhandlungen im Rahmen der Konferenz über Sicherheit und Zusammenarbeit in Europa (KSZE) von 1975, die ebenfalls Ausreiseerleichterungen zur Folge hatte“ (Miera 2007, S. 33)

- „Phase des politischen Aufbruchs in Polen Ende der 70er Jahre bis Dezember 1981 mit einer kurzen Phase der temporären Erwerbsmigration“ (ebd., S. 33f.)

- „erneut restriktive Zeit des Kriegsrechts bis zur beginnenden politischen Transformation" (ebd., S. 34)

Nachdem die Emigration Anfang der 1950er Jahre zunächst gestoppt worden war, wurde diese mit dem Tod Stalins und der damit einsetzenden ,Tauwetterperiode“ teilweise wieder ermöglicht. Sie erfolgte auf der Basis verschiedener Übereinkommen zwischen der ostdeutschen und der polnischen Regierung bzw. dem westdeutschen und dem polnischen Roten Kreuz und sollte vor allem Familienangehörige, die bei den früheren Bewegungen von 1945 bis 1950 getrennt worden waren, wieder zusammenführen. Doch erwies sich die Unterscheidung dessen, was ,polnisch“ und was ,deutsch'war, als schwierig. Dies zeigt sich an der Ausweitung der Kriterien, auf die sich die polnische Regierung einließ: So konnten auch Personen ,deutscher Abstammung ${ }^{*}$ eine Ausreisegenehmigung erhalten, die die polnische Staatsbürgerschaft hatten (vgl. Iglicka 2001a, S. 15, Miera 2007, S. 34). Laut Krystyna Iglicka (2001a, S. 15) wurde sogar ganzen Familien mit ,polnischer Nationalität ${ }^{\star}$ und Staatsbürgerschaft die Ausreise bewilligt. Damit habe die Regierung indirekt ,Fehler ${ }^{6}$ bei der ,Verifizierung ${ }^{6}$ der polnischen Staatsangehörigen eingeräumt (vgl. Miera 2007, S. 34). ${ }^{251}$

Das Entgegenkommen der polnischen Regierung bei der ,Familienzusammenführung' führt Miera (2007, S. 34) auf die schweren Versorgungsprobleme zurück, aufgrund derer es in dieser Zeit in Polen zu Aufständen und Streiks gekommen war. Von deutscher Seite wurde der ,Aussiedlerstatus' ebenfalls relativ bereitwillig verliehen. So emigrierten zwischen 1956 und 1958 über 300.000 Personen $^{252}$, obwohl Schätzungen von 1950 von nur 160.000 verbliebenen Deutschen in Polen ausgegangen waren (Iglicka 2001a, S. 15). Die ,Aktion Familienzusammenführung wurde 1956 durch die polnischen Behörden beendet. Danach gab es nur noch eine sehr begrenzte Auswanderung auf Basis der Familienzusammenführung (15.000 bis 30.000 jährlich) und fast gar keine Einwanderung mehr (vgl. Okólski 1998, S. 15).

\footnotetext{
${ }^{251}$ Bei dem im Mai 1945 eingeleiteten Verifizierungsverfahren sollte die „,urpolnische“ und teilweise ,zwangsgermanisierte ' Bevölkerung [...] von der ,tatsächlich deutschen' Bevölkerung getrennt und an der Ausreise aus Polen gehindert werden, während ,die Deutschen' ausgewiesen und gewaltsam vertrieben wurden“ (Miera 2007, S. 29). Um „,,als Landsleute rehabilitiert",, (ebd.) zu werden, mussten sie ihr ,Polentum' glaubhaft darlegen. Bemerkenswert ist, dass dieser, Verifizierung' die ,Deutsche Volksliste' (DVL) zugrunde gelegt wurde, die auf einem Erlass Heinrich Himmlers zur Erfassung und ,rassenpolitischen' Kategorisierung der ,deutschstämmigen' Bevölkerung in den besetzten Gebieten basierte (vgl. ebd.).

252 Im Jahr 1955 hatten nur 700 Personen das Land verlassen (vgl. Miera 2007, S. 34).
} 
Die polnischen Behörden beschieden von 1959 bis 1970538.000 Ausreiseanträge negativ. Während einerseits sowohl die Ausreise als auch die Wiedereinreise sehr restriktiv geregelt waren und oft in der Praxis noch restriktiver gehandhabt wurden, wurde angesichts innenpolitischer Krisen, unerwünschten' StaatsbürgerInnen die Ausreise mitunter nahegelegt bzw. in einem vereinfachten Verfahren ermöglicht (vgl. Miera 2007, S. 35).

Dies zeigt sich am Beispiel einer weiteren bedeutenden Auswanderungsbewegung besonders drastisch: Der Emigration - oder zutreffender: Vertreibung - von ca. 25.000 jüdischen PolInnen von 1968-1970 (vgl. Kosmala 2000) war eine antisemitische (nach offizieller Darstellung: antizionistische) Kampagne vorausgegangen, die Innenminister Mieczysław Moczar gestartet hatte. Sie sollte verhindern, dass die Studentenproteste, die 1968 auch in Polen stattfanden, sich zu einer Massenbewegung ausweiteten und wurde als nationaler Befreiungskampf gegen angeblich ,moskauhörige JüdInnen` in der Parteispitze deklariert. Entgegen der landläufigen Interpretation, es habe sich um eine vor allem von Moczar initiierte und von ,Moczaristen' getragene Kampagne gehandelt, die sich gegen die Führungsgruppe um Parteichef Gomułka innerhalb der kommunistischen Partei richtete, verweist Andrzej Szczypiorski (1998) auch auf den gesellschaftlich tief verankerten Antisemitismus. Dieser habe dazu beigetragen, dass die Kampagne in diesem Ausmaß stattfinden konnte. In der Folge mussten Tausende polnische BürgerInnen (vermeintlich) jüdischer Herkunft ihre Ämter in Partei, Wissenschaft und Kultur verlassen, vielen wurde die polnische Staatsbürgerschaft entzogen. Trotz der sonst vorherrschenden Ausreiserestriktionen erhielten sie problemlos eine Ausreiseerlaubnis und verließen das Land vor allem in Richtung Israel und USA.

In den 1970ern öffnete sich Polen etwas und ging wirtschaftliche Kooperationen mit westlichen Staaten ein. Im Zuge dessen wurden die Bedingungen für Auslandsreisen liberalisiert, mehr Tourismus und in gewissem Umfang Arbeitsmigration ermöglicht. Dies nutzten viele, um auszureisen und nicht mehr zurückzukehren (Okólski 1998, S. 15). Auch die Ausreise von Personen ,deutscher Volkszugehörigkeit' erhielt neuen Schwung, als diese im Zusammenhang mit dem Abschluss des Warschauer Vertrages von 1970 zeitweise vermehrt zugelassen wurde. In den 1970ern wurde die Ausreise von ,Aussiedlern` zudem immer stärker mit wirtschaftlichen Interessen verknüpft. In den Übereinkünften im Rahmen der KSZESchlussakte von 1975 wurde im Austausch für wirtschaftliche Unterstützung die Erteilung von Ausreiseerlaubnissen in Aussicht gestellt, was ExilpolInnen unter dem Schlagwort „Ludzie za marki“ (Menschen für Geld) als Menschenhandel kritisierten (Dziennik Polski vom 19.08.1975, zit. n. Miera 2007, S. 38). Im Jahre 1979 vereinbarten Polen und die Bundesrepublik (übrigens ungeachtet des Anwerbestopps in der Bundesrepublik) den Austausch von Arbeitskräften. Ende der 1970er Jahre bis zur Ausrufung des Kriegrechts reagierte die polnische Regierung auf die Unruhen und Proteste mit einer massiven Vereinfachung der Ausreise, im Rahmen derer temporäre Arbeitsmigration ermöglicht wurde (vgl. Miera 2007, 
S. 40). Viele reisten als TouristInnen aus und beantragten dann in der Bundesrepublik den Aussiedlerstatus und in zunehmendem Umfang auch Asyl.

In den 1980er Jahren gab es unter dem Einfluss der Wirtschaftskrise, der Verhängung des Kriegsrechts (1981-1983) und der Niederschlagung der SolidarnośćBewegung erneut eine große Auswanderungsbewegung bei gleichzeitig restriktiver Ausreisepolitik (vgl. Miera 2007, S. 43). Während diese Emigration in offiziellen Statistiken mit 271.000 Personen beziffert wurde, gehen Schätzungen von 1 bis 1,3 Millionen Auswanderern zwischen 1980 und 1989 aus (vgl. Currle 2004, S. 361; Okólski 1998, S. 16). Diese Bewegung war zum einen wiederum durch den Bezug auf den Aussiedlerstatus geprägt, zum anderen auch auf politische Gründe, bzw. wurde in der Bundesrepublik, wer nicht den Aussiedlerstatus beantragte, pauschal als politischer Flüchtling klassifiziert. Daraufhin wurde die Ausreise von polnischer Seite erschwert, die Erschwernisse wurden 1986 wieder gelockert. Mit dieser Emigrationsbewegung war ein starker ,Brain Drain` verbunden: 15 Prozent der polnischen WissenschaftlerInnen verließen Polen (vgl. Iglicka 2001a, S. 6; 2001b, S. 26). Zwar wurden die MigrantInnen in der Bundesrepublik nur in eine der beiden Kategorien ,AussiedlerInnen' oder ,politische Flüchtlinge` einsortiert, tatsächlich waren die Ursachen für ihre Emigration jedoch komplexer. Neben ökonomischen und politischen Motiven werden auch die Desillusionierung und Demoralisierung weiter Teile der Bevölkerung angesichts fehlender Selbstverwirklichungsmöglichkeiten und des Versagens des Staates in vielen seiner Aufgaben als Auswanderungsursachen gesehen. ${ }^{253}$ Die allein auf zwei Motive - politische Gründe oder deutsche Abstammung - reduzierte Wahrnehmung und Aufnahme der MigrantInnen spiegelt wiederum die Bedeutung der Kategorisierung von MigrantInnen für den politischen bzw. öffentlichen Diskurs und für die MigrantInnen selbst: Zwar hatten sie verschiedenste Gründe auszuwandern, dennoch ordneten sie sich der einen oder anderen Kategorie zu und wurden so von der Bundesrepublik aufgenommen, in der es zu diesem Zeitpunkt opportun war, einerseits durch Aufnahme von Flüchtlingen aus dem, Ostblock ${ }^{`}$ die Überlegenheit des eigenen Systems zu demonstrieren und andererseits, diskriminierten Deutschstämmigen' im Ausland die ,Heimkehr' zu ermöglichen.

Ab Ende der 1980er Jahre veränderte sich das Emigrationsgeschehen grundlegend. Bereits ab 1987 entwickelte sich die in den 1990er Jahren charakteristische Pendelmigration, da nun auch befristete Ausreisen vermehrt zugelassen wurden.

„Waren bis in die 80er Jahre in der BRD langfristige Migration und Ansiedlung und in der DDR eher quantitativ geringfügige, temporäre Erwerbsmigration dominant, so werden in den 90er Jabren die temporäre Pendelmigration und das Leben in transnationalen Räumen typisch. "(Miera 2007, S. 13)

${ }^{253}$ Ausführlicher zu diesem Erklärungsansatz vgl. Okólski 1999, S. 20 ff. 
Mitte 1988 hob die letzte kommunistische Regierung die noch bestehenden Beschränkungen der Reisefreiheit für polnische BürgerInnen auf und öffnete gleichzeitig die Grenzen für die Einreise von AusländerInnen (vgl. Okólski 1999, S. 22). Damit wandelten sich zum einen die Emigrationsmuster der PolInnen und zum anderen entwickelte sich Polen von einem Auswanderungsland mit restriktiver Auswanderungspolitik binnen kurzer Zeit auch zu einem Ziel von AusländerInnen: KleinhändlerInnen, temporären ArbeitsmigrantInnen, Flüchtlingen, dauerhaften EinwanderInnen, aber auch von RückkehrerInnen sowie TransitmigrantInnen.

Obwohl sie nun nicht mehr illegal stattfinden mussten, ist in den 1990er Jahren ein deutlicher Rückgang der Emigrationen aus Polen zu verzeichnen (vgl. Iglicka 2001b, S. 124; Okólski 1999, S. 27f.). Als also der Personenverkehr deutlich liberalisiert und die Migrationshürden seitens des polnischen Staates verringert wurden, sank die Zahl der polnischen EmigrantInnen. Die Ursachen dafür werden einerseits in den verschärften Einwanderungspolitiken der westeuropäischen Staaten gesehen - insbesondere in den höheren Barrieren für AussiedlerInnen und der nun für PolInnen nicht mehr Erfolg versprechenden Asylbeantragung. Andererseits habe „die Erwartung eines besseren Lebensstandards und besserer Entwicklungsperspektiven des eigenen Landes die Auswanderung unattraktiv“ gemacht (Orlowski 2001). Insbesondere für junge qualifizierte PolInnen in den Städten hätten sich neue Chancen in Polen ergeben (vgl. Iglicka 2001a; 2001b, S. 33). Dafür spricht auch, dass sich nun vermehrt weniger qualifizierte PolInnen auf den Weg ins Ausland machten:

„One of the most spectacular structural changes concerning emigration from Poland has been a shift from more to less educated migrants. "(Okólski 1999, S. 28)

Die gleichzeitig stattfindende Verschiebung von dauerhafter hin zu temporärer Migration (vgl. Iglicka 2001b, S. 124; Kaczmarczyk 2004, S. 68f.; Okólski 1999, S. 22) und zum „Leben in transnationalen Räumen“ (Miera 2007, S. 13) verweist auf gewandelte Migrationsstrategien angesichts der neuen Rahmenbedingungen. Da die Auswanderungsentscheidung nun nicht mehr zwangsläufig endgültigen Charakter haben musste und die Migrationswilligen nicht mehr vor der Entscheidung: „stay or go once and for all“ standen (Okólski 1999, S. 35), eröffneten sich ihnen flexiblere Migrationsoptionen. Diese ermöglichten enge Verbindungen zum Herkunftsland, für das perspektivisch eine prosperierende Entwicklung mit einer Angleichung der Lebensverhältnisse an diejenigen in den Zielländern der EmigrantInnen erwartet wurde.

Im Zuge dieses Wandels ging auch die Bedeutung der, ethnischen Herkunft ${ }^{\star}$ als Auswanderungsoption zurück. Die schwindende Popularität des Aussiedlerstatus illustriert den strategischen Umgang mit den politisch konstruierten gates of entry". Was auch immer die jeweiligen Gründe für die Auswanderung waren, die Bezugnahme auf die ,ethnische Herkunft' ermöglichte es, den Migrationswunsch 
umzusetzen. Obwohl sie bei der Aufnahme in der Bundesrepublik oft der zentrale Bezugspunkt war, war die, nationale Identität' nur einer von vielen Faktoren, die die individuelle Auswanderungsentscheidung beeinflusst haben. Daneben seien die sich nach dem Zweiten Weltkrieg wandelnde ökonomische Attraktivität der Bundesrepublik, die politische Einstellung zum kommunistischen Regime und soziale Netzwerke, die bei der Migration behilflich waren, für die Emigrationsentscheidung bedeutsam gewesen (vgl. Miera 2007, S. 29f.).

Da es aufgrund der ökonomischen Rezession zunehmend schwieriger wurde, in Deutschland, Großbritannien oder Frankreich Arbeit zu finden, vollzogen sich auch hinsichtlich der Auswahl der Zielländer einige Veränderungen. So orientierten sich polnische ArbeitsmigrantInnen auch in Richtung Italien und Griechenland, wo sie zum einen als TouristInnen vergleichsweise unkompliziert einreisen und zum anderen in der Schattenwirtschaft leicht Arbeit finden konnten (vgl. Iglicka 2001b, S. 50). ${ }^{254}$ Auch die mit der Migration verbundenen Ziele der ArbeitsmigrantInnen wandelten sich: Während durch die Arbeit im Ausland vor 1989 aufgrund der starken Kaufkraft der westlichen Währung der Lebensstandard von Familien in Polen entscheidend erhöht werden konnte, dienten die Rücküberweisungen der ArbeitsmigrantInnen später vor allem dazu, den bestehenden Lebensstandard zu halten (vgl. Iglicka 2001b, S. 46).

Über den genauen Umfang der Emigration aus Polen nach dem Zweiten Weltkrieg gibt es keine gesicherten quantitativen Erkenntnisse. Statistische Daten sind insofern unzuverlässig, als viele ihre Emigration vor allem aus Angst vor Repression nicht offiziell gemeldet haben:

„It became routine, that, unless required by immigration or naturalization procedures in the countries of destination, emigrating Polish citizens failed to report their departure to the local administration, and they left the country as ordinary tourists" (Okólski 1998, S. 13).

Dies zeigt sich vor allem beim Vergleich der Zahl derer, die laut offiziellen polnischen Statistiken ausgewandert sind und der Zahl derer, die in der Bundesrepublik oder anderen Zielländern als Ankömmlinge registriert wurden (vgl. Okólski 1998, S. 13).

Die Literatur zu Polen und Migration befasst sich auch heute noch zu einem bedeutenden Teil mit der Auswanderung aus Polen. Dabei ging es immer wieder um Prognosen über die Zahl der polnischen Auswanderer angesichts verschiede-

\footnotetext{
254 Während Anfang der 1980er Jahre PolInnen vor allem als Flüchtlinge nach Italien und Griechenland kamen und von dort vorwiegend nach Übersee weiterwanderten, erleichterten die so entstandenen MigrantInnen-Netzwerke Ende der 1980er Jahre den nun folgenden ArbeitsmigrantInnen den Zugang zu diesen beiden Staaten und ihren Arbeitsmärkten. Dabei handelte es sich vor allem um gering qualifizierte PolInnen mittleren Alters, die aus ländlichen Regionen Polens stammen und in Griechenland und Italien die Tätigkeiten übernahmen, die für GriechInnen und ItalienerInnen nicht mehr attraktiv waren (vgl. Iglicka 2001b).
} 
ner Stufen der Liberalisierung des Zugangs zum ,Westen‘. Mit Blick auf den Systemwechsel im Jahr 1989
„war die Vorstellung weit verbreitet, mit den Grenzen öffne sich quasi ein Ventil, durch das ein über Jahre angestauter Migrationsdruck entweichen könne, und es würden mas- senhaft Menschen aus Osteuropa in den Westen strömen. In verschiedenen Arbeiten wurde das in der Öffentlichkeit vorherrschende Gefühl einer neuen Bedrohung durch die Wanderung aus dem Osten mit fragwürdigen Prognosen und Szenarien scheinbar wissen- schaftlich untermauert. "(Miera 2007, S. 19)255

Dieser Fokus auf die Vorhersage künftiger Wanderungsbewegungen erhielt in den Jahren kurz vor und nach Polens Beitritt zur Europäischen Union erneut Aufwind. Es gab einen sprunghaften Anstieg der Zahl an Artikeln und Aufsätzen zu Prognosen darüber, wie viele PolInnen die neue Freizügigkeit nutzen und sich weiter westlich Arbeit suchen würden und welche Auswirkungen für die Volkswirtschaften der Zielländer zu erwarten seien (vgl. Brücker 2004; Fassmann/Münz 2003256; Kupiszewski 2002; Orlowski 2001257). Tatsächlich können die Jahre nach dem EU-Beitritt 2004 als weitere bedeutende Auswanderungsphase gesehen werden, die ihren Höhepunkt inzwischen bereits überschritten hat.

Gegenwärtig werden in Polen Maßnahmen eingefordert, um die Auswanderungsbereitschaft zu senken und die bereits Ausgewanderten zur Rückkehr zu

\footnotetext{
255 Ebenfalls kritisch dazu vgl. Angenendt et al. (1994).

256 Heinz Fassmann und Rainer Münz schlussfolgern, dass der „Bedarf an Zuwanderern“ in den alten EU-Mitgliedstaaten aufgrund der demografischen Entwicklung in Ostmitteleuropa und den damit verbundenen besseren Arbeitsmarktchancen der Jüngeren vor Ort nicht mit Arbeitskräften aus den Beitrittsländern gedeckt werden könne: „Auf jene Zuwanderung aus Ostmitteleuropa, vor der sich heute etliche fürchten, werden wir mittelfristig nicht einmal hoffen können." (Fassmann/Münz 2003, S. 33)

257 Orlowski (2001) diskutiert die Tragfähigkeit ökonomischer Prognosen: „Alle makroökonomischen Analysen und Prognosen über die Migration nehmen als Ausgangspunkt die Formulierung aus der Handelstheorie an, nach der die Integration von Staaten mit relativ hoher Relation der Arbeitskosten zum Kapital (d.h. von denjenigen, in denen vergleichsweise viel Kapital existiert und die Arbeit sehr teuer ist - in unserem Fall die jetzigen Mitgliedsstaaten) und Staaten mit relativ hoher Relation des Kapitalwerts zur Arbeit (dort, wo die Löhne niedrig sind, also in Mittelosteuropa) im Endeffekt einen Austausch von Produktionsfaktoren und eine veränderte Relation der Löhne zum Kapital in beiden Ländern mit sich bringt. Speziell wird Kapital in die Staaten Mittelosteuropas strömen und Personen nach Westeuropa einwandern. Als Folge davon werden sich nach einiger Zeit die Werte der Produktionsfaktoren zwischen den Ländern angleichen und der Wohlstand auf beiden Seiten zunehmen. Die Migration ist also ein positiver Effekt, der der Verbesserung der Wirtschaftsergebnisse der gesamten Region dient, allerdings auf Kosten der Arbeitslöhne in den wohlhabenderen Staaten. Empirische Untersuchungen zeigen hingegen, dass der Mechanismus, von dem hier die Rede ist, in der Praxis nur in einem begrenzten Zeitraum wirkt. Die Hindernisse für einen Austausch des Arbeitsfaktors, d.h. für die Lohnmigration, sind nicht nur rechtlichadministrativen Charakters. Trotz des unterschiedlichen Lohnniveaus, das zur Migration motiviert, treffen die Menschen eine solche Entscheidung deutlich seltener, als es die Theorie suggeriert.“
} 
bewegen. ${ }^{258}$ So hat zum Beispiel Wrocław, die viertgrößte Stadt Polens, die Kampagne, Wrocloves you' ins Leben gerufen. Unter anderem mit Anzeigen und Interviews in polnischen Zeitungen in Großbritannien soll der Kontakt zu ausgewanderten BürgerInnen aus Wrocław gehalten und diese zur Rückkehr animiert werden (vgl. Gazeta Wyborcza vom 24.11.2006). Laut einer Umfrage vom Juli 2007 plant die Hälfte der polnischen Auswanderer in Irland und Großbritannien entweder gar nicht oder frühestens nach fünf bis zehn Jahren nach Polen zurückzukehren (vgl. Gazeta Wyborcza vom 4.07.2007). Auf diesem Bereich liegt auch der Fokus der öffentlichen Aufmerksamkeit. Denn der erneute Exodus hat nicht nur zu einem allgemeinen Arbeitskräftemangel geführt, sondern bedeutet auch einen starken ,Brain Drain': Unter den EmigrantInnen sind HandwerkerInnen, ÄrztInnen, Pflegekräfte und andere gut Qualifizierte. Allerdings wird in diesem Zusammenhang neben den Rücküberweisungen auch auf die Weiterqualifikation verwiesen, die eine temporäre Migration vor allem hinsichtlich Fremdsprachenkenntnissen und anderen, soft skills ${ }^{6}$ bedeutet und die schließlich nach der Rückkehr für Polen wieder von Nutzen sein könne.

Die Versuche, die EmigrantInnen an das Herkunftsland Polen zu binden, gehen bis in die 1960er Jahre zurück. Während die endgültige Ausreise bis zum Ende der stalinistischen Ära als Landesverrat galt, wurde anschließend eher versucht, die Bindungen an Polen zu erhalten und die Rückkehr der EmigrantInnen zu fördern, wofür bereits 1955 die parteinahe Towarzystwo Polonia (Gesellschaft Polonia) gegründet wurde. ${ }^{259}$

Auch die aktuelle Auswanderungsbewegung nach Polens EU-Beitritt kann nicht exakt beziffert werden. Die Schwierigkeit einer genauen Quantifizierung ergibt sich unter anderem erneut daraus, dass sich viele nicht regulär abgemeldet haben und noch als arbeitsuchend in der Arbeitslosenstatistik geführt werden. In einer Studie über die Arbeitsemigration hat das polnische Wirtschaftsministerium die verschiedenen Schätzungen über das Ausmaß, die von 660.000 bis zu 4 Millionen reichen, in einer Übersicht zusammengefasst (vgl. Übersicht 2):

\footnotetext{
258 Vergleichbare Bestrebungen gibt es auch in Russland, das seine Rückholprogramme an ,deutschstämmige' RussInnen richtet, sowie in Tschechien, der Slowakei, Bulgarien und Rumänien (vgl. Handelsblatt vom 01.10.2007). Aber auch innerhalb der Bundesrepublik versuchen die von Abwanderung stark betroffenen ostdeutschen Bundesländer mit bildungs-, wirtschafts- und familienpolitischen Maßnahmen diesem Trend entgegenzusteuern (vgl. z.B. http://www.menschen-fuersachsen-anhalt.de). Die Stadt Magdeburg verschickte gar in einer öffentlichkeitswirksamen Kampagne ,Heimatschachteln' mit regionalen Produkten an Abgewanderte.

${ }^{259} \mathrm{Zu}$ den weiteren, politischen Funktionen dieser Gesellschaft Miera vgl. 2007, S. 36.
} 
Übersicht 2: Schätzungen über den Umfang der Arbeitsemigration aus Polen (2004-2006), entnommen aus: Ministerstwo Gospodarki 2007 (Übersetzung DM)

\begin{tabular}{|l|l|}
\hline Quelle der Schätzung & emigrierte Personen \\
\hline Ministerium für Arbeit und Soziales & 660.000 \\
\hline Katholische Kirche & 1.000 .000 \\
\hline Medien & 2.000 .000 bis 4.000 .000 \\
\hline Einheimische Experten & 1.200 .000 \\
\hline ECAS (European Citizen Action Service) -Report & 1.120 .000 \\
\hline
\end{tabular}

Die Verteilung auf die Zielländer zeigt die herausragende Bedeutung Deutschlands für die polnische Emigration (vgl. Tabelle $7 \mathrm{im}$ Anhang). Bereits während der gesamten Phase der sowjetkommunistischen Herrschaft und ein gutes Jahrzehnt danach war die Bundesrepublik das Hauptzielland für auswandernde PolInnen. Diese Kontinuität wurde erst mit dem EU-Beitritt Polens durchbrochen. Ab 2004 gewannen Irland, Großbritannien und die Niederlande signifikant an Bedeutung (vgl. Tabellen 7 und 8 im Anhang). Diese Konzentration der Emigration vor allem auf Großbritannien und Irland ${ }^{260}$ ist auf die im Zusammenhang mit der EUOsterweiterung 2004 festgelegten Übergangsregelungen für die Einwanderung aus den Beitrittsstaaten zurückzuführen. Danach kann jeder Mitgliedstaat den BürgerInnen der neuen Mitgliedstaaten die Arbeitnehmerfreizügigkeit für bis zu sieben Jahre verwehren (vgl. Brücker 2004). Die Arbeitnehmerfreizügigkeit wurde bereits beim EU-Beitritt Griechenlands 1981 sowie beim Beitritt Spaniens und Portugals 1986 übergangsweise eingeschränkt. Allerdings wurde die Entscheidung über die Anwendung der Übergangsregelungen 2004 erstmalig den einzelnen Mitgliedstaaten überlassen. So haben Dänemark, Großbritannien und Irland ihre Arbeitsmärkte mit einigen Auflagen, Schweden hat seinen Arbeitsmarkt vollständig für ArbeitnehmerInnen der beigetretenen Staaten geöffnet. ${ }^{261}$

Befürworter der Arbeitsmarktöffnung erwarten durch die unterschiedliche Inanspruchnahme der Übergangsregelungen durch die Mitgliedstaaten vor allem Verzerrungen im ,Kampf um die besten Köpfe'. So sieht das Deutsche Institut für

\footnotetext{
${ }^{260}$ Irland war über Jahrhunderte ein Auswanderungsland. Erst in den 1990er Jahren erlebte es einen intensiven wirtschaftlichen Aufschwung, im Zuge dessen auch die Nachfrage nach ausländischen Arbeitskräften stieg. Obwohl die zahlreichen polnischen EinwanderInnen offenbar überwiegend einen Arbeitsplatz gefunden haben, hat Irland seinen Arbeitsmarkt für Rumänen und Bulgaren nach deren EU-Beitritt nicht sofort vollständig geöffnet (vgl. Bröll 2007). In Großbritannien wird erwartet, dass die PolInnen die IrInnen bald als größte Ausländergemeinde ablösen (vgl. Frankfurter Allgemeine Zeitung vom 10.08.2006).

${ }^{261} \mathrm{Zu}$ den Details der Übergangsregelung und der unterschiedlichen Ausgestaltung in den einzelnen Mitgliedstaaten vgl. Brücker 2005.
} 
Wirtschaftsforschung in Berlin (DIW) Wettbewerbsvorteile für Großbritannien und Irland durch deren ,Brain Gain` aus den neuen Mitgliedstaaten:
„Die Reduzierung der Migration durch die Übergangsfristen für die Arbeitnehmer- freizügigkeit führt nach Simulationsrechnungen zu erheblichen gesamtwirtschaftlichen $V$ erlusten in der erweiterten EU. Am Ende könnte Deutschland von einer Umlenkung qualifizierter Migranten aus den neuen Mitgliedstaaten nach Großbritannien und in an- dere EU-Staaten negativ betroffen sein. " (Brücker 2005)

In den öffentlichen Debatten in den einzelnen Mitgliedstaaten wird dies jedoch häufig anders diskutiert. In Frankreich bspw. stand die Dienstleistungsrichtlinie in den Augen der GegnerInnen des Europäischen Verfassungsvertrages stellvertretend für die Gefahren einer erweiterten Union. Die Mobilisierung für ein ,Non“ bei der Volksabstimmung zum Verfassungsvertrag drehte sich stark um die zu niedrigen Löhnen arbeitenden Arbeitskräfte aus den Beitrittsstaaten, die im Falle der Dienstleistungsfreiheit in Konkurrenz zu den ArbeitnehmerInnen in den alten Mitgliedstaaten treten und ein Lohndumping auslösen würden. Der polnische Klempner (,plombier polonais) wurde in den Medien zum Symbol für diese Lohnkonkurrenz. ${ }^{262}$ Im Juni 2005 griff schließlich das polnische Tourismusministerium das negativ besetzte Bild auf und formulierte es mit einer originellen Kampagne für seine eigenen Zwecke um: Mit einem Plakat, auf dem ein polnischer Klempner ,in Gestalt eines muskelbepackten, jungen, kalifornisch anmutenden Beach Boys mit schwellendem Bizeps vor dem Hintergrund der malerischen Tatra“ (Maryniak 2007) oder städtischen Sehenswürdigkeiten posiert und dem Slogan ,Ich bleibe in Polen, kommt alle her' (,Je reste en Pologne. Venez nombreux") warben sie in Frankreich für touristische Reisen nach Polen (vgl. ebd.). „Die Kampagne erwies sich als der schlagkräftigste polnische Werbegag aller Zeiten“" und erzielte ein internationales Medienecho (vgl. ebd.). ${ }^{263}$

Die aktuelle Auswanderungsbewegung nach dem EU-Beitritt war (bzw. ist) ein öffentliches Thema in Polen. ${ }^{264}$ Unter anderem begann der polnische Ombuds-

\footnotetext{
262 Einige Stimmen grenzten sich jedoch von den Versuchen ab, die Kritik an der Absenkung sozialer Standards, wie sie die Richtlinie impliziert, durch eine Stigmatisierung der HandwerkerInnen aus den Beitrittstaaten aufzuladen. So verkündeten die französischen Sozialisten, dem polnischen Klempner zu Ehren eine Statue errichten zu wollen und die Schweizer Sozialisten entwarfen den Slogan ,Klempner aller Länder, vereinigt euch' (vgl. Maryniak 2007).

263 Piotr Adamski, der als Model auf dem Plakat den Klempner dargestellt hatte, tourte sogar durch Frankreich und posierte unter anderem auf dem Eiffelturm. Angeblich gab ihm der ehemalige polnische Staatspräsident Lech Wałęsa den Auftrag in Frankreich nachzufragen: „Why the heck for so many years they encouraged Poles to build capitalism when as it turns out they are Communists themselves" (zit. n. New York Times vom 26.06.2005).

264 Die linksliberale Gazeta Wyborcza veröffentlichte im September 2006 unter dem Titel „Przystanek Polska“ (Baustelle Polen) eine zweiwöchige Reihe, die Antworten auf die Frage finden sollte, warum die PolInnen massenhaft ins Ausland gehen und was geschehen muss, um sie aufzuhalten oder zur Rückkehr zu bewegen. Laut einer Umfrage unter 18-24jährigen PolInnen, die die Zeitung in Auftrag gegeben hatte, antworteten 19 Prozent auf die Frage ,Ziehst du die Möglichkeit in Be-
} 
mann ${ }^{265}$ eine Debatte über den Schutz polnischer Arbeiter im Ausland und schlug vor, bei den Arbeitsbehörden der Zielländer polnischer ArbeitsmigrantInnen Vertretungsbüros einzurichten. Im Wahlkampf zur polnischen Parlamentswahl im Oktober 2007 spielte das Thema ebenfalls eine Rolle: Die Partei des Wahlsiegers Donald Tusk (Platforma Obywatelska - Bürgerplattform) hatte die angekündigten Reformen in der Arbeitsmarkt-, Steuer- und Sozialpolitik auch damit begründet, die Auswanderung aus Polen stoppen zu wollen. Auch der unterlegende damalige Präsident Lech Kaczynski hatte angekündigt, der Emigration von ÄrztInnen, PflegerInnen, HandwerkerInnen und IngenieurInnen etwas entgegen setzen zu wollen. Die öffentliche Thematisierung der ,nationalen Erfahrung ${ }^{626}$ der Auswanderung ermöglichte gleichzeitig, auch Migration im Allgemeinen, und damit erstmals auch Immigration, breiter zu thematisieren.

\subsubsection{Einwanderung: „Polen ist der östlichste Westen““267}

„The new spatial mobility of persons in former Soviet bloc countries was initially perceived mainly as a threat, but later it turned out to have many positive effects" (Iglicka 2001a, S. 7)

Die Einwanderung nach Polen wird häufig als eine gänzlich neue Entwicklung dargestellt. Dies ist jedoch nur mit Blick auf die jüngere polnische Geschichte zutreffend. Denn zwischen dem 13. und dem 19. Jahrhundert war Polen schon einmal für lange Zeit das Ziel von (deutschen) Einwanderern, während die umgekehrte Bewegung von Polen Richtung Deutschland in diesem Zeitraum eine wesentlich geringere Rolle spielte (vgl. Borodziej/Lemberg 2004, S. 52):

„Seit dem Mittelalter siedelten sich in Polen Einwanderer aus Deutschland an. Auf der Suche nach Boden und Arbeit Kamen Bauern, Handwerker und Kaufleute in mehreren Wellen zwischen dem 13. und 19. Jahrbundert. Deutsche und Juden waren aus dem

tracht, Polen in den nächsten zwei Jahren zu verlassen, um im Ausland zu arbeiten?` mit ,ja‘, 69 Prozent mit ,nein'. Diejenigen, die die erste Frage mit ,ja beantwortet hatten, antworteten auf ,Hast du mit den Vorbereitungen für die Ausreise begonnen?` zu 69 Prozent mit ,ja‘, 24 Prozent mit ,nein‘. Auch unter den Älteren sei die Frage Thema: Jeder fünfte erwachsene Pole denke darüber nach. Bis zu 54 Prozent der Befragten haben in ihrer Familie jemanden, der nach dem 1. Mai 2004 das Land verlassen hat, um anderswo zu arbeiten, weitere 18 Prozent haben so jemanden in ihrem Bekanntenkreis. Dabei sei die Arbeitsemigration keine Spezifität bestimmter sozialer Gruppen oder auf eine einzelne Region im Land beschränkt. Einzig in großen Städten sei sie nicht ganz so stark ausgeprägt (vgl. Gazeta Wyborcza vom 03.09.2006).

265 Die Institution des Ombudsmanns ist in Art. 208 der polnischen Verfassung verankert. Unter anderem können Privatpersonen Beschwerden an den Ombudsmann richten, denen dieser im Interesse der Wahrung der BürgerInnenrechte nachzugehen hat.

266 Die Interviewpartnerin vom Central European Forum for Migration and Population Research in Warschau (CEFMR; Środkowoeuropejskie Forum Badań Migracyjnych i Ludnościowych) bezeichnete die Auswanderung aufgrund ihres Ausmaßes als „nationale Erfahrung“ (A S. 2).

267 O S. 3. 
Bild des mittelalterlichen Krakau ebenso wenig wegzudenken wie aus dem erst im 19. Jabrbundert entstandenen Industriezentrum Lod\%. Umgekehrt war die Siedlungsbewegung der polnischen Bevölkerung bis in das 19. Jabrbundert wesentlich schwächer; nur der Staat des Deutschen Ordens in Preußen, ab 1525 das Hervogtum Preußen, wurde unter Mitwirkung polnischer Bauern kolonisiert." (Borodziej/Lemberg 2004, S. 52)

Für die jüngste Geschichte lassen sich mit Blick auf die Einwanderungsprozesse nach Polen vereinfacht drei Phasen unterscheiden:

- die Phase vor 1989, in der nahezu keine Einwanderung stattfand,

- der Zeitraum von 1989 bis zum EU-Beitritt Polens im Jahr 2004, in dem verschiedene Formen vor allem temporärer Ein- und Transitwanderung nebeneinander existierten und in Vorbereitung auf den EU-Beitritt zunehmend erschwert wurden,

- die Zeit seit Polens EU-Beitritt 2004, in der es Bestrebungen gab, den Arbeitsmarktzugang für AusländerInnen zu erleichtern, um dem Arbeitskräftemangel entgegenzuwirken, der durch die starke Auswanderung entstanden war.

Während des Staatssozialismus spielte Einwanderung nach Polen so gut wie keine Rolle. Sie beschränkte sich auf einige VertragsarbeiterInnen und StudentInnen aus anderen sozialistischen Staaten - vor allem aus Vietnam - und kommunistische Kontingentflüchtlinge aus Griechenland und Chile.

Mit dem Wegfall der rigiden Beschränkung der Bewegungsfreiheit in den ,Ostblockstaaten' nahm einerseits die Auswanderung aus Polen ab und andererseits gewann die Einwanderung nach Polen in einem bislang unbekannten Aus$\mathrm{maß}$ an Bedeutung. ${ }^{268}$ Sowohl in der polnischen Öffentlichkeit und Politik als auch in der (inter-)nationalen Forschung ist diese Entwicklung jedoch lange unbeachtet geblieben. Zunächst handelte es sich weniger um MigrantInnen, die einwanderten und sich niederließen, als um grenzüberschreitende Mobilität von stark gewachsenem Ausmaß: „The key terms for describing migrations in Poland have been flow rather than stock and mobility rather than residence." (Kępińska/Stola 2004, S. 160) Die Zahl der Einreisen von AusländerInnen stieg von 6,2 Millionen 1988 auf 36,8 Millionen 1991 und 88,6 Millionen 1998. Dennoch habe sich die Politik bei ihren Entscheidungen kaum an dieser Erscheinungsform von Migration orientiert, sondern Politiken, Gesetzgebung und Verwaltungshandeln konzentrierten sich auf „less fluid and more manageable forms of migration“ (Kępińska/ Stola 2004, S. 160).

Für Iglicka (2001c) handelt es sich bei dieser Form der Mobilität um ein neues soziales Phänomen, das charakteristisch für die Transformationsphase in Mittelosteuropa sei. Sie zeichne sich dadurch aus, dass Menschen „constantly on the move“ (Iglicka 2001c, S. 507) seien. Sie bezeichnet diese Bewegung als „primitive

268 Vgl. Kępińska 2003, S. 5ff.: Übersicht 1: „Arrivals of Foreigners in Poland“ illustriert den Anstieg der Einreisen seit 1990 und einen Rückgang ab 1999. 
mobility“ (Iglicka 2001c). ${ }^{269}$ Diese sei durch verschiedene Faktoren bestimmt: den einfachen Zugang, den Menschen aus der früheren UdSSR zu Polen hatten, die geografische, kulturelle und sprachliche Nähe zwischen Polen und vielen der neuen unabhängigen Staaten der früheren UdSSR und starke historische Verbindungen - wie bspw. zur Ukraine. Darüber hinaus spiel(t)en unterschiedliche Lebensstandards und Unterschiede in Wechselkurs und Preisverhältnis sowie ökonomische Restrukturierung und Dualisierung von Arbeitsmärkten mit einem großen grauen Markt für billige, flexible ArbeitsmigrantInnen einerseits und der Nachfrage nach hochqualifizierten ExpertInnen anderseits eine große Rolle (Iglicka 2001b, S. 126f.). Die ProtagonistInnen der ,primitive mobility ${ }^{6}$ zwischen der früheren Sowjetunion und Polen sind nach Iglicka (2001c, S. 507) ,Profis' in ihrem Fach. Sie betrachteten also den grenzüberschreitenden Handel als ihre Haupttätigkeit und -einkommensquelle. Zudem sei die Häufigkeit der Grenzübertritte hoch und der Umfang der transferierten Produkte in der Summe groß.

Mit dieser Art der Mobilität eng verbunden sind die charakteristischen Freiluft-Märkte, die auch während des kommunistischen Regimes - wenn auch in geringerem Umfang - existiert hatten (vgl. Sword 1999). Entgegen den Erwartungen, dass der Basarhandel mit den ökonomischen Umwälzungen und der dann nicht mehr bestehenden Güterknappheit verschwinden würde, hat dieser nach 1990 eine enorme Ausweitung erfahren (vgl. Sword 1999, S. 146). Mit der Öffnung der Grenzen konnten die HändlerInnen nahezu ungehindert ein- und ausreisen und billigere Waren aus dem östlichen Ausland importieren (vgl. ebd.). Nachdem KleinhändlerInnen aus der früheren Sowjetunion zunächst billige Produkte importierten, auf polnischen Märkten verkauften und auf dem Rückweg Produkte aus Polen mitnahmen, die sich in ihren Herkunftsorten gewinnbringend verkaufen ließen, änderte sich dieser Handelsmodus im Laufe der 1990er Jahre. Nun kommen KleinhändlerInnen aus den östlichen Nachbarstaaten weniger nach Polen, um Waren zu verkaufen, sondern um Produkte für den Weiterverkauf auf lokalen Märkten im Osten einzukaufen (vgl. ebd., S. 147). Was auf den ersten Blick als unbedeutender Kleinhandel erscheint, erreichte beachtliches ökonomisches Gewicht: Die Märkte fungierten als Arbeitgeber für Zehntausende Arbeitskräfte, wie HändlerInnen, Sicherheitspersonal sowie die MitarbeiterInnen von Transportunternehmen und der Firmen, die die angebotenen Produkte herstellten. Vor allem im Textilsektor entstanden im Zuge der Expansion des Basarhandels zahlreiche kleine Firmen, die Waren für die Märkte produzierten und dafür PendelmigrantInnen aus dem östlichen Ausland zu niedrigen Löhnen beschäftigten (vgl. ebd., S. 160). Gleichzeitig ermöglichten die Märkte den in der Transformationsphase mit knappen Mitteln kalkulierenden polnischen Haushalten, günstige Einkäufe zu tätigen. Ein berühmtes Beispiel und einer der größten Märkte war der Warschauer Stadion Markt (Stadion Dziesieciolecia, Jarmark Europa). Der Markt galt lange

269 Andere Bezeichnungen sind „petty-trade migration, shuttle mobility, circular migration, or incomplete migration“. (Iglicka 2001c, S. 506). 
Zeit als einer der bedeutendsten Exporteure Polens (vgl. Sword 1999, S. 159). Er wurde täglich von 20.000 bis 30.000 Menschen besucht, fast 60 Prozent davon waren AusländerInnen (vgl. ebd., S. 158). Das Stadion musste jedoch Ende 2007 von den HändlerInnen verlassen werden und soll bis zur Fußball-Europameisterschaft 2012 in einen riesigen Sportkomplex umgewandelt werden.

Seit Ende der 1990er Jahre lässt sich schrittweise ein Übergang von dieser ,primitive mobility' hin zu einer mehr auf Dauer angelegten Einwanderung beobachten. So bleiben einige der früheren HändlerInnen für längere Zeiträume, um in Polen - legal oder illegal - zu arbeiten und wiederum als Vermittler für andere Landsleute aufzutreten, die ebenfalls im Handel aktiv werden wollen (vgl. ebd., S. 164). Dabei spielen auch die Netzwerke eine Rolle, die während der ersten Phase der grenzüberschreitenden Mobilität entstanden sind. Die bedeutendsten Herkunftsländer der EinwanderInnen ${ }^{270}$ in Polen sind neben Deutschland und den USA die Ukraine, Armenien und Vietnam. Hinsichtlich der hohen Bedeutung Deutschlands als Herkunftsland wird angenommen, dass es sich dabei einerseits um rückkehrende, einst ,Vertriebene' und andererseits um ehemalige polnische EmigrantInnen handelt (Currle 2004, S. 374). Letzteres gilt in ähnlicher Weise für die USA und Kanada als Herkunftsländer von EinwanderInnen nach Polen.

Die UkrainerInnen übernehmen in Polen die Arbeiten, die PolInnen in Deutschland, Großbritannien und Frankreich verrichten: in der Landwirtschaft, im Baugewerbe und in privaten Haushalten (vgl. Kindler 2005). Für ihren Aufenthalt als SaisonarbeiterInnen oder KleinhändlerInnen in Polen organisieren sie meist Pendelmigrationen - eine Strategie, die durch die Einführung der Visapflicht und die restriktivere Handhabung der Einreisekriterien an den Grenzen in Vorbereitung auf Polens Beitritt zum Schengen-Raum deutlich erschwert wurde. Sie können zudem auf Netzwerke zurückgreifen, die durch die ,alte‘ ukrainische Minderheit in Polen bestehen (Iglicka 2001b, S. 79f.). Die Zahl der Anträge auf Aufenthaltsgenehmigung und der ausgestellten Genehmigungen für UkrainerInnen ist seit 2000 kontinuierlich gestiegen (vgl. Kępińska 2006, S. 62).

Eine weitere wichtige Einwanderergruppe sind die VietnamesInnen. Wie bereits erwähnt, waren es vor allem StudentInnen aus Vietnam, die bereits vor 1989 mit Unterstützung der Regierungen Polens und Vietnams nach Polen kamen und nach Studienabschluss wieder nach Vietnam zurückkehrten. Nach 1989 liberalisierte Vietnam seine Auswanderungspolitik - woraufhin viele VietnamesInnen nach Polen auswanderten. Seit Ende 1993 nutzten sie die Möglichkeit der kombinierten Visa-Arbeitserlaubnis und bildeten damit die zweitgrößte Gruppe nach

\footnotetext{
270 In der polnischen Statistik gilt als Einwanderer, wer aus dem Ausland nach Polen kommt und sich dort niederlassen möchte, nachdem er oder sie dauerhaft in einem anderen Land gelebt hat und sich dementsprechend bei der zuständigen polnischen Behörde registriert hat. Diese Definition schließt also ausländische und polnische BürgerInnen ein - sofern diese vorher dauerhaft im Ausland gelebt haben. Diejenigen, die nicht die polnische Staatsbürgerschaft besitzen, müssen eine dauerhafte Aufenthaltserlaubnis erhalten, um als Einwanderer zu gelten (vgl. Kępińska 2006, S. 10).
} 
den UkrainerInnen, die auf diesem Wege einreiste. Ein Teil der VietnamesInnen lebt und arbeitet ,illegal' in Polen. Die VietnamesInnen werden sowohl in wissenschaftlichen Publikationen als auch von den InterviewpartnerInnen, mit denen ich im Rahmen meiner Recherchen gesprochen habe, stets als unauffällige und fleißige EinwanderInnen charakterisiert, die sich nach außen hin gut in die polnische Gesellschaft integrieren (A S. 10; B S. 19) ${ }^{271}$ :

„They send their children to Polish schools, learn the Polish language, read Polish papers and watch Polish TV. They carry on a lively economic activity, not only in the fields of small trade and gastronomy. "(Iglicka 2001b, S. 87)

Gleichzeitig pflegen sie nach innen ihre Traditionen und ihre Muttersprache. Dass die VietnamesInnen in einer starken Community leben, die zum Beispiel im Krankheitsfall auch eine Versorgung durch ÄrztInnen aus dieser Gemeinschaft sicherstellen kann (M S. 7), sowie die Tatsache, dass sie räumlich häufig in bestimmten Vierteln oder Wohnblocks zusammenleben, wird mitunter jedoch als Desintegration und Leben in „Ghettos“ (B S. 19) interpretiert. Diese Lesart verwundert nicht nur aufgrund der entgegen gesetzten vorangegangenen Beschreibung, sondern auch, da ein Teil der Gruppe aufgrund seines illegalen Status notwendigerweise auf den Rückhalt der Community angewiesen ist. Versuche, VietnamesInnen abzuschieben, gestalten sich nach Aussage eines Interviewpartners für die polnischen Behörden schwierig, da die vietnamesische Botschaft keine Dokumente ausstelle (B S. 19). Es wird vermutet, dass der vietnamesische Staat nicht auf die Rücküberweisungen seiner Landsleute verzichten möchte (B S. 10). Um diesem Problem zu begegnen, orientiere sich Polen an der deutschen Praxis und erwäge nun ebenfalls, die Ausstellung von Dokumenten durch Botschaftsvorführungen zu erreichen (B S. 20).

Bis zum Jahr 2003 war die Einwanderung von Arbeitskräften nach Polen aufgrund der Visafreiheit, die auf einem Abkommen aus sowjetkommunistischer Zeit mit Weißrussland, der Ukraine und Russland basierte, relativ einfach und fand daher in großem Maßstab statt:

„As a matter of fact, irregular migration to Poland is large and illegal migrants come mostly from Poland's eastern neighbours, but their illegality does not result from illegal border crossing or visa overstaying but from mass illegal employment. [...] reasonable estimates of annual flows of unregistered labour from the East reach into the hundreds of thousands. "(Kepinska/Stola 2004, S. 171f.)

\footnotetext{
${ }^{271}$ Die Kürzel beziehen sich auf die in Polen interviewten ExpertInnen. Im Anhang I findet sich eine Liste mit den Institutionen bzw. Organisationen, aus denen ein (mitunter leitendes) Mitglied interviewt wurde. Daraus kann entnommen werden, welche Einrichtung hinter dem Kürzel steht, die Namen und Positionen der einzelnen ExpertInnen werden jedoch zu deren Schutz nicht genannt. Die Seitenzahlen beziehen sich auf die Paraphrasen bzw. Transkripte der Interviews, die mir vorliegen.
} 
Die Behörden stünden dieser Einwanderung arbeitender BesucherInnen aus der früheren UdSSR eher tatenlos gegenüber. Ihre Aktivitäten konzentrierten sich hingegen auf rumänische Roma, ausländische Sexarbeiterinnen und TransitmigrantInnen auf dem Weg nach Westen (Kępińska/Stola 2004, S. 172). ${ }^{272}$ Die Toleranz gegenüber der umfangreichen illegalen Arbeitseinwanderung wird als fester Bestandteil der polnischen Migrationpolitik bzw. -praxis charakterisiert. Über die Gründe dafür wird gemutmaßt: Sie könnten in dem Unvermögen liegen, dagegen etwas zu unternehmen, in der geringen Priorität, die dem Thema beigemessen werde oder in einer bewussten Entscheidung, nicht dagegen vorzugehen. ${ }^{273}$

In der Phase seit 1989 vollzogen sich also die ersten Schritte hin zum Übergang Polens von einem der größten Entsendeländer in Mittelosteuropa zu einem Land, das Ziel von (vor allem kurzfristig) Einwandernden und Durchgangsstation für TransitmigrantInnen ist. Damit verbunden ist eine Erosion des mittel- und osteuropäischen Migrationssystems: Polen gehöre inzwischen nicht mehr nur dem mittel- und osteuropäischen, sondern gleichermaßen dem westeuropäischen Migrationssystem an (Iglicka 2001b, S. 132).

Mit der Einführung der Visa-Pflicht im Jahr 2003 für Weißrussland, die Ukraine und Russland erlebt diese Phase einen starken Einschnitt. Die Visapflicht war eine zentrale Voraussetzung für Polens EU-Beitritt und in diesem Politikbereich die umstrittenste. Nach ihrer Einführung waren die polnischen Konsulate in diesen Ländern mit der Durchführung der Bestimmungen überfordert. Nach wie vor reisen jedoch BewohnerInnen der Nachbarstaaten - nun mit Visum - nach Polen ein, um dort Handel zu betreiben oder irregulär zu arbeiten. Gleichzeitig waren mit dem Ausländergesetz von 1997 und seiner Reform 2001 die Hürden für einen legalen Aufenthalt sukzessive erhöht worden:

\section{„Looking at the 1997 Alien Act and its 2001 amendments, one notes that the periods a foreigner needs to reside in Poland in order to climb the bierarchy of legal statuses be- came more demanding. "(Kepińska/Stola 2004, S. 166)}

Die erhöhten Anforderungen an AntragstellerInnen, die eine Aufenthaltserlaubnis erhalten wollen, führten seit 1998 zu einem Rückgang der Antragszahlen. Kępińska und Stola (2004, S. 166) vermuten, dass diese Maßnahmen zum Schutz des polnischen Arbeitsmarktes vor allem zur Ausweitung des illegalen Arbeitsmarktes geführt haben.

\footnotetext{
272 „There were several major operations against illegal migrants, but they targeted overstaying Romanian Roma (Gypsies), foreign prostitutes and westward transit migrants. The authorities seem largely passive towards the main stream of migrants, consisting of illegally employed visitors from the ex-USSR.” (Kępińska/Stola 2004, S. 172)

273 „Alongside this rather restrictive migration regime is large-scale irregular labour migration. Tolerance of the latter, be it due to the state's inability to counteract it, its low priority or a conscious decision not to oppose it, is a stable part of domestic migration policy/practice. The termination of visa-free entry for visitors from the East is a major change in this area." (Kępińska/Stola 2004, S. 174)
} 
Zwar unterliegen die Visa-Regelungen aus Warschau der Interpretation und Umsetzung durch die polnischen Grenzbehörden:

„The border, the place where migration policy enforcement is the easiest, is therefore not used to enforce any consistent policy on labour migration. [...] Variations in border control scrutiny in different periods or at different checkpoints seem to depend more on the whims of local officers rather than on any general policy. " Kepinska/ Stola 2004, S. 172)

Die Varianzen im Umgang mit den Vorgaben aus Warschau änderten jedoch nichts am allgemeinen Trend der Verschärfung der Grenzkontrollen an der polnischen Ostgrenze, womit auch die Einreise von undokumentierten Arbeitskräften erschwert wurde. Nachdem das Innenministerium - offenbar vor dem Hintergrund der Forderung nach Einhaltung der Schengen-Kriterien seitens der EU im Sommer 2006 die Bedingungen für den Grenzübertritt weiter verschärft hatte, konnten viele illegale SaisonarbeiterInnen plötzlich nicht mehr einreisen. Dies führte zu einer Situation, die eine Interviewpartnerin anschaulich als die „Himbeerkrise“ bezeichnete: Polen ist in der EU führender Produzent von so genanntem Weichobst. Aufgrund der Restriktion der Einreisebedingungen fehlten in der Landwirtschaft tausende ErntehelferInnen. So blieben Himbeeren, Erdbeeren und Johannisbeeren ungepflückt. Die niedrigen Preise für Agrarprodukte liegen vor allem in den niedrigen Löhnen in diesem Bereich begründet. Viele PolInnen sind jedoch nicht mehr bereit, für diese Löhne zu arbeiten, weshalb die polnischen Bauern auf ausländische Saisonarbeitskräfte zurückgreifen. Angesichts der ,Himbeerkrise' im Sommer 2006 forderte daher der damalige Landwirtschaftsminister Andrzej Lepper den Grenzschutz öffentlich zu einer ,Politik des geschlossenen Auges' (przymykać oczy) auf. ${ }^{274}$ Das heißt, dass die SaisonarbeiterInnen über die Grenze gelassen werden sollten, egal mit welchen Papieren. ${ }^{275}$ Die Bauernlobby und Lobbyisten im Bausektor hatten sich im vorangegangenen Jahr für die Zulassung ausländischer (Saison-)Arbeitskräfte stark gemacht. Da das Landwirtschaftssowie das Arbeits- und Bauressort 2006 von der Samoobrona geleitet wurden, die vor allem die Interessen der Bauern vertritt, hatte diese Lobby-Arbeit zumindest für den Agrarbereich offenbar Erfolg. Dies nicht zuletzt, weil angesichts der Ab-

274 Andrzej Lepper (Vorsitzender der Bauernpartei Samoobrona/Selbstverteidigung) wurde am 09.7.2007 wegen Korruptionsvorwürfen als Vizeministerpräsident und Landwirtschaftsminister bereits zum zweiten Mal innerhalb einer Legislaturperiode - entlassen, wodurch eine Regierungskrise ausgelöst wurde, die zu vorgezogenen Neuwahlen im Oktober 2007 führte, die einen Regierungswechsel mit Donald Tusk als neuem Regierungschef nach sich zogen.

275 Vgl. Andrzej Lepper im Interview mit Radio Jedynka vom 07.07.2006. Diese Debatte war verbunden mit der Diskussion darüber, ob man nicht polnische Arbeitslose, die diese Arbeiten aufgrund der sehr niedrigen Löhne nicht machen wollen, dazu zwingen könne. In Deutschland hatte eine ähnliche Debatte zur Einführung der umstrittenen Erntehelferregelung geführt, nach der die Bauern einen bestimmten Prozentsatz ihrer ErntehelferInnen aus deutschen Arbeitslosen rekrutieren sollten. 
wanderung zahlreicher PolInnen nach dem EU-Beitritt in Polen Arbeitskräfte fehlten:

„Now that so many Poles have left home, people from struggling economies further east, Ukraine for example, are appearing in towns like Wroclaw to take the jobs that Poles are doing in London." (Maryniak 2007, o.S.)

Zudem hat Polen nach dem Beitritt Rumäniens und Bulgariens - im Gegensatz zum überwiegenden Teil der übrigen EU-Mitgliedstaaten - seinen Arbeitsmarkt für Arbeitskräfte aus diesen Ländern nicht verschlossen. Dies sei aber nach Aussage des polnischen Arbeitsministeriums lediglich eine politische Geste, ohne Auswirkungen auf den polnischen Arbeitsmarkt, da die dortigen Löhne für RumänInnen und BulgarInnen nicht attraktiv seien (vgl. Wirtualna Polska vom 30.01.2007). Für die Beseitigung des Arbeitskräftemangels ${ }^{276}$ im Agrarsektor, im Baugewerbe und Dienstleistungsbereich (vor allem private Haushalte) müssten stattdessen ArbeiterInnen außerhalb der EU angesprochen werden, vornehmlich in den Nachbarstaaten. ${ }^{277}$ Doch legale Arbeitserlaubnisse ${ }^{278}$ scheinen dort kaum auf Interesse zu stoßen: Von 10.304 Arbeitserlaubnissen im Jahr 2005 wurden nur 2.697 von UkrainerInnen und 610 von WeißrussInnen Anspruch genommen (vgl. International Herald Tribune vom 16.11.2006). Da UkrainerInnen vermehrt auch in anderen EU-Staaten Arbeit finden (zum Beispiel in Spanien und Portugal), wird legale Arbeit in Polen zunehmend uninteressant für sie (vgl. Wirtualna Polska vom 19.07.2007). ${ }^{279}$ Seit dem 20.07.2007 hat Polen die Beschränkungen für Arbeits-

276 Der Arbeitskräftemangel besteht insbesondere im Niedriglohnsektor - trotz einer im europäischen Vergleich hohen Arbeitslosenquote (rund 15 Prozent) in Polen. Die hohe Quote wird zum einen darauf zurückgeführt, dass viele Arbeitslose vor allem in den ländlichen Regionen Ostpolens nicht die benötigten Qualifikationen und die erforderliche Mobilität aufweisen. Zum anderen seien viele der Ausgewanderten in Polen als arbeitslos gemeldet, um weiterhin sozial- und vor allem krankenversichert zu sein. Diesem Argument, das vor allem das Arbeitsministerium im Zusammenhang mit der Anwerbepolitik anführt, stehen Berichte gegenüber, wonach viele Arbeitslose sich gar nicht registrieren lassen, da die Unterstützung so gering ist, dass es sich ohnehin nicht lohnt. Wer keine zusätzlichen Einkünfte angebe, werde unter Druck gesetzt. Daher würden viele Einkünfte angeben, die sie gar nicht haben, womit sich die finanzielle Unterstützung wiederum verringert. ,Schwarzarbeit` sei auch deshalb sehr verbreitet - sowohl unter PolInnen als auch unter MigrantInnen (vgl. Sikorski/Heuser 2005).

277 So hieß es aus dem Arbeitsministerium: „Będziemy musieli posiłkować się pracownikami z krajów trzecich, pozaunijnych [...] Naturalnymi kandydatami są w głównej mierze kraje sąsiadujące, przede wszystkim Ukraina" (Wir werden uns mit Arbeitern aus Drittstaaten außerhalb der EU behelfen müssen. Die natürlichen Kandidaten sind hauptsächlich in den Nachbarstaaten zu suchen, vor allem in der Ukraine) (vgl. Wirtualna Polska vom 30.01.2007).

278 Eine solche Arbeitserlaubnis ist drei Monate gültig und kostet 900 Zloty. Das sind rund 240 Euro. Da der Kurs über den Untersuchungszeitraum großen Schwankungen unterlag, wird im Folgenden bei der Umrechnung von Złoty in Euro der Durchschnittswert des Jahres 2007, das heißt ein Kurs von 3,79 Złoty zu 1 Euro, zugrunde gelegt (vgl. oanda currency converter o.J.).

$279 \mathrm{Vgl}$. auch das Statement von Krystyna Iglicka in der International Herald Tribune vom 16.11.2006: „Polish labor costs are too high [...] so Ukrainians prefer to go south to Spain and 
kräfte aus östlichen Nachbarländern nochmals gelockert. Demnach dürfen UkrainerInnen, WeißrussInnen und RussInnen jeweils drei Monate lang obne besondere Arbeitserlaubnis in Polen arbeiten (vgl. Gazeta Wyborcza vom 18.07.2007).

In Vorbereitung auf die Fußball-Europameisterschaft in Polen und der Ukraine 2012 sind umfangreiche Baumaßnahmen (Infrastruktur, Straßen, Flughäfen, Hotels, Stadien) notwendig, die den Arbeitskräftemangel im Bausektor zusätzlich verschärft haben. Daher sind die Aktivitäten zur Anwerbung von Arbeitskräften seit Sommer 2007 nicht mehr nur auf den Agrarsektor und die Arbeitskräfte aus den Nachbarstaaten beschränkt, sondern werden auch auf andere Sektoren und auf andere Herkunftsländer ausgeweitet (vgl. Gazeta Wyborcza vom 31.05.2007). Die damalige Arbeitsministerin Anna Kalata (Samoobrona) hat im Juni 2007 eine Vereinbarung mit der indischen Regierung getroffen, mit der indische Arbeiter angeworben werden sollen (vgl. The Guardian vom 25.06.2007; Hindustan Times vom 13.06.2007). Ähnliche Pläne existierten für eine Zusammenarbeit mit China. Eine entsprechende Gesetzesvorlage will darüber hinaus zum einen die Aktivitäten für die Rückkehr von ,ethnischen Polen' aus Weißrussland, der Ukraine und Kasachstan verstärken und zum anderen 20.000 Strafgefangene unter Aufsicht auf den Baustellen arbeiten lassen (vgl. International Herald Tribune vom 22.06.2007).

Insofern besteht auch in Polen ein segmentierter Arbeitsmarkt, der auf der einen Seite zunehmend attraktive Stellen bereithält, die vor allem jungen PolInnen in den Städten eine Perspektive bieten und sie von der Auswanderung abhalten. In diesem Segment findet auch hoch qualifizierte Einwanderung aus westlichen Ländern statt, die meist Rückkehrmigration von PolInnen ist, die als Kinder mit ihren Eltern in den 1980er Jahren ausgewandert sind. Diese Rückkehrbewegung wird als „return of innovation“ interpretiert (Iglicka 2001b, S. 132). Dabei ist Warschau die Stadt, deren Bilanz aus Auswanderung und Einwanderung ein Netto-Plus an Einwanderung verzeichnen kann. Auf der anderen Seite gibt es ein Segment, in dem teilweise auf informeller Basis - Arbeitskräfte gesucht werden, die unter ungeschützten und prekären Bedingungen niedrig qualifizierte Tätigkeiten übernehmen. Dem Mangel an Arbeitskräften wird dabei nur teilweise mit höheren Löhnen begegnet, wie es im Bausektor der Fall ist. Eine deutliche Erhöhung der Löhne wird durch einen erleichterten Arbeitsmarktzugang für ausländische Arbeitskräfte umgangen. Die Auswanderung vieler PolInnen und der damit verbundene eklatante Arbeitskräftemangel hat daher eine völlig neue Diskussion über legale Einwanderung eröffnet. Damit bahnt sich die Tatsache, dass Einwanderung nach Polen stattfindet und Arbeitskräfte sich zunehmend auch niederlassen, ihren Weg in das öffentliche Bewusstsein.

Nach wie vor befinden sich unter den verschiedenen Gruppen von MigrantInnen in Polen aber auch zahlreiche, die das Land eigentlich nur zum Transit nutzen. Dazu gehören teilweise die bereits erwähnten VietnamesInnen, von denen

Portugal. Employers here say it is now not worth their while to hire on the official labor market. Some resort to the black economy to employ Ukrainians, or Poles for that matter." 
angenommen wird, dass sie die Zeit in Polen nutzen, um genügend Geld zu verdienen, mit dem sie der nachfolgenden Generation die Weiterreise in den Westen finanzieren können (A S. 10). Zum anderen gilt dies insbesondere für Flüchtlinge, deren Situation im Folgenden ausführlicher beleuchtet wird. Sie verschwinden oft vor Ende ihres Asylverfahrens und versuchen, sich in ein anderes EUMitgliedsland durchzuschlagen. Aufgrund der Zuständigkeitsregelungen innerhalb der EU werden sie jedoch nach Polen zurückgeschoben und befinden sich dort in einer Art,Wartezone'. Die Weiterwanderung der Flüchtlinge wird von einigen meiner InterviewpartnerInnen als einfache Lösung für die mit der Einwanderung von Flüchtlingen einhergehenden Aufgaben gesehen.

„Wir haben die Idee, nett zu den AusländerInnen sein zu wollen. Aber eigentlich wollen wir, dass sie weiter gen Westen ziehen, weil wir für sie kein Geld haben. Wir wollen sie nicht behalten. "(F S. 2)

„Ihnen den Flüchtlingsstatus geben und in dem Moment wo sie den bekommen, gehen sie.

Wenn sie den Duldungsstatus kriegen, können sie nicht gehen und so bleiben sie." (N S. 4)

Die Zahl der Flüchtlinge ist im Verhältnis zu den irregulär zu Arbeitszwecken Einwandernden bzw. Pendelnden eher gering. Der Anstieg der Zahl der Anträge auf Flüchtlingsstatus seit Anfang der 1990er Jahre ist jedoch signifikant. ${ }^{280} \mathrm{Im}$ Kontrast zur zahlenmäßigen Bedeutung ist der Flüchtlingsbereich am stärksten gesetzlich reguliert.

\subsection{Keine Migrationspolitik in Polen?}

„Ich habe einige Zweifel an der Existenz einer polnischen Migrationspolitik, das ist ein Mysterium für mich, ob wir irgendeine Art von Migrationspolitik baben. "(D S. 10)

Die vorangegangenen Ausführungen haben gezeigt, dass Polen ein von Auswanderung und Rückkehr, von Transitmigration und zunehmend auch von Einwanderung geprägtes Land ist. Nun soll - bevor genauer auf die konkrete Ausgestaltung des hier interessierenden Teilbereichs Flucht und Asyl eingegangen wird kurz umrissen werden, wie sich die Migrationspolitik in Polen im Allgemeinen darstellt bzw. inwieweit eine solche überhaupt existiert. Denn wer die polnische Migrationspolitik untersuchen will, wird mitunter zunächst mit der Frage, welche Migrationspolitik?" konfrontiert. Unter polnischen WissenschaftlerInnen, aber auch anderen AkteurInnen, die sich in Polen mit Migration befassen, wird eine Debatte darüber geführt, wie die Migrationspolitik Polens zu bewerten ist, und

280 Vgl. Tabelle 9: Anträge auf Flüchtlingsschutz in Polen 1992 - 2007 im Anhang. 
manche bezweifeln, dass überhaupt von einer solchen gesprochen werden kann. ${ }^{281}$ Die meisten InterviewpartnerInnen waren der Ansicht, dass es in Polen keine Migrationspolitik gibt. ${ }^{282}$ Gleichzeitig zeigte sich, dass es auch eine Frage der Definition von Migrationspolitik ist, ob eine solche gesehen werden kann oder nicht. Die Verabschiedung von Gesetzen allein macht nach Ansicht einiger InterviewpartnerInnen noch keine kohärente Migrationspolitik aus. ${ }^{283}$ Demgegenüber steht die Position, dass eine Migrationspolitik auch dann bestehen könne, wenn es keine gesetzlichen Regelungen gibt (F S. 1; O S. 1). Aus der Sicht einiger interviewter ExpertInnen fehlen vor allem klare Prioritäten und Leitlinien: Wer soll aufgenommen werden, wie soll mit Abgelehnten umgegangen werden usw. (D S. 10, C S. 8, M S. 2; Kępińska/Stola 2004, S. 160) ${ }^{284}$. Anstelle einer Ausrichtung entlang von formulierten Leitlinien waren migrationspolitische Maßnahmen in Polen meist reaktiv: „A key feature of the emergence of the new Polish immigration regime was its reactive character." (Kępińska/Stola 2004, S. 160) Unter der Vielzahl an Gegenständen, die in den 1990er Jahren in Polen neu geregelt werden mussten, wurde der Regulierung von Migration kein sehr hoher Stellenwert beigemessen. Im Jahr 1997 wurden in Polen 190 Gesetze verabschiedet. Diese äuBerst hohe Zahl ging oft zulasten der Qualität der Regelungen: Viele Gesetze wurden von einzelnen Fachressorts ohne Absprache mit anderen Bereichen entworfen und dann verabschiedet. Dadurch ergaben sich zahlreiche Probleme, die unter anderem darin lagen, dass sich die alten und neuen, aber auch die neuen Gesetze untereinander als inkompatibel erwiesen (vgl. Currle 2004, S. 363).

281 So heißt es bei Kępińska/Stola (2004, S. 162): „[...] the practice of migration policy has differed from one regional office to another. As one official of the ORA noted, Poland had as many migration policies as it had regional offices (49 until the administrative reform in 1999, 16 at present)." Und: „Immigration is not regarded as one of the Poland's fundamental problems during its building of a new political and economic order. Nor has it been a big issue in political debates or in the media. The usual agents of anti-immigrant backlash, such as nationalist right-wing parties and nativist trade unions, do not seem much interested either. Thus, [...] defining a migration policy has not been a high priority. The emerging migratory regime has lacked clear objectives; migration policies have been reactive and lack long-term goals and guidelines.“ (ebd., S. 173)

282 „Es existiert keine Migrationspolitik in Polen.“ (B S. 15) „Meiner Meinung nach existiert in Polen keine Migrationspolitik.“ (M S. 2) „Das Problem in Polen ist, dass wir keinerlei Migrationspolitik haben.“ (K S. 5) „Es gibt nichts, was man als einheitliche Migrationspolitik bezeichnen könnte. Eher eine Politik hinsichtlich Grenzen und Sicherheit.“ (G S. 2) „Ich habe einige Zweifel an der Existenz einer polnischen Migrationspolitik, das ist ein Mysterium für mich, ob wir irgendeine Art von Migrationspolitik haben.“ (D S. 10)

283 „,...] es gibt zwar einige Gesetze, aber keine kohärente Migrationspolitik“ (K S. 5); „Wir haben Gesetze, aber wenn man es zusammenpackt, dann machen sie keine Migrationspolitik“ (C S. 7)

284 „Wenn wir eine Migrationspolitik hätten, wären einige Prioritäten klarer. Soweit ich das sehe, gibt es keine Prioritäten, wir haben uns in Polen nicht wirklich hingesetzt und entschieden, wen wir aufnehmen wollen. Nicht nur Flüchtlinge, sondern generell in Bezug auf Migration. Ich denke, dass wir eher reagieren auf das, was gerade vor sich geht." (D S. 10) 
Entgegen der überwiegenden Meinung, dass es keine eigenständige Migrationspolitik gebe, arbeitet Anna Kicinger (2005) eine etwas differenziertere These heraus und betont, dass durchaus von einer polnischen Migrationspolitik gesprochen werden könne:

„not ideal, not widely discussed, not extremely efficient, not very well articulated and presented and not comprehensive - yet still present and defending Polish interests; not only merely reacting to EU integration requirements" (ebd., S. 28).

Die Tatsache, dass keine offizielle Einwanderungspolitik existiert, bedeute nicht, dass es gar keine Politik in diesem Bereich gibt: ,although the quality and accuracy of the Polish migration policy might be subject to discussion, it cannot be the existence of thereof" (ebd., S. 29f.). So gebe es in der Migrationspolitik einige Bereiche, in denen die polnischen Interessen und deren Artikulation deutlich erkennbar seien ${ }^{285}$, während bei anderen der EU-Einfluss (Flucht und Asyl) bzw. der Einfluss von Regelungen westlicher Einwanderungsländer deutlich würden. Mit Cornelius/Tsuda (2004) argumentiert Kicinger, dass zur Erklärung dieser Konvergenz verschiedene Faktoren berücksichtigt werden müssten, die oft miteinander verknüpft seien. Regionale Integration stehe dabei als ein Einflussfaktor neben ,policy emulation' und paralleler Pfadentwicklung (vgl. Kicinger 2005, S. 28). Darüber hinaus sei auch zu berücksichtigen, dass polnische und die Interessen der EU bzw. anderer EU-Mitgliedstaaten sich mitunter nicht widersprechen (zum Beispiel hinsichtlich Sicherheit, Grenzschutz, Kampf gegen organisierte und transnationale Kriminalität, Schutz des Arbeitsmarktes). Allerdings sieht sie einen Widerspruch zwischen den polnischen Sicherheitsinteressen und den Verpflichtungen aus internationalen Menschenrechts-Abkommen, deren Beachtung aus dem Streben Polens nach einer Reputation als liberaler demokratischer Staat heraus erwachse (vgl. ebd.).

Die Betrachtung der bislang existierenden Regelungen und der mit der Migration befassten Institutionen und AkteurInnen zeigt, dass die Migrationspolitik in Polen geprägt ist durch die verschiedenen Wanderungsbewegungen, mit denen das Land konfrontiert ist: Ein-, Transit- und Auswanderung existieren in unterschiedlichem Ausmaß nebeneinander, verändern ihren Umfang im Zeitverlauf oder gehen zum Teil ineinander über. Zum Beispiel entwickelt sich Polen vom Transitland für Flüchtlinge zunehmend zum (unfreiwilligen) Endpunkt ihrer Migration. Die Regulierungen und Institutionen spiegeln dieses Nebeneinander wider. Bislang gibt es Regelungen zu vier Bereichen, die unterschiedlich umfangreich und ausdifferenziert sind: zur Repatriierung von Personen polnischer Herkunft, zur Aufnahme von Flüchtlingen, zum Arbeitsmarktzugang und zur Einwanderung

285 Sie nennt hier die Visapolitik, die an dem Ziel der Pflege guter Beziehungen zu den östlichen Nachbarstaaten ausgerichtet werde, die Repatriierungspolitik, den Schutz des polnischen Arbeitsmarktes und die Ermöglichung des Arbeitens polnischer Arbeitskräfte in anderen Ländern der EU sowie sicherheitspolitische Interessen und die Beschränkung von ,Asylmissbrauch“. 
von EU-BürgerInnen und ihrer Angehörigen. Die Repatriierung und die damit verknüpften Fragen nach Abstammung und ,Ethnizität' haben in Polen schon länger einen wichtigen Stellenwert. Die übrigen migrationspolitischen Bereiche werden erst seit jüngerer Zeit bearbeitet. Unter diesen sind die Regelungen zur Aufnahme von Flüchtlingen am meisten fortgeschritten und umfassend, während gesetzliche Initiativen, die einen legalen Arbeitsmarktzugang für Nicht-PolInnen ermöglichen sollen, die jüngste Entwicklung darstellen. Der Anstoß dazu wurde, wie oben geschildert, erstmals im Sommer 2006 von Andrzej Lepper ${ }^{286}$ angesichts fehlender Arbeitskräfte in der Landwirtschaft gegeben. Sicherheitspolitische Erwägungen fließen in all diese Regulierungen ein. Im Folgenden wird dieses Spektrum der migrationspolitischen Regelungen in Polen kurz skizziert, bevor der Teilbereich Flucht und Asyl genauer untersucht wird.

Die Repatriierung, ethnischer ${ }^{6}$ Pollnnen hat in Polen - wie schon angesprochen - bereits eine längere Tradition. Seit Anfang der 1990er Jahre hat das Thema immer wieder öffentliche Aufmerksamkeit auf sich gezogen. Dabei wird mit verschiedenen Maßnahmen versucht, die Rückkehr von Personen polnischer Herkunft und deren Nachkommen zu ermöglichen und zu fördern. Zielgruppe sind in erster Linie PolInnen, die in den 1930er und 1940er Jahren unter dem stalinistischen Regime von früheren ostpolnischen Territorien zwangsweise nach Osten, vor allem nach Zentralasien, deportiert und vertrieben wurden (vgl. Kępińska/ Stola 2004, S. 168ff.). Diese werden allerdings nicht als AusländerInnen betrachtet. Ähnlich wie bei den Sonderregelungen für ,AussiedlerInnen“ in Deutschland liegt den - vor allem durch die Kirche und rechte Parteien propagierten - Regelungen in Polen die Idee einer nationalen, auf gemeinsamer Abstammung 'beruhenden Gemeinschaft zugrunde. Daraus wird eine Verantwortung des polnischen Staates und der polnischen ,Nation“ gegenüber diesen Personen polnischer ,Abstammung' bzw. Herkunft und ihren Nachkommen abgeleitet. Die entsprechenden Regelungen, die 2000 in Gesetzesform gegossen wurden, konzentrieren sich auf die Rückkehr aus den asiatischen Republiken der früheren UdSSR und insbesondere aus Kasachstan. Die ,RückkehrerInnen' erhalten automatisch die polnische Staatsbürgerschaft sowie Zugang zu einigen Integrations-Angeboten (vgl. Iglicka 2005a, S. 10f.; Iglicka 1998). Faktisch ist das Repatriierungsprogramm jedoch nicht sehr erfolgreich: Laut Aussage von InterviewpartnerInnen sind nur 5.000 Personen nach Polen ,zurückgekehrt' (A S. 7; M S. 3). Das Konzept der Rückkehr - von EmigrantInnen im Allgemeinen - war bereits im Staatsbürgerschaftsgesetz von 1920 enthalten und fand auch Eingang in die Staatsbürgerschaftsregelungen während des Staatssozialismus. Damit ist es „an important example of continuity in Polish migration policy“ (Kicinger 2005, S. 23), womit Kicinger ihre These gestärkt sieht, dass Polen in der Migrationspolitik neben der Anpassung an EU-Vorgaben durchaus eigene Interessen verfolgt.

286 Vgl. zu Andrzej Lepper Fn. 274. 
Die Repatriierung ist eng mit Fragen der Staatsbürgerschaft und Nationalität verknüpft. Die Frage, die sich hier stellt, lautet: „what makes a person Polish“ (Kępińska/Stola 2004, S. 170)? Das polnische Staatsbürgerschaftsgesetz von 1962 kennt die Erlangung der polnischen Nationalität durch Geburt und NachGeburts-Verfahren. Die Erlangung der Staatsbürgerschaft durch Geburt orientiert sich am ,Blutsprinzip“ (ius sanguinis), das heißt ein Kind wird polnischer Staatsbürger, wenn mindestens ein Elternteil Pole ist. Das Nach-Geburts-Verfahren kennt drei Modi: die Verleihung, die Anerkennung und das vereinfachte Heiratsverfahren. Die Verleihung ist dabei das Verfahren mit dem größten Ermessenspielraum und gleichzeitig der schnellste Weg zur Staatsbürgerschaft. ImmigrantInnen, die sich in Polen niederlassen und die polnische Staatsbürgerschaft erhalten wollen, spielen jedoch quantitativ (noch) eine untergeordnete Rolle. Der überwiegende Teil sind Personen, die die polnische Staatsangehörigkeit wiedererlangen wollen und denen sie zum Beispiel aufgrund der Emigration entzogen wurde. Unter diese Gruppe fallen auch die Kinder von EmigrantInnen, die im Ausland geboren wurden und niemals in Polen waren. Viele wollen die Staatsbürgerschaft zurück, auch wenn sie nicht vorhaben, sich dauerhaft in Polen niederzulassen.

Obwohl die Interessen der polnischen Diaspora seit Anfang der 1990er Jahre kontinuierlich eine wichtige Rolle auf der politischen Agenda gespielt haben, hatte das 1962er Staatsbürgerschaftsgesetz bis nach der Jahrtausendwende Gültigkeit. Inzwischen wird eine Gesetzesreform verhandelt, die unter anderem das Verbot der doppelten Staatsbürgerschaft abschaffen und die Wiedererlangung der polnischen Staatsbürgerschaft erleichtern soll, wenn diese aus politischen Gründen während des Staatssozialismus aberkannt wurde. Die doppelte Staatsbürgerschaft war nach dem Gesetz von 1962 zwar nicht erlaubt. Dennoch gibt es zahlreiche BürgerInnen in Polen, die sowohl die deutsche als auch die polnische Staatsbürgerschaft besitzen. Schätzungen gehen von 300.000 bis 700.000 Personen mit doppelter Staatsbürgerschaft aus (vgl. Górny et al. 2007, S. 152; O S. 6).

Ein weiterer Gesetzentwurf befasst sich mit der Feststellung der polnischen Herkunft und dem Erhalt der polnischen Karte (Karta Polaka). Die polnische Karte bestätigt die Zugehörigkeit einer Person zur polnischen Nation. Um als Person polnischer Herkunft, als Pole im Ausland bzw. auswärtiger Pole anerkannt zu werden, müssen verschiedene Nachweise erbracht werden: So müssen mindestens ein Eltern- oder Großelternteil oder mindestens zwei Urgroßelternteile polnisch gewesen sein. Weiterhin muss sich die betreffende Person zu ihrer Zugehörigkeit zur polnischen Nation bekennen, die polnische Sprache wenigstens passiv beherrschen und polnische Traditionen pflegen. Darüber hinaus muss ein festes Einkommen nachgewiesen werden. Wenn diese Voraussetzungen erfüllt sind, wird ein (kostenloses) Mehrfach-Visum ausgestellt, mit der Erlaubnis, sich in Polen niederzulassen (vgl. Kępińska 2006). Die genannten Voraussetzungen müssen auch diejenigen erfüllen, die am Repatriierungsprogramm teilnehmen wollen - 
auch von ihnen wird der Nachweis ihrer polnischen ,Ethnizität' verlangt, es gilt also das Blutsprinzip (ius sanguinis). Wenn sie ein Repatriierungsvisum erhalten haben, wird ihnen bei ihrer Ankunft in Polen automatisch die polnische Staatsbürgerschaft verliehen.

Neben den genannten Regelungen sowie dem Flüchtlingsschutzgesetz und dem Ausländergesetz sind Regelungen, die die Rechte und Pflichten von AusländerInnen in Polen betreffen, auch in verschiedenen anderen Gesetzen zu finden: im Gesetz zur sozialen Unterstützung vom 12.03.2004, im Gesetz über öffentliche Leistungen zur Gesundheitsversorgung vom 27.08.2004, im Gesetz zu Familienleistungen vom 28.11.2003, im Ausbildungsgesetz vom 07.09.1991, im Gesetz für Höhere Bildung vom 12.09.1990, im Gesetz zur Arbeitsförderung und den Arbeitsmarktinstitutionen vom 20.04.2004, im Gesetz zur Freiheit der Geschäftstätigkeit vom 02.07.2004. Zwar sollen laut Arbeits- und Sozialministerium integrationspolitische Belange in Form eines Mainstreamings in andere Bereiche einfließen (Ministerstwo Polityki Społecznej 2005). Bis zur Umsetzung dieses Ziels scheint es jedoch noch ein weiter Weg zu sein - denn dies ist nicht nur eine Frage gesetzlicher Regulierung, sondern auch der Umsetzung auf behördlicher Ebene. ${ }^{287}$

Die Ausführungen zeigen, dass die Rede von der Nicht-Existenz der polnischen Migrationspolitik zu kurz greift, um die Entwicklungen zu erfassen. Wird die im Theoriekapitel vorgeschlagene Definition von Migrationspolitik angelegt (vgl. S. 18ff.), so kann durchaus von einer solchen auch in Polen gesprochen werden. Befragt man das Beispiel Polen hinsichtlich der einzelnen Punkte der Definition, können die Erkenntnisse des vorliegenden Kapitels folgendermaßen zusammengefasst werden: Auch in Polen wird versucht, grenzüberschreitende Mobilität zu regulieren - hinsichtlich des Zugangs zum polnischen Territorium und auch hinsichtlich des Status und der damit verbundenen Rechte innerhalb Polens. Die Aktivitäten in diesem Bereich sind in den vergangenen Jahren ausdifferenziert worden: Zahlreiche Instrumente zur Regelung der Einreise und des Aufenthaltsstatus wurden gesetzlich festgelegt, die entsprechenden Behörden und Verfahren geschaffen. Besonders gut zeigt sich am Beispiel Polens die Verwobenheit migrationspolitischer Interessen mit denen anderer Politikfelder: Der EU-Beitritt war treibende Kraft der Entwicklung vor allem im Flüchtlingsbereich, es lassen sich aber auch sicherheits- und arbeitsmarktpolitische Erwägungen identifizieren. Ersteres zeigt sich bspw. anhand der hohen Priorität von Sicherheitsaspekten im Flüchtlingsschutzgesetz und letzteres bei der Anwerbung von Arbeitskräften für die Landwirtschaft und den Bausektor. Mit der Prägung der Politiken durch EUVorgaben ist auch bereits die Supranationalisierung der Migrationspolitik in Polen angesprochen - zahlreiche Regelungen sind erst durch supranationale Einflüsse

\footnotetext{
287 So berichtete eine Interviewpartnerin, dass einer Frau mit Flüchtlingsstatus, die seit über zehn Jahren in Polen lebt, die zusätzlichen Hilfen, die im neuen Sozialgesetz für Alleinerziehende vorgesehen sind, nicht gewährt worden seien. Sie habe nicht nachweisen können, dass ihr Mann im Krieg gestorben sei. (C S. 10)
} 
entstanden. Ein Blick auf das AkteurInnenfeld zeigt eine wachsende Vielfalt und eine starke Bedeutung von NGOs (NGOisierung) sowie von Think Tanks, die durch Internationale Organisationen wie die IOM finanziert werden. Basisinitiativen und Flüchtlingsselbstorganisationen sind hingegen kaum vertreten. Während es schwierig ist, einen gap zwischen proklamierten Zielen und tatsächlichem Outcome der polnischen Migrationspolitik festzustellen, da klare Leitlinien bislang kaum ausgeprägt sind, lässt sich sehr wohl eine Kluft zwischen den gesetzlichen Regelungen und deren Umsetzung feststellen, zum Beispiel mit Blick auf die sozialen Rechte von Flüchtlingen. ${ }^{288}$ Der die Migrationspolitik in vielen Ländern begleitende Ressentiment-geladene Diskurs ist in Polen vorhanden, wenn auch schwach ausgeprägt. In Wahlkämpfen wurde das Thema bislang (noch) nicht aufgegriffen.

Schaut man sich die Situation durch die Brille der, drei Dimensionen des Politischen' an - policy, politics, polity - kann die Frage nach der Existenz einer polnischen Migrationspolitik ebenfalls bejaht werden. Insbesondere muss unterschieden werden zwischen verschiedenen Teilbereichen, die unterschiedlich stark reguliert und ausdifferenziert sind und in unterschiedlichem Ausmaß konkrete Leitlinien erkennen lassen (policy). Außerdem wird deutlich, dass sich - oft unabhängig vom Regulierungsgrad - nur für wenige Aspekte eine Politisierung beobachten lässt (politics): Willensbildungs-, Entscheidungs- und Implementierungsprozesse finden kaum im öffentlichen Raum, sondern vielmehr auf der Verwaltungsebene statt. So sei die polnische Migrationspolitik nach Aussage eines Interview-Partners gekennzeichnet durch ihre ,nicht-politische Entstehung und Existenz“ (O S. 3). Die Entwicklung der Gesetze fand vor allem auf der Verwaltungsebene, also in einem bürokratischen Verfahren statt und wurde vom Parlament lediglich abgesegnet. Immigration ist (noch) kein politischer Streitgegenstand und die entsprechenden Regelungen wurden kaum kontrovers diskutiert (vgl. Kępińska/Stola 2004, S. 162). So lassen sich die Zweifel an der Existenz einer Migrationspolitik auch mit dem geringen Politisierungsgrad des Themas erklären. Gleichwohl kann eine zunehmende institutionelle Ausgestaltung und Differenzierung beobachtet werden (polity).

Wie im Folgenden gezeigt wird, ist insbesondere der asylpolitische Bereich schon sehr weit ausdifferenziert - dies lässt sich nicht nur an den zahlreichen Regelungen in diesem Bereich und ihren realen Wirkungen auf die Situation von Schutzsuchenden ablesen, sondern zum Beispiel auch an seiner zunehmenden institutionellen und finanziellen Rahmung. Darüber hinaus kann bereits ein Wandel im Umgang mit Flüchtlingen festgestellt werden. So haben sich die Anerkennungszahlen von TschetschenInnen signifikant verringert. Dies interpretierte eine Interviewpartnerin dahingehend, dass hier eine explizit politische Entscheidung

288 Vgl. hierzu im Abschnitt 6.3.3, S. 277ff.: Soziale Rechte. 
getroffen worden sei. ${ }^{289}$ Während also der damalige polnische Präsident Lech Wałęsa noch 1992 auf bundesdeutsche Abschottungsforderungen antwortete: „Wir werden diese Menschen [aus Osteuropa und Asien, D.M.] nicht aufhalten, dazu sind wir gar nicht imstande. Wir werden ihnen ein Ehrenspalier aufstellen und sie zu Ihnen schicken." (vgl. Der Spiegel vom 20.01.1992), hat sich das Credo polnischer Politik gegenüber Flüchtlingen und MigrantInnen inzwischen spürbar gewandelt. Die Existenz einer Politik gegenüber Flüchtlingen kann kaum noch bestritten werden, es wird jedoch weiter auf die Einflussfaktoren, die zentralen AkteurInnen und den Politisierungsgrad einzugehen sein. Dies soll im letzten Abschnitt des Kapitels erörtert werden.

\title{
6.3 Kategorie Flucht und Asyl: AkteurInnen, Regulierungen und migrantische Strategien
}

\author{
„[...] vor 15 oder 20 Jabren haben wir in Polen nicht mal an Asylbewerber \\ gedacht. Jetzt haben wir 7.000 bis 8.000 pro Jahr. Was wird in zehn oder \\ in fünf Jahren sein?" (B S. 16)
}

Vor dem Hintergrund der in den vorigen Abschnitten vorgestellten Geschichte der Wanderungsbewegungen aus, durch und nach Polen soll im Folgenden das Hauptaugenmerk auf die Kategorie Flucht und Asyl gelegt werden. Dabei stehen drei Aspekte im Vordergrund: der Verlauf der Entwicklungen bei der Gestaltung des polnischen Flüchtlingsschutzregimes, die konkrete aktuelle Ausgestaltung und schließlich die Diskussion der zentralen Einflussfaktoren. Die Untersuchung der polnischen Flüchtlingspolitik, insbesondere ihrer Einflussfaktoren, ist deshalb schwierig, weil es in den ersten Jahren nach der, Wende' kaum offizielle Verlautbarungen zu diesem Bereich gab. Auch heute noch, da die Einwanderung und die Zahl der Asylsuchenden quantitativ an Bedeutung gewinnen, ist Einwanderungspolitik ein kaum umkämpftes Feld in Polen. Die Untersuchung der Flüchtlingspolitik muss daher auf Basis der Gesetzesänderungen erfolgen, die einen Einblick in die Intentionen der politischen AkteurInnen geben. Die Analyse der Gesetzgebung wird durch Informationen aus zahlreichen Interviews mit AkteurInnen ergänzt (vgl. Kapitel Methoden, S. 67ff.).

Vor den Umbrüchen von 1989/90 war Polen selbst Herkunftsland von Flüchtlingen: Viele PolInnen flohen aus politischen Gründen insbesondere nach

\footnotetext{
289 D S. 6: „Aber irgendwie hat sich dann, also ich gehe davon aus, dass sich die Regierungspolitik dann geändert hat, nach einer ziemlich kurzen Zeit. Und sie entschieden, dass es aus politischer Sicht vermutlich nicht die beste Idee ist, allen Tschetschenen Flüchtlingsstatus zu geben, denn das könnte Russland, sagen wir, weniger glücklich machen. Also irgendwie hat sich meiner Ansicht nach die Regierungspolitik, also wenn es überhaupt eine Regierungspolitik gibt, geändert und jetzt ist es für uns ziemlich schwierig, Flüchtlingsstatus für unsere Klienten zu bekommen.“
} 
der Verhängung des Kriegsrechts in den 1980er Jahren in den Westen, v. a. nach Deutschland und in die USA. Nach 1989/90 entwickelte sich Polen rasch zum Transitland für (undokumentierte) MigrantInnen aus Rumänien, Bulgarien und Ländern der ehemaligen UdSSR. Zu Beginn beantragten erst wenige Hundert Flüchtlinge Asyl, die aufgrund des Rückübernahmeabkommens aus Deutschland zurückgeschoben wurden. Inzwischen sind es zwischen 5.000 und 8.000 jährlich (vgl. Tabelle $9 \mathrm{im}$ Anhang). Polen hat eine über 1.000 Kilometer lange Grenze zur russischen Exklave Kaliningrad, zu Weißrussland und zur Ukraine und liegt damit auf einer der Hauptrouten von MigrantInnen, die auf dem Landweg versuchen, die EU zu erreichen. War es vor dem EU-Beitritt 2004 ein Transitland, das Flüchtlinge auf dem Weg in die EU durchquerten, ist es nun als EU-Mitglied selbst mit einer zunehmend hochgerüsteten Grenze zu seinen östlichen Nachbarstaaten bewehrt und hat seine Rolle als der EU vorgelagerte Pufferzone an die Nachbarn - vor allem die Ukraine, aber auch Weißrussland - abgegeben. Der vorläufige Höhepunkt bei der Integration Polens in den EU-,Raum der Freiheit, der Sicherheit und des Rechts' wurde Ende 2007 mit dem Beitritt Polens zum Schengener Abkommen und seiner Aufnahme in den Schengenraum erreicht. Damit hat es binnen weniger Jahre einen beachtlichen Wandel sowohl hinsichtlich seiner grenz- und migrationspolitischen Bedeutung im EU-Kontext als auch hinsichtlich seiner Bedeutung für MigrantInnen bzw. Flüchtlinge und ihre Routen durchlaufen.

Während, wie oben dargestellt, die Einreise von Arbeitskräften inzwischen ein politisches Thema ist, hat das Thema Flüchtlinge in Politik und Öffentlichkeit in Polen noch immer geringe Bedeutung:

„Ich denke, dass die Änderung der Politik [der Regierungswechsel, D.M.] keine Hauptrolle bei dem Problem der Flüchtlinge spielt. Denn es ist eine Tatsache, dass das Flüchtlingsthema und Migration im Allgemeinen kein sehr sehr großes Problem ist, wie in West-Europa. [...] Hier gibt es mehr Diskussionen wie man den Arbeitsmark.t öffnen kann für Leute aus der Ukraine, als das Problem mit den Flüchtlingen. "(J S. 3)

Das geringe öffentliche Interesse wird mit der Zahl der Flüchtlinge begründet (vgl. C S. 12), die sich zwar im Laufe der vergangenen Jahre stark erhöht hat, die jedoch im europäischen Vergleich immer noch niedrig ist. Die polnische Regierung habe andere bzw. dringendere Probleme (vgl. G S. 5; D S. 24). Dennoch war Polen seit Anfang der 1990er Jahre mit dem Thema konfrontiert und hat binnen weniger Jahre die entsprechenden Regelungen, Verfahren und Behörden geschaffen.

\subsubsection{AkteurInnen}

Wie am Anfang dieser Studie dargelegt, wird das Migrations(politische) Geschehen hier aus der Regime-Perspektive untersucht. Dies impliziert vor allem, den Blick auf die Vielzahl der AkteurInnen in dem Feld und ihr Verhältnis zueinander 
und insbesondere auf die mitunter „eigensinnigen“ (Benz/Schwenken 2005) Praxen von MigrantInnen zu richten. Aus dieser Perspektive werden im Folgenden die beteiligten AkteurInnen und die von diesen artikulierten Interessen vorgestellt.

Das polnische Flüchtlingsschutzregime ist im Vergleich zu dem in Deutschland noch sehr jung und (noch) weniger stark ausdifferenziert. Das AkteurInnenfeld umfasst im Wesentlichen MigrantInnen, staatliche AkteurInnen, NGOs, die EU sowie die IOM und wissenschaftliche Think Tanks. Wenn man davon ausgeht, dass „Migration selbst das dynamische Moment in einem Regime der Migration ist“ (Karakayalı/Tsianos 2005, S. 50) und „die soziale Bewegung der Migration“ als eine Größe im migrationspolitischen „Kräfteparallelogramm“ (ebd.) konzeptualisiert, so müssen die MigrantInnen im AkteurInnenfeld mitgedacht werden. Während die Migration an sich zunächst mehr eine „Abstimmung mit den Füßen“ (Hess/Tsianos 2004) ist und eine individuelle Strategie zur Erreichung bestimmter Ziele darstellt, die aufgrund der Zahl der individuellen AkteurInnen zu einem kollektiven Phänomen und einer sozialen Bewegung wird bzw. werden kann, artikulieren sich MigrantInnen auch explizit politisch. Etwa wenn sie Hungerstreiks in den Heimen organisieren und Forderungen nach einer ungehinderten Weiterreise stellen oder Selbstorganisationen gründen. Die Landschaft der flüchtlingspolitischen Selbstorganisationen in Polen ist allerdings (noch) nicht sehr ausdifferenziert. Außer der Flüchtlingsvereinigung (Stowarzyszenie Uchodźców w Rzeczypospolitej Polskiej) gibt es nur einige wenige weitere Selbstorganisationen nach Schätzungen eines Interviewpartners ca. zehn bis 15 (O S. 6). Diese beziehen sich in ihrem Selbstverständnis stark auf ihre Herkunftsländer - so gibt es zum Beispiel Zusammenschlüsse äthiopischer, sudanesischer und tschetschenischer MigrantInnen - und sind bislang kaum auf eine politische Artikulation von Flüchtlingsrechten ausgerichtet.

„Viele der Gruppen lassen sich von der Öffentlichkeit nicht unter menschenrechtlichen Aspekten, sondern unter dem Motto: ,Wir sind exotisch!" einordnen. Das ist gegenüber der Presse gut zu benutzen und zeigt sich besonders während des jährlichen Flüchtlingstags. " (O S. 7).

Eine formale Anerkennung der Vereinigungen ist schwierig, weil das polnische Recht eine Zulassung nur vorsieht, wenn der Vorsitzende des Vereins ein Pole ist. Die geringe Zahl von Selbstorganisationen lässt sich einerseits mit der vergleichsweise geringen Zahl von Flüchtlingen in Polen und andererseits mit der fehlenden Bleibeperspektive derer erklären, die in Polen einen Antrag gestellt oder eine Duldung erhalten haben.

Die wichtigsten staatlichen AkteurInnen sind

- der Sejm als gesetzgebende Instanz (einschließlich der Menschenrechtskommission des Parlaments),

- das Innenministerium, 
- die zentrale Ausländerbehörde (URIC/UdSC) ${ }^{290}$, die über Anträge auf Flüchtlingsschutz entscheidet, ,Dublin-Fälle“ bearbeitet, die Aufsicht über die Flüchtlingsheime hat und an der Erarbeitung von Gesetzentwürfen beteiligt ist,

- der so genannte ,Flüchtlingsrat ${ }^{`}$ - die erste Berufungsinstanz gegen ablehnende Entscheidungen des UdSC - sowie

- die (Verwaltungs-)Gerichte (zwei Instanzen - eine auf Woiwodschaftsebene und ein Oberverwaltungsgericht),

- der Grenzschutz,

- das Arbeitsministerium, das die Integration von Flüchtlingen koordiniert, und

- die Landkreise (Powiats), die für die Integration der anerkannten Flüchtlinge (Erstellung eines individuellen Integrationsprogramms) zuständig sind.

Nach Einschätzung von Interviewpartnern (M S. 3; O S. 5f.) spielte der polnische Grenæschutz in den 1990er Jahren eine entscheidende Rolle im migrationspolitischen Geschehen:

„Sie kannten sich aus und machten eine gute Pressearbeit bezüglich illegaler Migration. Eine sehr effektive Arbeitsweise binsicbtlich der Ausarbeitung eines restriktiven Gesetzes." (O S. 5)

Der Grenzschutz hat im Verlauf der letzten zwei Dekaden einschneidende Veränderungen bezüglich seiner Aufgaben und seiner Arbeitsweise erlebt, gleichzeitig haben sich die Bedeutung von Ost- und Westgrenze und die dortigen Aktivitäten jeweils grundlegend gewandelt.

Nach 1989/90 musste der Grenzschutz wesentlich umstrukturiert werden: sowohl hinsichtlich seines Selbstverständnisses als auch hinsichtlich seines Aufgabenbereichs und seiner personellen Struktur. Er sollte einerseits als einstiges wichtiges Instrument des früheren kommunistischen Regimes im Übergang zur demokratischen Ordnung grundlegend transformiert und andererseits für die neuen Herausforderungen fit gemacht werden (vgl. Latawski 1999, S. 90). Die entsprechenden gesetzlichen Grundlagen wurden 1990 verabschiedet und umfassten in erster Linie eine Abkehr von militärischen und die Übernahme polizeilicher Funktionen (vgl. Latawski 1999, S. 94).291 Zudem sollte der Grenzschutz, der bis 1989/90 eine „strong political role as an important pillar in the security apparatus of the communist party-state“ spielte und „Soviet models and practices“ (ebd., S. 92) entsprach, entpolitisiert werden. Von 22.000 früheren Grenzschützern verblieben nach der ,Verifizierung' nur 5.000 und diese sowie die neu Hinzugekommenen dürfen ausdrücklich während ihres Dienstes nicht Mitglied einer poli-

290 Das URIC (Urząd do spraw repatriacji i cudzoziemców / Amt für Repatriierungs- und Ausländerangelegenheiten) wurde im Jahr 2007 umbenannt (UdSC - Urząd do spraw cudzoziemców / Amt für Ausländerangelegenheiten) und in seinem Aufgabenbereich eingeschränkt - es ist nun nicht mehr für die Repatriierung und die Vergabe der Staatsbürgerschaft zuständig.

${ }^{291}$ Vgl. Law on the Protection of the State Border vom 19.11.1990 und Law Governing the Border Guard Service vom 19.11.1990. 
tischen Partei sein (vgl. ebd., S. 95). Während sich der Grenzschutz zu Zeiten der Blockkonfrontation besonders auf die Überwachung der Westgrenze konzentrierte und sich an der Ostgrenze auf die Arbeit der sowjetischen Grenzschützer verlassen konnte (vgl. ebd., S. 92), herrschte 1989/90 nach der Öffnung der einstmals geschlossenen Grenzen zur Sowjetunion „relative [...] Personenfreizügigkeit in Mittel- und Osteuropa“ (Alscher 2004, S. 222). Diese Phase war durch regen Grenzverkehr und vor allem grenzüberschreitenden Kleinhandel (,Ameisenhandel $^{\circ}$ ) gekennzeichnet. An der Westgrenze wurden die Hürden von Seiten der Bundesrepublik erhöht. Im Rahmen der Beitrittsvorbereitungen wurde der Übertritt der Westgrenze für polnische BürgerInnen vereinfacht und mit dem Beitritt zum Schengen-Raum vollkommen schrankenfrei. Der Fokus grenzüberwachender Maßnahmen verlagerte sich an die Ostgrenze, sie wurde zu einem zentralen Aktionsfeld restriktiver Maßnahmen: Obwohl die Einschränkung der Reisefreiheit in der Grenzregion auf Widerstand stieß, wurden bereits seit Anfang der 1990er Jahre und mit finanzieller Unterstützung aus der Bundesrepublik umfangreiche Investitionen für effektivere Kontrollen an der Ostgrenze getätigt. Die Technik wurde erneuert und Personal von der West- an die Ostgrenze verlegt, um die Kontrollen im Osten zu verschärfen (vgl. Der Spiegel vom 28.06.1993). Heute muss es nach Ansicht des Grenzschutzes darum gehen, vor allem illegale Migration abzuwehren und die Filterfunktion der Grenze zu verbessern:

„Nach dem Beitritt zur EU muss diese Art des Denkens geändert werden. Jetzt natürlich - sind die Hauptaufgaben der Grenzschützer die gleichen: Grenzkontrolle und Verkehrskontrolle, aber nicht, alles zu tun, um die Leute nicht in die EU einreisen zu lassen, aber alles zu tun, um seine/ihre Papiere gründlich zu prüfen. Und das ist alles. Und natürlich nicht in jedem Ausländer einen illegalen Migranten zu sehen. Es gibt eine dritte Aufgabe: illegale Migration bekämpfen. Die Zeit zeigt, dass diese Aufgabe die wichtigste werden wird in einigen Jahren. "(B S. 2)

Mit dem Beitritt zum Schengenraum müsse sich die Arbeit des Grenzschutzes weiter verändern:

„W Was heißt das [der Beitritt zum Schengenraum] für den Grenzschutz? Wir müssen unsere Art des Denkens verändern. Wir müssen uns darauf vorbereiten, andere Aktivitäten zu verfolgen/andere Dinge zu tun. Ich meine Kontrollen und Überprüfungen innerhalb des Landes; ich meine sich darauf vorzubereiten, bezüglich der illegalen Migrationsaktivitäten von Ausländern zu agieren; mit unseren Nachbarn zu kooperieren, insbesondere im Osten" (BS. 2)

Es geht also darum ,to find the correct balance between efficiently processing legitimate cross-border movement while providing an effective screen to block unlawful transborder activity“" (Latawski 1999, S. 91). Seit 2001 sind Grenzschutzoperationen nicht mehr nur auf die Grenzzone beschränkt, sondern in ganz Polen 
möglich. Mit der Vorbereitung auf den EU-Beitritt hat der Grenzschutz an Ressourcen und Macht gewonnen:

„While formally just a law-enforcing agency, the Border Guard plays an important role
in shaping migrations. [...] Thanks to increased government allocations and foreign
grants for the modernization of equipment, new infrastructure and training, the Border
Guard's capacity and efficiency have grown significantly. The Border Guard has secured
growing resources as well as growing powers. "(Kepinska/Stola 2004, S. 161)

Die von den Interviewpartnern genannte bedeutende Rolle des Grenzschutzes in migrationspolitischen Fragen in den 1990er Jahren kann darin gesehen werden, dass der Grenzschutz die durch die fehlenden politischen Leitlinien und Regelungen im Migrationsbereich entstandene Lücke im Austausch mit dem Innenministerium durch eigene Aktivitäten ausgefüllt hat. Andererseits kann aus den Stellungnahmen der Interviewpartner auch entnommen werden, dass der Grenzschutz durch Pressearbeit eigene Akzente bei der Entwicklung der Migrationspolitik gesetzt hat.

Das URIC bzw. UdSC ist in gewisser Hinsicht das polnische Pendant zum deutschen Bundesamt für Migration und Flüchtlinge (BAMF). Es ist für die Bearbeitung der Anträge auf Flüchtlingsschutz zuständig und - im Gegensatz zum BAMF - zusätzlich zweite Instanz bei der Entscheidung über sonstige Aufenthaltsstatus, die in erster Instanz die zuständige Woiwodschaft trifft. Darüber hinaus betreibt das UdSC die polnischen Flüchtlingsheime und zeichnet für die Pflege einer nationalen Datenbank mit Daten über AusländerInnen in Polen verantwortlich. In der Datenbank ist das UdSC mit den Woiwodschaften vernetzt, um mehrfache Antragstellungen in verschiedenen Woiwodschaften zu verhindern. Bis 2001 war das UdSC maßgeblich an der Erarbeitung des Flüchtlingsschutzgesetzes beteiligt, Mitarbeiter des UdSC arbeiteten an der Formulierung von Gesetzentwürfen mit. Hier manifestiert sich der bürokratische Charakter der Entstehung des Flüchtlingsschutzregimes: Behörden und Gesetzgebung sind eng miteinander verzahnt, was insbesondere dadurch bedeutsam wird, dass die behördlich ausgearbeiteten Gesetzentwürfe im Parlament - im Sejm - bislang unwidersprochen angenommen wurden.

Daneben hat sich in den vergangenen Jahren eine NGO-Landschaft entwickelt, die weiter im Wachsen begriffen ist. Dazu gehören beispielsweise die Helsinki Stiftung für Menschenrechte (Helsińska Fundacja Praw Człowieka), die Polnische Humanitäre Aktion (Polska Akcja Humanitarna), der Menschenrechtsverein Halina Niec (Stowarzyszenie Praw Człowieka im. Haliny Nieć), die Stiftung Ocalenie (Fundacja Ocalenie) und die Caritas. Einige davon befassen sich ausschließlich mit der Flüchtlingsthematik, einige beraten und unterstützen neben polnischen BürgerInnen auch Flüchtlinge. Die Rolle dieser Organisationen im polnischen Flüchtlingsschutzregime ist vielschichtig und mitunter ambivalent, denn neben Rechtsberatung und humanitärer Unterstützung nehmen sie zum Teil 
aktiv am Gesetzgebungsprozess teil. Die meisten Organisationen konzentrieren sich überwiegend auf Unterstützung, Beratung und humanitäre Hilfen. Sie füllen oft Lücken an sozialer Unterstützung und rechtlichem Beistand, die im polnischen Flüchtlingssystem, aber auch für polnische BürgerInnen bestehen. So gibt es beispielsweise von staatlicher Seite keine Angebote an kostenloser rechtlicher Beratung - für polnische BürgerInnen ebenso wenig wie für Flüchtlinge. Diese Lücke versuchen zum Beispiel die Helsinki-Stiftung, der Menschenrechtsverein Halina Niec oder die Legal Clinics an den Universitäten ${ }^{292} \mathrm{zu}$ füllen. Sie haben aber nicht genügend Kapazitäten, um der hohen Nachfrage gerecht zu werden (vgl. Koryś 2003, S. 61).

Zudem sind einige, wie die Helsinki Stiftung, als BeraterInnen eng in den Policy-Gestaltungsprozess einbezogen:

„The role of non-governmental organizations is worth mentioning. They were recognized as a partner in shaping the relevant legislation during the debate on the 1997 Alien Act and proved to be efficient and competent. In particular, the Helsinki Foundation for Human Rights and its legal experts have contributed to many provisions on foreigners (mainly refugees), especially from human rights perspective." (Kepinska/Stola 2004, S. 162)

Die InterviewpartnerInnen von der Helsinki Stiftung berichteten im Interview, dass sie als ExpertInnen bei allen bisherigen Gesetzesänderungen im Ausländerrecht beteiligt gewesen seien. ${ }^{293}$ Aus Sicht einer Mitarbeiterin der Helsinki Stiftung sei es besser, dass sie sich um die Inhalte des Flüchtlingsschutzgesetzes kümmern, als wenn populistische PolitikerInnen sich des Themas annähmen:

„,...] das mag nicht so demokratisch k.lingen - aber ich denke, es ist besser, dass wir sagen, welche Änderungen nötig sind und was getan werden muss, als wenn einige populistische Politiker nur Sachen sagen, die für ihre Wähler gut klingen mögen, die aber schädlich sein könnten. Denn sie haben keine Abnung, worüber sie reden, es klingt nur gut für die Leute, die sie wählen. "(J S. 13)

Der Menschenrechtsverein Halina Niec möchte ebenfalls in den Gesetzgebungsprozess eingebunden werden, war mit seinen diesbezüglichen Bemühungen bislang aber nicht erfolgreich (L S. 3). Auch sind einige Organisationen vom URIC angefragt worden, in den neuen Haftanstalten, die derzeit an der Ostgrenze entstehen, Rechtsberatung anzubieten (J S. 14).

Dieses Selbstverständnis als humanitäre Helfer und ExpertInnen im PolicyGestaltungsprozess sowie die mögliche Mitarbeit in den Haftanstalten statt einer

\footnotetext{
292 Die Legal Clinics arbeiten nach einem aus den USA entlehnten Konzept, wonach Bedürftige sich durch JurastudentInnen rechtlich beraten und vertreten lassen können.

293 „Was in dieser Hinsicht sehr wichtig für uns ist: Wir sind die Experten im Gesetzgebungsprozess (im Parlament). Wir waren und sind die Experten bei allen bisherigen Gesetzesänderungen im Ausländerrecht.“ (J S. 1f.)
} 
Kritik an der Inhaftierung von MigrantInnen spiegelt die starke, Innen'-Position polnischer NGOs im Flüchtlingsbereich wider, das heißt eine recht ausgeprägte Nähe zu staatlichen Institutionen. Im Gegensatz dazu finden sich weniger Kampagnen in der Öffentlichkeit, die von außen durch politischen Druck auf die Situation Einfluss zu nehmen versuchen. Die durchgeführten Kampagnen enthalten selten radikale Forderungen und präsentieren Flüchtlinge häufig als Opfer. Öffentliche Kampagnen werden zum Teil auch als Strategie abgelehnt, zum Beispiel wenn sich die Organisation auf die juristische Vertretung von Flüchtlingen - also auf Einzelfälle - spezialisiert hat (J S. 6).

Eine Ursache für diese Positionierung polnischer NGOs kann darin gesehen werden, dass sie nicht aus Basisbewegungen entstanden sind, sondern überwiegend auf Initiative von außen - zum Beispiel des UNHCR (wie bspw. die Helsinki Stiftung, die Halina Niec Organisation, aber auch die Legal Clinic). Viele Projekte von NGOs werden aus EU- und UNHCR-Mitteln finanziert, was unabhängige Positionierungen unwahrscheinlich macht. Als Pendant zu diesen NGOs finden sich kaum antirassistische Basisbewegungen oder Flüchtlingsselbstorganisationen.

Eine ähnliche flüchtlingspolitische NGO-Landschaft zeigt sich auch in anderen östlichen EU-Mitglieds- und Beitrittsstaaten. Bedingt durch ihre Installierung ,von oben' und entsprechende finanzielle Abhängigkeit nehmen die NGOs selten eine über niedrig schwellige, humanitär ausgerichtete Korrekturvorschläge hinausgehende kritische Perspektive ein. So übernehmen sie nicht selten Perspektive und Rhetorik der EU und treten zum Beispiel für die Implementierung der EURichtlinien ein. Die Folgen der Installierung der Asylregime für die MigrantInnen - eingeschränkte Bewegungsfreiheit, Festsitzen in den Ländern an der ,Peripheriec, Aufteilung in legitime und illegitime MigrantInnen, etc. - werden kaum thematisiert bzw. widerspruchslos übernommen. Ein Beispiel ist die Forderung einer serbischen NGO, dass es kein Rückübernahmeabkommen zwischen Serbien und der EU geben solle, solange in Serbien noch kein Asylsystem etabliert sei. ${ }^{294}$ Weder Rückübernahmeabkommen noch die Funktion von Asylsystemen werden damit infrage gestellt, sondern vielmehr als gegebene Institutionen gedacht, die lediglich menschenrechtlich abgesichert werden müssen. Der Vertreter des International Law and Research on Human Rights Monitoring Center (ILRHRMC) in Ungarn, Dr. Ejalu, vertrat die Ansicht, MigrantInnen sollten gestoppt und vor den Gefahren der Migration gewarnt werden. Sie sollten wissen, dass in Deutschland ,das Geld nicht von den Bäumen fällt'. Er stellte auch die Frage, welche Alternative es zu den Flüchtlingslagern gäbe, hier hätten die MigrantInnen wenigstens ein

\footnotetext{
${ }^{294}$ Eigene Mitschrift des Beitrages von Vladimir Petronijevic (grupa 484), gehalten auf dem Migreurop-Workshop in Radovljica, Slowenien am 28. und 29. Mai 2007. Gleichwohl war es zu dieser Zeit das Ziel der grupa 484 ,to create a world in which people will be free to choose where to live, and in which all their rights and differences will be respected.“ (grupa 484 o.J.)
} 
Dach über dem Kopf. ${ }^{295}$ Tatsächlich sind Wohnungen zum Beispiel in Polen für die meisten Flüchtlinge unerschwinglich und eine Unterkunft in einem der Heime deshalb oft die einzige Alternative zur Obdachlosigkeit. Diese Situation ist in vielen (süd)osteuropäischen Staaten vorzufinden. Dennoch kann es auch von dort kritische Stimmen zur Einrichtung von Lagern geben, wie das Beispiel des slowenischen Politologen Andrej Kurnik zeigt. Er illustrierte die widersprüchliche Rolle von NGOs bei der Normalisierung von Lageraufenthalten anhand des slowenischen Lagers Postojna. ${ }^{296}$ Indem sie nicht die Lager an sich infrage stellten, sondern lediglich die Einhaltung von Menschenrechten in den Lagern forderten, trügen die NGOs zur Normalisierung von Lagern bei und versperrten so den Raum für eine grundlegende Auseinandersetzung mit dem Lagerregime. Die Professorin Anna Krasteva aus Bulgarien ${ }^{297}$ fächerte das AkteurInnenfeld und die darin enthaltenen Perspektiven folgendermaßen auf: Die EU sei durch eine Sicherheitsperspektive gekennzeichnet, während die lokalen NGOs eine Konsumhaltung aufwiesen und die Flüchtlinge nicht als Partner, sondern als Objekte wahrnähmen. Die Konsumhaltung zeige sich einerseits in der finanziellen Abhängigkeit von EU-Mitteln und andererseits in der Übernahme von Argumenten und Begründungen aus dem EU-Repertoire. Schließlich fragte sie, wer angesichts dieser Akteurskonstellation die Menschenrechtsperspektive übernähme.

Bei der bisherigen Darstellung der AkteurInnen und ihrer Aktivitäten wird immer wieder auch die EU angesprochen. Da die EU nicht als einheitliche Akteurin betrachtet werden kann, sondern in sich eine relative Vielfalt von Interessen und AkteurInnen enthält, wird hier, wenn die Rede von ,der EU' ist, Bezug genommen auf die Entwicklungen, die von EU-Gremien während des Beitrittsprozesses und danach in Polen in Gang gesetzt wurden. Im Rahmen der Verpflichtung Polens, den Aquis communautaire, den gemeinschaftlichen Besitzstand, zu übernehmen, spielte auch der Bereich Justiz und Inneres eine wichtige Rolle. So verpflichtete sich Polen, die flüchtlingspolitischen Vorgaben, die die EU bis zum Mai 2004 in Richtlinien gegossen hatte und die darauf folgenden Änderungen in polnisches Recht umzusetzen, mit grenzpolitischen Maßnahmen an der Erfüllung der Schengen-Kriterien zu arbeiten, administrative Um- und Neustrukturierungen vorzunehmen und das entsprechende Personal zu schulen. Welche Bedeutung der EU bei der Entwicklung des polnischen Flüchtlingsschutzregimes beizumessen ist, wird im Abschnitt 6.3.5 Resümee der Einflussfaktoren näher dargestellt.

\footnotetext{
${ }^{295}$ Eigene Mitschrift von Beiträgen auf dem Migreurop Workshop in Radovljica, Slowenien am 28. und 29. Mai 2007.

${ }^{296}$ Eigene Mitschrift des Beitrages von Dr. Andrej Kurnik (Universität Ljubljana): Slovenia. Situation in the detention center in Postojna, Slovenia, gehalten auf dem Migreurop Workshop in Radovljica, Slowenien am 29. Mai 2007.

297 Eigene Mitschrift des Beitrages von Prof. Anna Krasteva (Universität Sofia): Bulgaria. Refugees and immigrants in Bulgaria: between securitarian and human rights concerns, gehalten auf dem Migreurop Workshop in Radovljica, Slowenien am 29. Mai 2007.
} 
Um die Akteurskonstellation zu vervollständigen sind noch die IOM und wissenschaftliche Think Tanks zu nennen. Beide sind stark miteinander verwoben, da die IOM in Polen insbesondere Forschung zum Thema Migration finanziert. Hier ist zum Beispiel das Central European Forum for Migration and Population Research (CEFMR) in Warschau zu nennen, das in der Migrationsforschung in Polen sehr aktiv ist und regelmäßig Working Papers zu Aus- und Einwanderungsfragen publiziert und ExpertInnen ausbildet.

Vor dem Hintergrund dieser Akteurskonstellation wird im Folgenden die Entwicklung des polnischen Asylregimes dargestellt.

\subsubsection{Phasen des asylpolitischen Wandels}

\section{0 - 2000: Schaffung erster asylrechtlicher Grundlagen}

Die polnische Verfassung enthielt bereits vor 1990 das Recht auf Asyl, dieses stand jedoch noch ganz im Zeichen der realsozialistischen polnischen Vergangenheit. In der polnischen Verfassung von 1952 hieß es in Artikel 75:

„Die Volksrepublik Polen gewährt denjenigen Bürgern anderer Staaten Asyl, die wegen
ihres Eintretens für die Interessen der Werktätigen, wegen ibres Kampfes für den gesell-
schaftlichen Fortschritt, wegen ibrer Tätigkeit für die Verteidigung des Friedens, wegen
ibres Kampfes für die nationale Befreiung oder wegen ibrer wissenschaftlichen Betätigung
verfolgt werden. "(Verfassung der Volksrepublik. Polen vom 22. 07.1952)

Im Jahre 1990 kamen die ersten Flüchtlinge nach der, Wende ${ }^{夭}$ nach Polen. Fast 600 Flüchtlinge waren 1990 aus Schweden nach Polen zurückgeschoben worden, da sie ohne die erforderlichen Visa in Schweden angekommen und über Polen mit polnischen Visa eingereist waren. Die polnischen Behörden brachten die Flüchtlinge in lokalen Ferienzentren an der Ostsee unter. Als die Zahl der Flüchtlinge anstieg, die Schweden nach Polen zurückschickte, erwies sich dieses Provisorium ebenso wie die Aufbringung der notwendigen finanziellen Mittel als Problem, mit dem die polnischen Behörden völlig unvorbereitet konfrontiert waren. Es entstand eine Kooperation mit dem UNHCR, die schließlich - zusammen mit Druck aus westlichen Ländern - in der Ratifikation der GFK und der Europäischen Menschenrechtskonvention 1991 mündete. Im November 1990 wurde eine Regierungskommission geschaffen und ein Generalbevollmächtigter des Innenministeriums für Flüchtlingsfragen eingesetzt. 1992 wurde das UNHCR-Verbindungsbüro in Warschau eröffnet, 1993 die Menschenrechtskonvention ratifiziert. Die Ratifikation der GFK bildete die Grundlage für die Entwicklung des polnischen Flüchtlingsrechts, die Garantien der GFK wurden in das polnische Ausländergesetz aufgenommen.

Im Zuge der Änderung des Asylrechts in Deutschland 1993 wurde Polen von deutscher Seite zum , sicheren Herkunftsstaat ${ }^{\star}$ und - mit Verweis auf die GFKRatifikation - zum, sicheren Drittstaat' erklärt. Deutschland vereinbarte im selben 
Jahr ein Rückübernahmeabkommen mit Polen. ${ }^{298}$ Polen wurde so dazu verpflichtet MigrantInnen zurückzunehmen, die von polnischem Territorium illegal nach Deutschland gekommen waren. Im Gegenzug wurden die Visa-Bedingungen für polnische StaatsbürgerInnen erleichtert. Diese Kombination von Rückübernahmegarantien einerseits und Visa-Erleichterungen andererseits hatte Modellcharakter für spätere Übereinkommen mit anderen Staaten. Nach Polens Beitritt zur Europäischen Union wurde dieses Abkommen durch die Dublin-II-Verordnung ersetzt.

Das Rückübernahmeabkommen mit Polen war Ausgangspunkt einer Kettenreaktion, im Zuge derer die Verantwortung für MigrantInnen sukzessive weiter östlich und nach außerhalb der EU verschoben wurde. So schloss Polen seinerseits zahlreiche Rückübernahmeabkommen mit fast allen Nachbarstaaten außer trotz zahlreicher Bemühungen - Russland und Belarus. ${ }^{299}$ Ziel sei es gewesen, „not to become a 'dumping ground' or a trap for illegal third-country migrants readmitted from Germany" (Kępińska/Stola 2004, S. 163). In diesem Sinne führte Polen auch eine Visapflicht für Herkunftsländer von Flüchtlingen ein. Beispielsweise wurden für MigrantInnen aus Jugoslawien im Jahr 1993 für die Einreise nach Polen Visa erforderlich. Dem war die Rückschiebung von 412 JugoslawInnen aus Schweden und Dänemark in der ersten Hälfte des Jahres 1993 vorausgegangen. Diese beiden Länder hatten die Visapflicht für Personen aus Jugoslawien bereits einen Monat früher eingeführt.

Mit diesen Maßnahmen gingen auch verstärkte Aktivitäten hinsichtlich der ,Grenzsicherung' einher. Insbesondere die Bundesrepublik Deutschland engagierte sich in diesem Bereich - sie konzentrierte sich anfangs auf die Sicherung der polnischen Westgrenze und verlegte ihren Fokus im Zuge des Beitrittsprozesses auf Polens Ostgrenze. Die Unterzeichnung des Rückübernahmeabkommens gratifizierte Deutschland mit 120 Millionen DM, die es zwischen 1993 und 1996 zweckgebunden vor allem für Grenzsicherung zur Verfügung stellte (vgl. Currle 2004, S. 362; Forschungsgesellschaft Flucht und Migration 1999).

In der ersten Hälfte der 1990er Jahre konzentrierten sich die polnischen Aktivitäten also auf den Bereich Einreise: Neben den Rückübernahmeabkommen mit Schengen- und Nachbarstaaten wurden Kontrollen an allen Grenzen eingeführt, ein erster institutioneller Rahmen für Asylverfahren geschaffen und der grenzüberschreitende (Handels-) Verkehr erleichtert (vgl. Iglicka 2005a). In der For-

\footnotetext{
${ }^{298} \mathrm{Zu}$ diesem Zeitpunkt gab es bereits ein Rückübernahmeabkommen zwischen Polen und den Schengen-Staaten von 1991.

299 Rückübernahmeabkommen wurden geschlossen mit: Bulgarien (24.08.1993), der Tschechischen Republik (10.05.1993), Kroatien (08.11.1994), Moldawien (21.11.1994), Rumänien (24.07.1993), der Slowakei (08.07.1993), Slowenien (28.08.1996), der Ukraine (24.05.1993), Ungarn (26.11.1994) und Litauen (13.07.1998) (in Kraft seit dem 09.01.2000). Des Weiteren vereinbarte Polen Rückübernahme bei Visafreiheit mit: Lettland (17.12.1992), Estland (26.02.1993). Mit Armenien, Kasachstan und Vietnam werden Verhandlungen über Rückübernahmeabkommen geführt (vgl. Forschungsgesellschaft Flucht und Migration 2005).
} 
schung über die polnische Migrationspolitik wird die Auffassung vertreten, dass in dieser Zeit sicherheitspolitische Erwägungen im Vordergrund standen, die vor allem von der Angst vor einem nie da gewesenen Anstieg von Migrationsbewegungen und Grenzüberschreitungen aus den destabilisierten, ehemaligen Sowjetrepubliken nach und durch Polen geleitet wurden (vgl. Kicinger 2005, S. 4f.). Dieses sicherheitspolitische Denken im Migrationsbereich war auch Teil der Abkehr vom rein militärischen Sicherheitsdenken während der Blockkonfrontation und der Identifizierung bzw. Konstruktion neuer Sicherheitsgefahren, zu denen auch die internationale Migration gezählt wurde (vgl. Kicinger 2005, S. 4).

Obwohl angesichts der Umbrüche Ende der 1980er Jahre und der Auslagerungsstrategien von deutscher Seite durch die ,sichere Drittstaatenregelung' die Einwanderung von MigrantInnen nach Polen zunahm, wurde das Ausländergesetz von 1963 in den Jahren 1991 und 1995 nur geringfügig und erst 1997 - ein Jahr vor Aufnahme der Beitrittsverhandlungen mit der EU - grundlegend novelliert. Im Vergleich zu seinen östlichen Nachbarstaaten ist Polen damit in migrationspolitischer Hinsicht ein Nachzügler (vgl. Currle 2004, S. 361). Die Regelungen des Gesetzes umfassen die Bereiche Einreise, Ausreise, Transit sowie Aufenthalt ausländischer Staatsbürger, das Asylverfahren sowie das Verfahren zur Gewährung des Flüchtlingsstatus und Vorgaben bezüglich Ausweisung und Abschiebung.

Die erste Änderung des Ausländergesetzes von 1963 trat 1992 in Kraft und beinhaltete in erster Linie eine Flüchtlingsdefinition, Regeln zur Gewährung des Flüchtlingsstatus sowie zur Inhaftierung von MigrantInnen, die abgeschoben werden sollen. Der ,Ombudsmann' klagte gegen das Abschiebungsverfahren vor dem Verfassungsgericht. Dieses stellte im Oktober 1992 fest, dass die Durchsetzung einer Abschiebung auf Basis einer Entscheidung der Woiwodschaft ein Verstoß gegen die Verfassung und die darin festgelegte Geltung völkerrechtlicher Verträge sei. Stattdessen könnten Abschiebungen nur durch ein Gericht oder durch die Staatsanwaltschaft angeordnet werden (vgl. Iglicka et al. 2003, S. 4).

Von 1995 bis 1997 wurde in parlamentarischen Kommissionen, durch Experten-Konsultationen und in drei parlamentarischen Lesungen die Änderung des Gesetzes vorbereitet und schließlich durch den Senat angenommen - zur gleichen Zeit wurde die neue Verfassung vorbereitet. Die Gesetzesnovelle trat Anfang 1998 in Kraft. Dabei lag nur ein Gesetzesvorschlag vor - der des Innenministeriums, der auf keinen nennenswerten Widerstand unter den ParlamentarierInnen der verschiedenen Parteien stieß. Er enthielt vor allem Bestimmungen zu Einreise, Aufenthalt und Transit und zur Umsetzung der Schengen-Vorgaben. Insbesondere wurden detaillierte Regelungen zur Ausweisung entwickelt und Instrumente wie die Haftung von Transportunternehmen für die Beförderung von Personen mit unzureichenden Papieren sowie die sichere Dritt- und Herkunftsstaatenregelung eingeführt. Darüber hinaus wurde der so genannte ,Flüchtlingsrat' (Rada do Spraw Uchodźców) als zweite Instanz im Asylverfahren eingeführt. Die Verabschiedung 
fiel in eine Phase, in der zahlreiche Gesetze auf den Weg gebracht wurden. Dies könnte eine Erklärung für den geringen Politisierungsgrad des Themas sein. Da sich die ,Unaufgeregtheit' bei entsprechenden Gesetzesänderungen in den kommenden Jahren fortsetzte, ist diese Erklärung jedoch nicht hinreichend. ${ }^{300}$ Das auf das Gesetz aufbauende Vorgehen von Polizei und Grenzschutz in der zweiten Hälfte des Jahres 1998 hatte hingegen für öffentliches Aufsehen gesorgt: Im Rahmen der ,Aktion Fremde، (Akcja Obcy) wurden zahlreiche Razzien durchgeführt, die vom polnischen Grenzschutz mit Hinweis auf den EU-Beitritt gerechtfertigt wurden:

„Wir setzen die Vorschriften des Ausländergesetzes sorgfältig um. Ziel der Aktion ist es, die Glaubwürdigkeit unseres Landes vor dem Einritt in die EU zu beweisen. Schließlich wird unsere östliche Grenze die Grenze des vereinigten Europa. " Mirostaw Szacitto in der Gazeta Wyborcza vom 29.10.1998, zit. n. Forschungsgesellschaft Flucht und Migration 1999)

Aufgrund der ,Aktion' stieg die Zahl der Abschiebungen 1998 auf über 6.000 Personen an. Das Fernsehen berichtete ausführlich über die ,Akcja Obcy ${ }^{6}$, die einen offenen Protestbrief von 79 polnischen Intellektuellen provozierte (vgl. Forschungsgesellschaft Flucht und Migration 1999).

\section{1 - 2003: Asylrechtliche Modifikationen in Vorbereitung auf den EU- Beitritt}

Mit der Gesetzesänderung von 2001 wurde das URIC (Urząd do spraw repatriacji i cudzoziemców) - die zentrale polnische Ausländerbehörde - geschaffen. Das URIC ist für die Durchführung der Asylverfahren zuständig und gleichzeitig Betreiber der (derzeit 16) Flüchtlingslager in Polen. Zudem wurde ein neues beschleunigtes Verfahren für AntragstellerInnen aus so genannten ,sicheren Drittstaaten' und mit ,offensichtlich unbegründeten' Anträgen installiert. Gleichzeitig wurden das Non-Refoulement-Gebot ${ }^{301}$ und ein temporärer Schutzstatus eingeführt.

Im Jahre 2003 wurde bereits die nächste Änderung verabschiedet: Zum einen fand eine Spezialisierung auf die verschiedenen Kategorien von AusländerInnen statt. Während 1997 noch alle AusländerInnen in einem Gesetzestext angesprochen wurden, wurden 2003 eigens ein Ausländergesetz (vgl. Act of 13 June 2003 on Aliens) und ein Ausländerschutzgesetz (vgl. Act of 13 June 2003 on granting protection to aliens) verabschiedet, die beide seit September 2003 in Kraft sind. Damit wurde die in westeuropäischen Staaten migrationspolitisch bedeutsame Trennung von MigrantInnen und Flüchtlingen auch im polnischen Gesetz deutlich hervorgehoben. Außerdem wurde für Abgelehnte der Duldungsstatus als

\footnotetext{
300 Auf die Analyse der Einflussfaktoren wird im Abschnitt 6.3.5 (vgl. S. 290ff.) näher eingegangen. 301 Vgl. Fußnote 31, S. 50.
} 
neuer Aufenthaltstitel eingeführt. Zum anderen wurden Inhaftierungsbestimmungen aufgenommen, die in ihrer Schärfe die anderer EU-Staaten deutlich übertreffen (siehe 6.3.3a). Kępińska und Stola (2004, S. 175) vergleichen die Inhaftierungsregelungen mit denen in Australien: „This is based on the Australian model and, in reality, is considered as one of the most restrictive systems in Europe." Darüber hinaus gab es Verschärfungen bei den Visa-Regelungen ${ }^{302}$ sowie Veränderungen bei den Bedingungen für eine befristete Aufenthalts- und die Niederlassungserlaubnis ${ }^{303}$ und für eine Niederlassungserlaubnis von ausländischen EhegattInnen polnischer BürgerInnen ${ }^{304}$.

Mit dem Gesetz von 2003 wurde überdies das erste Regularisierungsprogramm (abolicja) durchgeführt - bis dahin gab es keine gesetzlich geregelte Legalisierungsmöglichkeit in Polen. Wer sich zwischen September und Dezember 2003 meldete und die Bedingungen erfüllte, konnte eine befristete Aufenthaltserlaubnis für ein Jahr erhalten. Voraussetzung waren ein Aufenthalt von mindestens sechs Jahren und acht Monaten, eine Wohnung, ausreichende finanzielle Mittel zur Sicherung des Auskommens oder eine Anstellungsgarantie bei einem Arbeitgeber. Es wurden 3.218 Anträge eingereicht, die meisten von ArmenierInnen (45 Prozent) und VietnamesInnen (40,3 Prozent) (vgl. Kępińska 2003, S. 4). Der überwiegende Teil der AntragstellerInnen wohnte in der Woiwodschaft Mazowsze (Masowien, 43,9 Prozent), zu der auch Warschau gehört, gefolgt von Małopolska (Kleinpolen, 13,5 Prozent) (ebd.). Wer die Bedingungen nicht erfüllte, war aufgerufen, sich zwischen September und Oktober 2003 bei der Polizei oder dem Grenzschutz zu melden und sollte ohne weitere Konsequenzen das Land verlassen können. Auf dieser Basis verließen 282 AusländerInnen das Land, die meisten von ihnen UkrainerInnen (vgl. Kępińska 2003, S. 4f.).

Die Gesetzesänderungen in dieser Phase standen im Zeichen der Beitrittsverhandlungen, die für den Bereich ,Justiz und Inneres' im Jahr 2000 begonnen hatten. Die EU wird allgemein als Haupteinflussfaktor in diesem Politikbereich betrachtet. Es wird davon ausgegangen, dass die Anpassung der polnischen Gesetzgebung an den Acquis „practically eliminated any serious controversy“ (Iglicka et al. 2003, S. 6). ${ }^{305}$ Einzig die von der EU geforderte Einführung von Visa für die

\footnotetext{
302 Die Dauer des Aufenthalts auf Basis eines Visums wurde von sechs auf maximal drei Monate innerhalb eines halben Jahres gesenkt. Wer länger als drei Monate bleiben will, muss eine befristete Aufenthaltserlaubnis beantragen. Es gibt (mit einigen Ausnahmen) keine Möglichkeit mehr, Erstoder Folgevisa innerhalb Polens zu beantragen.

303 Während das Gesetz zuvor den Behörden einen großen Ermessensspielraum zuwies, wurden die Bedingungen mit der Änderung klarer definiert.

304 AusländerInnen, die PolInnen heiraten, qualifizieren sich nach zwei Jahren Aufenthalt in Polen für eine Niederlassungserlaubnis - der Zeitraum betrug früher fünf Jahre. Während dieser zwei Jahre mit befristeter Aufenthaltserlaubnis ist keine Arbeitserlaubnis vorgesehen. Es besteht also finanzielle Abhängigkeit von dem polnischen Ehepartner bzw. der polnischen Ehepartnerin.

305 Zur Rolle der EU vgl. die Abschnitte 6.3.1 AkteurInnen, S. 252 und 6.3.5 Resümee der Einflussfaktoren, S. 290.
} 
BürgerInnen der östlichen Nachbarstaaten Polens sorgte seit 1998 immer wieder für erwähnenswerte Auseinandersetzungen und Ansätze von Widerstand:

„Until then the argument that a given action is necessary from the point of view of adjusting Polish law to EU acquis was of indisputable strength and ended every discussion."

(Kicinger 2005, S. 18)

Die Visapflicht wurde von vielen als Gefährdung der nachbarschaftlichen Beziehungen insbesondere zur Ukraine sowie des grenzüberschreitenden Kleinhandels angesehen, der für die Existenzsicherung (nicht nur) der BewohnerInnen der strukturschwachen Grenzregionen einen zentralen Stellenwert hat (vgl. Alscher 2004, S. 220; Kępińska/Stola 2004, S. 171). Es wurde auf die engen historischen, kulturellen und ökonomischen Verbindungen zu den Nachbarn ebenso verwiesen wie auf die dort lebenden polnischen Communities. Um ein Visum zu erhalten, mussten von nun an ausreichende finanzielle Mittel oder eine notariell bestätigte Einladung von einer polnischen Institution bzw. Person nachgewiesen werden. Als am 01.10.2003 das neue Visa-Regime implementiert wurde und WeißrussInnen, UkrainerInnen und RussInnen nur noch mit Visum nach Polen einreisen durften, waren die polnischen Konsulate im Ausland zunächst mit der Bearbeitung der zahlreichen Visa-Anträge überfordert (vgl. Kępińska 2003, S. 5). Die Grenzübertritte an Polens Ostgrenze gingen mit der Einführung der Visapflicht signifikant zurück (ebd., S. 5ff.).

\section{4 - 2007: Nach dem Beitritt: Fortschreitende Restriktionen}

Der Beitritt Polens zur EU im Jahr 2004 hatte zum einen wichtige grenzpolitische Folgen. Die polnische Ostgrenze wurde neue Außengrenze der EU. Trotz jahrelanger Bemühungen, diese Grenze im Interesse der alten EU-Mitglieder aufzurüsten, wurden die Grenzkontrollen an der deutsch-polnischen Grenze zunächst noch aufrecht erhalten. Mit Polens Beitritt zum Schengen-Raum im Dezember 2007 entfielen diese und es fand erneut eine Verschiebung der Bedeutung der polnischen Grenzen statt: Eine Grenze, die während des ,Kalten Krieges` für PoIInnen unüberwindbar war, wird nun beinahe bedeutungslos ${ }^{306}$. Gleichzeitig wird die einst geschlossene, in der Transformationsphase aber offene und viel frequentierte Ostgrenze nun zu einer großen Hürde für diejenigen, die die EU-Kriterien für die Einreise nicht erfüllen.

Zum anderen passte Polen in den Jahren 2005 und 2006 seine Gesetzgebung durch weitere Novellierungen an die EU-Normen an, die nach seinem Beitritt verabschiedet wurden. Das Sozialhilfegesetz, das im März 2004 in Kraft trat, führte ein Integrationsprogramm für anerkannte Flüchtlinge ein. Auf dieser Grundlage

\footnotetext{
306 Wie im ersten Teil des Kapitels unter 6.1 erläutert, wird der Zugang polnischer StaatsbürgerInnen zu den Arbeitsmärkten der meisten alten Mitgliedstaaten durch Übergangsregelungen nach wie vor eingeschränkt.
} 
können anerkannte Flüchtlinge ein Jahr lang besondere finanzielle Unterstützungsleistungen, Hilfen bei der Wohnungssuche und Sprachkurse in Anspruch nehmen (vgl. Ministry of Labour and Social Policy 2005, S. 8ff.). Die zahlreichen Geduldeten sind von diesen Maßnahmen (bislang) ausgeschlossen. Für sie hat sich die Situation durch eine Gesetzesänderung vom Herbst 2006 sogar noch verschlechtert: Normalerweise muss, wer eine Duldung erhält, das Flüchtlingsheim verlassen. Aufgrund der schwierigen Situation auf dem polnischen Wohnungsund Arbeitsmarkt und der geringen Unterstützungsleistungen haben viele Geduldete keine Chance, eine eigene Wohnung zu mieten. Sie stellen deshalb vielfach Asylfolgeanträge, um weiterhin in den Heimen bleiben zu können. Dieser Strategie sollte mit der Gesetzesänderung ein Riegel vorgeschoben werden: Danach rechtfertigt ein Folgeantrag, der von einer Person mit Duldungsstatus eingereicht wird, keinen weiteren Aufenthalt in einem Flüchtlingsheim (vgl. Kępińska 2006, S. 3). Die Betroffenen versuchen nun, diese neue Regelung mit einem juristischen Trick zu umgehen: Sie weisen den Duldungsstatus zurück und stellen erneut einen Asylantrag (vgl. D S. 14). ${ }^{307}$ Diese Novelle kann allerdings nicht ohne Weiteres mit der Umsetzung von EU-Richtlinien erklärt werden.

Die Entwicklung der polnischen Gesetzgebung im Flüchtlingsbereich ist damit selbstverständlich nicht abgeschlossen. Auch im Jahr 2007 wurde ein Entwurf für ein verändertes Flüchtlingsschutzgesetz vorgelegt, die Verabschiedung verzögerte sich jedoch durch die vorgezogenen Neuwahlen im Oktober 2007 (vgl. Sunjic 2007).

\section{Zwischenfazit}

Der kurze Streifzug durch die flüchtlingspolitisch zentralen Stationen in den letzten zwei Dekaden in Polen macht deutlich, dass hier binnen weniger Jahre und zeitgleich mit grundlegenden Umstrukturierungen des polnischen Staates, seiner Institutionen und gesetzlichen Grundlagen erste flüchtlingspolitische Maßnahmen ergriffen und ein entsprechendes Fundament an Regelungen, Verfahren, Behörden und Einrichtungen gewissermaßen aus dem ,Nichts“ geschaffen wurde. Der Überblick über die Entwicklung der Regulierungen zeigt aber auch, dass je ausdifferenzierter und detailreicher die Regulierungen in diesem Bereich insbesondere ab 1997 wurden, umso unzugänglicher wurde Polen für Flüchtlinge, aber auch für andere MigrantInnen. Das Nachzeichnen der Regelungen offenbart eine sukzessive Restriktion der Zugangsbestimmungen. Eine Interviewpartnerin zitierte den Gründer des Helsinki-Komitees und ersten Präsidenten der Helsinki-Stiftung für Menschenrechte, Marek Nowicki, der dies auf einer Konferenz auf den Punkt gebracht habe, indem er sagte, die frühen 1990er Jahre seien eine „romantische Zeit" gewesen. Es habe gewissermaßen keine Regulierungen in diesem Bereich

\footnotetext{
${ }^{307} \mathrm{Zu}$ den Folgen dieser Regelungen vgl. auch die Ausführungen zum Duldungsstatus S. 282.
} 
gegeben, Polen sei jedermann zugänglich gewesen und es habe den Willen gegeben, jeden aufzunehmen (G S. 2).

Diese Entwicklung hin zu einem restriktiven Flüchtlingsschutzregime und die vor allem in der Praxis bestehenden Unzulänglichkeiten dieses Regimes wurden von den betroffenen MigrantInnen nicht unwidersprochen hingenommen. Viele versuch $(\mathrm{t})$ en, trotz gegenläufiger gesetzlicher Regeln dennoch weiter nach Westen zu wandern, andere haben direkt in Polen gegen ihre Situation protestiert. So hat es in den Heimen immer wieder spontane Proteste gegen die schlechten Lebensbedingungen und Forderungen nach ungehinderter Weiterreise gegeben - zum Beispiel im Januar 2005 in den Flüchtlingsheimen Linin, Moszna und Wołomin (vgl. Eßer et al. 2005, S. 13). Im Erstaufnahmezentrum Dębak haben sich im Dezember 2004 rund 200 Flüchtlinge zwei Wochen lang geweigert, dass Kantinenessen zu sich zu nehmen. In einer Petition kritisierten die Hungerstreikenden: "There is no hot water, food is not good, we have no warm clothes, there is no education for our children. Why does Poland accept us if it cannot help us?". Sie forderten: „We demand, that Poland opens safe passage to Western Europe“ (zit. n. Kosowicz 2004).

\section{Dublin II - Polen als Teil der ,Pufferzone' innerhalb der EU}

Nach wie vor ist Polen also in den Augen der meisten Flüchtlinge Transitstation sie müssen einen Antrag auf Flüchtlingsschutz stellen, um legal nach Polen - und damit in die EU - einzureisen oder stellen einen Antrag, wenn ihr undokumentierter Aufenthalt entdeckt wird. Diejenigen, die nicht von Anfang an geplant haben weiterzureisen, fassen diesen Entschluss häufig nach einiger Zeit des Aufenthalts in Polen, spätestens aber, wenn ihr Verfahren mit der Erteilung eines Duldungsstatus abgeschlossen wird und sie sich damit in einer äußerst prekären sozialen Situation wiederfinden. Die Weiterreise vieler Flüchtlinge zeigt sich in der Flüchtlingsstatistik: Viele Anträge werden nicht zu Ende verfolgt - die Flüchtlinge verschwinden, bevor eine Entscheidung gefällt wurde (vgl. Tabelle 10 im Anhang). Seit Polen Mitglied der EU ist, greift an dieser Stelle die Dublin-II-Verordnung (vgl. Asylpolitik der EU, S. 83ff.). Eine erneute Antragstellung in einem anderen EU-Mitgliedstaat ist nun nicht mehr möglich. Daher bestehen praktisch keine Chancen mehr auf einen legalen Aufenthalt in einem anderen EU-Mitgliedstaat, es sei denn, jemand kann eine Familienzusammenführung mit engsten Verwandten durchsetzen. Doch die Entscheidung darüber, ob aus Polen ein Überstellungsantrag an ein anderes EU-Mitglied gestellt wird, liegt beim UdSC. Viele der Flüchtlinge, die ich in polnischen Flüchtlingsheimen angetroffen habe, waren bereits mehrmals in einem weiter westlich gelegenen EU-Mitgliedstaat und sind wieder zurückgeschoben worden.

Die Ziele und Verfahren der Dublin-II-Verordnung wurden bereits im Kapitel zum europäischen Asylregime erläutert. Hier wird im Folgenden kurz die damit verbundene Praxis geschildert. Wer in Polen einen Antrag auf Flüchtlingsschutz 
stellt, ist verpflichtet, sich seine Fingerabdrücke abnehmen zu lassen. Diese werden in das Datenspeicherungssystem Eurodac aufgenommen. Bei erneuter Antragstellung in einem anderen EU-Land werden die Daten in Eurodac abgeglichen. Wenn sich zum Beispiel in Deutschland herausstellt, dass ein Asylbewerber bereits von polnischen Behörden registriert wurde, wird ein Übernahmegesuch an Polen gestellt. Auch ohne Eurodac-,Treffer ${ }^{6}$ können - wie im EU-Kapitel beschrieben - andere Indizien einen Übernahmeantrag rechtfertigen. Umgekehrt kann Polen auf Antrag der Flüchtlinge ein Übernahmegesuch an einen anderen Mitgliedstaat stellen - zum Beispiel um eine Familienzusammenführung zwischen einem Flüchtling in Polen mit seinen Angehörigen in Deutschland zu ermöglichen. Die Regelung hat in der Praxis zum Aufbau eines enormen bürokratischen Apparates geführt, der damit beschäftigt ist, die Hin- und Herschiebung von MigrantInnen zu verwalten und durchzuführen. Daten müssen erhoben und abgeglichen, Gesuche auf Übernahme müssen gestellt, angenommen und bearbeitet werden, Rückfragen werden hin und her gesendet. Manche dieser Verfahren ziehen sich über Monate hin. Um die anfallenden Aufgaben zu bewältigen, hat das URiC/UdSC nach dem EU-Beitritt Polens eine Abteilung für Dublin-Fälle eingerichtet und mehrere neue MitarbeiterInnen eingestellt.

Die MigrantInnen, die aufgrund der Dublin-II-Verordnung von Deutschland nach Polen zurückgeschoben werden, werden zunehmend inhaftiert - zuerst in Deutschland (zum Beispiel in Eisenhüttenstadt) und häufig nach der Rückschiebung auch in Polen. Wenn die Flüchtlinge nach Polen zurückkommen, läuft - je nach Dauer ihrer Abwesenheit - ihr erstes Verfahren noch oder sie müssen ein neues anstrengen. Die Weiterwanderung wird dann mitunter gegen sie verwendet (D S. 15; N S. 13). Laut Auskunft mehrerer InterviewpartnerInnen gehen die Behörden davon aus, dass diejenigen, die weiterwandern, ausschließlich ökonomische Migrationsmotive haben. Dadurch verringern sich nach der Rückschiebung ihre Chancen auf Anerkennung. Viele versuchen erneut Polen zu verlassen und lösen die Prozedur von neuem aus. Diesen Widerstand der MigrantInnen gegen die Verordnung illustriert der Interviewpartner der Grenzschutzbehörde folgendermaßen:
„Zum Beispiel ein Tschetschene, ein Tschetschene ist kein gutes Beispiel, denn es gibt Verfolgung, aber zum Beispiel ein Armenier kommt nach Polen, beantragt Asyl, über- quert dann illegal die polnisch-deutsche Grenze und beantragt in Deutschland Asyl. Seine Fingerabdrücke wurden natürlich genommen und er wird nach Polen zurïck- geschickt. Wenn er in Polen ist, versucht er dasselbe in einem Monat. Und er reist auf Kosten der EU und das heißt auf meine Kosten und Ibre Kosten. Er reist von Deutschland nach Polen, dann von den Niederlanden nach Polen, dann von Belgien nach Polen, drei Jahre, aber ist immer noch hier. "(B S. 14)

Zwar werden bei dem Zitat die TschetschenInnen ausgeklammert, in der Realität sind sie jedoch wie alle anderen von der Dublin-II-Verordnung betroffen. Flücht- 
linge, die die Verordnung umgehen wollen, müssen sich die notwendigen (gefälschten) Papiere besorgen und direkt in ihr Zielland fliegen:

„Die beste Lösung für sie ist ein Flugticket zu kaufen und direkt in eines der westeuropäischen Länder zu fliegen und dann Asyl zu beantragen. Nachdem sie zum Beispiel eineinhalb Jahre illegal dort geblieben sind, dann die Dokumente zu zerstören und Asyl zu beantragen. " (B S. 13)

Dieser ,Tipp' des Grenzschutzbeamten veranschaulicht die hohe Selektivität, die mit der Dublin-II-Verordnung verbunden ist: Netzwerke, finanzielle Ressourcen und Unabhängigkeit (denn eine Familie mit kleinen Kindern könnte nur unter großen Schwierigkeiten ein Leben in der Illegalität organisieren) sind notwendig, um die Verordnung zu umgehen. Mitunter setzt sich die Abschiebung aufgrund der sicheren Drittstaatenregelung aus Polen weiter nach Osten, zum Beispiel in die Ukraine, fort.

Um den beschriebenen bürokratischen Dublin-II-Apparat gruppieren sich darüber hinaus zahlreiche Organisationen und Initiativen, die ebenfalls mit hohem finanziellen und (vor allem ehrenamtlichen) Arbeitsaufwand versuchen, die Härten des Dublin-II-Regimes abzufedern. So vernetzen sich Initiativen und Beratungsstellen in Polen und Deutschland, um die betroffenen Flüchtlinge besser betreuen zu können. NGO-MitarbeiterInnen und Mitglieder von Initiativen aus Deutschland unternehmen Recherchereisen, um die Situation in Polen zu dokumentieren, AnwältInnen mit Informationen zu versorgen und zum Beispiel kranke behandlungsbedürftige Flüchtlinge vor der Rückschiebung nach Polen zu bewahren. Es werden Tagungen, Veranstaltungen und Pressekonferenzen durchgeführt, Broschüren herausgegeben usw., um über die Einzelfallhilfe hinaus Öffentlichkeit für die Auswirkungen der Verordnung zu schaffen. Auf Seiten polnischer NGOMitarbeiterInnen besteht eine hohe Frustration, wenn die Flüchtlinge, die von ihnen begleitet wurden, ,verschwinden' (L S. 5; I S. 11).

Aus der Perspektive der polnischen Behörden ist das Dublin-Verfahren Ausdruck der ungleichen Verteilung der Verantwortung für den Flüchtlingsschutz in der EU. Durch seine Lage an der EU-Außengrenze wird Polen zwangsweise das Aufnahmeland für alle auf dem Landweg Ankommenden. Mit der dadurch ansteigenden Zahl an AsylbewerberInnen wird es weitgehend allein gelassen. Die finanzielle Unterstützung der EU richtet sich vor allem auf Grenzsicherungs- und Infrastrukturmaßnahmen, für die soziale Versorgung der MigrantInnen ist Polen selbst verantwortlich. „In keinem anderen neuen EU-Mitgliedsstaat (abgesehen von der Slowakei), sind die Negativ-Folgen der Verordnung Dublin II jetzt bereits so deutlich greifbar wie in Polen“ (Eßer et al. 2005, S. 30). Nach Ansicht des Leiters der Zentralen Ausländerbehörde, Jan Węgrzyn, sollte in der EU ein „Lastenausgleich" geschaffen werden, und zwar insbesondere ein Aufnahmeprogramm für tschetschenische Flüchtlinge, in dessen Rahmen diese gerade auch nach dem Abschluss des Verfahrens - ob mit Flüchtlingsstatus oder mit Duldung - sozial 
unterstützt werden (vgl. ebd.). Der Interviewpartner vom polnischen Grenzschutz schlägt vor dem Hintergrund seiner oben dargestellten Äußerungen vor, dass das Asylverfahren in dem Land stattfinden soll, in dem der Flüchtling letztlich landet. Wird es negativ beschieden, soll die Person auf Kosten des Erstaufnahmestaates (zum Beispiel Polen) abgeschoben werden. Dadurch könne das teure Hin- und Herschieben vermieden werden (B S. 14).

Das Beispiel Polen zeigt, dass die Dublin-II-Verordnung - wenn man sie an ihren proklamierten Zielen misst - gescheitert ist. Vor allem läuft der zugrundeliegende Zuständigkeitsmechanismus dem proklamierten Ziel des ,Lastenausgleichs“ zwischen den Mitgliedstaaten zuwider. Um zu dieser Erkenntnis zu gelangen, ist kein Blick auf die Praxis erforderlich: Wenn diejenigen Länder für die Asylverfahren verantwortlich gemacht werden, die die Einreise der jeweiligen AsylbewerberInnen nicht verhindert haben, so führt das zu einer stärkeren Inanspruchnahme der Mitgliedstaaten an den Außengrenzen und nicht zu einem burden sharing zwischen allen Mitgliedstaaten. Doch die Praxis zeigt, dass es nicht zu umfassenden Rücküberstellungen kommt. Laut Statistik ist die Zahl der tatsächlichen Rücküberstellungen im Verhältnis zu den Überstellungsanträgen gering, da viele Flüchtlinge untertauchen (vgl. Dublin-II- und Eurodac-Verordnung, S. 104). Es stellt sich also die Frage, ob das Anliegen des burden sharing sich hier nicht auf andere Weise erledigt. Denn das zweite offizielle Ziel, asylum shopping zu erschweren, das nicht nur rhetorisch das Anliegen von MigrantInnen diffamiert, ihr Zielland selbst zu bestimmen, wird durch die Verordnung in der Tat erreicht: Eine eigene Entscheidung über das Zielland können die MigrantInnen fast nur noch um den Preis der Illegalisierung durchsetzen. Die Verordnung bringt in der Praxis ein strapaziöses ,Flüchtlingskarussell' hervor, das hohe Kosten verursacht - sowohl für die Flüchtlinge mit Blick auf ihre materiellen aber auch physischen und psychischen Ressourcen, als auch für die Staaten, die den damit verbundenen bürokratischen und polizeilichen Apparat finanzieren. Die offiziell proklamierten Ziele - burden sharing und die Bestimmung des Ziellands ,von oben' - werden so kaum erreicht. Das kostspielige Karussell, auf das die Flüchtlinge immer wieder aufspringen und von dem sie immer wieder - an unterschiedlichen Stellen in Europa - abgeworfen werden, mündet stattdessen einerseits in der Auslagerung des Flüchtlingsschutzes aus den Ländern im geografischen Kern der EU hin zu jenen an den Außengrenzen. Davon sind vor allem Flüchtlinge mit geringen Ressourcen und schwachen Netzwerken betroffen. Andererseits führt es analog zur Illegalisierung derer, die ausgestattet mit den notwendigen Ressourcen und/oder unter großen Risiken dennoch weiterwandern. Die Folge ist, dass MigrantInnen immer seltener als Asylsuchende und immer häufiger als Undokumentierte in Deutschland leben. Immer weniger können im Rahmen eines Asylverfahrens in Deutschland (eingeschränkte) soziale Rechte in Anspruch nehmen, immer mehr sind nun faktisch bar jeder Rechte. Darin liegt eine gewisse Funktionalität der Verordnung, die erklären würde, warum ihre Anwendung nicht infrage steht - trotz des hohen administrati- 
ven Aufwands und der Tatsache, dass MigrantInnen sich in großer Zahl über sie hinwegsetzen. Das komplexe Verhältnis proklamierter und tatsächlicher Ziele einerseits und der tatsächlichen Folgen andererseits verweist auch auf die Vielfalt der Interessen, die hier zusammenspielen.

\subsubsection{Flüchtlingsschutz zwischen Recht und Praxis}

Das polnische Flüchtlingsschutzgesetz vom 13. Juni 2003 sieht vier Status vor: den Flüchtlingsstatus, den Asylstatus, den Duldungsstatus (pobyt tolerowany) und den temporären Schutz. Im Folgenden werden die Regelungen des Flüchtlingsschutzgesetzes dargestellt und dabei insbesondere Abweichungen zwischen Recht und Praxis herausgearbeitet. Dabei wird ähnlich vorgegangen, wie bei der Darstellung der Situation in der Bundesrepublik Deutschland. Aufgrund der unterschiedlichen Bedeutung der Status in beiden Ländern steht hier jedoch das Verfahren zur Gewährung von Flüchtlingsschutz im Vordergrund. Das in der Verfassung enthaltene Asyl spielt in Polen nur eine untergeordnete Rolle und wird am Ende des folgenden Abschnitts kurz vorgestellt. Zudem enthalten die Ausführungen hier keinen eigenständigen Abschnitt zum Thema Abschiebung, da für Polen zu diesem Themenbereich nur wenige Informationen vorliegen. Diese wurden in den Abschnitt 6.3.3 unter Inhaftierung und Residenzpflicht (vgl. S. 284) integriert.

\section{Verfahren und Ausgestaltung des Flüchtlingsschutzes}

Die Verleihung des Flüchtlingsstatus wird an die Kriterien der Genfer Flüchtlingskonvention und des New York Protokolls geknüpft: Wer diese erfüllt, soll laut polnischem Flüchtlingsschutzgesetz den Status eines Flüchtlings erhalten (Art. 13). Anlass für die Ablehnung eines Antrages ist dessen ,offensichtliche Unbegründetheit‘ („oczywisty bezzasadny wniosek“) (Art. 14) oder das Bestehen einer inländischen Fluchtalternative (Art. 15). Ein Antrag ist ,offensichtlich unbegründet', wenn er keine Gründe enthält, die die Annahme einer wohlbegründeten Angst vor Verfolgung gemäß Art. 1 A der GFK rechtfertigen (Art. 14 Abs. 1 S. 1), wenn er darauf abzielt, die Behörden zu täuschen oder das Verfahren zu missbrauchen (Art. 14 Abs. 1 S. 2) oder wenn sich herausstellt, dass der/die AntragstellerIn aus einem sicheren Dritt- oder Herkunftsstaat eingereist ist (Art. 14 Abs. S. 3). Keine Gründe liegen insbesondere vor, wenn andere Gründe präsentiert werden als die Angst vor Verfolgung aufgrund von ,Rasse', Religion, Nationalität, Mitgliedschaft in einer bestimmten sozialen Gruppe oder aufgrund von politischen Überzeugungen (Art. 14 Abs. 2 S. 1). Des Weiteren sind die Gründe nichtig, wenn keine Angabe über die Umstände bezüglich der Angst vor Verfolgung gemacht werden (Art. 14 Abs. 2 S. 2) oder wenn die Angaben offensichtlich unglaubwürdig oder unwahr sind sowie inkohärent oder sich offensichtlich widersprechen (Art. 14 Abs. 2 S. 3). 
Von ,Täuschung' oder ,Missbrauch ' wird gesprochen, wenn die AntragstellerInnen die Behörden nicht über bereits früher gestellte Anträge informieren, falsche Angaben machen, Beweise vernichten oder den Antrag nur stellen, um einer Abschiebung zu entgehen, oder wenn sie ihren Pflichten - wie die Verpflichtung eine Aussage zu machen und Beweise zur Verfügung zu stellen - im Verfahren nicht nachkommen (Art. 14 Abs. 3).

Damit enthält das polnische Flüchtlingsschutzgesetz die wesentlichen Instrumente westlicher Asylregulierungen und der EU-Richtlinien: ,offensichtliche Unbegründetheit ${ }^{\star}$ des Antrages aufgrund der Einreise aus bzw. über einen, sicheren Dritt- oder Herkunftsstaat' sowie wegen ,Täuschung' und ,Missbrauch'.

Mit den Passagen zu Missbrauch und Täuschung sowie zur Glaubwürdigkeit der Aussagen wurde für die polnischen Behörden ein weiter Spielraum eröffnet, um Anträge negativ zu bescheiden. So bleiben die Kriterien für die Beurteilung der Glaubwürdigkeit im Dunkeln. Ebenso wie in der deutschen Gesetzgebung werden Erkenntnisse der Traumaforschung und -therapie durch diesen Passus ignoriert. So wird es als unglaubwürdig eingestuft, wenn erlittene Traumata nicht bei der ersten Anhörung, sondern erst später vorgetragen werden (gesteigertes Vorbringen'), obwohl dies auch ein Hinweis auf eine posttraumatische Belastungsstörung sein könnte. Vielen Flüchtlingen im polnischen Asylverfahren wird offenbar der Vorwurf gemacht, dass sich ihre erste Aussage, die meist direkt an der Grenze protokolliert wird, von der Aussage in der zweiten Anhörung unterscheide (N S. 10). Die erste werde in der Regel als die entscheidende gewertet (L S. 8). Gleichzeitig gibt es Berichte, dass GrenzbeamtInnen manipulativ Fragen stellen, sodass das ,Zauberwort' Asyl gar nicht genannt wird und die MigrantInnen sofort abgewiesen werden können. Wer zum Beispiel die Frage, ob er eine bestimmte Verletzung in Polen behandeln lassen wolle, mit ,ja ${ }^{a}$ beantwortet, kann mit der Begründung, dafür sei nur eine Einreise mit Visum möglich, abgelehnt werden (vgl. Flüchtlingsrat Schleswig-Holstein 2004, S. 18). Zudem werde häufig denjenigen, die nach Antragstellung in Polen weiter nach Westen gewandert sind und wieder zurückgeschoben wurden, die Glaubwürdigkeit abgesprochen - insbesondere erhärte sich dann der Verdacht, es handele sich um ,WirtschaftsmigrantInnen' (D S. 15).

$\mathrm{Zu}$ der Erwartung, dass erste und zweite Aussage identisch sind, kommen meist implizite Anforderungen an die Vortragsweise der AntragstellerInnen: Sie sollen das Erlebte kurz, detailliert und präzise darstellen, was zwar dem Umgang in hiesigen Behörden, aber nicht notwendig der kultur-, geschlechts- und auch schichtspezifisch geprägten Erzählweise der jeweiligen angehörten Person entspricht. Diese ethnozentrische Perspektive zeigt sich auch, wenn zum Beispiel nicht berücksichtigt wird, dass MigrantInnen und AnhörerInnen zentrale Begriffe unterschiedlich definieren - bspw. hinsichtlich dessen, was unter Verfolgung oder Folter verstanden wird (N S. 10). 
Auch die ,inländische Fluchtalternative' ist ein aus den Asylregulierungen der westlichen Nachbarn Polens bzw. aus der EU-Anerkennungsrichtlinie übernommenes Instrument. Eine solche liegt laut Gesetz vor, wenn sich die Verfolgung auf bestimmte Teile des Herkunftslandes beschränkt (Art. 15 Abs. 1 Satz 1). Dies muss aber nicht zwingend zur Ablehnung führen (Art. 15 Abs. 2). Anders als in der Bundesrepublik schiebt Polen bislang keine TschetschenInnen mit Verweis auf eine inländische Fluchtalternative nach Russland ab.

Die Ablehnung eines Antrages geht entweder mit der Erteilung des Duldungsstatus oder mit einer Ausreiseaufforderung (Verlassen des Landes innerhalb von maximal 30 Tagen) einher (Art. 16 Abs. 1). Wenn der/die AntragstellerIn gegen die Entscheidung Berufung einlegt, wird von der Berufungsinstanz eine neue Frist festgelegt, die 14 Tage nicht überschreitet (Art. 16 Abs. 2). Das bedeutet, dass der Antrag auf Flüchtlingsstatus mit einem Flüchtlingsstatus, dessen Ablehnung oder einer Duldung beschieden werden kann - es handelt sich also um ein Verfahren, an dessen Ende zwei unterschiedliche Status stehen können.

Die AntragstellerInnen sind zur Angabe verschiedener Daten zu ihrer Identität sowie zur Abgabe von Fingerabdrücken verpflichtet und müssen sich fotografieren und durchsuchen lassen (Art. 24 Flüchtlingsschutzgesetz). Wenn die Mitwirkung dabei verweigert wird, bleibt der Antrag unberücksichtigt (Art. 25). Diese Daten werden durch das Eurodac-System zwischen den Mitgliedstaaten ausgetauscht und dienen unter anderem der Anwendung der Zuständigkeitsregelungen innerhalb der EU. Außerdem sieht das polnische Gesetz vor, dass Jugendliche medizinisch untersucht werden können, wenn Zweifel an ihrem Alter bestehen. Wie in Deutschland werde das Alter von Jugendlichen mitunter angezweifelt (D S. 18), weil für Minderjährige aufgrund der EU-Vorgaben günstigere Regeln bestehen als für Erwachsene.

Für die Bearbeitung der Anträge auf Flüchtlingsschutz ist die zentrale Ausländerbehörde UdSC zuständig. MigrantInnen ohne gültige Einreisepapiere stellen ihren Antrag auf Flüchtlingsschutz direkt an der Grenze nach Polen, diejenigen mit Einreisepapieren richten ihren Antrag direkt an das UdSC in Warschau. Sie werden dann im Erstaufnahmelager Dębak, in der Nähe von Warschau, untergebracht und von dort aus in eine der anderen Asylbewerberunterkünfte umverteilt. MigrantInnen, die vom UdSC einen negativen Bescheid erhalten haben, können beim ,Flüchtlingsrat ${ }^{`}$ Berufung einlegen. Er besteht aus zwölf Mitgliedern, die vom Premierminister ernannt werden: vier auf Vorschlag des Innenministeriums, vier auf Vorschlag des Justizministeriums und vier Kandidaten des Premierministers. Als Verwaltungsorgan und aufgrund seiner Zusammensetzung ist der Flüchtlingsrat keine unabhängige Instanz. Zudem kann er zwar eine vollständige Überprüfung der UdSC-Entscheidung vornehmen und dafür eigene Recherchen anstellen, zum Beispiel durch Anhörungen. Laut Aussage eines interviewten Mitglieds des Flüchtlingsrats fehlen für eine vollständige Überprüfung der Fälle allerdings die Kapazitäten. Wenn der Flüchtlingsrat eine Entscheidung beanstande, erteile er 
nur in seltenen Fällen selbst einen Status, sondern gebe die Entscheidung an das UdSC zurück (N S. 2).

Eine Berufung gegen die Entscheidung des Flüchtlingsrats muss beim Verwaltungsgericht eingereicht werden, letzte Instanz ist das Oberverwaltungsgericht. Das Verwaltungsgericht hat in der Vergangenheit eine Vielzahl von Entscheidungen des Flüchtlingsrates beanstandet und an diesen zurückgewiesen. Die Berufung hat allerdings keine aufschiebende Wirkung. Dies ist nach Aussage von Interviewpartnerinnen insbesondere deshalb problematisch, weil Abgelehnte meist binnen 14 Tagen das Land verlassen sollen - das Verwaltungsgericht jedoch 30 Tage Zeit hat, eine Entscheidung zu fällen. Dies sei zwar im Einklang mit den EURichtlinien, verstoße aber gegen Art. 45 der polnischen Verfassung, der das Recht auf ein faires Verfahren enthält, sowie gegen entsprechende menschenrechtliche Maßgaben (D S. 6; G S. 8). Darüber hinaus agiere das Verwaltungsgericht mitunter zu legalistisch: Es verlange für alles Dokumente - Folter könne aber nicht durch Dokumente belegt werden (N S. 8f.).

Hinsichtlich des gesamten Verfahrens ist zudem von großer Bedeutung, dass es in Polen für Mittellose keine Möglichkeit gibt, einen kostenlosen Rechtsbeistand in Anspruch zu nehmen. Dieser wird ausschließlich durch karitative bzw. Nichtregierungs-Organisationen bereit gestellt sowie durch die Legal Clinics an den Universitäten, in deren Rahmen Bedürftige durch JurastudentInnen vertreten werden.

Kapitel 3 des Flüchtlingsschutzgesetzes befasst sich mit Regeln zum Umgang mit unbegleiteten Minderjährigen. Für sie sollen ein Vormund sowie ein Pfleger bestellt werden. Der Pfleger soll sich um Unterbringung und um Zugang zu Bildung sowie zum Gesundheitssystem des Minderjährigen kümmern (Art. 48). Während des Verfahrens, bei Anhörungen und Erklärungen soll Rücksicht auf die besondere Situation von Minderjährigen genommen werden (Art. 49), AnhörerInnen und EntscheiderInnen müssen bestimmte Qualifikationsanforderungen erfüllen. Hier klaffen Gesetz und Umsetzung offenbar weit auseinander: Zwar werden in dem Gesetz umfangreiche Rechte für minderjährige Asylsuchende festgeschrieben, faktisch werden jedoch kaum jugendliche MigrantInnen als Minderjährige anerkannt, wie bereits in den Ausführungen zu Artikel 21 angesprochen. Eine Mitarbeiterin der Legal Clinic schilderte im Interview, dass „Minderjährige, von denen wir denken, dass es Minderjährige sind [...] von unserer Regierung nicht als Minderjährige anerkannt werden.“ (D S. 18) „Die Psychologen sehen den Minderjährigen einmal während des ganzen Verfahrens und wissen nichts über den Minderjährigen." (D S. 18) Auch eine interviewte Juristin, die sich unter anderem auf Flüchtlingsrecht spezialisiert hat, sieht eine erhebliche Diskrepanz zwischen Recht und Praxis. So sollten Minderjährige zwar einen Vertreter und einen Pfleger zur Seite gestellt bekommen, tatsächlich haben sie zu diesen jedoch so gut wie keinen Kontakt. Zum Zeitpunkt des Interviews sei eine Person für 20 Minderjährige zuständig gewesen (insgesamt gebe es nur zehn SozialarbeiterInnen), weshalb die 
Minderjährigen nur einmal im Monat einen solchen Kontakt hätten (G S. 7). Obwohl sich die Behörden laut EU-Richtlinien bemühen sollen, die Angehörigen so schnell wie möglich ausfindig zu machen, wird dies von polnischer Seite unterlassen - offenbar um zu vermeiden, dass außerhalb der EU lebende Angehörige ein Recht auf Familienzusammenführung geltend machen (vgl. Flüchtlingsrat Schleswig-Holstein 2004, S. 32).

Ähnliche Differenzen bestehen zwischen den offiziellen Rechten für traumatisierte AntragstellerInnen und den Rechten, die sie tatsächlich in Anspruch nehmen können. Auch die Berücksichtigung der besonderen Bedürfnisse von Traumatisierten (Kap. 4, Art. 54 und 55) scheitert offensichtlich daran, dass sie nicht als solche anerkannt werden. In ihrer achtjährigen Praxis hat die interviewte Mitarbeiterin der Legal Clinic an der Universität Warschau von lediglich drei Personen gehört, die vom URIC bzw. UdSC als traumatisiert anerkannt wurden (D S. 16):

„Ich würde sagen, dass fast alle unsere Klienten traumatisiert sind, deshalb ist es so schwierig vom URIC anerkannt zu werden, denn sie denken, dass man wirklich wirklich sehr traumatisiert sein muss, um offiziell anerkannt zu werden. [...] wir haben Klienten, die gefoltert wurden und die die Zeichen der Folter tragen und sie sagen, es reicht nicht aus, um als Flüchtling anerkannt zu werden" (D S. 22).

In dem Gesetz wird dem Sicherheitsaspekt hohe Bedeutung beigemessen. Sicherheitsbedenken durchziehen das gesamte Gesetz und dienen gewissermaßen als Universalvorbehalt. Gleich zu Beginn bei den allgemeinen Regelungen im ersten Kapitel des Gesetzes wird festgelegt, dass eine Behörde eine Entscheidung, die sie aufgrund dieses Gesetzes gefällt hat, nicht begründen muss, wenn dies die staatliche Verteidigung oder Sicherheit oder die öffentliche Sicherheit und Ordnung erfordern (Art. 5) (im Folgenden: staatliche Sicherheit). Zudem werden Sicherheitserwägungen als legitime Begründung für die Einschränkung von Rechten angeführt. Beispielsweise kann das Recht auf persönlichen Kontakt mit UNHCRVertreterInnen aufgrund von Sicherheitsbedenken ausgehebelt werden (Art. 43). Außerdem kann Arrest mit dem Ziel der Ausweisung unter anderem dann stattfinden, wenn der Grenzschutz dies aus Gründen der staatlichen Sicherheit als notwendig erachtet (Art. 41). Auch bei den Regelungen zur Freilassung aus geschlossenen Zentren oder dem Arrest ist eine Ausnahme aufgrund von Sicherheitsbedenken vorgesehen (Art. 44 Abs. 2). Weiterhin soll vor der Vergabe eines Status überprüft werden, ob der Aufenthalt der jeweiligen Personen eine Gefahr für die staatliche Sicherheit darstellt (zum Beispiel Art. 87). Flüchtlingsstatus und Duldung können jeweils aberkannt werden, wenn der/die AntragstellerIn gegen die staatliche Sicherheit agiert oder eine Gefahr für die öffentliche Sicherheit darstellt (Art. 91 und 102). Auch temporärer Schutz soll nicht gewährt werden, wenn die Einreise der jeweiligen Person eine Sicherheitsgefahr oder eine Gefahr für polnische BürgerInnen darstellen würde (Art. 109). Zudem werden die im Zu- 
sammenhang mit den verschiedenen Schutzverfahren erhobenen Daten auch den Sicherheitsbehörden zugänglich gemacht (Art. 123). Mit dieser exponierten Stellung des Sicherheitsgedankens im Flüchtlingsschutzgesetz folgt Polen einem allgemeinen Trend in der Migrationspolitik der meisten Zielländer von Migration: Mit „Securitisation of Migration“ (vgl. Abiri 2000) wird in aktuellen Analysen vor allem eine diskursive Strömung umschrieben, aber auch ein wachsender Trend in Einwanderungspolitiken erfasst. Migration und Sicherheitsrisiken werden diskursiv miteinander verknüpft und legitimieren restriktive Politiken.

Neben dem Flüchtlingsstatus gemäß GFK gibt es in Polen auch ein in der Verfassung verankertes Recht auf Asyl. Mit der Neuformulierung der Verfassung wurde das bis dato realsozialistisch geprägte Asylrecht vollkommen neu gestaltet (vgl. 6.3.2a). So heißt es in Art. 56 Abs. 1 seit der Verfassungsänderung von 1997:

„Staatsangehörige anderer Staaten sowie Staatenlose können das Recht auf Asyl aufgrund der durch das Gesetz, bestimmten Prinzipien genießen";

Abs. 2: „Einem Ausländer, der in der Republik Polen Schutz vor Verfolgung sucht, kann der Flüchtlingsstatus in Einklang mit den für die Republik. Polen bindenden völkerrechtlichen Verträgen verliehen werden."

Im Flüchtlingsschutzgesetz von 2003 wird das Recht dahingehend konkretisiert, dass jemand Asyl erhalten soll, wenn dies für seinen/ihren Schutz unerlässlich ist (niezbędne do zapewnienia mu ochrony) und wenn ein wichtiges Interesse der Republik Polen dafür spricht (gdy przemawia za tym ważny interes Rzeczpospolitej Polskiej) (Art. 90). Der Status kann aberkannt werden, wenn die Asylgründe nicht mehr existieren oder er/sie gegen die staatliche Sicherheit agiert (Art. 91). Der Antrag auf Asyl kann sowohl auf polnischem Territorium als auch von auBerhalb gestellt werden. Auch AsylantragstellerInnen müssen bereit sein, sich Fingerabdrücke nehmen und fotografieren zu lassen, andernfalls bleibt ihr Antrag unberücksichtigt (Art. 92). Dabei gelten hinsichtlich der Verpflichtung zur Aussage und zur Vorlage von Beweisen die gleichen Verpflichtungen wie für Personen, die Flüchtlingsschutz beantragen. Auch die Regelungen zum Umgang mit unbegleiteten Minderjährigen und zur sozialen Unterstützung sind auf Asylsuchende anwendbar (Art. 93). Die Zuständigkeit für Asylanträge liegt ebenfalls beim URIC, Entscheidungen zur Zu- und Aberkennung des Asylstatus können mit dem AuBenminister abgestimmt werden. Ein/e Asylberechtigte/ $r$ darf nur nach Aberkennung seines/ihres Status ausgewiesen werden (Art. 96). Der Asylstatus hat - sicherlich aufgrund der voraussetzungsvollen Klausel des, wichtigen Interesses der Republik Polen` - in der Realität kaum eine Bedeutung. So wurden zum Beispiel in den Jahren 2003 bis 2005 insgesamt nur 26 Asylanträge gestellt und kein Antrag positiv beschieden (vgl. UdSC 2006, Tabelle 61 und 62).

Ähnliches gilt für den temporären Schutz, der entsprechend der EU-Richtlinie (vgl. 2001/55/EG) ausgestaltet ist, bisher jedoch noch keine praktische Bedeutung in Polen hatte. Er ist vorgesehen für den Fall, dass eine große Zahl von 
Flüchtlingen in Polen ankommt aufgrund von Besatzung, (Bürger-)Krieg, ethnischen Konflikten und Menschenrechtsverletzungen im Herkunftsland (Art. 106). Diese Ankunft kann spontan oder mit Hilfe der Republik Polen oder der Internationalen Gemeinschaft erfolgen. Der Schutz wird gewährt, bis eine Rückkehr möglich ist, höchstens jedoch für ein Jahr. Zusätzlich ist zwei Mal eine Verlängerung um ein halbes Jahr möglich. Den Schutzberechtigten werden medizinische Versorgung, Unterbringung und Nahrung gewährt (Art. 112). Sie dürfen arbeiten, ohne eine gesonderte Arbeitserlaubnis zu beantragen (Art. 116). Kinder haben das Recht auf Zugang zu Bildungseinrichtungen. Das URIC soll die Rückkehr nach Ablauf des temporären Schutzes organisieren (Art. 118). Wenn eine Rückkehr aus gesundheitlichen Gründen nicht möglich ist (insbesondere wenn dafür ein Krankenhausaufenthalt abgebrochen werden müsste), soll das URIC eine befristete Aufenthaltserlaubnis ausstellen (Art. 118 Abs. 3).

\section{Soziale Rechte}

AntragstellerInnen auf Flüchtlingsschutz und ihre Angehörigen können bei Nachweis ihrer Bedürftigkeit soziale Unterstützungsleistungen in Anspruch nehmen, sofern sie nicht in einem geschlossen Zentrum oder einem Arrest mit dem Ziel der Ausweisung untergebracht sind (Art. 56, vgl. auch weitere Ausnahmen: Art. 56 Abs. 4). Die Unterstützung umfasst Unterkunft im Heim oder finanzielle Unterstützung sowie medizinische Versorgung (Art. 57). Allerdings wird die Heimunterbringung nur dann durch finanzielle Hilfen ersetzt, wenn dies die Gesundheit oder Sicherheit erfordern (Art. 64). Der Unterbringung im Heim soll eine ärztliche Untersuchung vorausgehen (Art. 58). Die Bedingungen für diese ärztliche Untersuchung legt das Gesundheitsministerium fest, dabei soll vor allem einer Verbreitung ansteckender Krankheiten entgegengewirkt werden (Art. 59). Inzwischen müssen sich auf Initiative des URIC bzw. UdSC alle AntragstellerInnen einem HIV-Test unterziehen. Diese Regelung wurde nach einem Skandal um den „berühmtesten Flüchtling Polens“ eingeführt, der angeblich wissentlich über ein Dutzend Frauen mit dem Virus infiziert hat (Gazeta Wyborcza vom 17.04.2007).

Für diejenigen, die in Heimen untergebracht sind, umfasst die soziale Unterstützung Nahrungsmittel, Fahrscheine für Fahrten im Rahmen des Asylverfahrens, zu medizinischen Behandlungen oder in anderen ,gerechtfertigten` Fällen, Materialien für Schulkinder sowie finanzielle Unterstützung für Toilettenartikel und andere Ausgaben (Taschengeld). Grundsätzlich ist in den Heimen eine Fremdversorgung mit Essen vorgesehen. Die BewohnerInnen können jedoch unter bestimmten Bedingungen so genannte, Äquivalente', das heißt Geld statt Essen, beantragen. Dies gilt für Personen mit bestimmten Krankheiten, für Personen mit Kindern unter sieben Jahren und für Kinder, die in die Schule gehen. Viele Flüchtlinge versuchen, durch Beantragung von ,Äquivalenten` etwas Bargeld $z u$ erhalten. Des Weiteren soll eine einmalige Unterstützung für den Kauf von 
Kleidung und Schuhen gewährt und ein kostenloser Polnisch-Kurs angeboten werden (Art. 61).

Die gesetzliche Grundlage für die Sozialleistungen in Polen, aus der sich auch die Unterstützung der Flüchtlinge ableitet, ist das Sozialhilfegesetz (vgl. Ustawa z dnia 12 marca 2004 r. o pomocy spolecznej). Laut Flüchtlingsschutzgesetz wird jedoch die konkrete Höhe der Unterstützung für Flüchtlinge - im Übrigen auch der Umfang der täglichen Essensration - vom Innenministerium festgelegt. Die Verfügung des Innenministeriums soll auch angeben, inwieweit das Taschengeld erhöht werden kann, wenn ein Flüchtling sich im Heim oder für andere Bewohner einbringt (Art. 69). Anhand der konkreten Zahlen kann die soziale Situation von Flüchtlingen in Polen veranschaulicht werden: Laut der entsprechenden Verordnung des Innenministeriums (vgl. Rozporzadzenie Ministra Spraw Wewnętrznych i Administracji) erhalten AntragstellerInnen während des Heimaufenthalts pro Monat 20 Złoty (rund 5 Euro) für Hygieneartikel und 50 Złoty (rund 13 Euro) Taschengeld. Eine Erhöhung auf 100 Złoty (rund 26 Euro) ist möglich, wenn die Person im Heim mithilft - zum Beispiel beim Putzen oder bei Übersetzungen. Für Kleidung und Schuhe wird eine einmalige Beihilfe von 140 Złoty (rund 37 Euro) gezahlt. Als so genannte Äquivalente bekommen die Flüchtlinge auf Antrag ersatzweise 9 Złoty (rund 2,40 Euro) pro Tag. Außerhalb der Heime erhalten Alleinstehende 25 Złoty (rund 7 Euro) pro Tag - diese pro-Kopf-Summe verringert sich mit steigender Anzahl der Familienmitglieder schrittweise auf 12,50 Złoty (rund 3 Euro) pro Person und Tag für eine Familie mit vier und mehr Mitgliedern. Eine alleinstehende Person, die nicht in einem Heim lebt, erhält also im Monat rund 750 Złoty (ca. 200 Euro). Für 200 Euro findet man jedoch in Warschau kaum eine Ein-Zimmer-Wohnung. Auch die übrigen Lebenshaltungskosten sind in Warschau sehr hoch. In ländlichen Regionen sind Mieten und Lebenshaltungskosten zwar deutlich niedriger, die meisten Flüchtlingsheime befinden sich aber in der Region rund um Warschau, wo auch die meisten Flüchtlinge leben, die nicht mehr in Heimen untergebracht sind.

Zwar haben AntragstellerInnen auf Flüchtlingsschutz in Polen - anders als in vielen westlicheren Zielländern - auch während des Verfahrens das Recht zu arbeiten. Aufgrund der hohen Arbeitslosenquote in Polen ist es jedoch sehr schwierig eine reguläre Arbeit zu finden. Die interviewten ExpertInnen waren überzeugt, dass die Flüchtlinge stattdessen irregulärer Arbeit nachgehen (G S. 7, D S. 25, K S. 7). Insofern greifen die Probleme bei Arbeits- und Wohnungssuche ineinander, wie Eßer et al. (2005, S. 16) zusammenfassen: So ,,scheint es schwer zu sein eine bezahlbare und zumutbare Wohnung an einem Ort zu finden, wo zumindest eine minimale Chance auf einen Arbeitsplatz besteht.“

Keine soziale Unterstützung erhält, wer selbst über ausreichende Mittel verfügt oder wer nach Gewährung der Hilfe die Grenze überquert oder dies versucht hat oder wer zeitweilig eine Freiheitsstrafe zu verbüßen hatte. Bei wiederholtem Einbehalten der Unterstützung wegen illegaler Grenzüberquerung oder Regelverstoß 
kann die Unterstützung auf ein Drittel gekürzt werden (Art. 66). Zudem kann die Unterstützung bei grober Verletzung der Heimregeln einbehalten werden - die Entscheidung hierüber trifft die Heimleitung (Art. 65). Damit hat die Heimleitung eine starkes Instrument, um das Verhalten der Flüchtlinge zu sanktionieren. Meine Besuche in polnischen Flüchtlingsheimen haben gezeigt, dass der Umgang der Heimleitung mit Flüchtlingen stark von der jeweilig verantwortlichen Person abhängig ist. In einem Heim in Białystok konnte ich mich selbst von der Feindseligkeit überzeugen, mit der die Angestellten den Flüchtlingen - und uns als BesucherInnen, die sich für die Belange der Flüchtlinge interessierten - begegneten. Dort berichteten die Flüchtlinge auch davon, dass ihre Kinder von Heim-Angestellten angeschrien und geschlagen würden, wenn sie sich deren Anweisungen widersetzten. Zudem würden MitarbeiterInnen unangekündigt in die Zimmer der Familien eindringen und die Schränke durchsuchen. Die Verantwortung für die Heime trägt das UdSC, dieses kann diese Aufgabe auf andere übertragen (Art. 63). Die Zahl der Flüchtlingsheime in Polen variiert. Das UdSC, das für die Heime verantwortlich ist, pachtet je nach Höhe der Flüchtlingszahlen weitere Unterkünfte hinzu.

Die medizinische Versorgung soll derjenigen entsprechen, die auch andere Personen, die in Polen pflicht- oder freiwillig versichert sind, erhalten (Art. 67). Wie die bereits genannten Forderungen der HeimbewohnerInnen im Hungerstreik im Dezember 2004 gezeigt haben, ist die medizinische Versorgung der Flüchtlinge in Polen de facto jedoch äußerst begrenzt. In den Heimen gibt es keine zahnärztliche Behandlung, die Therapie besteht meist aus einfachen Schmerzmitteln. Wer einen Antrag auf Besuch eines Facharztes stellt, wird in der Regel mit der Begründung abgewiesen, dass die erforderlichen finanziellen Mittel dafür nicht zur Verfügung stünden. ${ }^{308}$ Auch die Versorgung mit psychologischer Betreuung ist vollkommen unzureichend (vgl. Flüchtlingsrat Schleswig-Holstein 2004, S. 13). Das polnische Gesundheitssystem ist insgesamt in einem sehr

\footnotetext{
308 Für Aufsehen im Ausland hat der Tod eines Tschetschenen mit Hepatitis C gesorgt. Diesem wurde, laut Angaben der Frankfurter Allgemeinen Zeitung vom 11.09.2007, keine Aufenthaltserlaubnis ausgestellt, obwohl er seit 1992 in Polen lebte. Er ließ sich 2005 nach Brüssel schmuggeln und stellte dort einen Asylantrag. ÄrztInnen diagnostizierten in Brüssel Hepatitis C und Verdacht auf TBC. Noch vor der Behandlung im Tropeninstitut in Antwerpen wurde er jedoch nach Polen zurückgeschoben und dort trotz der Nachweise über seine Krankheit nicht aus der Abschiebehaft in Lesznowola entlassen und nicht behandelt. Er starb drei Monate nach seiner Entlassung im Oktober 2006 an Hepatitis C. Auf Anfrage beim polnischen Ombudsmann für die Wahrung der BürgerInnenrechte wurde uns mitgeteilt, der Fall werde noch untersucht, die bisherigen Ergebnisse deuteten aber aus Sicht des Ombudsmanns darauf hin, dass der Betroffene die polnischen Behörden nicht über seine Erkrankung informiert und eine medizinische Behandlung abgelehnt habe (Q S. 8). Viele der Flüchtlinge aus Tschetschenien leiden an Hepatitis und TBC. Ich habe ebenfalls mit einer aus Deutschland nach Polen zurückgeschobenen Tschetschenin gesprochen, der mit der Begründung, man könne ihre Hepatitis und TBC auch noch in zehn Jahren behandeln, die Behandlung verweigert wurde. Auch der interviewte Mitarbeiter des Ombudsmanns räumte ein, dass Fälle bekannt seien, wo eine mangelnde Behandlung zu einer Verschlechterung des Krankheitsbildes und zu einer Verlängerung der Krankheitsdauer geführt hätten (Q S. 7).
} 
schlechten Zustand, es befindet sich nach Aussage eines Mitarbeiters des Ombudsmanns ,in einer Phase des totalen Kollapses“ (Q S. 10). Ähnlich wie bei der Situation auf dem Arbeits- und dem Wohnungsmarkt sehen sich die Flüchtlinge hier einer in Polen insgesamt schwierigen sozialen Situation gegenüber, die sie aber in vielerlei Hinsicht mit besonderer Härte trifft: Im Gegensatz zu den ebenfalls betroffenen polnischen BürgerInnen haben sie zusätzlich mit Sprachproblemen, Diskriminierung, Traumatisierung, Unkenntnis des polnischen Sozialsystems usw. zu kämpfen. Aufgrund der zum Teil abgelegenen Lage der Heime können sie die benötigten ÄrztInnen nur schwer erreichen, wenn überhaupt eine Überweisung zu einem Facharzt ausgestellt wurde. Zudem kommen viele von ihnen in einem gesundheitlich schlechten Zustand in Polen an. Vergleicht man die soziale Situation von Flüchtlingen der verschiedenen Status mit der polnischer BürgerInnen, muss man laut einer Interviewpartnerin aus dem Arbeitsministerium zwischen Stadt- und Landbevölkerung unterscheiden: Verglichen mit der oft armen Landbevölkerung im Osten Polens gehe es den Flüchtlingen in Polen gut. Verglichen mit der Stadtbevölkerung zum Beispiel in Warschau, seien sie in einer schlechten Situation (K S. 7). Angesichts dieser Situation erscheint es fragwürdig, wie die EU-Kommission zu der Einschätzung gelangt, dass die Gesundheitsversorgung für AsylbewerberInnen in Polen gewährleistet sei. In dem entsprechenden Bericht der Kommission vom November 2007 heißt es: „Zahlreiche Mitgliedstaaten haben den Zugang zur medizinischen Versorgung erweitert und gewähren mitunter den gleichen Zugang wie ihren eigenen Staatsangehörigen (CZ, NL, PL).“ (KOM(2007) 745, S. 8) Hier wurde offenbar nur der Wortlaut der gesetzlichen Implementierung der Richtlinienvorgaben berücksichtigt, während die tatsächliche Umsetzung vor Ort nicht überprüft wurde.

Außerdem sind Hilfen im Falle freiwilliger Rückkehr vorgesehen: Fahrscheine, Verwaltungsgebühren für Dokumente sowie teilweise das Essen während der Reise (Art. 68). Die Hilfen beziehen sich also lediglich auf die Reise selbst, nicht jedoch auf die Ankunft im Herkunftsland und die Möglichkeiten, sich eine Existenz aufzubauen.

Nach Aussage des URIC-Präsidenten Jan Węgrzyn wird die Versorgung der Flüchtlinge vollständig aus polnischen Mitteln bestritten, während die EUUnterstützung in die Infrastruktur fließe, wie etwa in Renovierungsarbeiten in den Heimen und den Erwerb von Software (vgl. Flüchtlingsrat Schleswig-Holstein 2004, S. 13). Derzeit gebe Polen sechs Millionen Euro jährlich für die Flüchtlingsversorgung aus - exklusive der Gehälter der MitarbeiterInnen in den Heimen und im URIC (ebd.).

Insbesondere hinsichtlich der Ansprüche auf soziale Unterstützung ist es entscheidend, welchen Aufenthaltsstatus ein Flüchtling erhalten hat. Wie oben bereits erwähnt, erhalten Anerkannte Unterstützung aus dem Integrationsprogramm. Die rechtlichen Grundlagen für die soziale Integration von anerkannten Flüchtlingen sind in Kapitel 5 des polnischen Gesetzes zur sozialen Unterstützung enthalten 
(vgl. Ministerstwo Polityki Społecznej 2005). Im Gegensatz zum weiter unten beschriebenen Duldungsstatus ist mit der Anerkennung als Flüchtling zumindest für ein Jahr eine etwas besser ausgestaltete soziale Unterstützung verbunden. Doch durch die zeitliche Beschränkung des Integrationsprogramms für Anerkannte verschiebt sich das Problem für diese lediglich, wie auch der interviewte Mitarbeiter des polnischen Ombudsmanns einräumt: „Nach einem Jahr befindet sich der Flüchtling in einer Situation, die sich mit ,Schwimm oder ertrinke!" umschreiben lässt, konfrontiert mit einer Situation, auf die er nicht vorbereitet wurde.“ (Q S. 6)

Die Kinder der AntragstellerInnen sind schulpflichtig. De facto gehen jedoch offenbar nicht alle Flüchtlingskinder regelmäßig zur Schule (M S. 4, I S. 9). In einem Bericht von Amnesty International aus dem Jahr 2007 heißt es: „Almost half of school-age children seeking asylum in Poland do not attend school" (Amnesty International 2007, S. 6). Einige Interviewte sehen die Gründe dafür vor allem bei den Eltern und deren Bildungsstand (I S. 10). Sie würden ihre Kinder lieber zuhause behalten, damit sie bei der Betreuung der jüngeren Geschwister helfen oder weil sie ohnehin nicht in Polen bleiben wollen (H S. 4) oder sie würden nicht für die notwendige Ruhe bei den Hausaufgaben sorgen (I S. 10). Letzteres dürfte schwierig sein, wenn sich - wie in den meisten Heimen - mindestens eine Familie (mit meist mindestens fünf Familienmitgliedern) ein Zimmer teilt. Außerdem wird angeführt, dass den Kindern einerseits die notwendigen Sprachkenntnisse fehlten (C S. 5) und sie andererseits einen sehr unterschiedlichen Bildungsstand hätten, der schwierig zu ermitteln sei (H S. 4). Zudem seien die Schulen bzw. LehrerInnen mit der Situation oft überfordert und würden mit den Problemen weitgehend allein gelassen (I S. 8). Das Argument der mangelnden Motivation der Eltern wird von FlüchtlingsberaterInnen in Deutschland nicht geteilt, die ebenfalls mit tschetschenischen Flüchtlingen zusammenarbeiten und hier häufig eine hohe Bildungsmotivation vorfinden (vgl. Eßer et al. 2005, S. 19). Da viele Flüchtlingskinder bereits in Tschetschenien nur begrenzten Zugang zu Bildungseinrichtungen hatten, stehe zu befürchten, dass „Bildungschancen verpasst werden“ (ebd.). Der Bericht der EU-Kommission über die Umsetzung der Aufnahmerichtlinie kritisiert in diesem Zusammenhang, dass unter anderem Polen den Zugang in Gewahrsam befindlicher Minderjähriger zum Bildungswesen verwehrt bzw. beschränkt (vgl. $\operatorname{KOM}(2007)$ 745, S. 9).

Darüber hinaus sind im Bereich der sozialen Rechte Inkongruenzen festzustellen, weil es zwar einerseits das Flüchtlingsrecht gibt, die Situation von Flüchtlingen aber andererseits in anderen, nicht direkt damit verbundenen Regelungen nicht mitgedacht wird. So sieht bspw. das Sozialgesetz Hilfen für Alleinstehende vor. Da Flüchtlingsfrauen aber in den meisten Fällen nicht nachweisen können, dass ihre Männer im Krieg gestorben sind, werden sie von den Behörden abgewiesen, wie im Fall einer Frau mit Flüchtlingsstatus, die schließlich Hilfe bei der Polska Akcja Humanitarna suchte: 
„Alles ist in diesem Gesetz drin, außer Flüchtlinge. Sie [die MitarbeiterInnen des Sozialamts] fragten [die Klientin] wo ist der Nachweis - denn sie ist eine Witwe - dass sie Witwe ist. Sie sagte: ,Es ist unmöglich, mein Ehemann wurde im Krieg getötet. Ich habe keinerlei Dokumente, es ist unmöglich, diese Dokumente beizubringen. Ich kann nicht meine Botschaft fragen.' Sie antworteten: ,Dann können wir Ihnen nichts geben.' [...] im Sozialgesetz, vergessen sie diese Leute einfach. "(C S. 10)

\section{Duldungsstatus}

Die soziale Situation der AntragstellerInnen und der Anerkannten unterscheidet sich von der der Geduldeten - insbesondere im Hinblick auf die Wohnsituation. Ein großer Teil der Flüchtlinge, die einen Antrag auf Flüchtlingsschutz in Polen stellen, erhält eine Duldung (pobyt tolerowany) (vgl. Tabelle 10 im Anhang). Diese wird ausgestellt, wenn eine Ausweisung nicht möglich ist, weil sie nur in ein Land stattfinden könnte, in dem

- das Recht auf Leben, Freiheit und persönliche Sicherheit gefährdet wäre oder

- die Gefahr der Folter, unmenschlicher oder erniedrigender Behandlung oder Bestrafung besteht oder

- andere Menschenrechtsverletzungen stattfinden, die die Menschenrechtskonvention verbietet (Zwangsarbeit, Vorenthaltung des Rechts auf ein faires Gerichtsverfahren oder Bestrafung ohne gesetzliche Grundlage) (Art. 97).

Weiterhin kann eine Duldung ausgestellt werden, wenn die Ausweisung aus anderen Gründen nicht möglich ist, die nicht von den Behörden oder dem Flüchtling herbeigeführt wurden. Personen mit Duldungsstatus haben die gleichen Rechte wie die InhaberInnen der befristeten Aufenthaltserlaubnis (Art. 98). Wer eine Duldung hat, darf nicht ausgewiesen werden (Art. 101). Wenn der Status aberkannt wurde, weil die Gründe nicht mehr bestehen, muss die Person Polen binnen 14 Tagen verlassen (Art. 102).

Die Duldung kann durch die Woiwodschaft, das URIC (bei Nicht-Gewährung des Flüchtlingsstatus), auf Antrag, wenn ein Gericht die Abschiebung für unzulässig erklärt hat, sowie auf Antrag der für Abschiebung zuständigen Behörden oder durch den Flüchtlingsrat gewährt werden (Art. 104).

Geduldete haben zwar formal Anspruch auf die gleichen finanziellen Unterstützungsleistungen wie polnische BürgerInnen, aber „,[w]ithout Polish language skills and specialized social support these allowances are not enough to start selfreliant life in a foreign country." (Kępińska 2006) Ihre Situation wurde durch eine Gesetzesänderung im Jahr 2006 noch verschärft. Seitdem haben Geduldete, die einen Folgeantrag auf Flüchtlingsstatus stellen, keinen Anspruch mehr auf die soziale Unterstützung, die sie als ErstantragstellerInnen erhalten würden. Das heißt vor allem, dass sie die Flüchtlingsheime verlassen müssen. ${ }^{309} \mathrm{Im}$ Gegensatz

309 „Pod koniec listopada ponad 1,5 tys. uciekinierów, głównie z Czeczenii, trafi z polskich ośrodków dla uchodźców na bruk. To efekt nowelizacji ustawy o ochronie cudzoziemców.“ (,Gegen Ende 
zur Bundesrepublik, wo viele Flüchtlinge versuchen, der Isolation und den schlechten Bedingungen in den Heimen zu entkommen, ist diese Form der Unterkunft für Flüchtlinge in Polen die einzige Wohnmöglichkeit. Dies ist - wie bereits erwähnt - bedingt durch den Mangel an Wohnungen und die Höhe der Mieten auf dem polnischen Wohnungsmarkt. Für Flüchtlinge ist eine eigene Wohnung überwiegend unerschwinglich. Darüber hinaus wird die Wohnungssuche durch rassistische Ressentiments von VermieterInnen stark erschwert - hier deckt sich die Einschätzung der ExpertInnen (K S. 13, M S. 8, Q S. 7) mit Aussagen von Flüchtlingen. Bei meinen eigenen Besuchen in den Heimen teilten sich mitunter zwei Familien ein Zimmer - nur eine hatte tatsächlich das Recht dort zu wohnen, die andere lebte, illegal' dort. Auch wurde immer wieder berichtet, dass Flüchtlinge, nachdem sie die Heime im Winter verlassen mussten, von den anderen nachts durch die Fenster wieder hereingelassen wurden. Bislang war der Folgeantrag für viele eine Möglichkeit, in den Heimen bleiben zu können. Diese Strategie spiegelte sich in der gewachsenen Zahl von Folgeanträgen (Kępińska 2006, S. 3). Ein interviewtes Mitglied des ,Flüchtlingsrates' (Berufungsinstanz gegen erstinstanzliche Entscheidungen) beschreibt die Situation so:

„,...] unser Hauptproblem waren Leute, die negative Entscheidungen erhielten und wieder und wieder und wieder kamen. Manchmal sechs bis sieben Mal, denn solange sie im Verfahren sind, können sie in den Flüchtlingsheimen bleiben. Das liegt am Mangel an ausreichendem Schutz, für Menschen, die den Duldungsstatus erhalten. Wir haben kein gutes System, was mit diesen Leuten geschehen soll. Sie erhalten eine minimale Unterstützung, die nicht adäquat ist. Faktisch kann man als Familie von sieben Personen bier keine Wohnung finden. " (N S. 3f.)

Bemerkenswert ist, dass ausgerechnet ein Mitarbeiter des ,Ombudsmanns ‘ sich das Argument zu Eigen macht, dass das Ziel der Gesetzesverschärfung sei, den Missbrauch sozialer Unterstützung zu verhindern. Er betonte im Gespräch:

„Man muss im Hinterkopf behalten, dass die praktischen Angelegenheiten dieser Regelung von den interessierten Personen missbraucht werden, als eine Verlängerung ihres kostenfreien Aufenthalts in Polen. "(QS. 9)

Ähnlich sieht es Jan Wegrzyn, der Direktor des UdSC: „Living in the welfare system made many foreigners not be able to find internal motivation for work or doing something with their life." (zit. n. Marczuk/Kosowicz 2006). Gleichzeitig haben die Flüchtlinge ohne Meldeadresse keine Möglichkeit, Sozialhilfe zu beantragen und können nur eine symbolische Unterstützung von monatlich 20 bis 70 Złoty (ca. 5 bis 18 Euro) erhalten (vgl. Eßer et al. 2005, S. 17). Inzwischen wird an einem Integrationsprogramm auch für Geduldete gearbeitet. Doch auch mit ei-

November werden über 1500 Flüchtlinge, hauptsächlich aus Tschetschenien, aus polnischen Flüchtlingsheimen auf die Straße gesetzt. Das ist das Ergebnis einer Novelle des Flüchtlingsschutzgesetzes.) (vgl. Gazeta Wyborcza vom 29.10.2006). 
nem einjährigen Integrationsprogramm wird das Problem lediglich zeitlich verschoben: Nach einem Jahr sind die Betroffenen wieder ohne Unterstützung.

\section{Inhaftierung und Residenzpflicht}

Kapitel II des Flüchtlingsschutzgesetzes enthält ausführliche Regelungen zur Inhaftierung von AntragstellerInnen. Der Art. 40 konstatiert, dass ein Antragsteller nicht inhaftiert werden soll. Die Ausnahmeregeln, die darauf mit ,chyba że' (,es sei denn') eingeleitet werden, sind jedoch so umfassend, dass sie auf eine routinemäBige Inhaftierung nahezu aller AntragstellerInnen hinauslaufen. Das heißt, ein Antragsteller soll nicht inhaftiert werden, es sei denn, er/sie hat kein Recht zur Einreise und stellt einen Antrag auf Flüchtlingsschutz während einer Grenzkontrolle oder während eines illegalen Aufenthalts in Polen (Art. 40 Abs. 1). Inhaftierung ist ebenfalls gerechtfertigt, wenn die Person vor der Antragstellung die Grenze illegal überquert oder dies versucht hat oder vor der Antragstellung bereits einen Ausweisungsbescheid erhalten hat (Art. 40 Abs. 2). Das Inhaftierungsinstrument ist damit in Polen eines der umfassendsten im europäischen Vergleich.

Die meisten Flüchtlinge stellen ihre Anträge direkt an der Grenze, um diese überhaupt überqueren zu können. Denn seit der Einführung der Visumspflicht ist es schwer geworden, legale Dokumente für eine Einreise nach Polen zu bekommen ${ }^{310}$ und viele TschetschenInnen - die seit Jahren die größte Flüchtlingsgruppe in Polen sind - müssen ohnehin den Landweg nutzen. Ohne gültige Einreisepapiere werden sie inhaftiert (vgl. Fagasinski 2007). Hinzu kommen Zurückgeschobene aus anderen EU-Ländern sowie Undokumentierte, die aus der Illegalität auftauchen wollen. Wenn diese sich dem UdSC ,stellen' und ein Flüchtlingsschutzverfahren beginnen wollen, werden sie zunächst inhaftiert: „Ins Gefängnis gehen [...] ist der Anfang jedweden Verfahrens zur Legalisierung ihres Aufenthalts." (C S. 17) Werden die Flüchtlinge in der Beratung bei der Polska Akcja Humanitarna (PAH) mit dieser Möglichkeit konfrontiert, ist die Reaktion der meisten: „,nein, wir wollen nicht ins Gefängnis‘. Aber manchmal gibt es keinen anderen Weg." (C S. 17) Meist werden Männer inhaftiert und dabei von ihren Familien getrennt; manche Frauen wissen tagelang nicht, wo sich ihre Angehörigen befinden (vgl. Flüchtlingsrat Schleswig-Holstein 2004, S. 18). Die Dauer der Haft kann bis zu zwölf Monate betragen, wenn der Antrag der jeweiligen Person negativ beschieden wird. Eine Freilassung erfolgt laut Gesetz, wenn eine hohe Wahrscheinlichkeit besteht, dass der Antrag auf Flüchtlingsschutz positiv beschieden wird. Trotz dieser umfassenden Inhaftierungspraxis wird Polen im Bericht der EU-Kommission über die Umsetzung der Aufnahmerichtlinie nicht als Land ge-

310 Um ein Visum für Polen zu erhalten, müssten TschetschenInnen nach Moskau reisen. Zudem erfüllen die meisten nicht die erforderlichen Kriterien. 
nannt, in dem eine ,allgemeine [...] Praxis des Gewahrsams aller Asylbewerber, die illegal in den Mitgliedstaat einreisen“ $(\operatorname{KOM}(2007) 745$, S. 8) vorherrscht. 311

Im Zusammenhang mit der Inhaftierung, zum Beispiel bei Berufung gegen negativ beschiedene Freilassungsanträge, gilt auch für MigrantInnen die Strafprozessordnung (Art. 44 Abs. 4). Zudem gilt die illegale Ausreise aus Polen in Richtung Westeuropa laut Strafgesetzbuch als strafrechtliches Delikt, das mit Geldund Haftstrafen geahndet wird (vgl. Eßer et al. 2005, S. 9). Schon mit der Inhaftierung bei der Einreise ohne Visum werden die AntragstellerInnen einem Verfahren unterzogen, das sonst nur für Personen vorgesehen ist, die strafrechtlich relevante Delikte in einem nennenswerten Ausmaß begangen haben. Mit der Subsumtion unter die Strafprozessordnung wird die Ineinssetzung von Migration bzw. Asylantragstellung mit Kriminalität unterstrichen, indem diese auch juristisch so verhandelt wird.

Ein Interviewpartner, der an der Erstellung des Flüchtlingsschutzgesetzes mitgewirkt hat, gab an, der Hintergrund für die umfassende Inhaftierungsregel sei, dass „Asylantragstellung die einfachste Art ist, nach Polen zu kommen“ (B S. 8). Deshalb würden ,alle Tschetschenen, oder fast alle, die Asyl beantragen, [...] inhaftiert" (B S. 8).

„Theoretisch wird niemand, der Angst vor Verfolgung hat, nach einem Visum fragen und dann nach Polen kommen und einen Asylantrag stellen. [...] Aber die Situation in Polen ist anders. Denn wir sind an der EU-Ostgrenze und [...] es ist ziemlich unmöglich, all diese Leute auf das europäische Territorium zu lassen, nur weil sie sagen ,Asyl:. [Wir sagen also zu ibnen:] Okay, ibr könnt das machen, aber seid euch im Klaren, dass ibr - Asylbewerber - in Haft genommen werdet. " (B S. 9)

Sehr offen kommt hier einerseits zum Ausdruck, dass die Inhaftierung von denen, die den Gesetzentwurf erarbeitet haben, klar als Abschreckungsstrategie gedacht ist. Andererseits wird mit dem Argument ebenso deutlich erklärt, dass bewusst in Kauf genommen wird, dass von dieser Strategie gerade MigrantInnen betroffen sind, die (vor Verfolgung) aus ihren Herkunftsländern geflohen sind und deshalb keine Gelegenheit hatten sich die notwendigen Papiere zu verschaffen. Es wird also hier gar nicht erst versucht, das Bild aufrecht zu erhalten, dass man , wirklich Verfolgten' bzw. ,echten Flüchtlingen' Schutz gewähren wolle. Dennoch wird auf Begrifflichkeiten geachtet: Flüchtlinge würden ,in Haft genommen. Das ist kein Arrest, sondern ein geschlossenes Zentrum“ (B S. 9). ${ }^{312}$

\footnotetext{
311 In dem Bericht wird die automatische Ingewahrsamnahme für unzulässig erklärt: „In Anbetracht der Tatsache jedoch, dass der Gewahrsam nach der Richtlinie eine Ausnahme von der Bewegungsfreiheit darstellt, die nur genutzt werden darf, ,wenn es notwendig ist', steht eine automatische Ingewahrsamnahme ohne jegliche Prüfung der Situation des Betroffenen im Widerspruch zur Richtlinie.“ (KOM(2007) 745, S. 8)

312 Die Interviewpassage bringt jedoch noch mehr zum Ausdruck: Der Interviewpartner verdeutlicht auch, dass Polen hier Verantwortung für die gesamte EU übernimmt. Wenn er sagt: „es ist ziemlich unmöglich, all diese Leute auf das europäische Territorium zu lassen“, dann geht es nicht ,nur
} 
Während noch Mitte der 1990er Jahre der Aufenthalt in einem geschlossenen Zentrum am Ende des Aufenthalts stehen und mit der Abschiebung enden sollte,

„[s]o hat sich zumindest Lesznowola in ein geschlossenes Aufnabmezentrum verwandelt, also in den Anfangspunkt des Asylverfahrens. Da gleichzeitig die Asyl-Verfahrensdauer gestrafft wurde, vermischen sich mehr und mehr Aufnabme und Abschiebehaftfunktionen." (Dietrich 2005, S. 384f.)

Diese Erweiterung der Inhaftierungsfunktionen und -ziele bestätigt die These des slowenischen Politologen Andrej Kurnik, wonach sich die Institution ,detention center ${ }^{6}$ selbst reproduziert, indem sie neue Funktionen kreiert: Menschen werden inhaftiert, obwohl sie nicht abgeschoben werden können; ja, sogar bevor über die Frage des Bleibens in irgend einer Form befunden wurde. ${ }^{313}$ Die Verklammerung von Aufnahme und Abschiebung durch die Inhaftierung vom Anfang bis zum Ende des Verfahrens ist Ausdruck neuer Strategien des ,Migrationsmanagements'. Wenn MigrantInnen an keinem Zeitpunkt ihres Aufenthaltes in der EU das Gelände geschlossener Lager verlassen, ist das Maximum an Kontrolle und (faktischer wie symbolischer) Exklusion erreicht.

Diese Demonstration von Kontrolle hat nach Migreurop, einem transnationalen Netzwerk, das über ,camps for foreigners' informiert, eine wichtige Legitimationsfunktion im Verhältnis der WählerInnen und EntscheidungsträgerInnen in den Zielländern. Die Kontrollaufgabe würde von den Staaten im geografischen Kern Europas an die Länder an den Außengrenzen delegiert:
„The internment of foreigners in Europe is not aimed (primarily) at punishing them. In- stead its goal is to demonstrate to the receiving state population that migrants, who must be controlled, are efficiently managed. It is a kind of tacit contract between State and soci- ety through which the State guarantees the security of its citizens. This is the legitimiza- tion of camps. Internment is part of a series of measures that are referred to as 'common migration and asylum policies' and aim at subcontracting the control of entry into the European Union to the states at the outer borders of Europe. "(Migreurop 2005)

Für die Interpretation der Situation in den neuen Mitgliedstaaten und also auch in Polen ist vor allem letzteres von Bedeutung: Die Rolle als Bewacherin der EUAußengrenze und der damit verbundene Druck aus anderen Mitgliedstaaten füh-

um Polen, sondern um die Bedeutung der polnischen Grenzkontrollen für die gesamte EU. Die Prämisse der gemeinsamen EU-Politik ist dabei für ihn klar: möglichst wenige hereinzulassen.

313 Eigene Mitschrift des Beitrages von Dr. Andrej Kurnik (Universität Ljubljana): Slovenia. Situation in the detention center in Postojna, Slovenia, gehalten auf dem Migreurop Workshop in Radovljica, Slowenien am 29. Mai 2007. Er berichtet zudem über die zweifelhafte Rolle der NGOs bei der Diskussion um ,detention centers'. Mit ihrer Forderung, innerhalb der Lager die Menschenrechte zu wahren, trügen sie zur Normalisierung der Inhaftierung von MigrantInnen bei. Für das Lager in Postojna sei die paradoxe Situation entstanden, dass rechte Gruppierungen gegen dessen Einrichtung protestiert hätten, während linke bzw. NGO-Gruppierungen dafür gewesen seien - sofern darin die Menschenrechte gewahrt würden. 
ren zum Import von derartigen Maßnahmen, während aufgrund des geringen Politisierungsgrades des Themas in Polen ein ,tacit contract between State and society “ hier - im Gegensatz zur Situation in den älteren Mitgliedstaaten - (noch) nicht angenommen werden kann.

Auch wenn aufgrund der Kritik bei der aktuellen Änderung des polnischen Flüchtlingsschutzgesetzes eine Überarbeitung der Inhaftierungsregeln vorgesehen war, werden gleichzeitig die Inhaftierungskapazitäten an der Grenze stark ausgebaut. Bislang erfolgte ein Großteil der Inhaftierungen in (grenz)polizeilichen Arrestzentren und in Arrestzellen lokaler Grenzpolizeiposten, die eigentlich für kurzfristige Arreste bzw. Gewahrsamnahmen angelegt sind (J S. 14). Daneben wurde 1996 mit finanzieller Hilfe aus Deutschland das geschlossene Zentrum Lesznowola - nahe des Warschauer Flughafens mitten im Wald gelegen - geschaffen, in dem bis zu 130 Personen inhaftiert werden können - auch Familien mit Kindern. ${ }^{314}$ Kurz vor Polens Beitritt zum Schengen-Raum im Dezember 2007 liefen die Vorbereitungen für die Eröffnung von vier weiteren geschlossenen Zentren direkt an Polens Ostgrenze auf Hochtouren. Im November 2007 wurde das erste in Przemyśl im Südosten Polens an der ukrainischen Grenze mit einer Kapazität von 138 Plätzen in Betrieb genommen. Die Kosten für den Umbau der Militärbaracken beliefen sich auf 11 Millionen Złoty (rund 2,93 Millionen Euro), wovon 85 Prozent von der EU beigesteuert wurden (vgl. onet.pl vom 08.11.2007). Drei weitere geschlossene Lager von ähnlicher Größe werden in Kętrzyn, Bialapodlaska und in Białystok eingerichtet. Das Zentrum in Kętrzyn, das 180 Personen aufnehmen soll, ist von einer vier Meter hohen Mauer umgeben. Um ,Nationalitätenkonflikte $^{6}$ zu vermeiden, würden die inhaftierten Personen zu verschiedenen Zeiten auf einen abgetrennten Platz ins Freie gelassen. Mit den Aufgaben rund um das Zentrum sind 150 BeamtInnen befasst - dies wird in der Öffentlichkeit als Schaffung neuer Arbeitsplätze dargestellt (vgl. TVP 3 Olsztyn vom 16.10.2007). Die neuen Lager werden dem Grenzschutz unterstellt sein. Zuvor wurden viele Flüchtlinge aufgrund der begrenzten Zahl der Haftplätze direkt in das Erstaufnahmezentrum in Dębak gebracht. Es ist zu erwarten, dass sich diese Praxis verändern wird, wenn die Kapazitäten mit der Eröffnung der neuen geschlossenen Lager erhöht werden.

Zur Frage, wer schließlich auf welche Weise aus Polen abgeschoben wird, liegen kaum konkrete Informationen vor. Die polnische Statistik über Ausweisungsentscheidungen lässt vermuten, dass es sich vor allem um ArbeitsmigrantInnen aus den östlichen Nachbarstaaten handelt (vgl. Tabelle 11 im Anhang). Zudem werden insbesondere VietnamesInnen und ArmenierInnen ausgewiesen. Wie bereits erwähnt, wird auch in Polen die Durchführung von Botschaftsanhörungen erwogen, um bspw. für VietnamesInnen die erforderlichen Dokumente zu erhalten und die Zahl der Abschiebungen nach Vietnam zu erhöhen (B S. 20). Inwie-

314 Zur Situation in Lesznowola vgl. Eßer et al. 2005, S. 9 ff. 
weit sich unter denjenigen, die nach Russland ausgewiesen werden, auch TschetschenInnen befinden, die in Polen mit Abstand die größte Flüchtlingsgruppe bilden, kann der Statistik nicht entnommen werden. Bislang schiebt Polen offiziell keine TschetschenInnen in die Russische Föderation ab. Allerdings werden zahlreiche MigrantInnen bzw. Flüchtlinge an der polnisch-ukrainischen Grenze auf der Basis eines bilateralen Rückübernahmeabkommens in die Ukraine zurückgeschoben. Der Menschenrechtskommisar des Europarates bezweifelt, dass hier das Non-Refoulement-Gebot eingehalten wird (vgl. Hammarberg 2007).

Abschließend sei noch auf das Instrument der ,Residenzpflicht' hingewiesen, das lange Zeit ein Spezifikum der Migrationspolitik in der Bundesrepublik Deutschland war. Auch dieses ist im polnischen Flüchtlingsschutzgesetz enthalten (Art. 45). Die Residenzpflicht kann für Personen gelten, die zwar freigelassen oder nicht inhaftiert wurden, aber eine der ,es sei denn'-Bedingungen des Art. 40 erfüllen. Sie können verpflichtet werden, einen bestimmten Wohnsitz zu nehmen, den sie - bis zur abschließenden Entscheidung - nicht ohne Zustimmung des URIC verlassen dürfen. Es kann angenommen werden, dass dieser Passus in dieser Form realiter relativ wenig Relevanz hat, da die meisten Asylsuchenden aufgrund der Wohnungsnot in den Flüchtlingsheimen leben und sich die Frage nach einer eigenen Wohnortwahl daher für die wenigsten stellt. Eine Interviewpartnerin, die im Arbeitsministerium mit der Integration von Flüchtlingen befasst ist, berichtet dazu:

„Sie [die Flüchtlinge] können auch fragen, ob sie außerhalb des Heims leben können, aber das passiert nicht oft, weil sie keinen Platz haben, wo sie sonst bleiben können. Sie bleiben in den Heimen. "(KS. 9)

Insofern besteht durch die Wohnungsknappheit und die zentrale Zuweisung zu den Flüchtlingsheimen durch das UdSC faktisch keine freie Wohnortwahl. Daher ist die Residenzpflicht bislang nur ein ,Papiertiger - eine Restriktion, die schon allein aufgrund der sozialen Bedingungen in Polen eine ganz andere Bedeutung hat als in der Bundesrepublik.

\subsubsection{Kennzeichen des polnischen Flüchtlingsschutzregimes}

Im vorangegangenen Abschnitt zur konkreten Ausgestaltung des Flüchtlingsschutzes wurde aufgezeigt, dass Polen ein vergleichsweise restriktives Flüchtlingsschutzgesetz hat. Wie dargelegt wurde, resultieren für MigrantInnen, die Flüchtlingsschutz in der EU erhalten wollen und die beabsichtigt oder unbeabsichtigt in Polen landen, zahlreiche Probleme direkt aus dieser Gesetzgebung. Sie sind aber auch mit zahlreichen Lücken konfrontiert, die zwischen dem Recht auf der einen und dessen Umsetzung auf der anderen Seite klaffen. Diese bestehen vor allem hinsichtlich der Gewährung sozialer Rechte. Hier laufen weniger restriktive Regelungen (zum Beispiel Gesundheitsversorgung entsprechend polnischen BürgerIn- 
nen) durch die Defizite bei der Gewährung dieser Rechte (zum Beispiel aufgrund der desolaten Situation des polnischen Gesundheitssystems) ins Leere.

Das polnische Asylregime ist derzeit durch folgende Merkmale geprägt:

- die geografische Lage an einer EU-Außengrenze und die damit verbundene Zuständigkeit für alle auf dem Landweg über diese Grenze in die EU einreisenden Flüchtlinge (Dublin-II-Verordnung),

- eine seit Anfang der 1990er Jahre steigende Zahl von Anträgen auf Flüchtlingsschutz, eine niedrige Anerkennungsquote und eine hohe Zahl Gedulde$\operatorname{ter}^{315}$,

- eine große Zahl an Flüchtlingen, die ihr Verfahren nicht zu Ende verfolgen (vgl. Iglicka 2001a, S. 10) und (unerlaubt) weiterwandern,

- die routinemäßige Inhaftierung fast aller AntragstellerInnen und den Ausbau der Haftkapazitäten an der polnischen Ostgrenze,

- soziale Probleme, die je nach Status (AntragstellerInnen, Geduldete oder anerkannte Flüchtlinge) unterschiedlich stark ausgeprägt sind: medizinische, psychologische und finanzielle Unterversorgung, Perspektivlosigkeit, fehlende Arbeitsmöglichkeiten sowie fehlender Wohnraum. AntragstellerInnen leben aufgrund mangelnder anderer erschwinglicher Wohnmöglichkeiten überwiegend in Heimen.

Die Erklärungen für die Probleme auf der Umsetzungsebene gehen auseinander: Während einerseits erklärt wird, Polen produziere trotz niedriger Antragszahlen einen ,Flüchtlingsnotstand ${ }^{6316}$, setze die schlechte Situation von Flüchtlingen in Polen also strategisch ein, wird andererseits postuliert, Ursache des gaps seien mangelnde finanzielle Möglichkeiten ${ }^{317}$. Letzteres lässt sich für viele soziale Aspekte bestätigen, hinsichtlich derer polnische BürgerInnen ebenfalls mit einer prekären Situation konfrontiert sind, wie zum Beispiel im Wohnungs- und Gesundheitssektor sowie beim Zugang zu kostenlosem Rechtsbeistand. Diese Auffassung wurde sowohl von den von mir interviewten polnischen GesprächspartnerInnen formuliert, als auch gegenüber Barbara Eßer et al. (2005, S. 30), die ebenfalls Interviews mit ExpertInnen in Polen durchgeführt haben:

„Unsere polnischen Gesprächspartner thematisieren offen die Defizite im polnischen Asylsystem. Zu den angegebenen Gründen für solche Defizite gehören an erster Stelle finanzielle Engpässe im überstrapaziierten polnischen Budget, die unzureichende Unterstützung durch europäische Förderprogramme [...], sowie insbesondere auch die rechtlichen Steuerungsmechanismen der europäischen Asylpolitik, die derzeit noch nicht im Sinne einer stabilen europäischen Schutzgewährung für Asylbewerber und Flüchtlinge greifen. "(ebd.)

\footnotetext{
315 Vgl. Tabelle 9 Anträge auf Flüchtlingsschutz in Polen seit 1992 im Anhang.

316 „Der polnische Staat eskaliert derzeit die Flüchtlingssituation“ und „praktiziert eine selektive Zuspitzung der Situation“ (Dietrich 2005, S. 389).

317 Diese Ansicht vertraten die meisten polnischen InterviewpartnerInnen.
} 
Um jenseits der Kluft zwischen Gesetz (Output) und Umsetzung (Outcome) auch von einer Kluft zwischen policy-Zielen (Input) und -Ergebnissen (Outcome) sprechen zu können, müssten zunächst einmal die Ziele polnischer Migrationspolitik identifizierbar sein. ${ }^{318}$ Solche konkreten Ziele lassen sich nach Ansicht eines Interviewpartners jedoch nur im Hinblick auf das Repatriierungsprogramm erkennen:

„Das Problem ist, dass man bezüglich der Gap-Theorie normalerweise auch von Zielen spricht. In der polnischen Migrationspolitik. haben wir aber diese Zielricbtung nicht. Meiner Meinung nach richtet sich der polnische Staat bei der Ausgestaltung der Migrationspolitik mehr nach internationalen Anforderungen. So wird geschaut, was die EU fordert und welches die polnischen Interessen sein könnten, dementsprechend wird anschließend gehandelt. Über eine wirklich ernsthafte, polnische Zielsetzung kann man eigentlich nur in Bezug auf das Repatriierungsprogramm sprechen. Da galt es möglichst viele, wenn nicht sogar alle ethnischen Polen, vor allem aus Kasachstan wieder zurïck ins Land zu bolen. Allerdings kebrten nur 5.000 Menschen zurück. Hier kann man einen Policy-Gap ausmachen. Meiner Meinung nach ist das die einzige Lücke: zwischen den hohen Erwartungen des Programms und den Ergebnissen. In Polen ist nach wie vor die Frage der Auswanderung weitaus wichtiger, als die der Einwanderung. "(O S. 4f.)

Die Frage nach den Zielen der polnischen Politik führt zur Untersuchung der Einflussfaktoren, die Gegenstand des folgenden Abschnitts sein soll.

\subsubsection{Resümee der Einflussfaktoren}

Ziel ist es im Folgenden, vor der Folie der vorangegangenen Ausführungen die Einflussfaktoren näher zu bestimmen, die die Entwicklung des polnischen Asylregimes präg(t)en. Angesichts der Komplexität politischer Systeme bzw. Regime und Entscheidungsstrukturen ist es naturgemäß schwer, die entscheidenden Einflussfaktoren herauszufiltern. Zudem gibt es weder die Interessen des polnischen Staates oder der polnischen Gesellschaft noch ein homogenes Gebilde ,EU': So fanden sich zum Beispiel auch in der - häufig auf den Widerstand reduzierten Diskussion um die Einführung der Visapflicht für BewohnerInnen der Nachbarstaaten Polens GegnerInnen und BefürworterInnen. Allerdings wird es dort, wo es keine Debatte gibt - wie in der polnischen Flüchtlingspolitik - schwierig, Interessen zu lokalisieren. Die Gefahr ist groß, Interessen zu unterstellen, die aus der ,Erfahrung' mit anderen Fällen herrühren. Voraussetzung für eine solide und plausible Darstellung von Erklärungsansätzen ist es daher, sich auf ein möglichst breit gefächertes Spektrum an Daten und Interpretationen zu stützen, möglichst unvoreingenommen auf das Material zu schauen und die Kompatibilität der Faktoren und Argumente zu prüfen.

318 Vgl. dazu auch 6.2 Keine Migrationspolitik in Polen?, S. 244. 
Die Flüchtlingspolitik in Polen ist gekennzeichnet durch einen sehr geringen Politisierungsgrad. ${ }^{319}$ Die Etablierung des Flüchtlingsschutzregimes war ein bürokratischer Akt (M S. 3), der von Juristen aus dem Innenministerium und dem URIC/UdSC sowie Mitarbeitern des Grenzschutzes vollzogen wurde. Vor diesem Hintergrund soll es nun um die Beantwortung der folgenden zwei Fragen gehen: Wie können 1) die fehlende politische Auseinandersetzung und 2) die - trotz fehlender Politisierung - vergleichsweise restriktive Ausgestaltung erklärt werden?

Wie die Ausführungen gezeigt haben, kann die (restriktive) Ausgestaltung des polnischen Flüchtlingsschutzregimes weder mit Erfahrungen aus der polnischen Geschichte von Aus-, Ein- und Transitwanderung begründet werden, die zum Beispiel eine besondere Art Polens im Umgang mit Flüchtlingen geprägt hätten. Die lange Geschichte Polens als Herkunftsland von EmigrantInnen und Flüchtlingen - also die ,eigene' Emigrationsgeschichte - haben jedenfalls nicht zu einer öffentlichen Debatte über den Umgang mit Flüchtlingen, die nun Schutz in Polen suchen, geführt und auch nicht zu einer liberalen Gesetzgebung im Flüchtlingsbereich. Ebenso wenig kann ein besonderer Handlungsdruck aufgrund einer hohen Zahl Schutz suchender Flüchtlinge oder eine polarisierte öffentliche Auseinandersetzung mit dem Thema, wie wir sie in westeuropäischen Einwanderungsländern vorfinden, festgestellt werden. Das vorliegende Datenmaterial - die gesetzlichen Regelungen und ihre Ähnlichkeit mit den EU-Vorgaben ${ }^{320}$ sowie die Interviews mit ExpertInnen, die an der Ausarbeitung ${ }^{321}$ und Implementierung ${ }^{322}$ der Politiken beteiligt waren, und die einschlägige (polnische) Literatur - bestätigen fast einstimmig die These, dass die EU-Vorgaben den entscheidenden Anstoß für die Entwicklung gaben und die konkrete Ausgestaltung des Flüchtlingsschutzregimes durch die übrigen dargestellten AkteurInnen vor Ort stark geprägt haben. ${ }^{323}$ In

\footnotetext{
319 Beispielhaft sei hier auch auf die Aussage eines Interviewpartners verwiesen: „Meiner Meinung nach ist das Thema in Polen kein politisches. Es spaltet nicht die politische Szene, wie es in Deutschland oder in Frankreich der Fall ist. [...] Ich mache das auch an der Entstehung des Ausländergesetztes von 1997 fest. Es gab dazu keine öffentliche oder politische Debatte. [...] Wenn ich auf die polnische Migrationspolitik schaue, würde ich diesen Fakt unterstreichen: die nichtpolitische Entstehung und Existenz.“ (O S. 5)

$320 \mathrm{Zu}$ nennen wären unter anderem: temporäre Aufenthaltserlaubnisse, Inverantwortungnahme der Beförderungsunternehmen für die Ausstattung ihrer Passagiere mit den erforderlichen Papieren sowie die sichere Dritt- und Herkunftsstaatenregelung, ,offensichtlich unbegründete` Anträge und beschleunigte Verfahren.

${ }^{321}$ H S. 1; J S. 2, 12.

322 G S. 2; G S. 1; O S. 6.

323 In der Literatur wird dies meist vertreten, ohne weitere Belege anzuführen oder andere Aspekte durchzudiskutieren. Einzige Ausnahme ist Kicinger 2005. Vgl. folgende Beispiele für den einstimmigen Tenor: „The development of the Polish asylum regime has been shaped by adoption of international standards on Poland's path to European Union“ (Kępińska/Stola 2004, S. 168); „At this stage, policy development has reflected mainly Poland's efforts for EU accession and related negotiations.“ (ebd., S. 173); „Adopting EU acquis turned out to be similar to following a moving target“ (ebd., S. 164, Hervorh. i. Orig.); „Zentraler Punkt der Beitrittsverhandlungen im Kapitel ,Justiz und Inneres“ war stets die Sicherung der Ostgrenze Polens gewesen, denn das größte migra-
} 
anderen migrationspolitischen Bereichen - wie zum Beispiel der Visapolitik - gab es durchaus Widerstände gegen die Forderungen der EU. Dabei zeigten sich polnische Interessen, welche zumindest nicht völlig widerstandslos dem Beitrittsziel untergeordnet wurden. In der Flüchtlingspolitik gab es jedoch keine Debatte obwohl mit dem Thema für Polen wichtige und mit Blick auf die polnische Geschichte geradezu ,klassische ${ }^{6}$ Themen wie Zugehörigkeit, Nationalismus und Identität verknüpft sind (vgl. Migrationsprozesse: Zusammenspiel von Auswanderung und Rückkehr, Einwanderung und Transitmigration, S. 219).

Die Gründe für den im Vergleich zur Visapolitik unaufgeregten Umgang mit dem Flüchtlingsthema sind vielfältig: Zum einen lassen sich Erklärungen finden, die nahelegen, dass der polnische Gesetzgeber mit dem Thema vor allem pragmatisch umgegangen ist. Dazu gehört, dass im Jahr 1997, während der ersten zentralen Novelle des Flüchtlingsschutzgesetzes insgesamt 190 Gesetze verabschiedet wurden. Eine öffentliche Diskussion aller Themen war so kaum möglich, ja angesichts dieses Regelungsvolumens aus Sicht des Gesetzgebers auch nicht wünschenswert. Zudem wird vielfach auf die geringe Zahl an Flüchtlingen verwiesen, die kaum die Aufmerksamkeit der Öffentlichkeit auf sich ziehen konnte. Auf der politischen Agenda in Polen standen daher anstelle des Flüchtlingsschutzes ganz andere Fragen im Mittelpunkt: Es galt, „[d]ie doppelte Aufgabe der politischen und ökonomischen Systemtransformation“ (Ziemer 1997, S. 42) zu bewältigen. Insofern betrafen die Auseinandersetzungen im Sejm und in der Öffentlichkeit vor allem die wirtschaftspolitische Ausrichtung und die sozialen Härten der Systemtransformation, aber auch das Verhältnis zwischen Kirche und Staat und hierbei insbesondere das bis zur, Wende' liberale Abtreibungsrecht sowie den Umgang mit der sozialistischen Vergangenheit (vgl. Matthes 2002, S. 99f.). Auch die Diskussion um die Verfassung band jahrelang Kräfte: Statt wie geplant 1991 konnte die neue Verfassung erst im Jahr 1997 nach einer acht Jahre dauernden Debatte in Kraft treten (vgl. Bos 2004). Der Flüchtlingsschutz war also kein besonders drängendes Thema und aufgrund von Polens Geschichte als Auswanderungsland gab es bis dato auch kaum Erfahrungen im Umgang mit migrationspolitischen Regulierungen und mit der Etablierung eines öffentlichen Diskurses in diesem Politikbereich.

tionspolitische Problem des Landes liegt in der über 1000 Kilometer langen, schwer zu sichernden Grenze zu Weißrussland und der Ukraine“ (Currle 2004, S. 359); EU-Beitritt als „external catalyst for legislative change“ (Iglicka et al. 2005, S. 2); „policy in the Central and Eastern European Countries has arisen almost entirely as a result of the requirements of EU accession and that EU policy models and ideas about borders, security and insecurity have been exported to CEE countries“ (Geddes 2003, S. 173); „the immigration policy frame adopted by the CEECs has been largely inspired by the requirements of accession to the EU and dominated by security concerns" (Geddes 2003, S. 184); „eine restriktive Migrationspolitik wurde in den EU-Staaten während der Beitrittsverhandlungen als Voraussetzung für den EU-Beitritt Polens gesehen" (Currle 2004, S. 362). 
So wurde in relativ kurzer Zeit weitgehend unbehelligt von der Öffentlichkeit ein Flüchtlingsregime eingerichtet, das nur zu einem Teil aus der Notwendigkeit der Regelung der Belange der zwar zunehmenden, aber im Vergleich noch immer geringen Zahl von Flüchtlingen erwuchs. Angesichts der fehlenden politischen Mobilisierung um das Thema innerhalb Polens muss der Haupteinflussfaktor außerhalb des Landes gesucht werden. Unter den externen Einflussfaktoren (zum Beispiel nachbarschaftliche Beziehungen mit Deutschland, aber auch Ukraine) war also der angestrebte EU-Beitritt Polens zentraler Motor für die Entstehung der flüchtlingspolitischen Regelungen. Bei den Beitrittsverhandlungen bildete der Bereich ,Justiz und Inneres' einen wichtigen Gegenstand. Dabei ging es insbesondere um die ,Sicherung` der über 1000 Kilometer langen Ostgrenze Polens zu Weißrussland und der Ukraine. Bis dahin hatte sich insbesondere Deutschland für grenz- und migrationspolitische Maßnahmen in Polen engagiert. Mit den Beitrittsverhandlungen trat die EU als entscheidende Akteurin in diesem Bereich auf und bot mit der Aussicht auf eine EU-Mitgliedschaft Polens einen gewichtigen Anreiz zur Kooperation. Die EU-Vorgaben im Flüchtlingsbereich wurden also mit Rücksicht auf den auf der Prioritätenskala ganz oben stehenden Beitrittsprozess übernommen. Kontroversen, langwierige Diskussionen und Widerstand gegen die EUVorgaben hätten zu einer Verzögerung des Beitrittsprozesses führen können. Nichtsdestotrotz sollte nicht ausgeblendet werden, dass Polen in anderen Bereichen zumindest rhetorisch häufig durch „Unnachgiebigkeit und Interessenpolitik“ (Lang 2006, S. 3) aufgefallen ist und dies nicht erst unter der Regierung der Kaczyńskis (vgl. ebd.). So wird Polen auch als „,solidarischer Egoist“ in der EU“ bezeichnet, der „Loyalität anmahnt, selbst aber eine Politik der Solidarität à la carte praktiziert.“ (ebd.) Doch selbst unter der Führung der Kaczyński-Zwillinge stand der EU-kritischen Rhetorik in verschiedenen Bereichen eine aktive praktische Zusammenarbeit gegenüber. Insbesondere im Bereich innere (Europäischer Haftbefehl) und äußere Sicherheit (EU-Mission im Kongo) zeigte sich Polen kooperativ (vgl. Lang 2006, S. 4). Das Thema Sicherheit wird schon seit den 1990er Jahren in Polen großgeschrieben (vgl. Kicinger 2005). Es spiegelt sich - wie oben gezeigt - auch in der Flüchtlingspolitik wider.

Insofern lassen sich - neben dem zentralen Beitrittsziel - zwei Aspekte identifizieren, die aus Sicht der polnischen EntscheidungsträgerInnen die Zustimmung zu den EU-Regularien im Flüchtlingsbereich erleichterten: Erstens verknüpft die Migrations-Agenda der EU Migration mit Sicherheitsfragen, die auch in der polnischen Politik eine bedeutende Rolle spielen. Zweitens ermöglichte die Implementierung von Flüchtlingsschutzgesetzen auf der Basis von EU-Regularien eine Positionierung Polens als Staat, der sich zu Demokratie und Menschenrechten bekennt und sich somit als Teil einer modernen europäischen Wertegemeinschaft präsentiert (G S. 1; O S. 6). Der große Druck, der von dem Beitrittsziel ausging, und die Dethematisierung in der Öffentlichkeit würden auch erklären, warum sich in der Entwicklung des polnischen Flüchtlingsschutzregimes kaum Bezüge zur 
polnischen Geschichte als Herkunftsland von Flüchtlingen und EmigrantInnen und auch zu seiner aktuellen Konstitution als Auswanderungsland finden lassen.

Der Einfluss der EU ist nicht nur ein direkter, das heißt er lässt sich nicht nur an der Vorgabe und Übernahme rechtlicher Leitlinien ablesen, sondern er macht sich auch indirekt bemerkbar - indem zum Beispiel AkteurInnen die Perspektive der EU oder der anderen Mitgliedstaaten übernehmen und entsprechend handeln. ${ }^{324}$ Hier wird es immer schwieriger, zwischen ,eigenen' polnischen und EUInteressen zu unterscheiden. Ein anschauliches Beispiel für die Problematik ist die Verschärfung der Regelungen für die Unterbringung der Geduldeten. Hierfür gibt es keine offizielle EU-Vorgabe und auch keinen, objektiven' Handlungsbedarf, da angesichts der zum Zeitpunkt der Verabschiedung der Änderung leicht zurückgegangenen Antragszahlen auf Flüchtlingsschutz genügend Plätze in den Heimen zur Verfügung standen. Es scheint also, als hätten sich unter den BürokratInnen Abschreckungsprämissen breit gemacht, die entweder eine Übernahme der restriktiven Vorgehensweise der westlichen Nachbarn und der EU darstellen oder aber im Gegenteil als Opposition gegen die Zumutungen als ,Türhüter ${ }^{\varsigma}$ der EU im Osten interpretiert werden können: Denn durch die Verschärfung der Situation der Geduldeten erhöht Polen die Wahrscheinlichkeit, dass sich diese auf den Weg nach Westen (oder zurück in die Herkunftsländer) machen. Die Äußerungen von Jan Węgrzyn, dem Leiter des UdSC, lassen beide Deutungen zu: Einerseits beanstandet er, die EU stelle zu wenig Hilfen bereit (vgl. Eßer et al. 2005, S. 30). Andererseits begründet er die Verschärfung der Unterbringungssituation mit der vermeintlichen Ausbeutung des Unterstützungssystems durch die Flüchtlinge (vgl. Marczuk/Kosowicz 2006) - eine Argumentation, die auch in der deutschen Asyldebatte populär ist.

Helmut Dietrich (2005, S. 389) vermutet:

„Es gibt offensichtlich eine breite Interessensübereinkunft, die vom polnischen Staat bis zu den EU-Think. Tanks und den maßgeblichen Internationalen Organisationen reicht: man will die Flüchtlings- und Migrationssituation nicht zu einem allgemeinpolitischen Hetzthema machen, sondern praktiziert eine selektive Zuspitzung der Situation."

Jedoch ist die politische Dethematisierung von Flüchtlingsbelangen nicht gleichbedeutend mit einem öffentlichen Desinteresse oder einer durchweg positiven Einstellung gegenüber AusländerInnen im Allgemeinen und Flüchtlingen im Besonderen. Die Untersuchungen von Ewa Nowicka geben Aufschluss darüber, inwieweit der Alltag speziell von Menschen aus afrikanischen Ländern, die in Polen leben, von rassistischen Vorfällen geprägt ist. Sie hat MigrantInnen aus verschiedenen afrikanischen Ländern über ihre Erfahrungen in Polen befragt: „Many Africans living in Warsaw and in other cities have had unpleasant experiences with the Poles.“ (Nowicka 2005, S. 282) Die von ihr befragten Männer berichten von

${ }^{324}$ Vgl. die bereits angeführte Interviewpassage mit dem Grenzschutz zu dessen Rolle. 
Anfeindungen oder Übergriffen auf der Straße sowie von häufig negativen Erfahrungen mit den Familien ihrer polnischen Ehefrauen (ebd., S. 282f.). Daneben gibt es in den Medien hin und wieder Berichte über rassistische Überfälle, wie im Juli 2006 auf SchauspielerInnen des ,Migrator'-Theaters. Zwar wurde über den Überfall auf die SchauspielerInnen in der Presse berichtet, das Ganze führte aber nicht zu einer weiteren Thematisierung der Problematik. ${ }^{325}$ Die Einstellungen von PolInnen gegenüber Flüchtlingen im Speziellen werden seit einigen Jahren im Kontext des Weltflüchtlingstages am 20. Juni im Auftrag des UNHCR erhoben (vgl. TNS OBOP 2004; TNS OBOP 2006). Dabei zeigt sich, dass der weit überwiegende Teil der befragten PolInnen es zwar angesichts der Geschichte Polens als Herkunftsland von Flüchtlingen als eine Verpflichtung sieht, dass das Land heute selbst Flüchtlinge aufnimmt (vgl. TNS OBOP 2004; TNS OBOP 2006). Allerdings steht dieser Einstellung wenig Bereitschaft zu praktischer Unterstützung für Flüchtlinge gegenüber: Nur wenige Befragte sind der Meinung, dass Flüchtlinge Hilfe bei der Arbeits- oder Wohnungssuche erhalten sollten (vgl. ebd.).

Die Europäisierung der Flüchtlingspolitiken ist eng verknüpft mit einer NGOisierung in diesem Politikbereich. Bei der Installierung der Regelungen in Polen spielten flüchtlingspolitische NGOs eine entscheidende Rolle, sie wirkten gleichsam als Katalysatoren: Als ExpertInnen (nicht nur für Menschenrechte im Allgemeinen, sondern auch für die EU-Vorgaben) nahmen sie Stellung zu Gesetzesentwürfen und erleichterten so die Arbeit der GesetzgeberInnen. Durch ihre direkte Arbeit mit Flüchtlingen zum Beispiel in der Rechtsberatung gehören sie nach eigener Einschätzung - zu den wenigen, die das notwendige Know How in Flüchtlingsfragen besitzen. Nach Darstellung der an prominenter Stelle in diesem Prozess vertretenen Helsinki Stiftung ging es in den entsprechenden Konsultationen zwar kontrovers zu, letztlich hätten sich aber einige Forderungen umsetzen lassen (J S. 2). Europäisierung und NGOisierung von Flüchtlingspolitiken verlaufen daher nicht nur parallel - sie sind vielmehr stark miteinander verflochten. Denn die NGOs finanzieren sich zu einem großen Teil über Projekte aus EUMitteln und leisten einen wichtigen Beitrag zur Legitimierung der EU-Politiken,

325 Eine viel breitere und stark aufgeladene öffentliche Auseinandersetzung fand stattdessen Anfang des Jahres 2007 statt, als - wie bereits oben kurz erwähnt - ein in Polen prominenter Flüchtling verdächtigt wurde, wissentlich mehrere Frauen mit dem HI-Virus infiziert zu haben. Das Thema schlug große Wellen in der Öffentlichkeit - Internet-Blogs füllten sich mit rassistischen Auslassungen, das Innenministerium erwog verpflichtende HIV-Tests für alle AsylbewerberInnen. Eine Diskussion über den individuellen Schutz vor dem Virus fand hingegen nicht statt, stattdessen hatte das Gesundheitsministerium einige Monate zuvor eine Gesetzesinitiative gegen die Verbreitung von Verhütungsmitteln unterstützt (vgl. Neue Zürcher Zeitung vom 15.01.2007). In der rassistisch gefärbten öffentlichen Diskussion meldeten sich die beiden großen liberalen Tageszeitungen - die Gazeta Wyborcza und die Rzeczpospolita - mit differenzierteren Wortmeldungen. In der Gazeta Wyborcza forderte Adam Leszczyński das Verbrechen zu verurteilen, nicht aber die Hautfarbe des mutmaßlichen Täters (vgl. Gazeta Wyborcza vom 10.01.2007). 
indem sie ihre menschenrechtliche Einbettung illustrieren. Eine Schlüsselposition kommt dabei dem UNHCR zu, der zwar keine NGO ist, aber gewissermaßen als flüchtlingspolitischer ,Entwicklungshelfer ${ }^{6}$ in allen osteuropäischen Staaten die Installierung von Flüchtlingsschutzregimen von Anfang an begleitete und als, Geburtshelfer für zahlreiche NGOs fungierte. Nach der Etablierung relativ stabiler Strukturen zog er sich aus dem Geschehen zurück. Eine ähnliche Rolle des UNHCR konstatieren Sabine Hess und Serhat Karakayalı (2007, S. 49) für die Türkei: „dem UNHCR [kam] die wichtige Rolle zu, das diskursive und politische Terrain zur Übernahme der EU-Migrationspolitik zu präparieren sowie ,Akteure‘ und Subjektpositionen (für) diese(r) Politik zu organisieren.“

MigrantInnen werden weder im Gesetzgebungsprozess noch - wie oben mit Bezug auf die Beobachtungen von Anna Krasteva dargestellt - in der Praxis der NGOs als AkteurInnen wahrgenommen oder gar einbezogen. Dennoch widersetzen sie sich vielfach den Zumutungen dieser Politik und insbesondere dem Verbot, den Zielort ihrer Wanderung bzw. Flucht selbst wählen zu dürfen, indem sie weiterwandern sowie den schlechten Lebensbedingungen in Polen, zum Beispiel durch Hungerstreiks. Die Weiterwanderung der Flüchtlinge wird von den politischen EntscheidungsträgerInnen als einfache Entlastungsmöglichkeit für Polen gesehen. Das interviewte Mitglied des ,Flüchtlingsrates' war der Ansicht, wenn Polen nicht bereit sei die Flüchtlinge aufzunehmen - wozu es seiner Ansicht nach bereit sein sollte - dann sollte doch einfach allen Flüchtlingen der GFK-Status verliehen werden:

\section{„Ihnen den Flücbtlingsstatus geben und in dem Moment, in dem sie den bekommen, gehen sie. Wenn sie den Duldungsstatus kriegen, können sie nicht gehen und so bleiben sie" (N S. 5).}

Wie sich die Regelungen letztlich konkret für die Flüchtlinge darstellen, ist von der Umsetzung durch die Behörden abhängig. Die Gesetze wurden in Einklang mit den EU-Regelungen gebracht - auf formaler Ebene wurde daher die geforderte Angleichung vollzogen, obgleich die EU-Kommission an einzelnen Regelungen Kritik übt. ${ }^{26}$ Doch wie es tatsächlich an der Grenze, bei den Anhörungen und in den Heimen und geschlossenen Lagern aussieht, darüber können die Gesetze keine Auskunft geben. Zwar verwiesen einige InterviewpartnerInnen auf den starken Zentralismus in Polen, der eine Lücke zwischen Gesetz und Praxis verhindere, indem zum Beispiel Innenministerium und URIC/UdSC sowohl das Gesetz ausgearbeitet haben als auch mit dessen Umsetzung betraut sind. Doch diese Sicht würde zum einen verkennen, dass die EU-Angleichung im Beitrittsprozess vor allem auf formaler Ebene stattfand und zum anderen, dass der Implementation durch die Behörden grundsätzlich eine Eigendynamik innewohnt, die von politi-

\footnotetext{
326 In der Evaluation der Umsetzung der Aufnahmerichtlinie wird kritisiert, dass die Richtlinie in Polen sowie einigen anderen Mitgliedstaaten nicht für inhaftierte Flüchtlinge gelte $(\operatorname{KOM}(2007)$ 745).
} 
schen Weisungen, den vorhandenen Ressourcen, einzelnen MitarbeiterInnen in Behörden und vielen weiteren Faktoren abhängig ist. Zunächst galt es also, die formale Angleichung an die EU-Vorgaben nachzuweisen, die Implementierung der Regelungen stand auf einem anderen Blatt. Diese ist stark durch finanzielle Restriktionen und Engpässe im Wohnungs- und Gesundheitssektor sowie auf dem Arbeitsmarkt geprägt, aber auch Gegenstand politischer Entscheidungen. So lässt die stark gesunkene Zahl von Anerkennungen tschetschenischer AntragstellerInnen bei gleichbleibender Ausgangslage auf ein politisches Umlenken schließen (D S. 8). ${ }^{327}$ Auch gibt es Vermutungen, dass die geringe Zahl von anerkannten afrikanischen Flüchtlingen auch auf rassistische Stereotype bei den Behörden zurückzuführen ist. ${ }^{328}$

Zusammenfassend bleibt also festzuhalten, dass sich in Polen nach Jahrzehnten als Auswanderungsland in den letzten zwei Dekaden eine Entwicklung beobachten lässt, die auf einen nicht allzu fernen Wandel zu einem Einwanderungsland hindeutet. Dies ist nicht zuletzt Folge der wirtschaftlichen Konsolidierung Polens und seines EU-Beitritts. Doch Polen wird nicht nur zunehmend attraktiv für MigrantInnen aus den östlichen Nachbarstaaten, die durch das Lohngefälle zwischen Polen und dem eigenen Herkunftsland im informellen Sektor in Polen ein höheres Einkommen erzielen können. Es ist aufgrund der Zuständigkeitsregelungen in der EU gleichzeitig auch unfreiwilliger Endpunkt der Wanderung zunehmend zahlreicherer MigrantInnen und Flüchtlinge geworden, die das Land bislang als Transitstation genutzt haben und eigentlich weiter nach Westen wollen. Für sie hat der grundlegende Wandel der Bedeutung und der Durchlässigkeit der polnischen Ostgrenze einschneidende Konsequenzen: Ihre Einreise ist regulär nur

327 „Ende der 90er, Anfang 2000 als es einige Tschetschenen gab, erinnere ich mich, dass alle Tschetschenen Flüchtlingsstatus erhalten haben. Soweit ich mich erinnere, war Polen eines der zwei Länder, die Tschetschenen Flüchtlingsstatus garantierten: Polen und Kanada. Und alle anderen waren unwillig, das zu tun. Aber irgendwie hat sich dann, also ich gehe davon aus, dass sich die Regierungspolitik dann geändert hat, nach einer ziemlich kurzen Zeit. Und sie entschieden, dass es aus politischer Sicht vermutlich nicht die beste Idee ist, allen Tschetschenen Flüchtlingsstatus zu geben, denn das könnte Russland, sagen wir, weniger glücklich machen. Also irgendwie hat sich meiner Ansicht nach die Regierungspolitik, also wenn es überhaupt eine Regierungspolitik gibt, geändert und jetzt ist es für uns ziemlich schwierig, Flüchtlingsstatus für unsere Klienten zu bekommen." (D S. 8)

328 „Aber auf der anderen Seite erhält fast keiner unserer afrikanischen Klienten Flüchtlingsstatus. Wir hatten sogar vor ein paar Wochen ein Treffen hier [...], der [...] jetzt im URIC arbeitet. Wir haben ihn eingeladen, damit er [...] über das Verfahren usw. erzählt. Und er [...] sagte, dass die meisten der afrikanischen Klienten keinen Flüchtlingsstatus bekommen. Und er hat gewissermaßen angedeutet, dass die Behörden den afrikanischen Klienten nicht glauben. Sie denken, dass im Grunde alle Afrikaner lügen, dass sie falsche Dokumente haben, dass sie nicht aus den Ländern kommen, von denen sie das behaupten; dass sie nur hier sind, um das Verfahren auszunutzen und einen Aufenthalt zu bekommen. [...] Es ist wirklich schwer zugunsten der Flüchtlinge zu argumentieren, wenn die Regierung eine solche negative Einstellung dieser bestimmten Gruppe gegenüber hat. Leute aus Somalia erhalten allerdings einfacher Flüchtlingsstatus als irgendeine andere afrikanische Gruppe.“ (D S. 11) 
noch möglich, wenn sie einen Antrag auf Flüchtlingsschutz direkt an der Grenze stellen. Während das in der Verfassung kodifizierte Recht auf Asyl praktisch keine Anwendung findet und der einfachgesetzlich geregelte Flüchtlingsstatus nach der Genfer Flüchtlingskonvention nur selten vergeben wird, erhält der überwiegende Teil der Flüchtlinge den aus sozialer Sicht hoch problematischen Duldungsstatus. Wer (es sich leisten) kann, versucht weiter nach Westen zu reisen, auch wenn die Dublin-II-Verordnung etwas anderes vorsieht. Die polnische Administration hat binnen kurzer Zeit ein Flüchtlingsschutzregime installiert, das sich eng an den Vorgaben der EU orientiert. Die EU spielt also im Hinblick auf MigrantInnen bzw. Flüchtlinge in Polen in dreierlei Hinsicht eine wichtige Rolle: Zum einen wurde die polnische Ostgrenze im Zuge von Polens EU-Beitritt zur EU-Außengrenze und hat dabei ihren Charakter einer einst relativ durchlässigen Grenze eingebüßt. Zum anderen muss(te) es die EU-Vorgaben aus den flüchtlingsrechtlichen Richtlinien umsetzen und ein Flüchtlingsschutzregime installieren, um die Voraussetzungen für den EU-Beitritt zu erfüllen. Und drittens wurde Polen durch die seit dem EU-Beitritt gültige Dublin-II-Verordnung für alle auf dem Landweg über Polen einreisenden Flüchtlinge als verantwortlich erklärt. Gleichzeitig ist die EU mit ihrem Europäischen Flüchtlingsfonds ein wichtiger Finanzier vieler Flüchtlingshilfeorganisationen. Diese starke Stellung der EU erklärt die geringe Politisierung und bürokratisch geprägte Regulierung der Flüchtlingspolitik in Polen, die bislang den Weg in den öffentlichen Diskurs nicht geschafft hat - trotz der langen Geschichte Polens als Auswanderungs- und Transitland und der groBen Bedeutung nationaler und ethnischer Zugehörigkeitskonstruktionen. 


\section{Flüchtlingsschutz in Deutschland und Polen}

Nach der eingehenden Untersuchung der Asylregime Deutschlands und Polens vor dem Hintergrund der Konstituierung eines Asylregimes auf EU-Ebene sollen die Entwicklungen in den beiden Ländern im Folgenden kontrastiert, miteinander in Beziehung gesetzt und zusammenfassend erläutert werden. Schließlich wird am Beispiel der Ukraine ein kurzer Ausblick auf die Entwicklungen gegeben, die sich in diesem Kontext weiter ,ostwärts' vollziehen.

Deutschland und Polen zeichnen sich zunächst durch unterschiedliche Positionen im europäischen Asylregime aus, und zwar hinsichtlich:

(a) ihrer Tradition als Aufnahmeländer von Asylsuchenden,

(b) der Ausdifferenzierung des AkteurInnenfelds,

(c) ihrer Position im europäischen Integrationsprozess,

(d) der Bedeutung des Themas Asyl im öffentlichen Diskurs, die sich wiederum aus den ersten beiden Punkten ableitet und

(e) der Bedeutung bzw. Funktion, die dem jeweils restriktiven Flüchtlingsrecht zukommt.

Gleichzeitig sind beide Länder beim Thema Einwanderung und Asyl stark miteinander verwoben. Während Deutschland seit Jahrzehnten zumindest faktisch ein Einwanderungsland ist, war und ist Polen in erster Linie durch Auswanderung geprägt. Entsprechend hat sich auch das deutsche Asylrecht über Jahrzehnte entwickelt: von einem relativ liberalen individuellen Grundrecht auf Asyl, das mit 
Blick auf menschenrechtliche Verpflichtungen nach dem Zweiten Weltkrieg verankert wurde, zu einem heute im Zugang zum Asylverfahren sehr restriktiven Asylrecht bei gleichzeitiger sozialer Schlechterstellung der Asylsuchenden. Im Zuge der Verschärfungen des Asylrechts und der Grundgesetzänderung umgab sich die Bundesrepublik mit ,sicheren Drittstaaten ${ }^{\text {` }}$ - einer davon ist Polen. Im Einwanderungs- bzw. Asylbereich entwickelte sich vor diesem Hintergrund nach 1990 eine spezifische Dynamik zwischen den beiden Ländern, wie sie für das Verhältnis zwischen den Staaten im geografischen ,Kern' der EU einerseits und denen an ihren Außengrenzen andererseits charakteristisch ist: Indem die Bundesrepublik Deutschland Polen als, sicheren Drittstaat ${ }^{\top}$ deklarierte und zur Umsetzung der Drittstaatenregelung ein Rückübernahmeabkommen vereinbarte, verwies sie die Flüchtlinge, die Polen bis dato nur als Transitland betrachtet hatten, auf die $\mathrm{Zu}$ ständigkeit des östlichen Nachbarn. Polen musste sich ad hoc auf die neue Rolle als Zielland von Asylsuchenden einstellen.

Die in Polen angesichts der ersten eingereichten Asylanträge ergriffenen Maßnahmen behielten ihren provisorischen Charakter, bis mit den Verhandlungen über einen EU-Beitritt Polens neuer Schwung in diesen Politikbereich kam. Die EU-Kommission legt bei Beitrittsverhandlungen großen Wert auf den Bereich ,Justiz und Inneres ${ }^{6}$ - insbesondere, wenn ein Mitgliedstaat eine derart lange Grenze mit Nicht-Mitgliedstaaten teilt: Polen wurde mit dem Beitritt zum ,Hüter eines beträchtlichen Teils der EU-Außengrenze. Die nach dem Ende des Kalten Krieges relativ frei passierbare über $1.000 \mathrm{~km}$ lange Ostgrenze Polens sollte im Zuge des Beitrittsprozesses und schließlich mit der vollständigen Mitgliedschaft Polens im Schengener Abkommen einen erheblichen Bedeutungswandel erleben. Sie wurde zu einer überwachten EU-Außengrenze umgestaltet, während die Grenze zwischen Deutschland und Polen an Bedeutung verlor. Die bilaterale Zusammenarbeit Deutschlands und Polens bei der Umgestaltung der Grenzen und der Umstellung Polens von einem Transit- zu einem Aufnahmeland von Asylsuchenden wurde durch die Beitrittsverhandlungen zwischen Polen und der EU teilweise durch eine neue Zusammenarbeit auf supranationaler Ebene abgelöst. Die ,Rücküberstellungen' von Asylsuchenden aus Deutschland finden nun nicht mehr im Rahmen der Drittstaatenregelung und Rückübernahmeabkommen, sondern als Teil des EU-weiten Dublin-II-Verfahrens statt. In diesem Rahmen stellte Deutschland im Jahr 2006455 Rückübernahmeersuchen an Polen, während dieses nur in 27 Fällen Ersuchen an Deutschland stellte (vgl. Bundesamt für Migration und Flüchtlinge 2006).

Die unterschiedliche Stellung der beiden Länder im europäischen Asylregime korrespondiert auch mit ihrer Position im europäischen Integrationsprozess. Im Rahmen der Beitrittsvorbereitungen musste Polen EU-Vorgaben umsetzen, an deren Aushandlung es noch nicht als Mitglied der Union beteiligt war, wohingegen Deutschland die Ausgestaltung des Asylregimes auf EU-Ebene wesentlich mitbestimmt - und kaum Anpassungsleistungen zu erbringen hatte. Dies illustriert 
ein starkes Machtgefälle, das zwischen den alten und den neuen Mitgliedstaaten besteht: Um den angestrebten Beitritt zu erreichen, müssen die Kandidaten zahlreiche Bedingungen erfüllen, die ihnen von den alten Mitgliedstaaten auferlegt werden. Mit dem Beitritt zum ,gemeinsamen Besitzstand' übernehmen sie schließlich einen Gesetzeskorpus, an dessen Erstellung sie zwar bereits beteiligt waren, jedoch nicht als gleichberechtigte Verhandlungspartner. Polens Gesetzgebung zum Flüchtlingsschutz basiert daher im Wesentlichen auf den EU-Vorgaben. Insbesondere die Zuständigkeitsregelungen, wie sie die Dublin-II-Verordnung enthält, erweisen sich für Polen als entscheidender Faktor bei der Bestimmung seiner Rolle im EU-Asylregime: Flüchtlinge, die das Land bislang als ,Durchgangsstation“ betrachteten, müssen nun hier ihr Asylverfahren betreiben. Angesichts ihrer unterschiedlichen geografischen Lage stellt sich das Dublin-Verfahren nicht als ,solidarische Lastenteilung' zwischen den Mitgliedstaaten dar, sondern weist den Ländern an den Außengrenzen eine Hauptrolle bei der Durchführung von Asylverfahren zu. Viele MigrantInnen wollen aus unterschiedlichen Gründen (bspw. Familienbindungen, Lebensbedingungen, Arbeitsmarktchancen, Aussichten auf einen dauerhaften Aufenthaltsstatus) nicht in den zugewiesenen Ländern bleiben und versuchen, sich über diese Zuständigkeiten hinwegzusetzen - sie reisen ,illegal' weiter. Eine Gegenüberstellung der Zahlen zeigt, dass einige Staaten ebenso viele Rücküberstellungsanträge an andere Staaten richten wie an sie selbst gestellt werden und letztlich nur ein Teil der MigrantInnen tatsächlich zurückgeschoben wird. ${ }^{329}$ Für die Dublin-II-Verordnung stellt sich also sprichwörtlich die ,Sinnfrage $^{6}$. Dennoch wird an dem aufwändigen Verfahren festgehalten.

Im Laufe der Entwicklungen seit Anfang der 1990er Jahre hat die Bedeutung Polens als Asylland zugenommen. Inzwischen verzeichnet das Land Asylantragszahlen, die denen in der Bundesrepublik bis Mitte der 1970er Jahre ähneln - damals waren die so gestiegenen Zahlen in Deutschland Ausgangspunkt der Asyldebatte, die fortan immer wieder auf der politischen Agenda auftauchte. Im Vergleich der aktuellen Zahlen (2006) kommen in Polen rein rechnerisch 0,1 AsylantragstellerInnen auf 1000 EinwohnerInnen, in Deutschland sind es 0,3. In absoluten Zahlen verzeichnete Polen 2006 zwar nur rund ein Fünftel der in Deutschland gestellten Asylanträge, allerdings stellt dies einen starken Zuwachs dar angesichts der Tatsache, dass Polen Anfang der 1990er Jahre noch so gut wie keine AntragstellerInnen zu verzeichnen hatte. Gleichzeitig verliert das Thema in der Bundesrepublik an Bedeutung - sowohl hinsichtlich der Zahlen, die seit Jahren rückläufig sind, als auch im öffentlichen Diskurs.

Obwohl sich Polen also mit Blick auf die AsylbewerberInnenzahlen in einer ähnlichen Situation befindet wie Deutschland Mitte der 1970er Jahre, ist das Thema in beiden Ländern sehr unterschiedlich im öffentlichen Diskurs positioniert: Während in Deutschland die Debatte um das Asylrecht und dessen ,Miss-

${ }^{329}$ Für das Beispiel Deutschland vgl. im Abschnitt 5.3.3, S. 185: Asylverfahren. 
brauch ' den Diskurs fortan über viele Jahre bestimmt und vor allem in Wahlkämpfen immer wieder eine prominente Rolle gespielt hat, wurde das Thema Asyl in Polen so gut wie gar nicht öffentlich thematisiert, sondern ausschließlich durch bürokratische AkteurInnen entwickelt und ohne weiteres Aufsehen gesetzlich verankert. Dies kann einerseits mit den unterschiedlichen Vorgeschichten der beiden Länder erklärt werden: Während Deutschland beim Aufkommen der Asyldebatte gleichzeitig damit beschäftigt war, die mit der ,Gastarbeiterära ' in Gang gesetzten Einwanderungsprozesse zu regulieren, findet die Steigerung der Asylantragszahlen in Polen vor dem Hintergrund weiter fortbestehender Auswanderung sowie Pendelmigrationen aus den östlichen Nachbarstaaten statt. Dabei scheint die unterschiedliche Bedeutung im Diskurs ein Indiz dafür zu sein, dass das Agenda-Setting in diesem Bereich stark von den politischen EntscheidungsträgerInnen ausgeht. Wie für die Bundesrepublik dargestellt, wurde das Thema Asyl in Wahlkämpfen präsentiert ,as a cause of manifold problems“ (Faist 1994, S. 52). Eine solche Aufladung des Gegenstands von Seiten der politischen AkteurInnen ist in Polen bislang ausgeblieben, und es stellt sich die Frage, warum das Thema angesichts der massiven Umstrukturierungen der polnischen Gesellschaft und den damit verbundenen sozialen Verwerfungen nicht auch hier von den AkteurInnen als eine Art diskursive Externalisierungsstrategie eingesetzt wurde. Wie die Ausführungen gezeigt haben, liegt dies darin begründet, dass die Installierung des Asylregimes in Polen eine zentrale Voraussetzung für den EU-Beitritt darstellte. Eine öffentliche Verhandlung des Themas oder gar Stimmungsmache wäre angesichts der klaren Zielstellung - der Umsetzung des Acquis Communautaire - und der knappen Zeit bei gleichzeitig umfangreichem Regelungsvolumen eher kontraproduktiv gewesen. Bei der bürokratischen Installierung der EU-Vorgaben im Interesse des Beitritts erwies sich zudem die zentralistische Organisation des polnischen Staates als hilfreich, durch die der Gesetzgebungsprozess mit einer geringeren Zahl an Veto-Spielern konfrontiert war. In Deutschland sind die ständige Aktivierung des Themas und die Verkomplizierung der gesetzgeberischen Aushandlungen demgegenüber auch auf die häufig unterschiedlichen politischen Konstellationen in Bund und Ländern und die hier quasi ständig stattfindenden Wahlkämpfe zurückzuführen. Denn das Thema hat sich wiederholt als geeignet erwiesen, um WählerInnenstimmen zu gewinnen.

Doch auch die, andere' Seite - die Pro-Flüchtlingsargumentation - ist im polnischen Diskurs recht schwach. Aufgrund der im Vergleich zu Deutschland kleinen ,Szene' von Flüchtlingsschutzorganisationen und der aufgezeigten starken Involvierung dieser zivilgesellschaftlichen AkteurInnen in den Policy-Gestaltungsprozess, die die grundlegenden Prämissen der europäischen bzw. polnischen Asylpolitik nicht infrage stellen, gibt es auch von dieser Seite kaum diskursive Interventionen. Während sich in der Bundesrepublik im Zeitverlauf angesichts der nach und nach vollzogenen Verschärfung des Asylrechts eine aktive flüchtlingspolitische ,Szene' entwickelt hat, die ein breites Spektrum an Positionen umfasst, ist 
dieses Korrektiv in Polen ebenfalls eher Top-Down mit Unterstützung des UNHCR und der EU installiert worden.

Parallel zur unterschiedlichen Gewichtung des Themas im öffentlichen Diskurs gestaltet sich auch die Art und Weise bzw. die Bedeutung der ergriffenen Maßnahmen: Die soziale Schlechterstellung der Flüchtlinge ist in Deutschland öffentlichkeitswirksam als Abschreckungspolitik inszeniert worden. In Polen befinden sich Asylsuchende in einer in vielerlei Hinsicht sozial deutlich prekäreren Situation, diese wird aber (noch) nicht durch weitere künstliche Repressalien wie Sachleistungsvorrang und Residenzpflicht verschärft, sondern lässt sich weitgehend mit dem ohnehin in Polen schwach ausgeprägten sozialen Sicherungssystem und der mangelnden Infrastruktur erklären. So ist zwar laut polnischem Flüchtlingsschutzgesetz vorgesehen, dass Flüchtlinge ein Recht auf die gleiche Gesundheitsversorgung haben wie polnische BürgerInnen, faktisch ist deren medizinische Versorgung jedoch aufgrund des maroden polnischen Gesundheitssystems desolat. In Deutschland hingegen wurde die Schlechterstellung von Flüchtlingen bei der medizinischen Versorgung aktiv herbeigeführt, indem die Ansprüche auf Gesundheitsversorgung ins Asylbewerberleistungsgesetz integriert und damit von den Leistungen, die deutsche StaatsbürgerInnen in Anspruch nehmen können, abgekoppelt wurden. Auch die Unterbringung der Flüchtlinge in Heimen hat in Polen (noch) eine andere Bedeutung als in der Bundesrepublik, wo dies Teil des auf Abschreckung zielenden Sachleistungsprinzips ist. In Polen hingegen sind die Flüchtlinge schon deshalb gezwungen in den Heimen zu leben, weil kaum andere erschwingliche Wohnmöglichkeiten zugänglich sind. Zudem setzt Polen das teure Sachleistungsprinzip (noch) nicht in so umfassendem Maße ein wie die Bundesrepublik. Ebensowenig gilt dort ein einjähriges Arbeitsverbot für Asylsuchende, wie es in Deutschland vorzufinden ist. Bei der konkreten Ausgestaltung der Situation von Asylsuchenden ist wiederum auf den Gegensatz von Föderalismus und Zentralismus zwischen beiden Ländern hinzuweisen. In Deutschland kommt den Ländern und ihren Innenministern und schließlich der politischen Konstellation in den Kommunen eine wichtige Stellung bei der Gestaltung der Situation vor Ort zu. In Polen ist beispielsweise die Situation in den Lagern zwar auch abhängig von den jeweils vor Ort Verantwortlichen, aus Warschau werden jedoch klare Leitlinien vorgegeben.

Obwohl die prekäre Situation in Polen bislang in Teilen noch als Policy-gap zwischen Gesetzeslage einerseits und mangelnden infrastrukturellen und finanziellen Möglichkeiten zur Umsetzung andererseits gedeutet werden kann, zeichnet sich Polen insgesamt betrachtet als ein Land mit einem restriktiven Asylregime aus mit einer Tendenz der fortschreitenden Restriktion. Mit dem Acquis wurde Polen ein Maßnahmenkatalog an die Hand gegeben, der sich nicht durch Offenheit gegenüber Schutzsuchenden auszeichnet. Das ohnehin stattfindende Policy-Learning, das zunehmend Konvergenzen zwischen den Zufluchtstaaten bedingt, wurde hier durch die Vereinheitlichungsaktivitäten auf EU-Ebene institutionalisiert. In Ver- 
bindung mit ebenfalls EU-initiierten Austauschen zwischen PraktikerInnen in diesem Feld wird der Transfer von Instrumenten und Maßnahmen beschleunigt.

Nach der formalen Übernahme der EU-Vorgaben zieht Polen nun langsam mit einer restriktiven Akzentsetzung mit Deutschland und anderen EU-Mitgliedstaaten gleich: Beispielhaft sei hier einerseits auf die im Verhältnis zu den Anträgen auf Flüchtlingsschutz stark zurückgegangene Zahl der anerkannten Flüchtlinge verwiesen, die mit der in Deutschland vergleichbar ist. Allerdings lassen sich auch hier kleinere Unterschiede feststellen: Während Deutschland durchaus tschetschenische Flüchtlinge in die Russische Förderation abschiebt, sind solche Fälle aus Polen (im Untersuchungszeitraum) nicht bekannt. Polen erkennt den TschetschenInnen zwar ebenfalls überwiegend keinen Flüchtlingsstatus zu, schiebt sie jedoch in der Regel nicht in die Russische Förderation ab.

Ein anderes Beispiel für eine restriktive Akzentsetzung ist die Verschärfung der sozialen Situation der Geduldeten in Polen. Diese kann nicht mehr allein mit fehlenden finanziellen Ressourcen erklärt werden: Die starre Begrenzung ihrer Wohnzeit in den Wohnheimen, die viele Geduldete in die Obdachlosigkeit bzw. auf irregulärem Wege weiter in andere EU-Mitgliedstaaten treibt, steht im Kontrast zu den aufgrund gesunkener Antragszahlen zunehmend freien Kapazitäten in den Unterkünften. Bemerkenswert an diesem Beispiel ist auch, dass die polnischen Behörden auf die teils kritische Auseinandersetzung einiger Tageszeitungen mit diesem Vorgehen mit einem in der Bundesrepublik altbekannten Argumentationsmuster reagierten: So rechtfertigte der Leiter der zentralen polnischen Ausländerbehörde UdSC die Maßnahmen damit, dass auf diese Weise der Ausnutzung des Unterstützungssystems durch die Geduldeten ein Riegel vorgeschoben werden solle. ${ }^{330}$ Die Missbrauchsargumentation wird hier eingesetzt, obwohl die schwierige Situation auf dem polnischen Wohnungsmarkt allseits bekannt ist. Und schließlich deutet die in Polen routinemäßig stattfindende Inhaftierung von AsylbewerberInnen auf eine stark restriktive Haltung hin: Sie ist im europäischen Vergleich beinah einzigartig und wird - wie die Interviews gezeigt haben - mit der geografischen Lage Polens an der EU-Außengrenze und daher notwendig werdenden verstärkten Abschreckung begründet, da sonst jeder, der Asyl beantrage, frei einreisen könne (vgl. im Abschnitt 6.3.3 Flüchtlingsschutz zwischen Recht und Praxis). Auch die zunehmende Vermischung von Aufnahme- und Abschiebefunktionen in den Lagern zeigt sich also in beiden Ländern - in Deutschland in den „multifunktionalen Großlagern“ (Pieper 2008), in Polen in den neuen, weitgehend aus EU-Mitteln finanzierten Haftanstalten an der Außengrenze und im inzwischen geschlossenen Aufnahmelager Lesznowola bei Warschau. Die Inhaftierung von MigrantInnen wird also zunehmend fester Bestandteil im Maßnahmen-Repertoire des ,Migrationsmanagements'.

${ }^{330}$ Vgl. hierzu im Polen-Kapitel S. 282 zum Duldungsstatus. 
Eine Bestimmung der Interessen und Ziele, die die beschriebenen Restriktionstendenzen in Deutschland und Polen begründen, gestaltet sich schwierig denn laut Stephen Castles ,ist es notwendig, vorgebliche Ziele in Frage zu stellen und nach versteckten Zielsetzungen zu suchen“" (Castles 2005, S. 12), da die tatsächlichen Ziele nicht immer offen gelegt werden. Neben der bereits erläuterten zentralen Stellung des EU-Beitritts in Polen, werden in beiden Ländern in diesem Zusammenhang sicherheitspolitische Interessen angesprochen - in Deutschland sowohl im öffentlichen Diskurs als auch in den gesetzlichen Regelungen, in Polen in erster Linie in der Gesetzgebung. In der Bundesrepublik kommen finanzpolitische Begründungen hinzu, die jedoch auch in Polen an Bedeutung gewinnen. Beide Aspekte erscheinen bei einer Konfrontation der formulierten Ziele mit den entsprechenden Maßnahmen jedoch fragwürdig. So ist zum einen zweifelhaft, inwieweit asylpolitische Abwehrmaßnahmen zu mehr Sicherheit in den Staaten beitragen können. Zum anderen hält das finanzpolitische Argument gerade in der Bundesrepublik nicht stand, da die Maßnahmen, die zur sozialen Schlechterstellung und Abschreckung von Asylsuchenden eingeleitet wurden (Sachleistungsprinzip, Arbeitsverbot, etc.), in erster Linie Kosten verursachen, die bei einer sozialrechtlichen Gleichstellung der AsylbewerberInnen nicht entstünden. Beide Zielstellungen haben also primär einen hohen symbolischen Charakter, was auch erklärt, warum sie in der Bundesrepublik angesichts der stärkeren Bedeutung des Themas im öffentlichen Diskurs eine noch stärkere Rolle spielen als in Polen. Wie ausgeführt wurde, kann auf diese Art und Weise sowohl Handlungsfähigkeit und -stärke demonstriert, als auch - durch die Stärkung eines exklusiven, Wir'-Gefühls - von anderen Themen abgelenkt werden. Für die Bundesrepublik konnte diese Rolle der „symbolischen Politik“" (Faist 1994, S. 52) in der vorliegenden Studie eindeutig aufgezeigt werden. Die staatlichen AkteurInnen suggerieren auf diese Weise, dass sie ,Herr der Lage' sind - sowohl was die Sicherheit der ,eigenen BürgerInnen angeht als auch bei der Auswahl derer, die an den knappen sozialen Gütern der nationalstaatlich definierten Gemeinschaft teilhaben dürfen. In Polen wird sich zeigen, inwieweit das Thema in Zukunft mit steigender Attraktivität des Landes für Einwanderer sowie wachsenden AsylbewerberInnenzahlen in ähnlicher Weise Eingang in den öffentlichen Diskurs findet. Anknüpfungspunkte dafür ergeben sich angesichts des ebenfalls als ethnokulturell homogen konstruierten polnischen Nationenverständnisses. Es bleibt daher abzuwarten, ob und welche Schlagwörter und Topoi hier auftauchen und inwiefern damit einhergehend eine weitere Schlechterstellung von Flüchtlingen stattfindet, die sich nicht mehr nur auf fehlende Finanzmittel und Infrastruktur zurückführen lässt; oder ob eine solchermaßen symbolische Politik hier auch künftig mit anderen Themen betrieben wird.

Neben diesen sicherheits- und finanzpolitischen Gesichtspunkten mit hohem Symbolgehalt spielen wirtschafts- und arbeitsmarktpolitische Interessen eine zentrale Rolle, die zumindest im Verlauf der einwanderungspolitischen Geschichte der Bundesrepublik eine starke Kontinuität aufweisen. Von Beginn der ,Gastarbei- 
terära' an über den Anwerbestopp bis zum Versuch das Asylrecht einzuschränken und schließlich Hochqualifizierte zu gewinnen, hatten einwanderungspolitische Regulierungen immer das Wohl des, Wirtschaftsstandorts' Deutschland im Blick. Diesem ist mit Flüchtlingen, die auf dem Weg der Asylantragstellung einreisen, kaum gedient - zumindest können dem völkerrechtlich vorgeschriebenen Kriterium der Verfolgung keine Anforderungen an das persönliche Qualifikationsprofil oder ein bestimmtes Investitionsvolumen hinzugefügt werden. Aus dieser Sichtweise heraus ist das Asylrecht also in erster Linie ein schwer steuerbarer Kostenfaktor, den es auf niedrigem Niveau zu halten gilt. Polen ist wirtschafts- und arbeitsmarktpolitisch mit Blick auf Migration damit beschäftigt, die in den vergangenen Jahren in großer Zahl abgewanderten Fachkräfte wieder zurückzugewinnen und die entstandenen Lücken auf dem Arbeitsmarkt unterdessen durch MigrantInnen aus den östlichen Nachbarstaaten und möglicherweise in Zukunft auch aus weiter entfernten Herkunftsländern zu füllen. Dabei wird auf Strategien zurückgegriffen, die in der Bundesrepublik wohlbekannt sind, wie Verhandlungen über Anwerbeabkommen mit Indien und anderen Herkunftsländern zeigen.

Auch wenn eine dahingehende Untersuchung im Rahmen dieser Studie nicht geleistet werden konnte, sei hier noch darauf hingewiesen, dass sich eine Fortsetzung der beschriebenen Dynamiken in Richtung Osten abzeichnet. So ist die Ukraine ebenfalls an einem EU-Beitritt interessiert. Da die EU vorerst keine neuen Mitglieder aufnimmt, aber dennoch eine enge Einbindung der Anrainerstaaten forciert, hat sie alternativ die Europäische Nachbarschaftsstrategie entwickelt, in die auch die Ukraine einbezogen ist. Mit Aktionsplänen werden die Nachbarstaaten animiert, verschiedene Anpassungsleistungen an die EU zu erbringen. Teil der Nachbarschaftspläne ist auch der Bereich Justiz und Inneres. Für die Ukraine, ein bedeutendes Transitland für MigrantInnen auf dem Weg in die EU, wurde im Jahr 2001 ein erster „EU-Aktionsplan für den Bereich Justiz und Inneres in der Ukraine“ entwickelt (2003/C 77/01). Darin wird die Ukraine angehalten, bereits vor den Toren der EU an der Verhinderung von Einwanderungsprozessen mitzuwirken, Grenzkontrollen auszubauen und ein Flüchtlingsschutzsystem einzurichten. Mit Verweis auf ein formal existierendes Flüchtlingsschutzsystem kann die Ukraine als sicherer Drittstaat klassifiziert werden - und nimmt so die Rolle ein, die Polen vor seinem EU-Beitritt innehatte. Obwohl die Ukraine bislang weder vom UNHCR noch von der EU als, sicherer Drittstaat ${ }^{`}$ eingestuft wird, wurde Ende 2007 auf der Basis des Nachbarschaftsplans von 2005 ein Rückübernahmeabkommen zwischen der EU und der Ukraine geschlossen. Innerhalb einer zweijährigen Übergangsfrist soll die Ukraine mit fachlicher und technischer Unterstützung der EU-Kommission mit Hochdruck an der Beseitigung ihrer bestehenden „Defizite' bei Migrationssteuerung und Grenzverwaltung arbeiten. Damit würde nach Ansicht des Innen- und Justizausschusses des Europäischen Parlaments der „Raum der Freiheit und des Rechts über die EU hinaus auf das Gebiet ihres unmittelbaren, äußerst wichtigen Nachbarn" ausgedehnt (Ausschuss für bürgerliche 
Freiheiten, Justiz und Inneres 2007). Als Anreiz für die Ukraine wurden gleichzeitig erleichterte Visabedingungen für ukrainische StaatsbürgerInnen vereinbart. Aufgrund bereits bestehender Rückübernahmeabkommen - unter anderem auch zwischen Polen und der Ukraine - und rechtswidriger Zurückweisungen aus Polen und der Slowakei stranden bereits heute Tausende Flüchtlinge in der Ukraine, wo sie unter extremen Bedingungen inhaftiert werden und kaum reelle Chancen auf die Stellung eines Asylantrags und eine Anerkennung als Flüchtling haben (vgl. Human Rights Watch 2005). Die Zahl der Inhaftierten hat in den vergangenen Jahren stetig zugenommen: von 25.539 in 2004 über 32.736 in 2005 bis hin zu $36.092 \mathrm{im} \mathrm{Jahr} 2006$ (vgl. Shapovalova 2007). Der Menschenrechtskommissar des Europarates kritisiert, dass in der Ukraine „[d]etention seems to be the main control procedure for migration“ (Hammarberg 2007). Würde ein Beitrittsverfahren mit der Ukraine eingeleitet, wäre sehr wahrscheinlich eine ähnliche Entwicklung wie die für Polen beschriebene zu erwarten - eine Übernahme des migrationsrelevanten Acquis, die im Interesse des übergeordneten Beitrittsziels in einem bürokratischen Verfahren ohne größere öffentliche Aufmerksamkeit vollzogen würde. 



\section{Fazit: Die Metamorphosen der Kategorie Flucht und Asyl}

Wie auch die Literaturlage zum Thema Migration widerspiegelt, hat sich das Migrationsgeschehen in der EU stark verändert. Sinkende Asylantragszahlen und eine steigende Zahl irregulärer MigrantInnen korrespondieren auch mit einer Verschiebung der wissenschaftlichen und öffentlichen Debatte: Das Thema Asyl fristet zunehmend ein Schattendasein, während die irreguläre Einwanderung die Debatten bestimmt. Im Völkerrecht vor dem Hintergrund der Geschehnisse während des Nationalsozialismus und aufgrund politischer Interessenlagen im ,Kalten Krieg' erst vor einigen Jahrzehnten verankert, verliert die Kategorie Flucht und Asyl als Einwanderungsmöglichkeit innerhalb der EU inzwischen wieder an Bedeutung. Angesichts dieser veränderten Konstellationen war es das Ziel der vorliegenden Untersuchung darzulegen, inwieweit sich Flüchtlingsschutz und Asyl in Europa in den vergangenen 20 Jahren gewandelt haben, wie dieser Wandel erklärt werden kann und in welchem Verhältnis er zu den Strategien von MigrantInnen steht. Um in einer Mehrebenenperspektive der Verflechtung der supranationalen, einzelstaatlichen und regionalen Ebenen in der EU gerecht zu werden, bildete die Analyse des Asylregimes auf EU-Ebene den Ausgangspunkt der empirischen Untersuchung. Denn die Ausgestaltung des Flüchtlingsschutzes der EU beeinflusst einerseits die Regime in den Mitgliedstaaten und ist andererseits von deren Konzepten inspiriert. Auf dieser Grundlage wurden in zwei Länderstudien die asylpoli- 
tischen Entwicklungen in Deutschland und Polen dargestellt und schließlich miteinander kontrastiert. Die Auswahl dieser beiden Länder ermöglichte eine eingehende Illustration der asylpolitischen Dynamiken und Verschiebungen, die sich zwischen unterschiedlich machtvollen und unterschiedlich geografisch positionierten Mitgliedstaaten der EU vollziehen. Dabei konnte die Auslagerung des Flüchtlingsschutzes aus dem geografischen Kern der EU an ihre Außengrenzen und weiter in die Nachbarstaaten als zentraler Modus des Wandels des europäischen Asylregimes identifiziert werden.

Vor dem Hintergrund beider Länderstudien und der erläuterten Entwicklungsdynamik, die sich hier zeigt, sollen im Folgenden einige Schlussfolgerungen über den Wandel des europäischen Asyl- und Migrationsregimes gezogen werden. Dabei werden die Ergebnisse der Studie auf die zu Beginn aufgestellten Thesen über das ,Verschwinden' der Flüchtlinge aus dem geografischen Kern der EU sowie über die Rolle migrationspolitischer Strategien rückbezogen. Die Kategorisierung von MigrantInnen wurde am Anfang der Untersuchung als zentraler Pfeiler migrationspolitischer Bestrebungen vorgestellt. Für die Kategorie Flucht und Asyl lässt sich feststellen, dass sich an der gängigen Flüchtlingsdefinition, wie sie nach Ende des Zweiten Weltkrieges entstanden war, kaum etwas geändert hat. Jedoch wurden Flüchtlingsschutz und Asyl mit verfahrensrechtlichen Restriktionen in Form von Zuständigkeitsregelungen und mit verschärften Visabedingungen verknüpft, die die Inanspruchnahme des Rechts auf Asyl erschweren und zu einer Auslagerung des Flüchtlingsschutzes aus der EU und einer Illegalisierung der dennoch stattfindenden Migrationsbewegungen führen. Diesen Entwicklungen liegt eine Explizierung einwanderungspolitischer Präferenzen in den meisten Zielländern der EU zugrunde: Es werden mehr und mehr Anstrengungen unternommen, um ,unerwünschte' Einwanderungsprozesse - also solche, die unter demografischen und arbeitsmarktpolitischen Gesichtspunkten als nicht nützlich qualifiziert werden $-\mathrm{zu}$ verhindern. Erhöhung von Wettbewerbsfähigkeit und Wirtschaftswachstum sind hier die leitenden Prämissen. Diese sind zwar nicht gänzlich neu, haben aber in den vergangenen 20 Jahren im Kontext der Einwanderungspolitik eine starke Fokussierung erfahren. Die verbliebenen Einwanderungsmöglichkeiten sind an dieser Zielstellung ausgerichtet und dabei höchst voraussetzungsvoll (wie bspw. Optionen für Hochqualifizierte) oder unattraktiv (wie die legalen Möglichkeiten zum Beispiel als Haushaltshilfe in der Bundesrepublik zu arbeiten). Seit den Anschlägen vom 11.09.2001 in den USA wurden diese Prämissen eng mit einer sicherheitspolitischen Debatte verknüpft, die dem ,Kampf gegen den Terror ${ }^{6}$ oberste Priorität einräumte und Einwanderung mehr und mehr in den Kontext von Terrorgefahren stellte. Die Betonung sicherheitspolitischer Interessen konnte sowohl in Deutschland als auch im polnischen Flüchtlingsschutzgesetz und in den Asylrichtlinien auf EU-Ebene festgestellt werden.

Der vielerorts postulierte Paradigmenwechsel, wonach das Paradigma der grundsätzlichen Verhinderung von Einwanderungsprozessen einer selektiven 
Öffnung unter Nützlichkeitsaspekten und verstärkten Integrationsbemühungen gewichen sei, schließt Flüchtlinge nicht mit ein. Sie gelten gemeinhin als ,unerwünscht'. Sie mögen im Einzelfall dem geforderten Qualifikationsprofil und damit dem Nützlichkeitsparadigma entsprechen, aufgrund der völkerrechtlichen Verankerung der Flüchtlingsdefinition lässt sich eine Auswahl der Flüchtlinge entlang von Nützlichkeitskriterien jedoch kaum steuern. Mit einer Vielzahl an Instrumenten und Maßnahmen und einer verstärkten gemeinsamen Koordination des Politikfelds auf supranationaler Ebene versuchen die Staaten daher anderweitig auf die Zahl der Asylsuchenden Einfluss zu nehmen. Dabei scheint sich das Ziel der Reduktion der AsylbewerberInnenzahl verselbstständigt zu haben. Denn den seit Jahren rückläufigen Zahlen zum Trotz wird, sofern das Thema auf der Agenda erscheint, weiterhin von der Notwendigkeit gesprochen, den ,Asylmissbrauch ${ }^{\text {zu }}$ verhindern - so zum Beispiel im Bericht der Unabhängigen Kommission Zuwanderung in der Bundesrepublik oder in der Variation des ,Asylum Shopping' in verschiedenen EU-Richtlinien. Die daran anknüpfenden immer weiter fortschreitenden Restriktionen im Asylbereich scheinen ebenfalls nicht im Zusammenhang mit der Entwicklung der AsylbewerberInnenzahlen zu stehen. Am Beispiel der zwei Länder und der EU-Aktivitäten zeigt sich, dass beim ,Verschwinden' der Flüchtlinge aus der Bundesrepublik und ihrem ,Auftauchen' in Polen und den EUNachbarstaaten die migrationspolitischen Regulierungen ein wirkungsmächtiger Faktor sind. Diese sind in den Mitgliedstaaten zwar zunehmend ähnlich, aber nicht gänzlich konvergent. Die beiden zentralen Hebel, an denen angesetzt wird, sind der Zugang zum Asylverfahren und die soziale und rechtliche Situation der Asylsuchenden im Verfahren.

Der Zugang zum Asylverfahren wurde mittels Zuständigkeitsregelungen erschwert. Diese sollen verhindern, dass MigrantInnen selbst über ihr Zielland entscheiden. Die eigenständige Wahl des Ziellandes wird in den EU-Dokumenten als ,Asylum Shopping diskreditiert. Mit der Festlegung, welcher Mitgliedstaat innerhalb der EU (Dublin-II-Verordnung) und welcher, Drittstaat' außerhalb der EU (sichere Drittstaatenregelung ${ }^{331}$ ) für ein Asylgesuch bzw. die Gewährung von Flüchtlingsschutz zuständig ist, wurde die oben angesprochene Dynamik zwischen Staaten im geografischen Kern und an den Außengrenzen der EU in Gang gesetzt. Diese Dynamik setzt sich weiter nach außen - zu den Anrainerstaaten - fort: Staaten wie Deutschland, die über keine Außengrenze verfügen, konnten so die Zahl der zu bearbeitenden Asylanträge deutlich senken, während die Verantwortung für die Durchführung der Asylverfahren den Staaten an den Außengrenzen - wie bspw. Polen - zugewiesen wird. Diese schließen Rückübernahmeabkommen mit den Transit- und Herkunftsstaaten, wie hier für das Beispiel Ukraine aufgezeigt wurde. Die MigrantInnen müssen ihre Anträge daher zunehmend bereits in den Transitstaaten stellen oder versuchen diese zu durchqueren, ohne dabei erfasst zu

331 Wie im Kapitel Asylpolitik der EU dargestellt, ist die Drittstaatenregelung auf EU-Ebene noch nicht in Kraft. Sie findet sich aber in den Gesetzgebungen der meisten Mitgliedstaaten. 
werden. Dies gestaltet sich schwierig, da die Zuständigkeitsregelungen durch grenzpolitische Maßnahmen flankiert werden. Durch die negativen Zuständigkeitsregelungen und die Aneinanderreihung von Rückübernahmeabkommen kann sich eine Kette von Abschiebungen ergeben, an deren Ende sich MigrantInnen bzw. Flüchtlinge in ihrem Herkunftsstaat wiederfinden, ohne dass ihr Asylantrag jemals geprüft wurde. Anstelle der Fluchtgründe entscheidet daher der Fluchtweg über die Chancen eines Asylantrags. Gleichzeitig werden so Ressourcen und Netzwerke der MigrantInnen mehr und mehr ausschlaggebend dafür, in welchem Land der Asylantrag gestellt werden kann.

,Migrationsmanagement ${ }^{6}$ und Grenzverwaltung werden also zunehmend ausgelagert und der Geltungs- und Wirkungsbereich grenz- und migrationspolitischer Strategien der EU weit über deren Territorium hinaus ausgedehnt. Mithilfe der Installation von Asylregimen an den neuen ,Rändern` und außerhalb der EU wurde das Kategorisierungsverfahren, das heißt die Filterung erwünschter bzw. berechtigter MigrantInnen und die Entrechtung der Übrigen, teilweise dorthin delegiert.

Trotz dieser Regelungen wandern viele Flüchtlinge weiter in die angestrebten Zielländer und versuchen ihr Glück mitunter mehrfach. Das zeigen unter anderem die Anträge auf Rückübernahme, die die Staaten im Rahmen des Dublin-IIVerfahrens aneinander stellen. Auch wenn hier mit einem ungeheuren bürokratischen Aufwand, der letztlich nur in einer geringen Zahl tatsächlicher Rücküberstellungen mündet, vor allem die Behörden beschäftigt werden, sind diese Regelungen nichtsdestotrotz in vielerlei Hinsicht wirkungsmächtig: Sie nehmen Einfluss auf die Bedingungen der Migration (zum Beispiel die Dauer, die Kosten, den Grad der Ungeschütztheit und Angewiesenheit auf ,Schlepper'), die individuellen Merkmale der Migrierenden (Ressourcen, Netzwerke, Geschlecht, Alter, Kinderzahl), die Form der Migration (regulär oder irregulär, mit oder ohne Papiere, auf dem Luft- oder Landweg) und den Status im Zielland. Eine alleinerziehende Mutter mit mehreren Kindern - zu denken ist etwa an die zahlreichen tschetschenischen Witwen, die in den polnischen Flüchtlingslagern leben - wird kaum über die notwendigen Ressourcen verfügen, um über Polen hinaus weiter westwärts zu gelangen. Wenn es ihr gelänge, wäre ein Leben in der Illegalität mit Kindern schwer zu bewältigen.

Die Verschlecbterung der sozialen Situation der AsylbewerberInnen als weiterer Hebel des Migrationsmanagements wurde vor allem in den ,alten' Mitgliedstaaten als Abschreckungsmittel betrachtet: So wurde zum Beispiel in der Bundesrepublik aktiv auf die Verschlechterung der sozialen Rahmenbedingungen hingewirkt. In den neuen Mitgliedstaaten an den Außengrenzen liegt die sozial prekäre Situation oft in fehlender Infrastruktur und mangelnden Ressourcen begründet, nichtsdestotrotz lässt sich bspw. auch in Polen eine zunehmend ähnliche Tendenz zum aktiven Einsatz von Restriktionsstrategien im sozialen Bereich identifizieren. 
Der Flüchtlingsbegriff der Genfer Flüchtlingskonvention (GFK) besteht also fort - es wird allerdings aufgrund der Zuständigkeitsregelungen immer schwieriger diesen in einem Asylverfahren innerhalb der EU geltend zu machen. Darüber hinaus greift die Flüchtlingsdefinition der GFK mit ihrem Fokus auf Verfolgung, Individualität und grenzüberschreitende Flucht ohnehin zu kurz und ist überdies inzwischen, in die Jahre gekommen'. Das heißt, zu den seit Jahrzehnten geäußerten Kritiken an der Engführung des Begriffs kommt auch seine mangelnde Anpassung an die diversifizierten und neuen Gründe der Schutzsuchenden - wie bspw. die Flucht vor den Folgen von Umweltschäden - weshalb die Definition der GFK mehr denn je nur einen kleinen Teil derer erfasst, die aus verschiedensten Gründen auf der Flucht sind. Doch um der eingangs postulierten Abstraktion von den politisch vorgegebenen Kategorisierungen gerecht zu werden ${ }^{332}$, ist es nicht Gegenstand dieser Untersuchung zu diskutieren, wie der Flüchtlingsbegriff an aktuelle Fluchtbewegungen angepasst werden kann. Denn faktisch können (und wollen) politische Kategorisierungen nicht das komplexe Spektrum an Migrationsmotiven erfassen - jegliche Kategorisierung produziert immer auch zugleich den Ausschluss derer, die die Kriterien nicht erfüllen. Vor allem die Abwertung wirtschaftlicher und sozialer Gründe von Migration bzw. Flucht als illegitimes Streben nach Teilhabe, die MigrantInnen aufgrund der nationalstaatlichen Verankerung der Teilhabekonzepte nicht zusteht, erweist sich als ,Pferdefuß ${ }^{`}$ der Flüchtlingskonzeption. Es wird weiter starr am ,Idealbild“ des politisch Verfolgten als einzig asylwürdigem Schutzsuchenden festgehalten, obwohl MigrantInnen sich mit einem komplexen Mix an Motiven auf den Weg machen, in dem wirtschaftliche und soziale Aspekte häufig eine wichtige Rolle spielen - mit diesen Motiven korrespondieren jedoch so gut wie keine Einwanderungsmöglichkeiten.

Vielmehr hat sich das Spektrum legaler Einwanderungsmöglichkeiten deutlich verkleinert. So wurden die Möglichkeiten eines legalen Zugangs zum Arbeitsmarkt in den ,alten' Mitgliedstaaten bereits in den 1970er Jahren stark begrenzt. Die Asylantragstellung, die anschließend als eines der wenigen gates of entry verblieben war, gleicht inzwischen nur mehr einem immer schmaler werdenden Nadelöhr. Ohne die Definition dessen, wer als schutzberechtigt gilt, grundlegend zu verändern, wurde die Kategorie Flucht und Asyl modifiziert und mit verschiedenen $\mathrm{Maßnahmen} \mathrm{verknüpft,} \mathrm{die} \mathrm{vor} \mathrm{allem} \mathrm{über} \mathrm{den} \mathrm{erschwerten} \mathrm{Zugang} \mathrm{zur} \mathrm{EU} \mathrm{und}$ damit über verringerte Chancen der Antragstellung eine Wirkung erzielen. Die Kategorie Flucht und Asyl ist also formal erhalten geblieben, kommt nun jedoch vor allem bereits in den Transitstaaten und den Mitgliedstaaten an den Außengrenzen zur Anwendung.

Aus der Kombination dieser beiden Aspekte - der Restriktion der Asylregime und anderer legaler Einwanderungswege einerseits und der fortbestehenden Motive der MigrantInnen andererseits - ergibt sich das in seiner Bedeutung wachsende

332 Vgl. hierzu das Kapitel 2 ,Flucht und Asyl' im Kontext von Migration und Migrationspolitik. 
Phänomen der irregulären Migration. Mit der Versperrung legaler Einwanderungsmöglichkeiten werden dennoch stattfindende Migrationsbewegungen neu definiert. Die „Figur“"333 (Karakayalı/Tsianos 2005, S. 417) des illegalen Migranten wurde mit den Restriktionen erst geschaffen. Sie ist eine Konstruktion, in der sich gleichsam das Scheitern der Kontrollpolitiken, aber auch ihre Wirkungsmächtigkeit anhand der Exklusion und Entrechtung der „Eigensinnigen“ (Benz/Schwenken 2005) manifestieren. ${ }^{334}$ Die zunehmende Illegalisierung zeigt daher einerseits die ,Produktivität' der Kategorisierungen im Sinne von Selektion, Prekarisierung und Entrechtung, andererseits aber auch ihr Scheitern hinsichtlich des Versuchs, Migration - also den Zugang zum Territorium - zu kontrollieren.

Insofern kann die schwindende Bedeutung von Flüchtlingsschutz und Asyl auf dem EU-Territorium „,nicht als Ende der Migrationen“ (Andrijasevic et al. 2005, S. 346f.) bzw. der Immigration in die EU gelesen werden, sondern es zeigt sich, dass sich Immigration durch das Verschließen dieses regulären Einwanderungsweges hin zu anderen, einschließlich irregulärer Strategien, sowie in die Staaten an den Außengrenzen und die Nachbar- bzw. Transitstaaten verschiebt. Doch auch vom „Ende [...] des Asylregimes“ (ebd.) kann kaum gesprochen werden - stattdessen ist seine Installierung in den neuen Mitgliedstaaten an den Außengrenzen und den EU-Nachbarstaaten zentraler Ausgangspunkt und Pfeiler der dort entstehenden Migrationsregime. Mit der wachsenden Bedeutung irregulärer Immigration und dem Wandel der neuen Mitgliedstaaten zu Zielländern von Migration vollzieht sich ein grundlegender Wandel der Migrationsregime in Europa.

Welche Schlussfolgerungen können daraus über die Plausibilität der eingangs erläuterten theoretischen Ansätze gezogen werden - der gap-Hypothese, der Konvergenz-Hypothese, der These vom Scheitern von Migrationskontrollpolitik sowie der Diskussion um Autonomie bzw. Eigensinnigkeit der Migration? Es lassen sich sowohl eine Reihe von gaps als auch Konvergenzen identifizieren. Besonders hervor sticht der gap zwischen Policy-Zielen und -Outcomes, der eng mit der Kluft zwischen den politisch konstruierten Kategorien bzw. Einwanderungsmöglichkeiten einerseits und den Zielen und Strategien der MigrantInnen andererseits zusammenhängt. Dies zeigt sich an der geringen Durchsetzbarkeit der Zuständigkeitsregelungen, die vor allem an der irregulären Weiterreise und der Vernichtung von Reisedokumenten scheitern. Auch in den deutschen ,Ausreisezentren' zeigt sich, dass das offiziell gesteckte Ziel, die Zahl der ,freiwilligen Ausreisen` zu erhöhen, kaum erreicht wird, sondern im Untertauchen der Betroffenen mündet. Dieses Beispiel verdeutlicht aber auch, dass die Ergebnisse, die sich aus den gaps erge-

\footnotetext{
333 Die „Figuren der Migration [...] repräsentieren weniger soziale Gruppen, als dass sie Migrationsverhältnisse begriffspolitisch reflektieren. Den ,Gastarbeiter', ,Flüchtling' oder ,Sans Papier' mag es zwar, immer schon' gegeben haben, aber in ihren jeweiligen Settings entfalten sie ihre ganze politische Wirkungsmacht als Begriffe, indem sie die Diskurse verknappen und bündeln.“ (Karakayalı/Tsianos 2005, S. 417)

334 Vgl. hierzu das Kapitel 2 ,Flucht und Asyl‘ im Kontext von Migration und Migrationspolitik.
} 
ben, nicht zwangsläufig dysfunktional sind - immerhin verschwinden die Betroffenen aus der Statistik der Ausreisepflichtigen.

Bei den Strategien im Umgang mit Flüchtlingsbewegungen lässt sich zudem eine zunehmende Konvergenz feststellen. Die Angleichungsprozesse, die sich zunächst als ,Domino-Effekt ${ }^{6}$ zwischen den Staaten dargestellt haben, die versuchen jeweils nicht durch liberalere Regelungen zum Anziehungspunkt für Flüchtlinge zu werden, sind inzwischen durch die Vereinheitlichungsbestrebungen auf EU-Ebene institutionalisiert worden. Die Zusammenarbeit bietet den EU-Mitgliedstaaten Vorteile auf verschiedenen Ebenen: bei der Legitimation von Maßnahmen in den Mitgliedstaaten (Brüsseler Diktat versus einzelstaatliche Durchsetzungsstärke) sowie bei der Erarbeitung (Austausch über Strategien) und Umsetzung der Maßnahmen (operative Zusammenarbeit und Datenaustausch). Durch die Supranationalisierung in diesem Feld wird nicht zuletzt versucht dem Migrationsgeschehen, das sich als grenzüberschreitendes Phänomen zeigt, nicht mehr nur mit Instrumenten auf nationalstaatlicher Ebene zu begegnen. Zudem ermöglicht die größere Machtfülle, die sich aus dem Zusammenschluss mehrerer Staaten ergibt, eine bessere Einflussnahme auf neue Mitglied- und Nachbarstaaten.

Die dargestellten gaps und die zunehmende Konvergenz bei gleichzeitiger Wirkungsmächtigkeit der Politiken stützen die eingangs formulierte Annahme, dass die These vom „Scheitern der Migrationspolitiken“ (Castles 2005) nicht für das gesamte Spektrum der Instrumente und Maßnahmen der Migrationspolitik Gültigkeit besitzt. Während man für die verschiedenen Steuerungs- und Kontrollstrategien jeweils in unterschiedlichem Ausmaß ,Erfolg' (Auslagerung) und ,Scheitern“ (Unterlaufen der Regelungen durch die MigrantInnen) konstatieren kann, sind sie in jedem Fall wirkungsmächtig. Das heißt, sie nehmen - wie oben erläutert - Einfluss auf die Bedingungen und die Form der Migration, die individuellen Merkmale der Migrierenden und deren Status im Zielland. Die These von der „Autonomie der Migration“ (Karakayal1/Tsianos 2005) durch die von der „Eigensinnigkeit“ (Benz/Schwenken 2005) zu ersetzen erscheint deshalb gerade mit Blick auf Flüchtlinge sinnvoll. Denn die Handlungsfähigkeit von MigrantInnen und ihre Möglichkeiten Kontroll- und Steuerungsversuche zu umgehen, hängen stark von den Ressourcen und Netzwerken ab, die sie mobilisieren können. Diese sind wiederum geschlechts- und herkunftsspezifisch und je nach zur Verfügung stehenden Alternativen ungleich verteilt. Die unterstellte Autonomie gegenüber den migrationspolitischen Regulierungen muss also entlang dieser Kategorien jeweils differenziert werden - sofern nicht nur eine Autonomie in der Formulierung der eigenen Ziele, sondern auch die konkreten Möglichkeiten und Chancen der tatsächlichen Umsetzung dieser Ziele gemeint sind. Damit wird nicht die Kategorisierung und Hierarchisierung von MigrantInnen reproduziert, sondern - im Gegenteil das Analyseinstrumentarium hinsichtlich der unter den MigrantInnen bestehenden Differenzen, die sich in jeweils unterschiedlicher Machtfülle fortsetzen, geschärft. Der Begriff der ,Eigensinnigkeit', wie ihn Benz und Schwenken angewendet ha- 
ben, bildet das Verhältnis von Zielen und der vor dem Hintergrund bestehender Restriktionen unterschiedlich verteilten Ermächtigungs- und Handlungsmöglichkeiten daher treffender ab als die Rede von der ,Autonomie bei Karakayalı und Tsianos.

Die Kategorienkonstruktion und die Hierarchisierung der Einwandererkategorien dienen jedoch nicht nur dem Versuch, Einwanderung zu steuern, sondern haben auch eine zentrale Funktion im Einwanderungsdiskurs - sowohl in der Bundesrepublik als auch auf EU-Ebene und zunehmend auch in Polen. Die Unterscheidung von ,echten Flüchtlingen' und ,Wirtschaftsflüchtlingen', mit der Unterstellung, letztere ,missbrauchen' das Asylrecht und die sozialstaatlichen Leistungen in der Bundesrepublik oder Polen, ist im Diskurs fest verankert und eine wichtige Legitimationsstrategie für restriktive Maßnahmen, aber auch um Handlungsstärke und die Bereitschaft und Fähigkeit zum ,Durchgreifen' zu demonstrieren. Eine nicht annähernd so prominente Rolle spielt hingegen die Frage nach der eigenen Verantwortung der Zielländer für die Migrationsursachen. Zwar wird immer wieder postuliert, man wolle die Fluchtursachen bekämpfen - dies verkennt jedoch einerseits erneut die Komplexität der Migrationsursachen, die nicht ausschließlich ökonomischer Natur und überdies nicht einfach abstellbar sind. Andererseits lassen sich konkrete Maßnahmen in diesem Bereich kaum finden. Im Gegenteil, zahlreiche Politiken der Zielländer und auch der EU laufen solchen Bestrebungen ausdrücklich entgegen, wenn man bspw. an die Agrarsubventionen und die Fischereipolitik der EU, an die Klimapolitik oder die Strukturanpassungsprogramme des IWF denkt. Indem jedoch im Diskurs Flüchtlinge jeweils kontextlos nur in ihrer Anwesenheit im Zielland und die Fluchtursachen als externer Faktor betrachtet werden, wird mit der Rede von ,Wirtschaftsflüchtlingen' ein Bild produziert, das die Ursachen und die Verantwortung für die Flucht den Herkunftsländern bzw. den Flüchtlingen selbst zuweist. Sehr eindrücklich wird das zum Beispiel mit der Überschrift eines Artikels von Otto Schily (2004) „Afrikas Probleme in Afrika lösen“" zur Einrichtung von Flüchtlingslagern auf dem afrikanischen Kontinent illustriert. Weist man die Gründe für die Migration mit solcher Selbstverständlichkeit den Herkunftsländern zu, lässt sich die Entscheidung über den Zugang zum Territorium als humanitärer Akt für die selbstverschuldet Hilfebedürftigen umdeuten.

Die Bedeutungslosigkeit, in die das Asylrecht derzeit in den Staaten der Europäischen Union manövriert wird und die Entrechtung derer, die nationalstaatliche Grenzen überschreiten, obwohl sie im Zielland offiziell als unerwünscht gelten, bestätigt einmal mehr die Thesen Hannah Arendts:

\footnotetext{
„Insofern die Französische Revolution die Menschbeit als eine Familie von Nationen begriff, richtete sich der Begriff des Menschen, der den Menschenrechten zugrunde lag, nach dem Volk. und nicht nach dem Individuum" (Arendt 2008, S. 604f.).
} 
Sie hat damit deutlich gemacht, dass die Menschenrechte de facto keineswegs voroder überstaatlich, sondern im Gegenteil, vom Willen nationalstaatlicher Regierungen zur Gewährung dieser Rechte abhängig sind. Die vorliegende Studie illustriert am Beispiel des Asylrechts anschaulich diese Fragilität vermeintlich universeller Rechte in einer Welt, in der Zugehörigkeit und Teilhabe in erster Linie nationalstaatlich definiert sind. Diese nationalstaatliche Definitionsmacht manifestiert sich für MigrantInnen einerseits in Entrechtung und Entmächtigung. Andererseits wird sie jedoch durch vielfältige Strategien der Selbstermächtigung infrage gestellt, wenn sich MigrantInnen über Grenzziehungen und Kategorisierungen hinwegsetzen oder sie strategisch für sich nutzen. Sie werden auf diese Weise nicht nur zur treibenden Kraft neuer Metamorphosen der Migrationsregime, sondern verweisen damit zugleich auf die Grenzen migrationspolitischer Definitionsmächtigkeit. 



\section{Anhang I: InterviewpartnerInnen}

Die InterviewpartnerInnen werden nicht namentlich genannt, nur die Institution bzw. Organisation, in der sie arbeiten, wird aufgeführt. Die Seitenangaben der indirekten Verweise auf InterviewpartnerInnen beziehen sich auf die paraphrasierten Interviewtexte, die mir vorliegen. Bei wörtlichen Zitaten beziehen sich die Seitenangaben auf das jeweilige Transkript. Die Interviews wurden im Sommer 2006 und im Frühjahr 2007 geführt.

A: Central European Forum for Migration and Population Research (Środkowoeuropejskie Forum Badań Migracyjnych i Ludnościowych), Warschau.

B: Ausländerabteilung im Hauptquartier der Grenzschutzbehörde, Warschau.

C: Polnische Humanitäre Aktion (Polska Akcja Humanitarna), Warschau.

D: Legal Clinic (Klinika Prawa), Fakultät für Recht und Verwaltung (Wydział Prawa i Administracji), Universität Warschau.

E: Verwaltungsgericht der Woiwodschaft Warschau, Warschau.

F: Center of Migration Research (Ośrodek Badań nad Migracjami), Warschau.

G: Fakultät für Internationales Öffentliches Recht und Europarecht (Katedra Prawa Międzynarodowego Publicznego i Prawa Europejskiego), Katowice.

H: Abteilung Internationale Beziehungen im URIC, Warschau.

I: One World Association (Stowarzyszenie Jeden Świat), Poznan.

J: Helsinki Foundation for Human Rights (Helsińska Fundacja Praw Człowieka), Warschau (an dem Gespräch waren drei MitarbeiterInnen der Stiftung beteiligt).

K: Ministerium für Arbeit und Sozialpolitik (Ministerstwo Pracy i Polityki Społecznej), Warschau.

L: Halina Niec Human Rights Association (Centrum Pomocy Prawnej im. Haliny Nieć), Kraków.

M: Caritas, Warschau.

N: Mitglied des Flüchtlingsrates (Rada do Spraw Uchodźców), Warschau.

O: Institut für Soziologie (Instytut Socjologii), Universität Warschau.

P: Flüchtlingsvereinigung (Stowarzyszenie Uchodźców w Rzeczypospolitej Polskiej), Warschau.

Q: Abteilung öffentliche Verwaltung, Gesundheit und Ausländerrecht beim Ombudsmann (Rzecznik Praw Obywatelskich), Warschau. 


\section{Anhang II: Tabellen und Diagramme}

\section{A) Deutschland}

Tabelle 1: Wanderungen zwischen Deutschland und dem Ausland 1995-2007335

\begin{tabular}{|r|r|r|r|}
\hline Jahr & Zugezogene & Fortgezogene & \multicolumn{1}{|c|}{ Saldo } \\
\hline 1995 & 1.096 .048 & 698.113 & 397.935 \\
\hline 1996 & 959.691 & 677.494 & 282.197 \\
\hline 1997 & 840.633 & 746.969 & 93.664 \\
\hline 1998 & 802.456 & 755.358 & 47.098 \\
\hline 1999 & 874.023 & 672.048 & 201.975 \\
\hline 2000 & 841.158 & 674.038 & 167.120 \\
\hline 2001 & 879.217 & 606.494 & 272.723 \\
\hline 2002 & 842.543 & 623.255 & 219.288 \\
\hline 2003 & 768.975 & 626.330 & 142.645 \\
\hline 2004 & 780.175 & 697.632 & 82.543 \\
\hline 2005 & 707.352 & 628.399 & 78.953 \\
\hline 2006 & 661.855 & 639.064 & 22.791 \\
\hline 2007 & 680.766 & 636.854 & 43.912 \\
\hline
\end{tabular}

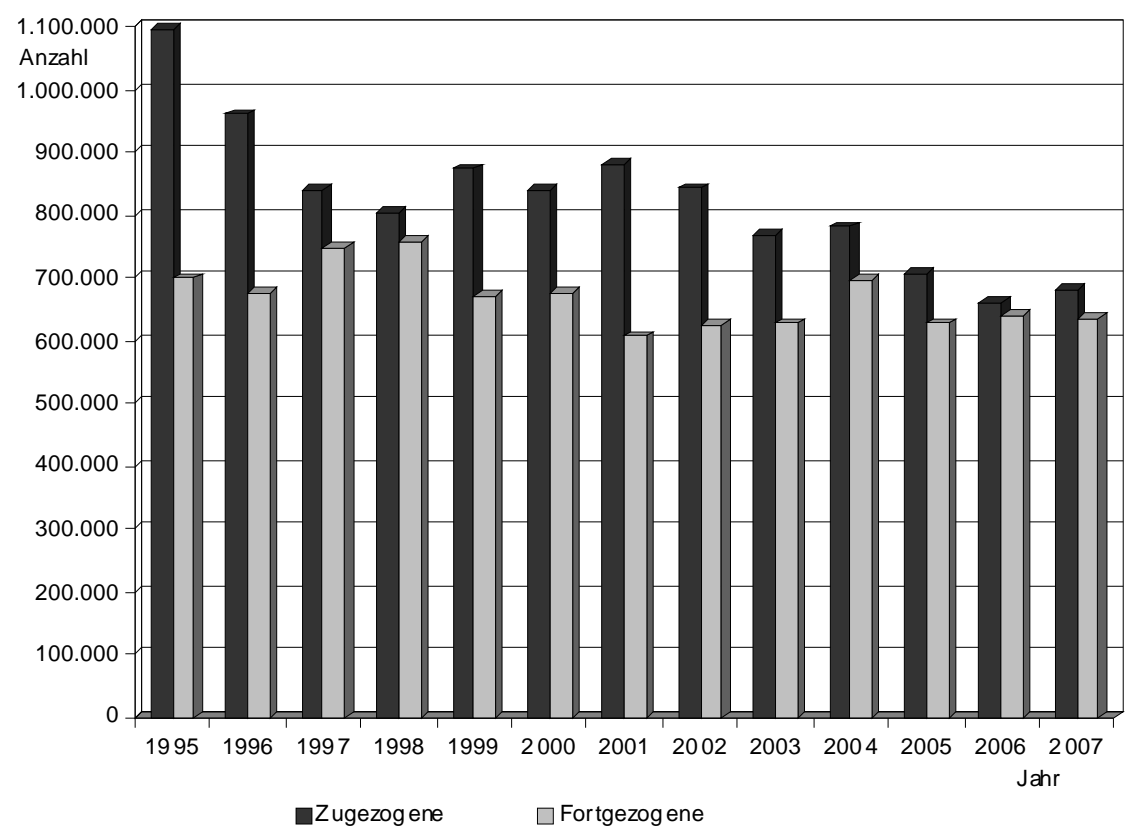

Abb. 1: Wanderungen zwischen Deutschland und dem Ausland 1995-2007.

335 Quelle: Statistisches Bundesamt (o.J.). 
Tabelle 2: Entwicklung der jährlichen Asylantragszahlen seit $1995^{336}$

\begin{tabular}{|c|c|c|c|}
\hline ZEITRAUM & $\begin{array}{c}\text { ASYLANTRÄGE } \\
\text { - insgesamt - }\end{array}$ & davon Erstanträge & davon Folgeanträge \\
\hline 1995 & 166.951 & 127.937 & 39.014 \\
\hline 1996 & 149.193 & 116.367 & 32.826 \\
\hline 1997 & 151.700 & 104.353 & 47.347 \\
\hline 1998 & 143.429 & 98.644 & 44.785 \\
\hline 1999 & 138.319 & 95.113 & 43.206 \\
\hline 2000 & 117.648 & 78.564 & 39.084 \\
\hline 2001 & 118.306 & 88.287 & 30.019 \\
\hline 2002 & 91.471 & 71.127 & 20.344 \\
\hline 2003 & 67.848 & 50.563 & 17.285 \\
\hline 2004 & 50.152 & 35.607 & 14.545 \\
\hline 2005 & 42.908 & 28.914 & 13.994 \\
\hline 2006 & 30.100 & 21.029 & 9.071 \\
\hline 2007 & 30.303 & 19.164 & 11.139 \\
\hline
\end{tabular}

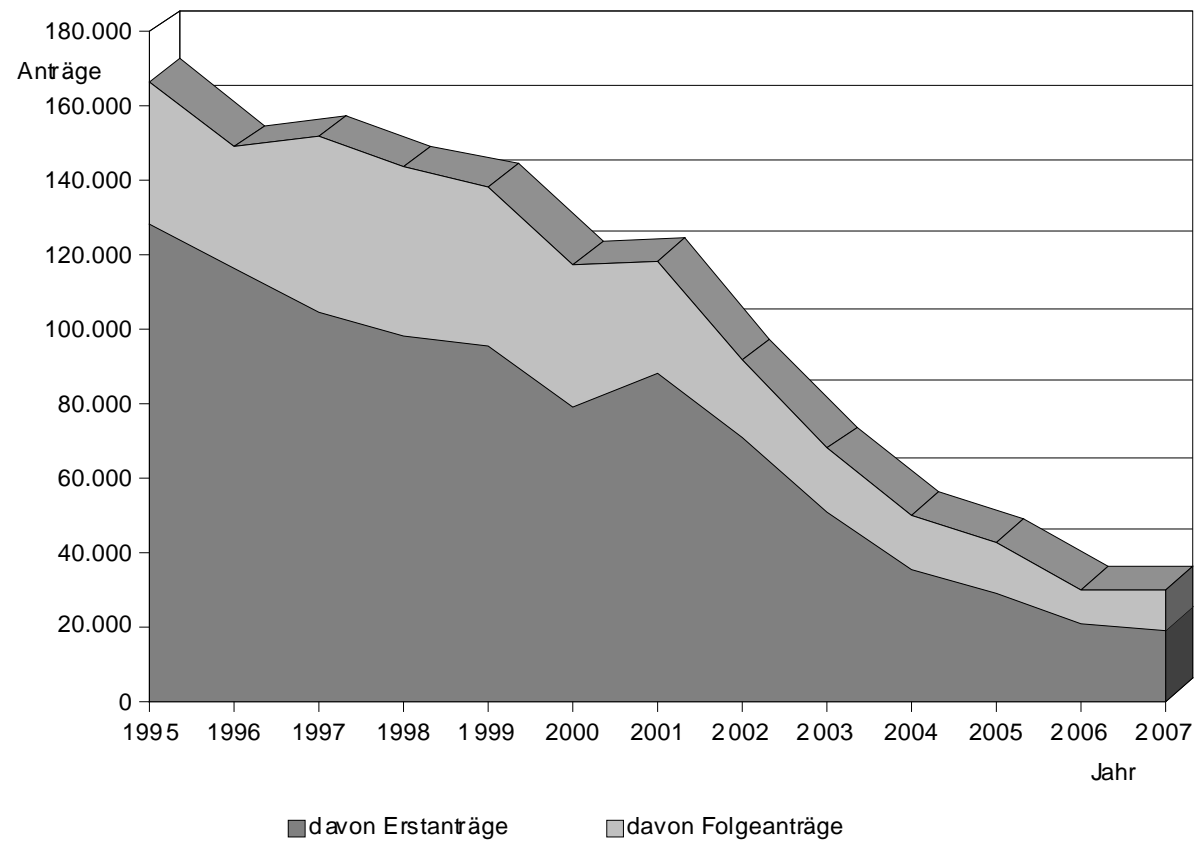

Abb. 2: Entwicklung der jährlichen Asylantragszablen seit 1995.

336 Quelle: Bundesamt für Migration und Flüchtlinge (2008), S. 11. 
Tabelle 3: Zahl der Asylsuchenden je 1000 Einwohner jährlich im Vergleich mit den übrigen EU-Mitgliedstaaten ${ }^{337}$

\begin{tabular}{|c|c|c|}
\hline Jahr & $\begin{array}{c}\text { Asylanträge/1000 Einwohner } \\
\text { in Deutschland }\end{array}$ & $\begin{array}{c}\text { Durchschnittsvergleichswert } \\
\text { aller jeweiligen EU-Mitgliedstaaten }\end{array}{ }^{338}$ \\
\hline 1998 & 1,20 & 1,04 \\
\hline 1999 & 1,16 & 1,27 \\
\hline 2000 & 0,96 & 1,49 \\
\hline 2001 & 1,1 & 1,66 \\
\hline 2002 & 0,9 & 1,78 \\
\hline 2003 & 0,6 & 1,58 \\
\hline 2004 & 0,4 & 1,44 \\
\hline 2005 & 0,4 & 1,17 \\
\hline 2006 & 0,3 & 0,88 \\
\hline 2007 & 0,2 & 0,98 \\
\hline
\end{tabular}

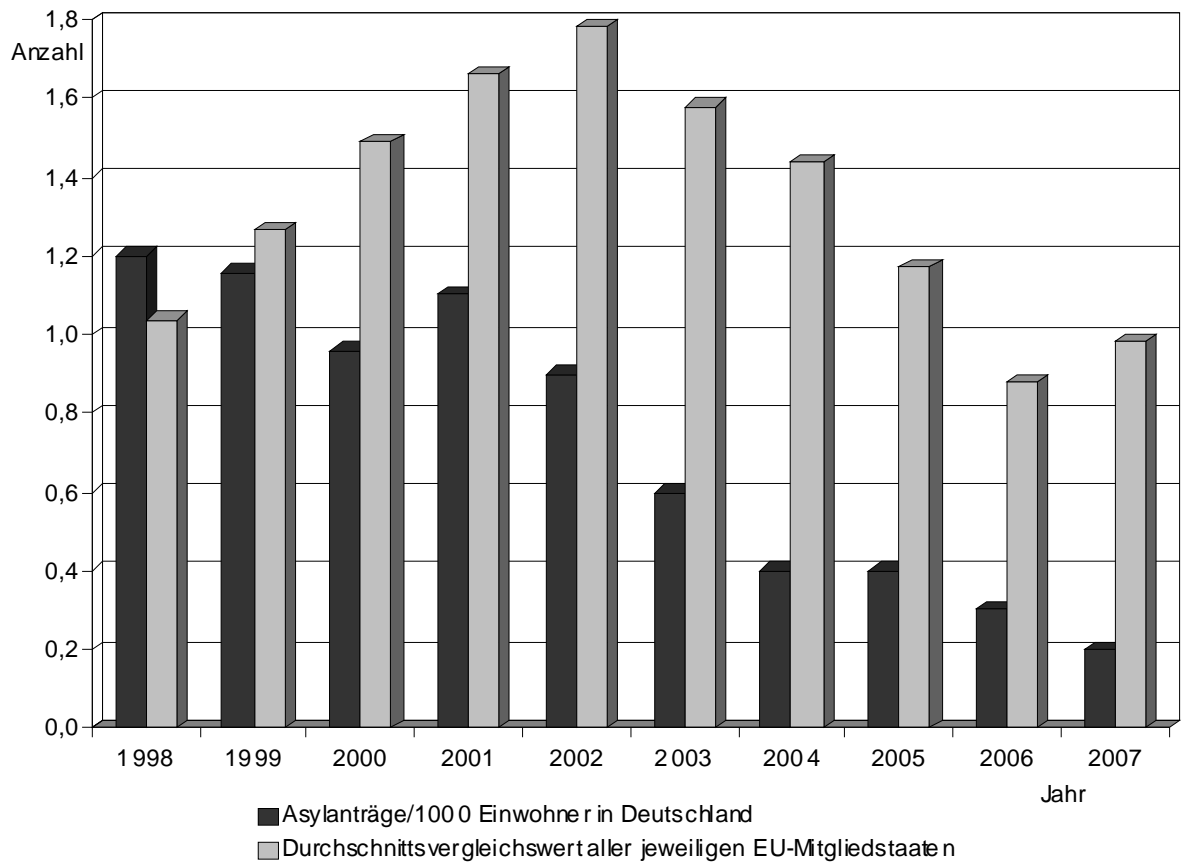

Abb. 3: Zabl der Asylsuchenden je 1000 Einwohner jährlich im Vergleich mit den übrigen EU-

Mitgliedstaaten.

337 Quelle: BT Drs. 16/8861.

338 Einschließlich Norwegen, das zwar nicht EU-Mitglied ist, aber Mitglied des Dublin-Systems. 
Tabelle 4: Asylerstanträge und Überstellungszustimmungen im Rahmen des Dublin Systems seit 1997339

\begin{tabular}{|c|c|c|c|}
\hline \multirow{2}{*}{ Jahr } & \multirow{2}{*}{$\begin{array}{c}\text { Asylerstanträge in } \\
\text { Deutschland }\end{array}$} & $\begin{array}{c}|c| \\
\text { der Mitgliedstaaten auf } \\
\text { Ersuchen Deutschlands }\end{array}$ & $\begin{array}{c}\text { Deutschlands auf Ersu- } \\
\text { chen der Mitgliedstaaten }\end{array}$ \\
\hline 1997 & 104.353 & 66 & 1.131 \\
\hline 1998 & 98.644 & 1.682 & 9.263 \\
\hline 1999 & 95.113 & 2.819 & 7.652 \\
\hline 2000 & 78.564 & 3.651 & 5.662 \\
\hline 2001 & 88.287 & 2.641 & 5.437 \\
\hline 2002 & 71.127 & 3.387 & 7.005 \\
\hline 2003 & 50.563 & 2.967 & 6.229 \\
\hline 2004 & 35.607 & 5.591 & 7.080 \\
\hline 2005 & 28.914 & 4.358 & 4.632 \\
\hline 2006 & 21.029 & 3.290 & 3.722 \\
\hline 2007 & 19.164 & 3.367 & 2.870 \\
\hline
\end{tabular}

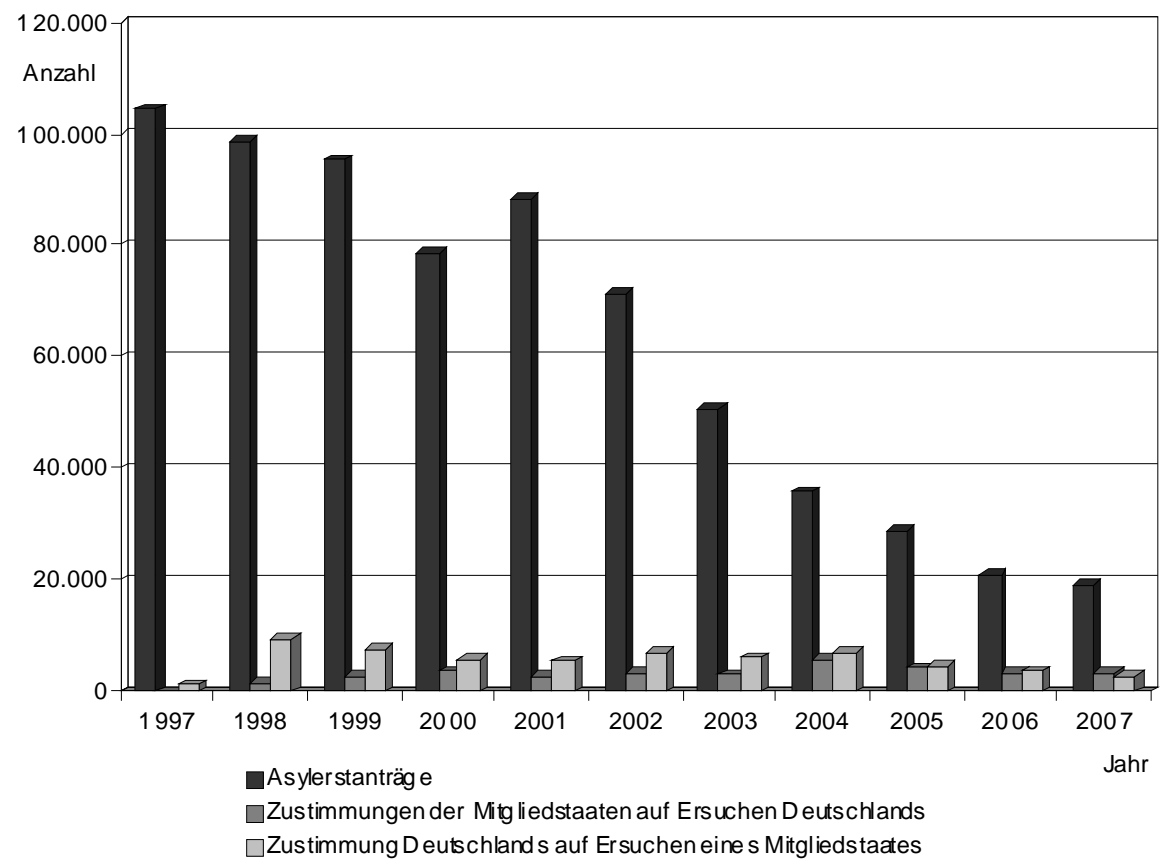

Abb. 4: Asylerstanträge und Überstellungszustimmungen im Rabmen des Dublin Systems seit 1997.

${ }^{339}$ Quelle: BT Drs. 16/8861. 
Tabelle 5: Verhältnis der von den Mitgliedstaaten positiv beantworteten Rückübernahmeersuchen und der tatsächlich ,Rücküberstellten'340

\begin{tabular}{|c|c|c|}
\hline Jahr & $\begin{array}{c}\text { Zustimmungen der Mitgliedstaaten } \\
\text { auf Übernahmeersuchen Deutsch- } \\
\text { lands }\end{array}$ & $\begin{array}{c}\text { Zahl der tatsächlich ,Rücküber- } \\
\text { stellten' nach der Dublin-II- } \\
\text { Verordnung }\end{array}$ \\
\hline 2004 & 5.591 & 2.765 \\
\hline 2005 & 4.358 & 2.516 \\
\hline 2006 & 3.290 & 1.921 \\
\hline 2007 & 3.367 & 1.913 \\
\hline
\end{tabular}

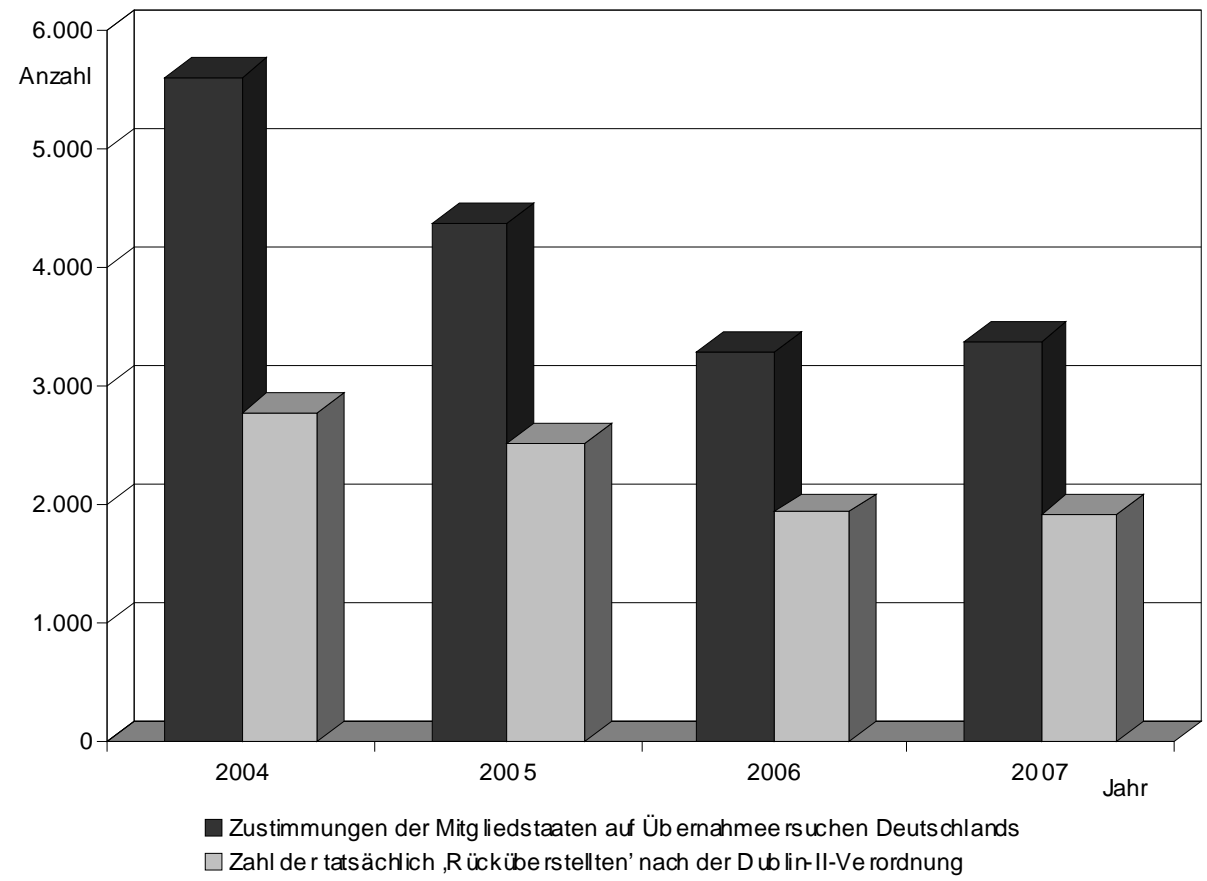

Abb. 5: Verhältnis der von den Mitgliedstaaten positiv beantworteten Rückübernahmeersuchen und der tatsächlich ,Rücküberstellten'.

${ }^{340}$ Quelle: BT Drs. 16/8861. 
Tabelle 6: Zahl der Abschiebungen aus Deutschland 1995 - $2006^{341}$

\begin{tabular}{|c|c|}
\hline Jahr & Abschiebungen \\
\hline 1995 & 36.455 \\
\hline 1996 & 31.761 \\
\hline 1997 & 38.205 \\
\hline 1998 & 38.479 \\
\hline 1999 & 32.929 \\
\hline 2000 & 35.444 \\
\hline 2001 & 27.902 \\
\hline 2002 & 29.036 \\
\hline 2003 & 26.487 \\
\hline 2004 & 23.334 \\
\hline 2005 & 17.773 \\
\hline 2006 & 13.894 \\
\hline
\end{tabular}

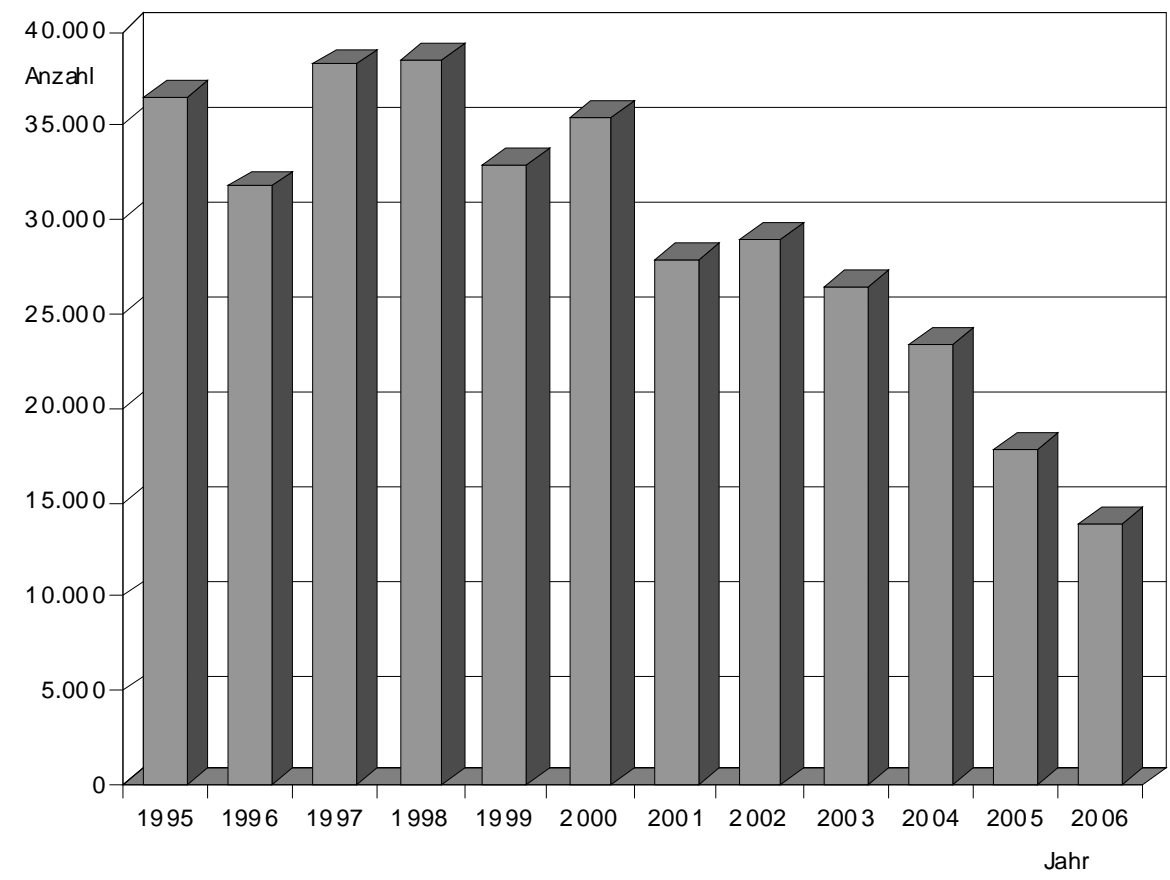

Abb. 6: Zabl der Abschiebungen aus Deutschland 1995 - 2006.

${ }^{341}$ Quelle: OECD (2008). 


\section{B) Polen}

Tabelle 7: Kumulierter Stand polnischer Migration in ausgewählte Länder Westund Mitteleuropas in den Jahren 1998-2006 [in Tsd. Personen] ${ }^{342}$

\begin{tabular}{|l|r|r|r|r|r|r|r|r|r|}
\hline Land Jahr & $\mathbf{1 9 9 8}$ & $\mathbf{1 9 9 9}$ & $\mathbf{2 0 0 0}$ & $\mathbf{2 0 0 1}$ & $\mathbf{2 0 0 2}$ & $\mathbf{2 0 0 3}$ & $\mathbf{2 0 0 4}$ & $\mathbf{2 0 0 5}$ & $\mathbf{2 0 0 6}$ \\
\hline Deutschland & 283,6 & 291,7 & 301,4 & 310,4 & 317,6 & 326,9 & 292,1 & 326,6 & 361,7 \\
\hline Italien & 23,6 & 29,5 & 30,4 & 32,9 & 35,0 & 64,9 & 50,8 & 60,8 & 72,5 \\
\hline Tschechien & 22,2 & 18,3 & 17,1 & 16,5 & 16,0 & 15,8 & 16,3 & 17,8 & 18,9 \\
\hline Schweden & 15,9 & 16,3 & 16,7 & 15,5 & 13,9 & 13,4 & 14,7 & 17,2 & 22,4 \\
\hline Griechenland & 6,7 & 10,4 & 11,2 & 13,5 & 14,1 & 15,9 & 17,0 & 16,1 & 16,6 \\
\hline Belgien & 6,3 & 6,7 & 6,9 & 8,9 & 10,4 & 11,6 & 14,0 & 18,0 & 23,2 \\
\hline Spanien & 6,3 & 7,8 & 12,9 & 18,1 & 23,9 & 26,5 & 34,6 & 43,3 & 58,3 \\
\hline Niederlande & 5,9 & 5,6 & 5,9 & 6,3 & 6,9 & 7,4 & 11,0 & 15,2 & 19,6 \\
\hline Dänemark & 5,5 & 5,6 & 5,5 & 5,7 & 5,7 & 5,8 & 6,2 & 7,4 & 9,7 \\
\hline Norwegen & 2,1 & 2,0 & 2,0 & 2,2 & 2,6 & 2,7 & 4,0 & 6,8 & 13,6 \\
\hline Großbritannien &.. &.. &.. & 34 & 24 & 34 & 48 & 110 & 209 \\
\hline
\end{tabular}

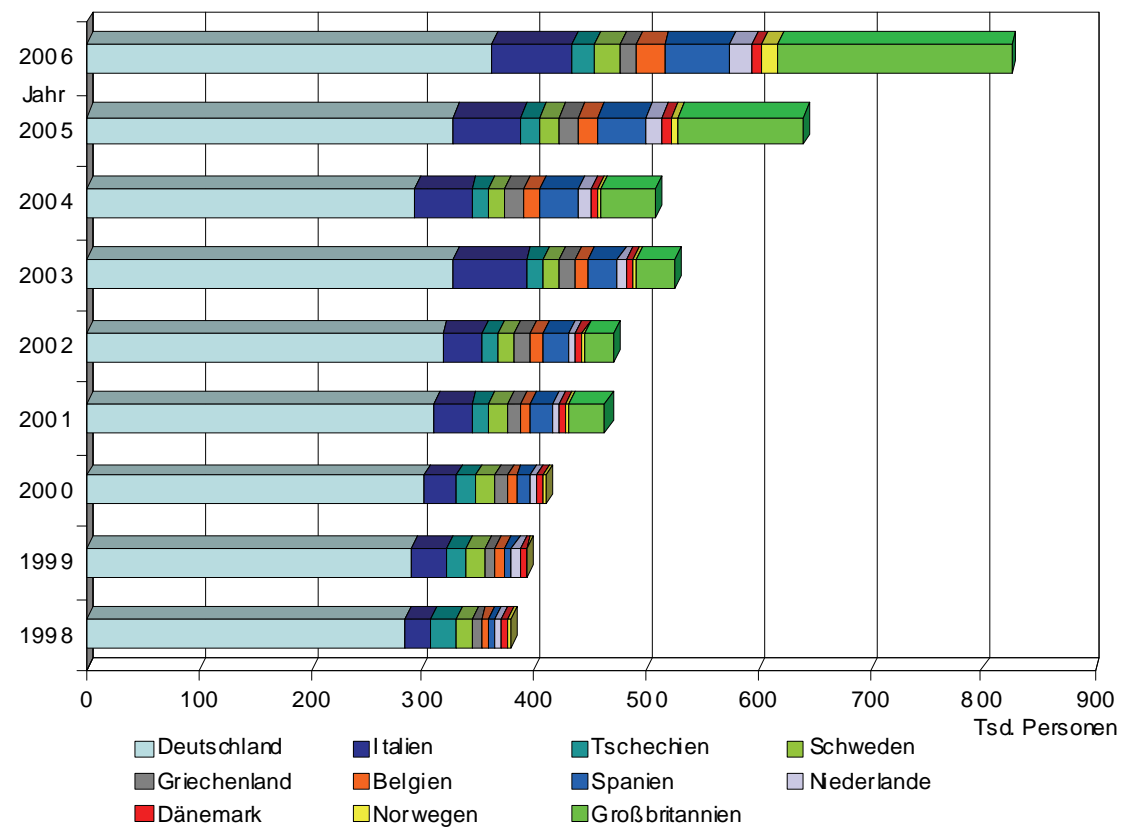

Abb. 7: Kumulierter Stand polnischer Migration in ausgewählte Länder West-und Mitteleuropas in den Jahren 1998-2006 [in Tsd. Personen].

${ }^{342}$ Quelle: OECD (2008). 
Tabelle 8: Zahl der an polnische BürgerInnen ausgestellten Genehmigungen zur Arbeitsaufnahme in ausgewählten Ländern des Europäischen Wirtschaftsraumes (Top 6) (in Tsd. Personen) ${ }^{343}$

\begin{tabular}{|l|r|r|r|}
\hline Staat & $\mathbf{2 0 0 3}^{\mathbf{3 4 4}}$ & $\mathbf{2 0 0 4}$ & $\mathbf{2 0 0 5}$ \\
\hline Deutschland & 274,9 & 392,6 & 321,6 \\
\hline Großbritannien & 1,4 & 75,5 & 129,4 \\
\hline Irland & 2,7 & 27,3 & 64,7 \\
\hline Italien & 45,7 & 37 & 33,5 \\
\hline Niederlande & 9,5 & 20,2 & 26,5 \\
\hline Norwegen & 15,3 & 13 & 23,8345 \\
\hline Gesamt & 401 & 610 & 645 \\
\hline
\end{tabular}

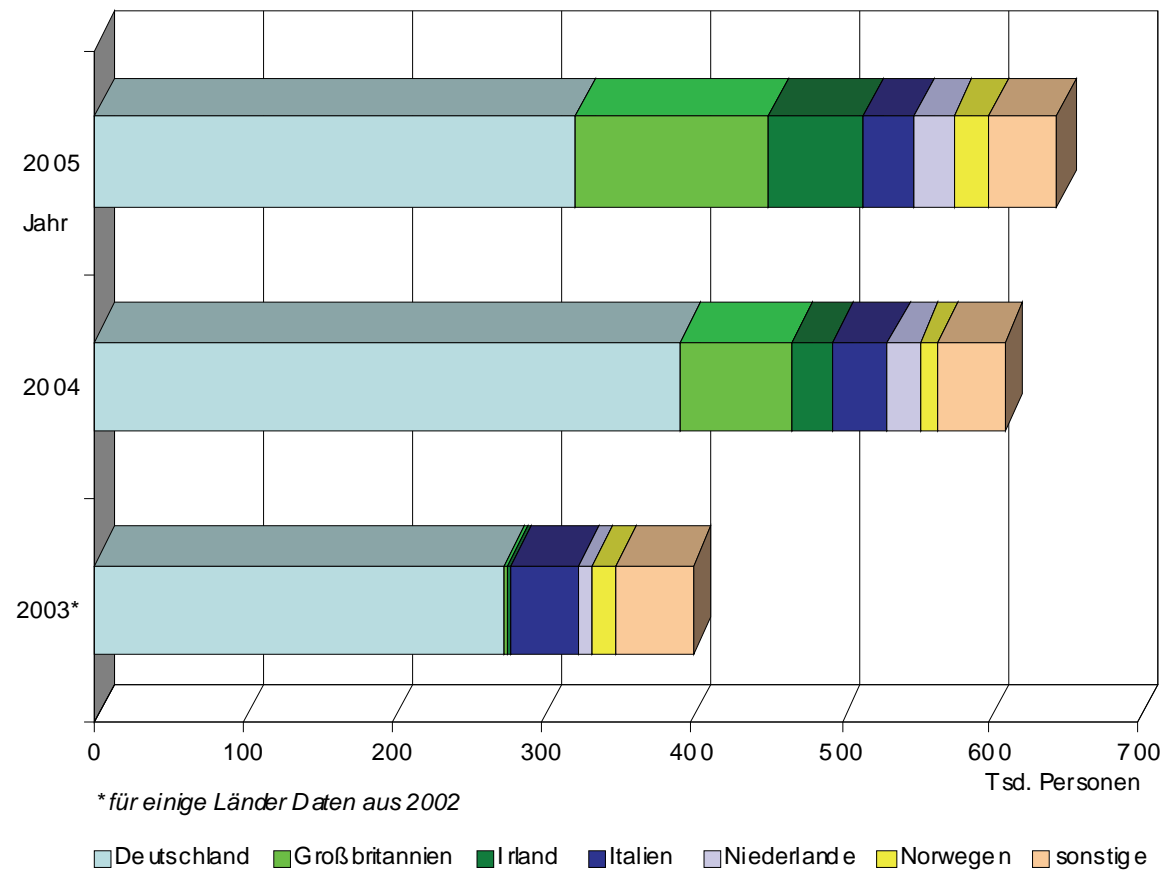

Abb. 8: Zabl der an polnische BürgerInnen ausgestellten Genehmigungen zur Arbeitsaufnabme in ausgewählten Ländern des Europä̈schen Wirtschaftsraumes (Top 6) (in Tsd. Personen).

343 Quelle: Ministerstwo Gospodarki (2007), S. 13.

344 Für einige Länder (bspw. Irland) Daten aus 2002.

345 Daten berücksichtigen Verlängerungen von Genehmigungen. 
Tabelle 9: Anträge auf Flüchtlingsschutz in Polen 1992 - 2007346

\begin{tabular}{|c|c|c|}
\hline Jahr & $\begin{array}{c}\text { ANTRÄGE } \\
\text { - insgesamt - }\end{array}$ & $\begin{array}{c}\text { Antragstellerlnnen } \\
\text { aus der Russischen } \\
\text { Föderation }\end{array}$ \\
\hline 1992 & 568 & 24 \\
\hline 1993 & 822 & 7 \\
\hline 1994 & 537 & 28 \\
\hline 1995 & 845 & 85 \\
\hline 1996 & 3210 & 63 \\
\hline 1997 & 3580 & 50 \\
\hline 1998 & 3423 & 52 \\
\hline 1999 & 3061 & 125 \\
\hline 2000 & 4662 & 1182 \\
\hline 2001 & 4529 & 1501 \\
\hline 2002 & 5170 & 3054 \\
\hline 2003 & 6906 & 5563 \\
\hline 2004 & 8079 & 7183 \\
\hline 2005 & 6860 & 6248 \\
\hline 2006 & 7093 & 6405 \\
\hline 2007 & 10048 & 9239 \\
\hline
\end{tabular}

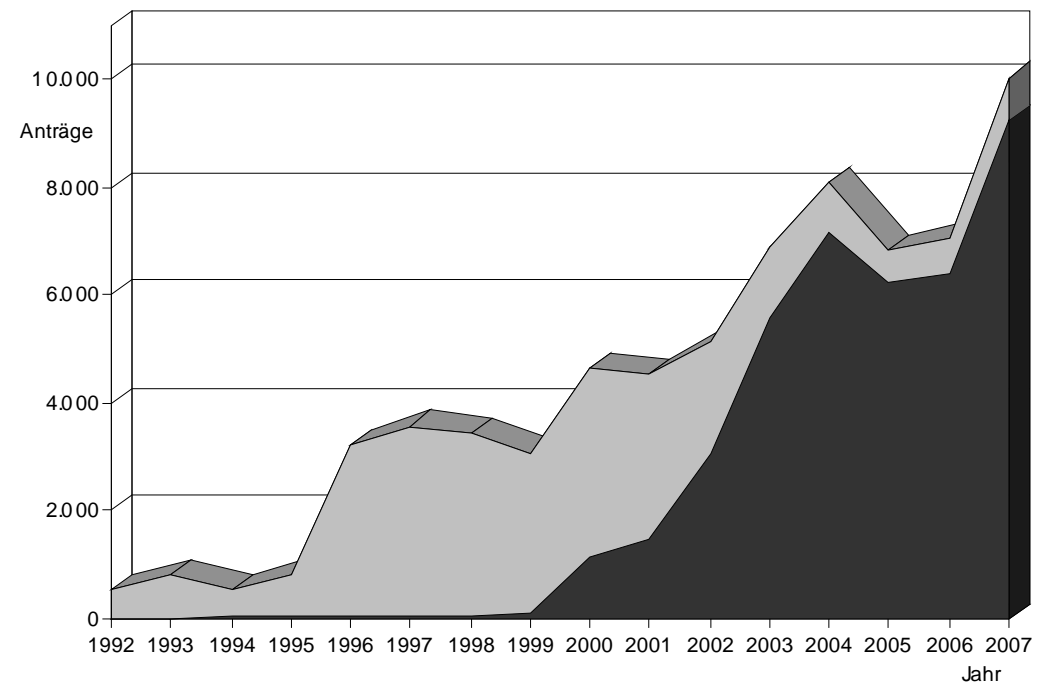

$\square$ Anteil der Antragstellerlnnen aus der Russischen Föderation $\quad \square$ insgesamt

Abb. 9: Anträge auf Flüchtlingsschutz. in Polen 1992 - 2007.

${ }^{346}$ Quelle: UdSC (2004), UdSC (2006), UdSC (2008), UdSC (o.J.). 
Tabelle 10: Entscheidungen über Erstanträge auf Flüchtlingsschutz in Polen 1992 - 2007347

\begin{tabular}{|c|c|c|c|c|c|}
\hline \multirow[b]{2}{*}{ Jahr } & \multirow{2}{*}{$\begin{array}{l}\text { ANTRÄGE } \\
\text { - insgesamt - }\end{array}$} & \multicolumn{4}{|c|}{ Entscheidungen } \\
\hline & & Positiv 348 & Negativ & $\begin{array}{l}\text { Duldungs- } \\
\text { status }\end{array}$ & $\begin{array}{l}\text { Antrag nicht zu Ende } \\
\text { verfolgt / Verfahren } \\
\text { eingestellt }\end{array}$ \\
\hline 1992 & 568 & 74 & 58 & & 1 \\
\hline 1993 & 822 & 61 & 135 & & 236 \\
\hline 1994 & 537 & 392 & 191 & & 363 \\
\hline 1995 & 845 & 105 & 199 & & 394 \\
\hline 1996 & 3210 & 120 & 374 & & 1461 \\
\hline 1997 & 3580 & 140 & 602 & & 3165 \\
\hline 1998 & 3423 & 55 & 1317 & & 1715 \\
\hline 1999 & 3061 & 39 & 1918 & & 790 \\
\hline 2000 & 4662 & 53 & 2527 & & 1217 \\
\hline 2001 & 4529 & 284 & 2864 & & 1830 \\
\hline 2002 & 5170 & 253 & 4729 & & 494 \\
\hline 2003 & 6906 & 219 & 3139 & 24 & 4366 \\
\hline 2004 & 8079 & 305 & 2002 & 826 & 2763 \\
\hline 2005 & 6860 & 312 & 2284 & 1832 & 4413 \\
\hline 2006 & 7093 & 423 & 939 & 2048 & 3875 \\
\hline 2007 & 10048 & 116 & 1727 & 2871 & 1102 \\
\hline
\end{tabular}

347 Quelle: UdSC (2004), UdSC (2006), UdSC (2008). Die verschiedenen Ausgaben dieser Statistik des UdSC enthalten leicht abweichende Daten. Hier liegen immer die Zahlen der jeweils aktuellsten Ausgabe zugrunde.

348 Ab 2002: GFK-Status. 


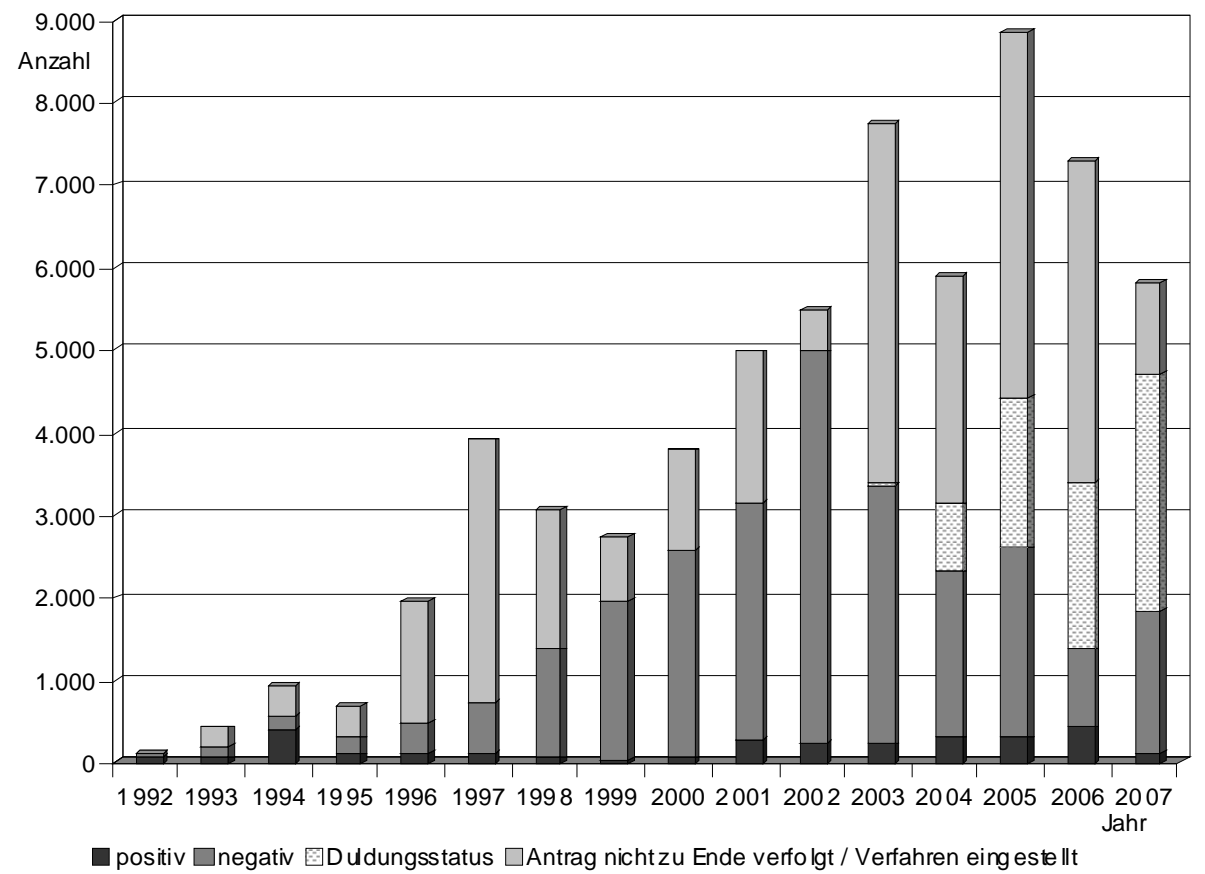

Abb. 10: Entscheidungen über Erstanträge auf Flüchtlingsschutz in Polen 1992 - 2007. 
Tabelle 11: Entscheidungen zur Abschiebung aus Polen 2001 - 2007349

\begin{tabular}{|l|r|r|r|r|r|r|r|}
\hline Nationalität & $\mathbf{2 0 0 1}$ & $\mathbf{2 0 0 2}$ & $\mathbf{2 0 0 3}$ & $\mathbf{2 0 0 4}$ & $\mathbf{2 0 0 5}$ & $\mathbf{2 0 0 6}$ & $\mathbf{2 0 0 7}$ \\
\hline Ukraine & & & & & & & \\
\hline Rumänien & 2322 & 1959 & 2885 & 3761 & 2518 & 2222 & 1541 \\
\hline Bulgarien & 972 & 318 & 298 & 128 & 77 & 182 & 3 \\
\hline Afghanistan & $\mathbf{7 3 8}$ & 936 & 650 & 392 & 189 & 261 & 3 \\
\hline Armenien & 633 & 709 & 246 & 32 & 8 & 11 & 3 \\
\hline $\begin{array}{l}\text { Russische } \\
\text { Föderation }\end{array}$ & 564 & 874 & 664 & 187 & 195 & 182 & 110 \\
\hline Weißrussland & 552 & 401 & 425 & 332 & 217 & 188 & 126 \\
\hline Vietnam & 529 & 696 & 593 & 276 & 331 & 414 & 215 \\
\hline Moldawien & 423 & 256 & 343 & 377 & 509 & 478 & 154 \\
\hline übrige Länder & 1104 & 1524 & 1768 & 934 & 511 & 622 & 456 \\
\hline Gesamt & 8497 & 8280 & 8410 & 6696 & 4898 & 4895 & 2833 \\
\hline
\end{tabular}

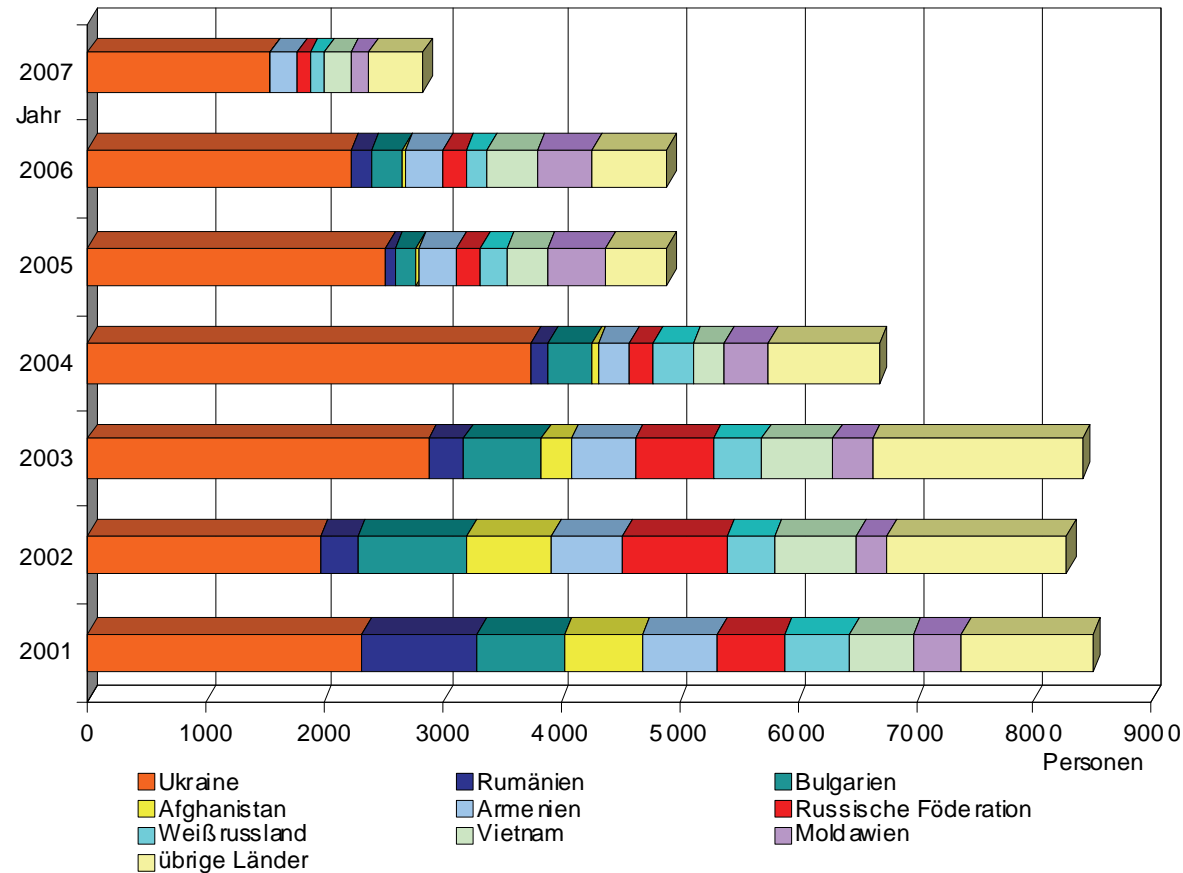

Abb. 11: Entscheidungen zur Abschiebung aus Polen 2001 - 2007.

${ }^{349}$ Quelle: UdSC (2004), UdSC (2006), UdSC (2008). 


\section{Literatur und Quellen}

\section{A) Literatur}

Aarebrot, Frank H./Bakka, Pal H. (2003): Die vergleichende Methode in der Politikwissenschaft, in: Berg-Schlosser, Dirk/Müller-Rommel, Ferdinand (Hrsg.): Vergleichende Politikwissenschaft, 4., überarb. u. erw. Aufl., Opladen, S. 57-76.

ABCDS et al. (2007): Offener Brief. In Marokko werden die Rechte auf eine menschenwürdige Behandlung von Männern und Frauen im Namen der Grenzsicherung Europas verletzt, Rabat, 4.01.2007;

online: www.fluechtlingsrat-

hamburg.de/content/offenerBriefRabat_040107_deu2.pdf, letzter Abruf: $<03.10 .2008>$.

Abels, Gabriele/Behrens, Maria (2005): ExpertInnen-Interviews in der Politikwissenschaft. Geschlechtertheoretische und politikfeldanalytische Reflexion einer Methode, in: Bogner, Alexander/Littig, Beate/Menz, Wolfgang (Hrsg.): Das Experteninterview. Theorie, Methode, Anwendung, 2. Auflage, Wiesbaden, S. 173190.

Abiri, Elisabeth (2000): The Securitisation of Migration. Towards an Understanding of Migration Policy Changes in the 1990s. The Case of Sweden, Göteborg.

Abkommen über die Rechtsstellung der Flüchtlinge vom 28. Juli 1951, in: BGB1. 1953 II S. 560; Protokoll über die Rechtsstellung der Flüchtlinge vom 31. Januar 1967, in: BGBl. 1969 II S. 1294.

Act of 13 June 2003 on Aliens, Journal of Laws of 2003, No. 128, item 1175, online: www.uric.gov.pl, letzter Abruf: <09.01.2009>.

Act of 13 June 2003 on granting protection to aliens within the territory of the Republic of Poland, Journal of Laws of 2003, No. 128, item 1176, online: www.uric.gov.pl, letzter Abruf: <09.01.2009>.

Abschiebehaftanstalt Neuss der JVA Düsseldorf, Selbstdarstellung, online: www.jva-duesseldorf.nrw.de/wir/behoerdenpraes/hh_neuss/index.php Rubrik, Wir über uns' - ,Behördenpräsentation“ - ,Abschiebehaft, letzter Abruf: <09.03.2009>.

Alliance2015 (2008): The EU's contribution to the Millenium Development Goals. Poverty Eradication: From Rhetoric to Results?, Kopenhagen.

Alscher, Stefan (2004): Grenzregion Ostpolen und die EU-Außengrenze: Brücke oder Trennlinie?, in: Banse, Christian/Stobbe, Holk (Hrsg.): Nationale Grenzen in Europa. Wandel der Funktion und Wahrnehmung nationaler Grenzen im Zuge der EU-Erweiterung, Frankfurt a.M., S. 215-234. 
Alscher, Stefan (2008): Länderprofil Polen, Fokus Migration Nr. 3, Januar 2008, online: http://www.focus-

migration.de/uploads/tx_wilpubdb/LP03_Polen_Update.pdf, letzter Abruf: <02.01.2009>.

Alt, Jörg (2003): Leben in der Schattenwelt. Problemkomplex ,illegale“ Migration, Karlsruhe.

Amnesty International (1998): ai gegen „Politik des Aushungerns Schutzbedürftiger“, Pressemitteilung vom 25.03.1998.

Amnesty International (2007): Poland. Submission to the UN Universal Periodic Review, First Session of the HRC UPR Working Group, 7.-18. April 2008, November 2007, AI Index: EUR 37/005/2007, London, online: http://www.amnesty.org, letzter Abruf: <25.09.2008>.

Andrijasevic, Rutvica/Bojadzijev, Manuela/Hess, Sabine/Karakayal1, Serhat/Panagiotidis, Efthimia/Tsianos, Vassilis (2005): Turbulente Ränder. Konturen eines neuen Migrationsregimes im Südosten Europas, in: Prokla 140, 35. Jg., Nr. 3, September 2005, S. 345-362.

Angenendt, Steffen (1997) (Hrsg.): Migration und Flucht. Aufgaben und Strategien für Deutschland, Europa und die internationale Gemeinschaft, Bonn.

Angenendt, Steffen (1999) (Hrsg.): Asylum and Migration Policies in the European Union, Bonn.

Angenendt, Steffen (2002a): Entwicklung und Perspektiven der europäischen Migrations- und Asylpolitik, in: Die Friedens-Warte. Journal of International Peace and Organization, 77. Jg., Nr. 1-2, S. 143-172.

Angenendt, Steffen (2002b): Einwanderungspolitik und Einwanderungsgesetzgebung in Deutschland 2000-2001, in: Bade, Klaus J./Bommes, Michael/Münz, Rainer (Hrsg.): Migrationsreport 2002, Fakten - Analysen - Perspektiven, Frankfurt a.M., S. 31-59.

Angenendt, Steffen (2006): Migrationspolitische Herausforderungen und Strategien in der EU: Kann die Nachbarschaftspolitik einen Beitrag zur Problemlösung leisten?, in: Koopmann, Martin/Lequesne, Christian (Hrsg.): Partner oder Beitrittskandidaten? Die Nachbarschaftspolitik der Europäischen Union auf dem Prüfstand, Baden-Baden, S. 181-206.

Angenendt, Steffen/Fischer, Andrea/Morokvasic, Mirjana (1994): Die OstWest-Wanderung als Thema der politischen und wissenschaftlichen Debatten in Frankreich und Deutschland, in: Morokvasic, Mirjana/Rudolph, Hedwig (Hrsg.): Wanderungsraum Europa. Menschen und Grenzen in Bewegung, Berlin, S. 81109.

Angenendt, Steffen/Kruse, Imke (2004): Migrations- und Integrationspolitik in Deutschland 2002-2003: der Streit um das Zuwanderungsgesetz, in: Bade, Klaus J./Bommes, Michael/Münz, Rainer (Hrsg.): Migrationsreport 2004, Fakten Analysen - Perspektiven, Frankfurt a.M., S. 175-202. 
Angenendt, Steffen/Parkes, Roderick (2007): Das Grünbuch zum EU-Asylsystem: Notwendig, aber nicht hinreichend, SWP-Aktuell 50, Oktober 2007, online: www.swp-berlin.org/common/get_document.php?asset_id=4381, letzter Abruf: $<09.12 .2008>$.

Arendt, Hannah (2008): Elemente und Ursprünge totaler Herrschaft. Antisemitismus, Imperialismus, totale Herrschaft, 12. Auflage, München.

Asyl in der Kirche (o.J.): 20 Jahre Asyl in der Kirche. Eine dokumentarische Ausstellung, online: www.kirchenasyl.de, letzter Abruf: <22.12.2008>.

Bade, Klaus J./Oltmer, Jochen (2007): Mitteleuropa - Deutschland, in: Bade, Klaus J./Emmer, Pieter C./Lucassen, Leo/Oltmer, Jochen (Hrsg.): Enzyklopädie Migration in Europa. Vom 17. Jahrhundert bis zur Gegenwart, Paderborn, S. 141170.

Balibar, Etienne (1991): Es Gibt Keinen Staat in Europa: Racism and politics in Europe today, in: New Left Review, Nr. 186, März/April 1991, S. 5-19.

Banse, Christian/Müller, Doreen/Stobbe, Holk (2007): Tödliche Vision Europa. Die grenz-, migrations- und asylpolitischen Komponenten der Europäischen Nachbarschaftspolitik, in: Fischer, Robert/Karrass, Anne/Kröger, Sandra (Hrsg.): Die Europäische Kommission und die Zukunft der EU. Ideenfabrik zwischen europäischem Auftrag und nationalen Interessen, Opladen und Farmington Hills, S. 179-201.

Barwig, Klaus/Schumacher, Christoph (2002): Migration/Migrationspolitik, in: Nohlen, Dieter (Hrsg.): Kleines Lexikon der Politik, 2. Auflage, München, S. 300305.

Basch, Linda/Glick Schiller, Nina/Szanton Blanc, Cristina (1994) (Hrsg.): Nations Unbound. Transnational Projects, Postcolonial Predicaments and Deterritorialized Nation-States, New York.

Beauftragte der Bundesregierung für Migration, Flüchtlinge und Integration (2004): Migrationsbericht der Integrationsbeauftragten im Auftrag der Bundesregierung, Berlin/Bonn, Januar 2004.

Becher, Ursula A. J./Borodziej, Włodzimierz/Maier, Robert (2005) (Hrsg.): Deutschland und Polen im 20. Jahrhundert. Analysen - Quellen - didaktische Hinweise, Bonn (Ausgabe der Bundeszentrale für politische Bildung).

Bem, Kazimierz (2003): Changes in Polish Refugee Law, in: European Journal of Migration and Law, Vol. 5, No. 3, S. 463-478.

Bendel, Petra (2006): Neue Chancen für die EU-Migrationspolitik? Die Europäische Union im Spagat zwischen Sicherheits-, Entwicklungs- und Außenpolitik, in: Butterwegge, Christoph/Hentges, Gudrun (Hrsg.): Zuwanderung im Zeichen der Globalisierung. Migrations-, Integrations- und Minderheitenpolitik, 3. aktualisierte Auflage, Wiesbaden, S. 123-134. 
Benedikt, Clemens (2004): Diskursive Konstruktion Europas. Migration und Entwicklungspolitik im Prozess der Europäisierung, Frankfurt a.M.

Benz, Martina/Schwenken, Helen (2005): Jenseits von Autonomie und Kontrolle: Migration als eigensinnige Praxis, in: Prokla 140, 35. Jg., Nr. 3, September 2005, S. 363-377.

Berg-Schlosser, Dirk (2003): Makro-Qualitative vergleichende Methoden, in: ders./Müller-Rommel, Ferdinand (Hrsg.): Vergleichende Politikwissenschaft, 4., überarb. u. erw. Auflage, Opladen, S. 103-125.

Bingen, Dieter (1992): Demokratisierung und Nationalismus in Polen, in: Mommsen, Margareta (Hrsg.): Nationalismus in Osteuropa. Gefahrvolle Wege in die Demokratie, München, S. 47-76.

Birkner, Martin/Foltin, Robert (2006): (Post-)Operaismus. Von der Arbeiterautonomie zur Multitude. Geschichte und Gegenwart, Theorie und Praxis. Eine Einführung, Stuttgart.

Birsl, Ursula (2004): Deutschland, in: Gieler, Wolfgang/Fricke, Dietmar (Hrsg.): Handbuch Europäischer Migrationspolitiken. Die EU-Länder und die Beitrittskandidaten, Münster, S. 31-50.

Birsl, Ursula (unter Mitarbeit von Doreen Müller) (2005): Migration und Migrationspolitik im Prozess der europäischen Integration?, Opladen.

Bogner, Alexander/Littig, Beate/Menz, Wolfgang (Hrsg.) (2005): Das Experteninterview. Theorie, Methode, Anwendung, 2. Auflage, Wiesbaden.

Bojadžijev, Manuela (2002): Antirassistischer Widerstand von Migrantinnen und Migranten in der Bundesrepublik: Fragen der Geschichtsschreibung, in: 1999. Zeitschrift für Sozialgeschichte des 20. und 21. Jahrhunderts, 17. Jg., Heft 1, März 2002, S. 125-152.

Böke, Karin (1997): Die „Invasion“ aus den „Armenhäusern Europas“. Metaphern im Einwanderungsdiskurs, in: Jung, Matthias/Wengeler, Martin/Böke, Karin (Hrsg.): Die Sprache des Migrationsdiskurses. Das Reden über „Ausländer““ in Medien, Politik und Alltag, Opladen, S. 164-193.

Bommes, Michael/Morawska, Ewa (2005) (Hrsg.): International Migration Research. Constructions, Omissions and the Promises of Interdisciplinarity, Aldershot.

Borodziej, Włodzimierz/Lemberg, Hans (2004): Migrationen: Arbeitswanderung, Emigration, Vertreibung, Umsiedlung, in: Becher, Ursula A. J./Borodziej, Włodzimierz/Maier, Robert (Hrsg.): Deutschland und Polen im 20. Jahrhundert. Analysen - Quellen - didaktische Hinweise, Bonn (Ausgabe der Bundeszentrale für politische Bildung), S. $52-54$.

Bos, Ellen (2004): Verfassungsgebung und Systemwechsel. Die Institutionalisierung von Demokratie im postsozialistischen Osteuropa, Wiesbaden.

Bosswick, Wolfgang (2000): Development of Asylum Policy in Germany, in: Journal of Refugee Studies, Vol. 13, No. 1, S. 43-60. 
Boswell, Christina (2002): The Liberal Dilemma in the Ethics of Refugee Policy, Paper prepared for the panel "Challenges to the 'Liberal Paradox' and 'Root Causes' Theory”, ISA, New Orleans, March 2002, online: http://isanet.ccit.arizona.edu/noarchive/boswell.html, letzter Abruf: $<01.09 .2010>$.

BR Drs. 799/06: Unterrichtung durch dasEuropäische Parlament: Entschließung des Europäischen Parlaments zur gemeinsamen Einwanderungspolitik der Europäischen Union, 25.10.2006.

BR Drs. 691/97: Entwurf eines Zweiten Gesetzes zur Änderung des Asylbewerberleistungsgesetzes - Antrag des Landes Berlin - Antrag des Landes Berlin gemäß \ 23 Abs. 3 i. V. m. $\int 15$ Abs. 1 GO BR.

Bröll, Claudia (2007): Das Guinness wird von Polen gezapft, in: Das Parlament Nr. 3, 15.01.2007.

Brubaker, Rogers (1992): Citizenship and Nationhood in France and Germany, Cambridge.

Brücker, Herbert (2004): EU-Osterweiterung: Effekte der Migration, in: Wochenbericht des DIW Berlin, 71. Jg., Heft 17, S. 223-228.

Brücker, Herbert (2005): EU-Osterweiterung: Übergangsfristen führen zu Umlenkung der Migration nach Großbritannien und Irland, in: Wochenbericht des DIW Berlin, 72. Jg., Heft 22, S. 353-359.

BT Drs. 12/4451: Entwurf eines Gesetzes zur Neuregelung der Leistungen an Asylbewerber (Asylbewerberleistungsgesetz).

BT Drs. 15/420: Gesetzentwurf der Bundesregierung. Entwurf eines Gesetzes zur Steuerung und Begrenzung der Zuwanderung und zur Regelung des Aufenthalts und der Integration von Unionsbürgern und Ausländern (Zuwanderungsgesetz).

B'T Drs. 16/1752: Antwort der Bundesregierung auf die Kleine Anfrage der Abgeordneten Silke Stokar von Neuforn, Wolfgang Wieland, Volker Beck (Köln), weiterer Abgeordneter und der Fraktion Bündnis 90/Die Grünen - Drucksache 16/1530 - Europäische Grenzschutzagentur.

BT Drs. 16/3446: Antwort der Bundesregierung auf die Kleine Anfrage der Abgeordneten Ulla Jelpke, Sevim Dağdelen, Jan Korte und der Fraktion DIE LINKE. - Drucksache 16/3239 - Zahl der geduldeten und asylsuchenden Personen in der Bundesrepublik Deutschland.

B'T Drs. 16/4523: Kleine Anfrage der Abgeordneten Ulla Jelpke, Sevim Dağdelen, Dr. Hakki Keskin, Jan Korte, Kersten Naumann und der Fraktion DIE LINKE. Abschiebungen im Jahr 2006.

BT Drs. 16/5498: Antwort der Bundesregierung auf die Kleine Anfrage der Abgeordneten Sevim Dağdelen, Ulla Jelpke, Karin Binder, weiterer Abgeordneter und der Fraktion DIE LINKE. - Drucksache 16/5201 - Geplante Regelungen zum Familiennachzug und Vermutungen zu „Scheinehen“, „Scheinlebenspartnerschaften“, „Zweckadoptionen“ und Zwangsverheiratungen. 
BT Drs. 16/7089: Antwort der Bundesregierung auf die Kleine Anfrage der Abgeordneten Ulla Jelpke, Petra Pau, Sevim Dağdelen, weiterer Abgeordneter und der Fraktion DIE LINKE. - Drucksache 16/6832 - Ergebnisse der Bleiberechtsregelung der Innenministerkonferenz.

BT Drs. 16/7365: Kleine Anfrage der Abgeordneten Josef Philip Winkler, Markus Kurth, Volker Beck (Köln), Kerstin Andreae, Britta Haßelmann, Monika Lazar, Brigitte Pothmer, Silke Stokar von Neuforn, Dr. Harald Terpe und der Fraktion Bündnis 90/Die Grünen. Sozialrechtliche Schlechterstellung von Flüchtlingen nach dem Asylbewerberleistungsgesetz.

BT Drs. 16/7574: Antwort der Bundesregierung auf die Kleine Anfrage der Abgeordneten Josef Philip Winkler, Markus Kurth, Volker Beck (Köln), weiterer Abgeordneter und der Fraktion Bündnis 90/Die Grünen - Drucksache 16/7365 Sozialrechtliche Schlechterstellung von Flüchtlingen nach dem Asylbewerberleistungsgesetz.

BT Drs. 16/8362: Antwort der Bundesregierung auf die Kleine Anfrage der Abgeordneten Ulla Jelpke, Petra Pau, Sevim Dağdelen und der Fraktion DIE LINKE. - Drucksache 16/8137 - Erste Bilanz der gesetzlichen Altfallregelung.

BT Drs. 16/8861: Antwort der Bundesregierung auf die Kleine Anfrage der Abgeordneten Ulla Jelpke, Sevim Dağdelen und der Fraktion DIE LINKE. - Drucksache 16/8722 - Bewertung des Dublin-Systems und Möglichkeiten der zeitweisen Aussetzung des Dublin-Verfahrens.

BT Drs. 16/9018: Antwort der Bundesregierung auf die Große Anfrage der Abgeordneten Ulla Jelpke, Sevim Dağdelen, Petra Pau, weiterer Abgeordneter und der Fraktion DIE LINKE. - Drucksache 16/7213 - Soziale Existenzsicherung nach dem Asylbewerberleistungsgesetz.

Bundesamt für Migration und Flüchtlinge (2006): Asyl in Zahlen, 15. Auflage, Nürnberg.

Bundesamt für Migration und Flüchtlinge (2008): Asyl in Zahlen 2007, Nürnberg. online:

http://www.bamf.de/cln_092/nn_442110/SharedDocs/Anlagen/DE/DasBA MF/Publikationen/broschuere-asyl-in-zahlen-2007.html, letzter Abruf: $<10.03 .2009>$.

Bundesministerium des Innern (1995) (Hrsg.): Bericht des Bundesministeriums des Innern zur Fortschreibung des Asyl-Erfahrungsberichts 1993 - AsylErfahrungsbericht 1994 -, Bonn.

Bundesministerium des Innern (2006a): Europa sicher leben. Arbeitsprogramm für die deutsche EU-Ratspräsidentschaft, Berlin, online: www.bmi.de, letzter Abruf: <27.08.2008>. 
Bundesministerium des Innern (2006b): Bericht zur Evaluierung des Gesetzes zur Steuerung und Begrenzung der Zuwanderung und zur Regelung des Aufenthalts und der Integration von Unionsbürgern und Ausländern (Zuwanderungsgesetz), online: http://www.bmi.bund.de, letzter Abruf: <21.09.2008>.

Bundesministerium des Innern (2006c): Praktiker Erfahrungsaustausch im Rahmen der Evaluierung des Zuwanderungsgesetzes am 30. und 31. März 2006 im Bundesministerium des Innern in Berlin, Anlage 1 zum Bericht zur Evaluierung des Zuwanderungsgesetzes, online: http://www.bmi.bund.de, letzter Abruf: <21.09.2008>.

Bundesministerium des Innern (2007a): Europäische Harmonisierung des Flüchtlings- und Migrationsrechts, publiziert durch die Internetredaktion des BMI über deren Newsletter am 14.02.2007 sowie online:

http://www.bmi.bund.de/DE/Themen/MigrationIntegration/AsylZuwanderun g/EURecht/EURecht.html, Abschnitt E. Europäische Rückführungspolitik, letzter Abruf: <01.04.2009>.

Bundesministerium des Innern (2007b): Innenminister wollen die Europäische Grenzschutzagentur Frontex weiter stärken und in Migrationsfragen eng mit den Herkunfts- und Transitländern zusammenarbeiten. Pressemitteilung vom 15.02.2007.

Butterwegge, Christoph (2003): Weltmarkt, Wohlfahrtsstaat und Zuwanderung, in: ders./Hentges, Gudrun (Hrsg.): Zuwanderung im Zeichen der Globalisierung, Migrations-, Integrations- und Minderheitenpolitik, 2. Auflage, Opladen, S. 53-91.

Butterwegge, Christoph (2006): Migrationsberichterstattung, Medienpädagogik und politische Bildung, in: ders./Hentges, Gudrun (Hrsg.): Massenmedien, Migration und Integration, Wiesbaden, S. 185-235.

Butterwegge, Christoph/Cremer, Janine/Häusler, Alexander/Hentges, Gudrun/Pfeiffer, Thomas/Reißlandt, Carolin/Salzborn, Samuel (2002) (Hrsg.): Themen der Rechten - Themen der Mitte. Zuwanderung, demografischer Wandel und Nationalbewusstsein, Opladen.

Butterwegge, Christoph/Hentges, Gudrun (2006) (Hrsg.): Massenmedien, Migration und Integration. Herausforderungen für Journalismus und politische Bildung, Wiesbaden.

BVerfGE 94, 49 vom 14.05.1996: Sichere Drittstaaten.

Byrne, Rosemary/Noll, Gregor/Vedsted-Hansen, Jens (2003): Understanding Refugee Law in an Enlarged European Union, IIIS Discussion Paper No. 11, online: www.forcedmigration.org/events/prague2004/byrne-paper.pdf, letzter Abruf: <01.09.2010>.

Calavita, Kitty (2006): Gender, Migration, and Law: Crossing Borders and Bridging Disciplines, in: International Migration Review, Vol. 40, No. 1, Spring 2006, S. 104-132. 
Cartagena Declaration on Refugees, Adopted by the Colloquium on the International Protection on Refugees in Central America, Mexico and Panama, held in Cartagena on 19 - 22 November 1984.

Castles, Stephen (2005): Warum Migrationspolitiken scheitern, in: Peripherie, 25. Jg., Nr. 97/98, S. 10-34.

CDU (2001): Zuwanderung steuern und begrenzen. Integration fördern. Beschluss des Bundesausschusses der CDU Deutschlands vom 7. Juni 2001 in Berlin, online:

www.grundsatzprogramm.cdu.de/doc/070601_zuwanderung_steuern.pdf, letzter Abruf: $<01.09 .2010>$.

CDU-Pressestelle (2001): Kommissionsbericht keine taugliche Grundlage für parteiübergreifenden Konsens, Stellungnahme der Union zum Bericht der Süßmuthkommission vom 04.07.2001.

Classen, Georg (1999): Menschenwürde mit Rabatt. Leitfaden und Dokumentation zum Asylbewerberleistungsgesetz, 2. überarbeitete Auflage, online: www.proasyl.de/lit/classen2/classen2-1.htm, letzter Abruf: <21.09.2008>.

COMECE (2008): Rückführungsrichtlinie: Große Bedenken von Kirchen und christlichen Organisationen, Pressemitteilung vom 05.06.2008, online: www.comece.org, letzter Abruf: <05.09.2008>.

Cornelius, Wayne A./Tsuda, Takeyuki (2004): Controlling Immigration: The Limits of Government Intervention, in: dies./Martin, Philip L./Hollifield, James F. (Hrsg.): Controlling Immigration. A Global Perspective, 2. Auflage, Stanford, S. 3-48.

Currle, Edda (2004): Migration in Europa - Daten und Hintergründe, Stuttgart.

Cuttitta, Paolo (2004): Das diskrete Sterben. Die Verlagerung europäischer Grenzkontrollen nach Nordafrika zeitigt schon jetzt in Wüste und Meer tödliche Folgen, Dokumentation, in: Frankfurter Rundschau vom 14.08.2004.

Cyrus, Norbert/Vogel, Dita (2003): Germany, in: Niessen, Jan/Schibel, Yongmi/Magoni, Raphaële (Hrsg.): EU and US approaches to the management of immigration, MPG-Paper, online: www.migpolgroup.com/public/docs/143.Germany_EUUSapproachestotheManagementofImmigration_2003.pdf, letzter Abruf: $<01.09 .2010>$.

Davy, Ulrike (2002): Das neue Zuwanderungsrecht: Vom Ausländergesetz zum Aufenthaltsgesetz, in: Zeitschrift für Ausländerrecht und Ausländerpolitik (ZAR), 22. Jg., Heft 5/6, S. 171-179.

Der Spiegel vom 09.03.2000: „Kinder statt Inder“. Rüttgers verteidigt verbalen Ausrutscher.

Der Spiegel vom 15.03.2004: „,Out of Germany“. Viele Green-Card-Inhaber verlassen Deutschland. 
Der Spiegel vom 20.01.1992: „Sie nehmen uns alles weg.“ Polens Präsident Lech Walesa über Revolution, Marktwirtschaft und die deutschen Nachbarn.

Der Spiegel vom 28.06.1993: Granate im Korb. Die verschärfte Bonner Asylpolitik macht Polen zum Fluchtpunkt. Deshalb will Warschau seine Ostgrenze besser abschirmen.

Der Standard vom 12.09.2007: Das andere Polen: Die Kinder der Solidarnosc.

Der Standard vom 21.12.2006: Frontex ist ein Sündenbock.

Deutscher Ärztetag (2004): Beschlussprotokoll des 107. Deutschen Ärztetages vom 18.-21. Mai 2004 in Bremen, online: www.bundesaerztekammer.de/downloads/Beschluesse107.pdf, letzter Abruf: <01.09.2010>.

Deutscher Ärztetag (2007): Beschlussprotokoll des 110. Deutschen Ärztetages vom 15.-18. Mai 2007 in Münster, online:

www.bundesaerztekammer.de/downloads/DAETBeschlussprotokoll20070822a. pdf, letzter Abruf: <20.12.2008>.

Dickel, Doris (2002): Einwanderungs- und Asylpolitik der Vereinigten Staaten von Amerika, Frankreichs und der Bundesrepublik Deutschland. Eine Vergleichsstudie der 1980er und 1990er Jahre, Opladen.

Die deutschen Bischöfe - Kommission für Migrationsfragen (2001): Leben in der Illegalität in Deutschland - eine humanitäre und pastorale Herausforderung, hrsg. vom Sekretariat der Deutschen Bischofskonferenz, Bonn, online: www.forum-illegalitaet.de/DBK_2001.pdf, letzter Abruf: <22.12.2008>.

Die Tageszeitung vom 18.04.2007: Linkspartei schiebt Flüchtlinge ab.

Die Welt vom 03.07.2008: Südamerika ist beleidigt. Mercosur droht EU wegen neuer Einwanderungsregeln mit Vergeltung.

Die Zeit vom 21.07.1995: Ein Innenminister und das Kirchenasyl. Heikles Angebot.

Die Zeit vom 28.10.1999: Das Asylrecht lässt sich nicht halten. Wie umgehen mit Flüchtlingen und Einwanderern? Was tun gegen Kriminelle? Gespräch mit Bundesinnenminister Otto Schily.

Dietrich, Helmut (2005): Polen: Flüchtlingsnotstand - aber wo sind die Flüchtlinge?, in: Prokla 140, 35. Jg., Nr. 3, S. 379-392.

Dittrich, Eckhard, J./Radtke, Frank-Olaf (1990) (Hrsg.): Ethnizität. Wissenschaft und Minderheiten, Opladen.

Düvell, Franck (2006): Europäische und internationale Migration. Einführung in historische, soziologische und politische Analysen, Hamburg. 
ECRE (2003): Information Note on the Council Directive 2003/9/EC of 27 January 2003, Juni 2003, online: www.ecre.org/resources/responses_recommendations/291, letzter Abruf: <27.08.2008>.

ECRE (2005): The EC Directive on the Reception of Asylum Seekers: Are asylum seekers in Europe receiving material support and access to employment in accordance with European legislation?, AD3/11/2005/EXT/SH, November 2005, online: www.ecre.org/files/Reception\%20Report_FINAL_Feb06.doc, letzter Abruf: <01.09.2010>.

ECRE (2006): Report on the Application of the Dublin-II-Regulation in Europe, März 2006, online: www.ecre.org/resources/Policy_papers/355, letzter Abruf: <01.09.2010>.

ECRE (2008): ECRE and Amnesty International urge MEPs not to accept the Returns Directive compromise text, Pressemitteilung vom 20.05.2008, online: www.ecre.org/resources/press_releases/1089, letzter Abruf: <01.09.2010>.

Eßer, Barbara/Gladysch, Barbara/Suwelack, Benita (2005): Die Situation tschetschenischer Asylbewerber und Flüchtlinge in Polen und Auswirkungen der EUVerordnung Dublin II, online: www.proasyl.info/texte/2005/Tschetschenien_04/TsFluechtlingePolen.pdf, letzter Abruf: <25.09.2008>.

Fagasinski, Maciej (2007): Flüchtlinge. In schlechter Gesellschaft, in: amnesty journal, Heft 1/2007, o.S., online: www.amnesty.de, letzter Abruf: <14.11.2008>.

Faist, Thomas (1994): How to Define a Foreigner? The Symbolic Politics of Immigration in German Partisan Discourse, 1978-1992, in: West European Politics, Vol. 17, No. 2, S. 50-71.

Falkner, Gerda (2003): Comparing Europeanisation Effects: From Metaphor to Operationalisation, in: European Integration online Papers (EIoP), Vol. 7, Nr. 13, online: http:/ /eiop.or.at/eiop/pdf/2003-013.pdf, letzter Abruf: <01.09.2010>.

Fassmann, Heinz/Münz, Rainer (1996): Europäische Migration - ein Überblick, in: dies. (Hrsg.): Migration in Europa. Historische Entwicklung, aktuelle Trends, politische Reaktionen, Frankfurt a.M./New York, S. 13-52.

Fassmann, Heinz/Münz, Rainer (2003): Auswirkungen der EU-Erweiterung auf die Ost-West-Wanderung, in: WSI Mitteilungen, 56. Jg., Heft 1, S. 25-32.

Finotelli, Claudia (2007): Illegale Einwanderung, Flüchtlingsmigration und das Ende des Nord-Süd-Mythos: Zur funktionalen Äquivalenz des deutschen und des italienischen Einwanderungsregimes, Berlin et al.

Flüchtlingsrat Schleswig-Holstein (2004): „Dann wird’s kritisch“ - Asyl in Polen nach dem Beitritt in die EU, Broschüre, Kiel. 
Focus vom 03.07.2000: Schröders Köder.

Forschungsgesellschaft Flucht und Migration (1999): Flüchtlings- und Migrationspolitik in Polen, in: Transdora Nr. 19, Februar 1999, S. 29-35.

Forschungsgesellschaft Flucht und Migration (2005): Europäische Migrationsgeografien, Polen, in: An Architektur 15/FFM Heft 11, Berlin.

Forschungsgruppe Transit Migration/Labor k3000 (o.J.): MigMap - Governing Migration. A Virtual Cartography of European Migration Policies, online: www.transitmigration.org/migmap/home_entry_d.html, letzter Abruf: <01.09.2010>.

Frankfurter Allgemeine Zeitung vom 18.01.1992: 1991 mehr als 300 Brandanschläge gegen Asylbewerber und Ausländer. Drei Viertel der Straftaten im Westen, zumeist in Nordrhein-Westfalen.

Frankfurter Allgemeine Zeitung vom 21.07.1995: „Kirche nicht Staat im Staat““ Politiker und Kirchenvertreter sprechen über Asyl.

Frankfurter Allgemeine Zeitung vom 30.03.1996: Innenminister einigen sich auf Härtefallregelung.

Frankfurter Allgemeine Zeitung vom 21.07.1997: „Nicht so zaghaft mit ausländischen Straftätern“. Schröder präsentiert sich als Kämpfer gegen die Kriminalität/Erbitterte Ablehnung bei den Grünen.

Frankfurter Allgemeine Zeitung vom 29.07.2006: Scheinvaterschaft. Schwerhörige Gesetzgeber.

Frankfurter Allgemeine Zeitung vom 10.08.2006: Großbritannien erlebt eine Einwanderungswelle.

Frankfurter Allgemeine Zeitung vom 11.09.2007: Tod vor der Abschiebung.

Frankfurter Rundschau vom 04.08.1990: Einig gegen die „Asylantenflut“. Stoiber und Lafontaine bestehen auf Änderung des Asylrechts.

Frankfurter Rundschau vom 21.03.1998: Offener Brief der Bischöfe Norddeutschlands.

Frankfurter Rundschau vom 29.04.1998: Berliner Aufruf gegen eine Politik des Aushungerns von Flüchtlingen in Deutschland.

Frankfurter Rundschau vom 05.02.2004: Pilot hielt Ageeb für „Mörder“. Schwere Vorwürfe gegen BGS im Prozess um Tod des Sudanesen.

Frankfurter Rundschau vom 30.03.2004: Vor Angriff auf globales Asylrecht gewarnt. Menschenrechtler und UNHCR starten vor EU-Beratungen zum Flüchtlingsschutz einen dramatischen Appell.

Frankfurter Rundschau vom 22.07.2004: Schily beharrt auf Lagern für afrikanische Flüchtlinge.

Frankfurter Rundschau vom 16.03.2007: Unfaire Absagen. Kritik an Asylverfahren. 
Frankfurter Rundschau vom 24.04.2008: Im Zweifel Abschiebung. Pakistaner trotz Ermittlungen ausgeflogen.

Freeman, Gary P. (2005): Political Science and Comparative Immigration Politics, in: Bommes, Michael/Morawska, Ewa (Hrsg.): International Migration Research. Constructions, Omissions and the Promises of Interdisciplinarity, Aldershot, S. 111-128.

Gazeta Wyborcza vom 03.09.2006: Co drugi młody Polak rozważa wyjazd z kraju (Jeder zweite junge Pole denkt darüber nach, das Land zu verlassen).

Gazeta Wyborcza vom 29.10.2006: Czeczeńcy niemile widziani (Tschetschenen nicht gern gesehen).

Gazeta Wyborcza vom 24.11.2006: Wrocloves you - wracaj, ziomku, do Wrocławia! (Wrocloves you - kehre zurück, Landsmann, nach Wrocław!).

Gazeta Wyborcza vom 10.01.2007: Osądźmy zbrodnię, a nie kolor skóry (Verurteilen wir das Verbrechen, nicht aber die Hautfarbe).

Gazeta Wyborcza vom 17.04.2007: Czy testy na HIV to dyskryminacja? (Sind HIVTests Diskriminierung?).

Gazeta Wyborcza vom 31.05.2007: Częściowo otworzymy rynek pracy dla Rosjan, Białorusinów i Ukraińców (Wir öffnen teilweise den Arbeitsmarkt für Russen, Weißrussen und Ukrainer).

Gazeta Wyborcza vom 04.07.2007: Emigranci: Nie wracamy (Emigranten: Wir kehren nicht zurück).

Gazeta Wyborcza vom 18.07.2007: Będziemy importować pracowników ze Wschodu (Wir werden Arbeiter aus dem Osten importieren).

Gazeta Wyborcza vom 18.07.2007: Od piątku pracownicy ze wschodu bez zezwoleń (Arbeiter aus dem Osten seit Freitag ohne Genehmigung).

Geddes, Andrew (2000): Immigration and European Union: Towards Fortress Europe?, Manchester.

Geddes, Andrew (2003): The Politics of Migration and Immigration in Europe, London/Thousand Oaks/Neu Delhi.

Geddes, Andrew (2005): Migration research and European Integration: The Construction and Institutionalization of Problems in Europe, in: Bommes, Michael/Morawska, Ewa (Hrsg.): International migration research: constructions, omissions, and the promises of interdisciplinarity, Aldershot, S. 265-285.

Germanwatch (1997): Neues EU-Fischereiabkommen mit Senegal wird Existenzbasis senegalesischer Kleinfischer gefährden: Drastischer Widerspruch zwischen EU-Fischerei- und Entwicklungspolitik, Pressemitteilung vom 10.04.97, Bonn, online: www.germanwatch.org/pubpress/p970410a.htm,

letzter Abruf: <01.09.2010>. 
Gesetz zur Steuerung und Begrenzung der Zuwanderung und zur Regelung des Aufenthalts und der Integration von Unionsbürgern und Ausländern (Zuwanderungsgesetz) vom 30.07.2004, BGBl. I S. 1950.

Gieler, Wolfgang/Fricke, Dietmar (2004) (Hrsg.): Handbuch europäischer Migrationspolitiken. Die EU-Länder und die Beitrittskandidaten, Münster.

Givens, Terri/Luedtke, Adam (2004): The Politics of European Union Immigration Policy: Institutions, Salience, and Harmonization, in: The Policy Studies Journal, Vol. 32, No. 1, S. 145-165.

Górny, Agata/Grzymała-Kazłowska, Aleksandra/Koryś, Piotr/Weinar, Agnieszka (2007): Selective Tolerance? Regulations, Practice and Discussions Regarding Dual Citizenship in Poland, in: Faist, Thomas (Hrsg.): Dual Citizenship in Europe. From Nationhood to Societal Integration, Burlington, S. 202-234.

Górny, Agata/Ruspini, Paolo (2004) (Hrsg.): Migration in the New Europe. EastWest Revisited, Basingstoke et al.

Green Cowles, Maria/Risse, Thomas (2001): Europeanization and domestic change: Conclusions, in: dies./Caporaso, James (Hrsg.): Europeanization and domestic change, Ithaca/New York, S. 217-237.

Green, Simon (2006): Zwischen Kontinuität und Wandel: Migrations- und Staatsangehörigkeitspolitik, in: Schmidt, Manfred G/Zohlnhöfer, Reimut (Hrsg.): Regieren in der Bundesrepublik Deutschland: Innen- und Außenpolitik seit 1949, S. 113-134.

grupa 484 o.J.: Mission, online: http://www.grupa484.org.rs, letzter Abruf: <21.07.2007>.

Hammarberg, Thomas (2007): Report by the Commissioner for Human Rights, Mr Thomas Hammarberg, on his Visit to Ukraine, 10-17 December 2006, Straßburg, CommDH(2007)15, online: https://wcd.coe.int, letzter Abruf: <15.01.2009>.

Han, Petrus (2000): Soziologie der Migration. Erklärungsmodelle - Fakten - Politische Konsequenzen - Perspektiven, Stuttgart.

Handelsblatt vom 01.10.2007: Werbekampagnen sollen Auswanderer zurücklocken.

Hatton, Timothy J. (2004): Seeking Asylum in Europe, in: Economic Policy, Vol. 19, No. 38, April 2004, S. 5-62.

Hatzinger, Katrin (2006): Evangelische Kirche und ihre Einschätzung des Flüchtlingsschutzes in Europa, in: Asyl in Europa: Chancen, Risiken und Perspektiven der gemeinsamen europäischen Asylpolitik, epd-Dokumentation des 6. Berliner Symposiums zum Flüchtlingsschutz in der Evangelischen Akademie zu Berlin am 19. Juni 2006, Nr. 32, Frankfurt a.M., S. 23-26.

Haug, Sonja (2000): Klassische und neuere Theorien der Migration. Arbeitspapiere Mannheimer Zentrum für Europäische Sozialforschung, Nr. 30, online: www.mzes.uni-mannheim.de/publications/wp/wp-30.pdf, letzter Abruf: <05.12.2008>. 
Haverland, Markus (2005): Does the EU cause domestic developments? The problem of case selection in Europeanization research, in: European Integration online Papers (EIoP), Vol. 9, No. 2, online: http://eiop.or.at/eiop/pdf/2005-002.pdf, letzter Abruf: <13.10.2008>.

Heitmeyer, Wilhelm (1992): Weshalb ist diese Gesellschaft angesichts von Fremdenfeindlichkeit und Gewalt so paralysiert?, in: Bielefelder Universitätszeitung, Nr. 168, vom 18.12.1992, S. 6-11.

Hentges, Gudrun (2002): Das Plädoyer für eine „deutsche Leitkultur“ - Steilvorlage für die extreme Rechte?, in: Butterwegge, Christoph/Cremer, Janine/Häusler, Alexander/Hentges, Gudrun/Pfeiffer, Thomas/Reißlandt, Carolin/Salzborn, Samuel (Hrsg.): Themen der Rechten - Themen der Mitte. Zuwanderung, demografischer Wandel und Nationalbewusstsein, Opladen, S. 95-121.

Herbert, Ulrich (2003): Geschichte der Ausländerpolitik in Deutschland, Bonn.

Héritier, Adrienne/Knill, Christoph/Mingers, Susanne (1996): Ringing the changes in Europe: Regulatory competition and the transformation of the state, Berlin.

Hess, Sabine/Karakayal1, Serhat (2007): New Governance oder: Die imperiale Kunst des Regierens. Asyldiskurs und Menschenrechtsdispositiv im neuen EUMigrationsmanagement, in: Transit Migration Forschungsgruppe (Hrsg.): Turbulente Ränder. Neue Perspektiven auf Migration an den Grenzen Europas, Bielefeld, S. 39-55.

Hess, Sabine/Tsianos, Vassilis (2003): Europeanizing transnationalism! Konturen des ,europäischen Grenzregime“, Vortrag gehalten auf der Konferenz „Arbeitsmigration. WanderarbeiterInnen auf dem Weltmarkt für Arbeitskraft", online: www.transitmigration.org/db_transit/ausgabe.php?inhaltID =6, letzter Abruf: <01.09.2010>.

Hess, Sabine/Tsianos, Vassilis (2004): „Killing me softly?“: „Festung Europa“ oder Grenzregime als soziales Kräfteverhältnis? Europäisierung der Migrationsund Grenzpolitiken, Vortrag bei der Heinrich Böll Stiftung Hessen e.V. in Frankfurt a.M. am 7.12.2004, online: www.hbs-hessen.de/archivseite/pol/Hess23_03-05.pdf, letzter Abruf: <01.09.2010> .

Hess, Sabine/Tsianos, Vassilis (2007): Europeanizing Transnationalism! Provincializing Europe! - Konturen eines neues Grenzregimes, in: Transit Migration Forschungsgruppe (Hrsg.): Turbulente Ränder. Neue Perspektiven auf Migration an den Grenzen Europas, Bielefeld, S. 23-38.

Hindustan Times vom 13.06.2007: Poland next hot destination for Indians. 
Hoffmann, Holger (2007): Auf zum 3. Änderungsgesetz - Anmerkungen zur Umsetzung der EU-Richtlinien zum Flüchtlings- und Asylrecht durch das „Richtlinienanpassungsgesetz“, Vortrag auf einer Tagung des „Netzwerks Flüchtlingshilfe in Niedersachsen“" am 23.11.2007 in Hannover, online: http://infonet-

frsh.de/fileadmin/infonet/pdf/Hoffmann_Aenderungsgesetz_Kommentar_11_ 2007.pdf, letzter Abruf: 21.09.2008.

Hoffmann-Nowotny, Hans-Joachim (1973): Soziologie des Fremdarbeiterproblems, Stuttgart.

Hollifield, James F. (1992): Immigrants, Markets and States: The Political Economy of Postwar Europe, Cambrigde, Mass.

Holz, Steffi (2007): Alltägliche Ungewissheit - Erfahrungen von Frauen in Abschiebehaft, Münster.

Holzberger, Mark (2003): Die Harmonisierung der europäischen Flüchtlingspolitik, in: Butterwegge, Christoph/Hentges, Gudrun (Hrsg.): Zuwanderung im Zeichen der Globalisierung. Migrations-, Integrations- und Minderheitenpolitik, 2. Auflage, Opladen, S. 111-121.

Holzer, Thomas/Schneider, Gerald (2002): Asylpolitik auf Abwegen. Nationalstaatliche und europäische Reaktionen auf die Globalisierung der Flüchtlingsströme, Opladen.

Hügel, Volker Maria (2004): Deutschland und seine humanitären Verpflichtungen die Umsetzung des Flüchtlingsschutzes im Zuwanderungsgesetz, in: Flüchtlingsrat. Zeitschrift für Flüchtlingspolitik in Niedersachsen, Ausgabe 5/2004, Heft 103, November 2004, S. 26-30.

Human Rights Watch (2005): Ukraine: On the Margins. Rights Violations against Migrants and Asylum Seekers at the New Eastern Border of the European Union, Vol. 17, No. 8(D), November 2005.

ICF - Information and Cooperation Forum (2005): Aufnahmebedingungen für AsylbewerberInnen in Deutschland, Österreich und die angrenzenden Beitrittsstaaten. Abschlußbericht, Frankfurt a.M.

Iglicka, Krystyna (1998): Are They Fellow Countrymen or Not? The Migration of Ethnic Poles from Kazakhstan to Poland, in: International Migration Review, Vol. 32, No. 4, Winter 1998, S. 995-1015.

Iglicka, Krystyna (2001a): Migration Movements from and into Poland in the Light of East-West European Migration, in: International Migration, Vol. 39, No. 1, S. 3-32.

Iglicka, Krystyna (2001b): Poland's Post-War Dynamic of Migration, Aldershot et al.

Iglicka, Krystyna (2001c): Shuttling from the former Soviet Union to Poland: from 'primitive mobility' to migration, in: Journal of Ethnic and Migration Studies, Vol. 27, No. 3, S. 505-518. 
Iglicka, Krystyna (2005a): Active Civic Participation of Immigrants in Poland. Country Report prepared for the European research project POLITIS, Oldenburg, online: www.uni-oldenburg.de/politis-europe/download/Poland.pdf, letzter Abruf: <01.09.2010>.

Iglicka, Krystyna (2005b): National Paradigms of Migration Research in Poland, in: Slany, Krystyna (Hrsg.): International Migration. A Multidimensional Analysis, Krakau, S. 49-63.

Iglicka, Krystyna/Kaźmierkiewicz, Piotr/Mazur-Rafał, Monika (2003): Poland, in: Niessen, Jan/Schibel, Yongmi/Magoni, Raphaële (Hrsg.): EU and US approaches to the management of immigration, online: www.migpolgroup.com, letzter Abruf: <01.09.2010>.

Iglicka, Krystyna/Kaźmierkiewicz, Piotr/Weinar, Agnieszka (2005): Poland, in: Niessen, Jan/Schibel, Yongmi/Thompson, Cressida (Hrsg.): Current Immigration Debates in Europe: A Publication of the European Migration Dialogue, online: www.isp.org.pl, letzter Abruf: <25.09.2008>.

International Herald Tribune vom 16.11.2006: Poland faces labor shortage as workers go west.

International Herald Tribune vom 22.06.2007: Growing concern about labor shortage in Poland.

Iz3w (2005): Debatte: Eine Frage der Rangordnung. Streitgespräch zwischen „Kanak Attak" und „Karawane " über unterschiedliche Ansätze antirassistischer Politik, iz3w, Nr. 284, April/Mai 2005, S. 18-20.

Jacobson, David (1996): Rights across Borders. Immigration and the Decline of Citizenship, Baltimore/London.

Jäger, Siegfried (1995): BrandSätze. Rassismus im Alltag, 4. Auflage, Duisburg, online: www.diss-

duisburg.de/Internetbibliothek/Buecher/Brandsaetze/Brandsaetze.htm, letzter Abruf: <01.09.2010>.

Jäger, Siegfried/Link, Jürgen (1993) (Hrsg.): Die vierte Gewalt. Rassismus und die Medien, Duisburg.

Jelpke, Ulla (2006): Zahl der Asylbewerber von Amts wegen nach oben geschraubt, Pressemitteilung vom 07.12.2006.

Joppke, Christian (1999): Immigration and the Nation State. The United States, Germany and Great Britain, New York.

Jung, Matthias/Wengeler, Martin/Böke, Karin (1997) (Hrsg.): Die Sprache des Migrationsdiskurses. Das Reden über „Ausländer“ in Medien, Politik und Alltag, Opladen.

Jungle World vom 25.10.2006: Heute bleibt die Kantine kalt. 
Kaczmarczyk, Paweł (2004): Future Westward Outflow from Accession Countries: the Case of Poland, in: Górny, Agata/Ruspini, Paolo (Hrsg.): Migration in the New Europe. East-West Revisited, Basingstoke et al., S. 65-92.

Kaczmarczyk, Paweł/Okólski, Marek (2005): International Migration in Central and Eastern Europe - Current and Future Trends, Paper prepared for the United Nations Expert Group Meeting on international Migration and Development, New York, online: http://www.un.org/esa/population/migration/turin/Symposium_Turin_ files/P12_Kaczmarczyk\&Okolski.pdf, letzter Abruf: <11.12.2008>.

Karakayal1, Serhat/Tsianos, Vasilis (2005): Die Figuren der Migration, in: Kölnischer Kunstverein et al. (Hrsg.): Projekt Migration, S. 416-421.

Karakayal1, Serhat/Tsianos, Vassilis (2005): Mapping the Order of New Migration. Undokumentierte Arbeit und die Autonomie der Migration, in: Peripherie, 25. Jg., Nr. 97/98, Mai 2005, S. 35-65.

Katzenstein, Peter J. (1987): Policy and Politics in West Germany. The Growth of a Semisovereign State, Philadelphia.

Kępińska, Ewa (2003): Recent trends in international migration. The 2003 SOPEMI Report for Poland, Warschau, online: http://pdc.ceu.hu/archive/00001898/01/052.pdf, letzter Abruf: $<25.09 .2008>$.

Kępińska, Ewa (2006): Recent trends in international migration. The 2006 SOPEMI Report for Poland, Warschau, online: www.migracje.uw.edu.pl/obm/pix/015_73.pdf, letzter Abruf: <25.09.2008>.

Kępińska, Ewa/Stola, Dariusz (2004): Migration Policies and Politics in Poland, in: Górny, Agata/Ruspini, Paolo (Hrsg.): Migration in the New Europe. East-West Revisited, Basingstoke et al., S. 159-176.

Kicinger, Anna (2005): Between Polish Interests and the EU Influence - Polish Migration Policy Development 1989-2004, CEFMR (Central European Forum For Migration Research) Working Paper 9/2005, Warschau.

Kimminich, Otto (1982): Asyl, das älteste Recht, in: Spaich, Herbert (Hrsg.): Asyl bei den Deutschen. Beiträge zu einem gefährdeten Grundrecht, S. 150-167.

Kindler, Marta (2005): Migrant domestic workers in Poland - balancing the risks, Vortrag gehalten auf der Internationalen Konferenz „Migration and Domestic Work in Global Perspective“, The Netherlands Institute for Advanced Studies, Wassenaar, online: www.nias.knaw.nl/en/news_forthcoming_activities/lutz/new_12/kindler.pdf, letzter Abruf: <25.09.2008>.

King, Gary/Keohane, Robert O./Verba, Sidney (1994): Designing Social Inquiry, Princeton. 
Klein, Anne (2007): A body, a passport, a soul. Flucht und Asyl in der Vorgeschichte der Shoah, in: Figatowski, Bartholomäus/Haile Gabriel, Kokebe/Meyer, Malte (Hrsg.):The Making of Migration - Repräsentationen/Erfahrungen/Analysen, Münster, S. 104-117.

Knill, Christoph/Lehmkuhl, Dirk (2002): The national impact of European Union regulatory policy: Three Europeanization mechanisms, in: European Journal of Political Research, Vol. 41, No. 2, S. 255-280.

Knill, Christoph/Lenschow, Andrea (1999): Neue Konzepte - alte Probleme? Die institutionellen Grenzen effektiver Implementation, in: Politische Vierteljahresschrift, 40. Jg., Nr. 4, S. 591-617.

Kohler-Koch, Beate (1989) (Hrsg.): Regime in den internationalen Beziehungen, Baden-Baden.

Kohler-Koch, Beate (1989): Zur Empirie und Theorie internationaler Regime, in: dies. (Hrsg.): Regime in den internationalen Beziehungen, Baden-Baden, S. 17-85.

Kok, Laura (2006): The Dublin II Regulation. A UNHCR Discussion Paper, Brussels, April 2006.

Koryś, Izabela (2003): Migration Trends in Selected EU Applicant Countries: Poland, CEFMR Working Paper 5/2003, online: www.cefmr.pan.pl/docs/cefmr_wp_2003-05.pdf, letzter Abruf: <25.09.2008>.

Koser, Khalid/Lutz, Helma (1998): The new Migration in Europe. Contexts, Constructions and Realities, in: dies. (Hrsg.): The new Migration in Europe. Social Constructions and Social Realities, London/Basingstoke, S. 1-20.

Kosmala, Beate (2000): Die Vertreibung der Juden aus Polen 1968. Antisemitismus und politisches Kalkül, Berlin.

Kosowicz, Agnieszka (2004): Hunger strike reveals strains in Polish asylum system, UNHCR News Stories, Genf/Warschau, online: www.unhcr.org, letzter Abruf: <01.11.2007>.

Kothen, Andrea (2002): Rassismus hat viele Gesichter: Die soziale Ausgrenzung von Flüchtlingen, in: Jäger, Margarete/Kauffmann, Heiko (Hrsg.): Leben unter Vorbehalt. Institutioneller Rassismus in Deutschland, Duisburg, S. 55-67.

Kreissl-Dörfler, Wolfgang (2006): Europäische Asylpolitik und ihre Umsetzung aus Sicht des Europäischen Parlamentes, in: Asyl in Europa: Chancen, Risiken und Perspektiven der gemeinsamen europäischen Asylpolitik, epd-Dokumentation des 6. Berliner Symposiums zum Flüchtlingsschutz in der Evangelischen Akademie zu Berlin am 19. Juni 2006, Nr. 32, Frankfurt a.M., S. 18-22.

Krzeminski, Adam (2004): Ankunft im Herzen Europas. Polens Beitritt zur Europäischen Union ist für das Land Erfüllung und Herausforderung zugleich, in: Frankfurter Rundschau vom 07.05.2004, Langfassung: www.fr-aktuell.de. 
Kühne, Peter/Rüßler, Harald (2000): Die Lebensverhältnisse der Flüchtlinge in Deutschland, Frankfurt a.M.

Kupiszewski, Marek (2002): How trustworthy are forecasts of international migration between Poland and the European Union?, Journal of Ethnic and Migration Studies, Vol. 28, No. 4, Oktober 2002, S. 627-645.

Lahav, Gallya/Guiraudon, Virginie (2006): Actors and Venue in Immigration Control: Closing the Gap between Political Demands and Policy Outcomes, in: West European Politics, Vol. 29, Nr. 2, March 2006, S. 201-223.

Lahlou, Mehdi (2006): „Europa erfasst Afrikas Probleme nicht“. Interview, in: die tageszeitung vom 11.07.2006.

Landman, Todd (2002): Comparative Politics and Human Rights, in: Human Rights Quarterly, Vol. 24, No. 4, November 2002, S. 890-923.

Lang, Kai-Olaf (2006): Doppelspitze in Warschau, SWP-Aktuell Nr. 37, August 2006.

Latawski, Paul (1999): Straz Graniczna: The Mission, Structure and Operations of Poland's Border Guard, in: Iglicka, Krystyna/Sword, Keith (Hrsg.): The Challenge of East-West Migration for Poland, Basingstoke et al., S. 90-103.

Lavenex, Sandra (2002): The Europeanisation of refguee policies: between human rights and internal security, 2 . Auflage, Aldershot.

Lavenex, Sandra (2006): Shifting up and out: the foreign policy of EU immigration control, in: West European Politics, Vol. 29, No. 2, S. 329-350.

Liebold, Renate/Trinczek, Rainer (2005): Qualitative Methoden der Organisationsforschung. Experteninterview, online: http://www.qualitative-research.net/organizations/or-exp-d.htm, letzter Abruf: <06.12.2008>.

Lijphart, Arend (1971): Comparative Politics and the Comparative Method, in: American Political Science Review, Vol. 65, No. 3, September 1971, S. 682-693.

Lochbihler, Barbara (2006): Menschenrechte und Abschiebungshaft, in: Katholische Stiftungsfachhochschule München und Bayerisches Aktionsbündnis gegen Abschiebungshaft: Studientag zur Abschiebungshaft. Dokumentation, online: www.abschiebungshaft-bayern.org/Doku_ms_301106.pdf, letzter Abruf: <25.11.2008>.

Loescher, Gil/Loescher, Ann Dull (1982): The World's Refugees: A Test of Humanity, New York.

Lutz, Helma (2005): Der Privathaushalt als Weltmarkt für weibliche Arbeitskräfte, in: Peripherie, 25. Jg., Nr. 97/98, Mai 2005, S. 65-87. 
Majcherek, Janusz A. (1999): Gewinner und Verlierer. Wie sich die Polen im ersten Jahrzehnt des neuen Systems verändert haben, in: Rzeczpospolita vom 15./16.

Mai 1999, übersetzte Version

online: www.dpg-brandenburg.de/nr_20/gewinner.shtml, letzter Abruf: <25.09.2008>.

Marczuk, Jarosław/Kosowicz, Agnieszka (2006): No More „Permanent Care Home“/Koniec z „domem nieustającej opieki“،, in: Refugee PL/Gazeta Uchodźców, 3/2006, hrsg. von der Polska Akcja Humanitarna, online: www.refugee.pl, letzter Abruf: <25.09.2008>.

Marfleet, Philip (2006): Refugees in a Global Era, Basingstoke.

Martini-Emden, Dietmar (2000): Problemstellung und Intention des Modellversuchs einer Landesunterkunft für Ausreisepflichtige in Rheinland-Pfalz, Manuskript einer Rede im Rahmen des 8. Migrationspolitischen Forums des „Forschungszentrums für Internationales und Europäisches Ausländer- und Asylrecht",

online:

www.ausreisezentren.de/az/index.php?link=Grundlagen/Rheinlandpfalz/Modell versuch_RhPf.htm, letzter Abruf: <20.09.2008>.

Maryniak, Irena (2007): Der polnische Klempner und das Spiel mit den Bildern, in: Wespennest Nr. 146, S. 4-13, online: www.eurozine.com/articles/article_2007-03-15-maryniak-de.html, letzter Abruf: $<01.09 .2010>$.

Matthes, Claudia-Yvette (2002): Polen - vom personalisierten zum rationalisierten Parlamentarismus, in: Kraatz, Susanne/von Steinsdorff, Silvia (Hrsg.): Parlamente und Systemtransformation im postsozialistischen Europa, Opladen, S. 87-109.

Messner, Dirk/Nuscheler, Franz (1996): Global Governance. Herausforderungen an die deutsche Politik an der Schwelle zum 21. Jahrhundert, Bonn.

Meuser, Michael/Nagel, Ulrike (1991): ExpertInneninterviews - vielfach erprobt, wenig bedacht. Ein Beitrag zur qualitativen Methodendiskussion, in: Garz, Detlef/Kraimer, Klaus (Hrsg.): Qualitativ-empirische Sozialforschung: Konzepte, Methoden, Analysen, Opladen, S. 441-471.

Meuser, Michael/Nagel, Ulrike (1997): Das ExpertInneninterview - Wissenssoziologische Voraussetzungen und methodische Durchführung, in: Friebertshäuser, Barbara/Prengel, Annedore (Hrsg.): Handbuch qualitative Forschungsmethoden in der Erziehungswissenschaft, Weinheim/München, S. 481-491.

Meyer, Evelyn (1997): Sprachgebrauch in der Asyldebatte, in: Jung, Matthias/Wengeler, Martin/Böke, Karin (Hrsg.): Die Sprache des Migrationsdiskurses. Das Reden über „Ausländer“ in Medien, Politik und Alltag, Opladen, S. 150-163.

Miera, Frauke (2007): Polski Berlin - Migration aus Polen nach Berlin: Integrationsund Transnationalisierungsprozesse 1945 bis Ende der 1990er Jahre, Münster. 
Migration und Bevölkerung (2006): EU: Kampagne zur Steigerung der Mobilität von Arbeitnehmern, Newsletter, Ausgabe 3/2006.

Migreurop (2005): From European Migration and Asylum Policies to Camps for Foreigners, Map of Foreigners' camps in Europe, online: www.migreurop.org, letzter Abruf: <25.09.2008>.

Mill, John Stuart (1874): A System of Logic. Ratiocinative and Inductive, New York.

Ministerstwo Gospodarki (2007): Wpływ emigracji zarobkowej na gospodarkę Polski (Einfluss der Arbeitsemigration auf die polnische Wirtschaft), Departament Analiz i Prognoz, Warschau, Februar 2007, online: www.mg.gov.pl/NR/rdonlyres/5254E5E7-B815-4E38-B6F9CEC6B1C1A44E/30274/migracja070301.pdf, letzter Abruf: <31.10.2008>.

Ministerstwo Polityki Społecznej (2005): Propozycje działań w celu stworzenia kompleksowej polityki integracji cudzoziemców w Polsce (Aktionsvorschläge mit dem Ziel der Etablierung einer Integrationspolitik für Ausländer in Polen), Warschau, Januar 2005.

Ministry of Labour and Social Policy (2005): A Guide for Refugees on selected legal provisons in Poland, Warschau.

Morales Ayma, Evo (2008): Negation der Freiheit. Offener Brief vom 05.06.2008, auf Deutsch erschienen in: Junge Welt vom 12.06.2008.

Morris, Lydia (2002): Managing Migration, Civic Stratification and Migrants' rights, London/New York.

Müller, Doreen (2004): Recht auf Gesundheit? Medizinische Versorgung illegalisierter MigrantInnen zwischen exklusiven Staatsbürgerrechten und universellen Menschenrechten, hrsg. v. Förderverein Niedersächsischer Flüchtlingsrat e.V., Flüchtlingsrat. Zeitschrift für Flüchtlingspolitik in Niedersachsen, Sonderheft 101, Sept. 2004.

Müller, Peter (2001): Von der Einwanderungskontrolle zum Zuwanderungsmanagement. Plädoyer für ein nationales Programm der Zuwanderungspolitik in Deutschland, in: ders./Bosbach, Wolfgang/Oberndörfer, Dieter: Zuwanderung und Integration, Zukunftsforum Politik Nr. 23, Broschürenreihe hrsg. von der Konrad-Adenauer-Stiftung, Sankt Augustin, März 2001, S. 5-30, online: www.kas.de/wf/doc/kas_190-544-1-30.pdf, letzter Abruf: <25.11.2008>.

Münch, Ursula (1992): Asylpolitik in der Bundesrepublik Deutschland: Entwicklung und Alternativen, Opladen.

Neue Zürcher Zeitung vom 15.01.2007: Wirbel um HIV-positiven Flüchtling in Polen.

Neumayer, Eric (2005): Asylum Recognition Rates in Western Europe. Their Determinants, Variation, and Lack of Convergence, in: Journal of Conflict Resolution, Vol. 49, No. 1, S. 43-66. 
New York Times vom 26.06.2005: Unlikely Hero in Europe’s Spat: The 'Polish Plumber'.

Newsletter Migration und Bevölkerung (2006): EU: Kampagne zur Steigerung der Mobilität von Arbeitnehmern, Nr. 3, April 2006, S. 4.

Niedersächsischer Flüchtlingsrat (2008): Innenminister Schünemann legt sich mit Kommunen an, Presserklärung vom 12.03.2008, online: www.nds-fluerat.org, letzter Abruf: <05.10.2008>.

Nohlen, Dieter (2002) (Hrsg.): Kleines Lexikon der Politik, 2. Auflage, München.

Nowicka, Ewa (2005): Identity, Adaptation, Integration: Africans in Warsaw, in: Slany, Krystyna (Hrsg.): International Migration. A Multidimensional Analysis, Krakau, S. 265-288.

Nuscheler, Franz (2004): Internationale Migration. Flucht und Asyl, 2. Auflage, Wiesbaden.

oanda currency converter (o.J.), online: www.oanda.com/convert/ fxhistory, letzter Abruf: $<01.09 .2010>$.

OAU Convention governing the specific aspects of refugee problems in Africa, concluded at Addis Ababa on 10 September 1969, United Nations Treaty Series, Nr. 14691, S. 45-52.

OECD (2008), The OECD Database on International Migration, online: http://stats.oecd.org/wbos/Index.aspx?datasetcode=MIG letzter Abruf: <10.03.2009>.

Ohlinger, Rainer; DOMiT (2004): Chronologie der Einwanderung nach Deutschland seit 1945, in: Motte, Jan/Ohlinger, Rainer (Hrsg.): Geschichte und Gedächtnis in der Einwanderungsgesellschaft. Migration zwischen historischer Rekonstruktion und Erinnerungspolitik, Essen, S. 323 - 332.

Okólski, Marek (1998): Poland's Population and Population Movements: An Overview, in: Frejka, Tomas/Okólski, Marek/Sword, Keith (Hrsg.): In-Depth Studies on Migration in Central and Eastern Europe: The Case of Poland, New York/Genf, S. 9-24.

Okólski, Marek (1999): Recent Migration in Poland: Trends and Causes, in: Iglicka, Krystyna/Sword, Keith (Hrsg.): The Challenge of East-West Migration for Poland, Basingstoke et al., S. 15-44.

Oltmer, Jochen (2003): Einführung: Steuerung und Verwaltung von Migration in Deutschland seit dem späten 19. Jahrhundert, in: ders. (Hrsg.): Migration steuern und verwalten, Göttingen, S. 9-56.

onet.pl vom 8.11.2007: Przemyśl: uruchomiono ośrodek dla uchodźców (Przemyśl: Inbetriebnahme des Zentrums für Ausländer).

Orlowski, Witold (2001): Warum sollten die Polen eigentlich massenhaft einwandern? Über Befürchtungen in Deutschland und tatsächliche empirische Befunde, Dokumentation, in: Frankfurter Rundschau vom 17.07.2001. 
Owczarek, Lidia (2002): Die Situation der nationalen Minderheiten in Polen während der Systemtransformation, in: UTOPIE kreativ, Heft 141/142, Juli/August 2002, S. 710-719.

Pelzer, Marei/Pennington, Alison (2006): Geschlechtsspezifische Verfolgung: Das neue Flüchtlingsrecht in der Praxis, in: Asylmagazin 5/2006, online: www.asyl.net/Magazin/5_2006b.html\#C1, letzter Abruf: <21.09.2008>.

Peter, Lothar (2006): Wozu noch Gesellschaftskritik?, in: Blätter für deutsche und internationale Politik, 51. Jg., Heft 5, Mai 2006, S. 587-599.

Pieper, Tobias (2008): Das Lager als Struktur bundesdeutscher Flüchtlingspolitik. Eine empirische Untersuchung zur politischen Funktion des bürokratischen Umgangs mit MigrantInnen in Gemeinschaftsunterkünften und Ausreiseeinrichtungen in Berlin, Brandenburg und Bramsche/Niedersachsen, online: www.diss.fu-berlin.de/2008/194, letzter Abruf: <21.09.2008>.

Plenarprotokoll 15/118: Deutscher Bundestag, Stenographischer Bericht, 118. Sitzung, Berlin, 01.07.2004.

Price, Matthew E. (2006): Persecution Complex: Justifying Asylum Law's Preference for Persecuted People, in: Harvard International Law Journal, Vol. 47, No. 2, Summer 2006, S. 413-466.

Pries, Ludger (2001): Internationale Migration, Bielefeld.

Pro Asyl (2004): Das Zuwanderungsgesetz. Überblick über die wichtigsten Neuerungen im Zuwanderungsgesetz, Frankfurt a.M.

Pro Asyl (2005): Widerrufsverfahren: Flüchtlingsschutz mit Verfallsdatum? Zahlen, Fakten \& Hintergründe, Rechtliche Grundlagen, Praktische Erfahrungen, online: www.proasyl.de/fileadmin/proasyl/fm_redakteure/Broschueren_pdf/B_Widerr ufe_internet_k.pdf, letzter Abruf: <21.09.2008>.

Pro Asyl (2006a): Ein Jahr Zuwanderungsgesetz. Stellungnahme zur Evaluierung des Zuwanderungsgesetzes, Frankfurt a.M., online: www.proasyl.de, letzter Abruf: <21.09.2008>.

Pro Asyl (2006b): Ein Jahr Zuwanderungsgesetz. Stellungnahme zur Evaluierung des Zuwanderungsgesetzes, Umsetzung des Zuwanderungsgesetzes unzureichend,

Pressemitteilung vom 7.03.2006, online: www.proasyl.de, letzter Abruf: <21.09.2008>.

Pro Asyl (2007): Informeller Rat in Dresden diskutiert Konzept von Schäuble und Sarkozy, PRO ASYL: „Rückwärtsgewandt und repressiv - Grenzschutz statt Flüchtlingsschutz“, Presseerklärung vom 15.01.2007, online: www.proasyl.de, letzter Abruf: <27.08.2008>.

Pro Asyl (2008): Flüchtlinge im Verschiebebahnhof EU. Die EU-

Zuständigkeitsverordnung „Dublin II“, Frankfurt a.M., online: www.proasyl.de, letzter Abruf: <21.09.2008>. 
Pro Asyl et al. (2005): Memorandum zur derzeitigen Situation des deutschen Asylverfahrens, Frankfurt a.M., online: www.proasyl.de/fileadmin/proasyl/Memorandum_II.pdf, letzter Abruf: <21.09.2008>.

Radio Jedynka vom 7.07.2006: W koalicji trzeszczy - Andrzej Lepper w Sygnałach Dnia (In der Koalition knackt es - Andrzej Lepper in Signale des Tages).

Ragin, Charles (1987): The Comparative Method. Moving beyond Qualitative and Quantitative Strategies, Berkeley et al.

Reißlandt, Carolin (2003): Ein neuer Anlauf nach dem Scheitern? - Die wechselhafte Geschichte des Zuwanderungsgesetzes, in: Butterwegge, Christoph/Hentges, Gudrun (Hrsg.): Zuwanderung im Zeichen der Globalisierung. Migrations-, Integrations- und Minderheitenpolitik, 2. aktualisierte Auflage, S. 123-148.

Richmond, Anthony H. (1988): Sociological Theories of International Migration: The Case of Refugees, in: Current Sociology, Vol. 36, No. 2, S. 7-25.

Richmond, Anthony H. (1993): Reactive Migration: Sociological Perspectives On Refugee Movements, in: Journal of Refugee Studies, Vol. 6, No. 1, S. 7-24.

Richmond, Anthony H. (2002): Globalization: implications for immigrants and refugees, in: Ethnic and Racial Studies, Vol. 25, No. 5, September 2002, S. $707-$ 727.

Riegert, Bernd (2005): Vernichtendes Urteil für die EU-Migrationspolitik, Deutsche Welle Kommentar, online: www.dw-world.de/dw/article/0,2144,1733520,00.htm, letzter Abruf: <01.09.2010>.

Roos, Alfred (1991): Flüchtlingspolitik und innenpolitische Debatte. Oder: 17 Jahre „Asylmissbrauch“ - (noch) kein Jubiläum, in: Vorgänge, Nr. 109, Heft 1, S. 46-60.

Rozporzadzenie Ministra Spraw Wewnętrznych i Administracji z dnia 14 sierpnia 2003 r. w sprawie wysokości pomocy dla cudzoziemców ubiegających się o nadanie statusu uchodźcy (Verordnung des Ministerums für innere und Verwaltungsangelegenheiten vom 14. August 2003 über die Höhe der Hilfe für Ausländer, die einen Antrag auf Verleihung des Flüchtlingsstatus gestellt haben), erschienen im: Dziennik Ustaw Rzeczypospolitej Polskiej (Gesetzblatt der Republik Polen) Nr. 146, poz. 1528 oraz z 2005 r. Nr 157, poz. 1322 vom 22.08.2003.

Rüschemeyer, Dietrich (2003): Can One or a Few Cases Yield Theoretical Gains?, in: ders./Mahoney, James (Hrsg.): Comparative Historical Analysis in the Social Sciences, Cambridge, S. 305-336.

Santel, Bernhard (1995): Migration in und nach Europa. Erfahrungen, Strukturen, Politik, Opladen.

Sassen, Saskia (1996): Losing Control? Sovereignty in an Age of Globalization, New York. 
Sassen, Saskia (2000): Migranten, Siedler, Flüchtlinge. Von der Massenauswanderung zur Festung Europa, 3. Auflage, Frankfurt a.M..

Schily, Otto (2004): Afrikas Probleme in Afrika lösen, Gastbeitrag, in: Frankfurter Allgemeine Zeitung vom 23.07.2004.

Schneider, Volker (2001): Institutional Reform in Telecommunications: The European Union in Transnational Policy Diffusion, in: Risse, Thomas/Green Cowles, Maria/Caporaso, James (Hrsg.): Transforming Europe. Europeanization and Domestic Change, Ithaca and London, S. 60-78.

Schönwälder, Karen (1999): „Ist nur Liberalisierung Fortschritt?“ Zur Entstehung des ersten Ausländergesetzes in der Bundesrepublik, in: Motte, Jan/Ohliger, Rainer/von Oswald, Anne (Hrsg.): 50 Jahre Bundesrepublik - 50 Jahre Einwanderung: Nachkriegsgeschichte als Migrationsgeschichte, Frankfurt a.M. et al., S. 127144.

Schönwälder, Karen (2006): Politikwandel in der (bundes-)deutschen Migrationspolitik, in: Davy, Ulrike/Weber, Albrecht (Hrsg.): Paradigmenwechsel in Einwanderungsfragen? Überlegungen zum neuen Zuwanderungsgesetz, Baden-Baden, S. 822.

Schuster, Liza (2003): The Use and Abuse of Political Asylum in Britain and Germany, London.

Schuth, Wolfgang (1988): Die Lebenssituation der Asylbewerber in der Bundesrepublik Deutschland, in: Thränhardt, Dietrich/Wolken, Simone (Hrsg.): Flucht und Asyl: Informationen, Analysen, Erfahrungen aus der Schweiz und der Bundesrepublik Deutschland, Freiburg im Breisgau, S. 171-189.

Schwäbisches Tagblatt vom 5.05.1982: Fast wie ein Gewerkschaftsredner. Für Arbeitsplatzkiller will der Ministerpräsident kein Geld ausgeben.

Schwarze, Susan (2001): Das Arenen-Verhandlungsmodell - Deutsche Asylpolitik im europäischen Kontext von 1989 bis 1993, Digitale Dissertation, FU Berlin, online: www.diss.fu-berlin.de/2001/202/, letzter Abruf: <21.09.2008>.

Schwenken, Helen (2006): Rechtlos, aber nicht ohne Stimme. Politische Mobilisierungen um irreguläre Migration in die Europäische Union, Bielefeld.

Shapovalova, Natalia (2007): Ukraine: a model of „buffer country“?, International Centre for Policy Studies, Kiew, Power-Point-Präsentation zum Vortrag, gehalten auf dem Migreurop Workshop in Radovljica, Slowenien am 29. Mai 2007, unveröffentlichtes Manuskript.

Sikorski, Tadeusz/Heuser, Marius (2005): Das soziale Elend in Polen. Eine Reportage aus dem schlesischen Zabrze, online: www.wsws.org/de/2005/jan2005/pol1-j27.shtml, letzter Abruf: <01.09.2010>. 
Sinn, Annette/Kreienbrink, Axel/von Loeffelholz, Hans Dietrich (2006): Illegal aufhältige Drittstaatenangehörige in Deutschland. Staatliche Ansätze, Profil und soziale Situation. Forschungsstudie 2005 im Rahmen des europäischen Migrationsnetzwerks, online: www.bamf.de, letzter Abruf: <18.12.2008>.

Skocpol, Theda/Somers, Margaret (1980): The Uses of Comparative History in Macrosocial Inquiry, in: Comparative Studies in Society and History, Vol. 22, No. 2, April 1980, S. 174-197.

Soysal, Yasemin Nuhoglu (1994): Limits of Citizenship. Migrants and Postnational Membership in Europe, Chicago/London.

Spaich, Herbert (1982) (Hrsg.): Asyl bei den Deutschen. Beiträge zu einem gefährdeten Grundrecht, Hamburg.

Spiegel online vom 14.03.2006: Der Holland-Test der eisernen Rita.

Statham, Paul (2003): Understanding Anti-Asylum Rhetoric: Restrictive Politics or Racist Publics?, in: Spencer, Sarah (Hrsg.): The Politics of Migration: Managing Opportunity, Conflict and Change, Oxford, S. 163-177.

Statistisches Bundesamt (o.J.): Wanderungen zwischen Deutschland und dem Ausland 1991 bis 2007 , online: www.destatis.de/jetspeed/portal/cms/Sites/destatis/Internet/DE/Content/Stat isti-

ken/Bevoelkerung/Wanderungen/Tabellen/Content50/WanderungenInsgesamt, letzter Abruf: <10.03.2009>

Stobbe, Holk (2004): Undokumentierte Migration in Deutschland und den Vereinigten Staaten. Interne Migrationskontrollen und die Handlungsspielräume von Sans Papiers, Göttingen.

Süddeutsche Zeitung vom 30.03.1996: Härtefallregelung für abgelehnte Asylbewerber.

Süddeutsche Zeitung vom 25.07.1998: CSU beugt sich in der Ausländerpolitik der CDU. Einwanderung nach Deutschland wird grundsätzlich akzeptiert /Zuzug soll aber nicht weiter verstärkt werden. Auch die Abschiebung straffällig gewordener Ausländer-Kinder samt Eltern findet sich nicht in dem Papier.

Süddeutsche Zeitung vom 02.08.2004: „Ich finde nichts Anstößiges daran, Menschen zurückzuführen“".

Sunjic, Melita (2007): New Asylum Laws. Bringing Good and Bad News for Refugees, UNHCR Regional Representation in Budapest, 6.09.2007, online: www.unhcr-rrbp.org/content/view/114/2/, letzter Abruf: <24.09.2008>.

Sword, Keith (1999): Cross-Border ,Suitcase Trade' and the Role of Foreigners in Polish Informal Markets, in: Iglicka, Krystyna/Sword, Keith (Hrsg.): The Challenge of East-West Migration for Poland, Basingstoke et al., S. 145-167. 
Szczypiorski, Andrzej (1998): Marzec i Polacy (Der März und die Polen), in: Gazeta Wyborcza vom 28./29.03.1998.

Tagesspiegel vom 30.10.2006: Unter Flüchtlingsstrom.

Tagesspiegel vom 05.06.2008: Umstrittene EU-Abschiebe-Richtlinie kommt.

Terre des femmes (2007): Regierung verwehrt Opfern von Zwangsheirat dringend benötigte Rechte, Pressemitteilung vom 25.11.2007, online: www.frauenrechte.de, letzter Abruf: $<06.10 .2008>$.

The Caravan (2004): Autonomie der Migration. Wo wir in dieser Debatte stehen, online: http://thecaravan.org/node/31, letzter Abruf: <05.12.2008>.

The Guardian vom 25.06.2007: With its workers over here, Poland turns east to get the country rebuilt.

Thränhardt, Dietrich (1997): Zuwanderungspolitik im europäischen Vergleich, in: Angenendt, Steffen (Hrsg.): Migration und Flucht. Aufgaben und Strategien für Deutschland, Europa und die internationale Gemeinschaft, Bonn, S. 137-153.

Thränhardt, Dietrich (2003): Der Nationalstaat als migrationspolitischer Akteur, in: ders./Hunger, Uwe (Hrsg.): Migration im Spannungsfeld von Globalisierung und Nationalstaat, Leviathan Sonderheft, Bd. 22, S. 8-31.

Thränhardt, Dietrich/Wolken, Simone (1988) (Hrsg.): Flucht und Asyl: Informationen, Analysen, Erfahrungen aus der Schweiz und der Bundesrepublik Deutschland, Freiburg im Breisgau.

TNS OBOP (2004): Poles about Refugees: results of a research poll performed two weeks after the World Refugee Day, Warschau, online: www.unhcr-budapest.org, letzter Abruf: <16.12.2008>.

TNS OBOP (2006): Poles about Refugees: results of a survey conducted 3 weeks after the World Refugee Day, Warschau, online: http://www.unhcr-budapest.org, letzter Abruf: <16.12.2008>.

Trampusch, Christine (2000): Arbeitsmarktpolitik, Gewerkschaften und Arbeitgeber. Ein Vergleich der Entstehung und Transformation der öffentlichen Arbeitsverwaltungen in Deutschland, Großbritannien und den Niederlanden zwischen 1909 und 1999, Dissertation, online: http://webdoc.sub.gwdg.de/diss/2000/trampusch/, letzter Abruf: $<21.12 .2008>$.

Transit Migration Forschungsgruppe (2007) (Hrsg.): Turbulente Ränder. Neue Perspektiven auf Migration an den Grenzen Europas, Bielefeld.

Transnational Newsletter Crossing Borders (2006): Bewegungen und Kämpfe der Migration, 1. Ausgabe, Oktober 2006, online: www.noborder.org/crossing_borders/newsletter01de.pdf, letzter Abruf: <01.09.2010>.

Treibel, Annette (2003): Migration in modernen Gesellschaften. Soziale Folgen von Einwanderung, Gastarbeit und Flucht, 3. Auflage, Weinheim/München. 
TVP 3 Olsztyn vom 16.10.2007: Ośrodek dla cudzoziemców (Zentrum für Ausländer), online: ww6.tvp.pl/7228,20071016581770.strona, letzter Abruf: <15.11.2008>.

UdSC (2004): Dane liczbowe dotyczące postępowań prowadzonych wobec cudzoziemców w latach 2001 - 2003 (Daten betreffend die Durchführung von Verfahren gegenüber Ausländern in den Jahren 2003 - 2005), Warschau, online: www.udsc.gov.pl/Zestawienia,roczne,233.html, letzter Abruf: <11.02.2009>.

UdSC (2006): Dane liczbowe dotyczące postępowań prowadzonych wobec cudzoziemców w latach 2003 - 2005 (Daten betreffend die Durchführung von Verfahren gegenüber Ausländern in den Jahren 2003 - 2005), Warschau, online: www.udsc.gov.pl/files/old_file/440426f016ddd_biuletyn_2003_2005.pdf, letzter Abruf: <10.01.2009>.

UdSC (2008): Dane liczbowe dotyczące statusu uchodźcy w roku 2007 (Daten betreffend den Flüchtlingsstatus im Jahr 2007),

online: www.udsc.gov.pl/files/statystyki/2007\%20status\%20uchodzcy.xls, letzter Abruf: <10.01.2009>.

UdSC (o.J.): Dane liczbowe złożonych w latach 1992 - 2000 wniosków oraz wydanych w tym okrezie decyzji w sprawie o nadanie statusu uchodźcy w RP (Zahlen der in den Jahren 1992 - 2000 eingereichten Anträge sowie in diesem Zeitraum erteilter Entscheidungen über die Flüchtlingsstatusvergabe in der Republik Polen), online: www.udsc.gov.pl/files/old_file/41206412b98bd_nsuch_gotowe.xls, letzter Abruf: <09.01.2009>.

UK Government (2003): New Vision for Refugees, 7.03.2003.

Ullrich, Peter (2006): Das explorative ExpertInneninterview - Modifikationen und konkrete Umsetzung der Auswertung von ExpertInnen-Interviews nach Meuser/Nagel, in: Engartner, Tim/Kuring, Diana/Teubl, Thorsten (Hrsg.): Die Transformation des Politischen. Analysen, Deutungen, Perspektiven, RosaLuxemburg-Stiftung, Manuskripte 66, Berlin, S. 100-109.

Unabhängige Kommission Zuwanderung (2001): Bericht: Zuwanderung gestalten - Integration fördern, 4. Juli 2001, online: www.bmi.bund.de, letzter Abruf: $<21.09 .2008>$.

UNHCR (2003): Stellungnahme zur Richtlinie 2001/55/EG des Rates vom 20. Juli 2001 über Mindestnormen für die Gewährung vorübergehenden Schutzes im Falle eines Massenzustroms, Berlin, August 2003.

UNHCR (2005): Kommentar des UNHCR zur Richtlinie 2004/83/EG, Genf, Mai 2005, deutsche Fassung: UNHCR-Vertretung Deutschland. 
UNHCR (2006): 2005 Global Refugee Trends. Statistical overview of populations of refugees, asylum-seekers, internally displaced persons, stateless persons, and others of concern to UNHCR, Genf, online: www.unhcr.org, letzter Abruf: <09.11.2008>.

UNHCR (2007): Asylum Levels and Trends in Industrialized Countries, 2006. Overview of asylum applications lodged in Europe and non-European industrialized countries in 2006, Genf, online: www.unhcr.org/statistics/STATISTICS/460150272.pdf, letzter Abruf: <01.12.2008.>

UNHCR (2008a): States Parties to the 1951 Convention relating to the Status of Refugees and the 1967 Protocol, online: www.unhcr.org/protect/PROTECTION/3b73b0d63.pdf, letzter Abruf: <01.12.2008>.

UNHCR (2008b): 2007 Global Trends: Refugees, Asylum-seekers, Returnees, Internally Displaced and Stateless Persons, o.O., online: www.unhcr.org/statistics.html, letzter Abruf: <15.12.2008>.

UNHCR (2008c): UNHCR Statement on the "Ceased Circumstances" Clause of the EC Qualification Directive, August 2008, o.O., online: www.unhcr.org/protect/PROTECTION/48a2f2a42.pdf, letzter Abruf: <19.09.2008>.

Unwort des Jahres (o.J.): Informationen zu einer sprachkritischen Aktion, online: www.unwortdesjahres.org, letzter Abruf: <08.03.2009>

Ustawa z dnia 12 marca 2004 r. o pomocy spolecznej (Gesetz vom 12. März 2004 über die Sozialleistungen), erschienen im: Dziennik Ustaw Rzeczypospolitej Polskiej (Gesetzblatt der Republik Polen) Nr. 64 vom 14.04.2004.

Verfassung der Volksrepublik Polen vom 22.07.1952 (Konstytucja Polskiej Rzeczypospolitej Ludowej), erschienen im: Dziennik Ustaw (Gesetzblatt) Nr. 33 vom 23.07.1952.

Vogel, Berthold (1995): „Wenn der Eisberg zu schmelzen beginnt...“ - Einige Reflexionen über den Stellenwert und die Probleme des Experteninterviews in der Praxis der empirischen Sozialforschung, in: Brinkmann, Christian/Deeke, Axel/Völkel, Brigitte (Hrsg.): Experteninterviews in der Arbeitsmarktforschung. Diskussionsbeiträge zu methodischen Fragen und praktischen Erfahrungen. Beiträge zur Arbeitsmarkt- und Berufsforschung Nr. 191, Nürnberg, S. 73-83.

von Rautenfeld, J. Erika (2007): Den neuen Europäer bilden. EU- Identitätspolitik an Universitäten, in: Belafi, Matthias/Krienke, Markus (Hrsg.): Identitäten in Europa - Europäische Identität, Wiesbaden, S. 103-126. 
Welge, Ines (2006): Untersuchung zur Entscheidungspraxis des Bundesamtes für Migration und Flüchtlinge in Fällen eritreischer Asylantragsteller, hrsg. von Pro Asyl et al., Frankfurt a.M., online: www.proasyl.de/fileadmin/proasyl/fm_redakteure/Newsletter_Anhaenge/122/ Eritrea_Studie.pdf, letzter Abruf: <21.09.2008>.

Wengeler, Martin (1997): Argumentation im Einwanderungsdiskurs. Ein Vergleich der Zeiträume 1970-1973 und 1980-1983, in: Jung, Matthias/Wengeler, Martin/Böke, Karin (Hrsg.): Die Sprache des Migrationsdiskurses. Das Reden über „Ausländer“ in Medien, Politik und Alltag, Opladen, S. 121-149.

Wichert, Frank (1998): Die konjunkturelle Entwicklung des Themas Asyl im Deutschen Bundestag, in: Jäger, Siegfried/Kretschmer, Dirk/Cleve, Gabriele/Griese, Birgit/Jäger, Margarete/Kellershohn, Helmut/Krüger, Coerw/Wichert, Frank (Hrsg.): Der Spuk ist nicht vorbei. Völkisch - nationalistische Ideologeme im öffentlichen Diskurs der Gegenwart, S. 102-124.

online: www.diss-duisburg.de/Internetbibliothek/Buecher/Der Spuk ist nicht vorbei.pdf, letzter Abruf: <01.09.2010>.

Wimmer, Andreas/Glick Schiller, Nina (2003): Methodological Nationalism, the Social Sciences, and the Study of Migration: An Essay in Historical Epistemology, in: International Migration Review, Vol. 36, No. 3, Fall 2003, S. 576-610.

Winkler, Josef Philip (2008): Humanitäre Standards bei Rückführungen achten, Bundestagsrede vom 29.03.2007, online: www.josef-winkler.de/diepresse/reden/artikel/423/7609870634/index.html, letzter Abruf: <01.09.2010>.

Wirtualna Polska vom 19.07.2007: Polska nie jest ziemią obiecaną dla pracowników ze Wschodu (Polen ist kein viel versprechendes Land für Arbeiter aus dem Osten).

Wirtualna Polska vom 30.01.2007: ,Jest praca w Polsce! Na razie dla Rumunów i Bułgarów - raport z ankiety“ (Es gibt Arbeit in Polen! Einstweilen für Rumänen und Bulgaren - Bericht mit Umfrage), online: http:/ / praca.wp.pl/drukuj.html?kat=\&wid=8690053, letzter Abruf: <16.03.2009>.

Wolf, Klaus Dieter (1994): Regimeanalyse, in: Boeckh, Andreas (Hrsg.): Lexikon der Politik, Band 6: Internationale Beziehungen, München, S. 422-429.

Wolken, Simone (1988): Das Grundrecht auf Asyl als Gegenstand der Innen- und Rechtspolitik in der Bundesrepublik Deutschland, Frankfurt a.M.

Wright, Steve (2005): Den Himmel stürmen. Eine Theoriegeschichte des Operaismus, Berlin/Hamburg. 
Ziemer, Klaus (1998): Zur Problematik nationaler Minderheiten in Polen und Deutschland, in: Transdora Nr. 18, Oktober 1998, S. 95-126, online: www.transodra-online.net, letzter Abruf: <13.11.2008>.

Zolberg, Aristide R. (1999): Matters of State: Theorizing Immigration Policy, in: Hirschman, Charles/Kasinitz, Philip/DeWind, Josh (Hrsg.): The Handbook of International Migration: The American Experience, New York, S. 71-93.

Zolberg, Aristide R./Suhrke, Astri/Aguayo, Sergio (1989): Escape from Violence. Conflict and the Refugee Crisis in the Developing World, New York/Oxford.

\section{B) EU-Dokumente}

2000/2725/EG: Verordnung Nr. 2725/2000 des Rates vom 11. Dezember 2000 über die Einrichtung von „Eurodac“ für den Vergleich von Fingerabdrücken zum Zwecke der effektiven Anwendung des Dubliner Übereinkommens.

2001/55/EG: Richtlinie des Rates vom 20. Juli 2001 über Mindestnormen für die Gewährung vorübergehenden Schutzes im Falle eines Massenzustroms von Vertriebenen und Maßnahmen zur Förderung einer ausgewogenen Verteilung der Belastungen, die mit der Aufnahme dieser Personen und den Folgen dieser Aufnahme verbunden sind, auf die Mitgliedstaaten.

2003/343/EG: Verordnung Nr. 343/2003 des Rates vom 18. Februar 2003 zur Festlegung der Kriterien und Verfahren zur Bestimmung des Mitgliedstaats, der für die Prüfung eines von einem Drittstaatsangehörigen in einem Mitgliedstaat gestellten Asylantrags zuständig ist (Dublin-II-Verordnung).

2003/86/EG: Richtlinie des Rates vom 22.09.2003 betreffend das Recht auf Familienzusammenführung.

2003/9/EG: Richtlinie des Rates vom 27.01.2003 zur Festlegung von Mindestnormen für die Aufnahme von Asylbewerbern in den Mitgliedstaaten.

2003/C 77/01: EU-Aktionsplan für den Bereich Justiz und Inneres in der Ukraine, vom Rat am 10.12.2001 genehmigt.

2004/114/EG: Richtlinie des Rates vom 13.12.2004 über die Bedingungen für die Zulassung von Drittstaatsangehörigen zur Absolvierung eines Studiums oder zur Teilnahme an einem Schüleraustausch, einer unbezahlten Ausbildungsmaßnahme oder einem Freiwilligendienst.

2004/2007/EG: Verordnung Nr. 2007/2004 des Rates vom 26. Oktober 2004 zur Errichtung der Europäischen Agentur für die operative Zusammenarbeit an den Außengrenzen der Mitgliedstaaten der Europäischen Union (Frontex).

2004/491/EG: Verordnung des Europäischen Parlaments und des Rates vom 10. März 2004 zur Errichtung eines Programms für die finanzielle und technische Hilfe für Drittländer im Migrations- und Asylbereich (AENEAS). 
2004/573/EG: Entscheidung des Rates vom 29.04.2004 betreffend die Organisation von Sammelflügen zur Rückführung von Drittstaatenangehörigen, die individuellen Rückführungsmaßnahmen unterliegen, aus dem Hoheitsgebiet von zwei oder mehr Mitgliedstaaten.

2004/83/EG: Richtlinie des Rates vom 29.04.2004 über Mindestnormen für die Anerkennung und den Status von Drittstaatsangehörigen oder Staatenlosen als Flüchtlinge oder als Personen, die anderweitig internationalen Schutz benötigen, und über den Inhalt des zu gewährenden Schutzes.

2005/71/EG: Richtlinie des Rates vom 12.10.2005 über ein besonderes Zulassungsverfahren für Drittstaatsangehörige zum Zwecke der wissenschaftlichen Forschung.

2005/85/EG: Richtlinie des Rates vom 1.12.2005 über Mindestnormen für Verfahren in den Mitgliedstaaten zur Zuerkennung und Aberkennung der Flüchtlingseigenschaft.

2005/C 53/01: Rat der Europäischen Union: Haager Programm zur Stärkung von Freiheit, Sicherheit und Recht in der Europäischen Union, 3.03.2005.

2007/575/EG: Entscheidung des Europäischen Parlaments und des Rates vom 23.05.2007 zur Einrichtung des Europäischen Rückkehrfonds für den Zeitraum 2008 bis 2013 innerhalb des Generellen Programms „Solidarität und Steuerung der Migrationsströme“.

Ausschuss für bürgerliche Freiheiten, Justiz und Inneres (2007): Bericht über den Vorschlag für einen Beschluss des Rates über den Abschluss des Rückübernahmeabkommens zwischen der Europäischen Gemeinschaft und der Ukraine, Plenarsitzungsdokument A6-0364/2007 vom 5.10.2007.

EuGH (2008): Pressemitteilung Nr. 29/08 vom 6. Mai 2008 zum Urteil des Gerichtshofs in der Rechtssache C-133/06: Der Gerichtshof erklärt verschiedene Bestimmungen der Richtlinie über die Zuerkennung und Aberkennung der Flüchtlingseigenschaft für nichtig, online:

http://curia.europa.eu/de/actu/communiques/cp08/aff/cp080029de.pdf, letzter Abruf: 27.08.2008.

EU-Pressemitteilung vom 10.10.2006: Einheitliche Asylbedingungen in der EU: Mitgliedstaaten versäumen fristgerechte Umsetzung der Kernvorschriften, IP/06/1345, Brüssel.

Europäisches Parlament (2008a): Europaparlament verabschiedet Rückführungsrichtlinie, Pressemitteilung vom 18.06.2008, online: www.europarl.europa.eu/news, letzter Abruf: <27.08.2008>. 
Europäisches Parlament (2008b): Legislative Entschließung vom 18. Juni $2008 \mathrm{zu}$ dem Vorschlag für eine Richtlinie des Europäischen Parlaments und des Rates über gemeinsame Normen und Verfahren in den Mitgliedstaaten zur Rückführung illegal aufhältiger Drittstaatsangehöriger (KOM(2005)0391 - C6-0266/2005 $-2005 / 0167(\mathrm{COD}))$.

KOM(2002) 175: Europäische Kommission: Grünbuch über eine Gemeinschaftspolitik zur Rückkehr illegal aufhältiger Personen, 10.04.2002.

$\operatorname{KOM}(2004)$ 401: Europäische Kommission: Mitteilung der Kommission an den Rat und das Europäische Parlament. Raum der Freiheit, der Sicherheit und des Rechts: Bilanz des Tampere-Programms und Perspektiven, 2.06.2004.

KOM(2004) 410: Europäische Kommission: Mitteilung zur Verbesserung des Zugangs zu dauerhaften Lösungen, 14.06.2004.

KOM(2005) 388: Europäische Kommission: Mitteilung über regionale Schutzprogramme, 1.09.2005.

$\operatorname{KOM}(2005)$ 669: Europäische Kommission: Strategischer Plan zur legalen Zuwanderung, 21.12.2005.

KOM(2007) 299: Europäische Kommission: Bericht der Kommission zur Bewertung des Dublin-Systems, 6.06.2007.

KOM(2007) 301: Europäische Kommission: Grünbuch über das künftige Gemeinsame Europäische Asylsystem, 6.06.2007.

KOM(2007) 745: Bericht der Kommission an den Rat und das Europäische Parlament über die Anwendung der Richtlinie 2003/9/EG des Rates vom 27. Januar 2003 zur Festlegung von Mindestnormen für die Aufnahme von Asylbewerbern in den Mitgliedstaaten, 26.11.2007.

KOM(2007) 745: Europäische Kommission: Bericht der Kommission an den Rat und das Europäische Parlament über die Anwendung der Richtlinie 2003/9/EG des Rates vom 27. Januar 2003 zur Festlegung von Mindestnormen für die Aufnahme von Asylbewerbern in den Mitgliedstaaten, 26.11.2007.

Rat der Europäischen Union (2002): Globaler Aktionsplan 6621/1/02 des Rates vom 28.02.2002 zur Bekämpfung der unerlaubten Einwanderung und des Menschenhandels.

SEK(2000) 522: Europäische Kommission: Überprüfung des Dubliner Übereinkommens: Ausarbeitung von Gemeinschaftsrechtsnormen zur Bestimmung des Mitgliedstaats, der für die Prüfung eines in einem Mitgliedstaat gestellten Asylantrags zuständig ist, 21.03.2000.

SN 200/99: Europäischer Rat: Schlussfolgerungen des Vorsitzes. Europäischer Rat (Tampere) 15./16.10.1999. 
$\mathrm{D}$ ie Zahl der Asylsuchenden ist in der Bundesrepublik Deutschland in den letzten Jahren stark zurückgegangen. Die Anerkennungsquote liegt bei rund einem Prozent. Wo sind die Flüchtlinge geblieben? Gibt es keine Gründe mehr, Asyl zu beantragen? Auf der Suche nach den Asylbewerberlnnen wird man in den neueren EU-Mitgliedstaaten an den Außengrenzen und in den EU-Anrainerstaaten fündig: Aufgrund von Zuständigkeitsregelungen stranden immer mehr Flüchtlinge an den Rändern der EU und können andere europäische Zielländer nur noch irregulär erreichen. Die Studie zeigt am Beispiel Deutschlands und Polens, wie sich der Wandel des Asylrechts in den vergangenen 20 Jahren vollzogen hat, wie er erklärt werden kann und in welchem Verhältnis er zu den Strategien von MigrantInnen steht. Sie erschließt damit das Feld der Asylpolitik in dreierlei Hinsicht neu: Zum einen wird die Asylpolitik im Kontext migrationspolitischer Kategorienkonstruktionen analysiert. Auf diese Weise wird die Bedeutung der Kategorisierung und Hierarchisierung von ,erwünschten“ und ,unerwünschten' Migrantlnnen als zentraler Bestandteil des ,Migrationsmanagements' aufgezeigt. Zum anderen liefern die beiden Länderstudien ein umfängliches Bild der Asylpolitik in Deutschland und Polen. Indem die beiden Fallstudien schließlich in die Entwicklungen auf EU-Ebene eingebettet und aufeinander bezogen werden, eröffnet die Studie einen neuen und umfassenden Einblick in die Dynamiken, die den Wandel des europäischen Asylregimes in den letzten zwei Jahrzehnten bestimmt haben. 